\title{
RELATIVE NONHOMOGENEOUS KOSZUL DUALITY
}

\author{
LEONID POSITSELSKI
}

\begin{abstract}
This paper contains a detailed exposition of the nonhomogeneous Koszul duality theory in the relative situation over a noncentral, noncommutative, nonsemisimple base ring, as announced in [27, Section 0.4]. We prove the Poincaré-Birkhoff-Witt theorem in this context and construct the triangulated equivalences of derived Koszul duality. The duality between the ring of differential operators and the de Rham DG-algebra, with the ring of functions as the base ring, is the thematic example. The moderate generality level makes the exposition in this paper more accessible than the very heavily technical [27, Chapter 11].
\end{abstract}

\section{Contents}

Introduction

1. Homogeneous Quadratic Duality over a Base Ring

2. Flat and Finitely Projective Koszulity

3. Relative Nonhomogeneous Quadratic Duality

4. The Poincaré-Birkhoff-Witt Theorem

5. Comodules and Contramodules over Graded Rings

6. Relative Nonhomogeneous Derived Koszul Duality: the Comodule Side

7. Relative Nonhomogeneous Derived Koszul Duality: the Contramodule Side

8. The Co-Contra Correspondence

9. Koszul Duality and Conversion Functor 174

10. Examples

References

\section{INTRODUCTION}

0.0. Let $A$ be an associative ring and $R \subset A$ be a subring. Derived Koszul duality is the functor $\operatorname{Ext}_{A}^{*}(-, R)$, or $\operatorname{Tor}_{*}^{A}(R,-)$, or $\operatorname{Ext}_{A}^{*}(R,-)$, enhanced to an equivalence of derived categories of modules.

The above definition raises many questions. To begin with, $R$ is not an $A$-module. So what does this Ext and Tor notation even mean? 
Secondly, let us consider the simplest example where $R=k$ is a field and $A=k[x]$ is the algebra of polynomials in one variable. Then $k$ indeed can be viewed as an $A$-module. There are many such module structures, indexed by elements $a$ of the field $k$ : given $a \in k$, one can let the generator $x \in A$ act in $k$ by the multiplication with $a$. Denote the resulting $A$-module by $k_{a}$.

To be specific, let us choose $k=k_{0}$ as our preferred $A$-module structure on $k$. Then the functors $\operatorname{Ext}_{A}^{*}\left(-, k_{0}\right)$, $\operatorname{Tor}_{A}^{*}\left(k_{0},-\right)$, and $\operatorname{Ext}_{A}^{*}\left(-, k_{0}\right)$ are indeed well-defined on the category of $A$-modules. But these functors are far from being faithful or conservative: all of them annihilate the $A$-modules $k_{a}$ with $a \neq 0$. How, then, can one possibly hope to enhance such cohomological functors to derived equivalences?

0.1. Koszul duality has to be distinguished from the comodule-contramodule correspondence, which is a different, though related, phenomenon.

In the simplest possible form, the comodule-contramodule correspondence is the functor $\operatorname{Ext}_{A}^{*}(-, A)$ enhanced to a derived equivalence (while Koszul duality is $\operatorname{Ext}_{A}^{*}(-, k)$, where $k$ is the ground field). In a more realistic covariant and relative situation, comparable to the discussion of Koszul duality in Section 0.0, the comodule-contramodule correspondence would be a derived equivalence enhancement of a functor like $\operatorname{Ext}_{A}^{*}\left(\operatorname{Hom}_{R}(A, R),-\right)$ or $\operatorname{Tor}_{*}^{A}\left(-, \operatorname{Hom}_{R}(A, R)\right)$.

0.2. In the present author's research, the desire to understand Koszulity and Koszul duality was the starting point. Then the separate existence and importance of comodule-contramodule correspondence was realized, particularly in the context of semi-infinite homological algebra [27]. The derived nonhomogeneous Koszul duality over a field was formulated as a "Koszul triality" picture, which is a triangle diagram of derived equivalences with the comodule-contramodule correspondence present as one side of the triangle and two versions of Koszul duality as two other sides [28].

The comodule-contramodule correspondence, its various versions, generalizations, and philosophy, are now discussed in several books and papers of the present author, including [27, 28, 30, 31, 35, 39] and others. On the other hand, the derived nonhomogeneous Koszul duality over a field attracted interest of a number of authors, starting from early works [11, 19, 17] and to very recent, such as [7, 21]; there is even an operadic version of it in [12].

Still, there is a void in the literature concerning relative nonhomogeneous Koszul duality. Presently, the only source of information on this topic known to this author is his own book [27], which contains an introductory discussion without proofs or details in [27, Section 0.4] and a heavily technical treatment in a very general and complicated setting in [27, Chapter 11]. (The memoir [29] represents a very different point of view.) The present paper is intended to fill the void by providing a reasonably accessible, detailed exposition on a moderate generality level.

Let us emphasize that relative nonhomogeneous Koszul duality is important. In addition to the presence of very natural examples such as the duality between the ring of differential operators and the de Rham DG-algebra (see Section 0.7 below), relative nonhomogeneous Koszul duality plays a crucial role in the semi-infinite (co)homology 
theory, as it was first pointed out in [1]. This idea was subsequently developed and utilized in [27, Section 11.9 and Appendix D].

The special case of triangulated equivalences between complexes of modules over the rings/sheaves of differential operators and DG-modules over the de Rham DG-algebra has been considered in [16] and [4, Section 7.2]. Our own treatment of it is presented in [28, Appendix B].

0.3. Let us start to explain the meaning of the terms involved. In the notation of Section 0.0 , relative means that $R$ is an arbitrary ring rather than simply the ground field. Homogeneous Koszul duality means that $A=\bigoplus_{n=0}^{\infty} A_{n}$ is a nonnegatively graded ring and $R=A_{0}$ is the degree-zero grading component. In this case, $R$ is indeed naturally both a left and a right $R$-module, so the meaning of the Ext and Tor notation in Section 0.0 is clear. Nonhomogeneous Koszul duality is the situation when there is no such grading on the ring $A$.

The main specific aspect of the homogeneous case is that one can consider graded $A$-modules with a bounding condition on the grading, that is, only positively graded or only negatively graded modules. If $M$ is a positively graded left $A$-module, then $R \otimes_{A} M=0$ implies $M=0$, while if $P$ is a negatively graded left $A$-module, then $\operatorname{Hom}_{A}(R, P)=0$ implies $P=0$. Hence the second problem described in Section 0.0 does not occur, either.

In the nonhomogeneous situation, the solution to the second problem from Section 0.0 is to consider derived categories of the second kind. This means that certain complexes or DG-modules are declared to be nonzero objects in the derived category even though their cohomology modules vanish.

As to the first problem, it may well happen that $R$ has a (left or right) $A$-module structure even though $A$ is not graded. When such a module structure (extending the natural $R$-module structure on $R$ ) has been chosen, one says that the $\operatorname{ring} A$ is augmented. In this case, the related Ext or Tor functor is well-defined. One wants to enhance it to a functor with values in DG-modules over a suitable DG-ring in such a way that it would induce a triangulated equivalence.

Generally speaking, the solution to the first problem is to consider curved $D G$-modules (CDG-modules), whose cohomology modules are undefined. So the Ext or Tor itself has no meaning, but the related curved DG-module has. In the augmented case, this DG-module becomes uncurved, and indeed computes the related Ext or Tor.

0.4. Let us now begin to state what our assumptions and results are. We assume that a ring $\widetilde{A}$ is endowed with an increasing filtration $R=F_{0} \widetilde{A} \subset F_{1} \widetilde{A} \subset F_{2} \widetilde{A} \subset$ ... which is exhastive $\left(\widetilde{A}=\bigcup_{n} F_{n} \widetilde{A}\right)$ and compatible with the multiplication in $\widetilde{A}$. Furthermore, the successive quotients $\operatorname{gr}_{n}^{F} \widetilde{A}=F_{n} \widetilde{A} / F_{n-1} \widetilde{A}$ are assumed to be finitely generated projective left $R$-modules. Finally, the associated graded $\operatorname{ring} A=\operatorname{gr}^{F} \widetilde{A}=$ $\bigoplus_{n} \operatorname{gr}_{n}^{F} \widetilde{A}$ has to be Koszul over its degree-zero component $A_{0}=R$; this means, in particular, that the ring $A$ is generated by its degree-one component $A_{1}$ over $A_{0}$ and defined by relations of degree 2 . 
In these assumptions, we assign to $(\widetilde{A}, F)$ a curved $D G$-ring $(C D G$-ring $)(B, d, h)$, which is graded by nonnegative integers, $B=\bigoplus_{n=0}^{\infty} B^{n}, B^{0}=R$, has a differential (odd derivation) $d: B^{n} \longrightarrow B^{n+1}$ of degree 1 , and a curvature element $h \in B^{2}$. The CDG-ring $(B, d, h)$ is defined uniquely up to a unique isomorphism of CDG-rings, which includes the possibility of change-of-connection transformations. The grading components $B^{n}$ are finitely generated projective right $R$-modules. In particular, one has $B^{1}=\operatorname{Hom}_{R}\left(A_{1}, R\right)$ and $A_{1}=\operatorname{Hom}_{R^{\text {op }}}\left(B^{1}, R\right)$.

Furthermore, to any left $\widetilde{A}$-module $P$ we assign a CDG-module structure on the graded left $B$-module $B \otimes_{R} P$, and to any right $\widetilde{A}$-module $M$ we assign a CDG-module structure on the graded right $B$-module $\operatorname{Hom}_{R^{\text {op }}}(B, M)$. These constructions are then extended to complexes of left and right $\widetilde{A}$-modules $P^{\bullet}$ and $M^{\bullet}$, assigning to them left and right CDG-modules $B \otimes_{R} P^{\bullet}$ and $\operatorname{Hom}_{R^{\text {op }}}\left(B, M^{\bullet}\right)$ over $(B, d, h)$. A certain (somewhat counterintuitive) way to totalize bigraded modules is presumed here. The resulting functors induce the derived equivalences promised in Section 0.0. The functor $P^{\bullet} \longmapsto B \otimes_{R} P^{\bullet}$ is a CDG-enhancement of the (possibly nonexistent) $\operatorname{Ext}_{\widetilde{A}}^{*}(R, P)$, and the functor $M^{\bullet} \longmapsto \operatorname{Hom}_{R^{\text {op }}}\left(B, M^{\bullet}\right)$ is a CDG-enhancement of the (possibly nonexistent) $\operatorname{Tor}_{*}^{\widetilde{A}}(M, R)$. However, there are further caveats.

0.5. One important feature of the nonhomogeneous Koszul duality over a field, as developed in the memoir [28, is that it connects modules with comodules or contramodules. In fact, the "Koszul triality" of [28] connects modules with comodules and contramodules. In the context of relative nonhomogeneous Koszul duality theory in the full generality of the present paper, the Koszul triality picture splits into two separate dualities. A certain exotic derived category of right $\widetilde{A}$-modules is equivalent to an exotic derived category of right $B$-comodules, while another exotic derived category of left $\widetilde{A}$-modules is equivalent to an exotic derived category of left B-contramodules. The triality picture is then restored under some additional assumptions (namely, two-sided locally finitely generated projectivity of the filtration $F$ and finiteness of homological dimension of the base ring $R$ ).

What are the "comodules" and "contramodules" in our context? First of all, we have complexes of $\widetilde{A}$-modules on the one side and CDG-modules over $B$ on the other side; so both the comodules and the contramodules are graded $B$-modules. In fact, the (graded) right $B$-comodules are a certain full subcategory in the graded right $B$-modules, and similarly the (graded) left $B$-contramodules are a certain full subcategory in the graded left $B$-modules.

Which full subcategory? A graded right $B$-module $N$ is called a graded right $B$-comodule if for every element $x \in N$ there exists an integer $m \geq 1$ such that $x B^{n}=0$ for all $n \geq m$. The definition of $B$-contramodules is more complicated and, as usually, involves certain infinite summation operations. A graded left $B$-module $Q$ is said to be a graded left $B$-contramodule if, for every integer $j$, every sequence of elements $q_{n} \in Q^{j-n}, n \geq 0$, and every sequence of elements $b_{n} \in B^{n}$, an element denoted formally by $\sum_{n=0}^{\infty} b_{n} q_{n} \in Q^{j}$ is defined. One imposes natural algebraic axioms 
on such infinite summation operations, and then proves that an infinite summation structure on a given graded left $B$-module $Q$ is unique if it exists.

In particular, this discussion implies that (somewhat counterintuitively), in the notation of Section 0.4, the bigraded module $\operatorname{Hom}_{R^{\text {op }}}\left(B, M^{\bullet}\right)$ has to be totalized by taking infinite direct sums along the diagonals (to obtain a graded right $B$-comodule), while the bigraded module $B \otimes_{R} P^{\bullet}$ needs to be totalized by taking infinite products along the diagonals (to obtain a graded left $B$-contramodule).

0.6. The explanation for the counterintuitive totalization procedures mentioned in Section 0.5, from our perspective, is that $B$ is a "fake" graded ring. It really "wants" to be a coring, but this point of view is hard to fully develop. It plays a key role, however, in (at least) one of our two proofs of the Poincaré-Birkhoff-Witt theorem for nonhomogeneous Koszul rings.

The graded coring in question is $C=\operatorname{Hom}_{R^{\text {op }}}(B, R)$, that is, the result of applying the dualization functor $\operatorname{Hom}_{R^{\text {op }}}(-, R)$ to the graded ring $B$. The point is that we have already done one such dualization when we passed from the $R$ - $R$-bimodule $A_{1}$ to the $R$ - $R$-bimodule $B^{1}=\operatorname{Hom}_{R}\left(A_{1}, R\right)$, as mentioned in Section 0.4 . The two dualization procedures are essentially inverse to each other, so the passage to the coring $C$ over $R$ returns us to the undualized world, depending covariantly-functorially on the $\operatorname{ring} A$.

Experience teaches that the passage to the dual vector space is better avoided in derived Koszul duality. This is the philosophy utilized in the memoir [28] and the book [27]. This philosophy strongly suggests that the graded coring $C$ is preferable to the graded ring $B$ as a Koszul dual object to a Koszul graded ring $A$.

The problem arises when we pass to the nonhomogeneous setting. In the context of the discussion in Section 0.4, the odd derivation $d$, which is a part of the structure of a CDG-ring $(B, d, h)$, is not $R$-linear. In fact, the restriction of $d$ to the subring $R=B^{0} \subset B$ may well be nonzero, and in the most interesting cases it is. This is a distinctive feature of the relative nonhomogeneous Koszul duality. So how does one apply the functor $\operatorname{Hom}_{R^{\text {op }}}(-, R)$ to a non- $R$-linear map?

0.7. The duality between the ring of differential operators and the de Rham DG-algebra of differential forms is the thematic example of relative nonhomogeneous Koszul duality. Let $X$ be a smooth affine algebraic variety over a field of characteristic 0 (or a smooth real manifold). Let $O(X)$ denote the ring of functions and $\operatorname{Diff}(X)$ denote the ring of differential operators on $X$. Endow the ring $\operatorname{Diff}(X)$ with an increasing filtation $F$ by the order of the differential operators. So the associated graded ring $\operatorname{Sym}_{O(X)}(T(X))=\operatorname{gr}^{F} \operatorname{Diff}(X)$ is the symmetric algebra of the $O(X)$-module $T(X)$ of vector fields on $X$.

In this example, $R=O(X)$ is our base ring, $\widetilde{A}=\operatorname{Diff}(X)$ is our nonhomogeneous Koszul ring over $R$, and $A=\operatorname{Sym}_{O(X)}(T(X))$ is the related homogeneous Koszul ring. The graded ring Koszul to $A$ over $R$ is the graded ring of differential forms $B=\Omega(X)$. There is no curvature in the CDG-ring $(B, d, h)$ (one has $h=0$; a nonzero curvature appears when one passes to the context of twisted differential operators, e. g., differential operators acting in the sections of a vector bundle $E$ 
over $X$; see [27, Section 0.4.7] or [28, Appendix B]). The differential $d: B \longrightarrow B$ is the de Rham differential, $d=d_{d R}$; so $(B, d)$ is a DG-algebra over $k$.

But the de Rham DG-algebra is not a DG-algebra over $O(X)$ (and neither the ring $\operatorname{Diff}(X)$ is an algebra over $O(X))$. In fact, the restriction of the de Rham differential to the subring $O(X) \subset \Omega(X)$ is quite nontrivial.

0.8. So the example of differential operators and differential forms is a case in point for the discussion in Section 0.6. In this example, $C=\operatorname{Hom}_{O(X)}(\Omega(X), O(X))$ is the graded coring of polyvector fields over the ring of functions on $X$. Certainly there is no de Rham differential on polyvector fields. What structure on polyvector fields corresponds to the de Rham differential on the forms?

Here is what we do. We adjoin an additional generator $\delta$ to the de Rham DG-ring $\left(\Omega(X), d_{d R}\right)$, or more generally to the underlying graded ring $B$ of a CDG-ring $(B, d, h)$. The new generator $\delta$ is subject to the relations $[\delta, b]=d(b)$ for all $b \in B$ (where the bracket denotes the graded commutator) and $\delta^{2}=h$. Then there is a new differential on the graded ring $\widehat{B}=B[\delta]$, which we denote by $\partial=\partial / \partial \delta$.

The differential $\partial$ is $R$-linear (and more generally, $B$-linear with signs), so we can dualize it, obtaining a coring $\widehat{C}=\operatorname{Hom}_{R^{\text {op }}}(\widehat{B}, R)$ with the dual differential $\operatorname{Hom}_{R_{\mathrm{op}}}(\partial, R)$. This is the structure that was called a quasi-differential coring in [27]. It plays a key role in the exposition in [27, Chapter 11].

Of course, the odd derivation $\partial=\partial / \partial \delta$ is acyclic, and so is the dual odd coderivation on the coring $\widehat{C}$. This may look strange; but in fact, this is how it should be. Recall that we started with a curved DG-ring $(B, d, h)$. Its differential $d$ does not square to zero, and its cohomology is undefined. So there is no cohomology ring in the game, and it is not supposed to suddenly appear from the construction.

0.9. Now, how does one assign a derived category of modules to the acyclic DG-ring $(\widehat{B}, \partial)$ ? The related constructions are discussed in [27, Section 11.7]. A quasidifferential module over $(\widehat{B}, \partial)$ is simply a graded $\widehat{B}$-module, without any differential. Such modules form a DG-category. In fact, a DG-module over $(\widehat{B}, \partial)$ is the same thing as a contractible object of the DG-category of quasi-differential modules. This point of view, adopted in [27, Chapter 11] in the context of quasi-differential comodules and contramodules over quasi-differential corings, is so counterintuitive that one is having a hard time with what otherwise are very simple constructions. We have none of that in this paper, using the equivalent, but much more tractable concept of a CDG-module over the CDG-ring $(B, d, h)$.

Some words about the coderived and contraderived categories are now in order. These are the most important representatives of the class of constructions known as the "derived categories of the second kind".

In the spirit of the discussion in Section 0.5, we consider right CDG-comodules and left CDG-contramodules over $(B, d, h)$. These are certain full subcategories in the DG-categories of, respectively, right and left CDG-modules over the CDG-ring $(B, d, h)$. Following the general definitions in [27, 28], the coderived category of right $C D G$-comodules over $(B, d, h)$ is constructed as the Verdier quotient category of the 
homotopy category of CDG-comodules by its minimal triangulated subcategory containing the total CDG-comodules of all the short exact sequences of CDG-comodules and closed under infinite direct sums. Similarly, the contraderived category of left $C D G$-contramodules over $(B, d, h)$ is the Verdier triangulated quotient category of the homotopy category of CDG-contramodules by its minimal triangulated subcategory containing the total CDG-contramodules of all the short exact sequences of CDG-contramodules over $(B, d, h)$ and closed under infinite products.

0.10. When the base ring $R$ has finite right homological dimension, our derived Koszul duality result simply states that the derived category of right $\widetilde{A}$-modules is equivalent to the coderived category of right CDG-comodules over $(B, d, h)$. When the ring $R$ has finite left homological dimension, one similarly has a natural equivalence between the derived category of left $\widetilde{A}$-modules and the contraderived category of left CDG-contramodules over $(B, d, h)$.

The situation gets more complicated when the homological dimension of $R$ is infinite. In this case, following the book [27] and the paper [32], one can consider the semiderived categories of right and left $\widetilde{A}$-modules, or more precisely the semicoderived category of right $\widetilde{A}$-modules relative to $R$ and the semicontraderived category of left $\widetilde{A}$-modules relative to $R$. These are defined as the Verdier quotient categories of the homotopy categories of complexes of right and left $\widetilde{A}$-modules by the triangulated subcategories of complexes that are, respectively, coacyclic or contraacylic as complexes of $R$-modules.

Then the derived Koszul duality theorem tells that the semicoderived category of right $\widetilde{A}$-modules is equivalent to the coderived category of right CDG-comodules over $(B, d, h)$; and the semicontraderived category of left $\widetilde{A}$-modules is equivalent to the contraderived category of left CDG-contramodules over $(B, d, h)$.

One can also describe the derived category of right $\widetilde{A}$-modules as the quotient category of the coderived category of right CDG-comodules over $(B, d, h)$ by its minimal triangulated subcategory closed under direct sums and containing all the CDG-comodules $\left(N, d_{N}\right)$ such that $N B^{i}=0$ for $i>0$ and $N$ is acyclic with respect to the differential $d_{N}$ (where $d_{N}^{2}=0$ since $N h=0$ ). Simlarly, the derived category of left $\widetilde{A}$-modules is equivalent to the quotient category of the contraderived category of left CDG-contramodules over $(B, d, h)$ by its minimal triangulated subcategory closed under products and containing all the CDG-contramodules $\left(Q, d_{Q}\right)$ such that $B^{i} Q=0$ for $i>0$ and $Q$ is acyclic with respect to the differential $d_{Q}$.

0.11. A basic fact of the classical theory of modules over the rings of differential operators $\operatorname{Diff}(X)$ is that the abelian categories of left and right $\operatorname{Diff}(X)$-modules are naturally equivalent to each other. This is a rather nontrivial equivalence, in that the ring $\operatorname{Diff}(X)$ is not isomorphic to its opposite ring.

The classical conversion functor $\operatorname{Diff}(X)-\bmod \longrightarrow \bmod -\operatorname{Diff}(X)$ assigns to a left $\operatorname{Diff}(X)$-module $M$ a natural right $\operatorname{Diff}(X)$-module structure on the tensor product $\Omega^{m}(X) \otimes_{O(X)} M$, where $m=\operatorname{dim} X$ and $\Omega^{m}(X)$ is the $O(X)$-module of global sections 
of the line bundle of differential forms of the top degree on $X$. The inverse conversion mod-Diff $(X) \longrightarrow \operatorname{Diff}(X)$-mod is performed by taking the tensor product over $O(X)$ with the (module of global sections of) the line bundle of top polyvector fields $\operatorname{Hom}_{O(X)}\left(\Omega^{m}(X), O(X)\right)=\Lambda_{O(X)}^{m}(T(X))$.

The present paper offers an interpretation of the conversion functor in the context of relative nonhomogeneous Koszul duality. Let $(B, d, h)$ be a nonnegatively graded CDG-ring with the (possibly noncommutative) degree-zero component $B^{0}=R$. Assume that the grading components of $B$ are finitely generated projective left and right $R$-modules, there is an integer $m \geq 0$ such that $B^{n}=0$ for $n>m$, the $R$ - $R$-bimodule $B^{m}$ is invertible, and the multiplication maps $B^{n} \otimes_{R} B^{m-n} \longrightarrow B^{m}$ are perfect pairings. Assume further that $B$ is a Koszul graded ring over $R$. Then we say that $B=(B, d, h)$ is a relatively Frobenius Koszul CDG-ring.

As the grading components of $B$ are finitely generated and projective over $R$ on both sides, there are two nonhomogeneous Koszul dual filtered rings to $(B, d, h)$, one on the left side and one on the right side; we denote them by $\widetilde{A}$ and $\widetilde{A} \#$. Then the claim is that, whenever $B$ is relatively Frobenius over $R$, the two rings $\widetilde{A}$ and $\widetilde{A}^{\#}$ are Morita equivalent. The tensor product with the invertible $R$ - $R$-bimodule $T=B^{m}$ transforms any left $\widetilde{A}$-module into a left $\widetilde{A}^{\#}$-module, and any right $\widetilde{A}^{\#}$-module into a right $\widetilde{A}$-module. The functors $\operatorname{Hom}_{R}(T,-)$ and $\operatorname{Hom}_{R^{\text {op }}}(T,-)$ provide the inverse transformations. (When the graded $\operatorname{ring} B$ is graded commutative and $h=0$, the $\operatorname{ring} \widetilde{A}^{\#}$ is simply the opposite ring to the $\operatorname{ring} \widetilde{A}$.)

In this context, assuming additionally that the ring $R$ has finite left homological dimension, we even obtain a "Koszul quadrality" picture. This means a commutative diagram of triangulated equivalences between four (conventional or exotic) derived categories: the derived category of left $\widetilde{A}^{\#}$-modules, the derived category of left $\widetilde{A}$-modules, the coderived category of left CDG-modules over $(B, d, h)$, and the contraderived category of left CDG-modules over $(B, d, h)$.

0.12. We discuss the homogeneous quadratic duality over a base ring in Section 1, flat and finitely projective Koszul graded rings over a base ring in Section 2, relative nonhomogeneous quadratic duality in Section 3 , and the Poincaré-Birkhoff-Witt theorem for nonhomogeneous Koszul rings over a base ring in Section 4. The discussion of comodules and contramodules over graded rings in Section 5 prepares ground for the derived Koszul duality for module categories, which is worked out on the comodule side in Section 6 and on the contramodule side in Section 7 . The comodule-contramodule correspondence, connecting the comodule and contramodule sides of the Koszul duality, is developed in Section 8. The interpretation of the conversion functor in terms of Koszul duality is discussed in Section 9.

Examples of relative nonhomogeneous Koszul duality are offered in Section 10. These are various species of differential operators, to which correspond the related species of differential forms. We consider algebraic differential operators over smooth affine varieties in characteristic 0 , crystalline differential operators over smooth affine varieties in arbitrary characteristic, differential operators acting in the sections of a 
vector bundle, and differential operators twisted with a chosen closed 2-form. Passing from the algebraic to the analytic setting, we discuss smooth differential operators on a smooth compact real manifold and $\bar{\partial}$-differential operators in the Dolbeault theory on a compact complex manifold. Returning to the algebraic context, we consider relative differential forms and differential operators for a morphism of commutative rings, Lie algebroids with their enveloping algebras and cohomological ChevalleyEilenberg complexes, and finally noncommutative differential forms for a morphism of noncommutative rings. In the latter situation, the related ring of "noncommutative differential operators" is simply the ring of all endomorphisms of the bigger ring as a module over the subring (endowed with the obvious two-step filtration). For the benefit of the reader, we have tried to make our exposition of these examples from various areas of algebra and geometry reasonably self-contained with many background details included.

Acknowledgment. Parts of the material presented in this paper go back more than a quarter century. This applies to the content of Sections 12 and the computations in Section 3 (with the notable exception of the 2-category story), which I worked out sometime around 1992. The particular case of duality over a field, which is much less complicated, was presented in the paper [25], and the possibility of extension to the context of a base ring was mentioned in [25, beginning of Section 4]. The main results in Sections 6 7 go back to Spring 2002. Subsequently, I planned and promised several times over the years to write up a detailed exposition. This paper partially fulfills that promise. The paper also contains some much more recent results; this applies, first of all, to the material of Section 5, which is largely based on [37, Section 6] or [36, Theorem 3.1]. I would like to thank all the people, too numerous to be mentioned here by name, whose help and encouragement contributed to my survival over the decades. Speaking of more recent events, I am grateful to Andrey Lazarev, Julian Holstein, and Bernhard Keller for stimulating discussions and interest to this work. The author was supported by research plan RVO: 67985840 and the GAČR project 20-13778S when writing the paper up.

\section{Homogeneous Quadratic Duality over a Base Ring}

All the associative rings in this paper are unital. We will always presume unitality without mentioning it; so all the left and ring modules over associative rings are unital, all the ring homomorphisms take the unit to the unit, all the subrings contain the unit, and all the gradings and filtrations are such that the unit element belongs to the degree-zero grading/filtration component.

Given an associative ring $R$, we denote by $R$-mod the abelian category of left $R$-modules and by mod- $R$ the abelian category of right $R$-modules.

Let $R, S$, and $T$ be three associative rings. For any left $R$-modules $L$ and $M$, we denote by $\operatorname{Hom}_{R}(L, M)$ the abelian group of all left $R$-module morphisms $L \longrightarrow M$. If $L$ is an $R$-S-bimodule and $M$ is an $R$-T-bimodule, then the group $\operatorname{Hom}_{R}(L, M)$ acquires a natural structure of $S$-T-bimodule. Similarly, for any right $R$-modules $Q$ 
and $N$, the abelian group of all right $R$-module morphisms $Q \longrightarrow N$ is denoted by $\operatorname{Hom}_{R^{\mathrm{op}}}(Q, N)$ (where $R^{\mathrm{op}}$ stands for the ring opposite to $R$ ). If $Q$ is an $S$ - $R$-bimodule and $N$ is a $T$-R-bimodule, then $\operatorname{Hom}_{R^{\text {op }}}(Q, N)$ is a $T$-S-bimodule.

In particular, for any $R$-S-bimodule $U$, the abelian $\operatorname{group} \operatorname{Hom}_{R}(U, R)$ is naturally an $S$-R-bimodule. If $U$ is a finitely generated projective left $R$-module, then $\operatorname{Hom}_{R}(U, R)$ is a finitely generated projective right $R$-module. Similarly, for any $S$ - $R$-bimodule $M$, the abelian group $\operatorname{Hom}_{R^{\text {op }}}(M, R)$ is naturally an $R$-S-bimodule. If $M$ is a finitely generated projective right $R$-module, then $\operatorname{Hom}_{R^{\text {op }}}(M, R)$ is a finitely generated projective left $R$-module.

For any $R$-S-bimodule $U$, there is a natural morphism of $R$-S-bimodules $U \longrightarrow$ $\operatorname{Hom}_{R^{\mathrm{op}}}\left(\operatorname{Hom}_{R}(U, R), R\right)$, which is an isomorphism whenever the left $R$-module $U$ is finitely generated and projective. For any $S$-R-bimodule $M$, there is a natural morphism of $S$-R-bimodules $M \longrightarrow \operatorname{Hom}_{R}\left(\operatorname{Hom}_{R^{\text {op }}}(M, R), R\right)$, which is an isomorphism whenever the right $R$-module $M$ is finitely generated and projective.

Let $U$ be an $R$-S-bimodule and $V$ be an $S$-T-bimodule. Then the left $R$-module $U \otimes_{S} V$ is projective whenever the left $R$-module $U$ and the left $S$-module $V$ are projective. The left $R$-module $U \otimes_{S} V$ is finitely generated whenever the left $R$-module $U$ and the left $S$-module $V$ are finitely generated. The similar assertions apply to the projectivity and finite generatedness on the right side.

Lemma 1.1. (a) Let $U$ be an $R$-S-bimodule and $V$ be an $S$-T-bimodule. Then there is a natural morphism of $T$-R-bimodules

$$
\operatorname{Hom}_{S}(V, S) \otimes_{S} \operatorname{Hom}_{R}(U, R) \longrightarrow \operatorname{Hom}_{R}\left(U \otimes_{S} V, R\right),
$$

which is an isomorphism whenever the left $S$-module $V$ is finitely generated and projective.

(b) Let $M$ be an $S$-R-bimodule and $N$ be a $T$-S-bimodule. Then there is a natural morphism of $R$-T-bimodules

$$
\operatorname{Hom}_{R^{\mathrm{op}}}(M, R) \otimes_{S} \operatorname{Hom}_{S^{\mathrm{op}}}(N, S) \longrightarrow \operatorname{Hom}_{R^{\mathrm{op}}}\left(N \otimes_{S} M, R\right),
$$

which is an isomorphism whenever the right $S$-module $N$ is finitely generated and projective.

Proof. Part (a): the desired map takes an element $g \otimes f \in \operatorname{Hom}_{S}(V, S) \otimes_{S} \operatorname{Hom}_{R}(U, R)$ to the map $U \otimes_{S} V \longrightarrow R$ taking an element $u \otimes v$ to the element $f(u g(v)) \in R$, for any $g \in \operatorname{Hom}_{S}(V, S), \quad f \in \operatorname{Hom}_{R}(U, R), u \in U$, and $v \in V$. The second assertion does not depend on the $T$-module structure on $V$, so one can assume $T=\mathbb{Z}$ and, passing to the finite direct sums and direct summands in the argument $V \in S$-mod, reduce to the obvious case $V=S$. Part (b): the desired map takes an element $h \otimes k \in \operatorname{Hom}_{R^{\text {op }}}(M, R) \otimes_{S} \operatorname{Hom}_{S^{\text {op }}}(N, S)$ to the map $N \otimes_{S} M \longrightarrow R$ taking an element $n \otimes m$ to the element $h(k(n) m) \in R$, for any $h \in \operatorname{Hom}_{R^{\text {op }}}(M, R), k \in \operatorname{Hom}_{S^{\text {op }}}(N, S)$, $n \in N$, and $m \in M$. The second assertion does not depend on the $T$-module structure on $N$, so it reduces to the obvious case $N=S$.

Let $R$ be an associative ring and $V$ be an $R$ - $R$-bimodule. The tensor ring of $V$ over $R$ (otherwise called the ring freely generated by an $R$ - $R$-bimodule $V$ ) is the graded ring 
$T_{R}(V)=\bigoplus_{n=0}^{\infty} T_{R, n}(V)$ with the components $T_{R, 0}(V)=R, T_{R, 1}(V)=V, T_{R, 2}(V)=$ $V \otimes_{R} V$, and $T_{R, n}(V)=V \otimes_{R} \cdots \otimes_{R} V$ ( $n$ factors) for $n \geq 2$. The multiplication in $T_{R}(V)$ is defined by the obvious rules $r\left(v_{1} \otimes \cdots \otimes v_{n}\right)=\left(r v_{1}\right) \otimes v_{2} \otimes \cdots \otimes v_{n}$, $\left(v_{1} \otimes \cdots \otimes v_{n}\right) s=v_{1} \otimes \cdots \otimes v_{n-1} \otimes\left(v_{n} s\right)$, and $\left(v_{1} \otimes \cdots \otimes v_{n}\right)\left(v_{n+1} \otimes \cdots \otimes v_{n+m}\right)=$ $v_{1} \otimes \cdots \otimes v_{n+m}$ for all $r, s \in R$ and $v_{i} \in V$.

Let $A=\bigoplus_{n=0}^{\infty} A_{n}$ be a nonnegatively graded ring with the degree-zero component $A_{0}=R$. Denote by $V$ the $R$ - $R$-bimodule $V=A_{1}$. Then there exists a unique homomorphism of graded rings $\pi_{A}: T_{R}(V) \longrightarrow A$ acting by the identity maps on the components of degrees 0 and 1 . The ring $A$ is said to be generated by $A_{1}$ (over $A_{0}$ ) if the map $\pi_{A}$ is surjective. Furthermore, denote by $J_{A}=\operatorname{ker}\left(\pi_{A}\right)$ the kernel ideal of the ring homomorphism $\pi_{A}$. Then $J_{A}$ is a graded ideal in $T_{R}(V)$, so we have $J_{A}=\bigoplus_{n=2}^{\infty} J_{A, n}$, where $J_{A, n} \subset T_{R, n}(V)$. Set $I_{A}=J_{A, 2} \subset V \otimes_{R} V$. A graded ring $A$ generated by $A_{1}$ over $A_{0}$ is said to be quadratic (over $R=A_{0}$ ) if the two-sided ideal $J_{A} \subset T_{R}(V)$ is generated by $I_{A}$, that is $J_{A}=\left(I_{A}\right)$, or explicitly

$$
J_{A, n}=\sum_{i=1}^{n-1} T_{R, i-1}(V) \cdot I_{A} \cdot T_{R, n-i-1}(V) \text { for all } n \geq 3 .
$$

Let $A=\bigoplus_{n=0}^{\infty} A_{n}$ be a quadratic graded ring with the degree-zero component $R=A_{0}$. We will say that $A$ is 2-left finitely projective if the left $R$-modules $A_{1}$ and $A_{2}$ are projective and finitely generated. Furthermore, $A$ is 3-left finitely projective if the same applies to the left $R$-modules $A_{1}, A_{2}$, and $A_{3}$. Similarly, a quadratic graded $\operatorname{ring} B=\bigoplus_{n=0}^{\infty} B_{n}$ with the degree-zero component $R=B_{0}$ is 2-right finitely projective if the right $R$-modules $B_{1}$ and $B_{2}$ are finitely generated projective, and $B$ is 3 -right finitely projective if the same applies to the right $R$-modules $B_{1}, B_{2}$, and $B_{3}$.

Now we fix an associative ring $R$ and consider the category of graded rings over $R$ $R$-rings gr defined as follows. The objects of $R$-rings gr $_{\text {are nonnegatively graded }}$ associative rings $A=\bigoplus_{n=0}^{\infty} A_{n}$ endowed with a fixed ring isomorphism $R \simeq A_{0}$.

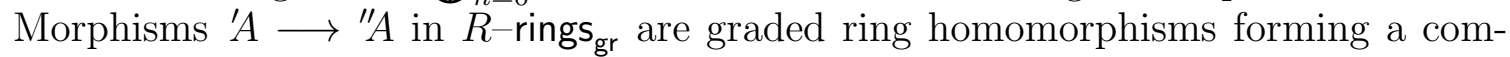
mutative triangle diagram with the isomorphisms $R \simeq{ }^{\prime} A_{0}$ and $R \simeq{ }^{\prime \prime} A_{0}$. Various specific classes of graded rings defined above in this section (and below in the next one) are viewed as full subcategories in $R$-rings gr $_{\text {. }}$

Proposition 1.2. There is an anti-equivalence between the categories of 2-left finitely projective quadratic graded rings $A$ over $R$ and 2-right finitely projective quadratic graded rings $B$ over $R$, called the quadratic duality and defined by the following rules. Given a ring $A$, the ring $B$ is constructed as $B=T_{R}\left(B_{1}\right) /\left(I_{B}\right)$, where $B_{1}=$ $\operatorname{Hom}_{R}\left(A_{1}, R\right)$ and $I_{B}=\operatorname{Hom}_{R}\left(A_{2}, R\right) \subset B_{1} \otimes_{R} B_{1}$. Then the R-R-bimodule $B_{2}$ is naturally isomorphic to $\operatorname{Hom}_{R}\left(I_{A}, R\right)$. Conversely, given a ring $B$, the ring $A$ is constructed as $A=T_{R}\left(A_{1}\right) /\left(I_{A}\right)$, where $A_{1}=\operatorname{Hom}_{R_{\mathrm{op}}}\left(B_{1}, R\right)$ and $I_{A}=\operatorname{Hom}_{R^{\mathrm{op}}}\left(B_{2}, R\right) \subset$ $A_{1} \otimes_{R} A_{1}$. Then the $R$-R-bimodule $A_{2}$ is naturally isomorphic to $\operatorname{Hom}_{R^{\mathrm{op}}}\left(I_{B}, R\right)$.

Proof. The category of quadratic graded rings $A$ over $R$ is equivalent to the category of $R$-R-bimodules $V=A_{1}$ endowed with a subbimodule $I=I_{A} \subset V \otimes_{R} V$. Here morphisms in the category of pairs $\left({ }^{\prime} V, I\right) \longrightarrow\left({ }^{\prime \prime} V,{ }^{\prime \prime} I\right)$ are defined as $R$ - $R$-bimodule morphisms $f:{ }^{\prime} V \longrightarrow{ }^{\prime \prime} V$ such that $(f \otimes f)\left({ }^{\prime} I\right) \subset{ }^{\prime \prime} I$. 
A quadratic graded ring $A$ is 2-left finitely projective if and only if in the related pair $(V, I)$ the left $R$-modules $V$ and $A_{2}=\left(V \otimes_{R} V\right) / I$ are finitely generated and projective. Assuming the former condition, the left $R$-module $V \otimes_{R} V$ is then finitely generated and projective, too, so the latter condition is equivalent to the $R$-R-subbimodule $I \subset V \otimes_{R} V$ being split as a left $R$-submodule.

Now we have a short exact sequence of $R$ - $R$-bimodules

$$
0 \longrightarrow I_{A} \longrightarrow A_{1} \otimes_{R} A_{1} \longrightarrow A_{2} \longrightarrow 0,
$$

which is split as a short exact sequence of left $R$-modules. Applying the functor $\operatorname{Hom}_{R}(-, R)$ and taking into account Lemma 1.1(a), we obtain a short exact sequence of $R$ - $R$-bimodules

$$
0 \longrightarrow \operatorname{Hom}_{R}\left(A_{2}, R\right) \longrightarrow \operatorname{Hom}_{R}\left(A_{1}, R\right) \otimes_{R} \operatorname{Hom}_{R}\left(A_{1}, R\right) \longrightarrow \operatorname{Hom}_{R}\left(I_{A}, R\right) \longrightarrow 0
$$

which is split as a short exact sequence of right $R$-modules. It remains to set $B_{1}=$ $\operatorname{Hom}_{R}\left(A_{1}, R\right)$ and $I_{B}=\operatorname{Hom}_{R}\left(A_{2}, R\right)$, so that $B_{2}=\operatorname{Hom}_{R}\left(I_{A}, R\right)$. According to the discussion in the beginning of this section, $B_{1}$ and $B_{2}$ are finitely generated projective right $R$-modules. It is clear that this construction is a contravariant functor between the categories of 2-left finitely projective and 2-right finitely projective quadratic graded rings over $R$, and that the similar construction with the left and right sides switched provides the inverse functor in the opposite direction.

The 2-left finitely projective quadratic ring $A$ and the 2-right finitely projective quadratic ring $B$ as in Proposition 1.2 are said to be quadratic dual to each other.

Proposition 1.3. The anti-equivalence of categories from Proposition 1.2 takes 3-left finitely projective quadratic graded rings to 3-right finitely projective quadratic graded rings and vice versa.

Proof. For any quadratic graded ring $A$, the grading component $A_{3}$ is the cokernel of the map $\left(A_{1} \otimes_{R} I_{A}\right) \oplus\left(I_{A} \otimes_{R} A_{1}\right) \longrightarrow A_{1} \otimes_{R} A_{1} \otimes_{R} A_{1}$ induced by the inclusion map $I_{A} \longrightarrow A_{1} \otimes_{R} A_{1}$. When the components $A_{1}$ and $A_{2}$ are projective as (say, left) $R$-modules, the maps $A_{1} \otimes_{R} I_{A} \longrightarrow A_{1} \otimes_{R} A_{1} \otimes_{R} A_{1}$ and $I_{A} \otimes_{R} A_{1} \longrightarrow A_{1} \otimes_{R} A_{1} \otimes_{R} A_{1}$ are injective, so we have a four-term exact sequence of $R$ - $R$-bimodules

$$
\begin{aligned}
0 \longrightarrow I_{A}^{(3)} \longrightarrow\left(A_{1} \otimes_{R} I_{A}\right) \oplus\left(I_{A} \otimes_{R} A_{1}\right) & \\
& \longrightarrow A_{1} \otimes_{R} A_{1} \otimes_{R} A_{1} \longrightarrow A_{3} \longrightarrow 0
\end{aligned}
$$

where $I_{A}^{(3)}=\left(A_{1} \otimes_{R} I_{A}\right) \cap\left(I_{A} \otimes_{R} A_{1}\right) \subset A_{1} \otimes_{R} A_{1} \otimes_{R} A_{1}$. When the component $A_{3}$ is a projective left $R$-module, too, we observe that all the terms of this exact sequence, except perhaps the leftmost one, are projective left $R$-modules. It follows that the sequence (2) splits as an exact sequence of left $R$-modules, and the leftmost term $I_{A}^{(3)}$ is a projective left $R$-module, too.

Furthermore, when $A_{1}, A_{2}$, and $A_{3}$ are finitely generated projective left $R$-modules, all the terms of the sequence (2) are also finitely generated projective left $R$-modules. Applying the functor $\operatorname{Hom}_{R}(-, R)$ to (2) , we obtain a four-term exact sequence of $R$ - $R$-bimodules 


$$
\begin{aligned}
0 \longrightarrow \operatorname{Hom}_{R}\left(A_{3}, R\right) & \longrightarrow B_{1} \otimes_{R} B_{1} \otimes_{R} B_{1} \\
& \longrightarrow\left(B_{1} \otimes_{R} B_{2}\right) \oplus\left(B_{2} \otimes_{R} B_{1}\right) \longrightarrow \operatorname{Hom}_{R}\left(I_{A}^{(3)}, R\right) \longrightarrow 0
\end{aligned}
$$

Now for any quadratic graded ring $B$, the cokernel of the map $B_{1} \otimes_{R} B_{1} \otimes_{R} B_{1} \longrightarrow$ $\left(B_{1} \otimes_{R} B_{2}\right) \oplus\left(B_{2} \otimes_{R} B_{1}\right)$ induced by the (surjective) multiplication map $B_{1} \otimes_{R}$ $B_{1} \longrightarrow B_{2}$ is the grading component $B_{3}$. Hence we have a natural isomorphism of $R$-R-bimodules $B_{3} \simeq \operatorname{Hom}_{R}\left(I_{A}^{(3)}, R\right)$, and it follows that $B_{3}$ is a finitely generated projective right $R$-module.

The statement similar to Proposition 1.3 does not hold in degrees higher than 3 for quadratic graded rings in general. It holds under the Koszulity assumption, though, as we will see in the next section.

Remark 1.4. The above discussion of the categories of nonnegatively graded and quadratic rings can be modified or expanded by including non-identity isomorphisms (in particular, automorphisms) in the degree-zero component. Denote by Rings ${ }_{\mathrm{gr}}$ the category whose objects are nonnegatively graded associative rings $A=\bigoplus_{n=0}^{\infty} A_{n}$, and morphisms are defined as follows. A morphism ' $A \longrightarrow{ }^{\prime \prime} A$ in Rings gr $_{\text {is a morphism }}$ of graded rings $f: ' A \longrightarrow{ }^{\prime \prime} A$ whose degree-zero component $f_{0}: A_{0} \longrightarrow{ }^{\prime \prime} A_{0}$ is an isomorphism. Then the same classes of 2 - and 3-left/right finitely projective quadratic rings as in Propositions 1.2 and 1.3 can be viewed as full subcategories in Rings . $_{\text {. }}$ The assertions of the two propositions remain valid with this modification.

The inclusion of the full subcategory of quadratic graded rings over $R$ into the category of (nonnegatively) graded rings over $R$ has a right adjoint functor, which we denote by $A \longmapsto \mathrm{q} A$. For any nonnegatively graded ring $A$, the quadratic graded ring $A^{\prime}=\mathrm{q} A$ together with the graded ring homomorphism $A^{\prime} \longrightarrow A$ is characterized by the properties that the maps $A_{0}^{\prime} \longrightarrow A_{0}$ and $A_{1}^{\prime} \longrightarrow A_{1}$ are isomorphisms and the map $A_{2}^{\prime} \longrightarrow A_{2}$ is injective. Explicitly, the ring q $A$ is constructed as the ring with degreeone generators and quadratic relations $\mathrm{q} A=T_{A_{0}}\left(A_{1}\right) /\left(I_{A}\right)$, where $I_{A} \subset A_{1} \otimes_{R} A_{1}$ is the kernel of the multiplication map $A_{1} \otimes_{R} A_{1} \longrightarrow A_{2}$.

\section{Flat and Finitely Projective Koszulity}

2.1. Graded and ungraded Ext and Tor. So far in this paper we denoted a graded ring by $A=\bigoplus_{n=0}^{\infty} A_{n}$ (and for the most part we will continue to do so in the sequel), but this is a colloquial abuse of notation. A graded abelian group $U$ is properly thought of as a collection of abelian groups $U=\left(U_{n}\right)_{n \in \mathbb{Z}}$. Then there are several ways to produce an ungraded group from a graded one.

Two of them are important for us in this section. One can take the direct sum of the grading components, which we denote by $\Sigma U=\bigoplus_{n \in \mathbb{Z}} U_{n}$; or one can take the product of the grading components, which we denote by $\Pi U=\prod_{n \in \mathbb{Z}} U_{n}$.

In particular, let $A=\left(A_{n}\right)_{n \in \mathbb{Z}}$ be a graded ring and $M=\left(M_{n}\right)_{n \in \mathbb{Z}}$ be a graded left $A$-module. Then $\Sigma A=\bigoplus_{n \in \mathbb{Z}} A_{n}$ is the underlying ungraded ring of $A$; and there are two underlying ungraded $\Sigma A$-modules associated with $M$. Namely, both 
the abelian groups $\Sigma M=\bigoplus_{n \in \mathbb{Z}} M_{n}$ and $\Pi M=\prod_{n \in \mathbb{Z}} M_{n}$ have natural structures of left $\Sigma A$-modules. Denoting the category of graded left $A$-modules by $A$ - $\bmod _{\mathrm{gr}}$, we have two forgetful functors $\Sigma$ and $\Pi: A-\bmod _{\mathrm{gr}} \longrightarrow \Sigma A$-mod.

The tensor product of a graded right $A$-module $N$ and a graded left $A$-module $M$ is naturally a graded abelian group $N \otimes_{A} M$, and applying the functor $\Sigma$ to $N \otimes_{A} M$ produces the tensor product of the ungraded $\Sigma A$-modules $\Sigma N$ and $\Sigma M$,

$$
\Sigma\left(N \otimes_{A} M\right) \simeq \Sigma N \otimes_{\Sigma A} \Sigma M .
$$

Similarly, for any graded left $A$-modules $L$ and $M$ one can consider the graded abelian group $\operatorname{Hom}_{A}(L, M)$ with the components $\operatorname{Hom}_{A, n}(L, M)$ consisting of all the homogeneous left $A$-module maps $L \longrightarrow M$ of degree $n$. The purpose of introducing the functor $\Pi$ above was to formulate the comparison between the graded and ungraded Hom, which has the form

$$
\Pi \operatorname{Hom}_{A}(L, M) \simeq \operatorname{Hom}_{\Sigma A}(\Sigma L, \Pi M) .
$$

Furthermore, the functor $\Sigma$ takes projective graded $A$-modules to projective $\Sigma A$-modules, while the functor $\Pi$ takes injective graded $A$-modules to injective $\Sigma A$-modules (as one can see from the description of projective and injective modules as the direct summands of the free and cofree modules, respectively). In addition, the functor $\Sigma$ takes flat graded $A$-modules to flat $\Sigma A$-modules (as one can see from the Govorov-Lazard description of flat modules as the filtered direct limits of finitely generated free modules). We define the graded versions of Tor and Ext as the derived functors of the graded tensor product and Hom, computed in the abelian categories of graded (right and left) modules.

So, for any graded right $A$-module $N$ and any graded left $A$-module $M$ there is a bigraded abelian group

$$
\operatorname{Tor}^{A}(N, M)=\left(\operatorname{Tor}_{i, j}^{A}(N, M)\right)_{i, j}, \quad i \geq 0, j \in \mathbb{Z},
$$

where $i$ is the usual homological grading and $j$ is the internal grading (induced by the grading of $A, N$, and $M)$. In order to compute the bigraded group $\operatorname{Tor}^{A}(M, N)$, one chooses a graded projective (or flat) resolution of one of the $A$-modules $M$ and $N$ and takes its tensor product over $A$ with the other module; then the grading $i$ is induced by the homological grading of the resolution and the grading $j$ comes from the grading of the tensor product of any two graded modules. In view of the above considerations concerning projective/flat graded modules, the formula (4) implies a similar formula for the Tor groups,

$$
\Sigma \operatorname{Tor}_{i}^{A}(N, M) \simeq \operatorname{Tor}_{i}^{\Sigma A}(\Sigma N, \Sigma M) \text { for every } i \geq 0,
$$

or more explicitly,

$$
\operatorname{Tor}_{i}^{\Sigma A}(\Sigma M, \Sigma N) \simeq \bigoplus_{j \in \mathbb{Z}} \operatorname{Tor}_{i, j}^{A}(M, N)
$$

Similarly, for any graded left $A$-modules $L$ and $M$ there is a bigraded abelian group

$$
\operatorname{Ext}_{A}(L, M)=\left(\operatorname{Ext}_{A, n}^{i}(L, M)\right)_{i, n}, \quad i \geq 0, n \in \mathbb{Z},
$$


where $i$ is the usual cohomological grading and $n$ is the internal grading. In order to compute the bigraded group $\operatorname{Ext}_{A}(L, M)$, one chooses either a graded projective resolution of the $A$-module $L$, or a graded injective resolution of the $A$-module $M$, and takes the graded Hom; then the grading $i$ is induced by the (co)homological grading of the resolution and the grading $n$ comes from the grading of the Hom groups.

In the context of the internal grading of the Ext, we will put $n=-j$ and use the notation $\operatorname{Ext}_{A}^{i, j}(L, M)=\operatorname{Ext}_{A,-j}^{i}(L, M)$. By abuse of terminology, the grading $j$ will be also called the internal grading of the Ext.

In view of the above considerations concerning projective and injective graded modules, the formula (5) implies a similar formula for the Ext groups,

$$
\Pi \operatorname{Ext}_{A}^{i}(L, M) \simeq \operatorname{Ext}_{\Sigma A}^{i}(\Sigma L, \Pi M) \text { for every } i \geq 0,
$$

or more explicitly,

$$
\operatorname{Ext}_{\Sigma A}^{i}(\Sigma L, \Pi M) \simeq \prod_{j \in \mathbb{Z}} \operatorname{Ext}_{A}^{i, j}(L, M)
$$

For any three graded left $A$-modules $K, L$, and $M$, there are natural associative, unital composition/multiplication maps

$$
\text { (8) } \operatorname{Ext}_{A}^{i^{\prime}, j^{\prime}}(L, M) \times \operatorname{Ext}_{A}^{i^{\prime}, j^{\prime}}(K, L) \longrightarrow \operatorname{Ext}_{A}^{i^{\prime}+i^{\prime \prime}, j^{\prime}+j^{\prime \prime}}(K, M), \quad i^{\prime}, i^{\prime \prime} \geq 0, j^{\prime}, j^{\prime \prime} \in \mathbb{Z}
$$

on the bigraded Ext groups. Whenever the graded $A$-module $L$ only has a finite number of nonzero grading components (so $\Sigma L=\Pi L$ ), the passage to the infinite products with respect to the internal gradings $j^{\prime}$ and $j^{\prime \prime}$ makes the multiplications (8) on the graded Ext groups agree with the similar multiplications on the ungraded Ext (between the $\Sigma A$-modules $\Sigma K, \Sigma L=\Pi L$, and $\Pi M$ ).

2.2. Relative bar resolution. Given an $R$ - $R$-bimodule $V$, we will use the notation $V^{\otimes_{R} n}=T_{R, n}(V)$ for the tensor product $V \otimes_{R} \cdots \otimes_{R} V$ ( $n$ factors).

Let $R \longrightarrow A$ be an injective homomorphism of associative rings. Denote by $A_{+}$the $R$ - $R$-bimodule $A / R$. Let $L$ be a left $A$-module. The reduced relative bar resolution of $L$ is the complex of left $A$-modules

(9) $\cdots \longrightarrow A \otimes_{R} A_{+} \otimes_{R} A_{+} \otimes_{R} L \longrightarrow A \otimes_{R} A_{+} \otimes_{R} L \longrightarrow A \otimes_{R} L \longrightarrow L \longrightarrow 0$

with the differential given by the standard formula $\partial\left(a_{0} \otimes \bar{a}_{1} \otimes \cdots \otimes \bar{a}_{n} \otimes l\right)=$ $a_{0} a_{1} \otimes a_{2} \otimes \cdots \otimes a_{n} \otimes l-a_{0} \otimes a_{1} a_{2} \otimes a_{3} \otimes \cdots \otimes l+\cdots+(-1)^{n} a_{0} \otimes a_{1} \otimes \cdots \otimes a_{n-1} \otimes a_{n} l$. One can easily check that the image of the right-hand side in $A \otimes_{R} A_{+}^{\otimes_{R} n-1} \otimes_{R} L$ does not depend on the arbitrary choice of liftings $a_{i} \in A$ of the given elements $\bar{a}_{i} \in A_{+}$, $1 \leq i \leq n$; so the differential is well-defined.

The complex (9) is contractible as a complex of left $R$-modules; the contracting homotopy is given by the formulas $t(l)=1 \otimes l, l \in L$, and $t\left(a_{0} \otimes \bar{a}_{1} \otimes \cdots \otimes \bar{a}_{n} \otimes l\right)=$ $1 \otimes \bar{a}_{0} \otimes \bar{a}_{1} \otimes \cdots \otimes \bar{a}_{n} \otimes l$, where $\bar{a}_{i} \in A_{+}, \quad 1 \leq i \leq n$, and $\bar{a}_{0} \in A_{+}$is the image of the element $a_{0} \in A$ under the natural surjection $A \longrightarrow A_{+}$. Hence it follows that the complex (9) is acyclic.

If both $L$ and $A_{+}$are flat left $R$-modules, then all the left $A$-modules $A \otimes_{R} A_{+}^{\otimes_{R}{ }^{n}} \otimes_{R}$ $L$ are flat, so (9) is a flat resolution of the left $A$-module $L$. Similarly, (9) is a projective 
resolution of the left $A$-module $L$ whenever both the left $R$-modules $L$ and $A_{+}$are projective.

Assume that $L$ and $A_{+}$are flat left $R$-modules, and let $N$ be an arbitrary right $A$-modules. Then one can use the flat resolution (9) of the left $A$-module $L$ in order to compute the groups $\operatorname{Tor}_{i}^{A}(N, L)$. Thus the groups $\operatorname{Tor}_{i}^{A}(N, L)$ are naturally isomorphic to the homology groups of the bar complex

$$
\cdots \longrightarrow N \otimes_{R} A_{+} \otimes_{R} A_{+} \otimes_{R} L \longrightarrow N \otimes_{R} A_{+} \otimes_{R} L \longrightarrow N \otimes_{R} L \longrightarrow 0 \text {. }
$$

Switching the roles of the left and right modules and using the reduced relative bar resolution of $N$, we conclude that the same bar complex (10) computes the groups $\operatorname{Tor}_{i}^{A}(N, L)$ whenever a right $A$-module $N$ is a flat right $R$-module, $A_{+}$is a flat right $R$-module, and $L$ is an arbitrary left $A$-module.

Let $M$ be a left $A$-module. The reduced relative cobar resolution of $M$ is the complex of left $A$-modules

$$
\begin{aligned}
0 \longrightarrow M \longrightarrow \operatorname{Hom}_{R}(A, M) \longrightarrow \operatorname{Hom}_{R}\left(A_{+} \otimes_{R} A, M\right) \\
\longrightarrow \operatorname{Hom}_{R}\left(A_{+} \otimes_{R} A_{+} \otimes_{R} A, M\right) \longrightarrow \cdots
\end{aligned}
$$

with the differential given by the formula $(\partial f)\left(\bar{a}_{n} \otimes \cdots \otimes \bar{a}_{1} \otimes a_{0}\right)=a_{n} f\left(a_{n-1} \otimes\right.$ $\left.\cdots \otimes a_{0}\right)-f\left(a_{n} a_{n-1} \otimes a_{n-2} \otimes \cdots \otimes a_{0}\right)+\cdots+(-1)^{n} f\left(a_{n} \otimes \cdots \otimes a_{2} \otimes a_{1} a_{0}\right)$, where $f \in \operatorname{Hom}_{R}\left(A_{+}^{\otimes_{R} n-1} \otimes_{R} A, M\right)$ and $a_{i} \in A$ are arbitrary liftings of elements $\bar{a}_{i} \in A_{+}$, $1 \leq i \leq n$. One easily checks that the expression in the right-hand side vanishes on the kernel of the natural surjection $A^{\otimes_{R} n+1} \longrightarrow A_{+}^{\otimes_{R} n} \otimes_{R} A$; so the differential is well-defined. The left $A$-module structure on $\operatorname{Hom}_{R}\left(A_{+}^{\otimes_{R} n} \otimes_{R} A, M\right)$ is induced by the right $A$-module structure on $A$.

The complex (11) is contractible as a complex of left $R$-modules; the contracting homotopy is given by the formula $t(f)\left(\bar{a}_{n-1} \otimes \cdots \otimes \bar{a}_{1} \otimes a_{0}\right)=(-1)^{n} f\left(\bar{a}_{n-1} \otimes \cdots \otimes\right.$ $\left.\bar{a}_{1} \otimes \bar{a}_{0} \otimes 1\right)$. In particular, it follows that the complex (11) is acyclic. If $M$ is an injective left $R$-module and $A_{+}$is a flat right $R$-module, then all the left $A$-modules $\operatorname{Hom}_{R}\left(A_{+}^{\otimes_{R} n} \otimes_{R} A, M\right), n \geq 0$, are injective; so (11) is an injective resolution of the left $A$-module $M$.

Let $L$ and $M$ be left $A$-modules. Assuming that $L$ is a projective left $R$-module and $A_{+}$is a projective left $R$-module, one can use the projective resolution (9) of the left $A$-module $L$ in order to compute the groups $\operatorname{Ext}_{A}^{i}(L, M)$. Thus the groups $\operatorname{Ext}_{A}^{i}(L, M)$ are naturally isomorphic to the cohomology groups of the cobar complex

$$
\begin{aligned}
0 \longrightarrow \operatorname{Hom}_{R}(L, M) \longrightarrow \operatorname{Hom}_{R}\left(A_{+} \otimes_{R} L, M\right) & \\
& \longrightarrow \operatorname{Hom}_{R}\left(A_{+} \otimes_{R} A_{+} \otimes_{R} L, M\right) \longrightarrow \cdots
\end{aligned}
$$

Alternatively, assuming that $M$ is an injective left $R$-module and $A_{+}$is a flat right $R$-module, one can use the injective resolution (11) of the left $A$-module $M$ in order to compute the groups $\operatorname{Ext}_{A}^{i}(L, M)$. Under these assumptions, one comes to the same conclusion that the groups $\operatorname{Ext}_{A}^{i}(L, M)$ are naturally isomorphic to the cohomology groups of the cobar complex (12). 
When $R$ and $A$ are graded rings, $R \longrightarrow A$ is a graded ring homomorphism, and $L$ is a graded left $A$-module, one can interpret (9) as a graded resolution of the graded $A$-module $L$. When $L$ and $A_{+}$are flat graded left $R$-modules, (9) is a graded flat resolution of the graded $A$-module $L$; and when $L$ and $A_{+}$are projective graded left $R$-modules, (9) is a graded projective resolution.

For a graded left $A$-module $M$, one can also interpret the Hom notation in (11) as the graded Hom, obtaning a graded resolution of the graded $A$-module $M$. When $M$ is an injective graded left $R$-module and $A_{+}$is a flat graded right $R$-module, (11) is a graded injective resolution.

It follows that, under the graded versions of the above flatness, projectivity, and/or injectivity assumptions, the graded bar complex (10) computes the bigraded $\operatorname{Tor}^{A}(N, L)$, and the graded version of the cobar complex (12) computes the bigraded $\operatorname{Ext}_{A}(L, M)$.

The functor $\Sigma$ transforms the graded versions of (9) and (10) (for graded rings $R$ and $A$ and graded modules $L$ and $N$ ) into the ungraded ones (for the ungraded rings $\Sigma R$ and $\Sigma A$ and the ungraded modules $\Sigma L$ and $\Sigma N)$. The functor $\Pi$ transforms the graded versions of (11) and (12) (for graded rings $R$ and $A$ and graded modules $L$ and $M$ ) into the ungraded ones (for the ungraded rings $\Sigma R$ and $\Sigma A$ and the ungraded modules $\Sigma L$ and $\Pi M)$.

The cobar complexes also compute the composition/multiplication on the Ext groups. Let $K, L$, and $M$ be left $A$-modules; assume that the left $R$-modules $K$, $L$, and $A_{+}$are projective. Then the natural composition/multiplication maps on the cobar complexes

$$
\operatorname{Hom}_{R}\left(A_{+}^{\otimes_{R} i^{\prime}} \otimes_{R} L, M\right) \times \operatorname{Hom}_{R}\left(A_{+}^{\otimes_{R} i^{\prime \prime}} \otimes_{R} K, L\right) \longrightarrow \operatorname{Hom}_{R}\left(A_{+}^{\otimes_{R} i^{\prime}+i^{\prime \prime}} \otimes_{R} K, M\right)
$$

agree with the cobar differentials and induce the Yoneda multiplication maps $\operatorname{Ext}_{A}^{i^{\prime}}(L, M) \times \operatorname{Ext}_{A}^{i^{\prime \prime}}(K, L) \longrightarrow \operatorname{Ext}_{A}^{i^{\prime}+i^{\prime \prime}}(K, M)$ on the Ext groups. In the case of graded rings $R$ and $A$ and graded $A$-modules $K, L$, and $M$, the same assertion applies to the graded Ext.

2.3. Diagonal Tor and Ext. Let $A=\bigoplus_{n=0}^{\infty} A_{n}$ be a nonnegatively graded ring with the degree-zero component $R=A_{0}$. Then the projection onto the degree-zero component is a graded ring homomorphism $A \longrightarrow R$. Using this homomorphism, one can consider $R$ as a left and right graded module over $A$.

So we can consider the bigraded Tor groups $\operatorname{Tor}_{i, j}^{A}(R, R)$ and the bigraded Ext ring $\operatorname{Ext}_{A}^{i, j}(R, R)$, with the (co)homological grading $i$ and the internal grading $j$. In fact, as $R$ is an $R$ - $A$-bimodule and an $A$ - $R$-bimodule, the groups $\operatorname{Tor}_{i, j}^{A}(R, R)$ have natural structures of $R$ - $R$-bimodules.

First of all, we notice the connection between the graded and ungraded Tor and Ext. As the graded (left or right) $A$-module $R$ is concentrated in the internal grading 0 , we have $\Sigma R=R=\Pi R$. Hence the formulas (6) and (7) reduce to

$$
\operatorname{Tor}_{i}^{\Sigma A}(R, R) \simeq \bigoplus_{j \in \mathbb{Z}} \operatorname{Tor}_{i, j}^{A}(R, R)
$$




$$
\operatorname{Ext}_{\Sigma A}^{i}(R, R) \simeq \prod_{j \in \mathbb{Z}} \operatorname{Ext}_{A}^{i, j}(R, R) .
$$

Proposition 2.1. Assume that the grading components $A_{n}, n \geq 1$, are flat left $R$-modules, and the multiplication map $A_{1} \otimes_{R} A_{1} \longrightarrow A_{2}$ is surjective with the kernel $I_{A}$. Then there are natural isomorphisms of $R$-R-bimodules

(a) $\operatorname{Tor}_{i, j}^{A}(R, R)=0$ whenever $i<0$, or $i=0$ and $j>0$, or $i>j$;

(b) $\operatorname{Tor}_{0,0}^{A}(R, R)=R, \operatorname{Tor}_{1,1}^{A}(R, R) \simeq A_{1}$, $\operatorname{Tor}_{2,2}^{A}(R, R) \simeq I_{A}$, and

$$
\operatorname{Tor}_{n, n}^{A}(R, R) \simeq \bigcap_{k=1}^{n-1} A_{1}^{\otimes_{R} k-1} \otimes_{R} I_{A} \otimes_{R} A_{1}^{\otimes_{R} n-k-1} \subset A_{1}^{\otimes_{R} n}, \quad n \geq 2 .
$$

Proof. The $R$ - $R$-bimodules $\operatorname{Tor}_{i, j}^{A}(R, R)$ can be computed as the homology bimodules of the bar complex (10) for $N=R=L$,

$$
\cdots \longrightarrow A_{+} \otimes_{R} A_{+} \otimes_{R} A_{+} \longrightarrow A_{+} \otimes_{R} A_{+} \longrightarrow A_{+} \stackrel{0}{\longrightarrow} R \longrightarrow 0 .
$$

More explicitly, this means that, for every fixed $n \geq 1$, the bimodules $\operatorname{Tor}_{i, n}^{A}(R, R)$ are the homology bimodules of the complex of $R$ - $R$-bimodules

$$
\begin{gathered}
0 \longrightarrow A_{1}^{\otimes_{R} n} \longrightarrow \bigoplus_{k=1}^{n-1} A_{1}^{\otimes_{R} k-1} \otimes_{R} A_{2} \otimes_{R} A_{1}^{\otimes_{R} n-k-1} \longrightarrow \\
\left.\cdots \longrightarrow A_{+}^{\otimes_{R} i}\right)_{n} \longrightarrow \cdots \\
\longrightarrow \bigoplus_{k=1}^{n-1} A_{k} \otimes_{R} A_{n-k} \longrightarrow A_{n} \longrightarrow 0
\end{gathered}
$$

The assertion (a) follows immediately; and to prove (b), it remains to compute, for every $1 \leq k \leq n-1$, the kernel of the map $A_{1}^{\otimes_{R} n} \longrightarrow A_{1}^{\otimes_{R} k-1} \otimes_{R} A_{2} \otimes_{R} A_{1}^{\otimes_{R} n-k-1}$ induced by the multiplication map $A_{1} \otimes_{R} A_{1} \longrightarrow A_{2}$.

Specifically, we have to check that the natural short sequence of $R$ - $R$-bimodules $0 \longrightarrow A_{1}^{\otimes_{R} k-1} \otimes_{R} I_{A} \otimes_{R} A_{1}^{\otimes_{R} n-k-1} \longrightarrow A_{1}^{\otimes_{R} n} \longrightarrow A_{1}^{\otimes_{R} k-1} \otimes_{R} A_{2} \otimes_{R} A_{1}^{\otimes_{R} n-k-1} \longrightarrow 0$ is exact. Indeed, by assumption we have a short exact sequence of $R$ - $R$-bimodules

$$
0 \longrightarrow I_{A} \longrightarrow A_{1} \otimes_{R} A_{1} \longrightarrow A_{2} \longrightarrow 0,
$$

whose terms are flat left $R$-modules. Furthermore, $A_{1}^{\otimes_{R}{ }^{n-k-1}}$ is a flat left $R$-module, too. It follows that tensoring (15) (with any right $R$-module, and in particular) with $A_{1}^{\otimes_{R}{ }^{k-1}}$ on the left does not affect exactness; and neither does tensoring (any exact sequence of right $R$-modules, and in particular) the resulting short sequence with $A_{1}^{\otimes_{R} n-k-1}$ on the right.

Concerning the Ext, our notation $\operatorname{Ext}_{A}(R, R)$ presumes, as above, that the Ext is taken in the category of left $R$-modules. So, in particular, the ring $\operatorname{Ext}_{A}^{0}(R, R)=$ $\operatorname{Hom}_{R}(R, R)=R^{\mathrm{op}}$ is the opposite ring to $R$.

Proposition 2.2. Assume that the grading components $A_{n}, n \geq 1$, are finitely generated projective left $R$-modules, and the multiplication map $A_{1} \otimes_{R} A_{1} \longrightarrow A_{2}$ is surjective with the kernel $I_{A}$. Then

(a) $\operatorname{Ext}_{A}^{i, j}(R, R)=0$ whenever $i<0$, or $i=0$ and $j>0$, or $i>j$; 
(b) the diagonal Ext ring $\bigoplus_{n=0}^{\infty} \operatorname{Ext}_{A}^{n, n}(R, R)$ is naturally isomorphic, as a graded ring, to the opposite ring $B^{\mathrm{op}}$ to the 2-right finitely projective quadratic dual ring $B=T_{R}\left(B_{1}\right) /\left(I_{B}\right), \quad B_{1}=\operatorname{Hom}_{R}\left(A_{1}, R\right), \quad I_{B}=\operatorname{Hom}_{R}\left(A_{2}, R\right)$ to the 2-left finitely projective quadratic ring $\mathrm{q} A=T_{R}\left(A_{1}\right) /\left(I_{A}\right)$.

Proof. Part (a) does not depend on the finite generatedness assumptions on the $R$-modules $A_{n}$, and requires only the projectivity assumptions. The bigraded ring $\operatorname{Ext}_{A}(R, R)$ can be computed as the cohomology ring of the DG-ring (12) for $L=$ $R=M$. The latter can be obtained by applying the functor $\operatorname{Hom}_{R}(-, R)$ to the bar complex (13),

$$
0 \longrightarrow R \stackrel{0}{\longrightarrow} \operatorname{Hom}_{R}\left(A_{+}, R\right) \longrightarrow \operatorname{Hom}_{R}\left(A_{+} \otimes_{R} A_{+}, R\right) \longrightarrow \cdots
$$

More specifically, for every fixed $n \geq 1$, the groups $\operatorname{Ext}_{A}^{i, n}(R, R)$ are the cohomology groups of the complex obtained by applying $\operatorname{Hom}_{R}(-, R)$ to the complex (14). This proves part (a).

When all the grading components $A_{j}$ are finitely generated and projective left $R$-modules, the complex obtained by applying the functor $\operatorname{Hom}_{R}(-, R)$ to the complex (14) can be computed using Lemma 1.1(a) as

$$
\begin{aligned}
& 0 \longrightarrow A_{n}^{\vee} \longrightarrow \bigoplus_{k=1}^{n-1} A_{n-k}^{\vee} \otimes_{R} A_{k}^{\vee} \longrightarrow \\
& \cdots \longrightarrow\left(A_{+}^{\vee \otimes_{R} i}\right)^{n} \longrightarrow \cdots \\
& \longrightarrow \bigoplus_{k=1}^{n-1} A_{1}^{\vee \otimes_{R} n-k-1} \otimes_{R} A_{2}^{\vee} \otimes_{R} A_{1}^{\vee \otimes_{R} k-1} \longrightarrow A_{1}^{\vee \otimes_{R} n} \longrightarrow 0,
\end{aligned}
$$

where the notation is $U^{\vee}=\operatorname{Hom}_{R}(U, R)$. Setting $B_{1}=A_{1}^{\vee}, \quad I_{B}=A_{2}^{\vee}$, and $B=T_{R}\left(B_{1}\right) /\left(I_{B}\right)$, we obtain the desired isomorphism $\operatorname{Ext}_{A}^{n, n}(R, R) \simeq B_{n}$. It follows immediately from the construction of the multiplication on the cobar complex that this is a graded ring isomorphism between $\bigoplus_{n} \operatorname{Ext}_{A}^{n, n}(R, R)$ and $B^{\mathrm{op}}$.

Remark 2.3. The bar complex $\operatorname{Bar}_{R}(A)$ computing $\operatorname{Tor}^{A}(R, R)$ (13) has a natural structure of a (coassociative, counital) $D G$-coring over the ring $R$, with the obvious maps of counit $\operatorname{Bar}_{R}(A) \longrightarrow R$ and a comultiplication $\operatorname{Bar}_{R}(A) \longrightarrow \operatorname{Bar}_{R}(A) \otimes_{R}$ $\operatorname{Bar}_{R}(A)$ compatible with the bar differential. However, as the functor of tensor product over $R$ is not left exact, this DG-coring structure, generally speaking, does not descend to a coring structure on the homology modules. When $A_{+}$is a flat left $R$-module and $\operatorname{Tor}^{A}(R, R)$ is a flat left $R$-module, the nonexactness problem does not interfere, and the (bi)graded $R$ - $R$-bimodule $\operatorname{Tor}^{A}(R, R)$ is a (coassociative, counital) coring over $R$. In particular, the diagonal Tor bimodule $C=\bigoplus_{n=0}^{\infty} \operatorname{Tor}_{n, n}^{A}(R)$ becomes a graded coring over $R$ in these assumptions, with the obvious counit $C \longrightarrow R$ and the induced comultiplication map $C \longrightarrow C \otimes_{R} C$.

2.4. Low-dimensional Tor, degree-one generators and quadratic relations. As in the previous section, we consider a nonnegatively graded ring $A=\bigoplus_{n=0}^{\infty} A_{n}$ with the degree-zero component $R=A_{0}$. 
Proposition 2.4. Assume that the grading components $A_{n}, n \geq 1$, are flat left $R$-modules. Then

(a) the graded ring $A$ is generated by $A_{1}$ over $R$ if and only if $\operatorname{Tor}_{1, j}^{A}(R, R)=0$ for all $j>1$;

(b) the graded ring $A$ is quadratic if and only if $\operatorname{Tor}_{1, j}^{A}(R, R)=0$ for all $j>1$ and $\operatorname{Tor}_{2, j}^{A}(R, R)=0$ for all $j>2$.

Proof. Part (a): folowing the proof of Proposition [2.1, the group $\operatorname{Tor}_{1, n}^{A}(R, R)$ can be computed as the rightmost homology group of the complex (14), that is, the cokernel of the multiplication map $\bigoplus_{k=1}^{n} A_{k} \otimes_{R} A_{n-k} \longrightarrow A_{n}$. Now it is clear that a nonnegatively graded ring $A$ is generated by $A_{1}$ over $R=A_{0}$ if and only if the latter map is surjective for all $n \geq 2$.

Part (b): by part (a), we can assume that $A$ is generated by $A_{1}$; then we have to show that $A$ is quadratic if and only if $\operatorname{Tor}_{2, j}^{A}(R, R)=0$ for all $j>2$. Once again, we compute the group $\operatorname{Tor}_{2, n}^{A}(R, R)$ using the complex (14). So we have to show that $A$ is quadratic if and only if the short sequence

$$
\bigoplus_{k+l+m=n}^{k, l, m \geq 1} A_{k} \otimes_{R} A_{l} \otimes_{R} A_{m} \longrightarrow \bigoplus_{k+l=n}^{k, l \geq 1} A_{k} \otimes_{R} A_{l} \longrightarrow A_{n} \longrightarrow 0
$$

is right exact for all $n \geq 3$.

Given an $R$ - $R$-bimodule $V$ and an $R$ - $R$-subbimodule $I \subset V \otimes_{R} V$, one can construct the quadratic ring $A^{\prime}=T_{R}(V) /(I)$ by the following inductive procedure. Set $A_{0}^{\prime}=R$, $A_{1}^{\prime}=V$, and $A_{2}^{\prime}=\left(V \otimes_{R} V\right) / I$; then there are the obvious multplication maps $A_{k}^{\prime} \times A_{l}^{\prime} \longrightarrow A_{k+l}^{\prime}$ for $k, l \geq 0, k+l \leq 2$. For every $n \geq 3$, set $A_{n}^{\prime}$ to be the cokernel of the $R$ - $R$-bimodule morphism

$$
\bigoplus_{k+l+m=n}^{k, l, m \geq 1} A_{k}^{\prime} \otimes_{R} A_{l}^{\prime} \otimes_{R} A_{m}^{\prime} \longrightarrow \bigoplus_{k+l=n}^{k, l \geq 1} A_{k}^{\prime} \otimes_{R} A_{l}^{\prime}
$$

Then we have biadditive multiplication maps $A_{k}^{\prime} \times A_{l}^{\prime} \longrightarrow A_{n}^{\prime}$ defined for all $k+l=n$, $k, l \geq 0$ and satisfying the associativity equations $(a b) c=a(b c)$ for all $a \in A_{k}^{\prime}, b=A_{l}^{\prime}$, $c \in A_{m}^{\prime}, \quad k+l+m=n, k, l, m \geq 0$. So we obtain a graded ring $A^{\prime}$. Obviously, $A^{\prime}$ is the graded associative ring freely generated by $A_{1}^{\prime}=V$ over $A_{0}^{\prime}=R$ with the relations $I \subset V \otimes_{R} V$; so in other words, $A^{\prime} \simeq T_{R}(V) /(I)$.

Returning to the original graded $\operatorname{ring} A$, put $V=A_{1}$ and let $I$ be the kernel of the multiplication map $A_{1} \otimes_{R} A_{1} \longrightarrow A_{2}$. Then there is a unique homomorphism of graded rings $A^{\prime} \longrightarrow A$ acting by the identity map in degrees 0 and 1 . The ring homomorphism $A^{\prime} \longrightarrow A$ is surjective by assumption. Arguing by induction in $n$, one can easily see that this ring homomorphism is an isomorphism if and only if the short sequence (18) is right exact for every $n \geq 3$.

2.5. First Koszul complex. Let $A=\bigoplus_{n=0}^{\infty} A_{n}$ and $B=\bigoplus_{n=0}^{\infty} B_{n}$ be two nonnegatively graded rings with the same degree-zero component $A_{0}=R=B_{0}$. Suppose that $B_{1}$ is a finitely generated projective right $R$-module, and that we are given an $R$ - $R$-bimodule morphism $\tau: \operatorname{Hom}_{R^{\text {op }}}\left(B_{1}, R\right) \longrightarrow A_{1}$. 
Equivalently, instead of a map $\tau$ one can consider an element of the tensor product $B_{1} \otimes_{R} A_{1}$ satisfying the equation spelled out in the next lemma (which is to be applied to the $R$ - $R$-bimodules $V=\operatorname{Hom}_{R^{\mathrm{op}}}\left(B_{1}, R\right)$ and $\left.U=A_{1}\right)$.

Lemma 2.5. Let $R$ and $S$ be assocative rings.

(a) Let $U$ be a left $R$-module and $V$ be a finitely generated projective left $R$-module. Then there is a natural isomorphism of abelian groups $\operatorname{Hom}_{R}(V, U) \simeq$ $\operatorname{Hom}_{R}(V, R) \otimes_{R} U$.

(b) Let $V$ and $U$ be two $R$-S-bimodules such that $V$ is a finitely generated projective left $R$-module. Then $R$-S-bimodule morphisms $f: V \longrightarrow U$ correspond to elements $e \in \operatorname{Hom}_{R}(V, R) \otimes_{R} U$ satisfying the equation se = es for all $s \in S$ under the isomorphism of part (a). In other words, there is a natural bijective correspondence between $R$-S-bimodule morphisms $f: V \longrightarrow U$ and $S$-S-bimodule morphisms $e: S \longrightarrow \operatorname{Hom}_{R}(V, R) \otimes_{R} U$.

Proof. Both the assertions are easy. Let us only specify that the correspondence between the maps $f: V \longrightarrow U$ and the elements $e=e(1) \in \operatorname{Hom}_{R}(V, R) \otimes_{R} U$ is given by the rule $f(v)=\langle v, e\rangle$. Here the pairing notation (cf. Section 3.4 below) stands for the map

$$
\langle,\rangle: V \times \operatorname{Hom}_{R}(V, R) \longrightarrow R, \quad\langle v, b\rangle=b(v),
$$

and, by extension, for the map

$$
\langle,\rangle: V \times \operatorname{Hom}_{R}(V, R) \otimes_{R} U \longrightarrow U
$$

given by the formulas $\langle v, b \otimes u\rangle=\langle v, b\rangle u=b(v) u$, where $v \in V, \quad u \in U$, and $b \in \operatorname{Hom}_{R}(V, R)$.

Denote by $K^{\tau}(B, A)$ the following bigraded $A$-B-bimodule endowed with an $A$ - $B$-bimodule endomorphism $\partial^{\tau}$. The bigrading components of $K^{\tau}(B, A)$ are

$$
K_{i, n}^{\tau}(B, A)=\operatorname{Hom}_{R^{\mathrm{op}}}\left(B_{i}, A_{n-i}\right), \quad i \geq 0, n \geq i .
$$

Here $i$ is interpreted as the homological grading and $n$ is the internal grading. The homogeneous $A$ - $B$-bimodule endomorphism $\partial^{\tau}: K^{\tau}(B, A) \longrightarrow K^{\tau}(B, A)$ of bidegree $(i, n)=(-1,0)$ is constructed as the composition

$$
\begin{aligned}
& \operatorname{Hom}_{R^{\mathrm{op}}}\left(B_{i}, A_{j}\right) \longrightarrow \operatorname{Hom}_{R^{\mathrm{op}}}\left(B_{i-1} \otimes_{R} B_{1}, A_{j}\right) \\
& \simeq \operatorname{Hom}_{R^{\mathrm{op}}}\left(B_{i-1}, \operatorname{Hom}_{R^{\mathrm{op}}}\left(B_{1}, A_{j}\right)\right) \simeq \operatorname{Hom}_{R^{\mathrm{op}}}\left(B_{i-1}, A_{j} \otimes_{R} \operatorname{Hom}_{R^{\mathrm{op}}}\left(B_{1}, R\right)\right) \\
& \stackrel{\tau}{\longrightarrow} \operatorname{Hom}_{R^{\mathrm{op}}}\left(B_{i-1}, A_{j} \otimes_{R} A_{1}\right) \longrightarrow \operatorname{Hom}_{R^{\mathrm{op}}}\left(B_{i-1}, A_{j+1}\right) .
\end{aligned}
$$

Here the map $\operatorname{Hom}_{R^{\text {op }}}\left(B_{i}, A_{j}\right) \longrightarrow \operatorname{Hom}_{R^{\text {op }}}\left(B_{i-1} \otimes_{R} B_{1}, A_{j}\right)$ is induced by the multiplication map $B_{i-1} \otimes_{R} B_{1} \longrightarrow B_{i}$, the map $\operatorname{Hom}_{R^{\text {op }}}\left(B_{i-1}, A_{j} \otimes_{R} \operatorname{Hom}_{R^{\text {op }}}\left(B_{1}, R\right)\right) \longrightarrow$ $\operatorname{Hom}_{R^{\text {op }}}\left(B_{i-1}, A_{j} \otimes_{R} A_{1}\right)$ is induced by the given map $\tau: \operatorname{Hom}_{R^{\text {op }}}\left(B_{1}, R\right) \longrightarrow A_{1}$, and the map $\operatorname{Hom}_{R^{\text {op }}}\left(B_{i-1}, A_{j} \otimes_{R} A_{1}\right) \longrightarrow \operatorname{Hom}_{R^{\mathrm{op}}}\left(B_{i-1}, A_{j+1}\right)$ is induced by the multiplication map $A_{j} \otimes_{R} A_{1} \longrightarrow A_{j+1}$. 
Lemma 2.6. Assume that both $B_{1}$ and $B_{2}$ are finitely generated projective right $R$-modules. Then the following conditions are equivalent:

(a) $\left(\partial^{\tau}\right)^{2}=0$ on the whole bigraded $A$-B-bimodule $K^{\tau}(B, A)$;

(b) the composition $\operatorname{Hom}_{R^{\mathrm{op}}}\left(B_{2}, R\right) \stackrel{\partial^{\tau}}{\longrightarrow} \operatorname{Hom}_{R^{\mathrm{op}}}\left(B_{1}, A_{1}\right) \stackrel{\partial^{\tau}}{\longrightarrow} \operatorname{Hom}_{R^{\mathrm{op}}}\left(R, A_{2}\right)=A_{2}$ vanishes;

(c) the composition of maps $\operatorname{Hom}_{R^{\text {op }}}\left(B_{2}, R\right) \longrightarrow \operatorname{Hom}_{R^{\text {op }}}\left(B_{1} \otimes_{R} B_{1}, R\right) \simeq$ $\operatorname{Hom}_{R^{\text {op }}}\left(B_{1}, R\right) \otimes_{R} \operatorname{Hom}_{R^{\text {op }}}\left(B_{1}, R\right) \stackrel{\tau \otimes \tau}{\longrightarrow} A_{1} \otimes_{R} A_{1} \longrightarrow A_{2}$ vanishes.

Proof. (a) $\Longrightarrow(\mathrm{b})$ is obvious.

(b) $\Longleftrightarrow$ (c) holds because the two maps in question are the same.

$(\mathrm{c}) \Longrightarrow(\mathrm{a})$ is straightforward, using the isomorphism $\operatorname{Hom}_{R^{\mathrm{op}}}\left(B_{2}, A_{j}\right) \simeq A_{j} \otimes_{R}$ $\operatorname{Hom}_{R^{\text {op }}}\left(B_{2}, R\right)$.

When the equivalent conditions of Lemma 2.6 hold, the bigraded $A$ - $B$-bimodule $K^{\tau}(B, A)$ with the differential $\partial^{\tau}$ can be viewed as a complex of graded left $A$-modules. We call it the first Koszul complex and denote by $K_{\bullet}^{\tau}(B, A)$. Assuming that $B_{i}$ is a finitely generated projective right $R$-module for every $i \geq 0$, the first Koszul complex $K_{\bullet}^{\tau}(B, A)$ has the form

$$
\cdots \longrightarrow A \otimes_{R} \operatorname{Hom}_{R^{\mathrm{op}}}\left(B_{2}, R\right) \longrightarrow A \otimes_{R} \operatorname{Hom}_{R^{\mathrm{op}}}\left(B_{1}, R\right) \longrightarrow A \longrightarrow 0 .
$$

Specifically, the following particular case is important. Let $A$ be a nonnegatively graded ring with the degree-zero component $R=A_{0}$. Assume that $A_{1}$ and $A_{2}$ are finitely generated projective left $R$-modules and the multiplication map $A_{1} \otimes_{R} A_{1} \longrightarrow$ $A_{2}$ is surjective. Denote the kernel of the latter map by $I_{A}$, and consider the 2-left finitely projective quadratic graded ring $\mathrm{q} A=T_{R}\left(A_{1}\right) /\left(I_{A}\right)$. Let $B$ be the 2-right finitely projective quadratic graded ring quadratic dual to $\mathrm{q} A$.

Then we have an isomorphism of $R$ - $R$-bimodules $\operatorname{Hom}_{R^{\text {op }}}\left(B_{1}, R\right) \simeq A_{1}$, which we use as our choice of the map $\tau$. The assumptions of Lemma 2.6 are satisfied, and condition (c) holds by the construction of quadratic duality. Therefore, the first Koszul complex $K_{\bullet}^{\tau}(B, A)$ is well-defined.

2.6. Dual Koszul complex. As in Section 2.5, we consider two nonnegatively graded rings $A=\bigoplus_{n=0}^{\infty} A_{n}$ and $B=\bigoplus_{n=0}^{\infty} B_{n}$ with the same degree-zero component $A_{0}=R=B_{0}$. Let $e \in B_{1} \otimes_{R} A_{1}$ be an element satisfying the equation $r e=e r$ for all $r \in R$, as in Lemma 2.5(b).

Denote by $K_{e}^{\vee}(B, A)$ the following bigraded $B$-A-bimodule endowed with a $B$ - $A$-bimodule endomorphism $d_{e}$. The bigrading components of $K_{e}^{\vee}(B, A)$ are

$$
K_{e}^{\vee i, n}(B, A)=B_{i} \otimes A_{n+i}, \quad i \geq 0, n \geq-i .
$$

Here $i$ is intepreted as the cohomological grading and $n$ is the internal grading. The homogeneous $B$-A-bimodule endomorphism $d_{e}: K_{e}^{\vee}(B, A) \longrightarrow K_{e}^{\vee}(B, A)$ of bidegree $(i, n)=(1,0)$ consists of the components $B_{i} \otimes_{R} A_{j} \longrightarrow B_{i+1} \otimes_{R} A_{j+1}$, which are constructed as the compositions

$$
B_{i} \otimes_{R} A_{j}=B_{i} \otimes_{R} R \otimes_{R} A_{j} \stackrel{e}{\longrightarrow} B_{i} \otimes_{R} B_{1} \otimes_{R} A_{1} \otimes_{R} A_{j} \longrightarrow B_{i+1} \otimes_{R} A_{j+1} .
$$


Here the map $B_{i} \otimes_{R} R \otimes_{R} A_{j} \longrightarrow B_{i} \otimes_{R} B_{1} \otimes_{R} A_{1} \otimes_{R} A_{j}$ is induced by the $R$ - $R$-bimodule map $e: R \longrightarrow B_{1} \otimes_{R} A_{1}$, while the map $B_{i} \otimes_{R} B_{1} \otimes_{R} A_{1} \otimes_{R} A_{j} \longrightarrow B_{i+1} \otimes_{R} A_{j+1}$ is the tensor product of the multiplication maps $B_{i} \otimes_{R} B_{1} \longrightarrow B_{i+1}$ and $A_{1} \otimes_{R} A_{j} \longrightarrow A_{j+1}$.

Lemma 2.7. Assume that both $B_{1}$ and $B_{2}$ are finitely generated projective right $R$-modules, and that an $R$-R-bimodule map $\operatorname{Hom}_{R^{\text {op }}}\left(B_{1}, R\right) \longrightarrow A_{1}$ and an element $e \in B_{1} \otimes_{R} A_{1}$ correspond to each other under the construction of Lemma 2.5. Then the following conditions are equivalent:

(a) $d_{e}^{2}=0$ on the whole bigraded $B$-A-bimodule $K_{e}^{\vee}(B, A)$;

(b) the element $d_{e}^{2}(1 \otimes 1) \in B_{2} \otimes_{R} A_{2}$ vanishes;

(c) the equivalent conditions of Lemma 2.6 hold.

Furthermore, the bigraded $A$-B-bimodule $K^{\tau}(B, A)$ with the endomorphism $\partial^{\tau}$ can be obtained by applying the functor $\operatorname{Hom}_{A^{\text {op }}}(-, A)$ to the bigraded $B$-A-bimodule $K_{e}^{\vee}(B, A)$ with the endomorphism $d_{e}$.

Proof. The equivalence $(\mathrm{a}) \Longleftrightarrow(\mathrm{b})$ does not depend on the assumptions of finite generatedness and projectivity of the right $R$-modules $B_{1}$ and $B_{2}$. The equivalence (b) $\Longleftrightarrow(c)$ appears to need these assumptions. The last assertion of the lemma is straightforward, and only needs the assumption of $B_{1}$ being a finitely generated projective right $R$-module.

(a) $\Longrightarrow$ (b) is obvious.

(b) $\Longrightarrow$ (a) holds because $d_{e}$ is a $B$ - $A$-bimodule map.

$(\mathrm{b}) \Longleftrightarrow$ (c) holds because the map $\operatorname{Hom}_{R^{\mathrm{op}}}\left(B_{2}, R\right) \longrightarrow A_{2}$ from Lemma 2.6(c) corresponds to the element $d_{e}^{2}(1 \otimes 1) \in B_{2} \otimes_{R} A_{2}$ under the correspondence of Lemma 2.5.

$(\mathrm{a}) \Longrightarrow(\mathrm{c})$ : the condition (a) implies the condition of Lemma 2.6(a) in view of the last assertion of the present lemma.

When the equivalent conditions of the lemma hold, the bigraded $B$ - $A$-bimodule $K_{e}^{\vee}(B, A)$ with the differential $d_{e}$ can be viewed as a complex of graded right $A$-modules. We call it the dual Koszul complex and denote by $K_{e}^{\vee} \bullet(B, A)$. The dual Koszul complex has the form

$$
0 \longrightarrow A \longrightarrow B_{1} \otimes_{R} A \longrightarrow B_{2} \otimes_{R} A \longrightarrow B_{3} \otimes_{R} A \longrightarrow \cdots .
$$

According to the lemma, we have

$$
K_{\bullet}^{\tau}(B, A)=\operatorname{Hom}_{A^{\text {op }}}\left(K_{e}^{\vee \bullet}(B, A), A\right) .
$$

The graded Hom group $\operatorname{Hom}_{R^{\text {op }}}(B, R)$ with the components $\operatorname{Hom}_{R^{\text {op }}}\left(B_{i}, R\right), \quad i \geq$ 0 , has natural structures of a graded right $B$-module (induced by the graded left $B$-module structure on $B$ ) and a graded left $R$-module (induced by the left $R$-module structure on $R$ ). We denote the tensor product $\operatorname{Hom}_{R^{\mathrm{op}}}(B, R) \otimes_{B} K_{e}^{\vee}(B, A)$ by

$$
{ }^{\tau} K(B, A)=\operatorname{Hom}_{R^{\mathrm{op}}}(B, R) \otimes_{B} K_{e}^{\vee}(B, A)=\operatorname{Hom}_{R^{\mathrm{op}}}(B, R) \otimes_{R} A .
$$

${ }^{\tau} K(B, A)$ is a bigraded $R$-A-bimodule with the bigrading components

$$
{ }^{\tau} K_{i, n}(B, A)=\operatorname{Hom}_{R^{\text {op }}}\left(B_{i}, R\right) \otimes_{R} A_{n-i}, \quad i \geq 0, n \geq i .
$$


Here $i$ is the homological grading and $n$ is the internal grading. Notice that there is no $B$-module structure on ${ }^{\tau} K(B, A)$.

The $B$-A-bimodule endomorphism $d_{e}: K_{e}^{\vee}(B, A) \longrightarrow K_{e}^{\vee}(B, A)$ induces an $R$-A-bimodule endomorphism of bidegree $(i, n)=(-1,0)$ on ${ }^{\tau} K(B, A)$, which we denote by ${ }^{\tau} \partial:{ }^{\tau} K(B, A) \longrightarrow{ }^{\tau} K(B, A)$. When the equivalent conditions of Lemma 2.7 hold, it follows that $\left({ }^{\tau} \partial\right)^{2}=0$. So the bigraded $R$ - $A$-bimodule ${ }^{\tau} K(B, A)$ can be viewed as a complex of graded right $A$-modules. We call it the second Koszul complex and denote by ${ }^{\tau} K_{\bullet}(B, A)$. The second Koszul complex ${ }^{\tau} K_{\bullet}(B, A)$ has the form

$$
\cdots \longrightarrow \operatorname{Hom}_{R^{\mathrm{op}}}\left(B_{2}, R\right) \otimes_{R} A \longrightarrow \operatorname{Hom}_{R^{\mathrm{op}}}\left(B_{1}, R\right) \otimes_{R} A \longrightarrow A \longrightarrow 0
$$

The particular case described at the end of Section 2.5 is important for us. In this case, $\mathrm{q} A$ is a 2-left finitely projective quadratic graded ring and $B$ is the quadratic dual 2-right finitely projective quadratic graded ring. Choosing $\tau$ to be the natural isomorphism $\operatorname{Hom}_{R^{\text {op }}}\left(B_{1}, R\right) \simeq A_{1}$, we see that the equivalent conditions of Lemma 2.7 are satisfied (because the condition of Lemma 2.6(c) holds). Therefore, the dual Koszul complex $K_{e}^{\vee \bullet}(B, A)$ and the second Koszul complex ${ }^{\tau} K \cdot(B, A)$ are well-defined.

Remark 2.8. Assume that $B_{n}$ is a finitely generated projective right $R$-module for every $n \geq 0$. Then the isomorphism of Lemma 1.1(b) provides the graded $R$-R-bimodule $C$ with the components $C_{n}=\operatorname{Hom}_{R^{\text {op }}}\left(B_{n}, R\right)$ with the structure of a graded coring over $R$. The comultiplication maps $C_{i+j} \longrightarrow C_{i} \otimes_{R} C_{j}$ are obtained by applying the functor $\operatorname{Hom}_{R^{\text {op }}}(-, R)$ to the multiplication maps $B_{j} \otimes B_{i} \longrightarrow B_{i+j}$.

By construction, we have $B_{n}=\operatorname{Hom}_{R}\left(C_{n}, R\right)$. Put ${ }^{\#} B_{n}=\operatorname{Hom}_{R^{\text {op }}}\left(C_{n}, R\right)$ (in the spirit of the notation in [1]). Then applying the functor $\operatorname{Hom}_{R^{\text {op }}}(-, R)$ to the comultiplication maps $C_{i+j} \longrightarrow C_{i} \otimes_{R} C_{j}$ and composing the resulting maps $\operatorname{Hom}_{R^{\mathrm{op}}}\left(C_{i} \otimes_{R} C_{j}, R\right) \longrightarrow \operatorname{Hom}_{R^{\mathrm{op}}}\left(C_{i+j}, R\right)$ with the natural $R$ - $R$-bimodule morphisms $\operatorname{Hom}_{R^{\text {op }}}\left(C_{j}, R\right) \otimes_{R} \operatorname{Hom}_{R^{\text {op }}}\left(C_{i}, R\right) \longrightarrow \operatorname{Hom}_{R^{\text {op }}}\left(C_{i+j}, R\right)$ from Lemma 1.1(b) produces multiplication maps ${ }^{\#} B_{j} \otimes_{R}{ }^{\#} B_{i} \longrightarrow{ }^{\#} B_{i+j}$. So ${ }^{\#} B=\bigoplus_{n=0}^{\infty}{ }^{\#} B_{n}$ is a nonnegatively graded ring with the degree-zero component ${ }^{\#} B_{0}=R$.

One easily observes that $C=\bigoplus_{n=-\infty}^{0} C_{-n}$ is a graded ${ }^{\#} B$ - $B$-bimodule. In fact, the left action map ${ }^{\#} B_{i} \otimes_{R} C_{i+j} \longrightarrow C_{j}$ can be constructed as the composition

$$
{ }^{\#} B_{i} \otimes_{R} C_{i+j} \longrightarrow{ }^{\#} B_{i} \otimes_{R} C_{i} \otimes_{R} C_{j} \longrightarrow{ }^{*} B_{i} \otimes_{R} C_{j}
$$

of the map ${ }^{\#} B_{i} \otimes_{R} C_{i+j} \longrightarrow{ }^{\#} B_{i} \otimes_{R} C_{i} \otimes_{R} C_{j}$ induced by the comultiplication map $C_{i+j} \longrightarrow C_{i} \otimes_{R} C_{j}$ with the map ${ }^{\#} B_{i} \otimes_{R} C_{i} \otimes_{R} C_{j} \longrightarrow{ }^{\#} B_{i} \otimes_{R} C_{j}$ induced by the evaluation map ${ }^{\#} B_{i} \otimes_{R} C_{i}=\operatorname{Hom}_{R^{\text {op }}}\left(C_{i}, R\right) \otimes_{R} C_{i} \longrightarrow R$.

Thus the tensor product ${ }^{\tau} K(B, A)=C \otimes_{B} K_{e}^{\vee}(B, A)=C \otimes_{R} A$ is naturally a graded ${ }^{\#} B$ - $A$-bimodule, and ${ }^{\tau} \partial:{ }^{\tau} K(B, A) \longrightarrow{ }^{\tau} K(B, A)$ is its graded ${ }^{*} B$ - $A$-bimodule endomorphism. So, instead of an action of the graded ring $B$, there is an action of the graded ring ${ }^{\#} B$ in ${ }^{\tau} K(B, A)$.

We refer to Section 8.7 for a further discussion of the two Koszul complexes. 
2.7. Distributive collections of subobjects. Let $\mathrm{C}$ be an abelian category in which all the subobjects of any given object form a set, and let $W \in \mathrm{C}$ be an object. A set $\Omega$ of subobjects of $W$ is said to be a lattice of subobjects if $0 \in \Omega, W \in \Omega$, and for any $X, Y \in \Omega$ one has $X \cap Y \in \Omega$ and $X+Y \in \Omega$. Any lattice of subobjects is modular, i. e., one has $(X+Y) \cap Z=X+(Y \cap Z)$ whenever $X, Y, Z \in \Omega$ and $X \subset Z$. A lattice of subobjects $\Omega$ is said to be distributive if the identity $(X+Y) \cap Z=X \cap Z+Y \cap Z$ holds for all $X, Y, Z \in \Omega$, or equivalently, the identity $(X+Y) \cap(X+Z)=X+(Y \cap Z)$ holds for all $X, Y, Z \in \Omega$.

Let $n \geq 2$ be an integer and $X_{1}, \ldots, X_{n-1} \subset W$ be a collection of $n-1$ subobjects in $W$. The lattice $\Omega$ of subobjects of $W$ generated by $X_{1}, \ldots, X_{n-1}$ consists of all the subobjects of $W$ that can be obtained from $X_{1}, \ldots, X_{n-1}$ by applying iteratively the operations of finite sum and finite intersection.

The collection of subobjects $\left(X_{1}, \ldots, X_{n-1}\right)$ in $W$ is said to be distributive if the lattice $\Omega$ of subobjects of $W$ generated by $X_{1}, \ldots, X_{n-1}$ is distributive. Any pair of subobjects $X_{1}, X_{2}(n=3)$ forms a distributive collection, but a triple of subobjects $X_{1}, X_{2}, X_{3}(n=4)$ does not need to be distributive. A collection of subobjects $\left(X_{1}, \ldots, X_{n-1}\right)$ in $W$ is said to be almost distributive if all its proper subcollections $\left(X_{1}, \ldots, \widehat{X}_{k}, \ldots, X_{n-1}\right), 1 \leq k \leq n-1$, are distributive.

The following two lemmas hold in any modular lattice $\Omega$, but we state them for lattices of subobjects only.

Lemma 2.9. A triple of subobjects $X, Y, Z \subset W$ is distributive if and only if $(X+Y) \cap Z=X \cap Z+Y \cap Z$, and if and only if $(X+Y) \cap(X+Z)=X+(Y \cap Z)$. Any permutation of $X, Y, Z$ replaces these equations by equivalent ones.

Proof. This is an easy exercise; see [14, Lemma 1] or [24, Lemma 6.1 in Chapter 1].

Lemma 2.10. An almost distributive collection of subobjects $\left(X_{1}, \ldots, X_{n-1}\right)$ in $W$ is distrubutive if and only if, for every $2 \leq k \leq n-2$, the triple of subobjects

$$
X_{1}+\cdots+X_{k-1}, X_{k}, X_{k+1} \cap \cdots \cap X_{n-1} \subset W
$$

is distributive.

Proof. This is the result of the paper [23], improving upon the previous work in [14]. See also [24, Theorem 6.3 in Chapter 1].

For any collection of subobjects $X_{1}, \ldots, X_{n-1} \subset W$, we consider the following three complexes in C. The bar complex $B_{\bullet}=B_{\bullet}\left(W ; X_{1}, \ldots, X_{n-1}\right)$ has the form

$$
\begin{aligned}
& W \longrightarrow \bigoplus_{t} W / X_{t} \longrightarrow \bigoplus_{t_{1}<t_{2}} W /\left(X_{t_{1}}+X_{t_{2}}\right) \longrightarrow \cdots \\
& \longrightarrow \bigoplus_{t_{1}<\cdots<t_{i}} W /\left(X_{t_{1}}+\cdots+X_{t_{i}}\right) \longrightarrow \cdots \longrightarrow W /\left(X_{1}+\cdots+X_{n-1}\right) .
\end{aligned}
$$

Here the indices $t_{s}$ range from 1 to $n-1$. The leftmost term $W$ is placed in the homological degree $n$, the term with the summation over $1 \leq t_{1}<\cdots<t_{i} \leq n-1$ is placed in the homological degree $n-i$, and the rightmost term $W /\left(X_{1}+\cdots+X_{n-1}\right)$ 
is placed in the homological degree 1 . The component of the differential acting from the direct summand $W /\left(X_{t_{1}}+\cdots+\widehat{X}_{t_{s}}+\cdots+X_{t_{i}}\right)$ in $B_{n-i+1}$ to the direct summand $W /\left(X_{t_{1}}+\cdots+X_{t_{i}}\right)$ in $B_{n-i}$ is the natural epimorphism taken with the sign $(-1)^{s-1}$.

The cobar complex $B^{\bullet}=B^{\bullet}\left(W ; X_{1}, \ldots, X_{n-1}\right)$ has the form

$$
\begin{aligned}
X_{1} \cap \cdots \cap X_{n-1} \longrightarrow \cdots \longrightarrow & \bigoplus_{1 \leq t_{1}<\cdots<t_{i} \leq n-1} X_{t_{1}} \cap \cdots \cap X_{t_{i}} \longrightarrow \cdots \\
& \longrightarrow \bigoplus_{1 \leq t_{1}<t_{2} \leq n-1} X_{t_{1}} \cap X_{t_{2}} \longrightarrow \bigoplus_{1 \leq t \leq n-1} X_{t} \longrightarrow W .
\end{aligned}
$$

Here the leftmost term $X_{1} \cap \cdots \cap X_{n-1}$ is placed in the cohomological degree 1 , the term with the summation over $1 \leq t_{1}<\cdots<t_{i} \leq n-1$ is placed in the cohomological degree $n-i$, and the rightmost term $W$ is placed in the cohomological degree $n$. The component of the differential acting from the direct summand $X_{t_{1}} \cap \cdots \cap X_{t_{i}}$ in $B^{n-i}$ to the direct summand $X_{t_{1}} \cap \cdots \cap \widehat{X}_{t_{s}} \cap \cdots \cap X_{t_{i}}$ in $B^{n-i+1}$ is the natural monomorphism taken with the sign $(-1)^{s-1}$.

The Koszul complex $K_{\bullet}\left(W ; X_{1}, \ldots, X_{n-1}\right)$ is

$$
\begin{aligned}
& X_{1} \cap \cdots \cap X_{n-1} \longrightarrow X_{2} \cap \cdots \cap X_{n-1} \longrightarrow X_{3} \cap \cdots \cap X_{n-1} / X_{1} \\
& \longrightarrow \cdots \longrightarrow\left(X_{i+1} \cap \cdots \cap X_{n-1}\right) /\left(X_{1}+\cdots+X_{i-1}\right) \longrightarrow \cdots \longrightarrow \\
& X_{n-1} /\left(X_{1}+\cdots+X_{n-3}\right) \longrightarrow W /\left(X_{1}+\cdots+X_{n-2}\right) \longrightarrow W /\left(X_{1}+\cdots+X_{n-1}\right) \text {. }
\end{aligned}
$$

Here the notation is $Y / Z=Y /(Y \cap Z)=(Y+Z) / Z$. The term $X_{1} \cap \cdots \cap X_{n-1}$ is placed in the homological degree $n$, and the term $W /\left(X_{1}+\cdots+X_{n-1}\right)$ is placed in the homological degree 0 .

Lemma 2.11. Let $X_{1}, \ldots, X_{n-1}$ be an almost distributive collection of subobjects of an object $W \in \mathrm{C}$. Then the following conditions are equivalent:

(a) the collection $\left(X_{1}, \ldots, X_{n-1}\right)$ is distributive;

(b) the Koszul complex $K_{\bullet}\left(W ; X_{1}, \ldots, X_{n-1}\right)$ is exact;

(c) the bar complex $B .\left(W ; X_{1}, \ldots, X_{n-1}\right)$ is exact everywhere except for its leftmost term $W$;

$\left(\mathrm{c}^{*}\right)$ the cobar complex $B^{\bullet}\left(W ; X_{1}, \ldots, X_{n-1}\right)$ is exact everywhere except for its rightmost term $W$.

Proof. This is [24, Proposition 7.2 in Chapter 1]. The assertion in [24] is stated for collections of subspaces in vector spaces, but the same argument applies to subobjects of any object in an abelian category.

Let $\mathrm{F} \subset \mathrm{C}$ be a class of objects closed under extensions and the passages to the kernels of epimorphisms. We will say that a lattice $\Omega$ of subobjects in an object $W$ is an $\mathrm{F}$-lattice if one has $Y / Z \in \mathrm{F}$ for any pair of subobjects $Y, Z \in \Omega$ such that $Z \subset Y$. In particular, existence of an F-lattice of subobjects in $W$ implies that $W=W / 0 \in \mathrm{F}$. A collection of subobjects $X_{1}, \ldots, X_{n-1} \subset W$ is said to be F-distributive if the lattice $\Omega$ of subobjects in $W$ generated by $X_{1}, \ldots, X_{n-1}$ is a distributive F-lattice. 
Lemma 2.12. A distributive collection of subobjects $X_{1}, \ldots, X_{n-1} \subset W$ is $\mathrm{F}$-distributive if and only if one has $W /\left(X_{t_{1}}+\cdots+X_{t_{i}}\right) \in \mathrm{F}$ for all $1 \leq t_{1}<\cdots<$ $t_{i} \leq n-1, \quad 0 \leq i \leq n-1$.

Proof. This is a generalization of [27, Lemma 11.4.3.2], and the same proof applies. Alternatively, one can argue by induction in $n$ in the following way. For any three subobjects $Z \subset Y$ and $X$ in $W$, there is a short exact sequence $0 \longrightarrow(X \cap Y) /(X \cap$ $Z) \longrightarrow Y / Z \longrightarrow(X+Y) /(X+Z) \longrightarrow 0$. Taking $Y, Z \in \Omega$ and $X=X_{n-1}$, we observe that the object $\left(X_{n-1}+Y\right) /\left(X_{n-1}+Z\right)$ belongs to $\mathrm{F}$ by the induction assumption applied to the collection of subobjects $\left(X_{t}+X_{n-1}\right) / X_{n-1} \subset W / X_{n-1}$, $1 \leq t \leq n-2$. Since $\mathrm{F}$ is closed under extensions, it suffices to show that $\left(X_{n-1} \cap\right.$ $Y) /\left(X_{n-1} \cap Z\right) \in \mathrm{F}$. Applying the same argument to $X=X_{n-2}$, etc., we reduce the question to showing that the object $X_{1} \cap X_{2} \cap \cdots \cap X_{n-1}$ belongs to $\mathrm{F}$. The argument finishes similarly to the proof in [27], by invoking Lemma 2.11(a) $\Rightarrow$ (c) and the assumption that the class $\mathrm{F}$ is closed under the kernels of epimorphisms.

We will say that a lattice $\Omega$ of subobjects in an object $W$ is split if for any pair of subobjects $Y, Z \in \Omega$ such that $Z \subset Y$ we have that $Z$ is a split subobject of $Y$. Equivalently, $\Omega$ is split if every $Z \in \Omega$ is a split subobject of $W$. A collection of subobjects $X_{1}, \ldots, X_{n-1} \subset W$ is said to be split distributive if the lattice $\Omega$ of subobjects in $W$ generated by $X_{1}, \ldots, X_{n-1}$ is split and distributive.

Lemma 2.13. A collection of subobjects $X_{1}, \ldots, X_{n-1} \subset W$ is split distributive if and only if there exists a finite direct sum decomposition $W=\bigoplus_{\eta} W_{\eta}$ of the object $W$ such that each of the subobjects $X_{i}$ is the sum of a set of subobjects $W_{\eta}$.

Proof. The proof of [24, Proposition $7.1(\mathrm{a}) \Leftrightarrow(\mathrm{b})$ in Chapter 1] is applicable.

2.8. Collections of subbimodules. Let $R$ and $S$ be associative rings and $W$ be an $R$-S-bimodule. Following the terminology of Section 2.7, we say that a collection of $R$-S-subbimodules $X_{1}, \ldots, X_{n-1} \subset W$ is distributive if the lattice of $R$-S-subbimodules $\Omega$ generated by $X_{1}, \ldots, X_{n-1}$ in $W$ is distributive. A collection of subbimodules $X_{1}, \ldots, X_{n-1} \subset W$ is said to be left flat distributive if it is distributive and for every pair of subbimodules $Y, Z \in \Omega$ such that $Z \subset Y$ the quotient bimodule $Y / Z$ is a flat left $R$-module.

Lemma 2.14. Let $R, S$, and $T$ be associative rings, $W$ be an $R$-S-bimodule, and $U$ be an $S$-T-bimodule. Let $X_{1}, \ldots, X_{n-1} \subset W$ be a left flat distributive collection of $R$-S-subbimodules, and let $Y_{1}, \ldots, Y_{m-1} \subset U$ be a left flat distributive collection of S-T-subbimodules. Then

$$
X_{1} \otimes_{S} U, \ldots, X_{n-1} \otimes_{S} U, W \otimes_{S} Y_{1}, \ldots, W \otimes_{S} Y_{m-1} \subset W \otimes_{S} U
$$

is a left flat distributive collection of $R$-T-subbimodules in the $R$-T-bimodule $W \otimes_{S} U$.

Proof. First of all, the map $X_{i} \otimes_{S} U \longrightarrow W \otimes_{S} U$ induced by the inclusion $X_{i} \longrightarrow W$ is injective for every $1 \leq i \leq n-1$, since $U$ is a flat left $S$-module. The map $W \otimes_{S} Y_{j} \longrightarrow W \otimes_{S} U$ induced by the inclusion $Y_{j} \longrightarrow U$ is also injective for every 
$1 \leq j \leq m-1$, since $U / Y_{j}$ is a flat left $S$-module. So $X_{i} \otimes_{S} U$ and $W \otimes_{S} Y_{j}$ are indeed subbimodules in $W \otimes_{S} U$.

Furthermore, arguing by induction in $n+m$, we can assume that our collection of $n+m-2$ subbimodules in $W \otimes_{S} U$ is almost distributive. Up to a homological shift by $[-1]$, the bar complex $B_{\bullet}=B_{\bullet}\left(W \otimes_{S} U ; X_{1} \otimes_{S} U, \ldots, X_{n-1} \otimes_{S} U\right.$, $\left.W \otimes_{S} Y_{1}, \ldots, W \otimes_{S} Y_{m-1}\right)$ (22) is isomorphic to the tensor product of two bar complexes $B_{\bullet}\left(W ; X_{1}, \ldots, X_{n-1}\right) \otimes_{S} B_{\bullet}\left(U ; Y_{1}, \ldots, Y_{m-1}\right)$. The only nonzero homology bimodule of the bar complex $B \cdot\left(U ; Y_{1}, \ldots, Y_{m-1}\right)$ is $Y_{1} \cap \ldots \cap Y_{m-1} \subset U$, and it is a flat left $S$-module by assumption. So are all the terms of the bar complex $B$. $\left(U ; Y_{1}, \ldots, Y_{m-1}\right)$. Thus the bar complex $B$. is exact everywhere except for its leftmost term $W \otimes_{S} U$. Applying Lemma 2.11(c) $\Rightarrow$ (a), we can conclude that out collection of $n+m-2$ subbimodules in $W \otimes_{S} U$ is distributive.

Finally, the quotient bimodule of $W \otimes_{S} U$ by the sum of any subset of $X_{1} \otimes_{S} U, \ldots$, $X_{n-1} \otimes_{S} U, W \otimes_{S} Y_{1}, \ldots, W \otimes_{S} Y_{m-1}$ is isomorphic to the tensor product of the quotient bimodules of $W$ and $U$ by the sums of the respective subsets of $X_{1}, \ldots, X_{n-1}$ and $Y_{1}, \ldots, Y_{n-1}$. As the tensor product of an $R$-flat $R$ - $S$-bimodule and an $S$-flat $S$-T-bimodule is an $R$-flat $R$-T-bimodule, any such quotient bimodule of $W \otimes_{S} U$ is a flat left $R$-module. It remains to apply Lemma 2.12 (for the class $\mathrm{F}$ of $R$-flat $R$-S-bimodules in the abelian category C of $R$-S-bimodules) in order to finish the proof of the lemma.

Lemma 2.15. In the context of Lemma 2.14, for any two subbimodules $X^{\prime}, X^{\prime \prime} \subset W$ belonging to the lattice of subbimodules generated by $X_{1}, \ldots, X_{n-1}$ in $W$ and any two subbimodules $Y^{\prime}, Y^{\prime \prime} \subset U$ belonging to the lattice of subbimodules generated by $Y_{1}, \ldots, Y_{m-1}$ in $U$, the following equations for subbimodules in $W \otimes_{S} U$ hold:
(a) $\left(X^{\prime}+X^{\prime \prime}\right) \otimes_{S} U=\left(X^{\prime} \otimes_{S} U\right)+\left(X^{\prime \prime} \otimes_{S} U\right)$;
(b) $\left(X^{\prime} \cap X^{\prime \prime}\right) \otimes_{S} U=\left(X^{\prime} \otimes_{S} U\right) \cap\left(X^{\prime \prime} \otimes_{S} U\right)$;
(c) $W \otimes_{S}\left(Y^{\prime}+Y^{\prime \prime}\right)=\left(W \otimes_{S} Y^{\prime}\right)+\left(W \otimes_{S} Y^{\prime \prime}\right)$;
(d) $W \otimes_{S}\left(Y^{\prime} \cap Y^{\prime \prime}\right)=\left(W \otimes_{S} Y^{\prime}\right) \cap\left(W \otimes_{S} Y^{\prime \prime}\right)$;
(e) $X^{\prime} \otimes_{S} Y^{\prime}=\left(W \otimes_{S} Y^{\prime}\right) \cap\left(X^{\prime} \otimes_{S} U\right)$;
(f) $\left(X^{\prime} \cap X^{\prime \prime}\right) \otimes_{S}\left(Y^{\prime} \cap Y^{\prime \prime}\right)=\left(X^{\prime} \otimes_{S} Y^{\prime}\right) \cap\left(X^{\prime \prime} \otimes_{S} Y^{\prime \prime}\right)$.

Proof. The maps $X^{\prime} \otimes_{S} U \longrightarrow W \otimes_{S} U$ and $W \otimes_{S} Y^{\prime} \longrightarrow W \otimes_{S} U$ induced by the inclusions $X^{\prime} \longrightarrow W$ and $Y^{\prime} \longrightarrow U$ are injective, as explained in the proof of Lemma 2.14. Furthermore, the map $X^{\prime} \otimes_{S} Y^{\prime} \longrightarrow W \otimes_{S} U$ is injective as the composition of injective maps $X^{\prime} \otimes_{S} Y^{\prime} \longrightarrow W \otimes_{S} Y^{\prime} \longrightarrow W \otimes_{S} U$ or $X^{\prime} \otimes_{S} Y^{\prime} \longrightarrow$ $X^{\prime} \otimes_{S} U \longrightarrow W \otimes_{S} U$. So these are indeed subbimodules in $W \otimes_{S} U$.

Now the equations (a) and (c) are obvious. The equation (b) holds since $U$ is flat left $S$-module. To prove (d), it suffices to consider the four-term exact sequence of flat left $S$-modules

$$
0 \longrightarrow Y^{\prime} \cap Y^{\prime \prime} \longrightarrow Y^{\prime} \oplus Y^{\prime \prime} \longrightarrow U \longrightarrow U /\left(Y^{\prime}+Y^{\prime \prime}\right) \longrightarrow 0
$$

and tensor it with $W$ over $S$ on the left.

To prove (e), one has to check that that the short sequence $0 \longrightarrow X^{\prime} \otimes_{S} Y^{\prime} \longrightarrow$ $W \otimes_{S} U \longrightarrow\left(W / X^{\prime} \otimes_{S} U\right) \oplus\left(W \otimes_{S} U / Y^{\prime}\right)$ is left exact. This follows from exactness of 
the sequences $0 \longrightarrow X^{\prime} \otimes_{S} Y^{\prime} \longrightarrow W \otimes_{S} Y^{\prime} \longrightarrow\left(W / X^{\prime}\right) \otimes_{S} Y^{\prime}$ and $0 \longrightarrow W \otimes_{S} Y^{\prime} \longrightarrow$ $W \otimes_{S} U \longrightarrow W \otimes_{S}\left(U / Y^{\prime}\right)$, and injectivity of the map $\left(W / X^{\prime}\right) \otimes_{S} Y^{\prime} \longrightarrow\left(W / X^{\prime}\right) \otimes_{S} U$. The equation (f) follows from (b), (d), and (e).

We will say that a collection of subbimodules $X_{1}, \ldots, X_{n-1} \subset W$ in an $R$-S-bimodule $W$ is left projective distibutive if it is distributive and for every pair of subbimodules $Y, Z \in \Omega$ such that $Z \subset Y$ the quotient bimodule $Y / Z$ is a projective left $R$-module. A collection of subbimodules $X_{1}, \ldots, X_{n-1} \subset W$ is said to be left split distributive if it is split distributive as a collection of submodules in the left $R$-module $W$ (in the sense of the definition in Section 2.7). Right projective distributive and right split distributive collections of subbimodules are defined similarly.

Clearly, a collection of subbimodules in an $R$-S-bimodule $W$ is left projective distributive if and only if it is left split distributive and the left $R$-module $W$ is projective.

Lemma 2.16. Let $R, S$, and $T$ be associative rings, $W$ be an $R$-S-bimodule, and $U$ be an $S$-T-bimodule. Let $X_{1}, \ldots, X_{n-1} \subset W$ be a left projective distributive collection of $R$-S-subbimodules, and let $Y_{1}, \ldots, Y_{m-1} \subset U$ be a left projective distributive collection of $S$-T-subbimodules. Then

$$
X_{1} \otimes_{S} U, \ldots, X_{n-1} \otimes_{S} U, W \otimes_{S} Y_{1}, \ldots, W \otimes_{S} Y_{m-1} \subset W \otimes_{S} U
$$

is a left projective distributive collection of subbimodules in the $R$-T-bimodule $W \otimes_{S} U$.

Proof. Similar to the proof of Lemma 2.14.

Lemma 2.17. Let $X_{1}, \ldots, X_{n-1} \subset W$ be a left split distributive collection of subbimodules in an $R$-S-bimodule $W$. Then $\operatorname{Hom}_{R}\left(W / X_{1}, R\right), \ldots, \operatorname{Hom}_{R}\left(W / X_{n-1}, R\right) \subset$ $\operatorname{Hom}_{R}(W, R)$ is a right split distributive collection of subbimodules in the $S$-R-bimodule $\operatorname{Hom}_{R}(W, R)$. If the left $R$-module $W$ is projective and finitely generated, then the above collection of subbimodules in $\operatorname{Hom}_{R}(W, R)$ is right projective distributive. In this case, the map $W \supset Y \longmapsto \operatorname{Hom}_{R}(W / Y, R) \subset \operatorname{Hom}_{R}(W, R)$ is an antiisomorphism between the lattice of subbimodules in $W$ generated by $X_{1}, \ldots, X_{n-1}$ and the lattice of subbimodules in $\operatorname{Hom}_{R}(W, R)$ generated by $\operatorname{Hom}_{R}\left(W / X_{1}, R\right), \ldots$, $\operatorname{Hom}_{R}\left(W / X_{n-1}, R\right)$ (i. e., a bijection of sets transforming the sums into the intersections and vice versa).

Proof. Follows easily from Lemma 2.13 (applied in the category of left $R$-modules $\mathrm{C}=R$-mod).

2.9. Left flat Koszul rings. Let $A=\bigoplus_{n=0}^{\infty} A_{n}$ be a nonnegatively graded ring with the degree-zero component $R=A_{0}$. The graded ring $A$ is said to be left flat Koszul if one of the equivalent conditions of the next theorem is satisfied. (Right flat Koszul graded rings are defined similarly.)

Theorem 2.18. The following three conditions are equivalent:

(a) $A_{n}$ is a flat left $R$-module for every $n \geq 1$ and $\operatorname{Tor}_{i, j}^{A}(R, R)=0$ for all $i \neq j$;

(b) $A_{n}$ is a flat left $R$-module for every $n \geq 1$, the graded ring $A$ is quadratic, and, setting $V=A_{1}$ and denoting by $I \subset V \otimes_{R} V$ the kernel of the multiplication map 
$A_{1} \otimes_{R} A_{1} \longrightarrow A_{2}$, for every $n \geq 4$ the collection of $n-1$ subbimodules

$$
I \otimes_{R} V \otimes_{R} \cdots \otimes_{R} V, V \otimes_{R} I \otimes_{R} V \otimes_{R} \cdots \otimes_{R} V, \ldots, V \otimes_{R} \cdots \otimes_{R} V \otimes_{R} I
$$

in the $R$-R-bimodule $W=V^{\otimes_{R} n}$ is distributive;

(c) $A_{1}$ and $A_{2}$ are flat left $R$-modules, the graded ring $A$ is quadratic, and for every $n \geq 1$ the collection of $R$-R-subbimodules $V^{\otimes_{R} k-1} \otimes_{R} I \otimes_{R} V^{\otimes_{R} n-k-1}, \quad 1 \leq k \leq n-1$ (25) in the $R$-R-bimodule $W=V^{\otimes_{R} n}$ is left flat distributive.

Proof. First of all, by Proposition 2.4(b), condition (a) implies that $A$ is quadratic. So we can assume that $A=T_{R}(V) /(I)$. Furthermore, any collection of less than three subobjects is distributive; so the distributivity condition in (b) is trivial for $n \leq 3$. It is clear from the formula (11) from Section 1 that the left flat distributivity condition in (c) implies flatness of the left $R$-modules $A_{n}$ for all $n \geq 1$. This suffices to prove the implication $(\mathrm{c}) \Longrightarrow(\mathrm{b})$.

To deduce $(\mathrm{b}) \Longrightarrow(\mathrm{c})$, we observe that the quotient bimodule of $V^{\otimes_{R} n}$ by any subset of the subbimodules (25) is isomorphic to the tensor product $A_{j_{1}} \otimes_{R} \cdots \otimes_{R} A_{j_{s}}$ for some $j_{1}, \ldots, j_{s} \geq 1, \quad j_{1}+\cdots+j_{s}=n$. Flatness of the left $R$-modules $A_{j}$ implies flatness of such tensor products (as left $R$-modules), and it remains to invoke Lemma 2.12 for the abelian category $\mathrm{C}$ of $R$ - $R$-bimodules and the class $\mathrm{F} \subset \mathrm{C}$ of all $R$ - $R$-bimodules that are flat as left $R$-modules.

It remains to prove the equivalence $(\mathrm{a}) \Longleftrightarrow(\mathrm{b})$. Arguing by induction in $n \geq 1$, we can assume that the collection of $j-1$ subbimodules (25) in the $R$ - $R$-bimodule $V^{\otimes_{R} j}$ is left flat distributive for all $1 \leq j \leq n-1$ and $\operatorname{Tor}_{i, j}^{A}(R, R)=0$ for all $i \neq j \leq n-1$. Under this induction assumption, we will prove the equivalence of conditions (a) and (b) for the fixed value of $j=n$.

By Lemma 2.14 (applied to the lattice of $k-1$ subbimodules in the $R$ - $R$-bimodule $W=V^{\otimes_{R}} k$ and the lattice of $n-k-1$ subbimodules in the $R$ - $R$-bimodule $U=$ $\left.V^{\otimes_{R} n-k}, \quad 1 \leq k \leq n-1\right)$, the induction assumption implies that the collection of $n-1$ subbimodules (25) in the $R$-R-bimodule $V^{\otimes_{R} n}$ is almost distributive.

Finally, the bar complex (14) computing the $R$ - $R$-bimodules $\operatorname{Tor}_{i, n}^{A}(R, R)$ is isomorphic to the lattice bar-complex $B .\left(W ; X_{1}, \ldots, X_{n-1}\right)$ (22) for the collection of $n-1$ subbimodules $X_{k}=V^{\otimes_{R} k-1} \otimes_{R} I \otimes_{R} V^{\otimes_{R} n-k-1}$ in the $R$ - $R$-bimodule $W=V^{\otimes_{R}{ }^{n}}$. Hence Lemma 2.11 (a) $\Leftrightarrow$ (c) implies the desired equivalence $(\mathrm{a}) \Longleftrightarrow(\mathrm{b})$.

The construction of the first Koszul complex in Section 2.5 was using double dualization: first we passed from $A_{1}$ to $B_{1}=\operatorname{Hom}_{R}\left(A_{1}, R\right)$, and then set $K_{1}^{\tau}(B, A)=$ $\operatorname{Hom}_{R^{\text {op }}}\left(B_{1}, A\right)$. Therefore, the assumption that $A_{1}$ is a finitely generated projective $R$-module was needed. A similar double dualization was used in the construction of the second Koszul complex in Section 2.6. The following alternative approach allows to produce the two Koszul complexes for any left flat Koszul ring.

Let $A$ be a left flat Koszul graded ring. Denote the kernel of the (surjective) multiplication map $A_{1} \otimes_{R} A_{1} \longrightarrow A_{2}$ by $I_{A} \subset A_{1} \otimes_{R} A_{1}$. Set $I_{A}^{(0)}=R, I_{A}^{(1)}=A_{1}$, $I_{A}^{(2)}=I_{A}$, and

$$
I_{A}^{(n)}=\bigcap_{k=1}^{n-1} A_{1}^{\otimes_{R} k-1} \otimes_{R} I_{A} \otimes_{R} A_{1}^{\otimes_{R} n-k-1} \subset A_{1}^{\otimes_{R} n} .
$$


So $I_{A}^{(n)}$ is an $R$ - $R$-subbimodule in $A_{1}^{\otimes_{R} n}$.

The intersection of all the subbimodules indexed by $k=2, \ldots, n-1$ (i. e., of all but the first one) in (26) is the subbimodule

$$
\bigcap_{k=2}^{n-1} A_{1}^{\otimes_{R} k-1} \otimes_{R} I_{A} \otimes_{R} A_{1}^{\otimes_{R} n-k-1}=A_{1} \otimes_{R} I_{A}^{(n-1)} \subset A_{1}^{\otimes_{R} n}
$$

by Lemma $2.15(\mathrm{~d})$. Hence we obtain an injective $R$ - $R$-bimodule morphism $I_{A}^{(n)} \longrightarrow$ $A_{1} \otimes_{R} I_{A}^{(n-1)}$, which is defined for all $n \geq 1$. For every $n \geq 2$, the image of the composition $I_{A}^{(n)} \longrightarrow A_{1} \otimes_{R} I_{A}^{(n-1)} \longrightarrow A_{1} \otimes_{R} A_{1} \otimes_{R} I_{A}^{(n-2)}$ is contained in the subbimodule $I_{A} \otimes_{R} I_{A}^{(n-2)} \subset A_{1} \otimes_{R} A_{1} \otimes_{R} I_{A}^{(n-2)}$.

Put $K_{i, n}^{\tau}(A)=A_{n-i} \otimes_{R} I_{A}^{(i)}$ for every $i \geq 0, n \geq i$ (where $\tau$ is just a placeholder or a notation for the identity map $\left.A_{1} \longrightarrow A_{1}\right)$. Define the differential $\partial: K_{i}^{\tau}(A) \longrightarrow$ $K_{i-1}^{\tau}(A)$ as the composition

$$
A_{j} \otimes_{R} I_{A}^{(i)} \longrightarrow A_{j} \otimes_{R} A_{1} \otimes_{R} I_{A}^{(i-1)} \longrightarrow A_{j+1} \otimes_{R} I_{A}^{(i-1)}
$$

of the map $A_{j} \otimes_{R} I_{A}^{(i)} \longrightarrow A_{j} \otimes_{R} A_{1} \otimes_{R} I_{A}^{(i-1)}$ induced by the map $I_{A}^{(i)} \longrightarrow A_{1} \otimes_{R} I_{A}^{(i-1)}$ and the map $A_{j} \otimes_{R} A_{1} \otimes_{R} I_{A}^{(i-1)} \longrightarrow A_{j+1} \otimes_{R} I_{A}^{(i-1)}$ induced by the multiplication map $A_{j} \otimes_{R} A_{1} \longrightarrow A_{j+1}$. Since the composition $I_{A} \longrightarrow A_{1} \otimes_{R} A_{1} \longrightarrow A_{2}$ vanishes, we have $\partial^{2}=0$. So we obtain a complex of graded left $A$-modules $K_{\bullet}^{\tau}(A)$ with the homological grading $i$ and the internal grading $n=i+j$.

Proposition 2.19. For any left flat Koszul graded ring A, the first Koszul complex $K_{\bullet}^{\tau}(A)$ is a graded flat resolution of the left $A$-module $R$.

Proof. The graded left $A$-modules $A \otimes_{R} I_{A}^{(i)}$ are flat, because the left $R$-modules $I_{A}^{(i)}$ are. Furthermore, for every $n \geq 1$ the internal degree $n$ component of the Koszul complex $K_{\bullet}^{\tau}(A)$ is isomorphic to the lattice Koszul complex $K_{\bullet}\left(W ; X_{1}, \ldots, X_{n-1}\right)$ (24) for the collection of $n-1$ subbimodules $X_{k}=A_{1}^{\otimes_{R} k-1} \otimes_{R} I_{A} \otimes_{R} A_{1}^{\otimes_{R} n-k-1}$ in the $R$-R-bimodule $W=A_{1}^{\otimes_{R}}$. Thus it remains to apply Lemma $2.11(\mathrm{a}) \Rightarrow(\mathrm{b})$.

Similarly, the intersection of all the subbimodules indexed by $k=1, \ldots, n-2$ (i. e., of all but the last one) in (26) is the subbimodule

$$
\bigcap_{k=1}^{n-2} A_{1}^{\otimes_{R} k-1} \otimes_{R} I_{A} \otimes_{R} A_{1}^{\otimes_{R} n-k-1}=I_{A}^{(n-1)} \otimes_{R} A_{1} \subset A_{1}^{\otimes_{R} n}
$$

by Lemma 2.15 (b). Hence we obtain an injective $R$ - $R$-bimodule morphism $I_{A}^{(n)} \longrightarrow$ $I_{A}^{(n-1)} \otimes_{R} A_{1}$, which is defined for all $n \geq 1$.

Put ${ }^{\tau} K_{i, n}(A)=I_{A}^{(i)} \otimes_{R} A_{n-i}$ for every $i \geq 0, n \geq i$. Define the differential $\partial:{ }^{\tau} K_{i}(A) \longrightarrow{ }^{\tau} K_{i-1}(A)$ as the composition

$$
I_{A}^{(i)} \otimes_{R} A_{j} \longrightarrow I_{A}^{(i-1)} \otimes_{R} A_{1} \otimes_{R} A_{j} \longrightarrow I_{A}^{(i-1)} \otimes_{R} A_{j+1} .
$$

Similarly to the construction above, we obtain a complex of graded right $A$-modules ${ }^{\tau} K_{\bullet}(A)$ with the homological grading $i$ and the internal grading $n=i+j$.

Recall the definition of a weakly $A / R$-flat right $A$-module for a morphism of associative rings $R \longrightarrow A$ such that $A$ is a flat left $R$-module [32, Section 5]. A 
right $A$-module $F$ is said to be weakly $A / R$-flat (or weakly flat relative to $R$ ) if the functor $F \otimes_{A}$ - takes short exact sequences of $R$-flat left $A$-modules to short exact sequences of abelian groups. For any right $R$-module $N$, the right $A$-module $N \otimes_{R} A$ is weakly flat relative to $R$. The main properties of the class of weakly $A / R$-flat right $A$-modules are listed in [32, Lemma 5.3(b)].

Proposition 2.20. For any left flat Koszul graded ring $A$, the second Koszul complex ${ }^{\tau} K_{\bullet}(A)$ is a graded weakly $A / R$-flat resolution of the right $A$-module $R$.

Proof. In view of the discussion above, the (graded) right $A$-modules $I_{A}^{(i)} \otimes_{R} A$ are (graded) weakly $A / R$-flat. This assertion does not require any assumptions about the right $R$-modules $I_{A}^{(i)}$. Furthermore, for every $n \geq 1$ the internal degree $n$ component of the Koszul complex ${ }^{\tau} K .(A)$ is isomorphic to the lattice Koszul complex $K_{\bullet}\left(W ; X_{1}, \ldots, X_{n-1}\right)$ (24) for the collection of $n-1$ subbimodules $X_{k}=$ $A_{1}^{\otimes_{R} n-k-1} \otimes_{R} I_{A} \otimes_{R} A_{1}^{\otimes_{R} k-1}$ in the $R$-R-bimodule $W=A_{1}^{\otimes_{R} n}$. This is the same collection of subbimodules as in the proof of Proposition 2.19, but numbered in the opposite order. So it remains to apply Lemma $[2.11(\mathrm{a}) \Rightarrow(\mathrm{b})$ in order to conclude that the internal degree $n$ component of the complex ${ }^{\tau} K_{\bullet}(A)$ is exact for $n>0$. Thus $H_{i}\left({ }^{\tau} K_{\bullet}(A)\right)=0$ for $i>0$ and $H_{0}\left({ }^{\tau} K_{\bullet}(A)\right)=R$.

2.10. Finitely projective Koszul rings. Let $A=\bigoplus_{n=0}^{\infty} A_{n}$ be a nonnegatively graded ring with the degree-zero component $R=A_{0}$. Assume that $A_{1}$ and $A_{2}$ are finitely generated projective left $R$-modules. When the multiplication map $A_{1} \otimes_{R}$ $A_{1} \longrightarrow A_{2}$ is surjective, we denote its kernel by $I \subset V \otimes_{R} V$, where $V=A_{1}$, and consider the 2-left finitely projective quadratic graded ring q $A=T_{R}(V) /(I)$ together with its quadratic dual 2-right finitely projective quadratic graded ring $B$.

As above in this paper, we denote by $\operatorname{Ext}_{A}(R, R)$ the bigraded Ext ring of the graded left $A$-module $R$, and by $\operatorname{Ext}_{B^{\mathrm{op}}}(R, R)$ the bigraded Ext ring of the graded right $B$-module $R$. The graded ring $A$ is said to be left finitely projective Koszul if one of the equivalent conditions of the next theorem is satisfied.

Theorem 2.21. For any nonnegatively graded ring $A$, the following four conditions are equivalent:

(a) $A_{n}$ is a finitely generated projective left $R$-module for every $n \geq 1$ and the graded ring $A$ is left flat Koszul;

(b) $A_{n}$ is a finitely generated projective left $R$-module for every $n \geq 1$, the multiplication map $A_{1} \otimes_{R} A_{1} \longrightarrow A_{2}$ is surjective, $B_{n}$ is a finitely generated projective right $R$-module for every $n \geq 1$, and $\operatorname{Ext}_{A}^{i, j}(R, R)=0$ for all $i \neq j$;

(c) the graded ring $A$ is quadratic, $A_{1}$ and $A_{2}$ are finitely generated projective left $R$-modules, and for every $n \geq 1$ the collection of $R$-R-subbimodules $V^{\otimes_{R} k-1} \otimes_{R}$ $I \otimes_{R} V^{\otimes_{R} n-k-1}, \quad 1 \leq k \leq n-1$ in the $R$-R-bimodule $W=V^{\otimes_{R}}$ is left projective distributive;

(d) $A_{1}$ and $A_{2}$ are finitely generated projective left $R$-modules, the multiplication map $A_{1} \otimes_{R} A_{1} \longrightarrow A_{2}$ is surjective, $B_{i}$ is a finitely generated projective right $R$-module for every $i \geq 1$, and the first Koszul complex $K_{\bullet}^{\tau}(B, A)$ (19) from Section 2.5 is exact in all the internal degrees $n \geq 1$; 
(e) $A_{n}$ is a finitely generated projective left $R$-module for every $n \geq 1$, the multiplication map $A_{1} \otimes_{R} A_{1} \longrightarrow A_{2}$ is surjective, and the second Koszul complex ${ }^{\tau} K .(B, A)$ (21) from Section 2.6 is exact in all the internal degrees $n \geq 1$.

Proof. (a) $\Longleftrightarrow$ (c) Take the condition of Theorem 2.18 (b) as the definition of left flat Koszulity and argue similarly to the proof of Theorem 2.18 (b) $\Leftrightarrow(\mathrm{c})$, using Lemma 2.12 for the abelian category $\mathrm{C}$ of $R$-R-bimodules and the class $\mathrm{F} \subset \mathrm{C}$ of all $R$ - $R$-bimodules that are projective as left $R$-modules.

$(\mathrm{a}) \Longleftrightarrow(\mathrm{b})$ Take the condition of Theorem 2.18(a) as the definition of left flat Koszulity. The $R$ - $R$-bimodules $\operatorname{Tor}_{i, n}^{A}(R, R)$ can be computed as the homology of the complex (14), while the $R$ - $R$-bimodules $\operatorname{Ext}_{A}^{i, n}(R, R)$ can be computed as the cohomology of the complex (17). Under (a), the graded ring $A$ is quadratic; so any one of the conditions (a) or (b) implies surjectivity of the multiplication map $A_{1} \otimes_{R} A_{1} \longrightarrow A_{2}$. By Proposition [2.2, we have $\operatorname{Ext}_{A}^{n, n}(R, R) \simeq B_{n}$.

Now the following lemma shows that, given a nonnegatively graded algebra $A$ with the degree-zero component $R=A_{0}$ such that $A_{j}$ is a finitely generated projective left $R$-module for every $j \geq 1$, and given a fixed $n \geq 1$, one has $\operatorname{Tor}_{i, n}^{A}(R, R)=0$ for all $1 \leq i \leq n-1$ if and only if $\operatorname{Ext}_{A}^{i, n}(R, R)=0$ for all $1 \leq i \leq n-1$ and the right $R$-module $\operatorname{Ext}_{A}^{n, n}(R, R)$ is projective.

Lemma 2.22. Let $0 \longrightarrow C_{n} \longrightarrow C_{n-1} \longrightarrow \cdots \longrightarrow C_{1} \longrightarrow 0$ be a complex of finitely generated projective left $R$-modules, and let $0 \longrightarrow \operatorname{Hom}_{R}\left(C_{1}, R\right) \longrightarrow$ $\cdots \longrightarrow \operatorname{Hom}_{R}\left(C_{n}, R\right) \longrightarrow 0$ be the dual complex of finitely generated projective right $R$-modules. Then the following four conditions are equivalent:

(a) $H_{i}\left(C_{\bullet}\right)=0$ for all $1 \leq i \leq n-1$;

$\left(\mathrm{a}^{\prime}\right)$ the complex of left $R$-modules $C$. is homotopy equivalent to the one-term complex $H_{n}\left(C_{\bullet}\right)[n]$;

(b) $H^{i}\left(\operatorname{Hom}_{R}\left(C_{\bullet}, R\right)\right)=0$ for all $1 \leq i \leq n-1$ and $H^{n}\left(\operatorname{Hom}_{R}\left(C_{\bullet}, R\right)\right)$ is a (finitely generated) projective right $R$-module;

$\left(\mathrm{b}^{\prime}\right)$ the complex of right $R$-modules $\operatorname{Hom}_{R}\left(C_{\bullet}, R\right)$ is homotopy equivalent to the one-term complex $H^{n}\left(\operatorname{Hom}_{R}\left(C^{\bullet}, R\right)\right)[-n]$.

If any one of these equivalent conditions holds, then $H_{n}\left(C_{\bullet}\right)$ is a finitely generated projective left $R$-module and $H^{n}\left(\operatorname{Hom}_{R}\left(C_{\bullet}, R\right)\right) \simeq \operatorname{Hom}_{R}\left(H_{n}\left(C_{\bullet}\right), R\right)$.

$(\mathrm{c}) \Longrightarrow(\mathrm{d})$, (e) Under (c), the results of Lemmas 2.15 and 2.17 are available for the collections of subbimodules $V^{\otimes_{R}{ }^{k-1}} \otimes_{R} I \otimes_{R} V^{\otimes_{R} j-k-1}$ in the $R$ - $R$-bimodules $V^{\otimes_{R} j}$, $1 \leq k \leq j-1, \quad j \geq 1$. This allows to compute the internal degree $n$ component of the Koszul complex $K_{\bullet}^{\tau}(B, A)$ as the lattice Koszul complex $K_{\bullet}\left(W ; X_{1}, \ldots, X_{n-1}\right)$ (24) for the collection of subbimodules $X_{k}=V^{\otimes_{R} k-1} \otimes_{R} I \otimes_{R} V^{\otimes_{R} n-k-1} \subset V^{\otimes_{R} n}=$ $W$. Similarly, the internal degree $n$ component of the Koszul complex ${ }^{\tau} K \bullet(B, A)$ is computed as the lattice Koszul complex for the collection of subbimodules $X_{k}=$ $V^{\otimes_{R} n-k-1} \otimes_{R} I \otimes_{R} V^{\otimes_{R} k-1} \subset V^{\otimes_{R} n}$ (the same subbimodules numbered in the opposite order). Then in both cases it remains to apply Lemma 2.11(a) $\Rightarrow$ (b).

$(\mathrm{d}) \Longrightarrow(\mathrm{a})$, (b) Under (d), the Koszul complex $K_{\bullet}^{\tau}(B, A)$ is a graded projective resolution of the graded left $A$-module $R$. Computing $\operatorname{Tor}^{A}(R, R)$ and $\operatorname{Ext}_{A}(R, R)$ in 
terms of this resolution immediately yields (a) and (b) (where (a) is interpreted as the condition of Theorem $2.18(\mathrm{a}))$.

(e) $\Longrightarrow\left(\right.$ a) Under (e), the Koszul complex ${ }^{\tau} K_{\bullet}(B, A)$ is a graded weakly $A / R$-flat resolution of the graded right $A$-module $R$, as explained in Section 2.9 (see the proof of Proposition 2.20). Since the graded left $A$-module $R$ is flat over $R$, one can compute $\operatorname{Tor}^{A}(R, R)$ in terms of this resolution (e. g., in view of [32, Lemma 5.3(b)]). This immediately implies (a) (intepreted as the condition of Theorem 2.18(a)).

The definition of right finitely projective Koszul graded ring is obtained from the above definition of a left finitely projective Koszul ring by switching the roles of the left and right sides.

Proposition 2.23. Let $A$ be a 2-left finitely projective quadratic graded ring with the degree-zero component $R=A_{0}$, and let $B$ be the 2-right finitely projective quadratic graded ring quadratic dual to $A$. Then the ring $A$ is left finitely projective Koszul if and only if the ring $B$ is right finitely projective Koszul. If this is the case, one has

$$
B \simeq \operatorname{Ext}_{A}(R, R)^{\mathrm{op}} \quad \text { and } \quad A \simeq \operatorname{Ext}_{B^{\mathrm{op}}}(R, R) .
$$

Proof. The first assertion is provable using the condition of Theorem 2.21(c) as the definition of projective Koszulity and the result of Lemma 2.17. Then, by Theorem 2.21(b), we have $\operatorname{Ext}_{A}^{i, j}(R, R)=0$ for $i \neq j$, and similarly, $\operatorname{Ext}_{B \text { op }}^{i, j}(R, R)=0$ for $i \neq j$. Finally, the graded ring isomorphism $B^{\mathrm{op}} \simeq \bigoplus_{n \geq 0} \operatorname{Ext}_{A}^{n, n}(R, R)$ is provided by Proposition 2.2. The graded ring isomorphism $A \simeq \bigoplus_{n \geq 0} \operatorname{Ext}_{B^{n o p}}^{n, n}(R, R)$ can be obtained by applying the same proposition to the ring $B^{\text {op }}$ and observing that the 2-left finitely projective quadratic graded ring $B^{\mathrm{op}}$ is quadratic dual to the 2-right finitely projective quadratic graded ring $A^{\text {op }}$.

Corollary 2.24. The anti-equivalences of categories from Propositions $1.2[1.3$ restrict to an anti-equivalence between the category of left finitely projective Koszul graded rings $A$ and the category of right finitely projective Koszul graded rings $B$ over any fixed base ring $R$.

Remark 2.25. Let us warn the reader that, even when the graded $\operatorname{rings} A$ and $B$ are Koszul (say, $A$ is left finitely projective Koszul and $B$ is right finitely projective Koszul, as above), the dual Koszul complex $K_{e}^{\vee \bullet}(B, A)$ (20) from Section 2.6 has no good exactness properties, generally speaking. In fact, in this case one has $K_{e}^{\vee \bullet}(B, A)=\operatorname{Hom}_{A}\left(K_{\bullet}^{\tau}(B, A), A\right)$, hence it follows from Theorem 2.21(d) that the complex $K_{e}^{\vee} \bullet(B, A)$ computes the graded $\operatorname{Ext}_{A}^{*}(R, A)$. This Ext can be quite complicated even for a Koszul algebra $A$ over a field $R=k$.

\section{Relative Nonhomogeneous Quadratic Duality}

Nonhomogeneous quadratic rings $\widetilde{A}$ can be informally described as rings defined by nonhomogeneous quadratic relations over a fixed base ring $R$. Not every system of nonhomogeneous quadratic relations is good enough to define a nonhomogeneous 
quadratic ring (see the general discussion in the introductions to [25, 24], [24, Section 5], and the counterexamples in [33, Section 5] and [25, Section 3.4]). For a system of nonhomogeneous quadratic relations to "make sense", its coefficients must, in turn, satisfy a certain system of equations, called the self-consistency equations.

Dualizing the degree-one and degree-zero parts of the nonhomogeneous quadratic relations and imposing the equations dual to the self-consistency equations on the relations' coefficients produces a curved DG-ring structure $(B, d, h)$ on the dual quadratic graded ring $B$ to the quadratic graded ring $A$ defined by the homogeneous quadratic parts of the nonhomogeneous quadratic relations.

3.1. Nonhomogeneous quadratic rings. Let $\widetilde{A}$ be an associative ring with a subring $R \subset \widetilde{A}$. Consider the $R$ - $R$-bimodule $\widetilde{A} / R$ and suppose that we have chosen a subbimodule $V \subset \widetilde{A} / R$. Denote by $\widetilde{V} \subset \widetilde{A}$ the full preimage of $V$ under the surjective $R$ - $R$-bimodule morphism $\widetilde{A} \longrightarrow \widetilde{A} / R$.

Consider the tensor ring $T_{R}(\widetilde{V})=\bigoplus_{n=0}^{\infty} T_{R, n}(\widetilde{V}), T_{R, n}(\widetilde{V})=\widetilde{V}^{\otimes_{R} n}$, as in Section 1, Then the $R$ - $R$-bimodule morphism of identity inclusion $\widetilde{V} \longrightarrow \widetilde{A}$ extends uniquely to a ring homomorphism $\pi_{\widetilde{A}}: T_{R}(\widetilde{V}) \longrightarrow \widetilde{A}$ forming a commutative triangle diagram with the subring inclusions $R \longrightarrow T_{R}(\widetilde{V})$ and $R \longrightarrow \widetilde{A}$.

Assume that the ring $\widetilde{A}$ is generated by its subgroup $\widetilde{V}$. In other words, the ring homomorphism $\pi_{\widetilde{A}}: T_{R}(\widetilde{V}) \longrightarrow \widetilde{A}$ is surjective. Let $\widetilde{J}_{\widetilde{A}} \subset T_{R}(\widetilde{V})$ be the kernel of $\pi_{\widetilde{A}}$.

Define an increasing filtration $F$ on the $\operatorname{ring} T_{R}(\widetilde{V})$ by the rule $F_{n} T_{R}(\widetilde{V})=$ $\bigoplus_{i=0}^{n} T_{R, i}(\widetilde{V}) \subset T_{R}(\widetilde{V})$. Furthermore, put $F_{n} \widetilde{A}=\pi_{\widetilde{A}}\left(F_{n} T_{R}(\widetilde{V})\right) \subset \widetilde{A}$. So we have $F_{0} T_{R}(\widetilde{V})=R$ and $F_{1} T_{R}(\widetilde{V})=R \oplus \widetilde{V}$, hence $F_{0} \widetilde{A}=R$ and $F_{1} \widetilde{A}=\widetilde{V} \subset \widetilde{A}$.

Clearly, $F$ is an exhaustive multiplicative filtration on the $\operatorname{ring} \widetilde{A}$, that is $\widetilde{A}=$ $\bigcup_{n \geq 0} F_{n} \widetilde{A}$ and $F_{n} \widetilde{A} \cdot F_{m} \widetilde{A} \subset F_{n+m} \widetilde{A}$. In fact, the filtration $F$ on $\widetilde{A}$ is generated by $F_{1}$, which means that the equation $F_{n} \widetilde{A} \cdot F_{m} \widetilde{A}=F_{n+m} \widetilde{A}$ holds for all $n, m \geq 0$. (Sometimes we will say that the filtration $F$ on $\widetilde{A}$ is generated by $F_{1} \widetilde{A}$ over $R$, which means that $F$ is generated by $F_{1}$ and $F_{0} \widetilde{A}=R$.) The filtration $F$ on the graded ring $T_{R}(\widetilde{V})$ has similar properties.

Put $\widetilde{I}_{\widetilde{A}}=F_{2} T_{R}(\widetilde{V}) \cap \widetilde{J}_{\widetilde{A}}$; so $\widetilde{I}_{\widetilde{A}}$ is an $R$-R-subbimodule in $F_{2} T_{R}(\widetilde{V})=R \oplus \widetilde{V} \oplus \widetilde{V}^{\otimes_{R}}{ }^{2}$. We will say that the ring $\widetilde{A}$ with a fixed subring $R \subset \widetilde{A}$ and a fixed $R$-R-subbimodule of generators $R \subset \widetilde{V} \subset \widetilde{A}$ is weak nonhomogeneous quadratic over $R$ if the ideal $\widetilde{J}_{\widetilde{A}} \subset T_{R}(\widetilde{V})$ is generated by its subgroup $\widetilde{I}_{\widetilde{A}}$.

Let ' $\widetilde{A}$ and " $\widetilde{A}$ be two weak nonhomogeneous quadratic rings over the same base ring $R$, and let $R \subset{ }^{\prime} \widetilde{V} \subset{ }^{\prime} \widetilde{A}$ and $R \subset{ }^{\prime \prime} \widetilde{V} \subset{ }^{\prime \prime} \widetilde{A}$ be their fixed subbimodules of generators. A morphism of weak nonhomogeneous quadratic rings $f:{ }^{\prime} \widetilde{A} \longrightarrow{ }^{\prime \prime} \widetilde{A}$ is a ring homomorphism forming a commutative triangle diagram with the subring inclusions $R \longrightarrow{ }^{\prime} \widetilde{A}$ and $R \longrightarrow{ }^{\prime \prime} \widetilde{A}$ and satisfying the condition that $f\left({ }^{\prime} \widetilde{V}\right) \subset{ }^{\prime \prime} \widetilde{V}$.

Conversely, suppose that we are given an associative ring $\widetilde{A}$ with an exhaustive multiplicative increasing filtration $0=F_{-1} \widetilde{A} \subset F_{0} \widetilde{A} \subset F_{1} \widetilde{A} \subset F_{2} \widetilde{A} \subset \cdots$. Then 
$R=F_{0} \widetilde{A}$ is a subring in $\widetilde{A}$. Suppose that the filtration $F$ on $\widetilde{A}$ is generated by $F_{1}$, and put $\widetilde{V}=F_{1} \widetilde{A}$. Then we have $R \subset \widetilde{V} \subset \widetilde{A}$ and the $\operatorname{ring} \widetilde{A}$ is generated by its subgroup $\widetilde{V}$.

Consider the associated graded $\operatorname{ring} \operatorname{gr}^{F} \widetilde{A}=\bigoplus_{n=0}^{\infty} \operatorname{gr}_{n}^{F} \widetilde{A}$, where $\operatorname{gr}_{n}^{F} \widetilde{A}=$ $F_{n} \widetilde{A} / F_{n-1} \widetilde{A}$. We will say that the filtered $\operatorname{ring} \widetilde{A}$ is nonhomogeneous quadratic if the $\operatorname{ring} A=\operatorname{gr}^{F} \widetilde{A}$ is quadratic (in the sense of Section 11).

Let $\left({ }^{A}, F\right)$ and (" $\left.\widetilde{A}, F\right)$ be two nonhomogeneous quadratic rings with the same degree-zero filtration component $F_{0}{ }^{\prime \prime} \widetilde{A}=R=F_{0}{ }^{\prime} \widetilde{A}$. A morphism of nonhomogeneous quadratic rings $f:{ }^{\prime} \widetilde{A} \longrightarrow{ }^{\prime \prime} \widetilde{A}$ is a morphism of filtered rings (that is, a ring homomorphism such that $f\left(F_{n}{ }^{\prime} \widetilde{A}\right) \subset F_{n}{ }^{\prime \prime} \widetilde{A}$ for all $\left.n \geq 0\right)$ forming a commutative triangle diagram with the subring inclusions $R \longrightarrow{ }^{\prime} \widetilde{A}$ and $R \longrightarrow{ }^{\prime \prime} \widetilde{A}$.

Lemma 3.1. The above construction assigning the subbimodule of generators $\widetilde{V}=$ $F_{1} \widetilde{A}$ to a filtration $F$ on a ring $\widetilde{A}$ defines a fully faithful functor from the category of nonhomogeneous quadratic rings to the category of weak nonhomogeneous quadratic rings (over any fixed base ring $R$ ). In particular, any nonhomogeneous quadratic ring is weak nonhomogeneous quadratic (so our terminology is consistent).

Proof. We will only prove the second assertion. Let $(A, F)$ be a nonhomogeneous quadratic ring. We have to show that the ideal $\widetilde{J}_{\widetilde{A}} \subset T_{R}(\widetilde{V})$ is generated by $\widetilde{I}_{\widetilde{A}}=F_{2} T_{R}(\widetilde{V}) \cap \widetilde{J}_{\widetilde{A}}$. Indeed, denote the ideal generated by $\widetilde{I}_{\widetilde{A}}$ by $\widetilde{J}^{\prime} \subset T_{R}(\widetilde{V})$, and consider the ring $\widetilde{A}^{\prime}=T_{R}(\widetilde{V}) / \widetilde{J}^{\prime}$. Then $\widetilde{J}^{\prime} \subset \widetilde{J}_{\widetilde{A}}$, so there is a unique surjective ring homomorphism $\widetilde{A}^{\prime} \longrightarrow \widetilde{A}$ forming a commutative triangle diagram with the surjective ring homomorphisms $T_{R}(\widetilde{V}) \longrightarrow \widetilde{A}^{\prime}$ and $T_{R}(\widetilde{V}) \longrightarrow \widetilde{A}$.

For every $n \geq 0$, denote by $F_{n} \widetilde{A}^{\prime} \subset \widetilde{A}^{\prime}$ the image of the subgroup $F_{n} T_{R}(\widetilde{V}) \subset T_{R}(\tilde{V})$ under the ring homomorphism $T_{R}(\widetilde{V}) \longrightarrow \widetilde{A}^{\prime}$. Then $F$ is an exhastive multiplicative filtration on the ring $\widetilde{A}^{\prime}$. Furthermore, the image of $F_{n} \widetilde{A}^{\prime}$ under the ring homomorphism $\widetilde{A}^{\prime} \longrightarrow \widetilde{A}$ coincides with $F_{n} \widetilde{A}$. It is clear that the maps $F_{n} \widetilde{A}^{\prime} \longrightarrow F_{n} \widetilde{A}$ are isomorphisms for $n=0$ and 1. Moreover, we have $\widetilde{I}_{\widetilde{A}} \subset F_{2} T_{R}(\widetilde{V}) \cap \widetilde{J}^{\prime}$ by construction, hence $F_{2} T_{R}(\widetilde{V}) \cap \widetilde{J}^{\prime}=F_{2} T_{R}(\widetilde{V}) \cap \widetilde{J}_{\widetilde{A}}$. Therefore, the map $F_{2} \widetilde{A}^{\prime} \longrightarrow F_{2} \widetilde{A}$ is an isomorphism, too.

We need to show that $\widetilde{J}^{\prime}=\widetilde{J}_{\widetilde{A}}$; equivalently, this means that the ring homomorphism $\widetilde{A}^{\prime} \longrightarrow \widetilde{A}$ is an isomorphism. It suffices to check that the induced homomorphism of graded rings $\operatorname{gr}^{F} \widetilde{A}^{\prime} \longrightarrow \operatorname{gr}^{F} \widetilde{A}$ is an isomorphism. Set $A^{\prime}=\operatorname{gr}^{F} \widetilde{A}^{\prime}$ and $A=\operatorname{gr}^{F} \widetilde{A}$. The graded ring $A^{\prime}$ is generated by $A_{1}^{\prime}$ over $A_{0}^{\prime}=R$, since the filtration $F$ on the ring $\widetilde{A}^{\prime}$ is generated by $F_{1}$ by construction. The graded ring $A$ is quadratic by assumption. The graded rings homomorphism $A^{\prime} \longrightarrow A$ is an isomorphism in the degrees 0,1 , and 2 , as we have shown. It remains to apply the next lemma.

Lemma 3.2. Let $f: A^{\prime} \longrightarrow A$ be a homomorphism of nonnegatively graded rings such that the maps $f_{n}: A_{n}^{\prime} \longrightarrow A_{n}$ are isomorphisms for $n=0,1$, and 2. Assume that 
the graded ring $A^{\prime}$ is generated by $A_{1}^{\prime}$ over $A_{0}^{\prime}$, while the graded ring $A$ is quadratic. Then the map $f$ is an isomorphism of graded rings.

Proof. Set $A_{0}^{\prime}=R=A_{0}$ and $A_{1}^{\prime}=V=A_{1}$. Then there is a unique graded ring homomorphism $\pi_{A^{\prime}}: T_{R}(V) \longrightarrow A^{\prime}$ acting by the chosen isomorphisms $R \simeq A_{0}$ and $V \simeq A_{1}^{\prime}$ on the components of degrees 0 and 1 , and a similar unique graded ring homomorphism $\pi_{A}: T_{R}(V) \longrightarrow A$. The triangle diagram of ring homomorphisms $T_{R}(V) \longrightarrow A^{\prime} \longrightarrow A$ is commutative, i. e., $\pi_{A}=f \pi_{A^{\prime}}$. Furthermore, both the graded rings $A$ and $A^{\prime}$ are generated by their degree-one components (over their degree-zero components) by assumption, hence both the maps $\pi_{A^{\prime}}$ and $\pi_{A}$ are surjective.

Denote by $J_{A^{\prime}}$ and $J_{A} \subset T_{R}(V)$ the kernels of the graded ring homomorphisms $\pi_{A^{\prime}}$ and $\pi_{A}$. Then we have $J_{A^{\prime}} \subset J_{A}$ and $J_{A^{\prime}, 2}=J_{A, 2}$, since the map $f_{2}: A_{2}^{\prime} \longrightarrow A_{2}$ is an isomorphism by assumption. The algebra $A$ is quadratic, so the ideal $J_{A}$ is generated by $I_{A}=J_{A, 2}$. It follows that $J_{A^{\prime}}=J_{A}$, hence $f_{n}$ is an isomorphism for all $n$.

Remark 3.3. We will see below in Section 4.6 that under the left finitely projective Koszulity assumption the classes of weak nonhomogeneous quadratic rings and nonhomogeneous quadratic rings coincide. Specifically, if $\widetilde{A}$ is a weak nonhomogeneous quadratic ring such that the quadratic graded $\operatorname{ring} \operatorname{qgr}^{F} \widetilde{A}$ is left finitely projective Koszul (where the filtration $F$ on $\widetilde{A}$ is generated by $F_{1} \widetilde{A}=\widetilde{V}$ over $F_{0} \widetilde{A}=R$, as above), then the graded $\operatorname{ring} \operatorname{gr}^{F} \widetilde{A}$ is quadratic (so the filtered ring $\widetilde{A}$ is nonhomogeneous quadratic).

3.2. Curved DG-rings. A $C D G$-ring (curved differential graded ring) $B=(B, d, h)$ is a graded associative ring $B=\bigoplus_{n \in \mathbb{Z}} B^{n}$ endowed with a sequence of additive maps $d_{n}: B^{n} \longrightarrow B^{n+1}, n \in \mathbb{Z}$, and an element $h \in B^{2}$ satisfying the following conditions:

(i) $d$ is an odd derivation of $B$, that is $d(b c)=d(b) c+(-1)^{|b|} b d(c)$ for all $b \in B^{|b|}$ and $c \in B^{|c|},|b|,|c| \in \mathbb{Z}$;

(ii) $d^{2}(b)=[h, b]$ for all $b \in B$ (where $[h, b]=h b-b h$ is the commutator);

(iii) $d(h)=0$.

In the context of the present paper, all CDG-rings will be nonnegatively graded, that is $B=\bigoplus_{n=0}^{\infty} B^{n}$. We denote the grading of $B$ by upper indices, because the differential $d$ has degree 1 .

Let ${ }^{\prime} B=\left({ }^{\prime} B, d^{\prime}, h^{\prime}\right)$ and ${ }^{\prime \prime} B=\left({ }^{\prime \prime} B, d^{\prime \prime}, h^{\prime \prime}\right)$ be two CDG-rings. A morphism of CDG-rings " $B \longrightarrow{ }^{\prime} B$ is a pair $(f, a)$ consisting of a morphism of graded rings $f:{ }^{\prime \prime} B \longrightarrow{ }^{\prime} B$ and an element $a \in{ }^{\prime} B^{1}$ such that

(iv) $f\left(d^{\prime \prime}(b)\right)=d^{\prime}(f(b))+[a, f(b)]$ for all $b \in \in^{\prime \prime} B^{|b|}$ (where $[x, y]=x y-(-1)^{|x||y|} y x$, $x \in{ }^{\prime} B^{|x|}, y \in{ }^{\prime} B^{|y|}$, is the graded commutator); and

(v) $f\left(h^{\prime \prime}\right)=h^{\prime}+d^{\prime}(a)+a^{2}$.

The composition of morphisms is defined by the formula $(f, a)(g, b)=(f g, a+f(b))$. The identity morphism is the morphism (id,0). These rules define the category of $C D G$-rings. We will denote the category of nonnegatively graded CDG-rings $(B, d, h)$ with the fixed degree-zero component $B^{0}=R$ by $R$-rings cdg. Morphisms $^{\prime \prime} B \longrightarrow{ }^{\prime} B$ in $R$-rings cdg $_{\text {are }}$ CDG-ring morphisms $(f, a):{ }^{\prime \prime} B \longrightarrow{ }^{\prime} B$ such that the graded ring 
homomorphism $f:{ }^{\prime \prime} B \longrightarrow{ }^{\prime} B$ forms a commutative triangle diagram with the fixed isomorphisms $R \simeq{ }^{\prime \prime} B^{0}$ and $R \simeq{ }^{\prime} B^{0}$.

The element $h \in B^{2}$ is called the curvature element. The element $a \in{ }^{\prime} B^{1}$ is called the change-of-connection element.

For any CDG-ring $B=(B, d, h)$ and any element $a \in B^{1}$, the triple ' $B=\left(B, d^{\prime}, h^{\prime}\right)$ with $d^{\prime}=d+[a,-]$ and $h^{\prime}=h+d(a)+a^{2}$ is also a CDG-ring. The CDG-rings $B$ and $' B$ are connected by the isomorphism (id, $a):{ }^{\prime} B \longrightarrow B$. Such isomorphisms will be called change-of-connection isomorphisms, while CDG-ring morphisms of the form $(f, 0)$ will be called strict morphisms. Any morphism of CDG-rings $(f, a)$ : ${ }^{\prime \prime} B=$ $\left({ }^{\prime \prime} B, d^{\prime \prime}, h^{\prime \prime}\right) \longrightarrow{ }^{\prime} B=\left({ }^{\prime} B, d^{\prime}, h^{\prime}\right)$ decomposes uniquely into a strict morphism followed by a change-of-connection isomorphism, $(f, a)=(\mathrm{id}, a)(f, 0)$.

Furthermore, one can define the 2-category of $C D G$-rings as follows. Let $(f, a)$ and $(g, b):{ }^{\prime \prime} B=\left({ }^{\prime \prime} B, d^{\prime \prime}, h^{\prime \prime}\right) \longrightarrow{ }^{\prime} B=\left({ }^{\prime} B, d^{\prime}, h^{\prime}\right)$ be two CDG-ring morphisms with the same domain and codomain. A 2-morphism $(f, a) \stackrel{z}{\longrightarrow}(g, b)$ is an invertible element $z \in{ }^{\prime} B^{0}$ satisfying the equations

(vi) $g(c)=z f(c) z^{-1}$ for all $c \in \in^{\prime \prime} B$; and

(vii) $b=z a z^{-1}-d^{\prime}(z) z^{-1}$.

The element $z \in{ }^{\prime} B^{0}$ is called the gauge transformation element.

The vertical composition of two 2-morphisms $\left(f^{\prime}, a^{\prime}\right) \stackrel{w}{\longrightarrow}\left(f^{\prime \prime}, a^{\prime \prime}\right) \stackrel{z}{\longrightarrow}\left(f^{\prime \prime \prime}, a^{\prime \prime \prime}\right)$ is the 2-morphism $\left(f^{\prime}, a^{\prime}\right) \stackrel{z w}{\longrightarrow}\left(f^{\prime \prime \prime}, a^{\prime \prime \prime}\right)$. The identity 2-morphism is the 2-morphism $(f, a) \stackrel{1}{\longrightarrow}(f, a)$. The horizontal composition of two 2-morphisms $\left(g^{\prime}, b^{\prime}\right) \stackrel{w}{\longrightarrow}$ $\left(g^{\prime \prime}, b^{\prime \prime}\right):\left(C, d_{C}, h_{C}\right) \longrightarrow\left(B, d_{B}, h_{B}\right)$ and $\left(f^{\prime}, a^{\prime}\right) \stackrel{z}{\longrightarrow}\left(f^{\prime \prime}, a^{\prime \prime}\right):\left(B, d_{B}, h_{B}\right) \longrightarrow$ $\left(A, d_{A}, h_{A}\right)$ is the 2-morphism $\left(f^{\prime} g^{\prime}, a^{\prime}+f^{\prime}\left(b^{\prime}\right)\right) \stackrel{z \circ w}{\longrightarrow}\left(f^{\prime \prime} g^{\prime \prime}, a^{\prime \prime}+f^{\prime \prime}\left(b^{\prime \prime}\right)\right):\left(C, d_{C}, h_{C}\right)$ $\longrightarrow\left(A, d_{A}, h_{A}\right)$ with the element $z \circ w=z f^{\prime}(w)=f^{\prime \prime}(w) z \in A^{0}$.

All the 2-morphisms of CDG-rings are invertible. If $(f, a):{ }^{\prime \prime} B \longrightarrow{ }^{\prime} B$ is a morphism of CDG-rings and $z \in{ }^{\prime} B^{0}$ is an invertible element, then the pair $(g, b)$ defined by the formulas (vi-vii) is also a morphism of CDG-rings $(g, b):{ }^{\prime \prime} B \longrightarrow{ }^{\prime} B$. The morphisms $(f, a)$ and $(g, b)$ are connected by the 2-isomorphism $(f, a) \stackrel{z}{\longrightarrow}(g, b)$.

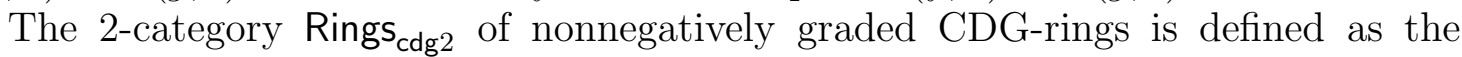
following subcategory of the 2-category of CDG-rings (cf. Remark 1.4). The objects of Rings $\mathrm{cdg}_{2}$ are nonnegatively graded CDG-rings $(B, d, h), \quad B=\bigoplus_{n=0}^{\infty} B^{n}$. Morphisms (" $\left.B, d^{\prime \prime}, h^{\prime \prime}\right) \longrightarrow\left({ }^{\prime} B, d^{\prime}, h^{\prime}\right)$ in Rings ${ }_{\mathrm{cdg} 2}$ are morphisms of CDG-rings $(f, a):\left({ }^{\prime \prime} B, d^{\prime \prime}, h^{\prime \prime}\right) \longrightarrow\left({ }^{\prime} B, d^{\prime}, h^{\prime}\right)$ such that the degree-zero component $f_{0}:{ }^{\prime \prime} B^{0} \longrightarrow$ ${ }^{\prime} B^{0}$ of the graded ring homomorphism $f$ is an isomorphism ${ }^{\prime \prime} B^{0} \simeq{ }^{\prime} B^{0}$. 2-morphisms $(f, a) \stackrel{z}{\longrightarrow}(g, b)$ in Rings $_{\mathrm{cdg} 2}$ between morphisms $(f, a)$ and $(g, b)$ belonging to Rings ${ }_{\mathrm{cdg} 2}$ are arbitrary 2-morphisms from $(f, a)$ to $(g, b)$ in the 2-category of CDG-rings.

3.3. Self-consistency equations. Let $\widetilde{A}$ be a weak nonhomogeneous quadratic ring over its subring $R \subset \widetilde{A}$ with the $R$-R-subbimodule of generators $\widetilde{V} \subset \widetilde{A}$. Consider the related increasing filtration $F$ on the ring $\widetilde{A}$, as constructed in Section 3.1, and let $A=\operatorname{gr}^{F} \widetilde{A}$ be the associated graded ring. Then $A$ is a nonnegatively graded 
ring generated by its degree-one component $A_{1}=V=\widetilde{V} / R$ over the degree-zero component $A_{0}=R$.

Denote by $I \subset V \otimes_{R} V$ the kernel of the multiplication map $A_{1} \otimes_{R} A_{1} \longrightarrow A_{2}$, and consider the quadratic graded ring $\mathrm{q} A=T_{R}(V) /(I)$. Then we have a natural (adjunction) homomorphism of graded rings $\mathrm{q} A \longrightarrow A$, which is an isomorphism in the degrees $n=0,1$, and 2 , and a surjective map in the degrees $n \geq 3$. We will say that a weak nonhomogeneous quadratic ring $\widetilde{A}$ is 3 -left finitely projective if such is the quadratic graded ring $\mathrm{q} A=\mathrm{qgr}^{F} \widetilde{A}$.

Let $\widetilde{A}$ be a weak nonhomogeneous quadratic ring. In the rest of this section, we will assume that the $R$ - $R$-bimodule $V=\widetilde{V} / R=A_{1}$ is projective as a left $R$-module and the $R$-R-bimodule $F_{2} \widetilde{A} / F_{1} \widetilde{A}=A_{2}$ is flat as a left $R$-module.

Then, in particular, in the short exact sequence of $R$ - $R$-bimodules $0 \longrightarrow R \longrightarrow$ $\widetilde{V} \longrightarrow V \longrightarrow 0$ all the bimodules are projective as left $R$-modules. Therefore, this sequence splits as a short exact sequence of left $R$-modules, and we can choose a splitting $V \longrightarrow \widetilde{V}$. Let $V^{\prime} \subset \widetilde{V}$ be the image of $V$ under such a splitting map; so $V^{\prime}$ is a left (but not a right) $R$-submodule in the $R$ - $R$-bimodule $\widetilde{V} \subset \widetilde{A}$ such that $\widetilde{V}=R \oplus V^{\prime}$ as a left $R$-module. We will call $V^{\prime}$ a submodule of strict generators of $\widetilde{A}$.

In the rest of this section, we will identify $V$ with $V^{\prime}$. Let $v \in V$ and $r \in R$ be two elements. Let $r v$ and $v r \in V$ denote the elements obtained by applying to $v \in V$ the left and right action of the element $r \in R$ in the $R$-R-bimodule $V$, and let $r * v$ and $v * r \in \widetilde{V}$ denote the products of the elements $v \in V \simeq V^{\prime} \subset \widetilde{V} \subset \widetilde{A}$ and $r \in R \subset \widetilde{A}$ in the $\operatorname{ring} \widetilde{A}$. Then we have

$$
r * v=r v \quad \text { and } \quad v * r=v r+q(v, r) \in \widetilde{V},
$$

where $q(v, r) \in R$ is a certain uniquely defined element. The map $q: V \times R \longrightarrow$ $R$ is obviously biadditive, so it can be (uniquely) extended to an abelian group homomorphism $q: V \otimes_{\mathbb{Z}} R \longrightarrow R$. Since $R$ is a subring in $\widetilde{A}$, we also have $r * s=r s$ (where the left-hand side denotes the product in $\widetilde{A}$ and the right-hand side is the product in $R$ ) for any pair of elements $r, s \in R$.

Furthermore, let $i \in I$ be an element. Consider the natural surjective map $V \otimes_{\mathbb{Z}}$ $V \longrightarrow V \otimes_{R} V$ from the tensor product of two copies of $V$ over the ring of integers $\mathbb{Z}$ to their tensor product over $R$, and denote by $\widehat{I} \subset V \otimes_{\mathbb{Z}} V$ the full preimage of the subbimodule $I \subset V \otimes_{R} V$ under the map $V \otimes_{\mathbb{Z}} V \longrightarrow V \otimes_{R} V$. Let $\hat{\imath} \in \widehat{I}$ denote some preimage of the element $i \in I$ under the natural surjective map $\widehat{I} \longrightarrow I$.

The element $i \in I \subset V \otimes_{R} V$ can be presented as a finite sum of decomposable tensors, $i=\sum_{\alpha} i_{1, \alpha} \otimes_{R} i_{2, \alpha}, i_{1, \alpha}, i_{2, \alpha} \in V$. We will suppress the notation for the sum over $\alpha$, and write simply $i=i_{1} \otimes i_{2}$. Similarly, the element $\hat{\imath} \in \widehat{I} \subset V \otimes_{\mathbb{Z}} V$ can be presented as a finite sum $\hat{\imath}=\sum_{\alpha} \hat{\imath}_{1, \alpha} \otimes_{\mathbb{Z}} \hat{\imath}_{2, \alpha}$, where $\hat{\imath}_{1, \alpha}, \hat{\imath}_{2, \alpha} \in V$. Once again, we will omit the notation for the sum over $\alpha$, and write $\hat{\imath}=\hat{\imath}_{1} \otimes \hat{\imath}_{2}$. All our formulas will be biadditive in $\hat{\imath}_{1}$ and $\hat{\imath}_{2}$ (or, as may be the case, appropriately $R$-bilinear in $i_{1}$ and $i_{2}$ ), so such simplification of the notation will be harmless. 
For any two elements $u$ and $v \in V$, we identify $u$ and $v$ with their images under the embedding $V \simeq V^{\prime} \hookrightarrow \widetilde{V} \subset \widetilde{A}$ and consider their product $u * v \in \widetilde{A}$ in the ring $\widetilde{A}$. The assignment of the element $u * v$ to a pair of elements $u$ and $v$ can be uniquely extended to well-defined homomorphism of abelian groups (or left $R$-modules) $V \otimes_{\mathbb{Z}} V \longrightarrow \widetilde{A}$, which does not, generally speaking, factorize through $V \otimes_{R} V$.

In particular, for any element $i=i_{1} \otimes i_{2} \in I$ and any its preimage $\hat{\imath}=\hat{\imath}_{1} \otimes \hat{\imath}_{2} \in$ $\widehat{I}$, we consider the element $\hat{\imath}_{1} * \hat{\imath}_{2} \in \widetilde{A}$. We have $\hat{\imath}_{1}, \hat{\imath}_{2} \in V^{\prime} \subset \widetilde{V}=F_{1} \widetilde{A}$, so $\hat{\imath}_{1} * \hat{\imath}_{2} \in F_{2} \widetilde{A}$. Moreover, the image of the element $\hat{\imath}_{1} * \hat{\imath}_{2}$ under the natural surjection $F_{2} \widetilde{A} \longrightarrow F_{2} \widetilde{A} / F_{1} \widetilde{A}$ is equal to the product $i_{1} i_{2}$ computed in the associated graded $\operatorname{ring} A=\operatorname{gr}^{F} \widetilde{A}$. Now we have $i_{1} i_{2}=0$ in $F_{2} \widetilde{A} / F_{1} \widetilde{A}=A_{2}$, since the composition $I \longrightarrow V \otimes_{R} V \longrightarrow A_{2}$ vanishes by construction. Thus $\hat{\imath}_{1} * \hat{\imath}_{2} \in F_{1} \widetilde{A}=\widetilde{V} \subset \widetilde{A}$. Therefore, there exist uniquely defined elements $p(\hat{\imath}) \in V$ and $h(\hat{\imath}) \in R$ such that

$$
\hat{\imath}_{1} * \hat{\imath}_{2}=p(\hat{\imath})-h(\hat{\imath}) \in \widetilde{V} \subset \widetilde{A},
$$

where the element $p(\hat{\imath}) \in V$ is identified with its image under the splitting $V \simeq V^{\prime} \hookrightarrow$ $\widetilde{V}$ and the element $h(\hat{\imath}) \in R$ is identified with its image under the subring inclusion $R \hookrightarrow \widetilde{V} \subset \widetilde{A}$.

Furthermore, denote by $I^{(3)}$ the intersection $I \otimes_{R} V \cap V \otimes_{R} I \subset V \otimes_{R} V \otimes_{R} V$. Notice that, since the left $R$-modules $V$ and $A_{2}=\left(V \otimes_{R} V\right) / I$ are flat by assumption, the tensor products $I \otimes_{R} V$ and $V \otimes_{R} I$ are subbimodules in the triple tensor product $V \otimes_{R} V \otimes_{R} V$ (see the proofs of Propositions 1.3 and 2.1).

We are also interested in "the intersection $\widehat{I} \otimes_{\mathbb{Z}} V \cap V \otimes_{\mathbb{Z}} \widehat{I} \subset V \otimes_{\mathbb{Z}} V \otimes_{\mathbb{Z}} V$ ", but here we cannot claim that $\widehat{I} \otimes_{\mathbb{Z}} V$ and $V \otimes_{\mathbb{Z}} \widehat{I}$ are subgroups in $V \otimes_{\mathbb{Z}} V \otimes_{\mathbb{Z}} V$. So the intersection as such is not well-defined. Instead, we have two abelian group homomorphisms $\widehat{I} \otimes_{\mathbb{Z}} V \longrightarrow V \otimes_{\mathbb{Z}} V \otimes_{\mathbb{Z}} V$ and $V \otimes_{\mathbb{Z}} \widehat{I} \longrightarrow V \otimes_{\mathbb{Z}} V \otimes_{\mathbb{Z}} V$ induced by the inclusion $\widehat{I} \longrightarrow V \otimes_{\mathbb{Z}} V$. We denote by $\widehat{I}^{(3)}$ the fibered product of the abelian groups $\widehat{I} \otimes_{\mathbb{Z}} V$ and $V \otimes_{\mathbb{Z}} \widehat{I}$ over the group $V \otimes_{\mathbb{Z}} V \otimes_{\mathbb{Z}} V$.

Lemma 3.4. The natural surjections $\widehat{I} \otimes_{\mathbb{Z}} V \longrightarrow I \otimes_{R} V$, $V \otimes_{\mathbb{Z}} \widehat{I} \longrightarrow V \otimes_{R} I$, and $V \otimes_{\mathbb{Z}} V \otimes_{\mathbb{Z}} V \longrightarrow V \otimes_{R} V \otimes_{R} V$ induce a surjective map $\widehat{I}^{(3)} \longrightarrow I^{(3)}$.

Proof. Denote by $\widehat{I}_{\bar{\otimes}_{\mathbb{Z}}} V$ and $V \bar{\otimes}_{\mathbb{Z}} \widehat{I} \subset V \otimes_{\mathbb{Z}} V \otimes_{\mathbb{Z}} V$ the images of the maps $\widehat{I} \otimes_{\mathbb{Z}} V \longrightarrow$ $V \otimes_{\mathbb{Z}} V \otimes_{\mathbb{Z}} V$ and $V \otimes_{\mathbb{Z}} \widehat{I} \longrightarrow V \otimes_{\mathbb{Z}} V \otimes_{\mathbb{Z}} V$, and let $\bar{I}^{(3)} \subset V \otimes_{\mathbb{Z}} V \otimes_{\mathbb{Z}} V$ stand for the intersection of $\widehat{I} \bar{\otimes}_{\mathbb{Z}} V$ and $V \bar{\otimes}_{\mathbb{Z}} \widehat{I}$. Then there is a natural surjective map $\widehat{I}^{(3)} \longrightarrow \bar{I}^{(3)}$. The surjection $V \otimes_{\mathbb{Z}} V \otimes_{\mathbb{Z}} V \longrightarrow V \otimes_{R} V \otimes_{R} V$ restricts to a map $\bar{I}^{(3)} \longrightarrow I^{(3)}$, and the map $\widehat{I}^{(3)} \longrightarrow I^{(3)}$ is equal to the composition $\widehat{I}^{(3)} \longrightarrow \bar{I}^{(3)} \longrightarrow I^{(3)}$. It remains to prove that the map $\bar{I}^{(3)} \longrightarrow I^{(3)}$ is surjective.

We have a short exact sequence of abelian groups $0 \longrightarrow \widehat{I} \longrightarrow V \otimes_{\mathbb{Z}} V \longrightarrow A_{2} \longrightarrow$ 0 . Hence the subgroups $X_{1}=\widehat{I}_{\otimes_{\mathbb{Z}}} V$ and $X_{2}=V \bar{\otimes}_{\mathbb{Z}} \widehat{I} \subset V \otimes_{\mathbb{Z}} V \otimes_{\mathbb{Z}} V$ are the kernels of the surjective maps $V \otimes_{\mathbb{Z}} V \otimes_{\mathbb{Z}} V \longrightarrow A_{2} \otimes_{\mathbb{Z}} V$ and $V \otimes_{\mathbb{Z}} V \otimes_{\mathbb{Z}} V \longrightarrow V \otimes_{\mathbb{Z}} A_{2}$, respectively. Denote by $Y_{1}$ and $Y_{2} \subset V \otimes_{\mathbb{Z}} V \otimes_{\mathbb{Z}} V$ the kernels of the natural surjective 
maps $V \otimes_{\mathbb{Z}} V \otimes_{\mathbb{Z}} V \longrightarrow V \otimes_{R} V \otimes_{\mathbb{Z}} V$ and $V \otimes_{\mathbb{Z}} V \otimes_{\mathbb{Z}} V \longrightarrow V \otimes_{\mathbb{Z}} V \otimes_{R} V$. Then we have $Y_{1} \subset X_{1}$ and $Y_{2} \subset X_{2} \subset V \otimes_{\mathbb{Z}} V \otimes_{\mathbb{Z}} V$.

Furthermore, $Y_{1}+Y_{2}$ is the kernel of the natural surjective map $V \otimes_{\mathbb{Z}} V \otimes_{\mathbb{Z}} V \longrightarrow$ $V \otimes_{R} V \otimes_{R} V$. Similarly, $X_{1}+Y_{2}$ is the kernel of the surjective map $V \otimes_{\mathbb{Z}} V \otimes_{\mathbb{Z}} V \longrightarrow$ $A_{2} \otimes_{R} V$, and $Y_{1}+X_{2}$ is the kernel of the surjective map $V \otimes_{\mathbb{Z}} V \otimes_{\mathbb{Z}} V \longrightarrow V \otimes_{R} A_{2}$. It follows that the subbimodule $I \otimes_{R} V \subset V \otimes_{R} V \otimes_{R} V$ is the image of $X_{1} \subset V \otimes_{\mathbb{Z}} V \otimes_{\mathbb{Z}} V$ under the map $V \otimes_{\mathbb{Z}} V \otimes_{\mathbb{Z}} V \longrightarrow V \otimes_{R} V \otimes_{R} V$, and the subbimodule $V \otimes_{R} I \subset$ $V \otimes_{R} V \otimes_{R} V$ is the image of $X_{2} \subset V \otimes_{\mathbb{Z}} V \otimes_{\mathbb{Z}} V$ under the same map.

The assertion that the map $\bar{I}^{(3)} \longrightarrow I^{(3)}$ is surjective is now expressed by the distributivity equation

$$
X_{1} \cap X_{2}+\left(Y_{1}+Y_{2}\right)=\left(X_{1}+Y_{2}\right) \cap\left(Y_{1}+X_{2}\right)
$$

on subgroups in the abelian group $W=V \otimes_{\mathbb{Z}} V \otimes_{\mathbb{Z}} V$. What we have here is two filtrations $0 \subset Y_{1} \subset X_{1} \subset W$ and $0 \subset Y_{2} \subset X_{2} \subset W$ of an abelian group $W$. It remains to observe that any two filtrations of an abelian category object generate a distributive lattice of its subobjects, which is a particular case of [14, Theorem 5] or [24, Corollary 6.4 in Chapter 1].

Let $j \in I^{(3)}$ be an element and $\hat{\jmath} \in \widehat{I}^{(3)}$ be one of its preimages. The element $j \in I^{(3)} \subset V^{\otimes_{R} 3}$ can be presented as a finite sum of decomposable tensors, $j=$ $\sum_{\alpha} j_{1, \alpha} \otimes_{R} j_{2, \alpha} \otimes_{R} j_{3, \alpha}, j_{1, \alpha}, j_{2, \alpha}, j_{3, \alpha} \in V$. As above, we will suppress the notation for the sum over $\alpha$, and write simply $j=j_{1} \otimes j_{2} \otimes j_{3}$. Moreover, the image of the element $\hat{\jmath} \in \widehat{I}^{(3)}$ under the natural map $\widehat{I}^{(3)} \longrightarrow V^{\otimes_{\mathbb{Z}} 3}$ can be presented as a finite sum $\sum_{\alpha} \hat{\jmath}_{1, \alpha} \otimes_{\mathbb{Z}} \hat{\jmath}_{2, \alpha} \otimes_{\mathbb{Z}} \hat{\jmath}_{3, \alpha}$, where $\hat{\jmath}_{1, \alpha}, \hat{\jmath}_{2, \alpha}, \hat{\jmath}_{3, \alpha} \in V$. We will write simply $\hat{\jmath}=\hat{\jmath}_{1} \otimes \hat{\jmath}_{2} \otimes \hat{\jmath}_{3}$, omitting the notation for the sum over $\alpha$ and ignoring the distinction between an element of $\widehat{I}^{(3)}$ and its image in $V^{\otimes_{\mathbb{Z}}}{ }^{3}$ in this notation.

For any three elements $u, v$, and $w \in V$, we will identify $u, v$, and $w$ with their images under the embedding $V \simeq V^{\prime} \hookrightarrow \widetilde{V} \subset \widetilde{A}$ and consider the triple product $(u * v) * w=u * v * w=u *(v * w) \in \widetilde{A}$ in the $\operatorname{ring} \widetilde{A}$. In particular, for any element $\hat{\jmath} \in \widehat{I}^{(3)}$, the element $\hat{\jmath}_{1} * \hat{\jmath}_{2} * \hat{\jmath}_{3} \in \widetilde{A}$ is well-defined.

Proposition 3.5. Let $\widetilde{A}$ be a weak nonhomogeneous quadratic ring such that the left $R$-module $V=A_{1}$ is projective and the left $R$-module $A_{2}$ is flat. Suppose that a left $R$-linear splitting $V \simeq V^{\prime} \hookrightarrow \widetilde{V}$ of the surjecitve $R$-R-bimodule map $\widetilde{V} \longrightarrow \widetilde{V} / R=V$ has been chosen. Then the maps $q: V \times R \longrightarrow R, p: \widehat{I} \longrightarrow V$, and $h: \widehat{I} \longrightarrow R$ defined above in (27 28) satisfy the following self-consistency equations:

(a) $q(r v, s)=r q(v, s)$ for all $r, s \in R$ and $v \in V$;

(b) $q(v, r s)=q(v r, s)+q(v, r) s$ for all $r, s \in R$ and $v \in V$;

(c) $p\left(r \hat{\imath}_{1} \otimes \hat{\imath}_{2}\right)=\operatorname{rp}\left(\hat{\imath}_{1} \otimes \hat{\imath}_{2}\right)$ for all $r \in R$ and $\hat{\imath} \in \widehat{I}$;

(d) $h\left(r \hat{\imath}_{1} \otimes \hat{\imath}_{2}\right)=r h\left(\hat{\imath}_{1} \otimes \hat{\imath}_{2}\right)$ for all $r \in R$ and $\hat{\imath} \in \widehat{I}$;

(e) $p(u \otimes r v-u r \otimes v)=q(u, r) v$ for all $r \in R$ and $u, v \in V$;

(f) $h(u \otimes r v-u r \otimes v)=0$ for all $r \in R$ and $u, v \in V$;

(g) $p\left(\hat{\imath}_{1} \otimes \hat{\imath}_{2} r\right)=p\left(\hat{\imath}_{1} \otimes \hat{\imath}_{2}\right) r-\hat{\imath}_{1} q\left(\hat{\imath}_{2}, r\right)$ for all $r \in R$ and $\hat{\imath}=\hat{\imath}_{1} \otimes \hat{\imath}_{2} \in \widehat{I}$; 
(h) $h\left(\hat{\imath}_{1} \otimes \hat{\imath}_{2} r\right)=h\left(\hat{\imath}_{1} \otimes \hat{\imath}_{2}\right) r-q\left(p\left(\hat{\imath}_{1} \otimes \hat{\imath}_{2}\right), r\right)+q\left(\hat{\imath}_{1}, q\left(\hat{\imath}_{2}, r\right)\right)$ for all $r \in R$ and $\hat{\imath} \in \widehat{I}$

(i) $p\left(\hat{\jmath}_{1} \otimes \hat{\jmath}_{2}\right) \otimes \hat{\jmath}_{3}-\hat{\jmath}_{1} \otimes p\left(\hat{\jmath}_{2} \otimes \hat{\jmath}_{3}\right) \in \widehat{I} \subset V \otimes_{\mathbb{Z}} V$ for all $\hat{\jmath}=\hat{\jmath}_{1} \otimes \hat{\jmath}_{2} \otimes \hat{\jmath}_{3} \in \widehat{I}^{(3)}$;

(j) $p\left(p\left(\hat{\jmath}_{1} \otimes \hat{\jmath}_{2}\right) \otimes \hat{\jmath}_{3}-\hat{\jmath}_{1} \otimes p\left(\hat{\jmath}_{2} \otimes \hat{\jmath}_{3}\right)\right)=h\left(\hat{\jmath}_{1} \otimes \hat{\jmath}_{2}\right) \hat{\jmath}_{3}-\hat{\jmath}_{1} h\left(\hat{\jmath}_{2} \otimes \hat{\jmath}_{3}\right)$ for all $\hat{\jmath} \in \widehat{I}^{(3)}$;

(k) $h\left(p\left(\hat{\jmath}_{1} \otimes \hat{\jmath}_{2}\right) \otimes \hat{\jmath}_{3}-\hat{\jmath}_{1} \otimes p\left(\hat{\jmath}_{2} \otimes \hat{\jmath}_{3}\right)\right)=q\left(\hat{\jmath}_{1}, h\left(\hat{\jmath}_{2} \otimes \hat{\jmath}_{3}\right)\right)$ for all $\hat{\jmath} \in \widehat{I}^{(3)}$.

Proof. All these equations follow, in one way or another, from the associativity of multiplication in the ring $\widetilde{A}$. The specific computations proving each of the formulas are presented below one by one.

Part (a): compare $r * v * s=(r * v) * s=(r v) * s=r v s+q(r v, s)$ with $r * v * s=$ $r *(v * s)=r *(v s+q(v, s))=r v s+r q(v, s)$.

Part (b): compare $v * r * s=v *(r * s)=v *(r s)=v r s+q(v, r s)$ with $v * r * s=$ $(v * r) * s=(v r+q(v, r)) * s=v r s+q(v r, s)+q(v, r) s$.

Parts (c) and (d): notice first of all that $\sum_{\alpha} r \hat{\imath}_{1, \alpha} \otimes \hat{\imath}_{2, \alpha} \in \widehat{I}$ whenever $r \in R$ and $\sum_{\alpha} \hat{\imath}_{1, \alpha} \otimes \hat{\imath}_{2, \alpha} \in \widehat{I}$. So the left-hand sides of both the equations are well-defined. To deduce the equations, compare

$$
r * \hat{\imath}_{1} * \hat{\imath}_{2}=\left(r * \hat{\imath}_{1}\right) * \hat{\imath}_{2}=\left(r \hat{\imath}_{1}\right) * \hat{\imath}_{2}=p\left(r \hat{\imath}_{1} \otimes \hat{\imath}_{2}\right)-h\left(r \hat{\imath}_{1} \otimes \hat{\imath}_{2}\right)
$$

with

$$
r * \hat{\imath}_{1} * \hat{\imath}_{2}=r *\left(\hat{\imath}_{1} * \hat{\imath}_{2}\right)=r *\left(p\left(\hat{\imath}_{1} \otimes \hat{\imath}_{2}\right)-h\left(\hat{\imath}_{1} \otimes \hat{\imath}_{2}\right)\right)=r p\left(\hat{\imath}_{1} \otimes \hat{\imath}_{2}\right)-r h\left(\hat{\imath}_{1} \otimes \hat{\imath}_{2}\right)
$$

and equate the terms belonging to $V \simeq V^{\prime} \subset \widetilde{V}$ separately and the terms belonging to $R \subset \widetilde{V}$ separately.

Parts (e) and (f): first of all, one has $u \otimes r v-u r \otimes v \in \widehat{I}$ for all $r \in R$ and $u$, $v \in V$. So the left-hand sides of the equations are well-defined. Furthermore,

$$
\begin{aligned}
0=u *(r * v)-(u * r) * v & =u *(r v)-(u r) * v-q(u, r) * v \\
& =p(u \otimes r v-u r \otimes v)-h(u \otimes r v-u r \otimes v)-q(u, r) v
\end{aligned}
$$

and it remains to equate separately the terms belonging to $V^{\prime}$ and to $R$.

Parts (g) and (h): we have $\sum_{\alpha} \hat{\imath}_{1, \alpha} \otimes \hat{\imath}_{2, \alpha} r \in \widehat{I}$ whenever $r \in R$ and $\sum_{\alpha} \hat{\imath}_{1, \alpha} \otimes \hat{\imath}_{2, \alpha} \in \widehat{I}$; so the left-hand sides of both the equations are well-defined. Now compare

$$
\begin{aligned}
\hat{\imath}_{1} * \hat{\imath}_{2} * r=\left(\hat{\imath}_{1} * \hat{\imath}_{2}\right) * r=\left(p\left(\hat{\imath}_{1} \otimes \hat{\imath}_{2}\right)-h\left(\hat{\imath}_{1} \otimes \hat{\imath}_{2}\right)\right) * r \\
=p\left(\hat{\imath}_{1} \otimes \hat{\imath}_{2}\right) r+q\left(p\left(\hat{\imath}_{1} \otimes \hat{\imath}_{2}\right), r\right)-h\left(\hat{\imath}_{1} \otimes \hat{\imath}_{2}\right) r
\end{aligned}
$$

with

$$
\begin{aligned}
\hat{\imath}_{1} * \hat{\imath}_{2} * r=\hat{\imath}_{1} *\left(\hat{\imath}_{2} * r\right)=\hat{\imath}_{1} * & \left(\hat{\imath}_{2} r+q\left(\hat{\imath}_{2}, r\right)\right) \\
= & p\left(\hat{\imath}_{1} \otimes \hat{\imath}_{2} r\right)-h\left(\hat{\imath}_{2} \otimes \hat{\imath}_{2} r\right)+\hat{\imath}_{1} q\left(\hat{\imath}_{2}, r\right)+q\left(\hat{\imath}_{1}, q\left(\hat{\imath}_{2}, r\right)\right)
\end{aligned}
$$

and equate separately the terms belonging to $V^{\prime}$ and to $R$.

Parts (i-k): given an element $j \in \widehat{I}^{(3)}$, we have $\left(\hat{\jmath}_{1} * \hat{\jmath}_{2}\right) * \hat{\jmath}_{3}=\hat{\jmath}_{1} * \hat{\jmath}_{2} * \hat{\jmath}_{3}=\hat{\jmath}_{1} *\left(\hat{\jmath}_{2} * \hat{\jmath}_{3}\right)$ in $F_{3} \widetilde{A} \subset \widetilde{A}$. By construction, the value of this triple product in $\widetilde{A}$ only depends on the image of the element $j$ in the group $V \otimes_{\mathbb{Z}} V \otimes_{\mathbb{Z}} V$. We will compute the value 
of $\left(\hat{\jmath}_{1} * \hat{\jmath}_{2}\right) * \hat{\jmath}_{3}$ in terms of the image of $j$ in $\widehat{I} \otimes_{\mathbb{Z}} V$ and the value of $\hat{\jmath}_{1} *\left(\hat{\jmath}_{2} * \hat{\jmath}_{3}\right)$ in terms of the image of $j$ in $V \otimes_{\mathbb{Z}} \widehat{I}$, and then equate the two expresssions.

Specifically, we have

$$
\left(\hat{\jmath}_{1} * \hat{\jmath}_{2}\right) * \hat{\jmath}_{3}=p\left(\hat{\jmath}_{1} \otimes \hat{\jmath}_{2}\right) * \hat{\jmath}_{3}-h\left(\hat{\jmath}_{1} \otimes \hat{\jmath}_{2}\right) * \hat{\jmath}_{3}
$$

and

hence

$$
\hat{\jmath}_{1} *\left(\hat{\jmath}_{2} * \hat{\jmath}_{3}\right)=\hat{\jmath}_{1} * p\left(\hat{\jmath}_{2} \otimes \hat{\jmath}_{3}\right)-\hat{\jmath}_{1} * h\left(\hat{\jmath}_{2} \otimes \hat{\jmath}_{3}\right),
$$

$$
p\left(\hat{\jmath}_{1} \otimes \hat{\jmath}_{2}\right) * \hat{\jmath}_{3}-\hat{\jmath}_{1} * p\left(\hat{\jmath}_{2} \otimes \hat{\jmath}_{3}\right)=h\left(\hat{\jmath}_{1} \otimes \hat{\jmath}_{2}\right) * \hat{\jmath}_{3}-\hat{\jmath}_{1} * h\left(\hat{\jmath}_{2} \otimes \hat{\jmath}_{3}\right) .
$$

Now, first of all, the right-hand side of (29) belongs to $\widetilde{V} \subset \widetilde{A}$, hence so does the left-hand side. Both summands in the left-hand side belong to $F_{2} \widetilde{A}$. So the image of the left-hand side in $A_{2}=F_{2} \widetilde{A} / F_{1} \widetilde{A}$ has to vanish, which means that the expression $p\left(\hat{\jmath}_{1} \otimes \hat{\jmath}_{2}\right) \otimes_{R} \hat{\jmath}_{3}-\hat{\jmath}_{1} \otimes_{R} p\left(\hat{\jmath}_{2} \otimes \hat{\jmath}_{3}\right)$ belongs to $I \subset V \otimes_{R} V$. This proves part (i).

It remains to compute both sides of (29) as

$$
\begin{aligned}
& p\left(\hat{\jmath}_{1} \otimes \hat{\jmath}_{2}\right) * \hat{\jmath}_{3}-\hat{\jmath}_{1} * p\left(\hat{\jmath}_{2} \otimes \hat{\jmath}_{3}\right) \\
& \quad=p\left(p\left(\hat{\jmath}_{1} \otimes \hat{\jmath}_{2}\right) \otimes \hat{\jmath}_{3}-\hat{\jmath}_{1} \otimes p\left(\hat{\jmath}_{2} \otimes \hat{\jmath}_{3}\right)\right)-h\left(p\left(\hat{\jmath}_{1} \otimes \hat{\jmath}_{2}\right) \otimes \hat{\jmath}_{3}-\hat{\jmath}_{1} \otimes p\left(\hat{\jmath}_{2} \otimes \hat{\jmath}_{3}\right)\right)
\end{aligned}
$$

and

$$
h\left(\hat{\jmath}_{1} \otimes \hat{\jmath}_{2}\right) * \hat{\jmath}_{3}-\hat{\jmath}_{1} * h\left(\hat{\jmath}_{2} \otimes \hat{\jmath}_{3}\right)=h\left(\hat{\jmath}_{1} \otimes \hat{\jmath}_{2}\right) \hat{\jmath}_{3}-\hat{\jmath}_{1} h\left(\hat{\jmath}_{2} \otimes \hat{\jmath}_{3}\right)-q\left(\hat{\jmath}_{1}, h\left(\hat{\jmath}_{2} \otimes \hat{\jmath}_{3}\right)\right) .
$$

Comparing and equating separately the terms belonging to $V^{\prime}$ and to $R$ produces the desired formulas $(\mathrm{j}-\mathrm{k})$.

3.4. The CDG-ring corresponding to a nonhomogeneous quadratic ring. Let $R$ and $S$ be associative rings, $U$ be an $R$-S-bimodule, and $U^{\vee}=\operatorname{Hom}_{R}(U, R)$ be the dual $S$-R-bimodule. We will use the notation

$$
\langle u, f\rangle=f(u) \in R \quad \text { for any } u \in U \text { and } f \in U^{\vee} .
$$

Then the condition that $f: U \longrightarrow R$ is a left $R$-module homomorphism is expressed by the identity

$$
\langle r u, f\rangle=r\langle u, f\rangle \quad \text { for all } r \in R, u \in U \text {, and } f \in U^{\vee},
$$

while the construction of the left $S$-module structure on $U^{\vee}$ is expressed by the identity

$$
\langle u, s f\rangle=\langle u s, f\rangle \quad \text { for all } u \in U, s \in S \text {, and } f \in U^{\vee} \text {, }
$$

and the construction of the right $R$-module structure on $U^{\vee}$ is expressed by

$$
\langle u, f r\rangle=\langle u, f\rangle r \quad \text { for all } u \in U, f \in U^{\vee} \text {, and } r \in R \text {. }
$$

Furthermore, given three rings $R, S$, and $T$, an $R$-S-bimodule $U$, and an $S$-T-bimodule $V$, the construction of the natural homomorphism of $T$ - $R$-bimodules

$$
\operatorname{Hom}_{S}(V, S) \otimes_{S} \operatorname{Hom}_{R}(U, R) \longrightarrow \operatorname{Hom}_{R}\left(U \otimes_{S} V, R\right),
$$


from Lemma 1.1(a) can be expressed by the formula

$$
\langle u \otimes v, g \otimes f\rangle=\langle u\langle v, g\rangle, f\rangle=\langle u,\langle v, g\rangle f\rangle
$$

for all $u \in U, v \in V, g \in \operatorname{Hom}_{S}(V, S)$, and $f \in \operatorname{Hom}_{R}(U, R)$.

Proposition 3.6. Let $\widetilde{A}$ be a 3-left finitely projective weak nonhomogeneous quadratic ring over its subring $R \subset \widetilde{A}$ with the $R$-R-bimodule of generators $R \subset \widetilde{V} \subset \widetilde{A}$. Denote by $B$ the 3-right finitely projective quadratic graded ring quadratic dual to the 3-left finitely projective quadratic graded ring $\mathrm{q} A=\mathrm{qgr}^{F} \widetilde{A}$. Suppose that a left $R$-linear splitting $V \simeq V^{\prime} \hookrightarrow \widetilde{V}$ of the surjective $R$-R-bimodule map $\widetilde{V} \longrightarrow \widetilde{V} / R=V$ has been chosen. Then the formulas

$$
\left\langle v, d_{0}(r)\right\rangle=q(v, r)
$$

and

$$
\left\langle i, d_{1}(b)\right\rangle=\left\langle p\left(\hat{\imath}_{1} \otimes \hat{\imath}_{2}\right), b\right\rangle-q\left(\hat{\imath}_{1},\left\langle\hat{\imath}_{2}, b\right\rangle\right)
$$

for all $r \in R, \quad v \in V, i \in I$, and $b \in B^{1}$, where the maps $q$ and $p$ are given by (27 28), specify well-defined abelian group homomorphisms $d_{0}: B^{0} \longrightarrow B^{1}$ and $d_{1}: B^{1} \longrightarrow B^{2}$. Furthermore, the map $h: \widehat{I} \longrightarrow R$ descends uniquely to a welldefined left $R$-linear map $I \longrightarrow R$, providing an element $h \in \operatorname{Hom}_{R}(I, R)=B^{2}$. The maps $d_{0}$ and $d_{1}$ satisfy the equations

(a) $d_{0}(r s)=d_{0}(r) s+r d_{0}(s)$ for all $r, s \in R$;

(b) $d_{1}(r b)=d_{0}(r) b+r d_{1}(b)$ for all $r \in R, \quad b \in B^{1}$;

(c) $d_{1}(b r)=d_{1}(b) r-b d_{0}(r)$ for all $r \in R, \quad b \in B^{1}$;

(d) $d_{1}\left(d_{0}(r)\right)=h r-r h$ for all $r \in R$;

(e) $\sum_{\alpha} d_{1}\left(e_{1, \alpha}\right) e_{2, \alpha}-\sum_{\alpha} e_{1, \alpha} d_{1}\left(e_{2, \alpha}\right)=0$ in $B^{3}$ for all tensors $e=\sum_{\alpha} e_{1, \alpha} \otimes_{R}$ $e_{2, \alpha} \in B^{1} \otimes_{R} B^{1}$ such that the image of e vanishes in $B^{2}$.

The formula

$$
d_{2}\left(e_{1} e_{2}\right)=d_{1}\left(e_{1}\right) e_{2}-e_{1} d_{1}\left(e_{2}\right) \quad \text { for all } e_{1}, e_{2} \in B^{1}
$$

specifies a well-defined abelian group homomorphism $d_{2}: B^{2} \longrightarrow B^{3}$, which satisfies the equations

(f) $d_{2}\left(d_{1}(b)\right)=h b-b h$ for all $b \in B^{1}$; and

(g) $d_{2}(h)=0$.

In other words, the maps $d_{0}$ and $d_{1}$ admit a unique extension to an odd derivation $d: B \longrightarrow B$ of degree 1 , and the triple $(B, d, h)$ is a $C D G$-ring.

Proof. Recall that, by the definition of quadratic duality, we have $B^{0}=R, B^{1}=$ $\operatorname{Hom}_{R}(V, R)$, and $B^{2}=\operatorname{Hom}_{R}(I, R)$ (where $I$ is the kernel of the surjective multiplication map $A_{1} \otimes_{R} A_{1} \longrightarrow A_{2} \simeq(\mathrm{q} A)_{2}$; the latter isomorphism holds since the graded ring $A$ is generated by $A_{1}$ over $R=A_{0}$ ). The grading on the ring $B$ was denoted by lower indices in Sections 112, but we denote it by upper indices here; the convention is $B^{n}=B_{n}$ for all $n \geq 0$ (and $B^{n}=0=B_{n}$ for $n<0$ ). 
Firstly we have to check that the maps $d_{0}$ and $d_{1}$ are well-defined by the formulas (30 31). Concerning $d_{0}$, it needs to be checked that $v \longmapsto\left\langle v, d_{0}(r)\right\rangle$ is a left $R$-linear map $V \longrightarrow R$ for every $r \in R$. Indeed, we have

$$
\left\langle s v, d_{0}(r)\right\rangle=q(s v, r)=s q(v, r)=s\left\langle v, d_{0}(r)\right\rangle
$$

for all $r, s \in R$ and $v \in V$ by Proposition 3.5(a).

Concerning $d_{1}$, it needs to be checked that, for every element $b \in B^{1}$, the map $\widehat{I} \longrightarrow R$ defined by the formula $\hat{\imath} \longmapsto\left\langle p\left(\hat{\imath}_{1} \otimes \hat{\imath}_{2}\right), b\right\rangle-q\left(\hat{\imath}_{1},\left\langle\hat{\imath}_{2}, b\right\rangle\right)$ descends to a left $R$-linear map $I \longrightarrow R$. Indeed, for all $u, v \in V$ and $r \in R$ we have

$$
\begin{aligned}
\langle p(u \otimes r v-u r \otimes v), b\rangle-q(u,\langle r v, b\rangle)+q(u r,\langle v, b\rangle) & \\
=\langle q(u, r) v, b\rangle & -q(u,\langle r v, b\rangle)+q(u r,\langle v, b\rangle) \\
& =q(u, r)\langle v, b\rangle-q(u, r\langle v, b\rangle)+q(u r,\langle v, b\rangle)=0
\end{aligned}
$$

by Proposition 3.5(e) and (b), the latter of which is being applied to the elements $u \in V$ and $r,\langle v, b\rangle \in R$. Since $\widehat{I} \longrightarrow I$ is a surjective map with the kernel spanned, as an abelian group, by the elements $u \otimes r v-u r \otimes v$, it follows that our map $\widehat{I} \longrightarrow R$ descends uniquely to a map $d_{1}(b): I \longrightarrow R$. To prove that the latter map is left $R$-linear, we compute

$$
\begin{aligned}
&\left\langle r i, d_{1}(b)\right\rangle=\left\langle p\left(r \hat{\imath}_{1} \otimes \hat{\imath}_{2}\right),\right.b\rangle-q\left(r \hat{\imath}_{1},\left\langle\hat{\imath}_{2}, b\right\rangle\right) \\
&=\left\langle r p\left(\hat{\imath}_{1} \otimes \hat{\imath}_{2}\right), b\right\rangle-r q\left(\hat{\imath}_{1},\left\langle\hat{\imath}_{2}, b\right\rangle\right) \\
& \quad=r\left\langle p\left(\hat{\imath}_{1} \otimes \hat{\imath}_{2}\right), b\right\rangle-r q\left(\hat{\imath}_{1},\left\langle\hat{\imath}_{2}, b\right\rangle\right)=r\left\langle i, d_{1}(b)\right\rangle
\end{aligned}
$$

using Proposition 3.5(c) and (a).

Similarly, the map $h: \widehat{I} \longrightarrow R$ descends to a well-defined map $I \longrightarrow R$ by Proposition 3.5(f), and the latter map is left $R$-linear by Proposition 3.5(d).

Now we have to prove the equations ( $\mathrm{a}-\mathrm{g})$. Part (a): for every element $v \in V$, one has

$$
\begin{aligned}
\left\langle v, d_{0}(r s)\right\rangle=q(v, r s)=q(v r, s)+q(v, r) s=\left\langle v r, d_{0}(s)\right\rangle+\left\langle v, d_{0}(r)\right\rangle s & \\
= & \left\langle v, r d_{0}(s)\right\rangle+\left\langle v, d_{0}(r) s\right\rangle=\left\langle v, r d_{0}(s)+d_{0}(r) s\right\rangle
\end{aligned}
$$

by Proposition 3.5(b).

Part (b): for every element $i \in I$ and its preimage $\hat{\imath} \in \widehat{I}$, one has

$$
\begin{array}{r}
\left\langle i, d_{1}(r b)\right\rangle=\left\langle p\left(\hat{\imath}_{1} \otimes \hat{\imath}_{2}\right), r b\right\rangle-q\left(\hat{\imath}_{1},\left\langle\hat{\imath}_{2}, r b\right\rangle\right)=\left\langle p\left(\hat{\imath}_{1} \otimes \hat{\imath}_{2}\right) r, b\right\rangle-q\left(\hat{\imath}_{1},\left\langle\hat{\imath}_{2} r, b\right\rangle\right) \\
=\left\langle\hat{\imath}_{1} q\left(\hat{\imath}_{2}, r\right), b\right\rangle+\left\langle p\left(\hat{\imath}_{1} \otimes \hat{\imath}_{2} r\right), b\right\rangle-q\left(\hat{\imath}_{1},\left\langle\hat{\imath}_{2} r, b\right\rangle\right) \\
=\left\langle\hat{\imath}_{1}\left\langle\hat{\imath}_{2}, d_{0}(r)\right\rangle, b\right\rangle+\left\langle i r, d_{1}(b)\right\rangle=\left\langle i, d_{0}(r) b\right\rangle+\left\langle i, r d_{1}(b)\right\rangle
\end{array}
$$

by Proposition 3.5(g).

Part (c): for every element $i \in I$ and its preimage $\hat{\imath} \in \widehat{I}$, one has

$$
\begin{gathered}
\left\langle i, d_{1}(b r)\right\rangle=\left\langle p\left(\hat{\imath}_{1} \otimes \hat{\imath}_{2}\right), b r\right\rangle-q\left(\hat{\imath}_{1},\left\langle\hat{\imath}_{2}, b r\right\rangle\right)=\left\langle p\left(\hat{\imath}_{1} \otimes \hat{\imath}_{2}\right), b\right\rangle r-q\left(\hat{\imath}_{1},\left\langle\hat{\imath}_{2}, b\right\rangle r\right) \\
=\left\langle p\left(\hat{\imath}_{1} \otimes \hat{\imath}_{2}\right), b\right\rangle r-q\left(\hat{\imath}_{1},\left\langle\hat{\imath}_{2}, b\right\rangle\right) r-q\left(\hat{\imath}_{1}\left\langle\hat{\imath}_{2}, b\right\rangle, r\right)
\end{gathered}
$$




$$
=\left\langle i, d_{1}(b)\right\rangle r-\left\langle\hat{\imath}_{1}\left\langle\hat{\imath}_{2}, b\right\rangle, d_{0}(r)\right\rangle=\left\langle i, d_{1}(b) r\right\rangle-\left\langle i, b d_{0}(r)\right\rangle
$$

by Proposition 3.5(b) applied to the elements $\hat{\imath}_{1} \in V$ and $\left\langle\hat{\imath}_{2}, b\right\rangle, r \in R$.

Part (d): for every element $i \in I$ and its preimage $\hat{\imath} \in \widehat{I}$, one has

$$
\begin{aligned}
\left\langle i, d_{1}\left(d_{0}(r)\right)\right\rangle=\left\langle p\left(\hat{\imath}_{1} \otimes \hat{\imath}_{2}\right), d_{0}(r)\right\rangle-q\left(\hat{\imath}_{1},\left\langle\hat{\imath}_{2}, d_{0}(r)\right\rangle\right) & \\
=q\left(p\left(\hat{\imath}_{1} \otimes \hat{\imath}_{2}\right), r\right)-q\left(\hat{\imath}_{1}, q\left(\hat{\imath}_{2}, r\right)\right) & =h\left(\hat{\imath}_{1} \otimes \hat{\imath}_{2}\right) r-h\left(\hat{\imath}_{1} \otimes \hat{\imath}_{2} r\right) \\
& =\langle i, h\rangle r-\langle i r, h\rangle=\langle i, h r\rangle-\langle i, r h\rangle
\end{aligned}
$$

by Proposition 3.5(h).

In order to prove parts $(\mathrm{e}-\mathrm{g})$, we recall the natural isomorphism $B^{3} \simeq$ $\operatorname{Hom}_{R}\left(I^{(3)}, R\right)$, which holds for a 3-left finitely projective quadratic graded ring $\mathrm{q} A$ and its quadratic dual 3-right finitely projective quadratic graded ring $B$ according to the proof of Proposition 1.3. In view of this isomorphism, in order to verify an equation in the group $B^{3}$, it suffices to evaluate it on every element $j \in I^{(3)}$ and check that the resulting equation in $R$ holds.

Furthermore, for any tensor $e=\sum_{\alpha} e_{1, \alpha} \otimes e_{2, \alpha}=e_{1} \otimes e_{2} \in B^{1} \otimes_{R} B^{1}$, and for every element $j \in I^{(3)}$ and its preimage $\hat{\jmath} \in \widehat{I}^{(3)}$, we compute

$$
\begin{gathered}
\left\langle j, d_{1}\left(e_{1}\right) e_{2}-e_{1} d_{1}\left(e_{2}\right)\right\rangle=\left\langle j_{1}\left\langle j_{2} \otimes j_{3}, d_{1}\left(e_{1}\right)\right\rangle, e_{2}\right\rangle-\left\langle j_{1} \otimes j_{2}\left\langle j_{3}, e_{1}\right\rangle, d_{1}\left(e_{2}\right)\right\rangle \\
=\left\langle\hat{\jmath}_{1}\left\langle p\left(\hat{\jmath}_{2} \otimes \hat{\jmath}_{3}\right), e_{1}\right\rangle, e_{2}\right\rangle-\left\langle\hat{\jmath}_{1} q\left(\hat{\jmath}_{2},\left\langle\hat{\jmath}_{3}, e_{1}\right\rangle\right), e_{2}\right\rangle \\
\quad-\left\langle p\left(\hat{\jmath}_{1} \otimes \hat{\jmath}_{2}\left\langle\hat{\jmath}_{3}, e_{1}\right\rangle\right), e_{2}\right\rangle+q\left(\hat{\jmath}_{1},\left\langle\hat{\jmath}_{2}\left\langle\hat{\jmath}_{3}, e_{1}\right\rangle, e_{2}\right\rangle\right) \\
=\left\langle\hat{\jmath}_{1}\left\langle p\left(\hat{\jmath}_{2} \otimes \hat{\jmath}_{3}\right), e_{1}\right\rangle, e_{2}\right\rangle-\left\langle p\left(\hat{\jmath}_{1} \otimes \hat{\jmath}_{2}\right)\left\langle\hat{\jmath}_{3}, e_{1}\right\rangle, e_{2}\right\rangle+q\left(\hat{\jmath}_{1},\left\langle\hat{\jmath}_{2}\left\langle\hat{\jmath}_{3}, e_{1}\right\rangle, e_{2}\right\rangle\right) \\
=\left\langle\hat{\jmath}_{1} \otimes p\left(\hat{\jmath}_{2} \otimes \hat{\jmath}_{3}\right)-p\left(\hat{\jmath}_{1} \otimes \hat{\jmath}_{2}\right) \otimes \hat{\jmath}_{3}, e_{1} \otimes e_{2}\right\rangle+q\left(\hat{\jmath}_{1},\left\langle\hat{\jmath}_{2} \otimes \hat{\jmath}_{3}, e_{1} \otimes e_{2}\right\rangle\right)
\end{gathered}
$$

by Proposition 3.5 (g) applied to the tensor $\hat{\imath}_{1} \otimes \hat{\imath}_{2}=\hat{\jmath}_{1} \otimes \hat{\jmath}_{2}$ and the element $r=\left\langle\hat{\jmath}_{3}, e_{1}\right\rangle$.

Now, tensors $e \in B^{1} \otimes_{R} B^{1}$ whose image vanishes in $B^{2}$ form the $R$ - $R$-bimodule $I_{B} \subset B^{1} \otimes_{R} B^{1}$ of quadratic relations in the quadratic graded ring $B$. By Proposition 1.2, we have $I_{B}=\operatorname{Hom}_{R}\left(A_{2}, R\right)=\operatorname{Hom}_{R}\left(\left(V \otimes_{R} V\right) / I, R\right)$. To prove part (e), it remains to observe that, in view of Proposition [3.5)(i), both the summands in the final expression in (32) involve the pairing of an element of $I \subset V \otimes_{R} V$ with the element $e$. Therefore, both the summands vanish for $e \in I_{B} \subset B^{1} \otimes_{R} B^{1}$.

Part (f): choose an element $e_{1} \otimes e_{2}=\sum_{\alpha} e_{1, \alpha} \otimes e_{2, \alpha} \in B^{1} \otimes_{R} B^{1}$ whose image under the multiplication map $B^{1} \otimes_{R} B^{1} \longrightarrow B^{2}$ is equal to $d_{1}(b)$. Then for every element $j \in I^{(3)}$ and its preimage $\hat{\jmath} \in \widehat{I}^{(3)}$ we have

$$
\begin{aligned}
& \left\langle j, d_{2}\left(d_{1}(b)\right)\right\rangle=\left\langle j, d_{1}\left(e_{1}\right) e_{2}-e_{1} d_{1}\left(e_{2}\right)\right\rangle \\
& =\left\langle\hat{\jmath}_{1} \otimes p\left(\hat{\jmath}_{2} \otimes \hat{\jmath}_{3}\right)-p\left(\hat{\jmath}_{1} \otimes \hat{\jmath}_{2}\right) \otimes \hat{\jmath}_{3}, e_{1} \otimes e_{2}\right\rangle+q\left(\hat{\jmath}_{1},\left\langle\hat{\jmath}_{2} \otimes \hat{\jmath}_{3}, e_{1} \otimes e_{2}\right\rangle\right) \\
& =\left\langle\hat{\jmath}_{1} \otimes p\left(\hat{\jmath}_{2} \otimes \hat{\jmath}_{3}\right)-p\left(\hat{\jmath}_{1} \otimes \hat{\jmath}_{2}\right) \otimes \hat{\jmath}_{3}, d_{1}(b)\right\rangle+q\left(\hat{\jmath}_{1},\left\langle\hat{\jmath}_{2} \otimes \hat{\jmath}_{3}, d_{1}(b)\right\rangle\right) \\
& =\left\langle p\left(\hat{\jmath}_{1} \otimes p\left(\hat{\jmath}_{2} \otimes \hat{\jmath}_{3}\right)-p\left(\hat{\jmath}_{1} \otimes \hat{\jmath}_{2}\right) \otimes \hat{\jmath}_{3}\right), b\right\rangle \\
& -q\left(\hat{\jmath}_{1},\left\langle p\left(\hat{\jmath}_{2} \otimes \hat{\jmath}_{3}\right), b\right\rangle\right)+q\left(p\left(\hat{\jmath}_{1} \otimes \hat{\jmath}_{2}\right),\left\langle\hat{\jmath}_{3}, b\right\rangle\right) \\
& +q\left(\hat{\jmath}_{1},\left\langle p\left(\hat{\jmath}_{2} \otimes \hat{\jmath}_{3}\right), b\right\rangle\right)-q\left(\hat{\jmath}_{1}, q\left(\hat{\jmath}_{2},\left\langle\hat{\jmath}_{3}, b\right\rangle\right)\right)
\end{aligned}
$$




$$
\begin{array}{r}
=\left\langle\hat{\jmath}_{1} h\left(\hat{\jmath}_{2} \otimes \hat{\jmath}_{3}\right), b\right\rangle-\left\langle h\left(\hat{\jmath}_{1} \otimes \hat{\jmath}_{2}\right) \hat{\jmath}_{3}, b\right\rangle+q\left(p\left(\hat{\jmath}_{1} \otimes \hat{\jmath}_{2}\right),\left\langle\hat{\jmath}_{3}, b\right\rangle\right)-q\left(\hat{\jmath}_{1}, q\left(\hat{\jmath}_{2},\left\langle\hat{\jmath}_{3}, b\right\rangle\right)\right) \\
=\left\langle\hat{\jmath}_{1} h\left(\hat{\jmath}_{2} \otimes \hat{\jmath}_{3}\right), b\right\rangle-h\left(\hat{\jmath}_{1} \otimes \hat{\jmath}_{2}\right)\left\langle\hat{\jmath}_{3}, b\right\rangle+q\left(p\left(\hat{\jmath}_{1} \otimes \hat{\jmath}_{2}\right),\left\langle\hat{\jmath}_{3}, b\right\rangle\right)-q\left(\hat{\jmath}_{1}, q\left(\hat{\jmath}_{2},\left\langle\hat{\jmath}_{3}, b\right\rangle\right)\right) \\
=\left\langle\hat{\jmath}_{1} h\left(\hat{\jmath}_{2} \otimes \hat{\jmath}_{3}\right), b\right\rangle-h\left(\hat{\jmath}_{1} \otimes \hat{\jmath}_{2}\left\langle\hat{\jmath}_{3}, b\right\rangle\right) \\
=\left\langle j_{1}\left\langle j_{2} \otimes j_{3}, h\right\rangle, b\right\rangle-\left\langle j_{1} \otimes j_{2}\left\langle j_{3}, b\right\rangle, h\right\rangle=\langle j, h b-b h\rangle
\end{array}
$$

by (32), (31), and Proposition 3.5(j) and (h), the latter of which is being applied to the tensor $\hat{\imath}_{1} \otimes \hat{\imath}_{2}=\hat{\jmath}_{1} \otimes \hat{\jmath}_{2}$ and the element $r=\left\langle\hat{\jmath}_{3}, b\right\rangle$.

Part (g): choose an element $h_{1} \otimes h_{2}=\sum_{\alpha} h_{1, \alpha} \otimes h_{2, \alpha} \in B^{1} \otimes_{R} B^{1}$ whose image under the multiplication map $B^{1} \otimes_{R} B^{1} \longrightarrow B^{2}$ is equal to $h$. Then for every element $j \in I^{(3)}$ and its preimage $\hat{\jmath} \in \widehat{I}^{(3)}$ we have

$$
\begin{aligned}
& \left\langle j, d_{2}(h)\right\rangle=\left\langle j, d_{1}\left(h_{1}\right) h_{2}-h_{1} d_{1}\left(h_{2}\right)\right\rangle \\
& \quad=\left\langle\hat{\jmath}_{1} \otimes p\left(\hat{\jmath}_{2} \otimes \hat{\jmath}_{3}\right)-p\left(\hat{\jmath}_{1} \otimes \hat{\jmath}_{2}\right) \otimes \hat{\jmath}_{3}, h_{1} \otimes h_{2}\right\rangle+q\left(\hat{\jmath}_{1},\left\langle\hat{\jmath}_{2} \otimes \hat{\jmath}_{3}, h_{1} \otimes h_{2}\right\rangle\right) \\
& \quad=h\left(\hat{\jmath}_{1} \otimes p\left(\hat{\jmath}_{2} \otimes \hat{\jmath}_{3}\right)-p\left(\hat{\jmath}_{1} \otimes \hat{\jmath}_{2}\right) \otimes \hat{\jmath}_{3}\right)+q\left(\hat{\jmath}_{1}, h\left(\hat{\jmath}_{2} \otimes \hat{\jmath}_{3}\right)\right)=0
\end{aligned}
$$

by (32) and Proposition 3.5(k).

Finally, for any quadratic graded ring $B$, any pair of maps $d_{0}: B^{0} \longrightarrow B^{1}$ and $d_{1}: B^{1} \longrightarrow B^{2}$ satisfying ( $\mathrm{a}-\mathrm{c}$ ) and (e) can be extended to an odd derivation $d: B \longrightarrow$ $B$ of degree 1 in a unique way. This assertion is provable, e. g., using the next lemma (where one takes $W=B^{0} \oplus B^{1}$ ). The equations (d) and (f) imply that $d(d(x))=[h, x]$ for all $x \in B$, since $B$ is generated by $B^{1}$ over $B^{0}$.

Lemma 3.7. (a) Let $W=W_{\overline{0}} \oplus W_{\overline{1}}$ be a $\mathbb{Z} / 2 \mathbb{Z}$-graded abelian group (where $\mathbb{Z} / 2 \mathbb{Z}=$ $\{\overline{0}, \overline{1}\}$ is the group of order 2 ), and let $T_{\mathbb{Z}}(W)=\bigoplus_{n=0}^{\infty} W^{\otimes_{\mathbb{Z}} n}$ denote the free associative ring spanned by $W$. Endow $T_{\mathbb{Z}}(W)$ with the $\mathbb{Z} / 2 \mathbb{Z}$-grading induced by that of $W$. Let $d_{W}: W \longrightarrow T_{\mathbb{Z}}(W)$ be an arbitrary odd homomorphism of $\mathbb{Z} / 2 \mathbb{Z}$-graded abelian groups. Then there exists a unique odd derivation $d_{T}: T_{\mathbb{Z}}(W) \longrightarrow T_{\mathbb{Z}}(W)$ of the free ring $T_{\mathbb{Z}}(W)$ extending the map $d_{W}$ from $W \subset T_{\mathbb{Z}}(W)$.

(b) Let $L \subset T_{\mathbb{Z}}(W)$ be a $\mathbb{Z} / 2 \mathbb{Z}$-homogeneous subgroup and $K \subset T_{\mathbb{Z}}(W)$ be the twosided $\mathbb{Z} / 2 \mathbb{Z}$-homogeneous ideal generated by $L$ in $T_{\mathbb{Z}}(W)$; so $K=(L)$. Suppose that $d_{T}(L) \subset K$. Then $d_{T}(K) \subset K$, and $d_{T}$ descends to a well-defined odd derivation $d$ of the quotient ring $T_{\mathbb{Z}}(W) / K$.

Proof. Part (a): put $d_{T}\left(w_{1} \otimes \cdots \otimes w_{n}\right)=d_{W}\left(w_{1}\right) \otimes w_{2} \otimes \cdots \otimes w_{n}+(-1)^{\left|w_{1}\right|} w_{1} \otimes$ $d_{W}\left(w_{2}\right) \otimes w_{3} \otimes \cdots \otimes w_{n}+\cdots+(-1)^{\left|w_{1}\right|+\cdots+\left|w_{n-1}\right|} w_{1} \otimes w_{2} \otimes \cdots \otimes w_{n-1} \otimes d_{W}\left(w_{n}\right)$ for all $w_{i} \in W_{\left|w_{i}\right|}, \quad 1 \leq i \leq n, \quad n \geq 0$. Part (b) is obvious.

3.5. Change of strict generators. Let $\widetilde{A}$ be a weak nonhomogeneous quadratic ring over a subring $R \subset \widetilde{A}$ with the $R$ - $R$-subbimodule of generators $\widetilde{V} \subset \widetilde{A}$. Assume that the $R$-R-bimodule $V=\widetilde{V} / R$ is projective as a left $R$-module and the $R$-R-bimodule $F_{2} \widetilde{A} / F_{1} \widetilde{A}$ is flat as a left $R$-module.

Furthermore, assume that we are given two left $R$-linear splittings $V \simeq V^{\prime \prime} \subset \widetilde{V}$ and $V \simeq V^{\prime} \subset \widetilde{V}$ of the surjective $R$-R-bimodule morphism $\widetilde{V} \longrightarrow V$. Given an element $v \in V$, we will denote by $v^{\prime \prime} \in V^{\prime \prime}$ and $v^{\prime} \in V^{\prime}$ its images under the two 
splittings. Then $v \longmapsto v^{\prime \prime}-v^{\prime}$ is a left $R$-linear map $V \longrightarrow R$, which we will denote by $a$. Conversely, given a left $R$-linear splitting $V \simeq V^{\prime} \subset \widetilde{V}$ and a left $R$-linear map $a: V \longrightarrow R$, one can construct a second splitting $V \simeq V^{\prime \prime} \subset \widetilde{V}$ by the rule

$$
V^{\prime \prime}=\left\{v^{\prime}+a(v) \mid v \in V\right\} \subset \widetilde{V} .
$$

Denote the maps $q, p$, and $h$ defined by the formulas (27 28) using the splitting $V^{\prime} \subset \widetilde{V}$ by

$$
q^{\prime}: V \times R \longrightarrow R, p^{\prime}: \widehat{I} \longrightarrow V, \text { and } h^{\prime}: \widehat{I} \longrightarrow R
$$

and the similar maps constructed using the splitting $V^{\prime \prime} \subset \widetilde{V}$ by

$$
q^{\prime \prime}: V \times R \longrightarrow R, p^{\prime \prime}: \widehat{I} \longrightarrow V \text {, and } h^{\prime \prime}: \widehat{I} \longrightarrow R \text {. }
$$

Proposition 3.8. The maps $q^{\prime \prime}, p^{\prime \prime}$, and $h^{\prime \prime}$ can be obtained from the maps $q^{\prime}, p^{\prime}$, and $h^{\prime}$ and the map $a: V \longrightarrow R$ by the formulas

(a) $q^{\prime \prime}(v, r)=q^{\prime}(v, r)+a(v) r-a(v r)$ for all $r \in R$ and $v \in V$;

(b) $p^{\prime \prime}\left(\hat{\imath}_{1} \otimes \hat{\imath}_{2}\right)=p^{\prime}\left(\hat{\imath}_{1} \otimes \hat{\imath}_{2}\right)+a\left(\hat{\imath}_{1}\right) \hat{\imath}_{2}+\hat{\imath}_{1} a\left(\hat{\imath}_{2}\right)$ for all tensors $\hat{\imath}=\hat{\imath}_{1} \otimes \hat{\imath}_{2} \in \widehat{I}$;

(c) $h^{\prime \prime}\left(\hat{\imath}_{1} \otimes \hat{\imath}_{2}\right)=h^{\prime}\left(\hat{\imath}_{1} \otimes \hat{\imath}_{2}\right)+a\left(p^{\prime}\left(\hat{\imath}_{1} \otimes \hat{\imath}_{2}\right)\right)-q^{\prime}\left(\hat{\imath}_{1}, a\left(\hat{\imath}_{2}\right)\right)+a\left(\hat{\imath}_{1} a\left(\hat{\imath}_{2}\right)\right)$ for all $\hat{\imath} \in \widehat{I}$.

Proof. In our new notation, the formulas (27 28) take the form

$$
\begin{aligned}
r * v^{\prime}=(r v)^{\prime} & \text { and } \quad r * v^{\prime \prime}=(r v)^{\prime \prime}, \\
v^{\prime} * r=(v r)^{\prime}+q^{\prime}(v, r) \quad \text { and } \quad & v^{\prime \prime} * r=(v r)^{\prime \prime}+q^{\prime \prime}(v, r),
\end{aligned}
$$

and

$$
\hat{\imath}_{1}^{\prime} * \hat{\imath}_{2}^{\prime}=p^{\prime}\left(\hat{\imath}_{1} \otimes \hat{\imath}_{2}\right)^{\prime}-h^{\prime}\left(\hat{\imath}_{1} \otimes \hat{\imath}_{2}\right) \quad \text { and } \quad \hat{\imath}_{1}^{\prime \prime} * \hat{\imath}_{2}^{\prime \prime}=p^{\prime \prime}\left(\hat{\imath}_{1} \otimes \hat{\imath}_{2}\right)^{\prime \prime}-h^{\prime \prime}\left(\hat{\imath}_{1} \otimes \hat{\imath}_{2}\right)
$$

for all $r \in R, v \in V$, and $\hat{\imath} \in \widehat{I}$. Here the elements $r v, v r \in V$ correspond to the elements $(r v)^{\prime},(v r)^{\prime} \in V^{\prime}$ and $(r v)^{\prime \prime},(v r)^{\prime \prime} \in V^{\prime \prime}$. Similarly, $p^{\prime}\left(\hat{\imath}_{1} \otimes \hat{\imath}_{2}\right), p^{\prime \prime}\left(\hat{\imath}_{1} \otimes \hat{\imath}_{2}\right) \in V$, while $p^{\prime}\left(\hat{\imath}_{1} \otimes \hat{\imath}_{2}\right)^{\prime} \in V^{\prime}$ and $p^{\prime \prime}\left(\hat{\imath}_{1} \otimes \hat{\imath}_{2}\right)^{\prime \prime} \in V^{\prime \prime}$.

Part (a): one has, on the one hand,

$$
v^{\prime} * r=(v r)^{\prime}+q^{\prime}(v, r)=(v r)^{\prime \prime}-a(v r)+q^{\prime}(v, r),
$$

and on the other hand,

$$
v^{\prime} * r=\left(v^{\prime \prime}-a(v)\right) * r=(v r)^{\prime \prime}+q^{\prime \prime}(v, r)-a(v) r
$$

since $v^{\prime}=v^{\prime \prime}-a(v)$ for all $v \in V$.

Parts $(\mathrm{b}-\mathrm{c})$ : we have, on the one hand

$$
\hat{\imath}_{1}^{\prime} * \hat{\imath}_{2}^{\prime}=p^{\prime}\left(\hat{\imath}_{1} \otimes \hat{\imath}_{2}\right)^{\prime}-h^{\prime}\left(\hat{\imath}_{1} \otimes \hat{\imath}_{2}\right)=p^{\prime}\left(\hat{\imath}_{1} \otimes \hat{\imath}_{2}\right)^{\prime \prime}-a\left(p^{\prime}\left(\hat{\imath}_{1} \otimes \hat{\imath}_{2}\right)\right)-h^{\prime}\left(\hat{\imath}_{1} \otimes \hat{\imath}_{2}\right),
$$

and on the other hand,

$$
\begin{aligned}
& \begin{aligned}
\hat{\imath}_{1}^{\prime} * \hat{\imath}_{2}^{\prime}=\left(\hat{\imath}_{1}^{\prime \prime}-a\left(\hat{\imath}_{1}\right)\right) *\left(\hat{\imath}_{2}^{\prime \prime}-a\left(\hat{\imath}_{2}\right)\right) \\
=\hat{\imath}_{1}^{\prime \prime} * \hat{\imath}_{2}^{\prime \prime}-a\left(\hat{\imath}_{1}\right) * \hat{\imath}_{2}^{\prime \prime}-\hat{\imath}_{1}^{\prime \prime} * a\left(\hat{\imath}_{2}\right)+a\left(\hat{\imath}_{1}\right) * a\left(\hat{\imath}_{2}\right)
\end{aligned} \\
& =p^{\prime \prime}\left(\hat{\imath}_{1} \otimes \hat{\imath}_{2}\right)^{\prime \prime}-h^{\prime \prime}\left(\hat{\imath}_{1} \otimes \hat{\imath}_{2}\right)-\left(a\left(\hat{\imath}_{1}\right) \hat{\imath}_{2}\right)^{\prime \prime}-\left(\hat{\imath}_{1} a\left(\hat{\imath}_{2}\right)\right)^{\prime \prime}-q^{\prime \prime}\left(\hat{\imath}_{1}, a\left(\hat{\imath}_{2}\right)\right)+a\left(\hat{\imath}_{1}\right) a\left(\hat{\imath}_{2}\right) .
\end{aligned}
$$


Comparing (34) with (35) and equating separately the terms belonging to $V^{\prime \prime} \subset \widetilde{V}$ and to $R \subset \widetilde{V}$, we obtain part (b) as well as the equation

$$
h^{\prime \prime}\left(\hat{\imath}_{1} \otimes \hat{\imath}_{2}\right)=h^{\prime}\left(\hat{\imath}_{1} \otimes \hat{\imath}_{2}\right)+a\left(p^{\prime}\left(\hat{\imath}_{1} \otimes \hat{\imath}_{2}\right)\right)-q^{\prime \prime}\left(\hat{\imath}_{1}, a\left(\hat{\imath}_{2}\right)\right)+a\left(\hat{\imath}_{1}\right) a\left(\hat{\imath}_{2}\right) .
$$

In order to deduce part (c), it remains to take into account the equation

$$
-q^{\prime \prime}\left(\hat{\imath}_{1}, a\left(\hat{\imath}_{2}\right)\right)+a\left(\hat{\imath}_{1}\right) a\left(\hat{\imath}_{2}\right)=-q^{\prime}\left(\hat{\imath}_{1}, a\left(\hat{\imath}_{2}\right)\right)+a\left(\hat{\imath}_{1} a\left(\hat{\imath}_{2}\right)\right)
$$

obtained by substituting $v=\hat{\imath}_{1}$ and $r=a\left(\hat{\imath}_{2}\right)$ into part (a).

Proposition 3.9. Let $\widetilde{A}$ be a 3-left finitely projective weak nonhomogeneous quadratic ring over its subring $R \subset \widetilde{A}$ with the $R$-R-bimodule of generators $R \subset \widetilde{V} \subset \widetilde{A}$. Let $V \simeq V^{\prime} \hookrightarrow \widetilde{V}$ and $V \simeq V^{\prime \prime} \hookrightarrow \widetilde{V}$ be two left $R$-linear splittings of the surjective $R$-R-bimodule map $\widetilde{V} \longrightarrow \widetilde{V} / R=V$. Denote by ${ }^{\prime} B=\left(B, d^{\prime}, h^{\prime}\right)$ and ${ }^{\prime \prime} B=\left(B, d^{\prime \prime}, h^{\prime \prime}\right)$ the two related $C D G$-ring structures on the 3 -right finitely projective graded ring $B$ quadratic dual to $\mathrm{q} A$, as constructed in Proposition 3.6. Let a $\in \operatorname{Hom}_{R}(V, R)=B^{1}$ be the element for which the two splittings $V^{\prime} \subset \widetilde{V}$ and $V^{\prime \prime} \subset \widetilde{V}$ are related by the rule $V^{\prime}=\left\{v^{\prime} \mid v \in V\right\}$ and $V^{\prime \prime}=\left\{v^{\prime \prime} \mid v \in V\right\}$ with $v^{\prime}, v^{\prime \prime} \longmapsto v$ under the map $\widetilde{V} \longrightarrow V$ and $v^{\prime \prime}=v^{\prime}+a(v)$ (33). Then the equations

(a) $d_{0}^{\prime \prime}(r)=d_{0}^{\prime}(r)+$ ar - ra for all $r \in R$;

(b) $d_{1}^{\prime \prime}(b)=d_{1}^{\prime}(b)+a b+b a$ for all $b \in B^{1}$; and

(c) $h^{\prime \prime}=h^{\prime}+d^{\prime}(a)+a^{2}$

hold in $B$, showing that (id, a): "B $\longrightarrow$ 'B is a CDG-ring isomorphism.

Proof. In our new notation, the formulas (30 31) take the form

$$
\begin{gathered}
\left\langle v, d_{0}^{\prime}(r)\right\rangle=q^{\prime}(v, r) \quad \text { and } \quad\left\langle v, d_{0}^{\prime \prime}(r)\right\rangle=q^{\prime \prime}(v, r), \\
\left\langle i, d_{1}^{\prime}(b)\right\rangle=\left\langle p^{\prime}\left(\hat{\imath}_{1} \otimes \hat{\imath}_{2}\right), b\right\rangle-q^{\prime}\left(\hat{\imath}_{1},\left\langle\hat{\imath}_{2}, b\right\rangle\right), \\
\left\langle i, d_{1}^{\prime \prime}(b)\right\rangle=\left\langle p^{\prime \prime}\left(\hat{\imath}_{1} \otimes \hat{\imath}_{2}\right), b\right\rangle-q^{\prime \prime}\left(\hat{\imath}_{1},\left\langle\hat{\imath}_{2}, b\right\rangle\right) .
\end{gathered}
$$

Part (a): for every element $v \in V$, one has

$$
\begin{aligned}
\left\langle v, d_{0}^{\prime \prime}(r)\right\rangle=q^{\prime \prime}(v, r) & =q^{\prime}(v, r)+a(v) r-a(v r) \\
& =\left\langle v, d_{1}^{\prime}(r)\right\rangle+\langle v, a\rangle r-\langle v r, a\rangle=\left\langle v, d_{1}^{\prime}(r)\right\rangle+\langle v, a r\rangle-\langle v, r a\rangle
\end{aligned}
$$

by Proposition 3.8(a).

Part (b): for every element $i \in I$ and its preimage $\hat{\imath} \in \widehat{I}$, one has

$$
\begin{gathered}
\left\langle i, d_{1}^{\prime \prime}(b)\right\rangle=\left\langle p^{\prime \prime}\left(\hat{\imath}_{1} \otimes \hat{\imath}_{2}\right), b\right\rangle-q^{\prime \prime}\left(\hat{\imath}_{1},\left\langle\hat{\imath}_{2}, b\right\rangle\right) \\
=\left\langle p^{\prime}\left(\hat{\imath}_{1} \otimes \hat{\imath}_{2}\right), b\right\rangle+\left\langle a\left(\hat{\imath}_{1}\right) \hat{\imath}_{2}, b\right\rangle+\left\langle\hat{\imath}_{1} a\left(\hat{\imath}_{2}\right), b\right\rangle \\
-q^{\prime}\left(\hat{\imath}_{1},\left\langle\hat{\imath}_{2}, b\right\rangle\right)-a\left(\hat{\imath}_{1}\right)\left\langle\hat{\imath}_{2}, b\right\rangle+a\left(\hat{\imath}_{1}\left\langle\hat{\imath}_{2}, b\right\rangle\right) \\
=\left\langle i, d_{1}^{\prime}(b)\right\rangle+\left\langle\hat{\imath}_{1}\left\langle\hat{\imath}_{2}, a\right\rangle, b\right\rangle+\left\langle\hat{\imath}_{1}\left\langle\hat{\imath}_{2}, b\right\rangle, a\right\rangle=\left\langle i, d_{1}^{\prime}(b)\right\rangle+\langle i, a b\rangle+\langle i, b a\rangle
\end{gathered}
$$

by Proposition 3.8(b) and (a), the latter of which is being applied to the elements $v=\hat{\imath}_{1}$ and $r=\left\langle\hat{\imath}_{2}, b\right\rangle$. 
Part (c): for every element $i \in I$ and its preimage $\hat{\imath} \in \widehat{I}$, one has

$$
\begin{aligned}
\left\langle i, h^{\prime \prime}\right\rangle=\left\langle i, h^{\prime}\right\rangle+\left\langle p^{\prime}\left(\hat{\imath}_{1} \otimes \hat{\imath}_{2}\right), a\right\rangle-q^{\prime}\left(\hat{\imath}_{1},\left\langle\hat{\imath}_{2}, a\right\rangle\right)+\left\langle\hat{\imath}_{1}\left\langle\hat{\imath}_{2}, a\right\rangle, a\right\rangle & \\
& =\left\langle i, h^{\prime}\right\rangle+\left\langle i, d_{1}^{\prime}(a)\right\rangle+\left\langle i, a^{2}\right\rangle
\end{aligned}
$$

by Proposition 3.8 (c).

Finally, the equations (a) and (b) imply that $d^{\prime \prime}(x)=d^{\prime}(x)+[a, x]$ for all $x \in B$, since $B$ is generated by $B^{1}$ over $B^{0}=R$.

3.6. The nonhomogeneous quadratic duality functor. Let $R$ be an associative ring. We denote by $R$-rings fil $_{\text {il }}$ the category of filtered rings $(\widetilde{A}, F)$ with increasing filtrations $F$ and the filtration component $F_{0} \widetilde{A}$ identified with $R$.

So the objects of $R$-rings fil $_{\text {il }}$ are associative rings $\widetilde{A}$ endowed with a filtration $0=$ $F_{-1} \widetilde{A} \subset F_{0} \widetilde{A} \subset F_{1} \widetilde{A} \subset F_{2} \widetilde{A} \subset \cdots$ such that $\widetilde{A}=\bigcup_{n=0}^{\infty} F_{n} \widetilde{A}$, the filtration $F$ is compatible with the multiplication in $\widetilde{A}$, and an associative ring isomorphism $R \simeq F_{0} \widetilde{A}$ has been chosen. Morphisms $\left({ }^{\prime} \widetilde{A}, F\right) \longrightarrow\left({ }^{\prime \prime} \widetilde{A}, F\right)$ in $R$-rings fil $_{\text {fil }}$ are ring homomorphisms $f:{ }^{\prime} \widetilde{A} \longrightarrow{ }^{\prime \prime} \widetilde{A}$ such that $f\left(F_{n}{ }^{\prime} \widetilde{A}\right) \subset F_{n}{ }^{\prime \prime} \widetilde{A}$ for all $n \geq 0$ and the ring homomorphism $F_{0} f: F_{0}{ }^{\prime} \widetilde{A} \longrightarrow F_{0}{ }^{\prime \prime} \widetilde{A}$ forms a commutative triangle diagram with the fixed isomorphisms $R \simeq F_{0}{ }^{\prime} \widetilde{A}$ and $R \simeq F_{0}{ }^{\prime \prime} \widetilde{A}$.

The category of 3 -left finitely projective weak nonhomogeneous quadratic rings over $R$, denoted by $R$-rings wnla $_{\text {, }}$, is defined as the full subcategory in $R$-rings fil $_{\text {il }}$ whose objects are the 3-left finitely projective weak nonhomogeneous quadratic rings $R \subset$ $\widetilde{V} \subset \widetilde{A}$ endowed with the filtration $F$ generated by $F_{1} \widetilde{A}=\widetilde{V}$ over $F_{0} \widetilde{A}=R$. In other words, this means that a morphism $\left({ }^{\prime} \widetilde{A},{ }^{\prime} \widetilde{V}\right) \longrightarrow\left({ }^{\prime \prime} \widetilde{A},{ }^{\prime \prime} \widetilde{V}\right)$ in $R$-rings wnlq $_{\text {is a }}$ ring homomorphism $f:{ }^{\prime} \widetilde{A} \longrightarrow{ }^{\prime \prime} \widetilde{A}$ forming a commutative triangle diagram with the inclusions $R \simeq F_{0}{ }^{\prime} \widetilde{A} \hookrightarrow{ }^{\prime} \widetilde{A}$ and $R \simeq F_{0}{ }^{\prime \prime} \widetilde{A} \hookrightarrow{ }^{\prime \prime} \widetilde{A}$ and satisfying the condition that $f\left({ }^{\prime} \widetilde{V}\right) \subset{ }^{\prime \prime} \widetilde{V}$ (cf. the discussion in Section 3.1) .

Furthermore, the category of 3-right finitely projective quadratic CDG-rings over $R$, denoted by $R$-rings cdg,rq $_{\text {,r }}$, is the full subcategory in the category $R$-rings ${ }_{\mathrm{cdg}}$ (as defined in Section 3.2) consisting of all the CDG-rings $(B, d, h)$ whose underlying nonnegatively graded ring $B$ is 3-right finitely projective quadratic over $R$.

Theorem 3.10. The constructions of Propositions 3.6 and 3.9 define a fully faithful contravariant functor

$$
\left(R \text {-rings }_{\text {wnlq }}\right)^{\text {op }} \longrightarrow R \text {-rings } \text { cdg, }, \mathrm{rq}
$$

from the category of 3-left finitely projective weak nonhomogeneous quadratic rings to the category of 3-right finitely projective quadratic CDG-rings over $R$.

Proof. Let $R$-rings $\mathrm{s}_{\mathrm{wnlq}}^{\mathrm{sg}}$ the category whose objects are 3-left finitely projective weak nonhomogeneous quadratic rings $R \subset \widetilde{V} \subset \widetilde{A}$ with a chosen submodule of strict generators $V^{\prime} \subset \widetilde{V}$. So $V^{\prime}$ is a left $R$-submodule in $\widetilde{V}$ such that $\widetilde{V}=R \oplus V^{\prime}$ as a left $R$-module. Morphisms $\left({ }^{\prime} \widetilde{A},{ }^{\prime} \widetilde{V},{ }^{\prime} V\right) \longrightarrow\left({ }^{\prime \prime} \widetilde{A},{ }^{\prime \prime} \widetilde{V},{ }^{\prime \prime} V\right)$ in $R$-rings ${ }_{\text {wnlq }}^{\text {sg }}$ are the same as morphisms $\left({ }^{\prime} \widetilde{A},{ }^{\prime} \widetilde{V}\right) \longrightarrow\left({ }^{\prime \prime} \widetilde{A},{ }^{\prime \prime} \widetilde{V}\right)$ in $R$-rings ${ }_{\text {wnlq }}^{\text {sg }}$; so a morphism in $R$-rings ${ }_{\text {wnlq }}^{\text {sg }}$ has 
to take ${ }^{\prime} \widetilde{V}$ into $" \widetilde{V}$, but it does not need to respect the chosen submodules of strict generators ${ }^{\prime} V \subset{ }^{\prime} \widetilde{V}$ and ${ }^{\prime \prime} V \subset{ }^{\prime \prime} \widetilde{V}$ in any way. Then the functor $R$-rings sulq $\longrightarrow$

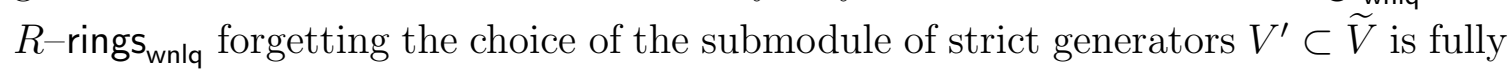
faithful and surjective on objects; so it is an equivalence of categories.

Furthermore, let $R$-rings $\mathrm{s}_{\text {wnlq }}^{\mathrm{sgsm}}$ be the subcategory in $R$-rings $\mathrm{s}_{\text {wnlq }}^{\mathrm{sg}}$ whose objects are all the objects of $R$-rings ${ }_{\text {wnlq }}^{\text {sg }}$ and whose morphisms are the morphisms $f:\left({ }^{\prime} \widetilde{A},{ }^{\prime} \widetilde{V},{ }^{\prime} V\right) \longrightarrow$ (" $\left.\widetilde{A},{ }^{\prime \prime} \widetilde{V},{ }^{\prime \prime} V\right)$ in $R$-rings swlq such that $f\left({ }^{\prime} V\right) \subset{ }^{\text {sg }} V$. The category $R-$ rings $_{\text {wnlq }}^{\text {sg }}$ can be called the category of 3-left finitely projective weak nonhomogeneous quadratic rings with strict generators chosen, while the category $R$-rings $\mathrm{s}_{\mathrm{wnlq}}^{\mathrm{sgsm}}$ is the category of 3-left finitely projective weak nonhomogeneous quadratic rings with strict generators and strict morphisms.

Finally, let $R$-rings sm denote the subcategory in $R$-rings ${ }_{c d g}$ whose objects are all the objects of $R$-rings cdg $_{\text {and }}$ and wose morphisms are the strict morphisms only, i. e., all morphisms of the form $(g, 0):\left({ }^{\prime \prime} B, d^{\prime \prime}, h^{\prime \prime}\right) \longrightarrow\left({ }^{\prime} B, d^{\prime}, h^{\prime}\right)$ in $R$-rings ${ }_{\mathrm{cdg}}$. We denote by $R$-rings $\mathrm{s}_{\mathrm{cdg}, \mathrm{rq}}^{\mathrm{sm}}$ the intersection $R$-rings $\mathrm{sdg}_{\mathrm{cdg}}^{\mathrm{sm}} \cap R$-rings ${ }_{\mathrm{cdg}, \mathrm{rq}} \subset R$-rings $\mathrm{s}_{\mathrm{cdg}}$, that is, the category of 3-right finitely projective quadratic CDG-rings over $R$ and strict morphisms between them.

Then the construction of Proposition 3.6 assigns an object $(B, d, h) \in R$-rings ${ }_{\mathrm{cdg}, \mathrm{rq}}$ to every object $\left(\widetilde{A}, \widetilde{V}, V^{\prime}\right) \in R$-rings wnlq $_{\text {sg }}$ in a natural way. Given a morphism $f:\left({ }^{\prime} \widetilde{A},{ }^{\prime} V,{ }^{\prime} V\right) \longrightarrow\left({ }^{\prime \prime} \widetilde{A},{ }^{\prime \prime} \widetilde{V},{ }^{\prime \prime} V\right)$ in $R$-rings so such that $f\left({ }^{\prime} V\right) \subset{ }^{\prime \prime} V$, we consider the induced morphism of 3-left finitely projective quadratic graded rings $\operatorname{qgr}^{F} f: \operatorname{qgr}^{F}{ }^{\prime} \widetilde{A} \longrightarrow \mathrm{qgr}^{F}{ }^{\prime \prime} \widetilde{A}$. According to Propositions [1.2 and 1.3, the morphism $\mathrm{qgr}^{F} f$ induces a morphism in the opposite direction between the quadratic dual 3-right finitely projective quadratic graded rings, $g:{ }^{\prime \prime} B \longrightarrow{ }^{\prime} B$.

Let $\left({ }^{\prime} B, d^{\prime}, h^{\prime}\right)$ and $\left({ }^{\prime \prime} B, d^{\prime \prime}, h^{\prime \prime}\right)$ denote the 3 -right finitely projective quadratic CDG-rings assigned to the 3-left finitely projective weak nonhomogeneous quadratic rings $\left({ }^{\prime} \widetilde{A},{ }^{\prime} \widetilde{V}\right)$ and $\left(" \widetilde{A},{ }^{\prime \prime} \widetilde{V}\right)$ with the submodules of strict generators ${ }^{\prime} V \subset{ }^{\prime} \widetilde{V}$ and " $V \subset{ }^{\prime \prime} \widetilde{V}$ by the construction of Proposition 3.6. Assigning the morphism $(g, 0):\left({ }^{\prime \prime} B, d^{\prime \prime}, h^{\prime \prime}\right) \longrightarrow\left({ }^{\prime} B, d^{\prime}, h^{\prime}\right)$ to the morphism $f:\left({ }^{\prime} \widetilde{A},{ }^{\prime} \widetilde{V},{ }^{\prime} V\right) \longrightarrow\left({ }^{\prime \prime} \widetilde{A},{ }^{\prime \prime} \widetilde{V},{ }^{\prime \prime} V\right)$, we obtain a contravariant functor

$$
\left(R-\text {-rings }_{\text {wnlq }}^{\mathrm{sgsm}}\right)^{\text {op }} \longrightarrow R \text { - } \text { rings }_{\mathrm{cdg}, \mathrm{rq}}^{\mathrm{sm}} \text {. }
$$

We still have to check that the functor (37) is well-defined, i. e., that $(g, 0)$ : $\left({ }^{\prime \prime} B, d^{\prime \prime}, h^{\prime \prime}\right) \longrightarrow\left({ }^{\prime} B, d^{\prime}, h^{\prime}\right)$ is indeed a morphism of CDG-rings. Simultaneously we will see that the functor (37) is fully faithful.

Indeed, specifying a morphism $f:\left({ }^{\prime} \widetilde{A},{ }^{\prime} \widetilde{V},{ }^{\prime} V\right) \longrightarrow\left({ }^{\prime \prime} \widetilde{A},{ }^{\prime \prime} \widetilde{V},{ }^{\prime \prime} V\right)$ in $R$-rings ${ }_{\text {wnlq }}^{\text {sgsm }}$ means specifying an $R$ - $R$-bimodule map $f_{1}:{ }^{\prime} \widetilde{V} / R \longrightarrow{ }^{\prime \prime} \widetilde{V} / R$ which, interpreted as a map ${ }^{\prime} V \longrightarrow{ }^{\prime \prime} V$ and taken together with the identity map $R \longrightarrow R$, extends (necessarily uniquely) to a ring homomorphism ' $\widetilde{A} \longrightarrow{ }^{\prime \prime} \widetilde{A}$. The latter condition is equivalent to the following two: 
(i) the map $\tilde{f}_{1}=\operatorname{id}_{R} \oplus f_{1}:{ }^{\prime \prime} \widetilde{V}=R \oplus^{\prime \prime} V \longrightarrow R \oplus^{\prime} V={ }^{\prime} \widetilde{V}$ agrees with the right $R$-module structures on " $\widetilde{V}$ and ' $\widetilde{V}$;

(ii) the tensor ring homomorphism $T_{R}\left(\tilde{f}_{1}\right): T_{R}\left({ }^{\prime} \widetilde{V}\right) \longrightarrow T_{R}\left({ }^{\prime \prime} \widetilde{V}\right)$ induced by the map $\tilde{f}_{1}:{ }^{\prime} \widetilde{V} \longrightarrow{ }^{\prime \prime} \widetilde{V}$ takes the $R$ - $R$-subbimodule $\widetilde{I} \widetilde{A} \subset R \oplus^{\prime} \widetilde{V} \oplus^{\prime} \widetilde{V}^{\otimes_{R}}{ }^{2}$ of nonhomogeneous quadratic relations in the ring ' $\widetilde{A}$ into the $R$ - $R$-subbimodule $\widetilde{I}_{\prime \prime} \subset R \oplus^{\prime \prime} \widetilde{V} \oplus^{\prime \prime} \widetilde{V}^{\otimes_{R} 2}$ of nonhomogeneous quadratic relations in the ring " $\widetilde{A}$ (see Section 3.1 for the notation). by

Denote the maps defined by the formulas (27-28) for $\left({ }^{\prime} \widetilde{A},{ }^{\prime} \widetilde{V},{ }^{\prime} V\right)$ and $\left({ }^{\prime \prime} \widetilde{A},{ }^{\prime \prime} \widetilde{V},{ }^{\prime \prime} V\right)$

$$
\begin{gathered}
q^{\prime}:{ }^{\prime} V \times R \longrightarrow R, \quad p^{\prime}:{ }^{\prime} \widehat{I} \longrightarrow{ }^{\prime} V, \quad h^{\prime}:{ }^{\top} \widehat{I} \longrightarrow R, \\
q^{\prime \prime}:{ }^{\prime \prime} V \times R \longrightarrow R, \quad p^{\prime \prime}:{ }^{\prime \prime} \widehat{I} \longrightarrow{ }^{\prime \prime} V, \quad h^{\prime \prime}:{ }^{\prime \prime} \widehat{I} \longrightarrow R,
\end{gathered}
$$

where the notation ${ }^{\prime} V={ }^{\prime} \widetilde{V} / R$ and ${ }^{\prime \prime} V={ }^{\prime \prime} \widetilde{V} / R$ is presumed, while ${ }^{\prime} \widehat{I} \subset{ }^{\prime} V \otimes_{\mathbb{Z}}{ }^{\prime} V$ and " $\widehat{I} \subset{ }^{\prime \prime} V \otimes_{\mathbb{Z}}{ }^{\prime \prime} V$ are the full preimages of the $R$ - $R$-subbimodules ${ }^{\prime} I \subset{ }^{\prime} V \otimes_{R}{ }^{\prime} V$ and " $I \subset{ }^{\prime \prime} V \otimes_{R}{ }^{\prime \prime} V$ of quadratic relations in the graded rings $' A=\operatorname{gr}^{F}, \widetilde{A}$ and $" A=\operatorname{gr}^{F}{ }^{\prime \prime} \widetilde{A}$, respectively. Then condition (i) is equivalent to the equation

$$
q^{\prime}(v, r)=q^{\prime \prime}\left(f_{1}(v), r\right) \quad \text { for all } v \in{ }^{\prime} V, r \in R .
$$

Assuming (i) or (38), condition (ii) is equivalent to the combination of the inclusion

$$
\left(f_{1} \otimes f_{1}\right)\left({ }^{\prime} I\right) \subset{ }^{\prime \prime} I
$$

with the equations

$$
\begin{gathered}
f_{1}\left(p^{\prime}\left(\hat{\imath}_{1} \otimes \hat{\imath}_{2}\right)\right)=p^{\prime \prime}\left(f_{1}\left(\hat{\imath}_{1}\right) \otimes f_{1}\left(\hat{\imath}_{2}\right)\right) \quad \text { for all } \hat{\imath}_{1} \otimes \hat{\imath}_{2} \in{ }^{\prime} \widehat{I} \\
h^{\prime}\left(i_{1}^{\prime} \otimes i_{2}^{\prime}\right)=h^{\prime \prime}\left(f_{1}\left(i_{1}\right) \otimes f_{1}\left(i_{2}\right)\right) \quad \text { for all } i_{1} \otimes i_{2} \in \in^{\prime} I .
\end{gathered}
$$

Finally, the inclusion (39) holds if and only if the $R$ - $R$-bimodule morphism $g_{1}=$ $\operatorname{Hom}_{R}\left(f_{1}, R\right):{ }^{\prime \prime} B^{1}=\operatorname{Hom}_{R}\left({ }^{\prime \prime} V, R\right) \longrightarrow \operatorname{Hom}_{R}\left({ }^{\prime} V, R\right)={ }^{\prime} B^{1}$ together with the identity map " $B^{0}=R \longrightarrow R={ }^{\prime} B^{0}$ can be extended to a graded ring homomorphism $g:{ }^{\prime \prime} B \longrightarrow{ }^{\prime} B$. Assuming (39) or (equivalently) the existence of $g=\left(g_{n}\right)_{n=0}^{\infty}$, the equations (38) and (40) are equivalent to the equations

$$
\begin{gathered}
d_{0}^{\prime}(r)=g_{1}\left(d_{0}^{\prime \prime}(r)\right) \quad \text { for all } r \in R, \\
d_{1}^{\prime}\left(g_{1}(b)\right)=g_{2}\left(d_{1}^{\prime \prime}(b)\right) \quad \text { for all } b \in{ }^{\prime \prime} B^{1}, \\
h^{\prime}=g_{2}\left(h^{\prime \prime}\right),
\end{gathered}
$$

which mean that $(g, 0):\left({ }^{\prime \prime} B, d^{\prime \prime}, h^{\prime \prime}\right) \longrightarrow\left({ }^{\prime} B, d^{\prime}, h^{\prime}\right)$ is a CDG-ring morphism.

Conversely, given a strict morphism $(g, 0):\left({ }^{\prime \prime} B, d^{\prime \prime}, h^{\prime \prime}\right) \longrightarrow\left({ }^{\prime} B, d^{\prime}, h^{\prime}\right)$ between 3-right finitely projective quadratic CDG-rings coming from 3-left finitely projective nonhomogeneous quadratic rings $\left({ }^{\prime} \widetilde{A},{ }^{\prime} V,{ }^{\prime} V\right)$ and $\left({ }^{\prime \prime} \widetilde{A},{ }^{\prime \prime} \widetilde{V},{ }^{\prime \prime} V\right)$, we put $f_{1}=$ $\operatorname{Hom}_{R^{\mathrm{op}}}\left(g_{1}, R\right):{ }^{\prime} V=\operatorname{Hom}_{R^{\text {op }}}\left({ }^{\prime} B^{1}, R\right) \longrightarrow \operatorname{Hom}_{R^{\mathrm{op}}}\left({ }^{\prime \prime} B^{1}, R\right)={ }^{\prime \prime} V$ and extend the map $f_{1}$ together with the identity map ${ }^{\prime} \widetilde{A} \supset R \longrightarrow R \subset$ " $\widetilde{A}$ to a ring homomorphism $f:{ }^{\prime} \widetilde{A} \longrightarrow " \widetilde{A}$. This can be done, since the combination of conditions (i) and (ii) 
is equivalent to the existence of a (necessarily unique) graded ring homomorphism $g:{ }^{\prime \prime} B \longrightarrow{ }^{\prime} B$ extending the given map $g_{1}:{ }^{\prime \prime} B^{1} \longrightarrow{ }^{\prime} B^{1}$ together with the identity map " $B^{0}=R \longrightarrow R={ }^{\prime} B^{0}$ and satisfying the equations (41).

Now we will construct a fully faithful functor

$$
\left(R-\text { rings }_{\text {wnlq }}^{\mathrm{sg}^{\mathrm{sg}}}\right)^{\text {op }} \longrightarrow R \text {-rings } \mathrm{cdg}_{\mathrm{cd}, \mathrm{rq}}
$$

extending the functor (37) to nonstrict morphisms. For a 3-left finitely projective weak nonhomogeneous quadratic ring $(\widetilde{A}, \widetilde{V}) \in R$-rings wnlq $_{\text {and }}$ any two choices of a submodule of strict generators $V^{\prime}, V^{\prime \prime} \subset \widetilde{V}$, we have two objects $\left(\widetilde{A}, \widetilde{V}, V^{\prime}\right)$ and $\left(\widetilde{A}, \widetilde{V}, V^{\prime \prime}\right) \in R$-rings sunlq connected by an isomorphism $\left(\widetilde{A}, \widetilde{V}, V^{\prime}\right) \longrightarrow\left(\widetilde{A}, \widetilde{V}, V^{\prime \prime}\right)$ corresponding to the identity map id: $\widetilde{A} \longrightarrow \widetilde{A}$. Let us call such isomorphisms in $R$-rings $\mathrm{s}_{\mathrm{wnlq}}^{\mathrm{sg}}$ the change-of-strict-generators isomorphisms.

Let $f:\left({ }^{\prime} \widetilde{A},{ }^{\prime},{ }^{\prime} V\right) \longrightarrow\left({ }^{\prime \prime} \widetilde{A},{ }^{\prime \prime} \widetilde{V},{ }^{\prime \prime} V\right)$ be an arbitrary morphism in $R$-rings ${ }_{\text {wnlq }}^{\text {sg }}$. Denote by ${ }^{\prime} V^{\prime} \subset{ }^{\prime} \widetilde{V}$ the full preimage of the left $R$-submodule ${ }^{\prime \prime} V \subset{ }^{\prime \prime} \widetilde{V}$ under the $R$ - $R$-bimodule morphism $F_{1} f:{ }^{\prime} \widetilde{V} \longrightarrow{ }^{\prime \prime} \widetilde{V}$. Then one has ${ }^{\prime} \widetilde{V}=R \oplus^{\prime} V^{\prime}$; so ${ }^{\prime} V^{\prime} \subset{ }^{\prime} \widetilde{V}$ is another choice of a submodule of strict generators in ' $\widetilde{A}$, alternative to ${ }^{\prime} V \subset{ }^{\prime} \widetilde{V}$. Any morphism $f:\left({ }^{\prime} \widetilde{A},{ }^{\prime} \widetilde{V},{ }^{\prime} V\right) \longrightarrow\left({ }^{\prime \prime} \widetilde{A},{ }^{\prime \prime} \widetilde{V},{ }^{\prime \prime} V\right)$ in $R$-rings sunlq decomposes uniquely into a change-of-strict-generators isomorphism $\left({ }^{\prime} \widetilde{A},{ }^{\prime} \widetilde{V},{ }^{\prime} V\right) \longrightarrow\left({ }^{\prime} \widetilde{A},{ }^{\prime} \widetilde{V},{ }^{\prime} V^{\prime}\right)$ followed by a strict morphism $\left({ }^{\widetilde{A}},{ }^{\prime} \widetilde{V},{ }^{\prime} V^{\prime}\right) \longrightarrow\left({ }^{\prime \prime} \widetilde{A},{ }^{\prime \prime} \widetilde{V},{ }^{\prime \prime} V\right)$.

The construction of Proposition 3.9 assigns a change-of-connection isomorphism in $R$-rings ${ }_{\text {cdg,rq }}$ to every change-of-strict-generators isomorphism in $R$-rings $\mathrm{s}_{\text {wnlq }}^{\mathrm{sg}}$. Decomposing any morphism in $R$-rings sg wnlq $^{\mathrm{sg}}$ into a change-of-strict-generators isomorphism followed by a strict morphism, one extends the functor (37) to a contravariant functor (42). We omit further details, which are straightforward.

We still have to show that the functor (42) is fully faithful. It is clear from the construction of Proposition 3.9 that this functor functor restricts to a fully faithful functor from the subcategory of change-of-strict-generators isomorphisms in $R$-rings $\mathrm{s}_{\mathrm{wnlq}}^{\mathrm{sg}}$ to the subcategory of change-of-connection isomorphisms in $R$-rings ${ }_{\mathrm{cdg}, \mathrm{rq}}$. Since the functor (37) is fully faithful as well, it follows that so is the functor (42).

In order to construct the desired fully faithful functor (36), it remains to choose any quasi-inverse functor to the category equivalence $R$-rings $\mathrm{s}_{\text {wnlq }}^{\mathrm{sg}} \longrightarrow R$-rings wnlq $_{\text {and }}$ compose it with the fully faithful functor (42).

Remark 3.11. The counterexample in [25, Section 3.4] shows that the fully faithful contravariant functor in Theorem 3.10 is not an anti-equivalence of categories (even when $R=k$ is the ground field). We will see below in Section 4.6 that this functor becomes an anti-equivalence when restricted to the full subcategories of, respectively, left and right finitely projective Koszul rings in $R$-rings wnlq $_{\text {and }} R$-rings, cdg,rq $_{\text {. }}$

3.7. Nonhomogeneous duality 2-functor. We define the 2-category of filtered rings Rings $_{\mathrm{fil} 2}$ as follows. The objects of Rings $_{\mathrm{fil} 2}$ are associative rings $\tilde{A}$ endowed with an exhastive increasing filtration $0=F_{-1} \widetilde{A} \subset F_{0} \widetilde{A} \subset F_{1} \widetilde{A} \subset F_{2} \widetilde{A} \subset \cdots$ compatible 
with the multiplication on $\widetilde{A}$. Morphisms $\left({ }^{\prime} \widetilde{A}, F\right) \longrightarrow\left({ }^{\prime \prime} \widetilde{A}, F\right)$ in Rings fil2 $_{\text {are }}$ ring homomorphisms $f:{ }^{\prime} \widetilde{A} \longrightarrow{ }^{\prime \prime} \widetilde{A}$ such that $f\left(F_{n}{ }^{\prime} \widetilde{A}\right) \subset F_{n}{ }^{\prime \prime} \widetilde{A}$ for all $n \geq 0$ and the map $F_{0} f: F_{0}{ }^{\prime} \widetilde{A} \longrightarrow F_{0}{ }^{\prime \prime} \widetilde{A}$ is an isomorphism. 2-morphisms $f \stackrel{z}{\longrightarrow} g$ between a pair of parallel morphisms $f, g:\left({ }^{\prime} \widetilde{A}, F\right) \longrightarrow\left({ }^{\prime \prime} \widetilde{A}, F\right)$ are invertible elements $z \in F_{0}{ }^{\prime \prime} \widetilde{A}$ such that $g(c)=z f(c) z^{-1}$ for all $c \in{ }^{\prime} \widetilde{A}$.

The vertical composition of two 2-morphisms $f^{\prime} \stackrel{w}{\longrightarrow} f^{\prime \prime} \stackrel{z}{\longrightarrow} f^{\prime \prime \prime}$ is the 2-morphism $f^{\prime} \stackrel{z w}{\longrightarrow} f^{\prime \prime \prime}$. The identity 2-morphism is the 2-morphism $f \stackrel{1}{\longrightarrow} f$. The horizontal composition of two 2-morphisms $g^{\prime} \stackrel{w}{\longrightarrow} g^{\prime \prime}:(\widetilde{A}, F) \longrightarrow(\widetilde{B}, F)$ and $f^{\prime} \stackrel{z}{\longrightarrow} f^{\prime \prime}:(\widetilde{B}, F) \longrightarrow$ $(\widetilde{C}, F)$ is the 2-morphism $f^{\prime} g^{\prime} \stackrel{z \circ w}{\longrightarrow} f^{\prime \prime} g^{\prime \prime}:(\widetilde{A}, F) \longrightarrow(\widetilde{C}, F)$ with the element $z \circ w=z f^{\prime}(w)=f^{\prime \prime}(w) z$.

All the 2-morphisms of filtered rings are invertible. If $f:\left({ }^{\prime} \widetilde{A}, F\right) \longrightarrow\left({ }^{\prime \prime} \widetilde{A}, F\right)$ is a morphism of filtered rings and $z \in F_{0}{ }^{\prime \prime} \widetilde{A}$ is an invertible element, then $g: c \longmapsto$ $z f(c) z^{-1}$ is also a morphism of filtered rings $g:\left({ }^{\prime} \widetilde{A}, F\right) \longrightarrow\left({ }^{\prime \prime} \widetilde{A}, F\right)$. The morphisms $f$ and $g$ are connected by the 2-isomorphism $f \stackrel{z}{\longrightarrow} g$.

The condition about the map $F_{0} f: F_{0}^{\prime} \widetilde{A} \longrightarrow F_{0}{ }^{\prime \prime} \widetilde{A}$ being an isomorphism could be harmlessly dropped from the above definition (which makes sense without this condition just as well), but we need it for the purposes of the next definition. The 2-category of 3-left finitely projective weak nonhomogeneous quadratic rings, denoted by Rings wnlq 2 , is defined as the following 2-subcategory in Rings fil $2_{2}$. The objects of Rings $_{\text {wnlq2 }}$ are the 3-left finitely projective weak nonhomogeneous quadratic rings $R \subset$ $\widetilde{V} \subset \widetilde{A}$ with the filtration $F$ generated by $F_{1} \widetilde{A}=\widetilde{V}$ over $F_{0} \widetilde{A}=R$. All morphisms in Rings $_{\text {fil } 2}$ between objects of Rings wnlq2 $_{2}$ are morphisms in Rings wnlq2 , and all 2-morphisms in Rings fil 2 between morphisms of Rings wnlq $2_{2}$ are 2-morphisms in Rings wnlq $2_{2}$.

Furthermore, the 2-category of 3-right finitely projective quadratic CDG-rings, denoted by Rings $s_{c d g 2, r q}$, is the following 2-subcategory in the 2-category Rings $s_{c d g 2}$ (as defined in Section [3.2). The objects of Rings ${ }_{\text {cdg2, rq }}$ are all the CDG-rings $(B, d, h)$ whose underlying nonnegatively graded ring $B$ is 3-right finitely projective quadratic (over its degree-zero component $B^{0}$ ). All morphisms in Rings ${ }_{\mathrm{cdg} 2}$ between objects of Rings $_{c d g 2, \text { rq }}$ are morphisms in Rings $s_{c d g 2, \text { rq }}$, and all 2-morphisms in Rings ${ }_{\mathrm{cdg} 2}$ between morphisms of Rings $_{c d g 2, r q}$ are 2-morphisms in Rings ${ }_{c d g 2, r q}$.

Lemma 3.12. Let $R \subset \widetilde{V} \subset \widetilde{A}$ be a 3-left finitely projective weak nonhomogeneous quadratic ring, and let ${ }^{\prime} V \subset \widetilde{V}$ be a submodule of strict generators of $\widetilde{A}$. Let $(B, d, h)$ be the 3-right finitely projective quadratic $C D G$-ring corresponding to $\left(\widetilde{A}, \widetilde{V},{ }^{\prime} V\right)$ under the construction of Proposition [3.6. Let $z \in R$ be an invertible element. Consider the conjugation morphism $f_{z^{-1}}: \widetilde{A} \longrightarrow \widetilde{A}$ taking any element $c \in \widetilde{A}$ to the element $f_{z^{-1}}(c)=z^{-1} c z$. Then the $C D G$-ring morphism $(B, d, h) \longrightarrow(B, d, h)$ corresponding to the morphism $f_{z^{-1}}$ under the duality functor of Theorem 3.10 is equal to

$$
\left(g_{z}, a_{z}\right):(B, d, h) \longrightarrow(B, d, h),
$$

where $g_{z}: B \longrightarrow B$ is the conjugation map taking any element $b \in B$ to the element $g_{z}(b)=z b z^{-1}$, and the element $a_{z} \in B^{1}$ is given by the formula $a_{z}=-d(z) z^{-1}$. 
Proof. Strictly speaking, the assertion of the lemma does not literally make sense as stated, and we need to make it more precise before proving it. The problem is that $f_{z^{-1}}: \widetilde{A} \longrightarrow \widetilde{A}$ is not a morphism in the category $R$-rings wnlq or $R$-rings fil $_{\text {fil }}$, as defined in Section 3.6, because it does not restrict to the identity map $\widetilde{A} \supset R \longrightarrow R \subset \widetilde{A}$, but rather to the map $R \longrightarrow R$ of conjugation with $z^{-1}$. For the same reason, $g_{z}: B \longrightarrow B$ is not a morphism in $R$-rings ${ }_{\mathrm{gr}}$, and consequently $\left(g_{z}, a_{z}\right):(B, d, h)$

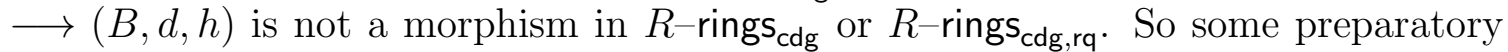
work is needed before the functor (36) could be applied to the map $f_{z^{-1}}$.

Denote by $t_{z}: R \longrightarrow R$ the conjugation map $r \longmapsto z r z^{-1}, r \in R$. Let $\left(\widetilde{A}^{(z)}, \widetilde{V}^{(z)}\right)$ denote the following 3-left finitely projective weak nonhomogeneous quadratic ring over $R$. As an associative ring, $\widetilde{A}^{(z)}$ coincides with $\widetilde{A}$. Denoting by $\iota: R \longrightarrow \widetilde{A}$ the embedding of the ring $R$ as a subring of the $\operatorname{ring} \widetilde{A}$, the map $\iota^{(z)}=\iota t_{z}: R \longrightarrow$ $\widetilde{A}=\widetilde{A}^{(z)}$ makes $R$ a subring of $\widetilde{A}^{(z)}$. For any element $c \in \widetilde{A}$, we will denote by $c^{(z)} \in \widetilde{A}^{(z)}$ the corresponding element of the $\operatorname{ring} \widetilde{A}^{(z)}$. So in particular, we have $\iota^{(z)}(r)=\left(z \iota(r) z^{-1}\right)^{(z)}$ for all $r \in R$. Furthermore, as a subgroup of $\widetilde{A}^{(z)}=\widetilde{A}$, the group $\widetilde{V}^{(z)}$ coincides with $\widetilde{V}$. Then the map $f_{z^{-1}}: \widetilde{A}^{(z)} \longrightarrow \widetilde{A}$ taking an element $c^{(z)} \in \widetilde{A}^{(z)}$ to the element $z^{-1} c z \in \widetilde{A}$ is a morphism in the category $R$-rings wnlq. $_{\text {. }}$.

Let $\left(B^{(z)}, d^{(z)}, h^{(z)}\right)$ denote the following 3-right finitely projective quadratic CDG-ring over $R$. As a graded associative ring, $B^{(z)}$ coincides with $B$; and both the differential $d^{(z)}: B^{(z)} \longrightarrow B^{(z)}$ and the curvature element $h \in B^{(z), 2}$ coincide with the differential $d$ and the curvature element $h$ in $B$. However, denoting by $\iota: R \longrightarrow B^{0}$ the identification of the ring $R$ with the degree-zero component of the graded ring $B$, the identification of the ring $R$ with the degree-zero component of the graded $\operatorname{ring} B^{(z)}$ is provided by the map $\iota^{(z)}=\iota t_{z}: R \longrightarrow B^{(z), 0}=B^{0}$.

For any element $b \in B$, we denote by $b^{(z)} \in B^{(z)}$ the corresponding element of the ring $B^{(z)}$. So, in particular, we have $d^{(z)}\left(b^{(z)}\right)=(d(b))^{(z)}$ for all $b \in B$, and our notation for the element $h^{(z)}$ is consistent: $h^{(z)} \in B^{(z), 2}$ is the element corresponding to $h \in B^{2}$. Then the map $g_{z}: B \longrightarrow B^{(z)}$ taking an element $b \in B$ to the element $\left(z b z^{-1}\right)^{(z)} \in B^{(z)}$ is a morphism in the category $R$-rings gr $_{\text {. Furthermore, }}$ let $a_{z}^{(z)} \in B^{(z), 1}$ be the element corresponding to the element $a_{z}=-d(z) z^{-1} \in B^{1}$ under the identity isomorphism $B=B^{(z)}$. Then the pair $\left(g_{z}, a_{z}^{(z)}\right)$ is a morphism of CDG-rings $(B, d, h) \longrightarrow\left(B^{(z)}, d^{(z)}, h^{(z)}\right)$, as one can readily check. Therefore, it is also a morphism in the category $R$-rings ${ }_{\mathrm{cdg}}$ and in the category $R$-rings cdg,rq $_{\text {. }}$.

Now the promised precise formulation of the lemma claims that the duality functor (36) takes the morphism $f_{z^{-1}}: \widetilde{A}^{(z)} \longrightarrow \widetilde{A}$ in the category $R$-rings wnlq to the morphism $\left(g_{z}, a_{z}^{(z)}\right):(B, d, h) \longrightarrow\left(B^{(z)}, d^{(z)}, h^{(z)}\right)$ in the category $R$-rings cdg,rq .

To be even more precise, we need to establish first that the functor (36) takes the object $\widetilde{A}^{(z)} \in R$-rings wnlq to the object $\left(B^{(z)}, d^{(z)}, h^{(z)}\right) \in R$-rings cdg,rq $_{\text {. }}$ Put $A^{(z)}=\operatorname{gr}^{F} \widetilde{A}^{(z)}$; then the identity isomorphism of rings $A_{0}^{(z)}=B^{(z), 0}$ commuting with 
the identifications $A_{0}^{(z)} \simeq R \simeq B^{(z), 0}$ together with the $A_{0}^{(z)}-A_{0}^{(z)}$-bimodule isomorphism $\operatorname{Hom}_{A_{0}^{(z)}}\left(A_{1}^{(z)}, A_{0}^{(z)}\right)=B^{(z), 1}$ allow to consider $B^{(z)}$ as the quadratic dual ring to $A^{(z)}$. Let the subgroup ${ }^{\prime} V^{(z)} \subset \widetilde{V}^{(z)}$ coincide with the subgroup ${ }^{\prime} V \subset \widetilde{V}$; then the construction of Proposition 3.6 takes the 3-left finitely projective weak nonhomogeneous quadratic ring $\left(\widetilde{A}^{(z)}, \widetilde{V}^{(z)}\right)$ with the submodule of strict generators ${ }^{\prime} V^{(z)} \subset \widetilde{V}^{(z)}$ to the 3-right finitely projective quadratic CDG-ring $\left(B^{(z)}, d^{(z)}, h^{(z)}\right)$.

At last, we can perform the computation proving the lemma. We have to check that the functor (42) takes the morphism $f_{z^{-1}}:\left(\widetilde{A}^{(z)}, \widetilde{V}^{(z)},{ }^{\prime} V^{(z)}\right) \longrightarrow\left(\widetilde{A}, \widetilde{V},{ }^{\prime} V\right)$ to the morphism $\left(g_{z}, a_{z}^{(z)}\right):(B, d, h) \longrightarrow\left(B^{(z)}, d^{(z)}, h^{(z)}\right)$.

Set ${ }^{\prime \prime} V^{(z)}=z^{\prime} V^{(z)} z^{-1} \subset \widetilde{V}^{(z)}$. Then our morphism $f_{z^{-1}}:\left(\widetilde{A}^{(z)}, \widetilde{V}^{(z)},{ }^{\prime} V^{(z)}\right) \longrightarrow$ $\left(\widetilde{A}, \widetilde{V},{ }^{\prime} V\right)$ decomposes into a change-of-strict-generators morphism $\left(\widetilde{A}^{(z)}, \widetilde{V}^{(z)},{ }^{\prime} V^{(z)}\right)$ $\longrightarrow\left(\widetilde{A}^{(z)}, \widetilde{V}^{(z)},{ }^{\prime \prime} V^{(z)}\right)$, which acts by the identity map on the underlying associative ring $\widetilde{A}^{(z)}$, followed by the strict morphism $f_{z^{-1}}:\left(\widetilde{A}^{(z)}, \widetilde{V}^{(z)},{ }^{\prime \prime} V^{(z)}\right) \longrightarrow\left(\widetilde{A}, \widetilde{V},{ }^{\prime} V\right)$. Denote by $\left(B^{(z)}, d_{(z)}, h_{(z)}\right)$ the CDG-ring assigned to the 3-left finitely projective weak nonhomogeneous quadratic ring $\left(\widetilde{A}^{(z)}, \widetilde{V}^{(z)}\right)$ with the submodule of strict generators " $V^{(z)} \subset \widetilde{V}^{(z)}$ by the construction of Proposition 3.6.

Let $q^{\prime}: V^{(z)} \times R \longrightarrow R$ be the map defined by the formula (27) using the splitting ${ }^{\prime} V^{(z)} \subset \widetilde{V}^{(z)}$ of the bimodule of generators of the 3-left finitely projective weak nonhomogeneous quadratic ring $\widetilde{A}^{(z)}$. Denoting by $u^{\prime} \in{ }^{\prime} V^{(z)}$ and $u^{\prime \prime} \in{ }^{\prime \prime} V^{(z)}$ the elements corresponding to an element $u=v^{(z)} \in V^{(z)}=\widetilde{V}^{(z)} / R$, we have

$$
u^{\prime \prime}=z *\left(z^{-1} u z\right)^{\prime} * z^{-1}=(u z)^{\prime} * z^{-1}=u^{\prime}+q^{\prime}\left(u z, z^{-1}\right) .
$$

Furthermore, by Proposition 3.5 (b),

$$
0=q^{\prime}(u, 1)=q^{\prime}\left(u, z z^{-1}\right)=q^{\prime}\left(u z, z^{-1}\right)+q^{\prime}(u, z) z^{-1},
$$

hence

$$
u^{\prime \prime}=u^{\prime}-q^{\prime}(u, z) z^{-1}=u^{\prime}-\left\langle u, d_{0}(z)\right\rangle z^{-1}=u^{\prime}-\left\langle u, d_{0}(z) z^{-1}\right\rangle=u^{\prime}+a_{z}^{(z)}(u) .
$$

By Proposition 3.9, it follows that (id, $\left.a_{z}^{(z)}\right):\left(B^{(z)}, d_{(z)}, h_{(z)}\right) \longrightarrow\left(B^{(z)}, d^{(z)}, h^{(z)}\right)$ is a change-of-connection morphism of CDG-rings over $R$. Hence one can compute that $d_{(z)}\left(z b^{(z)} z^{-1}\right)=z d^{(z)}\left(b^{(z)}\right) z^{-1}=z(d(b))^{(z)} z^{-1}$ for all $b \in B$, and $h_{(z)}=z h^{(z)} z^{-1}$. By construction, (id, $\left.a_{z}^{(z)}\right):\left(B^{(z)}, d_{(z)}, h_{(z)}\right) \longrightarrow\left(B^{(z)}, d^{(z)}, h^{(z)}\right)$ is the change-ofconnection morphism of CDG-rings over $R$ assigned to the change-of-strict-generators morphism $\left(\widetilde{A}^{(z)}, \widetilde{V}^{(z)},{ }^{\prime} V^{(z)}\right) \longrightarrow\left(\widetilde{A}^{(z)}, \widetilde{V}^{(z)},{ }^{\prime \prime} V^{(z)}\right)$ by the functor (42).

Finally, we need to show that the functor (37) takes the strict morphism $f_{z^{-1}}:\left(\widetilde{A}^{(z)}, \widetilde{V}^{(z)},{ }^{\prime \prime} V^{(z)}\right) \longrightarrow\left(\widetilde{A}, \widetilde{V},{ }^{\prime} V\right)$ to the strict morphism $\left(g_{z}, 0\right):(B, d, h) \longrightarrow$ $\left(B^{(z)}, d_{(z)}, h_{(z)}\right)$. For this purpose, it suffices to check that the morphism $\bar{f}_{z^{-1}}=$ $\operatorname{gr}^{F} f_{z^{-1}}: A^{(z)}=\operatorname{gr}^{F} \widetilde{A}^{(z)} \longrightarrow \operatorname{gr}^{F} \widetilde{A}=A$ corresponds to the morphism $g_{z}: B \longrightarrow B^{(z)}$ under the homogeneous quadratic duality of Propositions 1.2 and 1.3. All we need to do is to observe that

$$
\left\langle v^{(z)}, g_{z}(b)\right\rangle=\left\langle v^{(z)}, z b^{(z)} z^{-1}\right\rangle=z\left\langle z^{-1} v^{(z)} z, b^{(z)}\right\rangle z^{-1}
$$




$$
=t_{z}\left(\left\langle z^{-1} v^{(z)} z, b^{(z)}\right\rangle\right)=\left\langle z^{-1} v z, b\right\rangle=\left\langle\bar{f}_{z^{-1}}\left(v^{(z)}\right), b\right\rangle
$$

for all $v^{(z)} \in V^{(z)}=A_{1}^{(z)}$ and $b \in B^{1}$.

It remains to compute the image of the composition of our morphisms in the category $R$-rings $\mathrm{s}_{\mathrm{wnlq}}^{\mathrm{sg}}$

$$
\left(\widetilde{A}^{(z)}, \widetilde{V}^{(z)},{ }^{\prime} V^{(z)}\right) \longrightarrow\left(\widetilde{A}^{(z)}, \widetilde{V}^{(z)},{ }^{\prime \prime} V^{(z)}\right) \stackrel{f_{z-1}}{\longrightarrow}\left(\widetilde{A}, \widetilde{V},{ }^{\prime} V\right)
$$

under the functor (42). This is equal, by construction, to the composition of morphisms of CDG-rings

$$
\left(\mathrm{id}, a_{z}^{(z)}\right) \circ\left(g_{z}, 0\right)=\left(g_{z}, a_{z}^{(z)}\right),
$$

as desired.

In the context of the functor (44) below instead of the functor (36), the assertion of the lemma becomes literally true as stated, without the additional discussion in the first half of the above proof.

Theorem 3.13. The constructions of Theorem 3.10 and Lemma 3.12 define a fully faithful strict contravariant 2-functor

$$
\left(\text { Rings }_{\text {wnlq } 2}\right)^{\text {op }} \longrightarrow \text { Rings }_{\mathrm{cdg} 2, \mathrm{rq}}
$$

from the 2-category of 3-left finitely projective weak nonhomogeneous quadratic rings to the 2-category of 3-right finitely projective quadratic CDG-rings.

Proof. The 2-functor (43) is fully faithful in the strict sense: for any two objects $\left({ }^{\prime} \widetilde{A},{ }^{\prime}\right)$ and $\left({ }^{\prime \prime} \widetilde{A},{ }^{\prime \prime} \widetilde{V}\right) \in$ Rings $_{\text {wnlq2 }}$ and the corresponding CDG-rings $\left({ }^{\prime} B, d^{\prime}, h^{\prime}\right)$ and $\left(" B, d^{\prime \prime}, h^{\prime \prime}\right) \in$ Rings $_{\text {cdg2,rq }}$, the 2-functor (43) induces a bijection between morphisms $\left({ }^{\prime} \widetilde{A},{ }^{\prime} \widetilde{V}\right) \longrightarrow\left({ }^{\prime \prime} \widetilde{A},{ }^{\prime \prime} \widetilde{V}\right)$ in Rings wnlq2 and morphisms $\left({ }^{\prime \prime} B, d^{\prime \prime}, h^{\prime \prime}\right) \longrightarrow$ $\left({ }^{\prime} B, d^{\prime}, h^{\prime}\right)$ in Rings ${ }_{c d g 2, r q}$. Furthermore, for any pair of parallel morphisms $f^{\prime}$, $f^{\prime \prime}:\left({ }^{\prime} \widetilde{A},{ }^{\prime} \widetilde{V}\right) \longrightarrow\left({ }^{\prime \prime} \widetilde{A},{ }^{\prime \prime} \widetilde{V}\right)$ in Rings wnlq2 and the corresponding pair of parallel morphisms $g^{\prime}, g^{\prime \prime}:\left({ }^{\prime \prime} B, d^{\prime \prime}, h^{\prime \prime}\right) \longrightarrow\left({ }^{\prime} B, d^{\prime}, h^{\prime}\right)$ in Rings ${ }_{\mathrm{cdg} 2, \text { rq }}$, the 2-functor (43) induces a bijection between 2-morphisms $f^{\prime} \stackrel{z}{\longrightarrow} f^{\prime \prime}$ in Rings wnlq2 and 2-morphisms $g^{\prime \prime} \stackrel{w}{\longrightarrow} g^{\prime}$ in Rings $_{\text {cdg2 } 2 \text { rq }}$.

To construct the desired 2-functor, denote by Rings wnlq $\subset$ Rings $_{\text {wnlq } 2}$ the category whose objects are the objects of the 2-category Rings wnlq2 $_{2}$ and whose morphisms are the morphisms of the 2-category Rings wnlq2 $_{2}$ (but there are no 2-morphisms in Rings $\left._{\text {wnlq }}\right)$. Similarly, denote by Rings $s_{c d g, r q}$ the category whose objects are the objects of the 2-category Rings cdg2,rq $_{\text {rq }}$ and whose morphisms are the morphisms of the 2-category Rings ${ }_{\mathrm{cdg} 2, \mathrm{rq}}$ (but there are no 2-morphisms in Rings ${ }_{\mathrm{cdg}, \mathrm{rq}}$ ). Then essentially the same construction that was used to define the functor (36) in Theorem 3.10 provides a fully faithful contravariant functor

$$
\left(\text { Rings }_{\text {wnlq }}\right)^{\text {op }} \longrightarrow \text { Rings }_{\text {cdg, }, r q} \text {. }
$$

In order to extend the functor (44) to a 2-functor (43), we notice that, in the notation of the first paragraph of this proof, for every 2-morphism $f^{\prime} \stackrel{z}{\longrightarrow} f^{\prime \prime}$ in Rings $_{\text {wnlq } 2}$, both the morphisms $F_{0} f^{\prime}: F_{0}{ }^{\prime} \widetilde{A} \longrightarrow F_{0}{ }^{\prime \prime} \widetilde{A}$ and $F_{0} f^{\prime \prime}: F_{0}{ }^{\prime} \widetilde{A} \longrightarrow F_{0}{ }^{\prime \prime} \widetilde{A}$ 
are ring isomorphisms and the preimages of the element $z \in F_{0}{ }^{\prime \prime} \widetilde{A}$ under these two isomorphisms coincide, $\left(F_{0} f^{\prime}\right)^{-1}(z)=\left(F_{0} f^{\prime \prime}\right)^{-1}(z)$, because the element $z$ is preserved by the conjugation with $z$. Similarly, for any 2-morphism $g^{\prime \prime} \stackrel{w}{\longrightarrow} g^{\prime}$ in Rings ${ }_{c d g 2, \text { rq }}$, both the morphisms $g_{0}^{\prime}:{ }^{\prime \prime} B^{0} \longrightarrow{ }^{\prime} B^{0}$ and $g_{0}^{\prime \prime}:{ }^{\prime \prime} B^{0} \longrightarrow{ }^{\prime} B^{0}$ are ring isomorphisms and the preimages of the element $w \in{ }^{\prime} B^{0}$ under these two isomorphisms coincide, $g_{0}^{\prime-1}(w)=g_{0}^{\prime \prime-1}(w)$. By construction, we have ${ }^{\prime} B^{0}=F_{0}{ }^{\prime} \widetilde{A}$ and ${ }^{\prime \prime} B^{0}=F_{0}{ }^{\prime \prime} \widetilde{A}$. The maps $F_{0} f^{\prime}: F_{0}{ }^{\prime} \widetilde{A} \longrightarrow F_{0}{ }^{\prime \prime} \widetilde{A}$ and $g_{0}^{\prime}:{ }^{\prime \prime} B^{0} \longrightarrow{ }^{\prime} B^{0}$ are mutually inverse under this identification, and so are the two maps $F_{0} f^{\prime \prime}: F_{0}{ }^{\prime} \widetilde{A} \longrightarrow F_{0}{ }^{\prime \prime} \widetilde{A}$ and $g_{0}^{\prime \prime}:{ }^{\prime \prime} B^{0} \longrightarrow{ }^{\prime} B^{0}$, that is $g_{0}^{\prime}=\left(F_{0} f^{\prime}\right)^{-1}$ and $g_{0}^{\prime \prime}=\left(F_{0} f^{\prime \prime}\right)^{-1}$.

We assign a 2-morphism $g^{\prime \prime} \stackrel{w}{\longrightarrow} g^{\prime}$ in Rings cdg2,rq $_{\text {, }}$ to a 2-morphism $f^{\prime} \stackrel{z}{\longrightarrow} f^{\prime \prime}$ in Rings $_{\text {wnlq2 }}$ if $g_{0}^{\prime}(z)=w=g_{0}^{\prime \prime}(z)$, or equivalently, if $\left(F_{0} f^{\prime}\right)(w)=z=\left(F_{0} f^{\prime \prime}\right)(w)$. It only needs to be checked that $g^{\prime \prime} \stackrel{w}{\longrightarrow} g^{\prime}$ is a 2-morphism in Rings cdg2,rq $_{\text {, }}$ if and only if $f^{\prime} \stackrel{z}{\longrightarrow} f^{\prime \prime}$ is a 2 -morphism in Rings wnlq2 . Then the compatibility with the vertical and horizontal compositions of 2-morphisms will be clear from the construction of such compositions in the beginning of this section and in Section 3.2.

Let us define basic 2-morphisms in the 2-category Rings wnlq $2_{2}$ as 2-morphisms of the form $f_{z^{-1}} \stackrel{z}{\longrightarrow} \operatorname{id}_{(\widetilde{A}, \widetilde{V})}$, where $(\widetilde{A}, \widetilde{V})$ is an object of Rings wnlq,$\quad z \in F_{0} \widetilde{A}$ is an invertible element, $f_{z^{-1}}:(\widetilde{A}, \widetilde{V}) \longrightarrow(\widetilde{A}, \widetilde{V})$ is the morphism taking an element $c \in \widetilde{A}$ to the element $f_{z^{-1}}(c)=z^{-1} c z \in \widetilde{A}$, and $\operatorname{id}_{(\widetilde{A}, \widetilde{V})}:(\widetilde{A}, \widetilde{V}) \longrightarrow(\widetilde{A}, \widetilde{V})$ is the identity morphism. Then any 2-morphism in Rings wnlq2 decomposes uniquely as a morphism followed by a basic 2-morphism, and also as a basic 2-morphism followed by a morphism. Specifically, a 2-morphism $f^{\prime} \stackrel{z}{\longrightarrow} f^{\prime \prime}$ as above is the composition of the morphism $f^{\prime \prime}$ followed by the basic 2-morphism $f_{z^{-1}} \stackrel{z}{\longrightarrow} \operatorname{id}_{\left({ }^{\prime \prime} \widetilde{A},{ }^{\prime \prime}\right)}$; and it is also the composition of the basic 2-morphism $f_{w^{-1}} \stackrel{w}{\longrightarrow} \operatorname{id}_{\left({ }^{\prime}, \tilde{A}, \widetilde{V}\right)}$ followed by the 2-morphism $f^{\prime \prime}$.

Similarly, we define basic 2-morphisms in the 2-category Rings $_{\mathrm{cdg} 2, \mathrm{rq}}$ as 2-morphisms of the form $\left(\operatorname{id}_{B}, 0\right) \stackrel{z}{\longrightarrow}\left(g_{z}, a_{z}\right):(B, d, h) \longrightarrow(B, d, h)$, where $(B, d, h)$ is an object of Rings ${ }_{\text {cdg,rq }}, \quad z \in B^{0}$ is an invertible element, $g_{z}: B \longrightarrow B$ is the graded ring homomorphism taking an element $b \in B$ to the element $g_{z}(b)=z b z^{-1} \in B$, and $a_{z}=-d(z) z^{-1} \in B^{1}$. Then any 2 -morphism in Rings wnlq $2^{2}$ decomposes uniquely as a morphism followed by a basic 2-morphism, and also as a basic 2-morphism followed by a morphism. Specifically, a 2-morphism $g^{\prime \prime} \stackrel{w}{\longrightarrow} g^{\prime}$ as above is the composition of the morphism $g^{\prime \prime}$ followed by the basic 2-morphism $\left(\mathrm{id}_{B}, 0\right) \stackrel{w}{\longrightarrow}\left(g_{w}, a_{w}\right):\left({ }^{\prime} B, d^{\prime}, h^{\prime}\right) \longrightarrow$ $\left({ }^{\prime} B, d^{\prime}, h^{\prime}\right)$, and it is also the composition of the basic 2-morphism (id" $\left.{ }^{\prime}, 0\right) \stackrel{z}{\longrightarrow}$ $\left(g_{z}, a_{z}\right):\left({ }^{\prime \prime} B, d^{\prime \prime}, h^{\prime \prime}\right) \longrightarrow\left({ }^{\prime \prime} B, d^{\prime \prime}, h^{\prime \prime}\right)$ followed by the morphism $g^{\prime \prime}$.

In other words, $f^{\prime} \stackrel{z}{\longrightarrow} f^{\prime \prime}$ is a 2-morphism in Rings wnlq 2 if and only if $f^{\prime}=f_{z^{-1}} f^{\prime \prime}$, or equivalently, $f^{\prime}=f^{\prime \prime} f_{w^{-1}}$. Similarly, $g^{\prime \prime} \stackrel{w}{\longrightarrow} g^{\prime \prime}$ is a 2 -morphism in Rings ${ }_{\mathrm{cdg} 2 \text {, rq }}$ if and only if $g^{\prime}=\left(g_{w}, a_{w}\right) \circ g^{\prime \prime}$, or equivalently, $g^{\prime}=g^{\prime \prime} \circ\left(g_{z}, a_{z}\right)$. 
It remains to refer to Lemma 3.12 for the assertion that, for any object $(\widetilde{A}, \widetilde{V}) \in$ Rings $_{\mathrm{wnlq} 2}$ and the corresponding object $(B, d, h) \in \operatorname{Rings}_{\mathrm{cdg} 2, \mathrm{rq}}$, basic 2-morphisms $f_{z^{-1}} \stackrel{z}{\longrightarrow} \mathrm{id}_{(\widetilde{A}, \widetilde{V})}$ in Rings wnlq2 correspond to basic 2-morphisms $\left(\operatorname{id}_{B}, 0\right) \stackrel{z}{\longrightarrow}\left(g_{z}, a_{z}\right):(B, d, h) \longrightarrow(B, d, h)$ in Rings ${ }_{\mathrm{cdg} 2, \mathrm{rq}}$.

3.8. Augmented nonhomogeneous quadratic rings. Let $\widetilde{A}$ be an associative ring and $R \subset \widetilde{A}$ be a subring. A left augmentation of $\widetilde{A}$ over $R$ is a left ideal $\widetilde{A}^{+} \subset \widetilde{A}$ such that $\widetilde{A}=R \oplus \widetilde{A}^{+}$. Equivalently, a left augmentation is a left action of $\widetilde{A}$ in $R$ extending the regular left action of $R$ in itself.

Given a left augmentation ideal $\widetilde{A}^{+} \subset \widetilde{A}$, such a left action of $\widetilde{A}$ in $R$ is obtained by identifying $R$ with the quotient left $\widetilde{A}$-module $\widetilde{A} / \widetilde{A}^{+}=R$. Conversely, given a left augmentation action of $\widetilde{A}$ in $R$, the left augmentation ideal $\widetilde{A}^{+} \subset \widetilde{A}$ is recovered as the annihilator of the element $1 \in R$.

The category of left augmented rings over $R$, denoted by $R$-rings ${ }^{\text {laug }}$, is defined as follows. The objects of $R$-rings laug are associative rings $\widetilde{A}$ endowed with a subring identified with $R$ and a left augmentation ideal $\widetilde{A}^{+} \subset \widetilde{A}$. Morphisms $\left({ }^{\prime} \widetilde{A}, \widetilde{A}^{+}\right) \longrightarrow$ (" $\left.\widetilde{A},{ }^{\prime \prime} \widetilde{A}^{+}\right)$in $R$-rings laug are ring homomorphisms $f:{ }^{\prime} \widetilde{A} \longrightarrow{ }^{\prime \prime} \widetilde{A}$ forming a commutative triangle diagram with the embeddings $R \longrightarrow{ }^{\prime} \widetilde{A}$ and $R \longrightarrow{ }^{\prime \prime} \widetilde{A}$ and satisfying the condition of compatibility with the augmentations, namely, that $f\left({ }^{\prime} \widetilde{A}^{+}\right) \subset{ }^{\prime \prime} \widetilde{A}^{+}$. Equivalently, both the conditions on $f$ can be expressed by saying that the left action of ' $\widetilde{A}$ in $R$ coincides with the action obtained from the left action of " $\widetilde{A}$ in $R$ by the restriction of scalars via $f$.

Moreover, one can define the 2-category of left augmented rings, denoted by Rings $^{\text {laug2 }}$, in the following way. The objects of Rings aug2 are associative rings $\widetilde{A}$ endowed with a subring $F_{0} \widetilde{A} \subset \widetilde{A}$ and a left ideal $\widetilde{A}^{+} \subset \widetilde{A}$ such that $\widetilde{A}=F_{0} \widetilde{A} \oplus \widetilde{A}^{+}$. Morphisms (' $\left.\widetilde{A}, F_{0}{ }^{\prime} \widetilde{A},{ }^{\prime} \widetilde{A}^{+}\right) \longrightarrow\left({ }^{\prime \prime} \widetilde{A}, F_{0}{ }^{\prime \prime} \widetilde{A},{ }^{\prime \prime} \widetilde{A}^{+}\right)$in Rings ${ }^{\text {laug2 }}$ are ring homomorphisms $f:{ }^{\prime} \widetilde{A} \longrightarrow{ }^{\prime \prime} \widetilde{A}$ such that $f$ restricts to an isomorphism $F_{0} f: F_{0}{ }^{\prime} \widetilde{A} \longrightarrow F_{0}{ }^{\prime \prime} \widetilde{A}$ and $f\left({ }^{\prime} \widetilde{A}^{+}\right) \subset{ }^{\prime \prime} \widetilde{A}^{+} . \quad$ 2-morphisms $f \stackrel{z}{\longrightarrow} g$ between a pair of parallel morphisms $f$, $g:\left({ }^{\prime} \widetilde{A}, F_{0}{ }^{\prime} \widetilde{A},{ }^{\prime} \widetilde{A}^{+}\right) \longrightarrow\left({ }^{\prime \prime} \widetilde{A}, F_{0}{ }^{\prime \prime} \widetilde{A},{ }^{\prime \prime} \widetilde{A}^{+}\right)$are invertible elements $z \in F_{0}{ }^{\prime} \widetilde{A}$ such that $g(c)=f\left(z c z^{-1}\right)$ for all $c \in{ }^{\prime} \widetilde{A}$.

Notice that it follows from the latter condition that $z^{\prime} \widetilde{A}^{+} z^{-1}={ }^{\prime} \widetilde{A}^{+}$, or equivalently, ${ }^{\prime} \widetilde{A}^{+} z={ }^{\prime} \widetilde{A}^{+}$. Indeed, $z c z^{-1}=r+a$ for $c, a \in{ }^{\prime} \widetilde{A}^{+}$and $r \in F_{0}{ }^{\prime} \widetilde{A}$ implies $g(c)=$ $f\left(z c z^{-1}\right)=f(r)+f(a) \in{ }^{\prime \prime} \widetilde{A}$ with $f(r) \in F_{0}{ }^{\prime \prime} \widetilde{A}$ and $f(a) \in{ }^{\prime \prime} \widetilde{A}^{+}$, hence $f(r)=0$ and $r=0$. Similarly, $z^{-1} c z=r+a$ for $c, a \in{ }^{\prime} \widetilde{A}^{+}$and $r \in F_{0}^{\prime} \widetilde{A}$ implies $f(c)=g\left(z^{-1} c z\right)=$ $g(r)+g(a) \in{ }^{\prime \prime} \widetilde{A}$, hence $r=0$.

The vertical composition of two 2-morphisms $f^{\prime} \stackrel{w}{\longrightarrow} f^{\prime \prime} \stackrel{z}{\longrightarrow} f^{\prime \prime \prime}$ is the 2-morphism $f^{\prime} \stackrel{w z}{\longrightarrow} f^{\prime \prime \prime}$. The identity 2-morphism is the 2-morphism $f \stackrel{1}{\longrightarrow} f$. The horizontal composition of two 2-morphisms $g^{\prime} \stackrel{w}{\longrightarrow} g^{\prime \prime}:\left(\widetilde{A}, F_{0} \widetilde{A}, \widetilde{A}^{+}\right) \longrightarrow\left(\widetilde{B}, F_{0} \widetilde{B}, \widetilde{B}^{+}\right)$ and $f^{\prime} \stackrel{z}{\longrightarrow} f^{\prime \prime}:\left(\widetilde{B}, F_{0} \widetilde{B}, \widetilde{B}^{+}\right) \longrightarrow\left(\widetilde{C}, F_{0} \widetilde{C}, \widetilde{C}^{+}\right)$is the 2-morphism $f^{\prime} g^{\prime} \stackrel{z \circ w}{\longrightarrow}$ $f^{\prime \prime} g^{\prime \prime}:\left(\widetilde{A}, F_{0} \widetilde{A}, \widetilde{A}^{+}\right) \longrightarrow\left(\widetilde{C}, F_{0} \widetilde{C}, \widetilde{C}^{+}\right)$with the element $z \circ w=\left(F_{0} g^{\prime}\right)^{-1}(z) w=$ 
$w\left(F_{0} g^{\prime \prime}\right)^{-1}(z)$, where $\left(F_{0} g^{\prime}\right)^{-1},\left(F_{0} g^{\prime \prime}\right)^{-1}: F_{0} \widetilde{B} \longrightarrow F_{0} \widetilde{A}$ are the inverse maps to the ring isomorphisms $F_{0} g^{\prime}, F_{0} g^{\prime \prime}: F_{0} \widetilde{A} \longrightarrow F_{0} \widetilde{B}$.

All the 2-morphisms of left augmented rings are invertible. If $f:\left({ }^{\prime} \widetilde{A}, F_{0}{ }^{\prime} \widetilde{A},{ }^{\prime} \widetilde{A}^{+}\right) \longrightarrow$ (" $\left.\widetilde{A}, F_{0}{ }^{\prime \prime} \widetilde{A},{ }^{\prime \prime} \widetilde{A}^{+}\right)$is a morphism of left augmented rings and $z \in F_{0}{ }^{\prime} \widetilde{A}$ is an invertible element such that ${ }^{\prime} \widetilde{A}^{+} z={ }^{\prime} \widetilde{A}^{+}$, then $g: c \longmapsto f\left(z c z^{-1}\right)$ is also a morphism of left augmented rings $g:\left({ }^{\prime} \widetilde{A}, F_{0}{ }^{\prime} \widetilde{A},{ }^{\prime} \widetilde{A}^{+}\right) \longrightarrow\left({ }^{\prime \prime} \widetilde{A}, F_{0}{ }^{\prime \prime} \widetilde{A},{ }^{\prime \prime} \widetilde{A}^{+}\right)$. The morphisms $f$ and $g$ are connected by the 2-isomorphism $f \stackrel{z}{\longrightarrow} g$.

Let $(\widetilde{A}, F)$ be a filtered ring with an increasing filtration $0=F_{-1} \widetilde{A} \subset F_{0} \widetilde{A} \subset$ $F_{1} \widetilde{A} \subset F_{2} \widetilde{A} \subset \cdots$ (which, as above, is presumed to be exhastive and compatible with the multiplication in $\widetilde{A})$. The filtered $\operatorname{ring}(\widetilde{A}, F)$ is said to be left augmented if the ring $\widetilde{A}$ is left augmented over its subring $F_{0} \widetilde{A}$. In other words, this means that a left ideal $\widetilde{A}^{+} \subset \widetilde{A}$ is chosen such that $\widetilde{A}=F_{0} \widetilde{A} \oplus \widetilde{A}^{+}$.

We denote by $R$-rings $s_{\text {fil }}^{\text {lag }}$ the category of left augmented filtered rings with the filtration component $F_{0} \widetilde{A}$ identified with $R$. So the objects of $R$-rings faug are left augmented filtered rings $\left(\widetilde{A}, F, \widetilde{A}^{+}\right)$for which a ring isomorphism $R \simeq F_{0} \widetilde{A}$ has been chosen. Morphisms $\left({ }^{\prime} \widetilde{A}, F, \widetilde{A}^{+}\right) \longrightarrow\left({ }^{\prime \prime} \widetilde{A}, F,{ }^{\prime \prime} \widetilde{A}^{+}\right)$in $R$-rings fil $_{\text {laug }}$ are ring homomorphisms $f:{ }^{\prime} \widetilde{A} \longrightarrow{ }^{\prime \prime} \widetilde{A}$ such that $f\left(F_{n}{ }^{\prime} \widetilde{A}\right) \subset F_{n}{ }^{\prime \prime} \widetilde{A}$ for all $n \geq 0, f\left({ }^{\prime} \widetilde{A}^{+}\right) \subset{ }^{\prime \prime} \widetilde{A}^{+}$, and the ring homomorphism $F_{0} f: F_{0}{ }^{\prime} \widetilde{A} \longrightarrow F_{0}{ }^{\prime \prime} \widetilde{A}$ forms a commutative triangle diagram with the fixed isomorphisms $R \simeq F_{0}{ }^{\prime} \widetilde{A}$ and $R \simeq F_{0}{ }^{\prime \prime} \widetilde{A}$.

The definition of the 2-category of left augmented filtered rings, denoted by Rings fil $_{\text {faug2 }}$, is similar to the above. The objects of Rings faug2 are left augmented filtered $\operatorname{rings}\left(\widetilde{A}, F, \widetilde{A}^{+}\right)$. Morphisms $\left({ }^{\prime} \widetilde{A}, F, \widetilde{A}^{+}\right) \longrightarrow\left({ }^{\prime \prime} \widetilde{A}, F,{ }^{\prime \prime} \widetilde{A}^{+}\right)$are ring homomorphisms $f:{ }^{\prime} \widetilde{A} \longrightarrow{ }^{\prime \prime} \widetilde{A}$ such that $f\left(F_{n}{ }^{\prime} \widetilde{A}\right) \subset F_{n}{ }^{\prime \prime} \widetilde{A}$ for all $n \geq 0$, the map $F_{0} f: F_{0}{ }^{\prime} \widetilde{A} \longrightarrow F_{0}{ }^{\prime \prime} \widetilde{A}$ is an isomorphism, and $f\left({ }^{\prime} \widetilde{A}^{+}\right) \subset{ }^{\prime \prime} \widetilde{A}^{+}$. 2-morphisms $f \stackrel{z}{\longrightarrow} g$ between a pair of parallel morphisms $f, g:\left({ }^{\prime} \widetilde{A}, F,{ }^{\prime} \widetilde{A}^{+}\right) \longrightarrow\left({ }^{\prime \prime} \widetilde{A}, F,{ }^{\prime \prime} \widetilde{A}^{+}\right)$are invertible elements $z \in F_{0}\left(\widetilde{A}\right.$ such that $g(c)=f\left(z c z^{-1}\right)$ for all $c \in{ }^{\prime} \widetilde{A}$. The composition of 2 -morphisms in Rings $s_{\mathrm{fil}}^{\text {laug2 }}$ is defined in the same way as in the category Rings ${ }^{\text {laug2 }}{ }^{\text {; so }}$ there is an obvious forgetful strict 2-functor Rings $s_{\mathrm{fil}}^{\text {laug2 }} \longrightarrow$ Rings $^{\text {laug2 }}$.

A weak nonhomogeneous quadratic ring $R \subset \widetilde{V} \subset \widetilde{A}$ is said to be left augmented if the ring $\widetilde{A}$ is endowed with a left augmentation over its subring $R$. The category of 3-left finitely projective left augmented weak nonhomogeneous quadratic rings over $R$, denoted by $R$-rings saug , is defined as the full subcategory in $R$-rings $\mathrm{s}_{\text {fil }}^{\text {laug }}$ whose objects are the left augmented weak nonhomogeneous quadratic rings over $R$ that are 3-left finitely projective as weak nonhomogeneous quadratic rings.

The 2-category of 3-left finitely projective left augmented weak nonhomogeneous quadratic rings, denoted by Rings sugn $_{\text {la }}$, is defined as the following 2-subcategory in Rings $_{\mathrm{fil}}^{\text {laug2 }}$. The objects of Rings $s_{\mathrm{fil}}^{\text {laug2 }}$ are the 3-left finitely projective left augmented weak nonhomogeneous quadratic rings $R \subset \widetilde{V} \subset \widetilde{A} \supset \widetilde{A}^{+}$with the filtration $F$ generated by $F_{1} \widetilde{A}$ over $F_{0} \widetilde{A}$. All morphisms in Rings fil $_{\text {laug2 }}$ between objects of Rings saug2 
are morphisms in Rings $s_{\text {wnla }}^{\text {laug2 }}$, and all 2-morphisms in Rings $s_{\text {fil }}^{\text {laug2 }}$ between morphisms of Rings $s_{\text {wnlq }}^{\text {laug2 }}$ are 2-morphisms in Rings $s_{\text {wnlq }}^{\text {laug2 }}$.

A $D G$-ring $(B, d)$ is a graded associative $\operatorname{ring} B=\bigoplus_{n \in \mathbb{Z}} B^{n}$ endowed with an odd derivation $d: B \longrightarrow B$ of degree 1 such that $d^{2}=0$. In other words, one can say that a DG-ring is a CDG-ring $(B, d, h)$ with $h=0$. In this section, we consider nonnegatively graded DG-rings, that is $B=\bigoplus_{n=0}^{\infty} B^{n}$.

A morphism of $D G$-rings $f:\left({ }^{\prime \prime} B, d^{\prime \prime}\right) \longrightarrow\left({ }^{\prime} B, d^{\prime}\right)$ is a morphism of graded rings $f:{ }^{\prime \prime} B \longrightarrow{ }^{\prime} B$ such that $f d^{\prime \prime}=d^{\prime} f$. In other words, one can say that a morphism of DG-rings is a morphism of CDG-rings $(f, a):\left({ }^{\prime \prime} B, d^{\prime \prime}, 0\right) \longrightarrow\left({ }^{\prime} B, d^{\prime}, 0\right)$ with $a=0$. Notice that there exist CDG-ring morphisms $(f, a)$ with $a \neq 0$ both the domain and codomain of which are DG-rings. In other words, DG-rings form a subcategory in CDG-rings, but it is not a full subcategory.

We will denote the category of nonnegatively graded DG-rings $(B, d)$ with the

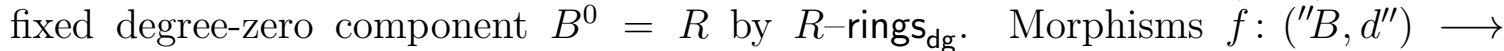
$\left({ }^{\prime} B, d^{\prime}\right)$ in $R$-rings ${ }_{\mathrm{dg}}$ are DG-ring morphisms such that the graded ring homomorphism $f:{ }^{\prime \prime} B \longrightarrow{ }^{\prime} B$ forms a commutative triangle diagram with the fixed isomorphisms $R \simeq{ }^{\prime \prime} B^{0}$ and $R \simeq{ }^{\prime} B^{0}$.

One can define the 2 -category of $D G$-rings as follows. Let $f, g:\left({ }^{\prime \prime} B, d^{\prime \prime}\right) \longrightarrow\left({ }^{\prime} B, d^{\prime}\right)$ be a pair of parallel morphisms of DG-rings. A 2-morphism $f \stackrel{z}{\longrightarrow} g$ is an invertible element $z \in \in^{\prime} B^{0}$ such that $d^{\prime}(z)=0$ and $g(c)=z f(c) z^{-1}$ for all $c \in{ }^{\prime \prime} B$.

The vertical composition of two 2-morphisms $f^{\prime} \stackrel{w}{\longrightarrow} f^{\prime \prime} \stackrel{z}{\longrightarrow} f^{\prime \prime \prime}$ is the 2-morphism $f^{\prime} \stackrel{z w}{\longrightarrow} f^{\prime \prime \prime}$. The identity 2-morphism is the 2-morphism $f \stackrel{1}{\longrightarrow} f$. The horizontal composition of two 2-morphisms $g^{\prime} \stackrel{w}{\longrightarrow} g^{\prime \prime}:\left(C, d_{C}\right) \longrightarrow\left(B, d_{B}\right)$ and $f^{\prime} \stackrel{z}{\longrightarrow}$ $f^{\prime \prime}:\left(B, d_{B}\right) \longrightarrow\left(A, d_{A}\right)$ is the 2-morphism $f^{\prime} g^{\prime} \stackrel{z \circ w}{\longrightarrow} f^{\prime \prime} g^{\prime \prime}:\left(C, d_{C}\right) \longrightarrow\left(A, d_{A}\right)$ with the element $z \circ w=z f^{\prime}(w)=f^{\prime \prime}(w) z \in A^{0}$.

All the 2-morphisms of DG-rings are invertible. If $f:\left({ }^{\prime \prime} B, d^{\prime \prime}\right) \longrightarrow\left({ }^{\prime} B, d^{\prime}\right)$ is a morphism of DG-rings and $z \in{ }^{\prime} B^{0}$ is an invertible element such that $d^{\prime}(z)=0$, then $g: c \longmapsto z f(c) z^{-1}$ is also a morphism of DG-rings $g:\left({ }^{\prime \prime} B, d^{\prime \prime}\right) \longrightarrow\left({ }^{\prime} B, d^{\prime}\right)$. The morphisms $f$ and $g$ are connected by the 2-isomorphism $f \stackrel{z}{\longrightarrow} g$.

It is clear from these definitions that the 2-category of DG-rings is a 2-subcategory of the 2-category of CDG-rings. Notice the difference, however: the 2-morphisms of CDG-rings correspond to arbitrary invertible elements $z \in{ }^{\prime} B^{0}$. The 2-morphisms of DG-rings correspond to invertible cocycles $z \in{ }^{\prime} B^{0}, d^{\prime}(z)=0$.

The 2-category Rings ${ }_{\mathrm{dg} 2}$ of nonnegatively graded DG-rings is defined as the following subcategory of the 2-category of DG-rings. The objects of Rings $\mathrm{dg}_{2}$ are nonnegatively graded DG-rings $(B, d), B=\bigoplus_{n=0}^{\infty} B^{n}$. Morphisms $f:\left({ }^{\prime \prime} B, d^{\prime \prime}\right) \longrightarrow\left({ }^{\prime} B, d^{\prime}\right)$ in Rings $_{\mathrm{dg} 2}$ are morphisms of DG-rings such that the map $f_{0}:{ }^{\prime \prime} B^{0} \longrightarrow{ }^{\prime} B^{0}$ is an isomorphism. 2-morphisms $f \stackrel{z}{\longrightarrow} g$ in Rings ${ }_{\mathrm{dg} 2}$ between morphisms $f$ and $g$ belonging to Rings $_{\mathrm{dg} 2}$ are arbitrary 2-morphisms from $f$ to $g$ in the 2-category of DG-rings.

The category of 3-right finitely projective quadratic DG-rings over $R$, denoted by $R$-rings dg,rq $_{\text {, }}$, is the full subcategory in the category $R$-rings dg $_{\mathrm{dg}}$ consisting of all the 
DG-rings $(B, d)$ whose underlying nonnegatively graded ring $B$ is 3-right finitely projective quadratic over $R$.

The 2-category of 3-right finitely projective quadratic DG-rings, denoted by Rings $_{\mathrm{dg} 2 \text {,rq }}$, is the similar 2-subcategory in the 2-category Rings ${ }_{\mathrm{dg} 2}$. The objects of Rings $_{\mathrm{dg} 2, \mathrm{rq}}$ are all the DG-rings $(B, d)$ whose underlying nonnegatively graded $\operatorname{ring} B$ is 3-right finitely projective quadratic over $B^{0}$. All morphisms in Rings ${ }_{\mathrm{dg} 2}$ between objects of Rings $_{\mathrm{dg} 2, \mathrm{rq}}$ are morphisms in Rings $\mathrm{dg}_{\mathrm{dg}, \mathrm{rq}}$, and all 2-morphisms in Rings $\mathrm{s}_{\mathrm{dg} 2}$ between morphisms of Rings ${ }_{\mathrm{dg} 2, \mathrm{rq}}$ are 2-morphisms in Rings ${ }_{\mathrm{dg} 2, \mathrm{rq}}$.

Theorem 3.14. The nonhomogeneous quadratic duality functor of Theorem 3.10 restricts to a fully faithful contravariant functor

$$
\left(R-\text { rings }_{\text {wnlq }}^{\text {laug }}\right)^{\text {op }} \longrightarrow R \text {-rings } \text { dg,rq }
$$

from the category of 3-left finitely projective left augmented weak nonhomogeneous quadratic rings to the category of 3-right finitely projective quadratic DG-rings over $R$.

Proof. It is clear from the above discussion that $R$-rings dg,rq $_{\text {is }}$ a subcategory in $R$-rings ${ }_{\text {cdg,rq }}$. Moreover, the category of 3-right finitely projective quadratic DG-rings $R$-rings $s_{\mathrm{dg}, \mathrm{rq}}$ is a full subcategory in the category $R$-rings $\mathrm{s}_{\mathrm{cdg}, \mathrm{rq}}^{\mathrm{sm}}$ of 3-right finitely projective quadratic CDG-rings over $R$ and strict morphisms between them (which was introduced in the proof of Theorem 3.10).

Similarly, we observe that the category of 3-left finitely projective left augmented weak nonhomogeneous quadratic rings $R$-rings saug is a full subcategory in the category $R$-rings snlq of $_{\text {shla }}^{\text {sgsm }}$-left finitely projective weak nonhomogeneous quadratic $\operatorname{rings}(\widetilde{A}, \widetilde{V})$ with a fixed submodule of strict generators $V^{\prime} \subset \widetilde{V}$ and morphisms $f:\left({ }^{\prime} \widetilde{A},{ }^{\prime} \widetilde{V},{ }^{\prime} V\right) \longrightarrow$ (" $\left.\widetilde{A},{ }^{\prime \prime} \widetilde{V}, " V\right)$ preserving the submodule of strict generators. Indeed, given a 3-left finitely projective left augmented weak nonhomogeneous quadratic $\operatorname{ring}\left(\widetilde{A}, \widetilde{V}, \widetilde{A}^{+}\right)$ over $R$, we choose the left $R$-submodule $V^{\prime}=\widetilde{A}^{+} \cap \widetilde{V} \subset \widetilde{V}$ as the submodule of strict generators of $\widetilde{A}$. The left augmentation ideal $\widetilde{A}^{+} \subset \widetilde{A}$ can be then recovered as the left ideal (equivalently, the subring without unit) generated by $V^{\prime}$ in $\widetilde{A}$.

Moreover, the essential image of the fully faithful functor $R$-rings wnlq $_{\text {aug }} \longrightarrow$

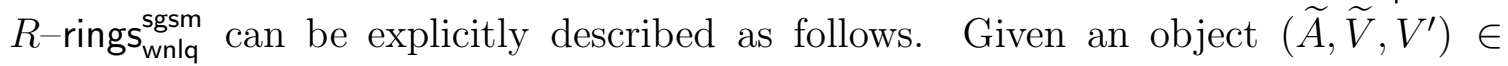
$R$-rings ${ }_{\text {wnlq }}^{\text {sgsm }}$, consider the related maps $q: V \times R \longrightarrow R, p: \widehat{I} \longrightarrow R$, and $h: \widehat{I} \longrightarrow R$ defined by the formulas (27, 28). Then the object $\left(\widetilde{A}, \widetilde{V}, V^{\prime}\right)$ corresponds to a (3-left finitely projective) left augmented weak nonhomogeneous quadratic ring if and only if one has $\hat{\imath}_{1} * \hat{\imath}_{2} \in V^{\prime} \subset \widetilde{V}$ for all $\hat{\imath} \in \widehat{I}$, that is, $h=0$.

Indeed, the "only if" assertion is obvious. To prove the "if", one observes that, for any weak nonhomogeneous quadratic ring $(\widetilde{A}, \widetilde{V})$ satisfying the assumptions of Section 3.3 and any chosen submodule of strict generators $V^{\prime} \subset \widetilde{V}$, the $\operatorname{ring} \widetilde{A}$ is generated by the ring $R$ and the abelian group $V^{\prime}$ with the defining relations (27 28). It is clear from the form of these relations that the left ideal generated by $V^{\prime}$ in $\widetilde{A}$ does not intersect $R$ whenever $h=0$. 
The latter condition means exactly that the quadratic CDG-ring $(B, d, h)$ assigned to $\left(\widetilde{A}, \widetilde{V}, V^{\prime}\right)$ by the functor (37) is a DG-ring. The desired fully faithful contravariant functor (45) can be now obtained as a restriction of the fully faithful contravariant functor (37) to the full subcategory $R$-rings wnlq $_{\text {laug }} \subset R$-rings wnlq $_{\text {ssm }}^{\text {s. }}$.

Theorem 3.15. The nonhomogeneous quadratic duality 2-functor of Theorem 3.13 restricts to a fully faithful strict contravariant 2-functor

$$
\left(\text { Rings }_{\text {wnlq }}^{\text {laug2 }}\right)^{\text {op }} \longrightarrow \text { Rings }_{\mathrm{dg} 2, \mathrm{rq}}
$$

from the 2-category of 3-left finitely projective left augmented weak nonhomogeneous quadratic rings to the 2-category of 3-right finitely projective quadratic DG-rings.

Proof. Similarly to the functor (43), the functor (46) is fully faithful in the strict sense. For any two objects $\left({ }^{\prime} \widetilde{A},{ }^{\prime} \widetilde{V}, \widetilde{A}^{+}\right)$and $\left(" \widetilde{A},{ }^{\prime \prime} \widetilde{V},{ }^{\prime \prime} \widetilde{A}^{+}\right) \in$ Rings $_{\text {wnlq }}^{\text {laug2 }}$ and the corresponding DG-rings $\left({ }^{\prime} B, d^{\prime}\right)$ and $\left({ }^{\prime \prime} B, d^{\prime \prime}\right) \in$ Rings $_{\mathrm{dg} 2, \mathrm{rq}}$, the 2-functor (46) induces a bijection between morphisms $\left({ }^{\prime} \widetilde{A},{ }^{\prime} \widetilde{V}, \widetilde{A}^{+}\right) \longrightarrow\left({ }^{\prime \prime} \widetilde{A},{ }^{\prime \prime} \widetilde{V},{ }^{\prime \prime} \widetilde{A}^{+}\right)$in Rings wnlq $_{\text {laug }}$ and morphisms $\left({ }^{\prime \prime} B, d^{\prime \prime}\right) \longrightarrow\left({ }^{\prime} B, d^{\prime}\right)$ in Rings ${ }_{\mathrm{dg} 2, \mathrm{rq}}$. Furthermore, for any pair of parallel morphisms $f^{\prime}, f^{\prime \prime}:\left({ }^{\prime} \widetilde{A},{ }^{\prime} \widetilde{V},{ }^{\prime} \widetilde{A}^{+}\right) \longrightarrow\left({ }^{\prime \prime} \widetilde{A},{ }^{\prime \prime} \widetilde{V},{ }^{\prime \prime} \widetilde{A}^{+}\right)$in Rings wnlq $_{\text {laug2 }}$ and the corresponding pair of parallel morphisms $g^{\prime}, g^{\prime \prime}:\left({ }^{\prime \prime} B, d^{\prime \prime}\right) \longrightarrow\left({ }^{\prime} B, d^{\prime}\right)$ in Rings dg2,rq $_{\text {, }}$, the 2-functor (46) induces a bijection between 2-morphisms $f^{\prime} \stackrel{z}{\longrightarrow} f^{\prime \prime}$ in Rings laug2 and 2-morphisms $g^{\prime \prime} \stackrel{z}{\longrightarrow} g^{\prime}$ in Rings ${ }_{\mathrm{dg} 2, \mathrm{rq}}$.

It is clear from the discussion above in this section that Rings $\mathrm{dg}_{\mathrm{dg}, \mathrm{rq}}$ is a 2-subcategory

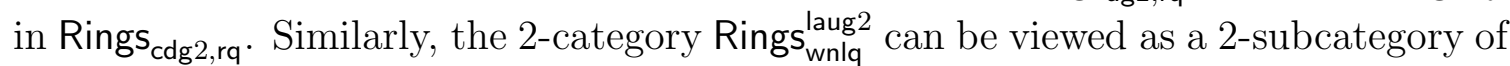
the 2-category Rings wnlq2 $_{2}$ in the following way. To any object $\left(\widetilde{A}, \widetilde{V}, \widetilde{A}^{+}\right) \in \operatorname{Rings}_{\text {wnlq }}^{\text {laug2 }}$ one assigns the object $(\widetilde{A}, \widetilde{V}) \in$ Rings $_{\text {wnlq } 2}$, and to any morphism $f:\left({ }^{\prime} \widetilde{A},{ }^{\prime} \widetilde{V},{ }^{\prime} \widetilde{A}^{+}\right) \longrightarrow$ (" $\left.\widetilde{A},{ }^{\prime \prime} \widetilde{V},{ }^{\prime \prime} \widetilde{A}^{+}\right)$in Rings wnlq $_{\text {laug2 }}$ one assigns the morphism $f:\left({ }^{\prime} \widetilde{A},{ }^{\prime} \widetilde{V}\right) \longrightarrow\left({ }^{\prime \prime} \widetilde{A},{ }^{\prime \prime} \widetilde{V}\right)$ in Rings $_{\text {wnlq2 }}$. Finally, to any 2-morphism $f \stackrel{z}{\longrightarrow} g:\left({ }^{\prime} \widetilde{A},{ }^{\prime} \widetilde{V},{ }^{\prime} \widetilde{A}^{+}\right) \longrightarrow\left({ }^{\prime \prime} \widetilde{A},{ }^{\prime \prime} \widetilde{V},{ }^{\prime \prime} \widetilde{A}^{+}\right)$in Rings wnlq $_{\text {laug2 }}$ one assigns the 2-morphism $f \stackrel{w}{\longrightarrow} g:\left({ }^{\prime} \widetilde{A},{ }^{\prime} \widetilde{V}\right) \longrightarrow\left({ }^{\prime \prime} \widetilde{A},{ }^{\prime \prime} \widetilde{V}\right)$ in Rings wnlq2 with the element $w=\left(F_{0} f\right)(z)=\left(F_{0} g\right)(z)$. Here $z \in F_{0}^{\prime} \widetilde{A}$ is an invertible element such that ' $\widetilde{A}^{+} z={ }^{\prime} \widetilde{A}^{+}$and $w \in F_{0}{ }^{\prime \prime} \widetilde{A}$ is an invertible element, while $F_{0} f$ and $F_{0} g: F_{0}{ }^{\prime} \widetilde{A} \longrightarrow F_{0}{ }^{\prime \prime} \widetilde{A}$ are two ring isomorphisms whose values coincide on the element $z$.

Denote by Rings laug $\subset$ Rings laug2 the category whose objects are the objects of the 2-category Rings aug2 and whose morphisms are the morphisms of the 2-category

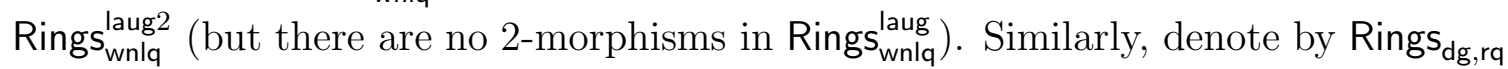
the category whose objects are the objects of the 2-category Rings ${ }_{d g 2, r q}$ and whose morphisms are the morphisms of the 2-category Rings ${ }_{\mathrm{dg} 2, \text { rq }}$ (but there are no 2-morphisms in Rings $\left.s_{d g, r q}\right)$. Then essentially the same argument that was used to restrict the functor (36) to the functor (45) in Theorem 3.14 shows that the fully faithful functor (44) restricts to a fully faithful functor

$$
\left(\text { Rings }_{\text {wnlq }}^{\text {laug }}\right)^{\text {op }} \underset{63}{\longrightarrow} \text { Rings }_{d g, r q} \text {. }
$$


To deduce the existence of a fully faithful functor (46) from the existence of the fully faithful functors (43) and (47), one can observe that both the embeddings of 2-categories Rings wnlq $_{\text {laug }} \longrightarrow$ Rings $_{\text {wnlq2 }}$ and Rings ${ }_{\mathrm{dg} 2, \mathrm{rq}} \longrightarrow$ Rings $_{\mathrm{cdg} 2, \mathrm{rq}}$ are fully faithful on the level of 2-morphisms. In other words, this means that any 2-morphism in

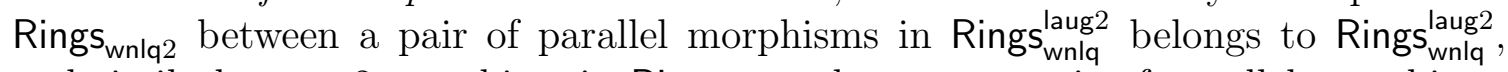
and similarly, any 2-morphism in Rings ${ }_{\mathrm{cdg} 2, \text { rq }}$ between a pair of parallel morphisms in Rings ${ }_{d g 2, r q}$ belongs to Rings ${ }_{d g 2, r q}$. (More generally, Rings $s_{f i l}^{\text {laug2 }}$ is a 2-subcategory in Rings $_{\text {fil2 }}$ such that any 2-morphism in Rings fil $2_{2}$ between a pair of parallel morphisms in Rings $s_{\text {fil }}^{\text {laug2 }}$ belongs to Rings fil $_{\text {fil }}$; and Rings $s_{\mathrm{dg} 2}$ is a 2-subcategory in Rings $\mathrm{s}_{\mathrm{cdg} 2}$ such that any 2-morphism in Rings $s_{\mathrm{cdg}}$ between a pair of parallel morphisms in Rings $\mathrm{dg}_{\mathrm{dg}}$ belongs to Rings $\mathrm{dg}_{2}$.) This suffices to prove the theorem.

Alternatively, one can construct the fully faithful strict 2-functor (46) in the way similar to the construction of the fully faithful strict 2-functor (43) in the proof of Theorem 3.13. For this purpose, one defines the "basic 2-morphisms" in the 2-categories Rings $s_{\text {wnlq }}^{\text {laug2 }}$ and Rings ${ }_{\mathrm{dg} 2 \text {,rq }}$, and observes that any 2-morphism in Rings $s_{\text {wnlq }}^{\text {laug2 }}$ decomposes uniquely as a basic 2-morphism followed by a morphism (but not in the other order), while any 2-morphism in Rings ${ }_{\mathrm{dg} 2, \mathrm{rq}}$ decomposes uniquely as a morphism followed by a basic 2-morphism (but not in the other order).

The reason is, essentially, that the cocycle equation $d^{\prime}(z)=0$ in the definition of a 2-morphism of DG-rings $f \stackrel{z}{\longrightarrow} g:\left({ }^{\prime \prime} B, d^{\prime \prime}\right) \longrightarrow\left({ }^{\prime} B, d^{\prime}\right)$ does not imply the equation $d^{\prime \prime}(w)=0$ for the element $w=f_{0}^{-1}(z)=g_{0}^{-1}(z)$. The latter equation is stronger than the former one, and does not need to hold. Similarly, the condition ' $\widetilde{A}^{+} z={ }^{\prime} \widetilde{A}^{+}$related to the definition a 2-morphism of left augmented rings $f \stackrel{z}{\longrightarrow} g:\left({ }^{\prime} \widetilde{A}, F_{0}{ }^{\prime} \widetilde{A},{ }^{\prime} \widetilde{A}^{+}\right) \longrightarrow$ (" $\left.\widetilde{A}, F_{0}{ }^{\prime \prime} \widetilde{A},{ }^{\prime \prime} \widetilde{A}^{+}\right)$does not imply the condition " $\widetilde{A}^{+} w={ }^{\prime \prime} \widetilde{A}^{+}$for the element $w=$ $\left(F_{0} f\right)(z)=\left(F_{0} g\right)(z)$. The latter condition is stronger than the former one, and does not need to hold.

\section{The Poincaré-Birkhoff-Witt Theorem}

4.1. Central element theorem. Let $\widehat{A}=\bigoplus_{n=0}^{\infty} \widehat{A}_{n}$ be a nonnegatively graded ring with the degree-zero component $R=\widehat{A}_{0}$, and let $t \in \widehat{A}_{1}$ be a central element. Let $A=\widehat{A} / \widehat{A} t$ denote the quotient ring of $\widehat{A}$ by the homogeneous ideal generated by $t$. So $A=\bigoplus_{n=0}^{\infty} A_{n}$ is also a nonnegatively graded ring with the degree-zero component $A_{0}=R$, the degree-one component $A_{1}=\widehat{A}_{1} / R t$, and the degree $n$ component $A_{n}=$ $\widehat{A}_{n} / \widehat{A}_{n-1} t$ for all $n \geq 1$. We will say that $t$ is a nonzero-divisor in $\widehat{A}$ if at $=0$ implies $a=0$ for any $a \in \widehat{A}_{n}, n \geq 0$.

Proposition 4.1. Let $\widehat{A}$ be a nonnegatively graded ring and $t \in \widehat{A}_{1}$ be a central element. Then

(a) the graded ring $\widehat{A}$ is generated by $\widehat{A}_{1}$ over $\widehat{A}_{0}$ if and only if the graded ring $A=\widehat{A} / \widehat{A} t$ is generated by $A_{1}$ over $A_{0}$; 
(b) assuming that $t$ is a nonzero-divisor in $\widehat{A}$, the graded ring $\widehat{A}$ is quadratic if and only if the graded ring $A=\widehat{A} / \widehat{A} t$ is quadratic.

Proof. Part (a): for any nonnegatively graded $\operatorname{ring} C=\bigoplus_{n=0}^{\infty} C_{n}$ generated by $C_{1}$ over $C_{0}$, and for any homogeneous ideal $H \subset C$, the quotient ring $A=C / H$ is generated by $A_{1}$ over $A_{0}$. This proves the implication "only if".

To prove the "if", suppose that we are given a nonnegatively graded ring $C$ and a homogeneous ideal $H \subset C$ which is generated, as a two-sided ideal, by its degree-one component $H_{1}$. Suppose further that the quotient $\operatorname{ring} A=C / H$ is generated by $A_{1}$ over $A_{0}$. Let $C^{\prime} \subset C$ denote the subring in $C$ generated by $C_{1}^{\prime}=C_{1}$ over $C_{0}^{\prime}=C_{0}$. Then the composition $C^{\prime} \longrightarrow C \longrightarrow A$ is surjective, so we have $C=C^{\prime}+H$. Arguing by induction, we will prove that $C_{n}^{\prime}=C_{n}$ for every $n \geq 2$. Indeed, assume that $C_{k}^{\prime}=C_{k}$ for all $k \leq n-1$. Then $H_{n}=\sum_{k=1}^{n} C_{k-1} H_{1} C_{n-k}=\sum_{k=1}^{n} C_{k-1}^{\prime} H_{1} C_{n-k}^{\prime} \subset C_{n}^{\prime}$, hence $C_{n}=C_{n}^{\prime}+H_{n}=C_{n}^{\prime}$. Thus $C^{\prime}=C$, so $C$ is generated by $C_{1}$ over $C_{0}$.

Part (b): for any quadratic graded ring $C=\bigoplus_{n=0}^{\infty} C_{n}$ and any homogeneous ideal $H \subset C$ that is generated, as a two-sided ideal, by its components $H_{1}$ and $H_{2}$, the quotient $\operatorname{ring} A=C / H$ is quadratic. This proves the implication "only if" (which does not depend on the assumption that $t$ is a nonzero-divisor).

To prove the "if", suppose that $t \in \widehat{A}_{1}$ is a central nonzero-divisor and the graded $\operatorname{ring} A=\widehat{A} / \widehat{A} t$ is quadratic. Then, by part (a), the graded ring $\widehat{A}$ is generated by $\widehat{A}_{1}$ over $R=\widehat{A}_{0}$.

Let $\widehat{I} \subset \widehat{A}_{1} \otimes_{R} \widehat{A}_{1}$ be the kernel of the multiplication map $\widehat{A}_{1} \otimes_{R} \widehat{A}_{1} \longrightarrow \widehat{A}_{2}$ and $\mathrm{q} \widehat{A}=T_{R}\left(\widehat{A}_{1}\right) /(\widehat{I})$ be the quadratic graded ring generated by $\widehat{A}_{1}$ with the relations $\widehat{I}$ over $R$. Then we have a unique surjective homomorphism of graded rings q $\widehat{A} \longrightarrow \widehat{A}$ acting by the identity maps on the components of degree 0 and 1 . By construction, the graded ring map $\mathrm{q} \widehat{A} \longrightarrow \widehat{A}$ is also an isomorphism in degree 2 .

The isomorphism $\mathrm{q} \widehat{A}_{1} \simeq \widehat{A}_{1}$ allows to consider $t$ as an element of the ring $\mathrm{q} \widehat{A}$. Moreover, $t \in \mathrm{q} \widehat{A}_{1}$ is a central element, since $\mathrm{q} \widehat{A}$ is generated by $\mathrm{q} \widehat{A}_{1}$ over $R$ and the relations of commutativity of $t$ with the elements of $R$ and q $\widehat{A}_{1}$ have degree $\leq 2$, so they hold in q $\widehat{A}$ whenever they hold in $\widehat{A}$.

Furthermore, by the "only if" assertion (which we have already explained) the quotient ring $A^{\prime}=\mathrm{q} \widehat{A} /(\mathrm{q} \widehat{A}) t$ is quadratic. We have the induced homomorphism of graded rings $A^{\prime}=\mathrm{q} \widehat{A} /(\mathrm{q} \widehat{A}) t \longrightarrow \widehat{A} / \widehat{A} t=A$. Since the map $\mathrm{q} \widehat{A} \longrightarrow \widehat{A}$ is an isomorphism in degree $\leq 2$, so is the map $A^{\prime} \longrightarrow A$. Since the graded $\operatorname{ring} A$ is quadratic by assumption, it follows by virtue of Lemma 3.2 that the map $A^{\prime} \longrightarrow A$ is an isomorphism of graded rings.

It follows that the kernel $H \subset \mathrm{q} \widehat{A}$ of the graded ring homomorphism $\mathrm{q} \widehat{A} \longrightarrow \widehat{A}$ is contained in $(\mathrm{q} \widehat{A}) t \subset \mathrm{q} \widehat{A}$. Now we will prove by induction in $n \geq 3$ that $H_{n}=0$. Indeed, let $h \in H_{n}$ be an element. Then $h=h^{\prime} t$ for some $h^{\prime} \in \mathrm{q} \widehat{A}_{n-1}$. The image of $h$ under the ring homomorphism $\mathrm{q} \widehat{A} \longrightarrow \widehat{A}$ vanishes, and since $t$ is a nonzero-divisor in $\widehat{A}$, it follows that the image of $h^{\prime}$ under the same homomorphism vanishes as well. Hence $h^{\prime} \in H_{n-1}=0$ by the induction assumption and $h=h^{\prime} t=0$. 
We have shown that $\mathrm{q} \widehat{A} \longrightarrow \widehat{A}$ is an isomorphism of graded rings, and it follows that the graded ring $\widehat{A}$ is quadratic.

Lemma 4.2. Let $\widehat{A}$ be a nonnegatively graded ring, $t \in \widehat{A}_{1}$ be a central nonzerodivisor, and $A=\widehat{A} / \widehat{A} t$ be the quotient ring. Let $n \geq 0$ be an integer. Assume that $A_{j}$ is a finitely generated projective (projective, or flat) left module over the ring $R=\widehat{A}_{0}=A_{0}$ for all $0 \leq j \leq n$. Then $\widehat{A}_{j}$ is a finitely generated projective (resp., projective or flat) left $R$-module for all $0 \leq j \leq n$.

Proof. Provable by induction in $n$ using the short exact sequences of $R$ - $R$-bimodules $0 \longrightarrow \widehat{A}_{n-1} \stackrel{t}{\longrightarrow} \widehat{A}_{n} \longrightarrow A_{n} \longrightarrow 0$.

The following theorem extends to the relative context a very specific particular case of the result of [26, second assertion of Theorem 6.1].

Theorem 4.3. Let $\widehat{A}$ be a nonnegatively graded ring and $t \in \widehat{A}_{1}$ be a central nonzerodivisor. Assume that $A_{n}=\widehat{A}_{n} / \widehat{A}_{n-1} t$ is a flat left $R$-module for every $n \geq 1$. Then the graded ring $\widehat{A}$ is left flat Koszul if and only if the graded ring $A$ is left flat Koszul.

Proof. For any homomorphism of (graded) rings $C \longrightarrow A$, any (graded) right $C$-module $N$, and any (graded) left $A$-module $M$, the isomorphism of left derived functors of tensor product

$$
\left(N \otimes_{C}^{\mathbb{L}} A\right) \otimes_{A}^{\mathbb{L}} M \simeq N \otimes_{C}^{\mathbb{L}} M
$$

on the derived categories of modules leads to a spectral sequence of (internally graded) abelian groups

$$
E_{p, q}^{2}=\operatorname{Tor}_{p}^{A}\left(\operatorname{Tor}_{q}^{C}(N, A), M\right) \Longrightarrow E_{p, q}^{\infty}=\operatorname{gr}_{p}^{F} \operatorname{Tor}_{p+q}^{C}(N, M)
$$

with the differentials $d_{p, q}^{r}: E_{p, q}^{r} \longrightarrow E_{p-r, q+r-1}^{r}$.

In particular, for any homomorphism of nonnegatively graded rings $C \longrightarrow A$ acting by the identity map on their degree-zero components $C_{0}=R=A_{0}$, we have a spectral sequence of internally graded $R$ - $R$-bimodules

$$
E_{p, q}^{2}=\operatorname{Tor}_{p}^{A}\left(\operatorname{Tor}_{q}^{C}(R, A), R\right) \Longrightarrow E_{p, q}^{\infty}=\operatorname{gr}_{p}^{F} \operatorname{Tor}_{p+q}^{C}(R, R) .
$$

In the situation at hand with $C=\widehat{A}$ and $A=\widehat{A} / t$, where $t \in \widehat{A}_{1}$ is a central nonzero-divisor, we have

$$
\operatorname{Tor}_{q}^{\widehat{A}}(R, A)= \begin{cases}R & \text { for } q=0 \\ R t & \text { for } q=1 \\ 0 & \text { for } q \geq 2\end{cases}
$$

where the $R$ - $R$-bimodule $\operatorname{Tor}_{0}^{\widehat{A}}(R, A)=R$ is situated in the internal degree 0 and the $R$ - $R$-bimodule $\operatorname{Tor}_{1}^{\widehat{A}}(R, A)=R t$ is situated in the internal degree 1 .

Furthermore, the assumption that $A_{n}$ is a flat left $R$-module for every $n \geq 1$ implies that $\widehat{A}_{n}$ is a flat left $R$-module as well, as one can show arguing by induction in $n$ and using the short exact sequences of $R$ - $R$-bimodules $0 \longrightarrow \widehat{A}_{n-1} \stackrel{t}{\longrightarrow} \widehat{A}_{n} \longrightarrow A_{n} \longrightarrow 0$. 
Now if $\operatorname{Tor}_{i, j}^{A}(R, R)=0$ for all $i \neq j$, then every term $E_{p, q}^{2}$ of the spectral sequence (48) is concentrated in the internal degree $p+q$; hence so is the term $E_{p, q}^{\infty}$. It follows immediately that $\operatorname{Tor}_{i, j}^{\widehat{A}}(R, R)=0$ for all $i \neq j$. So the conditions of Theorem 2.18(a) hold for $\widehat{A}$ whenever they hold for $A$. This proves the implication "if".

To prove the "only if", one can proceed by induction in $i$. Assume that the graded $R$ - $R$-bimodule $\operatorname{Tor}_{p}^{A}(R, R)$ is concentrated in the internal degree $j=p$ for all $p \leq i-1$. Then the terms $E_{p, q}^{2}$ are concentrated in the internal degree $p+q$ for all $p \leq i-1$. Furthermore, if the graded $R$ - $R$-bimodule $\operatorname{Tor}_{i}^{\widehat{A}}(R, R)$ is concentrated in the internal degree $i$, then so are the $R$ - $R$-bimodules $E_{p, q}^{\infty}$ for all $p+q=i$. In particular, the term $E_{i, 0}^{\infty}$ is concentrated in the internal degree $i$.

The only possibly nontrivial differentials passing through $E_{i, 0}^{r}$ with $r \geq 2$ are $d_{i, 0}^{2}: E_{i, 0}^{2} \longrightarrow E_{i-2,1}^{2}$. As the term $E_{i-2,1}^{2}$ is concentrated in the internal degree $i-1$ by the induction assumption and the above discussion, and the term $E_{i, 0}^{\infty}$ is concentrated in the internal degree $i$, it follows that the term $E_{i, 0}^{2}=\operatorname{Tor}_{i}^{A}(R, R)$ can only have nonzero components in the internal degrees $j=i-1$ and $i$. It remains to recall that $\operatorname{Tor}_{i, j}^{A}(R, R)=0$ for $j<i$ by Proposition [2.1(a). Thus the graded $R$ - $R$-bimodule $\operatorname{Tor}_{i}^{A}(R, R)$ is concentrated in the internal degree $j=i$.

The next result is a kind of Poincaré-Birkhoff-Witt theorem (see first proof of Theorem 4.19 in Section 4.6).

Theorem 4.4. Let $\widehat{A}$ be a quadratic graded ring and $t \in \widehat{A}_{1}$ be a central element. Assume that the left $R$-modules $\widehat{A}_{n}$ are flat for all $n \geq 1$ and the graded ring $A=\widehat{A} / \widehat{A} t$ is left flat Koszul. Assume further that the three maps $R \stackrel{t}{\longrightarrow} \widehat{A}_{1} \stackrel{t}{\longrightarrow} \widehat{A}_{2} \stackrel{t}{\longrightarrow} \widehat{A}_{3}$ are injectve. Then the central element $t$ is a nonzero-divisor in $\widehat{A}$.

Proof. For any graded module $M$ over a graded $\operatorname{ring} C$, let us denote by $M(1)$ the same module with the shifted grading, $M(1)_{n}=M_{n-1}$. Denote by $H \subset \widehat{A}$ the kernel of the multiplication map $\widehat{A} \stackrel{t}{\longrightarrow} \widehat{A}$. Then we have a four-term exact sequence of graded $\widehat{A}$ - $\widehat{A}$-bimodules

$$
0 \longrightarrow H(1) \longrightarrow \widehat{A}(1) \stackrel{t}{\longrightarrow} \widehat{A} \longrightarrow A \longrightarrow 0 .
$$

It follows that $\operatorname{Tor}_{2}^{\widehat{A}}(R, A) \simeq R \otimes_{\widehat{A}} H(1)$ and $\operatorname{Tor}_{0}^{\widehat{A}}\left(\operatorname{Tor}_{2}^{\widehat{A}}(R, A), R\right) \simeq R \otimes_{\widehat{A}} H(1) \otimes_{\widehat{A}} R$ as an internally graded $R$ - $R$-bimodule. We also have $\operatorname{Tor}_{1}^{\widehat{A}}(R, A)=R t \simeq R(1)$ and $\operatorname{Tor}_{0}^{\widehat{A}}(R, A)=R$.

By assumption, we have $H_{n}=0$ for $n \leq 2$. Arguing by induction in $n \geq 3$, we will prove that $H_{n}=0$ for all $n$. Assuming that $H_{n-1}=0$, the $R$ - $R$-bimodule $H_{n}$ is isomorphic to the degree $n$ component of the $R$ - $R$-bimodule $R \otimes_{\widehat{A}} H \otimes_{\widehat{A}} R$, or which is the same, the degree $n+1$ component of the $R$ - $R$-bimodule $R \otimes_{\widehat{A}} H(1) \otimes_{\widehat{A}} R$.

We will make use of the spectral sequence (48) for the graded ring homomorphism $C=\widehat{A} \longrightarrow A$. In the induction assumption as above, we need to check that the degree $n+1$ component of the term $E_{0,2}^{2}$ vanishes. Indeed, we have $\operatorname{Tor}_{2, j}^{\widehat{A}}(R, R)=0$ 
for $j \geq 3$ by Proposition 2.4(b) (since the graded ring $\widehat{A}$ is quadratic and its grading components are flat left $R$-modules by assumption), hence the term $E_{p, q}^{\infty}$ has no grading components in the internal degrees $j \geq 3$ when $p+q=2$. In particular, this applies to the term $E_{0,2}^{\infty}$.

The only possibly nontrivial differentials passing through $E_{0,2}^{r}$ with $r \geq 2$ are $d_{2,1}^{2}: E_{2,1}^{2} \longrightarrow E_{0,2}^{2}$ and $d_{3,0}^{3}: E_{3,0}^{3} \longrightarrow E_{0,2}^{3}$. Since the graded ring $A$ is left flat Koszul by assumption, we have $\operatorname{Tor}_{2, j}^{A}(R, R)=0$ for $j \geq 3$ and $\operatorname{Tor}_{3, j}^{A}(R, R)=0$ for $j \geq 4$ by Theorem 2.18(a). Therefore, both the terms $E_{2,1}^{2}$ and $E_{3,0}^{2}$ have no grading components in the internal degrees $j \geq 4$. It follows that the term $E_{0,2}^{2}$ cannot have a nonzero grading component in the degree $n+1 \geq 4$, and we are done.

4.2. Quasi-differential graded rings and CDG-rings. A quasi-differential graded ring $(\widehat{B}, \partial)$ is a graded associative ring $\widehat{B}=\bigoplus_{n \in \mathbb{Z}} \widehat{B}^{n}$ endowed with an odd derivation $\partial: \widehat{B} \longrightarrow \widehat{B}$ of degree -1 such that $\partial^{2}=0$ and the homology ring $H_{\partial}(\widehat{B})=\operatorname{ker} \partial / \operatorname{im} \partial$ vanishes. The latter condition holds (for an odd derivation $\partial$ of degree -1 satisfying $\left.\partial^{2}=0\right)$ if and only if the homology class of the unit element $1 \in \widehat{B}^{0}$ vanishes, that is, $1 \in \partial\left(\widehat{B}^{1}\right)$.

The underlying graded ring $B$ of a quasi-differential graded ring $(\widehat{B}, \partial)$ is defined as the kernel of the differential $\partial: \widehat{B} \longrightarrow \widehat{B}$, that is $B=\operatorname{ker} \partial \subset \widehat{B}$. A quasi-differential structure on a graded ring $B$ is the datum of a quasi-differential graded $\operatorname{ring}(\widehat{B}, \partial)$ together with a graded ring isomorphism $B \simeq \operatorname{ker} \partial \subset \widehat{B}$.

A morphism of quasi-differential graded rings $\hat{f}:\left({ }^{\prime \prime} \widehat{B}, \partial^{\prime \prime}\right) \longrightarrow\left({ }^{\prime} \widehat{B}, \partial^{\prime}\right)$ is a morphism of graded rings $\hat{f}:{ }^{\prime \prime} \widehat{B} \longrightarrow{ }^{\prime} \widehat{B}$ such that $\hat{f} \partial^{\prime \prime}=\partial^{\prime} \hat{f}$. The composition of morphisms of quasi-differential graded rings is defined in the obvious way. These rules define the category of quasi-differential graded rings.

A quasi-differential graded ring $(\widehat{B}, \partial)$ is said to be nonnegatively graded if its underlying graded ring $B$ is nonnegatively graded, $B=\bigoplus_{n=0}^{\infty} B^{n}$, or equivalently, the graded ring $\widehat{B}$ is nonnegatively graded, $\widehat{B}=\bigoplus_{n=0}^{\infty} \widehat{B}^{n}$. For a nonnegatively graded quasi-differential ring $(\widehat{B}, \partial)$, one has $B^{0}=\widehat{B}^{0}$.

We will denote the category of nonnegatively graded quasi-differential rings $(\widehat{B}, \partial)$ with the fixed degree-zero component $\widehat{B}^{0}=B^{0}=R$ by $R$-rings ${ }_{\mathrm{qdg}}$. Morphisms

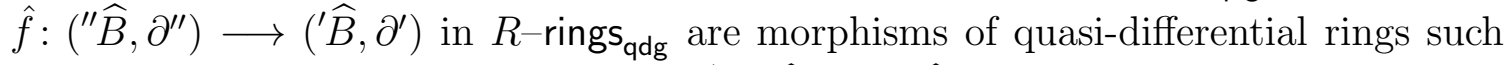
that the graded ring homomorphism $\hat{f}:{ }^{\prime \prime} \widehat{B} \longrightarrow{ }^{\prime} \widehat{B}$ forms a commutative triangle diagram with the fixed isomorphisms $R \simeq{ }^{\prime \prime} \widehat{B}^{0}$ and $R \simeq{ }^{\prime} \widehat{B}^{0}$, or equivalently, the induced homomorphism $f:{ }^{\prime \prime} B \longrightarrow{ }^{\prime} B$ between the graded rings ${ }^{\prime \prime} B=\operatorname{ker} \partial^{\prime \prime} \subset{ }^{\prime \prime} \widehat{B}$ and ' $B=\operatorname{ker} \partial^{\prime} \subset{ }^{\prime} \widehat{B}$ forms a commutative diagram with the fixed isomorphisms $R \simeq{ }^{\prime \prime} B^{0}$ and $R \simeq{ }^{\prime} B^{0}$.

Theorem 4.5. The category of quasi-differential graded rings is equivalent to the category of $C D G$-rings. In particular, for any fixed ring $R$, the category $R$-rings ${ }_{\text {qdg }}$ is equivalent to the category $R$-rings ${ }_{\mathrm{cdg}}$. 
Proof. To a CDG-ring $(B, d, h)$, one assigns the following quasi-differential graded ring $(\widehat{B}, \partial)$. The graded ring $\widehat{B}=B[\delta]$ is obtained by adjoining to the graded ring $B$ a new element $\delta \in \widehat{B}^{1}$ satisfying the relations

$$
[\delta, b]=\delta b-(-1)^{|b|} b \delta=d(b) \quad \text { for all } b \in B
$$

and

$$
\delta^{2}=h
$$

In other words, the elements of the grading component $\widehat{B}^{n}$ are all the formal expressions $b+c \delta$ with $b \in B^{n}$ and $c \in B^{n-1}$. The unit element in $\widehat{B}$ is $1_{\widehat{B}}=1_{B}+0 \delta$. The multiplication in $\widehat{B}$ is given by the formula

$$
\left(b^{\prime}+c^{\prime} \delta\right)\left(b^{\prime \prime}+c^{\prime \prime} \delta\right)=\left(b^{\prime} b^{\prime \prime}+c^{\prime} d\left(b^{\prime \prime}\right)+(-1)^{\left|c^{\prime \prime}\right|} c^{\prime} c^{\prime \prime} h\right)+\left(b^{\prime} c^{\prime \prime}+(-1)^{\left|b^{\prime \prime}\right|} c^{\prime} b^{\prime \prime}+c^{\prime} d\left(c^{\prime \prime}\right)\right) \delta .
$$

The odd derivation $\partial$ on $\widehat{B}$ can be described informally as the partial derivative $\partial=\partial / \partial \delta$. Explicitly, we put $\partial(b+c \delta)=(-1)^{|c|} c+0 \delta$. So the kernel ker $\partial \subset \widehat{B}$ clearly coincides with the subring $B \subset \widehat{B}$ embedded by the obvious rule $b \longmapsto b+0 \delta$.

Let $(\mathrm{id}, a):\left(B, d^{\prime \prime}, h^{\prime \prime}\right) \longrightarrow(B, d, h)$ be a change-of-connection isomorphism of CDG-rings; so $d^{\prime \prime}(b)=d^{\prime}(b)+[a, b]$ for all $b \in B$ and $h^{\prime \prime}=h^{\prime}+d^{\prime}(a)+a^{2}$. Let " $\widehat{B}=B\left[\delta^{\prime \prime}\right]$ and ' $\widehat{B}=B\left[\delta^{\prime}\right]$ denote the quasi-differential graded rings corresponding to $\left(B, d^{\prime \prime}, h^{\prime \prime}\right)$ and $\left(B, d^{\prime}, h^{\prime}\right)$, respectively. Then the rules $b \longmapsto b$ for all $b \in B$ and $\delta^{\prime \prime} \longmapsto \delta^{\prime}+a$ define an isomorphism of graded rings ${ }^{\prime \prime} \widehat{B} \simeq{ }^{\prime} \widehat{B}$ forming a commutative square diagram with the odd derivations $\partial^{\prime \prime}:{ }^{\prime \prime} \widehat{B} \longrightarrow{ }^{\prime \prime} \widehat{B}$ and $\partial^{\prime}:{ }^{\prime} \widehat{B} \longrightarrow{ }^{\prime} \widehat{B}$.

Generally, to a morphism of CDG-rings $(f, a):\left({ }^{\prime \prime} B, d^{\prime \prime}, h^{\prime \prime}\right) \longrightarrow\left({ }^{\prime} B, d^{\prime}, h^{\prime}\right)$ one assigns the morphism of quasi-differential graded rings $\hat{f}:\left({ }^{\prime \prime} \widehat{B}, \partial^{\prime \prime}\right) \longrightarrow\left({ }^{\prime} \widehat{B}, \partial^{\prime}\right)$, where the graded ring homomorphism $\hat{f}:{ }^{\prime \prime} \widehat{B}={ }^{\prime \prime} B\left[\delta^{\prime \prime}\right] \longrightarrow{ }^{\prime} B\left[\delta^{\prime}\right]={ }^{\prime} \widehat{B}$ takes any element $b \in{ }^{\prime \prime} B \subset{ }^{\prime \prime} \widehat{B}$ to the element $\hat{f}(b)=f(b) \in{ }^{\prime} B \subset{ }^{\prime} \widehat{B}$ and the element $\delta^{\prime \prime} \in{ }^{\prime \prime} \widehat{B}$ to the element $\hat{f}\left(\delta^{\prime \prime}\right)=\delta^{\prime}+a \in^{\prime} \widehat{B}$. This construction defines a functor from the category of CDG-rings to the category of quasi-differential graded rings, and one can easily see that this functor is fully faithful.

To construct the inverse functor, one needs to choose, for each quasi-differential graded ring $(\widehat{B}, \partial)$, an element $\delta \in \widehat{B}^{1}$ such that $\partial(\delta)=1$. Then the CDG-ring $(B, d, h)$ is recovered by the rules $B=\operatorname{ker} \partial \subset \widehat{B}, d(b)=[\delta, b]$ for all $b \in B \subset \widehat{B}$, and $h=\delta^{2}$. One has $\partial([\delta, b])=[\partial(\delta), b]-[\delta, \partial(b)]=[1, b]-[\delta, 0]=0$ for all $b \in B$ and $\partial\left(\delta^{2}\right)=[\partial(\delta), \delta]=[1, \delta]=0$, hence $[\delta, b] \in B$ and $\delta^{2} \in B$, as desired. The construction of the morphism of CDG-rings assigned to a morphism of quasidifferential graded rings is obvious from the above.

One can define the 2-category of quasi-differential graded rings in the way similar to the definition of the 2-category of DG-rings in Section 3.8. Let $\hat{f}$, $\hat{g}:\left({ }^{\prime \prime} \widehat{B}, \partial^{\prime \prime}\right) \longrightarrow\left({ }^{\prime} \widehat{B}, \partial^{\prime}\right)$ be a pair of parallel morphisms of quasi-differential graded rings. A 2-morphism $\hat{f} \stackrel{z}{\longrightarrow} \hat{g}$ is an invertible element $z \in{ }^{\prime} B^{0}=\operatorname{ker}\left(\partial_{0}:{ }^{\prime} \widehat{B}^{0} \rightarrow{ }^{\prime} \widehat{B}^{-1}\right)$ 
such that $\hat{g}(c)=z \hat{f}(c) z^{-1}$ for all $c \in \in^{\prime \prime} \widehat{B}$. The composition of 2-morphisms is defined in the same way as in Section 3.8.

The 2-category Rings ${ }_{\text {qdg2 }}$ of nonnegatively graded quasi-differential rings is defined as the following subcategory of the 2-category of quasi-differential graded rings. The objects of Rings qdg2 $_{2}$ are nonnegatively graded quasi-differential rings $(\widehat{B}, \partial)$. Morphisms $\hat{f}:\left({ }^{\prime \prime} \widehat{B}, \partial^{\prime \prime}\right) \longrightarrow\left({ }^{\prime} \widehat{B}, \partial^{\prime}\right)$ in Rings ${ }_{\text {qdg2 }}$ are morphisms of quasi-differential graded rings such that the map $\hat{f}_{0}:{ }^{\prime \prime} \widehat{B}^{0} \longrightarrow{ }^{\prime} \widehat{B}^{0}$ is an isomorphism. 2-morphisms $\hat{f} \stackrel{z}{\longrightarrow} \hat{g}$ in Rings ${ }_{\mathrm{qd} 2}$ between morphisms $\hat{f}$ and $\hat{g}$ belonging to Rings ${ }_{\mathrm{qdg} 2}$ are arbitrary 2-morphisms from $\hat{f}$ to $\hat{g}$ in the 2-category of quasi-differential graded rings.

Theorem 4.6. The equivalence of categories from Theorem 4.5 can be extended to a strict equivalence between the 2-category of CDG-rings and the 2-category of quasi-differential graded rings. In particular, the 2-category of nonnegatively graded $C D G$-rings Rings $_{\mathrm{cdg}_{2}}$ is strictly equivalent to the 2-category of quasi-differential graded rings Rings $_{\mathrm{qdg} 2}$.

Proof. Let $(f, a)$ and $(g, b):\left({ }^{\prime \prime} B, d^{\prime \prime}, h^{\prime \prime}\right) \longrightarrow\left({ }^{\prime} B, d^{\prime}, h^{\prime}\right)$ be a pair of parallel morphisms of CDG-rings and $\hat{f}, \hat{g}$ : ${ }^{\prime \prime} \widehat{B}={ }^{\prime \prime} B\left[\delta^{\prime \prime}\right] \longrightarrow{ }^{\prime} B\left[\delta^{\prime}\right]={ }^{\prime} \widehat{B}$ be the corresponding pair of parallel morphisms of quasi-differential graded rings. Let $z \in \in^{\prime} B^{0}$ be an invertible element. We leave it to the reader to check that $(f, a) \stackrel{z}{\longrightarrow}(g, b)$ is a 2-morphism in the 2-category of CDG-rings if and only if $\hat{f} \stackrel{z}{\longrightarrow} \hat{g}$ is a 2-morphism in the 2-category of quasi-differential graded rings.

4.3. Quadratic quasi-differential graded rings. We will say that a nonnegatively graded quasi-differential ring $(\widehat{B}, \partial)$ is quadratic if its underlying graded $\operatorname{ring} B=$ ker $\partial \subset \widehat{B}$ is quadratic.

Lemma 4.7. Let $(\widehat{B}, \partial)$ be a nonnegatively graded quasi-differential ring and $B=$ ker $\partial \subset \widehat{B}$ be its underlying graded ring. Then

(a) the graded ring $\widehat{B}$ is generated by $\widehat{B}^{1}$ over $\widehat{B}^{0}$ whenever the graded ring $B$ is generated by $B^{1}$ over $B^{0}$;

(b) the graded ring $\widehat{B}$ is quadratic whenever the graded ring $B$ is quadratic.

Proof. Part (a): following the proof of Theorem 4.5, we have $\widehat{B}=B[\delta]$, where $\delta \in \widehat{B}^{1}$. So the ring $\widehat{B}$ is generated by $B$ and $\delta$; and if $B$ is generated by $B^{1}$ over $B^{0}$, then it follows that $\widehat{B}$ is generated by $\widehat{B}^{1}$ over $\widehat{B}^{0}=B^{0}$.

Part (b): the ring $\widehat{B}=B[\delta]$ is generated by the ring $B$ and the adjoined element $\delta$ with the defining relations (49) 50). The relation (50) has degree 2; and it remains to observe that, whenever the $\operatorname{ring} B$ is generated by $B^{1}$ over $B^{0}$, it suffices to impose the relation (49) for elements $b \in B^{0}$ and $B^{1}$ only. This makes the ring $\widehat{B}$ defined by relations of degree $\leq 2$ between generators of degree $\leq 1$ provided that the ring $B$ can be so defined.

Lemma 4.8. Let $(\widehat{B}, \partial)$ be a nonnegatively graded quasi-differential ring and $B=$ ker $\partial \subset \widehat{B}$ be its underlying graded ring. 
(a) Assume that the graded ring $\widehat{B}$ is generated by $\widehat{B}^{1}$ over $\widehat{B}^{0}$. Then the graded ring $B$ is generated by $B^{1}$ over $B^{0}$ if and only if the component $B^{2}$ is generated by $B^{1}$, that is, the multiplication map $B^{1} \otimes_{B^{0}} B^{1} \longrightarrow B^{2}$ is surjective.

(b) Assume that the graded ring $B$ is generated by $B^{1}$ over $B^{0}$ and the graded ring $\widehat{B}$ is quadratic. Then the graded ring $B$ is quadratic if and only if it has no relations of degree 3 , that is, in other words, the natural homomorphism of graded rings $\mathrm{q} B \longrightarrow B$ is injective in degree 3 .

Proof. The "only if" assertion in both (a) and (b) is obvious. To prove the "if", choose an element $\delta \in \widehat{B}^{1}$ such that $\partial(\delta)=1$ and consider the CDG-ring $(B, d, h)$ corresponding to $(\widehat{B}, \partial)$ under the equivalence of categories from Theorem 4.5 .

Part (a): let ${ }^{\prime} B \subset B$ denote the graded subring in $B$ generated by ${ }^{\prime} B^{1}=B^{1}$ over ${ }^{\prime} B^{0}=B^{0}$. By assumption, we have ' $B^{2}=B^{2}$. Hence the curvature element $h \in B^{2}$ belongs to ${ }^{\prime} B$. Moreover, we have $d\left({ }^{\prime} B^{1}\right) \subset{ }^{\prime} B^{2}$, and it follows that $d\left({ }^{\prime} B\right) \subset{ }^{\prime} B \subset B$. Thus $\left({ }^{\prime} B,\left.d\right|^{\prime} B, h\right)$ is a CDG-ring. Let ${ }^{\prime} \widehat{B}={ }^{\prime} B[\delta]$ be the quasi-differential graded ring corresponding to the CDG-ring $\left({ }^{\prime} B,\left.d\right|^{\prime} B, h\right)$ under the construction of Theorem 4.5. Then ${ }^{\prime} \widehat{B}$ is naturally a graded subring in $\widehat{B}$. Furthermore, we have ${ }^{\prime} \widehat{B}^{0}=\widehat{B}^{0}$ and ${ }^{\prime} \widehat{B}^{1}=\widehat{B}^{1}$, since ${ }^{\prime} B^{0}=B^{0}$ and ${ }^{\prime} B^{1}=B^{1}$. Since the graded ring $\widehat{B}$ is generated by $\widehat{B}^{1}$ over $\widehat{B}^{0}$ by assumption, it follows that ${ }^{\prime} \widehat{B}=\widehat{B}$. In view of the equivalence of categories from Theorem 4.5, we can conclude that ${ }^{\prime} B=B$.

Part (b): the natural homomorphism of graded rings $\mathrm{q} B \longrightarrow B$, which we will denote by $f$, is surjective in our assumptions. If it is injective in degree 3 , this means that it is an isomorphism in degrees 2 and 3 . So we can consider the curvature element $h \in B^{2}$ as an element of $\mathrm{q} B^{2}$.

Furthermore, the components $d_{0}: B^{0} \longrightarrow B^{1}$ and $d_{1}: B^{1} \longrightarrow B^{2}$ of the odd derivation $d: B \longrightarrow B$ can be considered as maps $d_{0}^{\prime}: \mathrm{q} B^{0} \longrightarrow \mathrm{q} B^{1}$ and $d_{1}^{\prime}: \mathrm{q} B^{1} \longrightarrow \mathrm{q} B^{2}$. The possibility of extending these maps to an odd derivation $d^{\prime}: \mathrm{q} B \longrightarrow \mathrm{q} B$ presents itself if and only if they respect the defining relations of the graded ring $\mathrm{q} B$, which all live in the degrees $\leq 2$. The same relations hold in the graded ring $B$. Applying the maps $d_{0}^{\prime}$ and $d_{1}^{\prime}$ to the defining relations of $\mathrm{q} B$, one obtains equations for elements of the components $\mathrm{q} B^{1}, \mathrm{q} B^{2}$, and $\mathrm{q} B^{3}$. These are isomorphic to $B^{1}, B^{2}$, and $B^{3}$, respectively, via the graded ring homomorphism $f: \mathrm{q} B \longrightarrow B$. Since the odd derivation $d: B \longrightarrow B$ exists, it follows that one can indeed extend the maps $d_{0}^{\prime}$ and $d_{1}^{\prime}$ to an odd derivation $d^{\prime}: \mathrm{q} B \longrightarrow \mathrm{q} B$.

Finally, the equation $d^{\prime}(h)=0$ holds in $\mathrm{q} B^{3}$ since the equation $d(h)=0$ holds in $B^{3}$. Concerning the equation $d^{\prime}\left(d^{\prime}(b)\right)=[h, b]$ for all $b \in \mathrm{q} B$, it suffices to check it for $b \in \mathrm{q} B^{0}$ and $\mathrm{q} B^{1}$, since the graded ring $\mathrm{q} B$ is generated by $\mathrm{q} B^{1}$ over $\mathrm{q} B^{0}$. Once again, the equations need to hold in the components $\mathrm{q} B^{2}$ and $\mathrm{q} B^{3}$, which are isomorphic to $B^{2}$ and $B^{3}$ via the graded ring homomorphism $f$. Since $(B, d, h)$ is a CDG-ring, it follows that (q $\left.B, d^{\prime}, h\right)$ is a CDG-ring as well. We have a strict morphism of CDG-rings $(f, 0):\left(\mathrm{q} B, d^{\prime}, h\right) \longrightarrow(B, d, h)$.

Let ${ }^{\prime} \widehat{B}=(\mathrm{q} B)[\delta]$ denote the quasi-differential graded ring corresponding to the CDG-ring (q $\left.B, d^{\prime}, h\right)$ under the construction of Theorem 4.5: and let $\hat{f}:{ }^{\prime} \widehat{B} \longrightarrow \widehat{B}$ 
denote the morphism of quasi-differential graded rings corresponding to the CDG-ring morphism $(f, 0)$. Then the graded ring homomorphism $\hat{f}$ is an isomorphism in degrees 0,1 , and 2 , since so is the graded ring homomorphism $f$. Since the graded ring $\widehat{B}$ is quadratic by assumption and the graded ring ' $\widehat{B}$ is quadratic by Lemma 4.7, we can conclude that the graded ring homomorphism $\hat{f}:{ }^{\prime} \widehat{B} \longrightarrow \widehat{B}$ is an isomorphism (in all the degrees) by Lemma 3.2, In view of the equivalence of categories from Theorem 4.5, it follows that the graded ring homomorphism $f: \mathrm{q} B \longrightarrow B$ is an isomorphism, too. Thus the graded $\operatorname{ring} B$ is quadratic.

Lemma 4.9. Let $(\widehat{B}, \partial)$ be a nonnegatively graded quasi-differential ring and $B=$ ker $\partial \subset \widehat{B}$ be its underlying graded ring. Let $n \geq 0$ be an integer. Then $\widehat{B}^{j}$ is a finitely generated projective (projective, or flat) right module over the ring $R=\widehat{B}^{0}=B^{0}$ for all $0 \leq j \leq n$ if and only if $B^{j}$ is a finitely generated projective (resp., projective or flat) right $R$-module for all $0 \leq j \leq n$.

Proof. First we observe that, for any the odd derivation $\partial$ of degree -1 on a nonnegatively graded ring $\widehat{B}$, the component $\partial_{0}: \widehat{B}^{0} \longrightarrow \widehat{B}^{-1}$ vanishes, since $\widehat{B}^{-1}=0$. In other words, we have $\partial(R)=0$; hence $\partial$ is an $R$ - $R$-bimodule map.

Returning to the situation at hand, the case $n=0$ is obvious. Arguing by induction in $n$, it suffices to assume that $B^{n-1}$ is a finitely generated projective (resp., projective or flat) right $R$-module, and prove that $\widehat{B}^{n}$ is a finitely generated projective (resp., projective or flat) right $R$-module if and only if $B^{n}$ is. This is clear from the short exact sequence of $R$ - $R$-bimodules $0 \longrightarrow B^{n} \longrightarrow \widehat{B}^{n} \stackrel{\partial}{\longrightarrow} B^{n-1} \longrightarrow 0$.

The following theorem is the version of a very specific particular case of [26, Corollary $6.2(\mathrm{~b})]$.

Theorem 4.10. Let $(\widehat{B}, \partial)$ be a nonnegatively graded quasi-differential ring and $B=$ ker $\partial \subset \widehat{B}$ be its underlying graded ring. Then

(a) the graded ring $\widehat{B}$ is right flat Koszul whenever the graded ring $B$ is;

(b) the graded ring $\widehat{B}$ is right finitely projective Koszul whenever the graded ring $B$ is.

Proof. In view of Lemma 4.9, it suffices to prove part (a). Then, by the same lemma, we know that $\widehat{B}^{n}$ is a flat right $R$-module for every $n \geq 0$.

In the notation of the proof of Theorem 4.4, we have a short exact sequence of $R$ - $B$-bimodules $0 \longrightarrow B \longrightarrow \widehat{B} \stackrel{\partial}{\longrightarrow} B(1) \longrightarrow 0$. So the graded right $B$-module $\widehat{B}$ is projective (in fact, free). Hence the spectral sequence (48) for the morphism of graded rings $B \longrightarrow \widehat{B}$ (with the roles of the left and right sides switched) degenerates to an isomorphism

$$
\operatorname{Tor}_{i}^{B}(R, R) \simeq \operatorname{Tor}_{i}^{\widehat{B}}\left(R, \operatorname{Tor}_{0}^{B}(\widehat{B}, R)\right) \text { for all } i \geq 0 .
$$

Furthermore, the internally graded $R$ - $R$-bimodule $\operatorname{Tor}_{0}^{B}(\widehat{B}, R)$ can be computed as

$$
\widehat{B} / \widehat{B} B^{+}=\operatorname{Tor}_{0}^{B}(\widehat{B}, R)=R \oplus R(1),
$$


where $B^{+}=\bigoplus_{n=1}^{\infty} B^{n}$. The $R$ - $R$-bimodule direct summand $R(1) \subset \operatorname{Tor}_{0}^{B}(\widehat{B}, R)$ is a left $\widehat{B}$-subbimodule, since the ring $\widehat{B}$ is nonnegatively graded. So we have a short exact sequence of graded $\widehat{B}$ - $R$-bimodules

$$
0 \longrightarrow R(1) \longrightarrow \operatorname{Tor}_{0}^{B}(\widehat{B}, R) \longrightarrow R \longrightarrow 0,
$$

where $\widehat{B}$ acts in $R(1)$ and in $R$ via the augmentation map $B \longrightarrow B / B^{+}=R$. We are arriving to a long exact sequence of $R$ - $R$-bimodules

$$
\begin{aligned}
\cdots \longrightarrow \operatorname{Tor}_{i, j-1}^{\widehat{B}}(R, R) \longrightarrow & \operatorname{Tor}_{i, j}^{B}(R, R) \\
& \longrightarrow \operatorname{Tor}_{i, j}^{\widehat{B}}(R, R) \longrightarrow \operatorname{Tor}_{i-1, j-1}^{\widehat{B}}(R, R) \longrightarrow \cdots
\end{aligned}
$$

where the index $i \geq 0$ denotes the homological grading, while the index $j \geq 0$ denotes the internal grading of the Tor induced by the grading of $B$ and $\widehat{B}$.

Now if $\operatorname{Tor}_{i, j}^{B}(R, R)=0$ for $i \neq j$ and $\operatorname{Tor}_{i-1, j-1}^{\widehat{B}}(R, R)=0$, then it follows that $\operatorname{Tor}_{i, j}^{\widehat{B}}(R, R)=0$. Arguing by induction in $i \geq 0$ (or in $j \geq 0$ ), one proves that $\operatorname{Tor}_{i, j}^{\widehat{B}}(R, R)=0$ for $i \neq j$.

4.4. Central elements and acyclic derivations. Let $\widehat{A}=\bigoplus_{n=0}^{\infty} \widehat{A}_{n}$ be a 2-left finitely projective quadratic graded ring with the degree-zero component $R=\widehat{A}_{0}$, and let $\widehat{B}=\bigoplus_{n=0}^{\infty} \widehat{B}^{n}, \widehat{B}^{0}=R$, be the 2-right finitely projective quadratic graded ring quadratic dual to $\widehat{A}$. The aim of this section is to establish and study a correspondence between central elements $t \in \widehat{A}_{1}$ and odd derivations $\partial: \widehat{B} \longrightarrow \widehat{B}$ of degree -1 .

First of all, to every element $t \in A_{1} \simeq \operatorname{Hom}_{R^{\text {op }}}\left(\widehat{B}^{1}, R\right)$ we assign a right $R$-module morphism $\partial_{1}: \widehat{B}^{1} \longrightarrow R$, and vice versa. This is done in the obvious way except for a sign rule. In the pairing notation of Section 3.4, we put

$$
\partial_{1}(b)=-\langle t, b\rangle \in R \quad \text { for all } b \in \widehat{B}^{1} .
$$

Lemma 4.11. For any element $t \in \widehat{A}_{1}$, consider the related right $R$-module morphism $\partial_{1}: \widehat{B}^{1} \longrightarrow R$. Then

(a) the element $t$ commutes with all the elements of $\widehat{A}_{0}=R$ in $\widehat{A}$ if and only if the map $\partial_{1}$ is an $R$-R-bimodule morphism;

(b) the element $t$ is central in $\widehat{A}$ if and only if the map $\partial_{1}$ can be (uniquely) extended to an odd derivation $\partial: \widehat{B} \longrightarrow \widehat{B}$ of degree -1 .

Proof. Part (a): we have $\partial_{1}(b)=-\langle t, b\rangle$ for all $b \in \widehat{B}^{1}$. Furthermore,

$$
\langle r t, b\rangle=r\langle t, b\rangle \quad \text { and } \quad\langle t r, b\rangle=\langle t, r b\rangle \quad \text { for all } r \in R \text { and } b \in \widehat{B}^{1} .
$$

Hence one has $r t=t r$ for all $r \in R$ if and only if $r \partial_{1}(b)=\partial_{1}(r b)$ for all $r \in R$ and $b \in \widehat{B}^{1}$, i. e., if and only if $\partial_{1}$ is a left $R$-module map.

Part (b): any odd derivation $\partial: \widehat{B} \longrightarrow \widehat{B}$ of degree -1 is an $R$ - $R$-bimodule map, as it was explained in the proof of Lemma 4.9. Moreover, the odd derivation $\partial$ is uniquely determined by its component $\partial_{1}$, since the graded ring $\widehat{B}$ is generated by 
$\widehat{B}^{1}$ over $\widehat{B}^{0}$. Similarly, the element $t$ is central in $\widehat{A}$ if and only if it commutes with all the elements of $\widehat{A}_{0}$ and $\widehat{A}_{1}$, since the graded ring $\widehat{A}$ is generated by $\widehat{A}_{1}$ over $\widehat{A}_{0}$.

So we can assume that both the equivalent conditions of part (a) hold, and it remains to prove that the element $t$ commutes with all the elements of $\widehat{A}_{1}$ if and only if the $R$ - $R$-bimodule map $\partial_{1}: \widehat{B}^{1} \longrightarrow \widehat{B}^{0}$ can be extended to an odd derivation of degree -1 . The latter condition can be equivalently restated as follows. Consider the $R$ - $R$-bimodule morphism

$$
\tilde{\partial}_{2}: \widehat{B}^{1} \otimes_{R} \widehat{B}^{1} \longrightarrow \widehat{B}^{1}
$$

defined by the formula

$$
\tilde{\partial}_{2}\left(b_{1} \otimes b_{2}\right)=\partial_{1}\left(b_{1}\right) b_{2}-b_{1} \partial_{1}\left(b_{2}\right) \quad \text { for all } b_{1}, b_{2} \in \widehat{B}^{1} \text {. }
$$

For any $R$ - $R$-bimodule map $\partial_{1}: \widehat{B}^{1} \longrightarrow R$, the corresponding map $\tilde{\partial}_{2}$ is well-defined. Since the graded ring $\widehat{B}$ is quadratic, the map $\partial_{1}$ extends to an odd derivation of $\widehat{B}$ if and only if the map $\tilde{\partial}_{2}$ annihilates the kernel $\widehat{J} \subset \widehat{B}^{1} \otimes_{R} \widehat{B}^{1}$ of the surjective multiplication map $\widehat{B}^{1} \otimes_{R} \widehat{B}^{1} \longrightarrow \widehat{B}^{2}$. This is provable using Lemma 3.7.

On the other hand, the condition that the element $t$ commutes with all the elements of $\widehat{A}_{1}$ can be restated as follows. Consider the $R$ - $R$-bimodule morphism

$$
\tilde{t}: \widehat{A}_{1} \longrightarrow \widehat{A}_{1} \otimes_{R} \widehat{A}_{1}
$$

defined by the formula

$$
\tilde{t}(a)=a \otimes t-t \otimes a .
$$

Then the element $t \in \widehat{A}_{1}$ commutes with all the elements of $\widehat{A}_{1}$ if and only if the composition of the map $\tilde{t}$ with the surjective multiplication map $\widehat{A}_{1} \otimes_{R} \widehat{A}_{1} \longrightarrow \widehat{A}_{2}$ vanishes. In order to prove the desired equivalence, it remains to recall and observe that the contravariant functor $\operatorname{Hom}_{R}(-, R)$ transforms the $R$-R-bimodule $\widehat{A}_{1}$ into the $R$ - $R$-bimodule $\widehat{B}^{1}$, the $R$ - $R$-bimodule $\widehat{A}_{1} \otimes_{R} \widehat{A}_{1}$ into the $R$ - $R$-bimodule $\widehat{B}^{1} \otimes_{R} \widehat{B}^{1}$, the map $\tilde{t}$ into the map $-\tilde{\partial}_{2}$, the $R$-R-bimodule $\widehat{A}_{2}$ into the $R$ - $R$-bimodule $\widehat{J}$, and the surjection $\widehat{A}_{1} \otimes_{R} \widehat{A}_{1} \longrightarrow \widehat{A}_{2}$ into the inclusion $\widehat{J} \longrightarrow \widehat{B}^{1} \otimes_{R} \widehat{B}^{1}$. Besides, the $R$ - $R$-bimodules $\widehat{A}_{1}, \widehat{A}_{1} \otimes_{R} \widehat{A}_{1}$, and $\widehat{A}_{2}$ are finitely generated and projective as left $R$-modules, while the $R$ - $R$-bimodules $\widehat{B}^{1}, \widehat{B}^{1} \otimes_{R} \widehat{B}^{1}$, and $\widehat{J}$ are finitely generated and projective as right $R$-modules; so the contravariant functor $\operatorname{Hom}_{R^{\text {op }}}(-, R)$ performs the inverse transformation.

Lemma 4.12. Let $\partial: \widehat{B} \longrightarrow \widehat{B}$ be an odd derivation of degree -1 . Then

(a) $\partial^{2}=0$;

(b) the homology ring $H_{\partial}(\widehat{B})$ vanishes if and only if, for the central element $t \in \widehat{A}_{1}$ corresponding to $\partial$, the multiplication map $\widehat{A}_{0} \stackrel{t}{\longrightarrow} \widehat{A}_{1}$ is injective and its cokernel $A_{1}=\widehat{A}_{1} / \widehat{A}_{0} t$ is a projective left $R$-module.

Proof. Part (a): the compositions $\partial_{-1} \partial_{0}: \widehat{B}^{0} \longrightarrow \widehat{B}^{-1} \longrightarrow \widehat{B}^{-2}$ and $\partial_{0} \partial_{1}: \widehat{B}^{1} \longrightarrow$ $\widehat{B}^{0} \longrightarrow \widehat{B}^{-1}$ vanish, since $\widehat{B}^{-1}=0$. Since the square of an odd derivation is a derivation and the graded ring $\widehat{B}$ is generated by $\widehat{B}^{1}$ over $\widehat{B}^{0}$, it follows that $\partial^{2}=0$ 
on the whole graded ring $\widehat{B}$. Part (b) is a particular case of the assertion that a morphism of finitely generated projective left $R$-modules $t: \widehat{A}_{0} \longrightarrow \widehat{A}_{1}$ is injective with a projective cokernel if and only if the dual morphism of finitely generated projective right $R$-modules $-\partial_{1}: \widehat{B}^{1} \longrightarrow \widehat{B}^{0}$ is surjective.

Proposition 4.13. Let $\widehat{A}$ be a 2-left finitely projective quadratic graded ring and $\widehat{B}$ be the quadratic dual 2-right finitely projective quadratic graded ring. Let $t \in \widehat{A}_{1}$ be a central element and $\partial: \widehat{B} \longrightarrow \widehat{B}$ be the corresponding odd derivation of degree -1 (see Lemma 4.11(b)). Assume that the equivalent conditions of Lemma 4.12(b) are satisfied. Let $B=\operatorname{ker} \partial \subset \widehat{B}$ be the underlying graded ring of the quasi-differential graded ring $(\widehat{B}, \partial)$, and let $A=\widehat{A} / \widehat{A} t$ be the quotient ring. Then

(a) the graded ring $B$ is generated by $B^{1}$ over $B^{0}$ if and only if the multiplication map $\widehat{A}_{1} \stackrel{t}{\longrightarrow} \widehat{A}_{2}$ is injective and its cokernel $A_{2}=\widehat{A}_{2} / \widehat{A}_{1} t$ is a projective left $R$-module;

(b) if the equivalent conditions of part (a) hold, then the 2-right finitely projective quadratic graded ring $\mathrm{q} B$ is quadratic dual to the 2-left finitely projective quadratic graded ring $A$. The composition of graded ring homomorphisms $\mathrm{q} B \longrightarrow B \longrightarrow \widehat{B}$ is the morphism of 2-right finitely projective quadratic graded rings corresponding to the surjective morphism of 2-left finitely projective quadratic graded rings $\widehat{A} \longrightarrow A$ under the equivalence of categories from Proposition 1.2.

Proof. Part (a): according to Lemma 4.8(a), the graded ring $B$ is generated by $B^{1}$ over $B^{0}$ if and only if the multiplication map $B^{1} \otimes_{R} B^{1} \longrightarrow B^{2}$ is surjective. In the notation of the proof of Lemma 4.11(b), we have

$$
B^{1} \otimes_{R} B^{1} \subset \operatorname{ker} \tilde{\partial}_{2} \subset \widehat{B}^{1} \otimes_{R} \widehat{B}^{1} .
$$

Here the map $B^{1} \otimes_{R} B^{1} \longrightarrow \widehat{B}^{1} \otimes_{R} \widehat{B}^{1}$ induced by the inclusion of the $R$ - $R$-subbimodule $B^{1}$ into the $R$ - $R$-bimodule $\widehat{B}^{1}$ is injective, because the right $R$-module $\widehat{B}^{1}$ is projective and the quotient bimodule $\widehat{B}^{1} / B^{1}$, being naturally isomorphic to $R$ via the surjective $R$ - $R$-bimodule map $\partial_{1}: \widehat{B}^{1} \longrightarrow \widehat{B}^{0}$, is also projective as a right $R$-module. The inclusion $B^{1} \otimes_{R} B^{1} \subset$ ker $\tilde{\partial}_{2}$ is clear from the construction of the map $\tilde{\partial}_{2}$.

Furthermore, following the proof of Lemma 4.11(b), we have $\widehat{J} \subset \operatorname{ker} \tilde{\partial}_{2}$, since $\partial$ is an odd derivation of $\widehat{B}$ by assumption. The two $R$ - $R$-bimodule maps

$$
\widehat{B}^{1} \otimes_{R} \widehat{B}^{1} \rightrightarrows \widehat{B}^{1}, \quad b_{1} \otimes_{R} b_{2} \longmapsto \partial\left(b_{1}\right) b_{2} \text { and } b_{1} \partial\left(b_{2}\right)
$$

restrict to one and the same map $u$ : ker $\tilde{\partial}_{2} \longrightarrow \widehat{B}_{1}$. The subbimodule $B^{1} \otimes_{R} B^{1} \subset$ ker $\tilde{\partial}_{2}$ is the kernel of the map $u$.

The map $\tilde{\partial}_{2}$ is equal to the composition of the surjective multiplication map $\widehat{B}^{1} \otimes_{R}$ $\widehat{B}^{1} \longrightarrow \widehat{B}^{2}$ with the map $\partial_{2}: \widehat{B}^{2} \longrightarrow \widehat{B}^{1}$. By the definition, $B^{2} \subset \widehat{B}^{2}$ is the kernel of the latter map. It follows that the multiplication map $B^{1} \otimes_{R} B^{1} \longrightarrow B^{2}$ is surjective if and only if one has ker $\tilde{\partial}_{2}=\widehat{J}+B^{1} \otimes_{R} B^{1}$. The latter condition holds if and only if the map

is surjective.

$$
\bar{u}=\left.u\right|_{\widehat{J}}: \widehat{J} \longrightarrow \widehat{B}^{1}
$$


It remains to observe that the functor $\operatorname{Hom}_{R}(-, R)$ transforms the map $\widehat{A}_{1} \stackrel{t}{\longrightarrow} \widehat{A}_{2}$ into the map $-\bar{u}$. Thus the map $\bar{u}$ is surjective if and only if the map $t$ is injective and its cokernel $A_{2}$ is a projective left $R$-module.

In part (b), the graded ring $A$ is quadratic by (the proof of) Proposition 4.1(b) and 2-left finitely projective by assumptions; while the quadratic graded ring $\mathrm{q} B$ is 2-right finitely projective by Lemma 4.9. Furthermore, whenever the equivalent conditions of Lemma 4.12(b) hold, the functor $\operatorname{Hom}_{R}(-, R)$ transforms the $R$-R-bimodule $A_{1}$ into the $R$-R-bimodule $B^{1}$. This is clear from the proof of Lemma 4.12(b). Finally, whenever the equivalent conditions of part (a) of the present proposition hold, the functor $\operatorname{Hom}_{R}(-, R)$ transforms the $R$ - $R$-bimodule $A_{2}$ into the $R$ - $R$-bimodule

$$
\operatorname{ker} \bar{u}=\widehat{J} \cap\left(B^{1} \otimes_{R} B^{1}\right) \subset \widehat{B}^{1} \otimes_{R} \widehat{B}^{1} .
$$

The latter $R$-R-bimodule is the kernel of the surjective multiplication map $B^{1} \otimes_{R}$ $B^{1} \longrightarrow B^{2}$. Then one has to check that the same functor transforms the surjective multiplication map $A_{1} \otimes_{R} A_{1} \longrightarrow A_{2}$ into the inclusion map ker $\bar{u} \longrightarrow B^{1} \otimes_{R} B^{1}$.

Proposition 4.14. Let $\widehat{A}$ be a 3-left finitely projective quadratic graded ring and $\widehat{B}$ be the quadratic dual 3-right finitely projective quadratic graded ring. Let $t \in$ $\widehat{A}_{1}$ be a central element and $\partial: \widehat{B} \longrightarrow \widehat{B}$ be the corresponding odd derivation of degree -1 . Assume that the equivalent conditions of Lemma 4.12(b) are satisfied and the equivalent conditions of Proposition 4.13(a) are satisfied as well. Then the graded ring $B$ is quadratic if and only if the multiplication map $\widehat{A}_{2} \stackrel{t}{\longrightarrow} \widehat{A}_{3}$ is injective and its cokernel $A_{3}=\widehat{A}_{3} / \widehat{A}_{2} t$ is a projective left $R$-module.

Proof. By Lemma 4.9, the right $R$-module $B^{3}$ is projective in our assumptions. So, if the graded ring $B$ is quadratic, then it is 3-right finitely projective quadratic. Since $\mathrm{q} B$ is quadratic dual to $A$ by Proposition 4.13 (b), it then follows by virtue of Proposition 1.3 that the quadratic graded ring $A$ is 3 -left finitely projective. Thus we can assume that $A_{3}$ is a projective left $R$-module in all cases.

Then, again by Propositions 4.13(b) and 1.3, it follows that the quadratic graded ring $\mathrm{q} B$ is 3-right finitely projective. According to Lemma 4.8(b), the graded ring $B$ is quadratic if and only if the surjective homomorphism of graded rings $\mathrm{q} B \longrightarrow B$ is an isomorphism in degree 3. Equivalently, this means that the composition $\mathrm{q} B^{3} \longrightarrow$ $B^{3} \longrightarrow \widehat{B}^{3}$ is an injective map. Furthermore, since both $\mathrm{q} B^{3}$ and $B^{3}$ are projective right $R$-modules, and since the right $R$-module $\widehat{B}^{3} / B^{3} \simeq B^{2}$ is projective as well, we can conclude that the map q $B^{3} \longrightarrow B^{3}$ is an isomorphism if and only if applying the functor $\operatorname{Hom}_{R^{\text {op }}}(-, R)$ to the composition $\mathrm{q} B^{3} \longrightarrow B^{3} \longrightarrow \widehat{B}^{3}$ produces a surjective $\operatorname{map} f: \operatorname{Hom}_{R^{\text {op }}}\left(\widehat{B}^{3}, R\right) \longrightarrow \operatorname{Hom}_{R^{\text {op }}}\left(\mathrm{q} B^{3}, R\right)$.

Denote by $I \subset A_{1} \otimes_{R} A_{1}$ and $\widehat{I} \subset \widehat{A}_{1} \otimes_{R} \widehat{A}_{1}$ the kernels of the multiplication maps $A_{1} \otimes_{R} A_{1} \longrightarrow A_{1}$ and $\widehat{A}_{1} \otimes_{R} \widehat{A}_{1} \longrightarrow \widehat{A}_{1}$. Both the graded rings $A$ and $\widehat{A}$ are 3 -left finitely projective quadratic in our assumptions; q $B$ and $\widehat{B}$, respectively, are their quadratic dual 3-right finitely projective quadratic rings. Put

$$
I^{(3)}=\left(I \otimes_{R} A_{1}\right) \cap\left(A_{1} \otimes_{R} I\right) \subset A_{1} \otimes_{R} A_{1} \otimes_{R} A_{1}
$$


and

$$
\widehat{I}^{(3)}=\left(\widehat{I} \otimes_{R} \widehat{A}_{1}\right) \cap\left(\widehat{A}_{1} \otimes_{R} \widehat{I}\right) \subset \widehat{A}_{1} \otimes_{R} \widehat{A}_{1} \otimes_{R} \widehat{A}_{1} .
$$

Following the proof of Proposition [1.3, we have natural isomorphisms of $R$ - $R$-bimodules $\operatorname{Hom}_{R^{\text {op }}}\left(\mathrm{q} B^{3}, R\right) \simeq I^{(3)}$ and $\operatorname{Hom}_{R^{\text {op }}}\left(\widehat{B}^{3}, R\right) \simeq \widehat{I}^{(3)}$. The map

$$
f: \widehat{I}^{(3)}=\operatorname{Hom}_{R^{\mathrm{op}}}\left(\widehat{B}^{3}, R\right) \longrightarrow \operatorname{Hom}_{R^{\mathrm{op}}}\left(\mathrm{q} B^{3}, R\right)=I^{(3)}
$$

that we are interested in is induced by the surjective morphism of quadratic graded rings $\widehat{A} \longrightarrow A$ (which is quadratic dual to the morphism of quadratic graded rings $\mathrm{q} B \longrightarrow \widehat{B}$ according to Proposition $4.13(\mathrm{~b})$ ).

We have shown that the graded ring $B$ is quadratic if and only if the natural map $f: \widehat{I}^{(3)} \longrightarrow I^{(3)}$ is surjective. Let us show that the latter condition holds if and only if the multiplication map $\widehat{A}_{2} \stackrel{t}{\longrightarrow} \widehat{A}_{3}$ is injective.

Consider the three-term complex

$$
\widehat{A}_{1} \otimes_{R} \widehat{A}_{1} \otimes_{R} \widehat{A}_{1} \longrightarrow\left(\widehat{A}_{1} \otimes_{R} \widehat{A}_{2}\right) \oplus\left(\widehat{A}_{2} \otimes_{R} \widehat{A}_{1}\right) \longrightarrow \widehat{A}_{3} \longrightarrow 0
$$

and denote it by $\widehat{C}_{\text {. }}$. For the sake of certainty of notation, let us place the complex $\widehat{C}$. in the homological degrees 1,2 , and 3 , so that $\widehat{C}_{3}=\widehat{A}_{1}^{\otimes_{R}} 3$ and $\widehat{C}_{1}=\widehat{A}_{3}$. Endow the graded ring $\widehat{A}$ with a multiplicative decreasing filtration by homogeneous ideals $\widehat{A}=$ $G^{0} \widehat{A} \supset G^{1} \widehat{A} \supset G^{2} \widehat{A} \supset \cdots$ defined by the rule $G^{p} \widehat{A}=t^{p} \widehat{A}$, and endow the complex $\widehat{C}$. with the induced decreasing filtration. So, in particular, one has $\widehat{A} / G^{1} \widehat{A}=A$, and the complex $\widehat{C}_{\bullet} / G^{1} \widehat{C}_{\bullet}$ is isomorphic to the complex

$$
A_{1} \otimes_{R} A_{1} \otimes_{R} A_{1} \longrightarrow\left(A_{1} \otimes_{R} A_{2}\right) \oplus\left(A_{2} \otimes_{R} A_{1}\right) \longrightarrow A_{3} \longrightarrow 0,
$$

which we denote by $C_{\bullet}=C_{\bullet}(A)$.

Since the graded rings $\widehat{A}$ and $A$ are quadratic, we have $H_{i}\left(\widehat{C}_{\bullet}\right)=0=H_{i}\left(C_{\bullet}\right)$ for all $i \neq 3$, while $H_{3}\left(\widehat{C}_{\bullet}\right)=\widehat{I}^{(3)}$ and $H_{3}\left(C_{\bullet}\right)=I^{(3)}$. It is clear from the homological long exact sequence related to the short exact sequence of complexes

$$
0 \longrightarrow G^{1} \widehat{C}_{\bullet} \longrightarrow \widehat{C}_{\bullet} \longrightarrow C_{\bullet} \longrightarrow 0
$$

that the natural map $f: \widehat{I}^{(3)} \longrightarrow I^{(3)}$ is surjective if and only if $H_{2}\left(G^{1} \widehat{C}_{\bullet}\right)=0$.

Consider the nonnegatively graded ring $\mathbb{Z}[\bar{t}]$ of polynomials in one variable $\bar{t}$ of degree 1 with integer coefficients. Let $A[\bar{t}]=A \otimes_{\mathbb{Z}} \mathbb{Z}[\bar{t}]$ be the tensor product of the graded rings $A$ and $\mathbb{Z}[t]$, taken over the $\operatorname{ring} \mathbb{Z}$ and endowed with the induced grading. Obviously, the graded ring $A[\bar{t}]$ is quadratic. Furthermore, there is a natural morphism of graded rings

$$
A[\bar{t}] \longrightarrow \operatorname{gr}_{G} \widehat{A}=\bigoplus_{p=0}^{\infty} G^{p} \widehat{A} / G^{p+1} \widehat{A}
$$

whose restriction to $A \subset A[\bar{t}]$ is equal to the inclusion $A \simeq \widehat{A} / G^{1} \widehat{A} \hookrightarrow \operatorname{gr}_{G} \widehat{A}$ and which takes the element $\bar{t} \in A[\bar{t}]$ to the coset $t+G^{2} \widehat{A} \in G^{1} \widehat{A} / G^{2} \widehat{A}$.

One easily observes that the map (52) is surjective. Furthermore, the map $A[\bar{t}]_{n} \longrightarrow\left(\operatorname{gr}_{G} \widehat{A}\right)_{n}$ is injective (equivalently, an isomorphism) if and only if the map $\widehat{A}_{j-1} \stackrel{t}{\longrightarrow} \widehat{A}_{j}$ is injective for all $j \leq n$. In our assumptions, we know that 
this injectivity holds in the internal degrees $j=1$ and 2 , and we are interested in knowing whether it holds for $j=3$. So the map $\widehat{A}_{2} \stackrel{t}{\longrightarrow} \widehat{A}_{3}$ is injective if and only if the map $A[\bar{t}]_{3} \longrightarrow \operatorname{gr}_{G} \widehat{A}_{3}$ is.

Put $\widetilde{A}=A[\bar{t}]$ and consider the three-term complex

$$
\widetilde{A}_{1} \otimes_{R} \widetilde{A}_{1} \otimes_{R} \widetilde{A}_{1} \longrightarrow\left(\widetilde{A}_{1} \otimes_{R} \widetilde{A}_{2}\right) \oplus\left(\widetilde{A}_{2} \otimes_{R} \widetilde{A}_{1}\right) \longrightarrow \widetilde{A}_{3} \longrightarrow 0,
$$

denoted by $\widetilde{C}_{\bullet}=C_{\bullet}(\widetilde{A})$. Since the graded ring $A$ is 2-left finitely projective, the complex $\operatorname{gr}_{G} \widehat{C}$. is isomorphic to the complex $C \cdot\left(\operatorname{gr}_{G} \widehat{A}\right)$. The surjective morphism of graded rings $\widetilde{A} \longrightarrow \operatorname{gr}_{G} \widehat{A}$ induces a surjective morphism of complexes $\widetilde{C} \bullet \longrightarrow \operatorname{gr}_{G} \widehat{C}_{\bullet}$.

Moreover, the maps $\widetilde{C}_{i} \longrightarrow \operatorname{gr}_{G} \widehat{C}_{i}$ are isomorphisms for $i \neq 1$, since the map $\widetilde{A}_{j} \longrightarrow \operatorname{gr}_{G} \widehat{A}_{j}$ is an isomorphism for $j<3$. Furthermore, the complex $\widetilde{C}_{i}$ is acyclic in the homological degrees $i \neq 3$, since the graded ring $\widetilde{A}=A[\bar{t}]$ is quadratic. Since the map $\widetilde{C}_{3} \longrightarrow \operatorname{gr}_{G} \widehat{C}_{3}$ is surjective, it follows that the map $A[\bar{t}]_{3} \longrightarrow \operatorname{gr}_{G} \widehat{A}_{3}$ is an isomorphism if and only if $H_{2}\left(\operatorname{gr}_{G} \widehat{C}_{\bullet}\right)=0$.

It remains to show that $H_{2}\left(G^{1} \widehat{C}_{\bullet}\right)=0$ if and only if $H_{2}\left(\operatorname{gr}_{G} \widehat{C}_{\bullet}\right)=0$. The implication "if" is obvious. To prove the "only if", we assume that $H_{2}\left(G^{1} \widehat{C}_{\bullet}\right)=0$, the map $f: \widehat{I}^{(3)} \longrightarrow I^{(3)}$ is surjective, and the graded ring $B$ is quadratic.

Consider the additional grading $p$ on the $\operatorname{ring} \operatorname{gr}_{G} \widehat{A}$ and the complex $\operatorname{gr}_{G} \widehat{C}$. induced by the indexing of the filtration $G$. The related grading $p$ on the ring $\widetilde{A}=A[\bar{t}]$ and the complex $\widetilde{C}_{\bullet}=C_{\bullet}(\widetilde{A})$ is induced by the grading of the ring $\mathbb{Z}[\bar{t}]$. The additional grading $p$ on the rings $\operatorname{gr}_{G} \widehat{A}$ and $\widetilde{A}$ takes values in the monoid of nonnegative integers $p \geq 0$. On the complexes $\operatorname{gr}_{G} \widehat{C}_{\bullet}$ and $\widetilde{C}_{\bullet}$, the additional grading takes values $0 \leq p \leq 3$.

In the additional grading $p=0$, the morphism of graded rings $A[t] \longrightarrow \operatorname{gr}_{G} \widehat{A}$ is an isomorphism, since $A=\widehat{A} / \widehat{A} t$. Therefore, the morphism of complexes $\widetilde{C} \bullet \longrightarrow \operatorname{gr}_{G} \widehat{C}$. is an isomorphism in the additional grading $p=0$. Any possible homology classes in $H_{2}\left(\operatorname{gr}_{G} \widehat{C}_{\bullet}\right)$ would occur in the additional grading $p=1,2$, or 3 .

We have $H_{1}\left(\operatorname{gr}_{G} \widehat{C}_{\bullet}\right)=0$, as the graded ring $\operatorname{gr}_{G} \widehat{A}$ is generated by its component $A \oplus R[\bar{t}]$ of degree $n=1$ over its component $R$ of degree $n=0$. Let us compute the $p$-graded abelian group $\left(R\right.$ - $R$-bimodule) $\widetilde{I}^{(3)}=H_{3}\left(\widetilde{C}_{\bullet}\right)=H_{3}\left(\operatorname{gr}_{G} \widehat{C}_{\bullet}\right)$.

The quadratic graded ring $\widetilde{A}=A[\bar{t}]$ (in the grading $n$ ) is 3-left finitely projective, and its quadratic dual 3-right finitely projective graded ring $\widetilde{B}$ can be computed as the graded ring $\widetilde{B}=B[\bar{\delta}]=B \otimes_{\mathbb{Z}}^{-1} \mathbb{Z}[\bar{\delta}]$ obtained by adjoining to $B$ a generator $\bar{\delta}$ of degree $n=1$ (and $p=1$ ) with the relations $\bar{\delta} b+b \bar{\delta}=0$ for all $b \in B^{1}$ and $\bar{\delta}^{2}=0$. Here the notation $B \otimes_{\mathbb{Z}}^{q} \mathbb{Z}[\bar{\delta}]$ stands for the relations $\bar{\delta} b=q b \bar{\delta}, b \in B^{1}$, with $q=-1$ [24, Section 1 of Chapter 3]. Consequently, $\widetilde{B}^{3}=B^{3} \oplus B^{2} \bar{\delta}$ and $\widetilde{I}^{(3)} \simeq \operatorname{Hom}_{R^{\text {op }}}\left(\widetilde{B}^{3}, R\right)=I^{(3)} \oplus \bar{\epsilon} I$, where $\bar{\epsilon}$ is a variable dual to $\bar{\delta}$. The direct summand $I^{(3)}$ of the $p$-graded group $\widetilde{I}^{(3)}$ sits in the additional grading $p=0$ and the direct summand $\bar{\epsilon} I \simeq I$ sits in the additional grading $p=1$. 
Thus we have

$$
H_{3}\left(G^{p} \widehat{C} \cdot / G^{p+1} \widehat{C} \bullet\right)= \begin{cases}I^{(3)} & \text { for } p=0 \\ \bar{\epsilon} I & \text { for } p=1 \\ 0 & \text { for } p \geq 2\end{cases}
$$

In view of the spectral sequence connecting the homology groups of the complexes $G^{1} \widehat{C}_{\bullet}$ and $\operatorname{gr}_{G} \widehat{C}^{\bullet}$, it remains to check that the map

$$
H_{3}\left(G^{1} \widehat{C}_{\bullet}\right) \longrightarrow H_{3}\left(G^{1} \widehat{C}_{\bullet} / G^{2} \widehat{C}_{\bullet}\right)=\bar{\epsilon} I
$$

is surjective (or equivalently, an isomorphism). Here the group $H_{3}\left(G^{1} \widehat{C}_{\bullet}\right)$ is the kernel of the natural map $f: \widehat{I}^{(3)} \longrightarrow I^{(3)}$ (see short exact sequence (51)).

Following the discussion in the beginning of this proof, the map $f: \widehat{I}^{(3)} \longrightarrow I^{(3)}$ can be obtained by applying the functor $\operatorname{Hom}_{R^{\text {op }}}(-, R)$ to the composition of maps $\mathrm{q} B^{3} \longrightarrow B^{3} \longrightarrow \widehat{B}^{3}$. In our present assumptions, the map $f$ is surjective and the graded ring $B$ is quadratic. The kernel of the map $f$ is $\operatorname{ker}(f) \simeq \operatorname{Hom}_{R^{\text {op }}}\left(\widehat{B}^{3} / B^{3}, R\right)=$ $\operatorname{Hom}_{R^{\text {op }}}\left(B^{2} \bar{\delta}, R\right) \simeq \bar{\epsilon} I$, as desired. Notice that existence of a well-defined odd derivation $\partial=\partial / \partial \delta$ on $\widehat{B}$ ensures injectivity (equivalently, bijectivity) of the multiplication $\operatorname{map} B^{2} \stackrel{\bar{\delta}}{\longrightarrow} \widehat{B}^{3} / B^{3}$.

4.5. Nonhomogeneous quadratic duality via quasi-differential graded rings. The construction of the nonhomogeneous quadratic duality functors

$$
\left(R-\text { rings }_{\text {wnlq }}\right)^{\text {op }} \longrightarrow R \text {-rings } \text { cdg, }, \mathrm{q} \text { and }\left(\text { Rings }_{\text {wnlq } 2}\right)^{\text {op }} \longrightarrow \text { Rings }_{\mathrm{cdg} 2, \mathrm{rq}}
$$

in Theorems 3.10 and 3.13 are based on the computations in Sections 3.3 3.5, which are beautiful, but quite involved. The definitions and results above in Section 4 allow to produce the nonhomogeneous duality functors in a more conceptual fashion.

A nonnegatively graded quasi-differential ring $(\widehat{B}, \partial)$ is said to be 3 -right finitely projective quadratic if its underlying graded $\operatorname{ring} B=\operatorname{ker} \partial \subset \widehat{B}$ is 3-right finitely projective quadratic. The results of Section 4.3 explain how such properties of the graded ring $B$ are related to the similar properties of the graded ring $\widehat{B}$.

Let $R$ be an associative ring. The category of 3-right finitely projective quadratic quasi-differential graded rings over $R$, denoted by $R$-rings ${ }_{\mathrm{qdg}, \mathrm{rq}}$, is the full subcategory

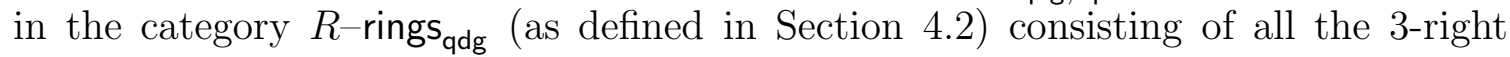
finitely projective quadratic quasi-differential graded rings.

Furthermore, the 2-category of 3-right finitely projective quadratic quasidifferential graded rings, denoted by Rings $_{\mathrm{qdg} 2, \mathrm{rq}}$, is the following 2-subcategory in the 2-category Rings ${ }_{\mathrm{qdg} 2}$ (which was also defined in Section 4.2). The objects of Rings $_{\text {qdg2,rq }}$ are all the 3-right finitely projective quadratic quasi-differential graded rings. All morphisms in Rings ${ }_{\mathrm{qdg} 2}$ between objects of Rings ${ }_{\mathrm{qdg} 2, \mathrm{rq}}$ are morphisms in Rings $_{\mathrm{qdg} 2, \mathrm{rq}}$, and all 2-morphisms in Rings ${ }_{\mathrm{qdg} 2}$ between morphisms of Rings ${ }_{\mathrm{qdg} 2, \mathrm{rq}}$ are 2-morphisms in Rings ${ }_{\mathrm{qdg} 2, \mathrm{rq}}$.

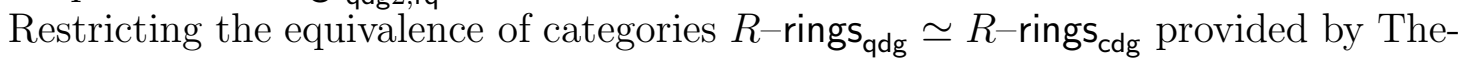
orem 4.5 to the full subcategories of 3-right finitely projective quadratic CDG-rings 
and quasi-differential graded rings over $R$, we obtain an equivalence of categories

$$
R \text {-rings }{ }_{\mathrm{qdg}, \mathrm{rq}} \simeq R \text {-rings }{ }_{\mathrm{cdg}, \mathrm{rq}} .
$$

Similarly, a restriction of the strict equivalence of 2 -categories $\operatorname{Rings}_{\mathrm{qdg}_{2}} \simeq \operatorname{Rings}_{\mathrm{cdg} 2}$ provided by Theorem 4.6 produces a strict equvalence between the 2-categories of 3-right finitely projective quadratic CDG-rings and quasi-differential graded rings,

$$
\text { Rings }_{\mathrm{qdg} 2, \mathrm{rq}} \simeq \text { Rings }_{\mathrm{cdg} 2, \mathrm{rq}} \text {. }
$$

Let $(\widetilde{A}, F)$ be a filtered ring with an increasing filtration $0=F_{-1} \widetilde{A} \subset F_{0} \widetilde{A} \subset F_{1} \widetilde{A} \subset$ $F_{2} \widetilde{A} \subset \cdots$. Consider two graded rings related to such a filtration: the associated graded ring $A=\operatorname{gr}^{F} \widetilde{A}$ and the Rees ring $\widehat{A}=\bigoplus_{n=0}^{\infty} F_{n} \widetilde{A}$.

The unit element $1 \in F_{0} \widetilde{A}$, viewed as an element of $F_{1} \widetilde{A}$, represents a central nonzero-divisor $t \in \widehat{A}_{1}$. The quotient $\operatorname{ring} \widehat{A} / \widehat{A} t$ is naturally isomorphic to the associated graded ring $A$ of the filtration $F$ on the $\operatorname{ring} \widetilde{A}$. By Proposition 4.1, the graded ring $\widehat{A}$ is generated by $\widehat{A}_{1}$ over $\widehat{A}_{0}$ if and only if the graded $\operatorname{ring} A$ is generated by $A_{1}$ over $A_{0}$; and moreover, the graded ring $\widehat{A}$ is quadratic if and only if the graded ring $A$ is.

Let $\widetilde{A}$ be a weak nonhomogeneous quadratic ring over a subring $R \subset \widetilde{A}$ with the $R$-R-bimodule of generators $\widetilde{V} \subset \widetilde{A}$. Let $F$ be the related increasing filtration on the $\operatorname{ring} \widetilde{A}$, as defined in Section 3.1. Then the graded ring $\widehat{A}$ is generated by $\widehat{A}_{1}$ over $\widehat{A}_{0}$, and the graded ring $A$ is generated by $A_{1}$ over $A_{0}$. The graded rings $\widehat{A}$ and $A$ do not need to be quadratic (as we only assume $\widetilde{A}$ be a weak nonhomogeneous quadratic ring); so let us consider the quadratic graded rings $\mathrm{q} \widehat{A}$ and $\mathrm{q} A$.

Lemma 4.15. Let $\widehat{A}$ be a nonnegatively graded ring, and let $t \in \widehat{A}$ be a central element. Let $A=\widehat{A} / \widehat{A} t$ be the quotient ring. Then

(a) $t \in \mathrm{q} \widehat{A}_{1}=\widehat{A}_{1}$ is a central element in the quadratic graded ring $\mathrm{q} \widehat{A}$;

(b) for each $n=1,2$, or 3 , the multiplication map $\mathrm{q} \widehat{A}_{n-1} \stackrel{t}{\longrightarrow} \mathrm{q} \widehat{A}_{n}$ is injective whenever the multipication map $\widehat{A}_{n-1} \stackrel{t}{\longrightarrow} \widehat{A}_{n}$ is;

(c) there is a natural isomorphism of quadratic graded rings $\mathrm{q} \widehat{A} /(\mathrm{q} \widehat{A}) t \simeq \mathrm{q} A$.

Proof. In part (a), the element $t \in \mathrm{q} \widehat{A}_{1}$ commutes with all the elements of $\mathrm{q} \widehat{A}_{0}$ and $\mathrm{q} \widehat{A}_{1}$, since the graded ring homomorphism $\mathrm{q} \widehat{A} \longrightarrow \widehat{A}$ is an isomorphism in degree $n \leq 1$ and a monomorphism in degree $n=2$, and the element $t \in \widehat{A}_{1}$ is central in $\widehat{A}$. Since the graded ring $\mathrm{q} \widehat{A}$ is generated by $\mathrm{q} \widehat{A}_{1}$ over $\mathrm{q} \widehat{A}_{0}$, it follows that the element $t \in \mathrm{q} \widehat{A_{1}}$ is central in $\mathrm{q} \widehat{A}$.

Part (b) follows from injectivity of the map q $\widehat{A}_{n-1} \longrightarrow \widehat{A}_{n-1}$ for $n \leq 3$ and commutativity of the square diagram $\mathrm{q} \widehat{A}_{n-1} \stackrel{t}{\longrightarrow} \mathrm{q} \widehat{A}_{n} \longrightarrow \widehat{A}_{n}, \mathrm{q} \widehat{A}_{n-1} \longrightarrow \widehat{A}_{n-1} \stackrel{t}{\longrightarrow} \widehat{A}_{n}$.

In part (c), the graded ring homomorphism $\mathrm{q} \widehat{A} \longrightarrow \widehat{A}$ induces a graded ring homomorphism between the quotient rings $\mathrm{q} \widehat{A} /(\mathrm{q} \widehat{A}) t \longrightarrow \widehat{A} / \widehat{A} t=A$. Since the graded ring q $\widehat{A} /(\mathrm{q} \widehat{A}) t$ is quadratic by Proposition 4.1, the latter morphism, in turn, induces the desired graded ring homomorphism $f: \mathrm{q} \widehat{A} /(\mathrm{q} \widehat{A}) t \longrightarrow \mathrm{q} A$. The map $f$ is 
an isomorphism in the degrees $n \leq 2$ by construction, hence it is an isomorphism of quadratic graded rings by Lemma 3.2 .

Proposition 4.16. Let $R \subset \widetilde{V} \subset \widetilde{A}$ be a 3-left finitely projective weak nonhomogeneous quadratic ring. Consider the corresponding graded rings $\widehat{A}=\bigoplus_{n=0}^{\infty} F_{n} \widetilde{A}$ and $A=\operatorname{gr}^{F} \widetilde{A}$. Then the quadratic graded rings $\mathrm{q} \widehat{A}$ and $\mathrm{q} A$ are 3 -left finitely projective.

Let $\widehat{B}$ be the 3-right finitely projective quadratic graded ring quadratic dual to $\mathrm{q} \widehat{A}$, and let $\partial: \widehat{B} \longrightarrow \widehat{B}$ be the odd derivation of degree -1 corresponding to the central element $t \in \widehat{A}_{1}$, as in Lemma $4.11(b)$. Then $(\widehat{B}, \partial)$ is a quasi-differential graded ring. The underlying graded ring $B=\operatorname{ker} \partial \subset \widehat{B}$ is 3-right finitely projective quadratic and quadratic dual to $\mathrm{q} A$.

Proof. The quadratic graded ring $\mathrm{q} A$ is 3-left finitely projective by the definition of what it means for a weak nonhomogeneous quadratic ring $\widetilde{A}$ to be 3-left finitely projective (see Section 3.3). To prove that the left $R$-module q $\widehat{A}_{n}$ is finitely generated projective for $n \leq 3$, one argues by induction in $0 \leq n \leq 3$, using the short exact sequences of $R$ - $R$-bimodules $0 \longrightarrow \mathrm{q} \widehat{A}_{n-1} \stackrel{t}{\longrightarrow} \mathrm{q} \widehat{A}_{n} \longrightarrow \mathrm{q} A_{n} \longrightarrow 0$ provided by Lemma 4.15. Here the multiplication maps $\mathrm{q} \widehat{A}_{n-1} \stackrel{t}{\longrightarrow} \mathrm{q} \widehat{A}_{n}$ are injective for $n \leq 3$ by Lemma $4.15(\mathrm{~b})$, since $t$ is a nonzero-divisor in $\widehat{A}$.

The pair $(\widehat{B}, \partial)$ is a quasi-differential graded ring by Lemma 4.12 (applied to the quadratic graded ring q $\widehat{A}$ with its central element $t$ ). The graded $\operatorname{ring} B=\operatorname{ker} \partial \subset \widehat{B}$ is generated by $B^{1}$ over $B^{0}$ by Proposition 4.13(a) and quadratic by Proposition 4.14. The quadratic graded ring $B$ is 3-right finitely projective by Lemma 4.9, It is quadratic dual to $\mathrm{q} A$ by Proposition 4.13 (b).

Theorem 4.17. The construction of Proposition 4.16 defines a fully faithful contravariant functor

$$
(R \text {-rings } \text { wnlq })^{\text {op }} \longrightarrow R \text {-rings } \text { qdg, } r q
$$

from the category of 3-left finitely projective weak nonhomogeneous quadratic rings to the category of 3-right finitely projective quasi-differential quadratic graded rings over $R$. The functor (56) forms a commutative triangle diagram with the fully faithful contravariant functor (36) and the equivalence of categories (54).

The same construction also defines a fully faithful strict contravariant 2-functor

$$
\left(\text { Rings }_{\text {wnlq } 2}\right)^{\text {op }} \longrightarrow \text { Rings }_{\text {qdg2,rq }}
$$

from the 2-category of 3-left finitely projective weak nonhomogeneous quadratic rings to the 2-category of 3-right finitely projective quasi-differential quadratic graded rings. The strict 2-functor (57) forms a commutative triangle diagram with the fully faithful strict contravariant 2-functor (43) and the strict equivalence of 2-categories (55).

Proof. The proof is straightforward. 
4.6. PBW theorem. The Poincaré-Birkhoff-Witt theorem in nonhomogeneous quadratic duality tells that, when restricted to the left/right finitely projective Koszul rings on both sides, the fully faithful contravariant nonhomogeneous quadratic duality functors from Theorems [3.10, 3.13, 3.14, 3.15, and 4.17 become anti-equivalences of categories (or strict anti-equivalences of 2-categories). In other words, every right finitely projective Koszul CDG-ring (or quasi-differential graded ring) arises from a left finitely projective nonhomogeneous Koszul ring.

A (weak) nonhomogeneous quadratic ring $R \subset \widetilde{V} \subset \widetilde{A}$ is said to be left finitely projective Koszul if the quadratic graded $\operatorname{ring} \mathrm{q} A=\operatorname{qgr}^{F} \widetilde{A}$ is left finitely projective Koszul (in the sense of the definition in Section 2.10). A nonnegatively graded CDG-ring $(B, d, h)$ is said to be right finitely projective Koszul if the nonnegatively graded ring $B$ is right finitely projective Koszul. A nonnegatively graded quasidifferential ring $(\widehat{B}, \partial)$ is said to be right finitely projective Koszul if its underlying graded ring $B=\operatorname{ker} \partial \subset \widehat{B}$ is right finitely projective Koszul.

The following result was mentioned in Remark 3.3 in Section 3.1 .

Theorem 4.18. If a weak nonhomogeneous quadratic ring is left finitely projective Koszul, then it is nonhomogeneous quadratic.

So we will call such filtered rings as in Theorem 4.18 left finitely projective nonhomogeneous Koszul rings. Notice that a filtered $\operatorname{ring}(\widetilde{A}, F)$ with an increasing filtration $F$ such that $A_{n}=\operatorname{gr}_{n}^{F} \widetilde{A}$ is a projective left $A_{0}$-module for all $n \geq 0$ is left finitely projective Koszul (i. e., the graded $\operatorname{ring} A=\operatorname{gr}^{F} \widetilde{A}$ is left finitely projective Koszul) if and only if the Rees ring $\widehat{A}=\bigoplus_{n=0}^{\infty} F_{n} \widetilde{A}$ is left finitely projective Koszul. This is clear from Lemma 4.2 and Theorem 4.3 .

The proof of Theorem 4.18 will be given at the end of Section 4.6.

Theorem 4.19. Every right finitely projective Koszul CDG-ring arises from a left finitely projective nonhomogeneous Koszul ring via the construction of Proposition [3.6. Equivalently, every right finitely projective Koszul quasi-differential graded ring arises from a left finitely projective nonhomogeneous Koszul ring via the construction of Proposition 4.16 .

The two assertions in Theorem 4.19 are equivalent in view of Theorems 4.5 and 4.17 . We will prove the second assertion.

First proof of Theorem 4.19. Let $(\widehat{B}, \partial)$ be a right finitely projective Koszul quasidifferential graded ring. Then the graded ring $B$ is right finitely projective Koszul by definition and the graded ring $\widehat{B}$ is right finitely projective Koszul by Theorem 4.10 (b). Let $\widehat{A}$ be the left finitely projective Koszul ring quadratic dual to $\widehat{B}$ (see Proposition [2.23), and let $t \in \widehat{A}_{1}$ be the central element corresponding to the odd derivation $\partial: \widehat{B} \longrightarrow \widehat{B}$ (see Lemma 4.11). Let $A=\widehat{A} / \widehat{A} t$ be the quotient ring.

By Lemma 4.12, the multiplication map $\widehat{A}_{0} \stackrel{t}{\longrightarrow} \widehat{A}_{1}$ is injective and $A_{1}$ is a projective left $R$-module. By Proposition 4.13 (a), the multiplication map $\widehat{A}_{1} \stackrel{t}{\longrightarrow} \widehat{A}_{2}$ is injective and $A_{2}$ is a projective left $R$-module. By Proposition 4.13 (b), the right 
finitely projective Koszul ring $B$ is quadratic dual to the 2-left finitely projective quadratic ring $A$. Therefore, the quadratic graded ring $A$ is left finitely projective Koszul. By Proposition 4.14, the multiplication map $\widehat{A}_{2} \stackrel{t}{\longrightarrow} \widehat{A}_{3}$ is injective.

Applying Theorem 4.4, we conclude that $t$ is a nonzero-divisor in $\widehat{A}$. It follows that $\widehat{A}$ is the Rees ring of the filtered ring $\widetilde{A}=\widehat{A} / \widehat{A}(t-1)$ with the filtration $F_{n} \widetilde{A}=\widehat{A}_{n}+\widehat{A}(t-1)$, and $A$ is the associated graded ring, $A \simeq \operatorname{gr}^{F} \widetilde{A}$. We have obtained the desired left finitely projective nonhomogeneous Koszul ring $\widetilde{A}$.

Second proof of Theorem 4.19. This is a particular case of the argument in [27, proof of Theorem 11.6]. Let $(\widehat{B}, \partial)$ be a right finitely projective Koszul quasi-differential graded ring and $B=\operatorname{ker} \partial \subset \widehat{B}$ be its underlying right finitely projective Koszul graded ring. Then the right $R$-modules $B^{n}$ are finitely generated projective by definition and the right $R$-modules $\widehat{B}^{n}$ are finitely generated projective by Lemma 4.9.

The (essentially) spectral sequence argument below is to be compared with, and distinguished from, a quite different (and simpler) spectral sequence argument proving the Poincaré-Birkhoff-Witt theorem for nonhomogeneous quadratic algebras over the ground field in [25, Section 3.3] and [24, Proposition 7.2 in Chapter 5].

Put $D_{n}=\operatorname{Hom}_{R^{\text {op }}}\left(B^{n}, R\right)$. Then $D=\bigoplus_{n=0}^{\infty} D_{n}$ is a graded coring over the ring $R$ with the counit map $\varepsilon: D \longrightarrow D_{0} \simeq R$ dual to the inclusion map $R \simeq B^{0} \longrightarrow B$ and the comultiplication maps $\mu_{i, j}: D_{i+j} \longrightarrow D_{i} \otimes_{R} D_{j}$ obtained by dualizing the multiplication maps $B^{j} \otimes_{R} B^{i} \longrightarrow B^{i+j}$ in the graded ring $B$,

$$
\begin{aligned}
D_{i+j}=\operatorname{Hom}_{R^{\mathrm{op}}}\left(B^{i+j}, R\right) \longrightarrow & \operatorname{Hom}_{R^{\mathrm{op}}}\left(B^{j} \otimes_{R} B^{i}, R\right) \\
& \simeq \operatorname{Hom}_{R^{\mathrm{op}}}\left(B^{i}, R\right) \otimes_{R} \operatorname{Hom}_{R^{\mathrm{op}}}\left(B^{j}, R\right)=D_{i} \otimes_{R} D_{j} .
\end{aligned}
$$

In the pairing notation of Section [3.4, we have $\left\langle\mu(f), b_{1} \otimes b_{2}\right\rangle=\left\langle f, b_{1} b_{2}\right\rangle$ for all $f \in D$ and $b_{1}, b_{2} \in B$.

Similarly, we set $\widehat{D}_{n}=\operatorname{Hom}_{R^{\text {op }}}\left(\widehat{B}^{n}, R\right)$; so $\widehat{D}=\bigoplus_{n=0}^{\infty} \widehat{D}_{n}$ is also a graded coring over $R$. The odd derivation $\partial: \widehat{B} \longrightarrow \widehat{B}$ is an $R$ - $R$-bilinear map, so it dualizes to an $R$ - $R$-bilinear map $\operatorname{Hom}_{R^{\text {op }}}(\partial, R): \widehat{D} \longrightarrow \widehat{D}$, which we denote for brevity also by $\partial$. There is a sign rule involved: in the pairing notation, we put $\langle\partial(f), b\rangle=$ $(-1)^{|f|+1}\langle f, \partial(b)\rangle$ for $f \in \widehat{D}$ and $b \in \widehat{B}$. The map $\partial: \widehat{D} \longrightarrow \widehat{D}$ is an odd coderivation of degree 1 on the coring $\widehat{D}$, in the sense that its components act as $\partial_{n}: \widehat{D}_{n} \longrightarrow \widehat{D}_{n+1}$ and, in the symbolic notation $\mu(f)=\mu_{1}(f) \otimes \mu_{2}(f)$ for the comultiplication, one has

$$
\partial(\mu(f))=\partial \mu_{1}(f) \otimes \mu_{2}(f)+(-1)^{\left|\mu_{1}(f)\right|} \mu_{1}(f) \otimes \partial \mu_{2}(f) \text { for all } f \in \widehat{D} .
$$

The odd coderivation $\partial$ on the graded coring $\widehat{D}$ has zero square, $\partial^{2}=0$ (so one can say that $\widehat{D}$ is a $D G$-coring over $R$ ). Furthermore, the odd coderivation $\partial$ on $\widehat{D}$ has vanishing cohomology, $H_{\partial}(\widehat{D})=0$. The cokernel of $\partial$ is the quotient coring $D$ of the coring $\widehat{D}$, that is $\widehat{D} \rightarrow \operatorname{coker}(\partial) \simeq D$.

Consider the bigraded $R$-R-bimodule $K$ with the components $K^{p, q}=\widehat{D}_{q-p}$ for $p \leq 0, q \leq 0$, and $K^{p, q}=0$ otherwise. The $R$-R-bimodule $K$ can be viewed as a bigraded coring over $R$ with the comultiplication inherited from the comultiplication 
of $\widehat{D}$. Considered as a graded coring in the total grading $p+q$, the coring $K$ has an odd coderivation $\partial_{K}$ of degree 1 with the components $\partial_{K}^{p, q}: K^{p, q} \longrightarrow K^{p, q+1}$ given by the rule $\partial_{K}^{p, q}=\partial_{q-p}: \widehat{D}_{q-p} \longrightarrow \widehat{D}_{q-p+1}$. So for every fixed $p=-n, n \geq 0$, the components $K^{p, q}$ with varying $q$ form a complex of $R$ - $R$-bimodules

$$
0 \longrightarrow K^{p, p} \stackrel{\partial_{K}^{p, p}}{\longrightarrow} K^{p, p+1} \stackrel{\partial_{K}^{p, p+1}}{\longrightarrow} \cdots \stackrel{\partial_{K}^{p,-2}}{\longrightarrow} K^{p,-1} \stackrel{\partial_{K}^{p,-1}}{\longrightarrow} K^{p, 0} \longrightarrow 0
$$

isomorphic to

$$
0 \longrightarrow \widehat{D}_{0} \stackrel{\partial_{0}}{\longrightarrow} \widehat{D}_{1} \stackrel{\partial_{1}}{\longrightarrow} \cdots \stackrel{\partial_{n-2}}{\longrightarrow} \widehat{D}_{n-1} \stackrel{\partial_{n-1}}{\longrightarrow} \widehat{D}_{n} \longrightarrow 0 .
$$

The only nontrivial cohomology bimodule of this complex occurs at its rightmost term $(q=0)$ and is isomorphic to $D_{n}$. So there is a surjective morphism of bigraded corings $K \longrightarrow D$ inducing an isomorphism of the bimodules/corings of cohomology, where the coring $D$ is placed in the bigrading $D^{p, 0}=D_{n}=D_{-p}$ and endowed with the zero differential.

Denote by $K_{+}$the cokernel of the inclusion $R \simeq K^{0,0} \longrightarrow K$. Consider the tensor ring $T_{R}\left(K_{+}\right)$of the bigraded $R$-R-bimodule $K_{+}$. By the definition, $T_{R}\left(K_{+}\right)$is a trigraded ring with the gradings $p \leq 0$ and $q \leq 0$ inherited from the bigrading of $K_{+}$ and the additional grading $r \geq 0$ by the number of tensor factors. We will consider $T_{R}\left(K_{+}\right)$as a graded ring in the total grading $p+q+r$. The graded ring $T_{R}\left(K_{+}\right)$is endowed with three odd derivations of total degree 1 , which we will now introduce.

For any (graded) $R$ - $R$-bimodule $V$, derivations of the tensor ring $T(V)$ (say, odd derivations with respect to the total parity on $T(V)$ ) annihilating the subring $R \subset$ $T(V)$ are uniquely determined by their restriction to the subbimodule $V \subset T(V)$, which can be an arbitrary $R$ - $R$-bilinear map $V \longrightarrow T(V)$ (that is homogeneous of the prescribed degrees). In the situation at hand, we let $\partial_{T}$ be the only odd derivation of $T_{R}\left(K_{+}\right)$which preserves the subbimodule $K_{+} \subset T_{R}\left(K_{+}\right)$and whose restriction to $K_{+}$is equal to $-\partial_{K}$. Let $d_{T}$ be the only odd derivation of $T_{R}\left(K_{+}\right)$which maps $K_{+}$ into $K_{+} \otimes_{R} K_{+}$by the comultiplication map $\mu$ with the sign rule

$$
d_{T}(k)=(-1)^{p_{1}+q_{1}} \mu_{1}(k) \otimes \mu_{2}(k), \quad k \in K_{+}, \mu_{1}(k) \in K^{p_{1}, q_{1}} .
$$

Finally, let $\delta_{T}$ be the only odd derivation of $T_{R}\left(K_{+}\right)$whose restiction to $K_{+}$is the identity map of the component $K^{-1,-1}=R$ to the unit component $T_{R}\left(K_{+}\right)^{0,0,0}=R$ and zero on all the remaining components of $K_{+}$.

All the three derivations are constructed to be odd derivations in the parity $p+q+r$. The derivations $\partial_{T}, d_{T}$, and $\delta_{T}$ have tridegrees $(0,1,0),(0,0,1)$, and $(1,1,-1)$, respectively, in the trigrading $(p, q, r)$ of the tensor ring $T_{R}\left(K_{+}\right)$. All the three derivations have zero squares, and they pairwise anti-commute.

There is an increasing filtration $F$ on the graded ring $T_{R}\left(K_{+}\right)$whose component $F_{n} T_{R}\left(K_{+}\right)$is the direct sum of all the trigrading components $T_{R}\left(K_{+}\right)^{p, q, r}$ with $-p \leq$ $n$. This filtration is compatible with the differentials $\partial_{T}, d_{T}$, and $\delta_{T}$; the graded ring $\operatorname{gr}^{F} T_{R}\left(K_{+}\right)=\bigoplus_{n=0}^{\infty} F_{n} T_{R}\left(K_{+}\right) / F_{n-1} T_{R}\left(K_{+}\right)$with the differential induced by $\partial_{T}+d_{T}+\delta_{T}$ is naturally isomorphic to $T_{R}\left(K_{+}\right)$with the differential $\partial_{T}+d_{T}$. 
Let us also consider the tensor ring $T_{R}\left(D_{+}\right)$of the $R$ - $R$-bimodule $D_{+}=D / R$. Similarly to the above, we endow $T_{R}\left(D_{+}\right)$with the grading $p$ coming from the grading $D^{p}=D_{n}=D_{-p}$ of $D_{+}$and the grading $r$ by the number of tensor factors. As to the grading $q$, we set it to be identically zero on $T_{R}\left(D_{+}\right)$. We will consider $T_{R}\left(D_{+}\right)$as a graded ring in the total grading $p+r$. Let $d_{T}^{\prime}$ be the only odd derivation of $T_{R}\left(D_{+}\right)$ which maps the subbimodule $D_{+} \subset T_{R}\left(D_{+}\right)$to $D_{+} \otimes_{R} D_{+}$by the comultiplication map $\mu$ with the sign rule similar to the above.

The DG-ring $T_{R}\left(D_{+}\right)$is naturally isomorphic to the cobar complex (16) (with the roles of the left and right sides switched) computing the $\operatorname{bigraded} \operatorname{Ext}_{B^{\text {op }}}(R, R)$. There is some sign rule involved in this isomorphism, which is discussed in [27, proof of Theorem 11.6]. By Proposition 2.23, it follows that the bigraded ring of cohomology of the DG-ring $T_{R}\left(D_{+}\right)$is isomorphic to the left finitely projective Koszul graded ring $A=\bigoplus_{n=0}^{\infty} A_{n}$ quadratic dual to $B$, placed in the diagonal bigrading $-p=r=n$. In the total grading $p+r$, the cohomology ring of the DG-ring $T_{R}\left(D_{+}\right)$is the whole ring $A$ placed in the degree $p+r=0$.

Consider the morphism of DG-rings $\left(T_{R}\left(K_{+}\right), \partial_{T}+d_{T}\right) \longrightarrow\left(T_{R}\left(D_{+}\right), d_{T}^{\prime}\right)$ induced by the surjective morphism of graded corings $K \longrightarrow D$. The morphism of DG-corings $K \longrightarrow D$ is a quasi-isomorphism, so the induced morphism of DG-rings is a quasi-isomorphism, too, due to the right flatness/projectivity conditions imposed on the $R$ - $R$-bimodule $D$ and the presence of a nonpositive (essentially, negative) internal grading $p$. The fact that the components of fixed grading $p$ in the DG-coring $K$ are finite complexes (of projective right $R$-modules, with projective right $R$-modules of cohomology) is relevant here. Thus we have $H_{\partial_{T}+d_{T}}^{0}\left(T_{R}\left(K_{+}\right)\right) \simeq A$ and $H_{\partial_{T}+d_{T}}^{i}\left(T_{R}\left(K_{+}\right)\right)=0$ for $i \neq 0$.

Finally, we put $\widetilde{A}=H_{\partial_{T}+d_{T}+\delta_{T}}^{0}\left(T_{R}\left(K_{+}\right)\right)$. Then the $\operatorname{ring} \widetilde{A}$ is endowed with an increasing filtration $F$ induced by the filtration $F$ of the DG-ring $\left(T_{R}\left(K_{+}\right), \partial_{T}+d_{T}+\right.$ $\left.\delta_{T}\right)$. Since $H_{\partial_{T}+d_{T}}^{i}\left(T_{R}\left(K_{+}\right)\right)=0$ for $i \neq 0$, we can conclude that the associated graded ring of the $\operatorname{ring} \widetilde{A}$ is naturally isomorphic to the graded $\operatorname{ring} A$, that $\operatorname{is}_{\operatorname{gr}} F \widetilde{A} \simeq A$, while $H_{\partial_{T}+d_{T}+\delta_{T}}^{i}\left(T_{R}\left(K_{+}\right)\right)=0$ for $i \neq 0$.

Since the graded $\operatorname{ring} A=\operatorname{gr}^{F} \widetilde{A}$ is left finitely projective Koszul, the graded ring $\widehat{A}=\bigoplus_{n=0}^{\infty} F_{n} \widetilde{A}$ is left finitely projective Koszul as well (by Lemma 4.2 and Theorem 4.3 ). Let $\widehat{B}^{\prime}$ be the right finitely projective Koszul graded ring quadratic dual to $\widehat{A}$. By Proposition 4.16, the graded ring $\widehat{B}^{\prime}$ is endowed with an odd derivation $\partial^{\prime}: \widehat{B}^{\prime} \longrightarrow \widehat{B}^{\prime}$ of degree -1 , making it a right finitely projective Koszul quasidifferential graded ring. The underlying graded ring $B^{\prime}=\operatorname{ker} \partial^{\prime} \subset \widehat{B}^{\prime}$ of the quasidifferential graded ring $\left(\widehat{B}^{\prime}, \partial^{\prime}\right)$ is quadratic dual to $A$, so we have $B^{\prime} \simeq B$. It remains to construct a natural isomorphism of quasi-differential graded rings $\left(\widehat{B}^{\prime}, \partial^{\prime}\right) \simeq(\widehat{B}, \partial)$.

For this purpose, let us consider the dual graded corings $D^{\prime}=\operatorname{Hom}_{R^{\text {op }}}\left(B^{\prime}, R\right)$ and $\widehat{D}^{\prime}=\operatorname{Hom}_{R^{\text {op }}}\left(\widehat{B}^{\prime}, R\right)$. As in the beginning of this proof, we have an odd coderivation $\partial^{\prime}: \widehat{D}^{\prime} \longrightarrow \widehat{D}^{\prime}$ of degree 1 dual to the odd derivation $\partial^{\prime}: \widehat{B}^{\prime} \longrightarrow \widehat{B}^{\prime}$. It suffices to construct a natural isomorphism $(\widehat{D}, \partial) \longrightarrow\left(\widehat{D}^{\prime}, \partial^{\prime}\right)$ of DG-corings over $R$. 
The embedding of the component $\widehat{D}_{1}=T_{R}\left(K_{+}\right)^{-1,0,1} \longrightarrow T_{R}\left(K_{+}\right)$induces an isomorphism of $R$ - $R$-bimodules $\widehat{D}_{1} \simeq F_{1} \widetilde{A}$. The composition $\widehat{D}_{2} \longrightarrow \widehat{D}_{1} \otimes_{R} \widehat{D}_{1} \simeq$ $F_{1} \widetilde{A} \otimes_{R} F_{1} \widetilde{A} \longrightarrow F_{2} \widetilde{A}$ of the comultiplication and multiplication maps vanishes, being killed by the differential $\left(\partial_{T}+d_{T}+\delta_{T}\right)^{-2,0,1}=d_{T}^{-2,0,1}: T_{R}\left(K_{+}\right)^{-2,0,1} \longrightarrow T_{R}\left(K_{+}\right)^{-2,0,2}$. So there is a natural morphism of graded corings $\widehat{D} \longrightarrow \widehat{D}^{\prime}$. Since the embedding $R=$ $F_{0} \widetilde{A} \longrightarrow F_{1} \widetilde{A}$ corresponds to the map $\partial_{0}: R=\widehat{D}_{0} \longrightarrow \widehat{D}_{1}$ under the isomorphisms $F_{0} \widetilde{A}=R=\widehat{D}_{0}$ and $F_{1} \widetilde{A} \simeq \widehat{D}_{1}$, the graded coring morphism $\widehat{D} \longrightarrow \widehat{D}^{\prime}$ forms a commutative square diagram with the differentials $\partial$ on $\widehat{D}$ and $\partial^{\prime}$ on $\widehat{D}^{\prime}$.

The induced morphism coker $(\partial) \longrightarrow \operatorname{coker}\left(\partial^{\prime}\right)$ coincides with the natural isomorphism $D \longrightarrow D^{\prime}$ on the components of degree 1 , and hence on the other components as well. Therefore, the morphism of corings $\widehat{D} \longrightarrow \widehat{D}^{\prime}$ is also an isomorphism.

Proof of Theorem 4.18. Let $R \subset \widetilde{V} \subset \widetilde{A}$ be a weak nonhomogeneous quadratic ring and $A=\operatorname{gr}^{F} \widetilde{A}$ be its associted graded ring with respect to the filtration $F$ generated by $F_{1} \widetilde{A}=\widetilde{V}$ over $F_{0} \widetilde{A}=R$. Assume that the quadratic graded $\operatorname{ring} \mathrm{q} A=\operatorname{qgr}^{F} \widetilde{A}$ is left finitely projective Koszul. Let $(B, d, h)$ be the CDG-ring corresponding to $\widetilde{A}$ under the construction of Proposition [3.6. Equivalently, one can consider the quasi-differential graded ring $(\widehat{B}, \partial)$ with the underlying graded ring $B=\operatorname{ker} \partial \subset \widehat{B}$ corresponding to $\widetilde{A}$ under the construction of Proposition 4.16.

Whichever one of these two points of view one takes, the graded ring $B$ is quadratic and quadratic dual to the quadratic graded ring $\mathrm{q} A$ by construction. Hence the graded ring $B$ is right finitely projective Koszul by Proposition 2.23. Applying Theorem 4.19, we see that the CDG-ring $(B, d, h)$ or the quasi-differential ring $(\widehat{B}, \partial)$ comes from a left finitely projective nonhomogeneous Koszul ring $\left(\widetilde{A}^{\prime}, F\right)$. Following either one of the two proofs of Theorem 4.19, the graded ring $\operatorname{gr}^{F} \widetilde{A}^{\prime}$ is quadratic and left finitely projective Koszul.

It remains to observe that the nonhomogeneous quadratic duality functor assigns the same CDG-ring $(B, d, h)$ (or the same quasi-differential graded ring $(\widehat{B}, \partial))$ to the two 3-left finitely projective (weak) nonhomogeneous quadratic rings $\widetilde{A}$ and $\widetilde{A^{\prime}}$. Since the nonhomogeneous quadratic duality functor is fully faithful by Theorem 3.10 or 4.17, it follows that the two (weak) nonhomogeneous quadratic rings $(\widetilde{A}, F)$ and $\left(\widetilde{A^{\prime}}, F\right)$ are isomorphic. Hence the graded $\operatorname{ring} A=\operatorname{gr}^{F} \widetilde{A} \simeq \operatorname{gr}^{F} \widetilde{A^{\prime}}$ is quadratic (and left finitely projective Koszul). In other words, the weak nonhomogeneous quadratic ring $R \subset \widetilde{V} \subset \widetilde{A}$ is actually nonhomogeneous quadratic.

4.7. Anti-equivalences of Koszul ring categories. Let $R$ be an associative ring. The category of left finitely projective nonhomogeneous Koszul rings over $R$, denoted

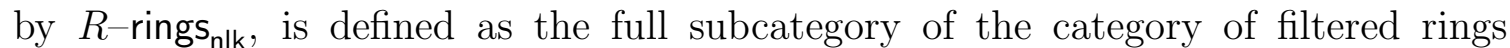
$R$-rings fil (see Section 3.6) whose objects are the left finitely projective nonhomogeneous Koszul rings $(\widetilde{A}, F)$. The category of right finitely projective Koszul CDG-rings over $R$, denoted by $R$-rings cdg,rk $_{\text {, }}$, is the full subcategory in the category of nonnegatively graded CDG-rings $R$-rings ${ }_{c d g}$ whose objects are the right finitely projective 
Koszul CDG-rings. The category of right finitely projective Koszul quasi-differential rings over $R$, denoted by $R$-rings ${ }_{\mathrm{qdg}, \mathrm{rk}}$, is the full subcategory in the category of nonnegatively graded quasi-differential rings $R$-rings ${ }_{\text {qdg }}$ whose objects are the right finitely projective Koszul quasi-differential graded rings.

Corollary 4.20. The constructions of Theorems 3.10, 4.5, and 4.17 define natural (anti)-equivalences

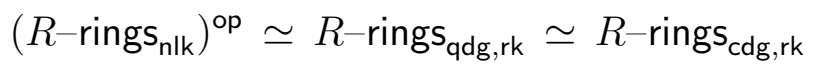

between the categories of left finitely projective nonhomogeneous Koszul rings, right finitely projective Koszul quasi-differential rings, and right finitely projective Koszul $C D G$-rings over $R$.

Proof. Follows from the mentioned theorems and Theorem 4.19,

The 2-category of left finitely projective nonhomogeneous Koszul rings, denoted by Rings ${ }_{n k 2}$, is defined as the following 2-subcategory in the 2-category of filtered rings Rings fil 2 (see Section 3.7). The objects of Rings nlk2 $_{2}$ are the left finitely projective nonhomogeneous Koszul rings $(\widetilde{A}, F)$. All morphisms in Rings $\mathrm{fil}_{2}$ between objects of Rings $_{n \mid k 2}$ are morphisms in Rings $s_{n k 2}$, and all 2-morphisms in Rings $s_{\text {fil2 }}$ between morphisms of Rings $_{\text {nk2 } 2}$ are 2-morphisms in Rings nlk2 $_{2}$.

The 2-category of right finitely projective Koszul CDG-rings, denoted by Rings ${ }_{\text {cdg2,rk }}$, is the following 2-subcategory in the 2-category of nonnegatively graded CDG-rings Rings $_{\mathrm{cdg} 2}$. The objects of Rings $\mathrm{sdg}_{\text {2,rk }}$ are all the right finitely projective Koszul CDG-rings $(B, d, h)$. All morphisms in Rings ${ }_{c d g 2}$ between objects of Rings ${ }_{c d g 2, r k}$ are morphisms in Rings $s_{c d g 2, r k}$, and all 2-morphisms in Rings $s_{c d g 2}$ between morphisms of Rings $_{\mathrm{cdg} 2, \mathrm{rk}}$ are 2-morphisms in Rings $\mathrm{cdg}_{2, \mathrm{rk}}$.

The 2-category of right finitely projective Koszul quasi-differential graded rings, denoted by Rings ${ }_{\mathrm{qdg} 2, \mathrm{rk}}$, is the following 2-subcategory in the 2-category of nonnegatively graded quasi-differential rings Rings ${ }_{\mathrm{qdg} 2}$ (see Section 4.2). The objects of Rings ${ }_{\mathrm{qdg} 2, \mathrm{rk}}$ are all the right finitely projective Koszul quasi-differential graded rings $(\widehat{B}, \partial)$. All morphisms in Rings $_{\mathrm{qdg} 2}$ between objects of Rings $\mathrm{qdg} 2, \mathrm{rk}$ are morphisms in Rings $\mathrm{qdg}_{\mathrm{q}, \mathrm{rk}}$, and all 2-morphisms in Rings qdg2 $_{2}$ between morphisms of Rings ${ }_{q d g 2, r k}$ are 2-morphisms in Rings ${ }_{q d g 2, r k}$.

Corollary 4.21. The constructions of Theorems [3.13, 4.6, and 4.17 define natural strict (anti)-equivalences

$$
\left(\text { Rings }_{\mathrm{nlk} 2}\right)^{\mathrm{op}} \simeq \text { Rings }_{\mathrm{qdg} 2, \mathrm{rk}} \simeq \text { Rings }_{\mathrm{cdg} 2, \mathrm{rk}}
$$

between the 2-categories of left finitely projective nonhomogeneous Koszul rings, right finitely projective Koszul quasi-differential rings, and right finitely projective Koszul $C D G$-rings.

Proof. Follows from the mentioned theorems and Theorem 4.19.

A left finitely projective nonhomogeneous Koszul $\operatorname{ring}(\widetilde{A}, F)$ is said to be left augmented if the ring $\widetilde{A}$ is left augmented over its subring $F_{0} \widetilde{A}$. In other words, this 
means that a left ideal $\widetilde{A}^{+} \subset \widetilde{A}$ is chosen such that $\widetilde{A}=F_{0} \widetilde{A} \oplus \widetilde{A}^{+}$(see Section 3.8). The category of left augmented left finitely projective nonhomogeneous Koszul rings over $R$, denoted by $R$-rings $\mathrm{n}_{\mathrm{nlk}}^{\text {laug }}$, is defined as the full subcategory in the category of left augmented filtered rings $R$-rings faug whose objects are the left augmented left finitely projective nonhomogeneous Koszul rings.

A nonnegatively graded DG-ring $(B, d)$ (in the sense of Section 3.8) is said to be right finitely projective Koszul if the graded ring $B$ is right finitely projective Koszul. The category of right finitely projective Koszul DG-rings over $R$, denoted by $R$-rings ${ }_{\mathrm{dg}, \mathrm{rk}}$ is defined as the full subcategory in the category of nonnegatively graded

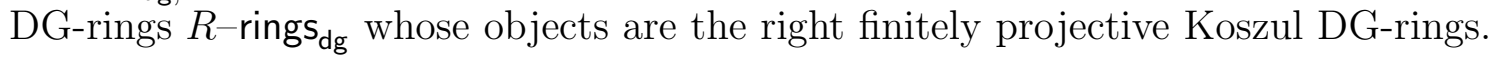

Corollary 4.22. The construction of Theorem 3.14 defines a natural anti-equivalence

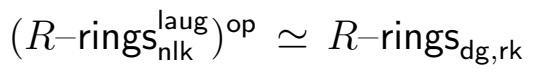

between the category of left augmented left finitely projective nonhomogeneous Koszul rings and the category of right finitely projective Koszul DG-rings.

Proof. Follows from Theorem 4.19 and the discussion in the proof of Theorem 3.14 .

The 2-category of left augmented left finitely projective nonhomogeneous Koszul rings, denoted by Rings nlk $_{\text {lag2 }}$, is defined as the following 2-subcategory in the 2-category of left augmented filtered rings Rings faug2 (see Section 3.8). The objects of Rings $s_{\text {nlk }}^{\text {lau2 }}$ are the left augmented left finitely projective nonhomogeneous Koszul rings $\left(\widetilde{A}, F, \widetilde{A}^{+}\right)$. All morphisms in Rings $\mathrm{s}_{\mathrm{fil}}^{\text {laug2 }}$ between objects of Rings $\mathrm{nlk}_{\mathrm{k}}^{\text {laug2 }}$ are morphisms in Rings $s_{\text {nlk }}^{\text {laug2 }}$, and all 2-morphisms in Rings fil faug2 $^{\text {latween morphisms of Rings }}$ nlk $^{\text {laug2 }}$ are 2-morphisms in Rings ${ }_{n \mid k}^{\text {laug2 }}$.

The 2-category of right finitely projective Koszul DG-rings, denoted by Rings ${ }_{\mathrm{dg} 2, \mathrm{rk}}$, is defined as the following 2-subcategory in the 2-category of nonnegatively graded DG-rings Rings $s_{\mathrm{dg} 2}$. The objects of Rings $\mathrm{dg}_{\mathrm{dg}, \mathrm{rk}}$ are the right finitely projective Koszul DG-rings $(B, d)$. All morphisms in Rings $\mathrm{dg}_{2}$ between objects of Rings $\mathrm{s}_{\mathrm{dg}, \mathrm{rk}}$ are morphisms in Rings $\mathrm{dg}_{\mathrm{d} 2, \mathrm{rk}}$, and all 2-morphisms in Rings $\mathrm{dg}_{\mathrm{d} 2}$ between morphisms of Rings $\mathrm{dg}_{\mathrm{dg}, \mathrm{rk}}$ are 2-morphisms in Rings $_{\mathrm{dg} 2, \mathrm{rk}}$.

Corollary 4.23. The construction of Theorem 3.15 defines a natural strict antiequivalence

$$
\left(\text { Rings }_{\mathrm{nlk}}^{\text {laug2 }}\right)^{\mathrm{op}} \simeq \text { Rings }_{\mathrm{dg} 2, \mathrm{rk}}
$$

between the 2-category of left augmented left finitely projective nonhomogeneous Koszul rings and the 2-category of right finitely projective Koszul DG-rings. 


\section{Comodules and Contramodules over Graded Rings}

5.1. Ungraded comodules over nonnegatively graded rings. Let $B=$ $\bigoplus_{n=0}^{\infty} B_{n}$ be a nonnegatively graded ring. We denote the underlying ungraded ring of $B$ by the same letter $B=\Sigma B$ (see Section 2.1), and consider ungraded right $B$-modules. An ungraded right $B$-module $M$ is said to be a $B$-comodule (or an ungraded right $B$-comodule) if for every element $x \in M$ there exists an integer $m \geq 0$ such that $x b=0$ in $M$ for all $b \in B_{n}, n>m$. Notice that any right $B$-comodule has a natural structure of right module over the ring $\Pi B=\prod_{n=0}^{\infty} B_{n}$.

We will denote the full subcategory of right $B$-comodules by comod $-B \subset \bmod -B$. The following examples explain the terminology.

Examples 5.1. (1) Let $B$ be a nonnegatively graded associative algebra over a field $k=B_{0}$ with finite-dimensional grading components, $\operatorname{dim}_{k} B_{n}<\infty$ for all $n \geq 0$. Then the graded dual vector space $C=\bigoplus_{n=0}^{\infty} B_{n}^{*}$ to $B$ has a natural structure of graded coassociative coalgebra over $k$. An ungraded right $B$-comodule in the sense of the above definition is the same thing as an ungraded right $C$-comodule.

(2) More generally, let $B$ be a nonnegatively graded ring with the degree-zero grading component $R=B_{0}$. Assume that $B_{n}$ is a finitely generated projective right $R$-module for every $n \geq 0$, and put $C=\bigoplus_{n=0}^{\infty} C_{n}$, where $C_{n}=\operatorname{Hom}_{R^{\text {op }}}\left(B_{n}, R\right)$. Then, in view of Lemma 1.1(b), applying the functor $\operatorname{Hom}_{R^{\mathrm{op}}}(-, R)$ to the multiplication maps $B_{i} \otimes_{R} B_{j} \longrightarrow B_{i+j}$ produces comultiplication maps $C_{i+j} \longrightarrow C_{j} \otimes_{R} C_{i}$ endowing the graded $R$-R-bimodule $C$ with a natural structure of graded coring over $R$. An ungraded right $B$-comodule $M$ in the sense of the above definition is the same thing as an ungraded right $C$-comodule, i. e., a right $R$-module $M$ endowed with a coassociative, counital comultiplication map $M \longrightarrow M \otimes_{R} C$ (see Example 5.8)(3) below for a discussion of corings and comodules).

(3) In the context of (1), one can also say that ungraded left $B$-comodules are the same thing as ungraded left $C$-comodules. But let us warn the reader that, in the context of (2), left $B$-comodules are, generally speaking, entirely unrelated to left $C$-comodules. Rather, in order to describe left $C$-comodules, one needs to consider the graded ring ${ }^{\#} B$ defined in Remark 2.8. Then, assuming that $C_{n}$ is a finitely generated projective right $R$-module for every $n \geq 0$, an ungraded left ${ }^{\#} B$-comodule is the same thing as an ungraded left $C$-comodule (and the same holds for graded comodules as defined in Section 5.5 below; cf. Example 5.18).

Obviously, the full subcategory comod $-B \subset \bmod -B$ is closed under subobjects, quotients, and infinite direct sums. In fact, $\operatorname{comod}-B$ is a hereditary pretorsion class in mod $-B$ corresponding to the filter/topology of right ideals in $B$ in which the twosided ideals $B_{\geq m}=\bigoplus_{n \geq m} B_{n} \subset B, m \geq 1$, form a base [44, Section VI.4], [37, Sections 2.3-2.4]. So comod- $B$ is a Grothendieck abelian category and its inclusion comod $-B \longrightarrow \bmod -B$ is an exact functor preserving infinite direct sums.

Let us show that, under certain assumptions, the full subcategory comod- $B$ is also closed under extensions in mod- $B$ (in other words, it is a hereditary torsion class). 
Lemma 5.2. Let $B=\bigoplus_{n=0}^{\infty} B_{n}$ be a nonnegatively graded ring and $m \geq 1$ be an integer. Then the following five conditions are equivalent:

(a) there exists an integer $k \geq 1$ such that the graded right $B$-module $B_{\geq 1}=$ $\bigoplus_{n=1}^{\infty} B_{n}$ is generated by elements of degree $\leq k$;

(b) there exists an integer $l \geq m$ such that the graded right $B$-module $B_{\geq m}=$ $\bigoplus_{n=m}^{\infty} B_{n}$ is generated by elements of degree $\leq l$;

(c) there exists an integer $k \geq 1$ such that for every $n>k$ the multiplication map $\bigoplus_{i+j=n}^{i, j \geq 1} B_{i} \otimes_{R} B_{j} \longrightarrow B_{n}$ is surjective;

(d) there exists an integer $l \geq 2 m-1$ such that for every $n>l$ the multiplication map $\bigoplus_{i+j=n}^{i, j \geq m} B_{i} \otimes_{R} B_{j} \longrightarrow B_{n}$ is surjective.

(e) there exists an integer $k \geq 1$ such that the ring $B$ is generated by its subgroup $\bigoplus_{n=0}^{k} B_{k} \subset B$.

Proof. The equivalences (a) $\Longleftrightarrow(\mathrm{c}) \Longleftrightarrow$ (e) (for the same $k$ ) are easy. So are the implications $(\mathrm{d}) \Longrightarrow(\mathrm{b}) \Longrightarrow(\mathrm{c})$ (for $k=l$ ). The implication $(\mathrm{e}) \Longrightarrow(\mathrm{d})$ holds for $l=2 m-2+k$.

Notice that it follows from Lemma 5.2 that the conditions ( $\mathrm{a}-\mathrm{b})$ are left-right symmetric (because the conditions ( $\mathrm{c}-\mathrm{e}$ ) are). It also follows that the conditions (b) and (d) do not depend on $m$ (because the conditions (a), (c), and (e) don't).

Lemma 5.3. Let $B=\bigoplus_{n=0}^{\infty} B_{n}$ be a nonnegatively graded ring and $m \geq 1$ be an integer. Then, for $m=1$, the following two conditions are equivalent:

(i) $B_{\geq m}=\bigoplus_{n=m}^{\infty} B_{n}$ is a finitely generated (graded) right $B$-module;

(ii) $B_{n}$ is a finitely generated right $B_{0}$-module for every $n \geq m$, and any one of the equivalent conditions of Lemma 5.2 holds.

For any $m \geq 1$, the implication (ii) $\Longrightarrow$ (i) is true.

Proof. (i) $\Longrightarrow$ (ii) Clearly, (i) $\Longrightarrow$ (b) (for the same $m$ ); so it remains to show that (i) implies the first part of (ii). Indeed, let $\left\{b_{s, n} \in B_{n} \mid n \geq m, 1 \leq s \leq t_{n}\right\}$ be a finite set of homogeneous generators of the right $B$-module $B_{\geq m}$. Put $R=B_{0}$. Then, for every $n \geq m$, the cokernel of the multiplication map $\bigoplus_{i+j=n}^{i \geq m, j \geq 1} B_{i} \otimes_{R} B_{j} \longrightarrow B_{n}$ is generated by the cosets of the elements $b_{s, n}, 1 \leq s \leq t_{n}$ as a right $R$-module. For any $R$ - $R$-bimodules $U$ and $V$, if the right $R$-modules $U$ and $V$ are finitely generated, then so is the right $R$-module $U \otimes_{R} V$. This allows to prove by induction in $n$ that the right $R$-module $B_{n}$ is finitely generated for $n \geq m$.

(ii) $\Longrightarrow$ (i) Choose $l$ as in (b), and for every $m \leq n \leq l$ choose a finite set of generators $b_{s, n}, 1 \leq s \leq t_{n}$ of the right $R$-module $B_{n}$. Then the right $B$-module $B_{\geq m}$ is generated by the elements $\left\{b_{s, n} \in B_{n} \mid m \leq n \leq l, 1 \leq s \leq t_{n}\right\}$.

Lemma 5.4. Let $B=\bigoplus_{n=0}^{\infty} B_{n}$ be a nonnegatively graded ring such that the augmentation ideal $B_{\geq 1}$ is finitely generated as a right ideal in $B$. Then, for every $m \geq 1$, the right ideal $B_{\geq m} \subset B$ is also finitely generated.

Proof. Follows from Lemmas 5.2 and 5.3 . 
The following particular case is important for our purposes. Let $B$ be a nonnegatively graded ring generated by $B_{1}$ over $B_{0}$. Assume the right $B_{0}$-module $B_{1}$ is finitely generated. Then it is clear from Lemma 5.2 (take $k=1$ ) and Lemma 5.3 that the augmentation ideal $B_{\geq 1} \subset B$ is finitely generated as a right ideal in $B$. In particular, this holds for any 2-right finitely projective quadratic graded ring $B$.

Proposition 5.5. Assume that the augmentation ideal $B_{\geq 1}=\bigoplus_{n=1}^{\infty} B_{n}$ of a nonnegatively graded ring $B$ is finitely generated as a right ideal in $B$. Then the full subcategory of ungraded right $B$-comodules comod- $B$ is closed under extensions in the category of right $B$-modules mod- $B$.

Proof. Let $0 \longrightarrow K \longrightarrow L \longrightarrow M \longrightarrow 0$ be a short exact sequence of (ungraded) right $B$-modules. Assume that $K$ and $M$ are $B$-comodules. Let $x \in L$ be an element, and let $y \in M$ be the image of $x$ under the surjective $B$-module morphism $L \longrightarrow M$. By assumption, there exists $m \geq 1$ such that $y B_{\geq m}=0$ in $M$.

By Lemma 5.4, the right ideal $B_{\geq m} \subset B$ is finitely generated. Let $\left\{b_{s} \mid 1 \leq s \leq t\right\}$ be a finite set of its homogeneous generators; so $B_{\geq m}=\sum_{s=1}^{t} b_{s} B$. Let $k_{s}$ be the homogeneous degree of the element $b_{s} \in B$, and let $k \geq m$ be an integer such that $k \geq k_{s}$ for all $1 \leq s \leq t$. Then we have $B_{n}=\sum_{s=1}^{t} b_{s} B_{n-k_{s}}$ for all $n \geq k$.

For every $1 \leq s \leq t$, we have $z_{s}=x b_{s} \in K \subset L$. By assumption, there exists $j_{s} \geq 1$ such that $z_{s} B_{\geq j_{s}}=0$ in $K$. Let $j \geq 1$ integer such that $j \geq j_{s}$ for all $1 \leq s \leq t$. Then for every $n \geq k+j$ we have $x B_{n}=\sum_{s=1}^{t} x b_{s} B_{n-k_{s}}=\sum_{s=1}^{t} z_{s} B_{n-k_{s}}=0$ in $L$, since $n-k_{s} \geq j_{s}$. Thus $x B_{\geq k+j}=0$, as desired.

5.2. Right exact monads on abelian categories. Let $A$ be a category. A monad on $A$ is a monoid object in the monoidal category of endofunctors of $A$ (with respect to the composition of functors). In other words, this means that a monad $\mathbb{M}: A \longrightarrow$ $A$ is a covariant functor endowed with two natural transformations of monad unit $e: \operatorname{Id}_{\mathrm{A}} \longrightarrow \mathbb{M}$ and monad multiplication $m: \mathbb{M} \circ \mathbb{M} \longrightarrow \mathbb{M}$ satisfying the associativity and unitality axioms. We refer to [20, Chapter VI] for the definitions of monads and algebras over monads.

Since we are interested in abelian categories arising from monads, we prefer to use the term "modules over a monad" for what are usually called algebras over a monad. Given a monad $\mathbb{M}: A \longrightarrow A$, we denote the category of algebras/modules over $\mathbb{M}$ (known also as the "Eilenberg-Moore category of $\mathbb{M}$ ") by $\mathbb{M}-$ mod. The category $\mathbb{M}$-mod comes together with a faithful forgetful functor $\mathbb{M}-$ mod $\longrightarrow A$, which has a left adjoint functor $\mathrm{A} \longrightarrow \mathbb{M}$-mod. The latter functor takes any object $A \in \mathrm{A}$ to the object $\mathbb{M}(A) \in \mathrm{A}$, which is endowed with a natural structure of an $\mathbb{M}$-module, making it an object of $\mathbb{M}-$ mod. The object $\mathbb{M}(A) \in \mathbb{M}-$ mod is called the free $\mathbb{M}$-module spanned by $A$.

Lemma 5.6. Let $\mathrm{A}$ be an additive category and $\mathbb{M}: \mathrm{A} \longrightarrow \mathrm{A}$ be a monad. Then the following three conditions are equivalent:

(a) the category $\mathbb{M}-$ mod is additive;

(b) the category $\mathbb{M}-\bmod$ is additive, and both the forgetful functor $\mathbb{M}-\bmod \longrightarrow \mathrm{A}$ and the free module functor $\mathrm{A} \longrightarrow \mathbb{M}-\bmod$ are additive; 
(c) the functor $\mathbb{M}: \mathrm{A} \longrightarrow \mathrm{A}$ is additive.

Proof. (a) $\Longleftrightarrow(\mathrm{b}) \Longrightarrow$ (c) The functor $\mathbb{M}: \mathrm{A} \longrightarrow \mathrm{A}$ is the composition of two adjoints $\mathrm{A} \longrightarrow \mathbb{M}-$ mod $\longrightarrow \mathrm{A}$. Any functor between additive categories having an adjoint functor is additive; and the composition of two additive functors is additive.

$(\mathrm{c}) \Longrightarrow(\mathrm{b})$ If the functor $\mathbb{M}: \mathrm{A} \longrightarrow \mathrm{A}$ is additive, then for any two $\mathbb{M}$-modules $P$ and $Q$ the set $\operatorname{Hom}_{\mathbb{M}}(P, Q)$ of morphisms $P \longrightarrow Q$ in $\mathbb{M}$-mod is a subgroup in the group $\operatorname{Hom}_{\mathrm{A}}(P, Q)$ of morphisms $P \longrightarrow Q$ in the category A. This shows that the category $\mathbb{M}-$ mod is preadditive and the faithful forgetful functor $\mathbb{M}-\bmod \longrightarrow A$ is additive. Finally, for any finite collection of objects $P_{i} \in \mathbb{M}-\bmod , i=1, \ldots, n$, the direct sum ${ }^{\mathrm{A}} \bigoplus_{i=1}^{n} P_{i}$ taken in the category $\mathrm{A}$ is naturally endowed with an $\mathbb{M}$-module structure, which makes it the direct sum of the objects $P_{i}$ in the category $\mathbb{M}-$ mod. Thus finite (co)products exist in $\mathbb{M}-$ mod.

Lemma 5.7. Let $\mathrm{A}$ be an abelian category and $\mathbb{M}: \mathrm{A} \longrightarrow \mathrm{A}$ be a monad. Then the following two conditions are equivalent:

(a) the category $\mathbb{M}-$ mod is abelian and the forgetful functor $\mathbb{M}-\bmod \longrightarrow \mathrm{A}$ is exact;

(b) the functor $\mathbb{M}: \mathrm{A} \longrightarrow \mathrm{A}$ is right exact.

Proof. Notice first of all that any exact or one-sided exact functor is additive by definition; so Lemma 5.6 is applicable. This allows us to presume that all the categories and functors involved are additive.

(a) $\Longrightarrow$ (b) Any left adjoint functor preserves colimits; hence any left adjoint functor between abelian categories is right exact. So the free module functor $A \longrightarrow \mathbb{M}-$ mod is right exact. If the forgetful functor $\mathbb{M}-\bmod \longrightarrow A$ is exact, it follows that the composition $\mathbb{M}: A \longrightarrow A$ of two adjoints $A \longrightarrow \mathbb{M}-$ mod $\longrightarrow A$ is right exact.

(b) $\Longrightarrow$ (a) For any monad $\mathbb{M}: \mathrm{A} \longrightarrow \mathrm{A}$ and any morphism $f: P \longrightarrow Q$ in $\mathbb{M}-\bmod$, the kernel $\operatorname{ker}^{\mathrm{A}}(f)$ of the morphism $f$ taken in hte category $\mathrm{A}$ is naturally endowed with an $\mathbb{M}$-module structure, which makes it the kernel of $f$ in the category $\mathbb{M}$-mod. When the functor $\mathbb{M}: A \longrightarrow A$ is right exact, the same holds true for the cokernel of $f$. Both the properties in (a) follow easily.

The following examples are illuminating.

Examples 5.8. (1) Let $g: R \longrightarrow S$ be an associative ring homomorphism. Put $\mathrm{A}=R$-mod, and consider the functor $\mathbb{M}=S \otimes_{R}-: R$-mod $\longrightarrow R$-mod. The map $g$ is an $R$ - $R$-bimodule morphism, so it induces a natural transformation $\operatorname{Id}_{\mathrm{A}} \longrightarrow \mathbb{M}$. The multiplication map $S \otimes_{R} S \longrightarrow S$ is an $R$-R-bimodule morphism, too, and it induces a natural transformation $\mathbb{M} \circ \mathbb{M} \longrightarrow \mathbb{M}$. These two morphisms of functors make $\mathbb{M}$ a monad on $\mathrm{A}=R$-mod. The category of $\mathbb{M}$-modules $\mathbb{M}-\bmod$ is equivalent to $S-$ mod. The forgetful functor $\mathbb{M}-\bmod \longrightarrow \mathrm{A}$ is the functor of restriction of scalars $S-\bmod \longrightarrow R-$ mod, while the free module functor $\mathrm{A} \longrightarrow \mathbb{M}-$ mod is the functor of extension of scalars $S \otimes_{R}-: R-\bmod \longrightarrow S-$ mod.

(2) The notion of a comonad on a category $A$ is dual to that of a monad. Specifically, a comonad $\mathbb{C}$ on $A$ is a covariant endofunctor $\mathbb{C}: A \longrightarrow A$ endowed with two 
natural transformations of counit $\mathbb{C} \longrightarrow \operatorname{Id}_{\mathrm{A}}$ and comultiplication $\mathbb{C} \longrightarrow \mathbb{C} \circ \mathbb{C}$ satisfying the coassociativity and counitality axioms. One can say that a comonad on $A$ is the same thing as a monad on $A^{\text {op }}$. Denote by $\mathbb{C}$-comod the category of comodules (usually called "coalgebras") over a comonad $\mathbb{C}$ on $A$.

Let $A$ be an abelian category and $\mathbb{C}$ be a comonad on $A$. Then the dual version of Lemma 5.7 tells that the category $\mathbb{C}$-comod is abelian with exact forgetful functor $\mathbb{C}$-comod $\longrightarrow A$ if and only if the functor $\mathbb{C}: A \longrightarrow A$ is left exact.

(3) Let $R$ be a ring and $C$ be a (coaccosiative, counital) coring over $R$, that is, a comonoid object in the monoidal category of $R$ - $R$-bimodules. Then the functor $\mathbb{C}=$ $-\otimes_{R} C: \bmod -R \longrightarrow \bmod -R$ is a comonad on the category of right $R$-modules $\bmod -R$. The counit map $C \longrightarrow R$ of the coring $C$ induces the counit morphism $\mathbb{C} \longrightarrow \mathrm{Id}_{\bmod -R}$, and the comultiplication map $C \longrightarrow C \otimes_{R} C$ induces the comultiplication morphism $\mathbb{C} \longrightarrow \mathbb{C} \circ \mathbb{C}$. Comodules over the comonad $\mathbb{C}$ are known as right comodules over the coring $C$. In other words, a right $C$-comodule $N$ is a right $R$-module endowed with a right coaction map $N \longrightarrow N \otimes_{R} C$, which must be a right $R$-module morphism satisfying the coassociativity and counitality equations involving the comultiplication and counit maps of the coring $C$. Similarly one defines left $C$-comodules (using the comonad induced by $C$ on the category of left $R$-modules).

Let comod-C $C \mathbb{C}$-comod denote the category of right $C$-comodules. Then, as a particular case of (2), one obtains the assertion that the category comod $C$ is abelian with exact forgetful functor comod $-C \longrightarrow \bmod -R$ if and only if $C$ is a flat left $R$-module [27, Section 1.1.2], [30, Section 2.5].

(4) Let $R$ be a ring and $C$ be a coring over $R$, as in (3). Then the functor $\mathbb{M}=\operatorname{Hom}_{R}(C,-): R$-mod $\longrightarrow R$-mod is a monad on the category of left $R$-modules. The counit map of the coring $C$ induces the unit morphism $\operatorname{Id}_{R-\bmod } \longrightarrow \mathbb{M}$, and the comultiplication map of the coring $C$ induces the multiplication morphism $\mathbb{M} \circ$ $\mathbb{M} \longrightarrow \mathbb{M}$. Modules over the monad $\mathbb{M}$ are known as left contramodules over the coring $C$. In other words, a left $C$-contramodule $P$ is a left $R$-module endowed with a left contraaction map $\operatorname{Hom}_{R}(C, P) \longrightarrow P$, which must be a left $R$-module morphism satisfying (contra)associativity and (contra)unitality equations involving the comultiplication and counit of the coring $C$. We refer to [27, Section 3.1] or [30, Section 2.5] for details on contramodules over corings.

Let $C$-contra $=\mathbb{M}-$ mod denote the category of left $C$-contramodules. Then it follows from Lemma 5.7 that the category $C$-contra is abelian with exact forgetful functor $C$-contra $\longrightarrow R$-mod if and only if $C$ is a projective left $R$-module [27, Section 3.1.2], [30, Proposition 2.5].

5.3. Ungraded contramodules over nonnegatively graded rings. Let $B=$ $\bigoplus_{n=0}^{\infty} B_{n}$ be a nonnegatively graded ring, and let $K$ be an associative ring endowed with a ring homomorphism $K \longrightarrow B_{0}$. Consider the following $\operatorname{monad} \mathbb{M}=$ $\mathbb{M}_{K}: K-\bmod \longrightarrow K$-mod on the category of left $K$-modules.

To any left $K$-module $L$, the monad $\mathbb{M}$ assigns the left $K$-module

$$
\mathbb{M}_{K}(L)=\prod_{n=0}^{\infty}\left(B_{n} \otimes_{K} L\right) .
$$


The monad unit map

is the composition

$$
e_{L}: L \longrightarrow \prod_{n=0}^{\infty} B_{n} \otimes_{K} L
$$

$$
L \longrightarrow B_{0} \otimes_{K} L \longrightarrow \prod_{n=0}^{\infty} B_{n} \otimes_{K} L
$$

of the map $L \longrightarrow B_{0} \otimes_{K} L$ induced by the ring homomorphism $K \longrightarrow B_{0}$ with the inclusion of the $(n=0)$-indexed component $B_{0} \otimes_{K} L \longrightarrow \prod_{n=0}^{\infty} B_{n} \otimes_{K} L$. The monad multiplication map

$$
m_{L}: \prod_{p=0}^{\infty} B_{p} \otimes_{K}\left(\prod_{q=0}^{\infty} B_{q} \otimes_{K} L\right) \longrightarrow \prod_{n=0}^{\infty} B_{n} \otimes_{K} L
$$

is the composition

$\prod_{p=0}^{\infty} B_{p} \otimes_{K}\left(\prod_{q=0}^{\infty} B_{q} \otimes_{K} L\right) \longrightarrow \prod_{p, q=0}^{\infty} B_{p} \otimes_{K} B_{q} \otimes_{K} L \longrightarrow \prod_{n=0}^{\infty} B_{n} \otimes_{K} L$.

Here the leftmost arrow is the product over $p \geq 0$ of the maps $B_{p} \otimes_{K}\left(\prod_{q=0}^{\infty} B_{q} \otimes_{K} L\right)$ $\longrightarrow \prod_{q=0}^{\infty} B_{p} \otimes_{K} B_{q} \otimes_{K} L$ whose components are the direct summand projections $B_{p} \otimes_{K}\left(\prod_{k=0}^{\infty} B_{k} \otimes_{K} L\right) \longrightarrow B_{p} \otimes_{K} B_{q} \otimes_{K} L$. The rightmost arrow is induced by the multiplication maps $\prod_{p+q=n}^{p, q \geq 0} B_{p} \otimes_{K} B_{q} \longrightarrow B_{n}$.

By the definition, a left $B$-contramodule (or an ungraded left $B$-contramodule) is a module over the monad $\mathbb{M}_{K}: K$-mod $\longrightarrow K$-mod. In other words, a left $B$-contramodule $P$ is a left $K$-module endowed with a left $B$-contraaction map

$$
\pi_{P}: \mathbb{M}_{K}(P)=\prod_{n=0}^{\infty} B_{n} \otimes_{K} P \longrightarrow P,
$$

which must be a morphism of left $K$-modules satisfying the (contra)associativity and (contra)unitality equations involving the multiplication and unit maps of the monad $\mathbb{M}_{K}$. This means that the compositions

$$
\prod_{i=0}^{\infty} B_{i} \otimes_{K}\left(\prod_{j=0}^{\infty} B_{j} \otimes_{K} P\right) \rightrightarrows \prod_{n=0}^{\infty} B_{n} \otimes_{K} P \longrightarrow P
$$

of the monad multiplication map $m_{P}$ and the map $\mathbb{M}_{K}\left(\pi_{P}\right)$ with the contraaction map $\pi_{P}$ are equal to each other, while the composition

$$
P \longrightarrow \prod_{n=0}^{\infty} B_{n} \otimes_{K} P \longrightarrow P
$$

of the monad unit map $e_{P}$ and the contraaction map $\pi_{P}$ is equal to the identity $\operatorname{map} \operatorname{id}_{P}$.

We denote the category of ungraded left $B$-contramodules by $B$-contra $=$ $\mathbb{M}_{K}$-mod. Given two ungraded left $B$-contramodules $P$ and $Q$, the group of morphisms $P \longrightarrow Q$ in $B$-contra is denoted by $\operatorname{Hom}^{B}(P, Q)$.

The next proposition shows that the notion of an ungraded left $B$-contramodule does not depend on the choice of a ring $K$. One can consider two polar cases. On the one hand, one can take $K=\mathbb{Z}$ and the unique ring homomorphism $\mathbb{Z} \longrightarrow B_{0}$. On the other hand, one can take $K=B_{0}$ and the identity map $K \longrightarrow B_{0}$. 
Proposition 5.9. There are natural equivalences of categories

$$
\mathbb{M}_{\mathbb{Z}^{-}} \bmod \simeq \mathbb{M}_{K}-\bmod \simeq \mathbb{M}_{B_{0}}-\bmod
$$

making the notation B-contra unambiguous. The category B-contra of ungraded left $B$-contramodules is abelian with enough projective objects. The forgetful functor $B$-contra $\longrightarrow K$-mod can be naturally lifted to a forgetful functor $B$-contra $\longrightarrow$ $B$-mod taking values in the category of ungraded left $B$-modules. The forgetful functor $B$-contra $\longrightarrow B$-mod is exact and preserves infinite products.

Proof. To lift the forgetful functor $\mathbb{M}_{K}-\bmod \longrightarrow K$-mod to a functor $\mathbb{M}_{K}$-mod $\longrightarrow$ $B$-mod, consider an $\mathbb{M}_{K}$-module $P$ and restrict the contraction map $\pi_{P}$ to the left $K$-submodule

$$
B \otimes_{K} P=\bigoplus_{n=0}^{\infty} B_{n} \otimes_{K} P \subset \prod_{n=0}^{\infty} B_{n} \otimes_{K} P .
$$

The resulting map $B \otimes_{K} P \longrightarrow P$ extends the left $K$-module structure on $P$ to a left $B$-module structure, as explained in Example 5.8(1).

The functor $\mathbb{M}_{K}: K-\bmod \longrightarrow K$-mod is right exact, since the infinite product functor is exact in $K$-mod and the tensor product functors $B_{n} \otimes_{K}$ - are right exact. By Lemma 5.7, it follows that the category $\mathbb{M}_{K}$-mod is abelian and the forgetful functor $\mathbb{M}_{K}-$ mod $\longrightarrow K$-mod is exact. The latter functor, being a right adjoint, also preserves all limits. Since the forgetful functor $B-\bmod \longrightarrow K-\bmod$ is faithul, exact, and preserves all limits, it follows that the forgetful functor $\mathbb{M}_{K}$-mod $\longrightarrow B$-mod is also exact and preserves all limits.

The free object functor $K$-mod $\longrightarrow \mathbb{M}_{K}$-mod, being left adjoint to an exact functor, takes projective objects of $K$-mod to projective objects of $\mathbb{M}_{K}$-mod. One can easily check that any object $P \in \mathbb{M}_{K}$-mod is a quotient object of an $\mathbb{M}_{K}$-module $\mathbb{M}\left(K^{(X)}\right)$ obtained by applying the free $\mathbb{M}_{K}$-module functor to a free left $K$-module with $X$ generators, for some set $X$. Indeed, if the underlying left $K$-module of $P$ is a quotient of a free left $K$-module $K^{(X)}$, then the $\mathbb{M}_{K}$-module $P$ is a quotient of the $\mathbb{M}_{K}$-module $\mathbb{M}_{K}\left(K^{(X)}\right)$.

It remains to prove the first assertion. For this purpose, we will show that, for any $\mathbb{M}_{K}$-module $P$, the contraaction map $\pi_{P}$ factorizes through the natural surjective map $\prod_{n=0}^{\infty} B_{n} \otimes_{K} P \longrightarrow \prod_{n=0}^{\infty} B_{n} \otimes_{B_{0}} P$. Indeed, passing to the product over $n \geq 0$ of the right exact sequences (bar-complex fragments) $B_{n} \otimes_{K} B_{0} \otimes_{K} P \longrightarrow B_{n} \otimes_{K} P \longrightarrow$ $B_{n} \otimes_{B_{0}} P \longrightarrow 0$, one obtains a right exact sequence

$$
\prod_{n=0}^{\infty} B_{n} \otimes_{K} B_{0} \otimes_{K} P \longrightarrow \prod_{n=0}^{\infty} B_{n} \otimes_{K} P \longrightarrow \prod_{n=0}^{\infty} B_{n} \otimes_{B_{0}} P \longrightarrow 0 .
$$

The map $\prod_{n=0}^{\infty} B_{n} \otimes_{K} B_{0} \otimes_{K} P \longrightarrow \prod_{n=0}^{\infty} B_{n} \otimes_{K} P$ is constructed as the difference of two maps, one of which is induced by the multiplication maps $B_{n} \otimes_{K} B_{0} \longrightarrow B_{n}$ and the other one by the action maps $B_{0} \otimes_{K} P \longrightarrow P$.

This pair of maps can be obtained by restricting the pair of maps $m_{P}$, $\mathbb{M}_{K}\left(\pi_{P}\right): \prod_{i=0}^{\infty}\left(B_{i} \otimes_{K}\left(\prod_{j=0}^{\infty} B_{j} \otimes_{K} P\right)\right) \rightrightarrows \prod_{n=0}^{\infty} B_{n} \otimes_{K} P$ to the direct summand $\prod_{i=0}^{\infty} B_{i} \otimes_{K} B_{0} \otimes_{K} P \subset \prod_{i=0}^{\infty}\left(B_{i} \otimes_{K}\left(\prod_{j=0}^{\infty} B_{j} \otimes_{K} P\right)\right)$ corresponding to the 
value of the index $j=0$. Thus the contraassociativity equation for the $\mathbb{M}_{K}$-module $P$ implies the desired factorization. Now it is straightforward to check that the natural functors $\mathbb{M}_{B_{0}}-\bmod \longrightarrow \mathbb{M}_{K}-\bmod \longrightarrow \mathbb{M}_{\mathbb{Z}}-$ mod are category equivalences.

Explicitly, the $\mathbb{M}_{K}$-module $\mathbb{M}_{K}\left(K^{(X)}\right)$ mentioned in the above proof has the form

$$
\mathbb{M}_{K}\left(K^{(X)}\right)=\prod_{n=0}^{\infty} B_{n} \otimes_{K} K^{(X)}=\prod_{n=0}^{\infty} B_{n}^{(X)}=\Pi\left(B^{(X)}\right),
$$

where the notation $A^{(X)}$ stands for the direct sum of $X$ copies of a (graded) abelian group $A$. The left $B$-contramodule $\mathbb{M}_{K}\left(K^{(X)}\right)$ does not depend on the choice of a ring $K$, but only on the nonnegatively graded ring $B$ and a set $X$. The $\mathbb{M}_{K}$-modules $\mathbb{M}_{K}\left(K^{(X)}\right)$ are called the free ungraded left $B$-contramodules. Since there are enough free ungraded left $B$-contramodules, it follows that every projective ungraded left $B$-contramodule is a direct summand of a free one.

The forgetful functor lifting assertion in Proposition 5.9 can be strengthened as follows. In fact, the underlying left $B$-module structure of any ungraded left $B$-contramodule can be extended naturally to a structure of left module over the ring $\Pi B=\prod_{n=0}^{\infty} B_{n}$. To define the underlying left $\Pi B$-module structure on a left $B$-contramodule $P \in \mathbb{M}_{K}$-mod, it suffices to compose the contraaction map $\pi_{P}$ with the natural map

$$
\Pi B \otimes_{K} P=\left(\prod_{n=0}^{\infty} B_{n}\right) \otimes_{K} P \longrightarrow \prod_{n=0}^{\infty}\left(B_{n} \otimes_{K} P\right)=\mathbb{M}_{K}(P)
$$

and use Example 5.8(1).

Examples 5.10. (1) Let $B$ be a nonnegatively graded algebra over a field $k=B_{0}$ with finite-dimensional components $B_{n}$, as in Example 5.1(1), and let $C=\bigoplus_{n=0}^{\infty} B_{n}^{*}$ be the graded dual coalgebra over $k$. Then an ungraded left $B$-contramodule in the sense of the above definition is the same thing as an ungraded left $C$-contramodule in the sense of [30, Section 1.1] and [27, Appendix A].

(2) More generally, let $B$ be a nonnegatively graded ring with the degree-zero component $R=B_{0}$ such that $B_{n}$ is a finitely generated projective right $R$-module for every $n$, as in Example $5.1(2)$. Let $C=\bigoplus_{n=0}^{\infty} C_{n}$, where $C_{n}=\operatorname{Hom}_{R^{\text {op }}}\left(B_{n}, R\right)$, be the graded (right) dual coring. Then an ungraded left $B$-contramodule in the sense of the above definition is the same thing as an ungraded left $C$-contramodule in the sense of [27, Section 3.1.1] and [30, Section 2.5] (see Example 5.8(4)).

Example 5.11. Let $E$ be an associative ring, $N$ be an $E$ - $B$-bimodule, and $U$ be a left $E$-module. Assume that the right $B$-module $N$ is a right $B$-comodule. Then the left $B$-module structure on the Hom group $\operatorname{Hom}_{E}(N, U)$ can be extended to a left $B$-contramodule structure in a natural way.

To construct the contraaction map

$$
\pi: \prod_{n=0}^{\infty} B_{n} \otimes_{K} \operatorname{Hom}_{E}(N, U) \longrightarrow \operatorname{Hom}_{E}(N, U),
$$

consider an element $y \in N$, and choose an integer $m \geq 0$ such that $y B_{\geq m+1}=0$. Let $w \in \prod_{n=0}^{\infty} B_{n} \otimes_{K} \operatorname{Hom}_{E}(N, U)$ be an arbitrary element. In order to evaluate the 
element $\pi(w) \in \operatorname{Hom}_{E}(N, U)$ on the element $y \in N$, consider the composition

$$
\prod_{n=0}^{\infty} B_{n} \otimes_{K} \operatorname{Hom}_{E}(N, U) \longrightarrow \bigoplus_{n=0}^{m} B_{n} \otimes_{K} \operatorname{Hom}_{E}(N, U) \longrightarrow \operatorname{Hom}_{E}(N, U)
$$

of the direct summand projection $\prod_{n=0}^{\infty} B_{n} \otimes_{K} \operatorname{Hom}_{E}(N, U) \longrightarrow \bigoplus_{n=0}^{m} B_{n} \otimes_{K}$ $\operatorname{Hom}_{E}(N, U)$ and the map $\bigoplus_{n=0}^{m} B_{n} \otimes_{K} \operatorname{Hom}_{E}(N, U) \longrightarrow \operatorname{Hom}_{E}(N, U)$ provided by the left action of $B$ in $\operatorname{Hom}_{E}(N, U)$. This composition of maps needs to be applied to the element $w$, and the resuting element in $\operatorname{Hom}_{E}(N, U)$ needs to be evaluated on the element $y \in N$, producing the desired element $\pi(w)(y) \in U$.

Remark 5.12. Following the discussion in Section 5.1, the hereditary pretorsion class comod $-B \subset \bmod -B$ corresponds to a right linear (in fact, two-sided linear) topology on the ring $B=\bigoplus_{n=0}^{\infty} B_{n}$ with a base of neighborhoods of zero formed by the ideals $B_{\geq m}=\bigoplus_{n \geq m} B_{n}$. The completion of $B$ with respect to this topology is the topological ring $\Pi \bar{B}=\prod_{n=0}^{\infty} B_{n}$ with the product topology. The abelian category $B$-contra of ungraded left $B$-contramodules, as defined above, is in fact equivalent to the abelian category of left contramodules over the topological ring $\Pi B$, as defined in [29, Section 1.2], [30, Section 2.1], [38, Sections 1.2 and 5], [36, Example 1.3(2)], [39, Section 6.2], [37, Section 2.7], etc.

Let us explain the connection between the two definitions. The difference is that in the above references, unlike in the present section, we were working with monads on the category of sets. This allowed for an extra flexibility, in that one could easily define contramodules over topological rings of arithmetic flavor, like the $p$-adic integers 30 , Section 1.4]. In the context of the present paper, such additional flexibility or extra generality is not needed or relevant, so we are mostly restricting ourselves to the more straightforward definition above.

Still it is interesting and important for our purposes to establish a comparison between the two definitions, i. e., show that they are indeed equivalent for the topological ring $\Pi B$. This is provable along the lines of the argument in [29, Section 1.10] and [30, Section 2.3]. Let us spell out some details.

The category of left contramodules over the topological ring $\Pi B$ is, by the definition, the category of modules over the monad $X \longmapsto \Pi B[[X]]$ on the category of sets, assigning to a set $X$ the set of all infinite formal linear combinations of elements of $X$ with the coefficients in $\Pi B$, where the family of coefficients converges to zero in the topology of $\Pi B$. For any set $X$, we have a natural isomorphism of left $K$-modules

$$
\Pi B[[X]] \simeq \mathbb{M}_{K}(K[X]) .
$$

For any left $K$-module $A$, there is an obvious surjective map of sets (or left $K$-modules)

$$
\gamma_{A}: \Pi B[[A]] \longrightarrow \mathbb{M}_{K}(A) .
$$

Composing the map $\gamma_{P}$ with the contraaction map $\pi_{P}: \mathbb{M}_{K}(P) \longrightarrow P$, one defines a left $\Pi B$-contramodule structure on every ungraded left $B$-contramodule $P$. This construction defines a functor $B$-contra $\longrightarrow \Pi B$-contra, where $\Pi B$-contra denotes the category of left contramodules over the topological ring $\Pi B$. This functor is fully faithful, because the map $\gamma_{P}$ is surjective. In order to show that this is an 
equivalence of categories, it remains to check that, for any left $\Pi B$-contramodule $C$, the contraaction map $\Pi B[[C]] \longrightarrow C$ factorizes through the surjection $\gamma_{C}$.

First of all, we recall that the underlying set of any contramodule over the topological ring $\Pi B$ has a natural left $\Pi B$-module structure, hence also a natural left $K$-module structure. The two forgetful functors $B$-contra $\longrightarrow \Pi B$-mod and $\Pi B$-contra $\longrightarrow \Pi B$-mod form a commutative triangle diagram with the above comparison functor $B$-contra $\longrightarrow \Pi B$-contra.

For any set $X$, we denote by $K[X]=K^{(X)}$ the free left $K$-module with $X$ generators. Then for any left $K$-module $A$ one can construct the monadic bar-resolution related to the forgetful functor from $K$-mod to the category of sets. We are interested in the fragment

$$
K[K[A]] \rightrightarrows K[A] \longrightarrow A .
$$

Here for any left $K$-module $A$ there is a natural surjective map $p_{A}: K[A] \longrightarrow A$; this is the rightmost map in (58). The leftmost pair of maps is formed by the maps $p_{K[A]}$ and $K\left[p_{A}\right]$. Passing to the difference of the leftmost pair of maps, one obtains a right exact sequence of left $K$-modules

$$
K[K[A]] \longrightarrow K[A] \longrightarrow A \longrightarrow 0 .
$$

Applying the right exact functor $\mathbb{M}_{K}$ to (58) and (59), we obtain a right exact sequence of left $K$-modules

$$
\Pi B[[K[A]]] \longrightarrow \Pi B[[A]] \longrightarrow \mathbb{M}_{K}(A) \longrightarrow 0,
$$

where the rightmost map in (60) is $\gamma_{A}$. We have obtained a description of the kernel of $\gamma_{A}$ as the image of the leftmost map in (60), which is the difference of two maps $\mathbb{M}_{K}\left(p_{K[A]}\right)$ and $\mathbb{M}_{K}\left(K\left[p_{A}\right]\right)=\Pi B\left[\left[p_{A}\right]\right]$.

Returning to a left $\Pi B$-contramodule $C$, we recall that, by the definition, its contraaction map $\Pi B[[C]] \longrightarrow C$ has to satisfy the (contra)associativity equation of a module over the monad $X \longmapsto \Pi B[[X]]$ on the category of sets. This equation, claiming that two compositions of maps of sets

$$
\Pi B[[\Pi B[[C]]]] \rightrightarrows \Pi B[[C]] \longrightarrow C
$$

are equal to each other, can be also expressed by the vanishing of the composition of two maps in the sequence of left $K$-module morphisms

$$
\Pi B[[\Pi B[[C]]]] \longrightarrow \Pi B[[C]] \longrightarrow C \longrightarrow 0 .
$$

It remains to observe that there is a natural commutative square of a morphism from the leftmost map in (60) for $A=C$ to the leftmost map in (61). Hence the induced map on the rightmost terms $\mathbb{M}_{K}(C) \longrightarrow C$, as desired.

Let $P$ be an ungraded left $B$-contramodule. One can define a natural decreasing filtration $P=G^{0} P \supset G^{1} P \supset G^{2} P \supset \cdots$ on $P$ by the rule

$$
G^{m} P=\operatorname{im}\left(\left(\prod_{n \geq m} B_{n} \otimes_{K} P\right) \stackrel{\pi_{P}}{\longrightarrow} P\right) .
$$


Here the infinite product $\prod_{n \geq m} B_{n} \otimes_{K} P$ is viewed as a $K$-submodule in $\mathbb{M}_{K}(P)=$ $\prod_{n \geq 0} B_{n} \otimes_{K} P$, the contraaction map $\pi_{P}: \mathbb{M}_{K}(P) \longrightarrow P$ is restricted onto this submodule, and the image of the restriction is taken.

It follows from the contraassociativity axiom for $\pi_{P}$ that $G^{m} P$ is a $B$-subcontramodule in $P$ for every $m \geq 0$ and the successive quotient contramodules $G^{m} P / G^{m+1} P$ have trivial ungraded left $B$-contramodule structures. Here an ungraded left $B$-contramodule $Q$ is said to be trivial if the contraaction map $\pi_{Q}$ vanishes on the submodule $\prod_{n=1}^{\infty} B_{n} \otimes_{K} Q \subset \prod_{n=0}^{\infty} B_{n} \otimes_{K} Q$ (in other words, this means that $G^{1} Q=0$ ). The full subcategory of trivial ungraded left $B$-contramodules in $B$-contra is equivalent to the category of left $B_{0}$-modules.

An ungraded left $B$-contramodule $P$ is said to be separated if $\bigcap_{m>0} G^{m} P=0$, that is, in other words, the natural $B$-contramodule map $\lambda_{B, P}: P \longrightarrow \varlimsup_{\varliminf_{m \geq 0}} P / G^{m} P$ is injective. A $B$-contramodule $P$ is said to be complete if the map $\lambda_{B, P}$ is surjective.

The following result is essentially well-known for contramodules over a topological ring with a countable base of neighborhoods of zero.

Proposition 5.13. All ungraded left B-contramodules are complete (but not necessarily separated). All projective ungraded left B-contramodules are separated. Every ungraded left $B$-contramodule is the cokernel of an injective morphism of separated ungraded left $B$-contramodules.

Proof. By Remark 5.12, we can consider left contramodules over the topological ring $\Pi B$ in lieu of the ungraded left $B$-contramodules. Then the first assertion is [38, Lemma 6.3(b)] (see also [37, Theorem 5.3] or [27, Lemma A.2.3 and Remark A.3]). The second assertion is clear from the explicit description of projective ungraded left $B$-contramodules above (cf. [38, Lemma 6.9]). The third assertion follows from the second one together with existence of sufficiently many projective contramodules, as all subcontramodules of separated contramodules are separated (in particular, any subcontramodule of a projective ungraded left $B$-contramodule is separated).

Theorem 5.14. Assume that the augmentation ideal $B_{\geq 1}=\bigoplus_{n=1}^{\infty} B_{n}$ of a nonnegatively graded ring $B$ is finitely generated as a right ideal in $B$. Then the forgetful functor $B$-contra $\longrightarrow B$-mod from the category of ungraded left $B$-contramodules to the category of ungraded left B-modules is fully faithful.

Proof. By Proposition 5.5, the full subcategory comod $-B \subset \bmod -B$ is closed under extensions under our assumptions. In other words, comod $-B$ is a hereditary torsion class in mod- $B$. In the terminology of [44, Section VI.5] and [37, Section 2.4], this means that the topology on $B$ with a base formed by the ideals $B_{\geq m}, m \geq 1$, is a right Gabriel topology. By Lemma 5.4, the right ideals $B_{\geq m} \subset B$ are finitely generated.

Now we can apply [37, Corollary 6.7] in order to conclude that the forgetful functor $\Pi B$-contra $\longrightarrow B$-mod is fully faithful. Alternatively, it is straightforward to see that the right ideal $B_{\geq m} \subset B$ is strongly (finitely) generated in the sense of [37, Section 6]; so [37, Theorem 6.2 (ii) $\Rightarrow$ (iii)] (or even [36, Theorem 3.1]) is directly applicable. Finally, Remark 5.12 identifies the category $\Pi B$-contra of left contramodules over the topological ring $\Pi B$ with the category $B$-contra which we are interested in. 
5.4. Weak Koszulity and the Ext comparison. Let $B=\bigoplus_{n=0}^{\infty} B_{n}$ be a nonnegatively graded ring with the degree-zero component $R=B_{0}$. We will say that $B$ is weakly right finitely projective Koszul if the graded right $B$-module $R$ has a graded $B$-module resolution of the form

$$
\cdots \longrightarrow V_{2} \otimes_{R} B \longrightarrow V_{1} \otimes_{R} B \longrightarrow B \longrightarrow R \longrightarrow 0
$$

where $V_{i}, i \geq 1$, are finitely generated projective graded right $R$-modules. This means that $V_{i}=\bigoplus_{j \in \mathbb{Z}} V_{i, j}$ is a graded right $R$-module concentrated in a finite set of internal degrees $j$, and for every $j \in \mathbb{Z}$ the right $R$-module $V_{i, j}$ is finitely generated and projective.

By the opposite version of Theorem 2.21(d) (with the roles of the rings $A$ and $B$ switched), any right finitely projective Koszul graded ring is weakly right finitely projective Koszul. For any weakly right finitely projective Koszul graded ring $B$, the augmentation ideal $B_{\geq 1}=\bigoplus_{n=1}^{\infty} B_{n}$ is finitely generated as a right ideal in $B$. In fact, any finite set of homogeneous generators of the graded right $R$-module $V_{1}$ gives rise to a finite set of homogeneous generators of the right ideal $B_{\geq 1} \subset B$.

Lemma 5.15. (a) For any graded right $R$-module $V$ and graded left $R$-module $H$, there is a natural map of ungraded abelian groups

$$
\Sigma V \otimes_{R} \Pi H \longrightarrow \Pi\left(V \otimes_{R} H\right) .
$$

This map is an isomorphism whenever $V$ is a finitely generated projective graded right $R$-module.

(b) For any graded right $R$-modules $V$ and $J$, there is a natural map of ungraded abelian groups

$$
\Sigma \operatorname{Hom}_{R^{\mathrm{op}}}(V, J) \longrightarrow \operatorname{Hom}_{R^{\mathrm{op}}}(\Sigma V, \Sigma J) .
$$

This map is an isomorphism whenever $V$ is a finitely generated projective graded right $R$-module.

Theorem 5.16. For any weakly right finitely projective Koszul graded ring $B=$ $\bigoplus_{n=0}^{\infty} B_{n}$, the exact, fully faithful forgetful functor of abelian categories $B$-contra $\longrightarrow$ $B$-mod induces isomorphisms on all the Ext groups.

Proof. According to the discussion above, the augmentation ideal $B_{\geq 1} \subset B$ is finitely generated as a right ideal in $B$. By Theorem 5.14, it follows that the forgetful functor $B$-contra $\longrightarrow B$-mod is indeed fully faithful. We have to show that it also induces isomorphisms of the groups $\operatorname{Ext}^{i}$ for $i>0$.

There are enough projective objects in the abelian category $B$-contra. Hence it suffices to prove that $\operatorname{Ext}_{B}^{i}(P, Q)=0$ for all projective objects $P \in B$-contra, all objects $Q \in B$-contra, and all $i>0$ (where, as usually, Ext ${ }_{B}^{*}$ denotes the Ext functor in the category $B$-mod). By Proposition 5.13, any object of $B$-contra is the cokernel of an injective morphism of separated contramodules. Hence we can assume that $Q$ is a separated ungraded left $B$-contramodule.

Any separated ungraded left $B$-contramodule $Q$ is an infinitely iterated extension, in the sense of projective limit, of trivial ungraded left $B$-contramodules $G^{m} Q / G^{m+1} Q$ 
(because, by the same proposition, all ungraded left $B$-contramodules are complete). In view of the dual Eklof lemma [9, Proposition 18], we can assume that $Q$ is a trivial ungraded left $B$-contramodule (that is, just a left $R$-module in which the augmentation ideal $B_{\geq 1} \subset B$ acts by zero).

Now the left $R$-module $Q$ has a resolution by injective left $R$-modules of the form $F^{+}=\operatorname{Hom}_{\mathbb{Z}}(F, \mathbb{Q} / \mathbb{Z})$, where $F$ ranges over free right $R$-modules. This reduces the question to the case of a left $R$-module of the form $Q=F^{+}$. Furthermore, we have $\operatorname{Ext}_{B}^{i}\left(P, F^{+}\right) \simeq \operatorname{Tor}_{i}^{B}(F, P)^{+}$(where $B_{\geq 1}$ acts by zero in $F$ ). Thus it suffices to show that $\operatorname{Tor}_{i}^{B}(R, P)=0$ for all projective objects $P \in B$-contra and all integers $i>0$ (where $R$ is viewed as a right $B$-module with $B_{\geq 1}$ acting by zero).

We have done a chain of reductions for an object $Q \in B$-contra; now we need to deal with a projective object $P \in B$-contra. According to the discussion after Proposition 5.9 in Section 5.3, the underlying left $B$-module of $P$ is a direct summand of an ungraded left $B$-module $\Pi H$ for some free graded left $B$-module $H=B^{(X)}$ with generators in degree zero.

Finally, we compute the groups $\operatorname{Tor}_{i}^{B}(R, \Pi H)$ using the projective resolution (62) of the right $B$-module $R$. More precisely, we use the ungraded projective resolution obtained by applying $\Sigma$ to (62). We obtain the complex of ungraded abelian groups

$$
\cdots \longrightarrow \Sigma V_{2} \otimes_{R} \Pi H \longrightarrow \Sigma V_{1} \otimes_{R} \Pi H \longrightarrow \Pi H \longrightarrow 0
$$

computing $\operatorname{Tor}_{*}^{B}(R, \Pi H)$. According to Lemma 5.15), the complex (63) is isomorphic to

$$
\cdots \longrightarrow \Pi\left(V_{2} \otimes_{R} H\right) \longrightarrow \Pi\left(V_{1} \otimes_{R} H\right) \longrightarrow \Pi(H) \longrightarrow 0 .
$$

The complex (64) can be obtained by applying $\Pi$ to the complex

$$
\cdots \longrightarrow V_{2} \otimes_{R} H \longrightarrow V_{1} \otimes_{R} H \longrightarrow H \longrightarrow 0
$$

computing the graded abelian groups $\operatorname{Tor}_{*}^{B}(R, H)$. It remains to observe that $\operatorname{Tor}_{i}^{B}(R, H)=0$ for $i>0$ and the functor $\Pi$ is exact. $\operatorname{Thus~}_{\operatorname{Tor}_{i}^{B}}(R, \Pi H) \simeq$ $\Pi \operatorname{Tor}_{i}^{B}(R, H)=0$ for $i>0$ under our assumptions.

The assertion of the next theorem can be equivalently restated by saying that, under its assumptions, the full subcategory of right $B$-comodules comod- $B$ is a weakly stable torsion class in $\bmod -B$ in the sense of the paper [46].

Theorem 5.17. For any weakly right finitely projective Koszul graded ring $B=\bigoplus_{n=0}^{\infty} B_{n}$, the exact, fully faithful inclusion functor of abelian categories comod- $B \longrightarrow \bmod -B$ induces isomorphisms on all the Ext groups.

Proof. There are enough injective objects in a Grothendieck abelian category of ungraded right $B$-comodules comod- $B$. Hence it suffices to prove that $\operatorname{Ext}_{B^{\text {op }}}^{i}(M, L)=0$ for all objects $M \in \operatorname{comod}-B$, all injective objects $L \in \operatorname{comod}-B$, and all $i>0$ (where Ext $_{B^{\text {op }}}^{*}$ denotes the Ext functor in the category mod- $B$ ).

Any object $M \in$ comod- $B$ has a natural increasing filtration $0=G_{-1} M \subset G_{0} M \subset$ $G_{1} M \subset G_{2} M \subset \cdots$ by right $B$-submodules $G_{m} M \subset M$, where $G_{m} M$ is the subset of all elements $x \in M$ such that $x B_{\geq m+1}=0$. By the definition, we have $M=$ 
$\bigcup_{m>0} G_{m} M$. The successive quotient modules $G_{m} M / G_{m-1} M$ are trivial ungraded right $B$-comodules, i. e., right $B$-modules in which the augmentation ideal $B_{\geq 1}$ acts by zero. The category of trivial ungraded right $B$-comodules is equivalent to the category of right $R$-modules $\bmod -R$.

In view of the Eklof lemma [9, Lemma 1], we can assume that $M$ is a trivial ungraded right $B$-comodule. Now the right $R$-module $M$ has a resolution by free right $R$-modules, which reduces the question to the case of a free right $R$-module $M=R^{(X)}$ (with $B_{\geq 1}$ acting by zero in $M$ ). Thus it suffices to show that $\operatorname{Ext}_{B \text { op }}^{i}(R, L)=0$ for all injective objects $L \in$ comod $-B$ and all integers $i>0$ (where $R$ is viewed as a right $B$-module with $B_{\geq 1}$ acting by zero).

Injective objects of the category comod- $B$ can be described as follows. The inclusion functor comod $-B \longrightarrow \bmod -B$ has a right adjoint functor $\Gamma_{B}: \bmod -B \longrightarrow$ comod- $B$ assigning to an ungraded right $B$-module $N$ its submodule $\Gamma_{B}(N) \subset N$ consisting of all the elements $x \in N$ for which there exists $m \geq 1$ such that $x B_{\geq m}=0$. The injective objects of comod $-B$ are precisely the direct summands of the objects $\Gamma_{B}(N)$, where $N$ ranges over the injective objects of $\bmod -B$.

Injective ungraded right $B$-modules can be further described as follows. Let $H=B^{(X)}$ be a free graded left $B$-module with generators in degree zero, and let $H^{+}=\operatorname{Hom}_{\mathbb{Z}}\left(B^{(X)}, \mathbb{Q} / \mathbb{Z}\right)$ be the graded character module of $H$. So $H^{+}$is a graded right $B$-module concentrated in the nonpositive degrees. Then $\Pi\left(H^{+}\right)$is an injective ungraded right $B$-module, and all injective right $B$-modules are direct summands of $B$-modules of this form. One can easily see that $\Gamma_{B}\left(\Pi\left(H^{+}\right)\right)=\Sigma\left(H^{+}\right)$.

Finally, we compute the groups $\operatorname{Ext}_{B^{\text {op }}}^{i}\left(R, \Sigma\left(H^{+}\right)\right)$using the projective resolution (62) of the right $B$-module $R$. More precisely, we use the projective resolution of ungraded right $B$-module $R$ obtained by applying $\Sigma$ to (62). We obtain a complex of ungraded abelian groups

$$
0 \longrightarrow \Sigma\left(H^{+}\right) \longrightarrow \operatorname{Hom}_{R^{\mathrm{op}}}\left(\Sigma V_{1}, \Sigma\left(H^{+}\right)\right) \longrightarrow \operatorname{Hom}_{R_{\mathrm{op}}}\left(\Sigma V_{2}, \Sigma\left(H^{+}\right)\right) \longrightarrow \cdots
$$

computing $\operatorname{Ext}_{B^{\text {op }}}^{*}\left(R, \Sigma\left(H^{+}\right)\right)$. According to Lemma 5.15(b), the complex (66) is isomorphic to

$$
0 \longrightarrow \Sigma\left(H^{+}\right) \longrightarrow \Sigma \operatorname{Hom}_{R^{\mathrm{op}}}\left(V_{1}, H^{+}\right) \longrightarrow \Sigma \operatorname{Hom}_{R^{\mathrm{op}}}\left(V_{2}, H^{+}\right) \longrightarrow \cdots
$$

The complex (67) can be obtained by applying $\Sigma$ to the complex of graded abelian groups

$$
0 \longrightarrow H^{+} \longrightarrow \operatorname{Hom}_{R^{\mathrm{op}}}\left(V_{1}, H^{+}\right) \longrightarrow \operatorname{Hom}_{R^{\mathrm{op}}}\left(V_{2}, H^{+}\right) \longrightarrow \cdots
$$

computing the graded $\operatorname{Ext}_{B^{\text {op }}}^{*}\left(R, H^{+}\right)$(that is, the derived functor of graded Hom of graded right $B$-modules). It remains to observe that $H^{+}$is an injective object of the category of graded right $B$-modules and the functor $\Sigma$ is exact. Thus $\operatorname{Ext}_{B^{\text {op }}}^{i}\left(R, \Sigma\left(H^{+}\right)\right) \simeq \Sigma \operatorname{Ext}_{B^{\text {op }}}^{i}\left(R, H^{+}\right)=0$ for $i>0$.

5.5. Graded comodules over nonnegatively graded rings. Let $B=\bigoplus_{n=0}^{\infty} B_{n}$ be a nonnegatively graded ring. We consider graded right $B$-modules $M=\bigoplus_{n \in \mathbb{Z}} M_{n}$. A graded right $B$-module $M$ is said to be a $B$-comodule (or a graded right 
$B$-comodule) if its underlying ungraded right $B$-module $\Sigma M$ is an ungraded right $B$-comodule. Equivalently, this means that for every (homogeneous) element $x \in M$ there exists an integer $m \geq 0$ such that $x b=0$ in $M$ for all $b \in B_{n}, n>m$.

Clearly, any nonpositively graded right $B$-module $M=\bigoplus_{n=-\infty}^{0} M_{n}$ is a $B$-comodule. But the free graded right $B$-module $B$ is usually not a $B$-comodule (in fact, $B$ is a $B$-comodule if and only if $B_{n}=0$ for $n \gg 0$ ).

We denote the category of graded right $B$-modules by $\bmod _{\mathrm{gr}}-B$ and the full subcategory of graded right $B$-comodules by $\operatorname{comod}_{\mathrm{gr}}-B \subset \bmod _{\mathrm{gr}}-B$. Obviously, the full subcategory comodgr $-B \subset \bmod _{\mathrm{gr}}-B$ is closed under subobjects, quotients, and infinite direct sums (in other words, $\operatorname{comod}_{\mathrm{gr}}-B$ is a hereditary pretorsion class in $\left.\bmod _{\mathrm{gr}}-B\right)$. Hence $\operatorname{comod}_{\mathrm{gr}}-B$ is a Grothendieck abelian category and its inclusion $\operatorname{comod}_{\mathrm{gr}}-B \longrightarrow \bmod _{\mathrm{gr}}-B$ is an exact functor preserving infinite direct sums.

Example 5.18. Let $B$ be a nonnegatively graded ring with the degree-zero grading component $R=B_{0}$. Assume that $B_{n}$ is a finitely generated projective right $R$-module for every $n \geq 0$, and consider the graded right dual coring $C$ as in Example 5.1 (2). Then a graded right $B$-comodule in the sense of the above definition is the same thing as a graded right $C$-comodule.

Proposition 5.19. Assume that the augmentation ideal $B_{\geq 1}=\bigoplus_{n=1}^{\infty} B_{n}$ of a nonnegatively graded ring $B$ is finitely generated as a right ideal in $B$. Then the full subcategory of graded right $B$-comodules comod $_{\mathrm{gr}}-B$ is closed under extensions in the category of graded right $B$-modules $\bmod _{\mathrm{gr}}-B$.

Proof. This is a graded version of Proposition 5.5. It can be either proved by the same computation, or deduced formally from the ungraded version.

5.6. Graded contramodules over nonnegatively graded rings. Let $B=$ $\bigoplus_{n=0}^{\infty} B_{n}$ be a nonnegatively graded ring, and let $K$ be an associative ring endowed with a ring homomorphism $K \longrightarrow B_{0}$. Consider the following monad $\mathbb{M}=\mathbb{M}_{K}^{\mathrm{gr}}: K-\bmod _{\mathrm{gr}} \longrightarrow K-\bmod _{\mathrm{gr}}$ on the category of graded left $K$-modules $K-\bmod _{\mathrm{gr}}$ (where $K$ is viewed as a graded ring concentrated in the grading 0 ).

To any graded left $K$-module $L=\left(L_{j}\right)_{j \in \mathbb{Z}}$, the monad $\mathbb{M}$ assigns the graded left $K$-module with the components

$$
\mathbb{M}_{K}^{\mathrm{gr}}(L)_{j}=\prod_{n=0}^{\infty}\left(B_{n} \otimes_{K} L_{j-n}\right) .
$$

The monad unit map $e_{L}: L \longrightarrow \mathbb{M}_{K}^{\text {gr }}(L)$ is the graded $K$-module morphism whose degree $j$ component is the composition

$$
L_{j} \longrightarrow B_{0} \otimes_{K} L_{j} \longrightarrow \prod_{n=0}^{\infty} B_{n} \otimes_{K} L_{j-n}
$$

of the map $L_{j} \longrightarrow B_{0} \otimes_{K} L_{j}$ induced by the ring homomorphism $K \longrightarrow B_{0}$ with the inclusion of the $(n=0)$-indexed summand $B_{0} \otimes_{K} L_{j} \longrightarrow \prod_{n=0}^{\infty} B_{n} \otimes_{K} L_{j-n}$. The monad multiplication map $m_{L}: \mathbb{M}_{K}^{\mathrm{gr}}\left(\mathbb{M}_{K}^{\mathrm{gr}}(L)\right) \longrightarrow \mathbb{M}_{K}^{\mathrm{gr}}(L)$ is the graded $K$-module 
morphism whose degree $j$ component is the composition

$$
\prod_{p=0}^{\infty} B_{p} \otimes_{K}\left(\prod_{q=0}^{\infty} B_{q} \otimes_{K} L_{j-p-q}\right) \longrightarrow \prod_{p, q=0}^{\infty} B_{p} \otimes_{K} B_{q} \otimes_{K} L_{j-p-q} \longrightarrow \prod_{n=0}^{\infty} B_{n} \otimes_{K} L_{j-n} .
$$

Here the leftmost arrow is the product over $p \geq 0$ of the maps

$$
B_{p} \otimes_{K}\left(\prod_{q=0}^{\infty} B_{q} \otimes_{K} L_{j-p-q}\right) \longrightarrow \prod_{q=0}^{\infty} B_{p} \otimes_{K} B_{q} \otimes_{K} L_{j-p-q}
$$

whose $q$-components are the direct summand projections $B_{p} \otimes_{K}\left(\prod_{k=0}^{\infty} B_{k} \otimes_{K} L_{j-p-k}\right)$ $\longrightarrow B_{p} \otimes_{K} B_{q} \otimes_{K} L_{j-p-q}$. The rightmost arrow is induced by the multiplication maps $\prod_{p+q=n}^{p, q \geq 0} B_{p} \otimes_{K} B_{q} \longrightarrow B_{n}$.

By the definition, a graded left $B$-contramodule is a module over the monad $\mathbb{M}_{K}^{\mathrm{gr}}: K-\bmod _{\mathrm{gr}} \longrightarrow K-\bmod _{\mathrm{gr}}$. In other words, a graded left $B$-contramodule $P$ is a graded left $K$-module endowed with a left $B$-contraaction map

$$
\pi_{P}: \mathbb{M}_{K}^{\mathrm{gr}}(P) \longrightarrow P
$$

which must be a morphism of graded left $K$-modules satisfying the (contra)associativity and (contra)unitality equations involving the multiplication and unit maps of the monad $\mathbb{M}_{K}^{\text {gr }}$. This means that, in every degree $j \in \mathbb{Z}$, the two compositions

$$
\prod_{p=0}^{\infty} B_{p} \otimes_{K}\left(\prod_{q=0}^{\infty} B_{q} \otimes_{K} P_{j-p-q}\right) \rightrightarrows \prod_{n=0}^{\infty} B_{n} \otimes_{K} P_{j-n} \longrightarrow P_{j}
$$

of the degree $j$ components of the monad multiplication map $m_{P}$ and the map $\mathbb{M}_{K}^{\mathrm{gr}}\left(\pi_{P}\right)$ with the degree $j$ component of the contraaction map $\pi_{P}$ are equal to each other, while the composition

$$
P_{j} \longrightarrow \prod_{n=0}^{\infty} B_{n} \otimes_{K} P_{j-n} \longrightarrow P_{j}
$$

of the degree $j$ components of the monad unit map $e_{P}$ and the contraaction map $\pi_{P}$ is equal to the identity map $\mathrm{id}_{P_{j}}$.

We denote the category of graded left $B$-contramodules by $B$-contra $a_{\mathrm{gr}}=\mathbb{M}_{K}^{\mathrm{gr}}-$ mod. The category of graded left $B$-modules $M=\bigoplus_{j \in \mathbb{Z}} M_{j}$ is denoted by $B$ - $\bmod _{\mathrm{gr}}$.

For any graded left $B$-contramodule $P$, the ungraded left $K$-module $\Pi P=\prod_{j \in \mathbb{Z}} P_{j}$ has a natural structure of ungraded left $B$-contramodule. Indeed, the functors $\mathbb{M}_{K}^{\mathrm{gr}}: K-\bmod _{\mathrm{gr}} \longrightarrow K-\bmod _{\mathrm{gr}}$ and $\mathbb{M}_{K}: K-\bmod \longrightarrow K$-mod form a commutative square diagram with the functor $\Pi: K-\bmod _{\mathrm{gr}} \longrightarrow K$-mod; and the monad multiplication and unit maps of the two monads agree. So we obtain a faithful functor of forgetting the grading

$$
\Pi: B \text { - } \text { contra }_{\mathrm{gr}} \longrightarrow B \text {-contra. }
$$

It is clear from the next proposition that the functor $\Pi: B$-contra ${ }_{\mathrm{gr}} \longrightarrow B$-contra is exact and preserves infinite products (because the functor $\Pi: K-\bmod _{\mathrm{gr}} \longrightarrow K-\bmod$ has such properties). 
Proposition 5.20. There are natural equivalences of categories

$$
\mathbb{M}_{\mathbb{Z}}^{\mathrm{gr}}-\bmod \simeq \mathbb{M}_{K}^{\mathrm{gr}}-\bmod \simeq \mathbb{M}_{B_{0}}^{\mathrm{gr}}-\bmod
$$

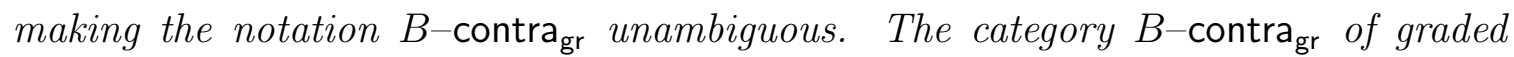
left $B$-contramodules is abelian with enough projective objects. The forgetful functor $B$-contra ${ }_{\mathrm{gr}} \longrightarrow K-\bmod _{\mathrm{gr}}$ can be naturally lifted to a forgetful functor $B$-contra $\mathrm{gr} \longrightarrow$ $B$ - $\bmod _{\mathrm{gr}}$ taking values in the category of graded left $B$-modules. The forgetful functor $B$-contra $\mathrm{gr} \longrightarrow B-$ mod $_{\mathrm{gr}}$ is exact and preserves infinite products.

Proof. This is a graded version of Proposition 5.9. To prove the first assertion, notice that for every graded left $B_{0}$-module $L$ there is a natural surjective map of graded left $K$-modules $\mathbb{M}_{K}^{\text {gr }}(L) \longrightarrow \mathbb{M}_{B_{0}}^{\text {gr }}(L)$, and for every graded left $K$-module $L$ there is a natural surjective map of graded abelian groups $\mathbb{M}_{\mathbb{Z}}^{\mathrm{gr}}(L) \longrightarrow \mathbb{M}_{K}^{\mathrm{gr}}(L)$. Using these maps, one constructs a natural $\mathbb{M}_{K}^{\text {gr }}$-module structure on the underlying graded left $K$-module of every $\mathbb{M}_{B_{0}}^{\text {gr }}$-module, and a natural $\mathbb{M}_{\mathbb{Z}}^{\mathrm{gr}}$-module structure on the underlying graded abelian group of every $\mathbb{M}_{K}^{\text {gr }}$-module. The resulting functors $\mathbb{M}_{B_{0}}^{\text {gr }}-\bmod \longrightarrow \mathbb{M}_{K}^{\text {gr }}-\bmod \longrightarrow \mathbb{M}_{\mathbb{Z}}^{\text {gr }}$-mod are fully faithful, since the above maps of graded modules are surjective.

To prove these functors are essentially surjective, it remains to show that, for every $\mathbb{M}_{K}^{\mathrm{gr}}$-module $P$, the contraaction map $\pi_{P}: \mathbb{M}_{K}^{\mathrm{gr}}(P) \longrightarrow P$ factorizes through the natural surjection $\mathbb{M}_{K}^{\text {gr }}(P) \longrightarrow \mathbb{M}_{B_{0}}^{\text {gr }}(P)$. This can be either checked directly in the way similar to the proof of the ungraded version in Proposition 5.9, or deduced formally from the ungraded version. The latter approach works as follows: the map $\Pi \pi_{P}: \Pi \mathbb{M}_{K}^{\mathrm{gr}}(P) \longrightarrow \Pi P$ is naturally identified with the contraaction map $\pi_{\Pi P}: \mathbb{M}_{K}(\Pi P) \longrightarrow \Pi P$. The latter map factorizes though $\mathbb{M}_{B_{0}}(\Pi P)$, as explained in the proof of Proposition 5.9, this means that the map $\Pi \pi_{P}$ factorizes though $\Pi \mathbb{M}_{B_{0}}^{\mathrm{gr}}(P)$. Hence the map $\pi_{P}$ factorizes through $\mathbb{M}_{B_{0}}^{\mathrm{gr}}(P)$.

The remaining assertions are provable in the way similar to Proposition 5.9. It is important that the functor $\mathbb{M}_{K}^{\mathrm{gr}}: K-\bmod _{\mathrm{gr}} \longrightarrow K-\bmod _{\mathrm{gr}}$ is right exact (as a combination of tensor products and direct products). We skip the obvious details, and will only explain what the projective objects of the category $B$-contragr are. Enough of these can be obtained by applying the free $\mathbb{M}_{K}^{\mathrm{gr}}$-module functor $K-\bmod _{\mathrm{gr}} \longrightarrow \mathbb{M}_{K}^{\mathrm{gr}}-$ mod to free graded left $K$-modules (with generators possibly sitting in all the degrees).

Explicitly, let $X$ be a graded set, which means a sorted set with the sorts indexed by the integers, or equivalently, a set presented as a disjoint union $X=\coprod_{i \in \mathbb{Z}} X_{i}$. Let $K[X]$ denote the graded left $K$-module with the components $K[X]_{i}=K^{\left(X_{i}\right)}$. Then, for every $j \in \mathbb{Z}$, the degree $j$ component of the graded left $B$-contramodule $\mathbb{M}_{K}^{\mathrm{gr}}(K[X])$ has the form

$$
\mathbb{M}_{K}^{\mathrm{gr}}(K[X])_{j}=\prod_{n=0}^{\infty} B_{n} \otimes_{K} K[X]_{j-n}=\prod_{n=0}^{\infty} B_{n}^{\left(X_{j-n}\right)}
$$

The graded left $B$-contramodule $\mathbb{M}_{K}(K[X])$ does not depend on the choice of a ring $K$, but only on the nonnegatively graded $\operatorname{ring} B$ and a graded set $X$. The $\mathbb{M}_{K}^{\text {gr }}$-module $\mathbb{M}_{K}(K[X])$ is called the free graded left $B$-contramodule spanned by a 
graded set $X$. Since there are enough free graded left $B$-contamodules, it follows that every projective graded left $B$-contramodule is a direct summand of a free one.

Examples 5.21. (1) Let $B$ be a nonnegatively graded algebra over a field $k=B_{0}$ with finite-dimensional components $B_{n}$, as in Examples [5.1(1) and 5.10(1), and let $C=\bigoplus_{n=0}^{\infty} B_{n}^{*}$ be the graded dual coalgebra over $k$. Then a graded left $B$-contramodule in the sense of the above definition is the same thing as a graded left $C$-contramodule in the sense of [28, Section 2.2]. (We ignore the issue of the sign rule, which only becomes important when one considers differentials on coalgebras, comodules, and contramodules.)

(2) More generally, let $B$ be a nonnegatively graded ring with the degree-zero component $R=B_{0}$ such that $B_{n}$ is a finitely generated projective right $R$-module for every $n$, and let $C=\bigoplus_{n=0}^{\infty} \operatorname{Hom}_{R^{\text {op }}}\left(B_{n}, R\right)$ be the graded (right) dual coring, as in Examples [5.1(2) and 5.10)(2). Then a graded left $B$-contramodule in the sense of the above definition is the same thing as a graded left $C$-contramodule in the sense of [27, Section 11.1.1].

Examples 5.22. (1) Any nonnegatively graded left $B$-module $P=\bigoplus_{j=0}^{\infty} P_{j}$ has a unique graded left $B$-contramodule structure compatible with its $B$-module structure. The point is that the relevant direct products are essentially finite in this case, and therefore coincide with the similar direct sums,

$$
\prod_{n=0}^{\infty} B_{n} \otimes_{K} P_{j-n}=\bigoplus_{n=0}^{\infty} B_{n} \otimes_{K} P_{j-n} \quad \text { for all } j \in \mathbb{Z} .
$$

(2) For the same reason, there is no difference between graded left $B$-modules and graded left $B$-contramodules when $B_{n}=0$ for $n \gg 0$. (There is also no difference between ungraded left $B$-modules and ungraded left $B$-contramodules in this case; and similarly for graded or ungraded right $B$-comodules.)

(3) However, outside of the degenerate case (2), the cofree graded left $B$-module $B^{+}=\operatorname{Hom}_{\mathbb{Z}}(B, \mathbb{Q} / \mathbb{Z})$ usually does not admit an extension of its graded $B$-module structure to a graded $B$-contramodule structure. In order to prove this for a concrete graded ring $B$, it suffices to show that the cofree ungraded left $B$-module $\Pi\left(B^{+}\right)$does not admit an exension of its $B$-module structure to an ungraded $B$-contramodule structure. Let us give a specific example.

Choose a field $k$, and let $B$ be the polynomial algebra $B=k[x]$ in one variable $x$ of the degree $\operatorname{deg} x=1$. Then the (graded or ungraded) $B$-contramodules are the same thing as the (respectively, graded or ungraded) contramodules over the coalgebra $C$ over $k$ graded dual to $B$ (as in Examples 5.10(1) and 5.21(1)). Ungraded $C$-contramodules are described in [27, Remark A.1.1], [30, Sections 1.3 and 1.6], [29, Theorem B.1.1], and [34, Theorem 3.3] as $k[x]$-modules $P$ such that $\operatorname{Hom}_{k[x]}\left(k\left[x, x^{-1}\right], P\right)=0=\operatorname{Ext}_{k[x]}^{1}\left(k\left[x, x^{-1}\right], P\right)$. In particular, it follows that $P$ has no nonzero $x$-divisible $k[x]$-submodules [34, Lemma 3.2]. Therefore, the $k[x]$-module $\Pi\left(B^{+}\right)$is not a $C$-contramodule, because it is nonzero and $x$-divisible (i. e., the operator $x: \Pi\left(B^{+}\right) \longrightarrow \Pi\left(B^{+}\right)$is surjective $)$. 
Example 5.23. Let $E=\bigoplus_{n \in \mathbb{Z}} E_{n}$ be a graded associative ring, $N=\bigoplus_{n \in \mathbb{Z}} N_{n}$ be a graded $E$ - $B$-bimodule, and $U=\bigoplus_{n \in \mathbb{Z}} U_{n}$ be a graded left $E$-module. Assume that the graded right $B$-module $N$ is a graded right $B$-comodule. Then the graded left $B$-module structure on the graded Hom group $\operatorname{Hom}_{E}(N, U)$ can be extended to a graded left $B$-contramodule structure in a natural way. The construction of the contraaction is similar to that in Example 5.11.

Specifically, for every $j \in \mathbb{Z}$ we need to construct the degree $j$ component of the contraaction map

$$
\pi_{j}: \prod_{n=0}^{\infty} B_{n} \otimes_{K} \operatorname{Hom}_{E}(N, U)_{j-n} \longrightarrow \operatorname{Hom}_{E}(N, U)_{j} .
$$

Let $w \in \prod_{n=0}^{\infty} B_{n} \otimes_{K} \operatorname{Hom}_{E}(N, U)_{j-n}$ be an arbitrary element. Consider an element $y \in N_{i}, \quad i \in \mathbb{Z}$, and choose an integer $m \geq 0$ such that $y B_{\geq m+1}=0$. In order to evaluate element $\pi_{j}(w) \in \operatorname{Hom}_{E}(N, U)_{j}$ on the element $y \in N_{i}$, consider the composition

$$
\prod_{n=0}^{\infty} B_{n} \otimes_{K} \operatorname{Hom}_{E}(N, U)_{j-n} \longrightarrow \bigoplus_{n=0}^{m} B_{n} \otimes_{K} \operatorname{Hom}_{E}(N, U)_{j-n} \longrightarrow \operatorname{Hom}_{E}(N, U)_{j}
$$

of the direct summand projection $\prod_{n=0}^{\infty} B_{n} \otimes_{K} \operatorname{Hom}_{E}(N, U)_{j-n} \longrightarrow \bigoplus_{n=0}^{m} B_{n} \otimes_{K}$ $\operatorname{Hom}_{E}(N, U)_{j-n}$ and the map $\bigoplus_{n=0}^{m} B_{n} \otimes_{K} \operatorname{Hom}_{E}(N, U)_{j-n} \longrightarrow \operatorname{Hom}_{E}(N, U)_{j}$ provided by the left action of $B$ in $\operatorname{Hom}_{E}(N, U)$. This composition of maps has to be applied to the element $w$, and the resulting element in $\operatorname{Hom}_{E}(N, U)_{j}$ has to be evaluated on the element $y \in N_{i}$, producing the desired element $\pi_{j}(w)(y) \in U_{i+j}$.

Let $P$ be a graded left $B$-contramodule. The natural decreasing filtration $P=$ $G^{0} P \supset G^{1} P \supset G^{2} P \supset \cdots$ on $P$ is defined in the following way. For every $m \geq 0$, the graded left $K$-submodule $G^{m} P \subset P$ has the grading components

$$
G^{m} P_{j}=\operatorname{im}\left(\left(\prod_{n \geq m} B_{n} \otimes_{K} P_{j-n}\right) \stackrel{\pi_{P}}{\longrightarrow} P_{j}\right) .
$$

Here the degree $j$ component of the contraaction map $\pi_{P, j}: \mathbb{M}_{K}^{\mathrm{gr}}(P)_{j} \longrightarrow P_{j}$ is restricted onto the $K$-submodule (in fact, a direct summand) $\prod_{n \geq m} B_{n} \otimes_{K} P_{j-n} \subset$ $\prod_{n=0}^{\infty} B_{n} \otimes_{K} P_{j-n}$, and the image of the restriction is taken.

The key observation is that $\bigoplus_{n>m} B_{m}$ is a homogeneous two-sided ideal in $B$. Hence it follows from the contraassociativity axiom for $\pi_{P}$ that $G^{m} P$ is a graded $B$-subcontramodule in $P$ for every $m \geq 0$ and the successive quotient graded contramodules $G^{m} P / G^{m+1} P$ have trivial graded left $B$-contramodule structures. Here a graded left $B$-contramodule $Q$ is said to be trivial if $G^{1} Q=0$. The full subcategory of trivial graded left $B$-contramodules in $B$-contra is equivalent to the category of graded left $B_{0}$-modules $B_{0}-\bmod _{\mathrm{gr}}$.

A graded left $B$-contramodule $P$ is said to be separated if $\bigcap_{m \geq 0} G^{m} P=0$, that is, in other words, the natural graded $B$-contramodule map $\lambda_{B, P}: P \longrightarrow \bigsqcup_{m \geq 0} P / G^{m} P$ is injective. Here the projective limit is taken in the category of graded left $K$-modules, which agrees with the projective limit in the category of graded left 
$B$-contramodules. A graded left $B$-contramodule $P$ is said to be complete if the map $\lambda_{B, P}$ is surjective.

The functor $\Pi: B$-contra gr $\longrightarrow B$-contra takes the canonical decreasing filtration $G$ on a graded left $B$-contramodule $P$ to the canonical decreasing filtration $G$ on the ungraded left $B$-contramodule $\Pi P$ (which was constructed in Section 5.3). Consequently, the functor $\Pi: B$-contragr $\longrightarrow B$-contra takes the morphism $\lambda_{B, P}$ to the morphism $\lambda_{B, \Pi P}$ constructed in Section 5.3 . Hence a graded left $B$-contramodule $P$ is separated (resp., complete) if and only if the ungraded left $B$-contramodule $\Pi P$ is separated (resp., complete).

Proposition 5.24. All graded left B-contramodules are complete (but not necessarily separated). All projective graded left B-contramodules are separated. Every graded left $B$-contramodule is the cokernel of an injective morphism of separated graded left $B$-contramodules.

Proof. This is a graded version of Proposition 5.13, The first assertion follows from the ungraded version in view of the discussion above. The second assertion is clear from the explicit description of projective graded contramodules as direct summands of the free ones (similarly to the ungraded version). The third assertion is provable similarly to the ungraded version explained in Proposition 5.13.

Example 5.25. Here is an example of a nonseparated graded contramodule. It is a modification of a now-classical ungraded example, which appeared in various guises in [42, Example 2.5], [47, Example 3.20], and [27, Section A.1.1] (see [30, Section 2.5] and [34, Example 2.7(1)] for a further discussion).

Let $B=k[x]$ be the polynomial algebra in one variable $x$ of the degree $\operatorname{deg} x=1$ over a field $k$. Let $E$ be the free graded $B$-contramodule with a countable graded set of generators $e_{1}, e_{2}, e_{3}, \ldots$, all of them of the degrees $\operatorname{deg} e_{i}=0$, and let $F$ be the free graded $B$-contramodule with a countable set of generators $f_{1}, f_{2}, f_{3}, \ldots$ of degrees $\operatorname{deg} f_{i}=-i$. Let $g: E \longrightarrow F$ be the unique graded $B$-contramodule morphism taking $e_{i}$ to $x^{i} f_{i}$ for every $i \geq 1$. Consider the quotient contramodule $P=F / g(E)$. Then the formal expression $f=\sum_{i=1}^{\infty} x^{i} f_{i}$ defines an element of degree 0 in $F$ whose image $p$ in $P$ belongs to $\bigcap_{m \geq 0} G^{m} P$. Still $f \notin g(E)$, hence $p \neq 0$ in $P$.

Theorem 5.26. Assume that the augmentation ideal $B_{\geq 1}=\bigoplus_{n=1}^{\infty} B_{n}$ of a nonnegatively graded ring $B$ is finitely generated as a right ideal in $B$. Then the forgetful functor $B$-contra $\mathrm{gr} \longrightarrow B$ - mod $_{\mathrm{gr}}$ from the category of graded left $B$-contramodules to the category of graded left B-modules is fully faithful.

Proof. Let $P$ and $Q$ be graded left $B$-contramodules, and let $f: P \longrightarrow Q$ be a graded left $B$-module morphism. Consider the ungraded left $B$-contramodules $\Pi P$ and $\Pi Q$, and the morphism of ungraded left $B$-modules $\Pi f: \Pi P \longrightarrow \Pi Q$. By Theorem 5.14, $\Pi f$ is a morphism of ungraded left $B$-contramodules. It follows easily that $f$ is a morphism of graded left $B$-contramodules.

5.7. The graded Ext comparison. Let $K$ be a ring. Given a graded right $K$-module $M$ and a graded left $K$-module $L$, we will denote by $M \otimes_{K}^{\Pi} L$ the graded 
abelian group with the grading components

$$
\left(M \otimes_{K}^{\Pi} L\right)_{n}=\prod_{p+q=n} M_{p} \otimes_{K} L_{q} .
$$

Similarly, given two graded right $K$-modules $L$ and $M$, we denote by $\operatorname{Hom}_{K^{\text {op }}}^{\Sigma}(L, M)$ the graded abelian group with the grading components

$$
\operatorname{Hom}_{K^{\mathrm{op}}}^{\Sigma}(L, M)_{n}=\bigoplus_{p-q=n} \operatorname{Hom}_{K^{\mathrm{op}}}\left(L_{q}, M_{p}\right) .
$$

When one of the graded modules involved is concentrated in a finite set of degrees (so there is no difference between the direct sum and product), we will drop the superindices $\Pi$ and $\Sigma$ from the above notation. The superindices $\Pi$ and $\Sigma$ will be also absent when the usual totalization rule is applied (i. e., the bigraded group of tensor products is totalized using the direct sums, and the bigraded Hom group is totalized using the direct products).

Lemma 5.27. (a) Let $K$ and $R$ be associative rings, $V$ be a graded right $R$-module, $D$ be a graded $K$-R-bimodule, and $H$ be a graded left $K$-module. Then there is a natural map of graded abelian groups

$$
V \otimes_{R}\left(D \otimes_{K}^{\Pi} H\right) \longrightarrow\left(V \otimes_{R} D\right) \otimes_{K}^{\Pi} H .
$$

This map is an isomorphism whenever $V$ is a finitely generated projective graded right $R$-module.

(a) Let $K$ and $R$ be associative rings, $V$ be a graded right $R$-module, $D$ be a graded $R$-K-bimodule, and $J$ be a graded right $K$-module. Then there is a natural map of graded abelian groups

$$
\operatorname{Hom}_{K^{\mathrm{op}}}^{\Sigma}\left(V \otimes_{R} D, J\right) \longrightarrow \operatorname{Hom}_{R^{\mathrm{op}}}\left(V, \operatorname{Hom}_{K^{\mathrm{op}}}^{\Sigma}(D, J)\right) .
$$

This map is an isomorphism whenever $V$ is a finitely generated projective graded right $R$-module.

Let $B=\bigoplus_{n=0}^{\infty} B_{n}$ be a nonnegatively graded ring with the degree-zero component $R=B_{0}$. In the proofs below, we denote by $\operatorname{Tor}_{*}^{B}$ and $\operatorname{Ext}_{B}^{*}$ the (bi)graded Tor and Ext of graded $B$-modules (see Section 2.1 for a discussion). So for any graded left $B$-module $L$ and graded right $B$-module $M$ we have $\operatorname{Tor}_{i}^{B}(M, L)=$ $\left.\operatorname{Tor}_{i}^{B}(M, L)_{n}\right)_{n \in \mathbb{Z}}$, and similarly, for any graded left $B$-modules $L$ and $M$ we have $\operatorname{Ext}_{B}^{i}(L, M)=\left(\operatorname{Ext}_{B}^{i}(L, M)_{n}\right)_{n \in \mathbb{Z}}$. In particular, the degree-zero components

$$
\operatorname{Ext}_{B}^{i}(L, M)_{0}=\operatorname{Ext}_{B-\bmod _{\mathrm{gr}}}^{i}(L, M) \text {. }
$$

are the Ext groups in the abelian category of graded left $B$-modules $B$-modgr. More generally, for every $n \in \mathbb{Z}$,

$$
\operatorname{Ext}_{B}^{i}(L, M)_{n}=\operatorname{Ext}_{B-\bmod _{\mathrm{gr}}}^{i}(L, M(-n)),
$$

where $M(-n)$ denotes the left $B$-module $M$ with the shifted grading, $M(-n)_{j}=$ $M_{j+n}$.

Similarly, as above in this Section [5, given a graded module/abelian group $F$, the notation $F^{+}=\operatorname{Hom}_{\mathbb{Z}}(F, \mathbb{Q} / \mathbb{Z})$ stands for the graded module/abelian group with 
the components $F_{n}^{+}=\operatorname{Hom}_{\mathbb{Z}}\left(F_{-n}, \mathbb{Q} / \mathbb{Z}\right)$. In particular, the natural isomorphism of graded abelian groups

$$
\operatorname{Ext}_{B}^{i}\left(L, M^{+}\right) \simeq \operatorname{Tor}_{i}^{B}(M, L)^{+}
$$

holds for any graded right $B$-module $M$ and graded left $B$-module $L$.

Theorem 5.28. Assume that $B$ is a weakly right finitely projective Koszul graded ring. Then the exact, fully faithful forgetful functor of abelian categories $B-$ contra $_{\mathrm{gr}} \longrightarrow B-$ mod $_{\mathrm{gr}}$ induces isomorphisms on all the Ext groups.

Proof. This is a graded version of Theorem 5.16. The forgetful functor $B$-contragr $\longrightarrow$ $B-$ modgr $_{\text {gr }}$ fully faithful by Theorem 5.26 . We have to show that it induces isomorphisms of the groups $\mathrm{Ext}^{i}$ for $i>0$.

There are enough projective objects in the abelian category $B$-contra gr $_{\text {. Hence it }}$ suffices to prove the graded Ext group vanishing $\operatorname{Ext}_{B}^{i}(P, Q)=0$ for all projective objects $P \in B$-contragr, all objects $Q \in B$-contragr, and all $i>0$.

By Proposition 5.24, any graded left $B$-contramodule is the cokernel of an injective morphism of separated graded left $B$-contramodules. Furthermore, any separated graded left $B$-contramodule $Q$ is an infinitely iterated extension, in the sense of the projective limit, of trivial graded left $B$-contramodules $G^{m} Q / G^{m+1} Q$. Using a graded version of the dual Eklof lemma [9, Proposition 18] (see [38, Lemma 4.5] for a farreaching generalization), we can reduce the question to the case when $Q$ is a trivial graded left $B$-contramodule. In other words, $Q$ is just a graded left $R$-module in which the augmentation ideal $B_{\geq 1} \subset B$ acts by zero.

The graded left $R$-module $Q$ has a resolution by injective graded left $R$-modules of the form $F^{+}=\operatorname{Hom}_{\mathbb{Z}}(F, \mathbb{Q} / \mathbb{Z})$, where $F$ ranges over free graded right $R$-modules. Arguing further as in the proof of Theorem 5.16, we see that it suffices to prove the graded Tor group vanishing $\operatorname{Tor}_{i}^{B}(R, P)=0$ for all projective objects $P \in B$-contragr and all integers $i>0$ (where $R$ is viewed as a graded right $B$-module with $B_{\geq 1}$ acting by zero).

A description of projective graded left $B$-contramodules was given in the proof of Proposition 5.20. In the notation above, we can rephrase it as follows. The projective graded left $B$-contramodules are precisely the direct summands of graded left $B$-contramodules of the form $\mathbb{M}_{K}^{\mathrm{gr}}(H)=B \otimes_{K}^{\Pi} K[X]$, where $K[X]$ is the free graded left $K$-module spanned by a graded set $X$. We put $H=K[X]$ for brevity.

Now we compute the graded groups $\operatorname{Tor}_{i}^{B}\left(R, B \otimes_{K}^{\Pi} H\right)$ using the graded projective resolution (62) of the graded right $B$-module $R$. Taking the tensor product of (62) with $B \otimes_{K}^{\Pi} H$ over $B$, we obtain the complex of graded abelian groups

$$
\cdots \longrightarrow V_{2} \otimes_{R}\left(B \otimes_{K}^{\Pi} H\right) \longrightarrow V_{1} \otimes_{R}\left(B \otimes_{K}^{\Pi} H\right) \longrightarrow B \otimes_{K}^{\Pi} H \longrightarrow 0 .
$$

According to Lemma 5.27(a), the complex (69) is isomorphic to

$$
\cdots \longrightarrow\left(V_{2} \otimes_{R} B\right) \otimes_{K}^{\Pi} H \longrightarrow\left(V_{1} \otimes_{R} B\right) \otimes_{K}^{\Pi} H \longrightarrow B \otimes_{K}^{\Pi} H \longrightarrow 0 .
$$

The complex (70) can be obtained by applying the exact functor $-\otimes_{K}^{\Pi} H$ to the resolution (62). Thus $\operatorname{Tor}_{i}^{B}\left(R, B \otimes_{K}^{\Pi} H\right)=0$ for $i>0$, as desired. 
Theorem 5.29. Assume that $B$ is a weakly right finitely projective Koszul graded ring. Then the exact, fully faithful inclusion functor of abelian categories comod $_{\mathrm{gr}}-B \longrightarrow \bmod _{\mathrm{gr}}-B$ induces isomorphisms on all the Ext groups.

Proof. This is a graded version of Theorem 5.17. There are enough injective objects in a Grothendieck abelian category $\operatorname{comod}_{\mathrm{gr}}-B$. Hence it suffices to prove the graded Ext group vanishing $\operatorname{Ext}_{B^{\text {op }}}^{i}(M, L)=0$ for all objects $M \in \operatorname{comod}_{\mathrm{gr}}-B$, all injective objects $L \in \operatorname{comod}_{\mathrm{gr}}-B$, and all $i>0$.

Every graded right $B$-comodule $M$ has a natural increasing filtration $0=G_{-1} M \subset$ $G_{0} M \subset G_{1} M \subset G_{2} M \subset \cdots$ by graded right $B$-submodules $G_{m} M \subset M$ with the grading component $G_{m} M_{j} \subset M_{j}$ consisting of all the elements $x \in M_{j}$ such that $x B_{\geq m+1}=0$ in $M$. By the definition, we have $M=\bigcup_{m>0} G_{m} M$. The successive quotient modules $G_{m} M / G_{m-1} M$ are trivial graded right $B$-comodules, that is graded right $B$-modules in which $B_{\geq 1}$ acts by zero. The category of trivial graded right $B$-comodules is equivalent to the category of graded right $R$-modules $\bmod _{\mathrm{gr}}-R$.

Using a graded version of the Eklof lemma [9, Lemma 1], we can assume that $M$ is a trivial graded right $B$-comodule. Then the graded right $R$-module $M$ has a resolutions by free graded right $R$-modules. Similarly to the proof of Theorem 5.17, we see that it suffices to prove the graded Ext group vanishing $\operatorname{Ext}_{B^{\circ \mathrm{p}}}^{i}(R, L)=0$ for all injective objects $L \in \operatorname{comod}_{\mathrm{gr}}-B$ and all integers $i>0$ (where $R$ is viewed as a graded right $R$-module with $B_{\geq 1}$ acting by zero).

Injective objects of the category comod $\operatorname{gr}-B$ can be described as follows. The inclusion functor comod $\operatorname{gr}-B \longrightarrow \bmod _{\mathrm{gr}}-B$ has a right adjoint functor $\Gamma_{B}: \bmod _{\mathrm{gr}}-B \longrightarrow$ comod $_{\text {gr }}-B$ assigning to a graded right $B$-module $N$ its graded submodule $\Gamma_{B}(N) \subset N$ whose component $\Gamma_{B}(N)_{j}$ consists of all elements $x \in N_{j}$ for which there exists $m \geq 1$ such that $x B_{\geq m}=0$. The injective objects of $\operatorname{comod}_{\mathrm{gr}}-B$ are precisely the direct summands of the objects $\Gamma_{B}(N)$, where $N$ ranges over the injective objects of $\bmod _{\mathrm{gr}}-B$.

The following description of injective graded right $B$-modules is convenient for our purposes. Let $H=K[X]$ be the free graded left $K$-module spanned by a graded set $X$, and let $J=K[X]^{+}$be the graded character module of $H$. Then $J$ is an injective graded right $K$-module, and all injective graded right $K$-modules are direct summands of modules of this form. The graded $\operatorname{Hom}$ module $\operatorname{Hom}_{K^{\mathrm{op}}}(B, J)$ is an injective graded right $B$-module, and all injective graded right $B$-modules are direct summands of modules of this form. One can easily check that $\Gamma_{B}\left(\operatorname{Hom}_{K^{\mathrm{op}}}(B, J)\right)=$ $\operatorname{Hom}_{K^{\text {op }}}^{\Sigma}(B, J)$ (see Lemma 6.6 below).

Now we compute the graded groups $\operatorname{Ext}_{B^{\text {op }}}^{i}\left(R, \operatorname{Hom}_{K^{\text {op }}}^{\Sigma}(B, J)\right)$ using the graded projective resolution (62) of the graded right $B$-module $R$. Taking the graded right $B$-module Hom from (62) to $\operatorname{Hom}_{K^{\text {op }}}^{\Sigma}(B, J)$, we obtain the complex of graded abelian groups

$$
\begin{aligned}
0 \longrightarrow \operatorname{Hom}_{K^{\mathrm{op}}}^{\Sigma}(B, J) & \longrightarrow \operatorname{Hom}_{R^{\mathrm{op}}}\left(V_{1}, \operatorname{Hom}_{K^{\mathrm{op}}}^{\Sigma}(B, J)\right) \\
& \longrightarrow \operatorname{Hom}_{R^{\mathrm{op}}}\left(V_{2}, \operatorname{Hom}_{K^{\mathrm{op}}}^{\Sigma}(B, J)\right) \longrightarrow \cdots
\end{aligned}
$$

According to Lemma 5.27(b), the complex (71) is isomorphic to 


$$
\begin{aligned}
0 \longrightarrow \operatorname{Hom}_{K^{\mathrm{op}}}^{\Sigma}(B, J) \longrightarrow \operatorname{Hom}_{K^{\mathrm{op}}}^{\Sigma}\left(V_{1} \otimes_{R} B, J\right) \\
\longrightarrow \operatorname{Hom}_{K^{\mathrm{op}}}^{\Sigma}\left(V_{2} \otimes_{R} B, J\right) \longrightarrow \cdots
\end{aligned}
$$

The complex (72) can be obtained by applying the exact functor $\operatorname{Hom}_{K^{\text {op }}}^{\Sigma}(-, J)$ to the resolution (62). Thus $\operatorname{Ext}_{B^{\text {op }}}^{i}\left(R, \operatorname{Hom}_{K^{\text {op }}}^{\Sigma}(B, J)\right)=0$ for $i>0$, as desired.

\section{Relative Nonhomogeneous Derived Koszul Duality: The Comodule Side}

6.1. CDG-modules. Let $(B, d, h)$ be a curved DG-ring, as defined in Section 3.2 , and let $(\widehat{B}, \partial)$ be the related quasi-differential graded ring constructed in Theorem 4.5. A left $C D G$-module over $(B, d, h)$ can be simply defined as a graded left $\widehat{B}$-module.

Equivalently, a left CDG-module $\left(M, d_{M}\right)$ over $(B, d, h)$ is a graded left $B$-module $M=\bigoplus_{n \in \mathbb{Z}} M^{n}$ endowed with a sequence of additive maps $d_{M, n}: M^{n} \longrightarrow M^{n+1}$, $n \in \mathbb{Z}$, satisfying following equations:

(i) $d_{M}$ is an odd derivation of the graded left module $M$ compatible with the odd derivation $d$ on the graded ring $B$, that is $d_{M}(b x)=d(b) x+(-1)^{|b|} b d_{M}(x)$ for all $b \in B^{|b|}$ and $x \in M^{|x|},|b|,|x| \in \mathbb{Z}$;

(ii) $d_{M}^{2}(x)=h x$ for all $x \in M$.

A right $C D G$-module over $(B, d, h)$ can be simply defined as a graded right $\widehat{B}$-module. Equivalently, a right CDG-module $\left(N, d_{N}\right)$ over $(B, d, h)$ is a graded right $B$-module $N=\bigoplus_{n \in \mathbb{Z}} N^{n}$ endowed with a sequence of additive maps $d_{N, n}: N^{n} \longrightarrow N^{n+1}, n \in \mathbb{Z}$, satisfying the following equations:

(i) $d_{N}$ is an odd derivation of the graded right module $N$ compatible with the odd derivation $d$ on the graded ring $B$, that is $d_{N}(y b)=d_{N}(y) b+(-1)^{|y|} y d(b)$ for all $b \in B^{|b|}$ and $y \in N^{|y|},|b|,|y| \in \mathbb{Z}$;

(ii) $d_{N}^{2}(y)=-y h$ for all $y \in N$.

Notice that the graded $B$ - $B$-bimodule $B$ with its odd derivation $d$ is neither a left nor a right CDG-module over $(B, d, h)$, because the formula (ii) for $d^{2}(b), \quad b \in B$ in Section 3.2 does not agree with the formulas (ii) in the two definitions above. However, the diagonal graded $B$ - $B$-bimodule $B$ is naturally a $C D G$-bimodule over the CDG-ring $(B, d, h)$, in the sense of the following definition.

Let $B=\left(B, d_{B}, h_{B}\right)$ and $C=\left(C, d_{C}, h_{C}\right)$ be two CDG-rings. A $C D G$-bimodule $K$ over $B$ and $C$ is a graded $B$-C-bimodule $K=\bigoplus_{n \in \mathbb{Z}} K^{n}$ endowed with a sequence of additive maps $d_{K, n}: K^{n} \longrightarrow K^{n+1}, n \in \mathbb{Z}$, satisfying the following conditions:

(i) $d_{K}$ is both an odd derivation of the graded left $B$-module $K$ compatible with the odd derivation $d_{B}$ on the graded ring $B$ and an odd derivation of the graded right $C$-module $K$ compatible with the odd derivation $d_{C}$ on the graded ring $C$;

(ii) $d_{K}^{2}(z)=h_{B} z-z h_{C}$ for all $z \in K$. 
Notice that a CDG-bimodule over $B$ and $C$ is not the same thing as a graded $\widehat{B}-\widehat{C}$-bimodule. We leave it to the reader to construct a CDG-ring structure on the graded ring $B \otimes_{\mathbb{Z}} C^{\text {op }}$ for which a CDG-bimodule over $B$ and $C$ would be the same thing as a left CDG-module over $B \otimes_{\mathbb{Z}} C^{\text {op }}$.

Given a graded left $B$-module $M$ and an integer $n \in \mathbb{Z}$, we denote by $M[n]$ the graded left $B$-module with the grading components $M[n]^{i}=M^{n+i}, \quad i \in \mathbb{Z}$, and the left action of $B$ transformed with the sign rule $b(x[n])=(-1)^{n|b|}(b x)[n]$ for any element $x \in M^{n+i}$ and the corresponding element $x[n] \in M[n]^{i}$. Given a graded right $B$-module $N$ and an integer $n \in \mathbb{Z}$, we let $N[n]$ denote the graded right $B$-module $N$ with the components $N[n]^{i}=N^{n+i}$ and the right action of $B$ unchanged, $(y[n]) b=(y b)[n]$ for $y \in N^{n+i}$ and $y[n] \in N[n]^{i}$. If a differential $d_{M}$ or $d_{N}$ is given, a grading shift transforms it with the usual sign rule $d_{M[n]}(x[n])=(-1)^{n}\left(d_{M}(x)\right)[n]$ or $d_{N[n]}(y[n])=(-1)^{n}\left(d_{N}(y)\right)[n]$. These sign rules are best understood with the hint that we write the shift-of-degree symbol $[n]$ to the right of our modules and their elements, as it is traditionally done, but tacitly presume it to be a left operator (as all our operators, such as the differentials, homomorphisms, etc., are generally left operators). So one may want to write $[n] x \in[n] M$ instead of $x[n] \in M[n]$ for $x \in M$ and similarly $[n] y \in[n] N$ for $y \in N$, which would explain the signs.

Given two graded left $B$-modules $L$ and $M$, we denote by $\operatorname{Hom}_{B}^{n}(L, M)$ the abelian group of all graded left $B$-module homomorphisms $L \longrightarrow M[n]$. For any two left CDG-modules $L$ and $M$ over a CDG-ring $(B, d, h)$, there is a natural differential $d$ on the graded abelian group $\operatorname{Hom}_{B}(L, M)$ defined by the usual rule $d(f)(l)=d_{M}(f(l))-$ $(-1)^{|f|} f\left(d_{L}(l)\right)$ for all $f \in \operatorname{Hom}_{B}^{|f|}(L, M), \quad|f| \in \mathbb{Z}$, and $l \in L$. Similarly, given two graded right $B$-modules $K$ and $N$, we denote by $\operatorname{Hom}_{B^{\text {op }}}^{n}(K, N)$ the abelian group of all graded right $B$-module homomorphisms $K \longrightarrow N[n]$. For any two right CDG-modules $K$ and $N$ over a CDG-ring $(B, d, h)$, there is a natural differential $d$ on the graded abelian group $\operatorname{Hom}_{B^{\text {op }}}(K, N)$ defined by the formula $d(g)(k)=d_{N}(g(k))-$ $(-1)^{|g|} g\left(d_{K}(k)\right)$ for all $g \in \operatorname{Hom}_{B^{\text {op }}}^{\mid g}(K, N),|g| \in \mathbb{Z}$, and $k \in K$.

One easily checks that $d^{2}(f)=0=d^{2}(g)$ for all $f$ and $g$ in the notation above. Hence for any two left CDG-modules $L$ and $M$ over $B$, the complex of morphisms $\operatorname{Hom}_{B}(L, M)$ is defined; and similarly, for any two right CDG-modules $K$ and $N$ over $B$, there is the complex of morphisms $\operatorname{Hom}_{B \text { op }}(K, N)$. These constructions produce the $D G$-category of left $C D G$-modules $\mathrm{DG}(B$-mod) and the similar DG-category of right $C D G$-modules $\mathrm{DG}(\bmod -B)$ over $B$. We refer to [28, Sections 1.2 and 3.1] for a discussion of these DG-categories and their triangulated homotopy categories $\operatorname{Hot}(B-\bmod )=H^{0} \mathrm{DG}(B-\bmod )$ and $\operatorname{Hot}(\bmod -B)=H^{0} \mathrm{DG}(\bmod -B)$.

Given a right graded $B$-module $N$ and a left graded $B$-module $M$, the graded tensor product $N \otimes_{B} M$ is a graded abelian group constructed in the usual way. So we consider the bigraded abelian group $N \otimes_{\mathbb{Z}} M$ with the components $N^{i} \otimes_{\mathbb{Z}} M^{j}$, totalize it by taking infinite direct sums along the diagonals $i+j=n$, and consider the graded quotient group by the graded subgroup spanned by the elements $y b \otimes x-y \otimes b x$, where $y \in N, \quad b \in B$, and $x \in M$. For any right CDG-module $N$ and any left CDG-module $M$ over $(B, d, h)$, the tensor product $N \otimes_{B} M$ is naturally a complex 
of abelian groups with the differential $d(y \otimes x)=d_{N}(y) \otimes x+(-1)^{|y|} y \otimes d_{M}(x)$. One can easily check that $d^{2}(y \otimes x)=0$ in $N \otimes_{B} M$.

More generally, let $\left(A, d_{A}, h_{A}\right),\left(B, d_{B}, h_{B}\right)$, and $\left(C, d_{C}, h_{C}\right)$ be three CDG-rings. Given a graded $C$-B-bimodule $N$ and a graded $B$ - $A$-bimodule $K$, the graded $C$-A-bimodule structure on the tensor product $N \otimes_{B} K$ is defined by the usual rule $c(y \otimes z) a=(c y) \otimes(z a)$ for all $c \in C, \quad y \in N, z \in K$, and $a \in A$. Now let $N$ be a CDG-bimodule over $C$ and $B$, and let $K$ be a CDG-bimodule over $B$ and $A$. Then the graded $C$-A-bimodule $N \otimes_{B} K$ with the differential $d(y \otimes z)=d_{N}(y) \otimes z+(-1)^{|y|} y \otimes d_{K}(z)$, where $y \in N^{|y|}$ and $z \in K^{|z|}$, is a CDG-bimodule over $C$ and $A$.

Given a graded $B$ - $A$-bimodule $K$ and a graded $B$-C-bimodule $L$, the graded $A$-C-bimodule structure on the graded abelian $\operatorname{group} \operatorname{Hom}_{B}(K, L)$ is defined by the formula $(a f c)(z)=(-1)^{|a||f|+|a||z|+|c||z|} f(z a) c$ for all $a \in A^{|a|}, \quad c \in C^{|c|}, \quad z \in K^{|z|}$, and $f \in \operatorname{Hom}_{B}^{|f|}(K, L)$. Now let $K$ be a CDG-bimodule over $B$ and $A$, and let $L$ be a CDG-bimodule over $B$ and $C$. Then the graded $A$-C-bimodule $\operatorname{Hom}_{B}(K, L)$ with the differential $d(f)(z)=d_{L}(f(z))-(-1)^{|f|} f\left(d_{K}(z)\right)$ is a CDG-bimodule over $A$ and $C$.

Given a graded $B$-A-bimodule $K$ and a graded $C$-A-bimodule $M$, the graded $C$ - $B$-bimodule structure on the graded abelian group $\operatorname{Hom}_{A^{\text {op }}}(K, M)$ is defined by the formula $(c g b)(z)=c g(b z)$ for all $c \in C, b \in B, z \in K$, and $g \in \operatorname{Hom}_{A^{\text {op }}}(K, M)$. Now let $K$ be a CDG-bimodule over $B$ and $A$, and let $M$ be a CDG-bimodule over $C$ and $A$. Then the graded $C$-B-bimodule $\operatorname{Hom}_{A^{\text {op }}}(K, M)$ with the differential $d(g)(z)=d_{M}(g(z))-(-1)^{|g|} g\left(d_{K}(z)\right)$, where $z \in K^{|z|}$ and $g \in \operatorname{Hom}_{A^{\text {op }}}^{|g|}(K, M)$, is a CDG-bimodule over $C$ and $B$.

Let $A, B, C$, and $D$ be four CDG-rings. Then for any CDG-bimodule $L$ over $A$ and $B$, any CDG-bimodule $M$ over $B$ and $C$, and any CDG-bimodule $N$ over $C$ and $D$, the natural isomorphism of graded $A$ - $D$-bimodules

$$
\left(L \otimes_{B} M\right) \otimes_{C} N \simeq L \otimes_{B}\left(M \otimes_{C} N\right)
$$

is a closed isomorphism of CDG-bimodules over $A$ and $D$.

Similarly, for any CDG-bimodule $L$ over $B$ and $A$, any CDG-bimodule $M$ over $C$ and $B$, and any CDG-bimodule $N$ over $C$ and $D$, the natural isomorphism of graded $A$ - $D$-bimodules

$$
f^{\prime} \in \operatorname{Hom}_{C}\left(M \otimes_{B} L, N\right) \simeq \operatorname{Hom}_{B}\left(L, \operatorname{Hom}_{C}(M, N)\right) \ni f^{\prime \prime}
$$

given by the rule $f^{\prime \prime}(z)(x)=(-1)^{|x||z|} f^{\prime}(x \otimes z)$ for all $z \in L^{|z|}$ and $x \in M^{|x|}$ is a closed isomorphism of CDG-bimodules over $A$ and $D$.

For any CDG-bimodule $L$ over $A$ and $B$, any CDG-bimodule $M$ over $B$ and $C$, and any CDG-bimodule $N$ over $D$ and $C$, the natural isomorphism of graded $D$ - $A$-bimodules

$$
g^{\prime} \in \operatorname{Hom}_{C \text { op }}\left(L \otimes_{B} M, N\right) \simeq \operatorname{Hom}_{B^{\circ \mathrm{op}}}\left(L, \operatorname{Hom}_{C^{\mathrm{op}}}(M, N)\right) \ni g^{\prime \prime}
$$

given by the rule $g^{\prime \prime}(z)(x)=g^{\prime}(z \otimes x)$ for all $z \in L$ and $x \in M$ is a closed isomorphism of CDG-bimodules over $D$ and $A$. 
In particular, let $K$ be a CDG-bimodule over $B$ and $A$. Then the DG-functor

$$
K \otimes_{A}-: \mathrm{DG}(A-\mathrm{mod}) \longrightarrow \mathrm{DG}(B-\mathrm{mod})
$$

is left adjoint to the DG-functor

$$
\operatorname{Hom}_{B}(K,-): \mathrm{DG}(B-\bmod ) \longrightarrow \mathrm{DG}(A-\bmod ) .
$$

Here the DG-functor $K \otimes_{A}-$ acts on morphisms by the rule $\left(K \otimes_{A} f\right)(k \otimes l)=$ $(-1)^{|f||k|} k \otimes f(l)$ for all $L, M \in \mathrm{DG}(A-\bmod ), \quad f \in \operatorname{Hom}_{A}^{|f|}(L, M), \quad k \in K^{|k|}$, and $l \in L^{|l|}$. The DG-functor $\operatorname{Hom}_{B}(K,-)$ acts on morphisms by the rule $\operatorname{Hom}_{B}(K, f)(g)(k)=f(g(k))$ for all $L, M \in \mathrm{DG}(B-\bmod ), \quad f \in \operatorname{Hom}_{B}(L, M)$, $g \in \operatorname{Hom}_{B}(K, L)$, and $k \in K$.

Hence the induced triangulated functors $K \otimes_{A}-$ and $\operatorname{Hom}_{B}(K,-)$ between the homotopy categories $\operatorname{Hot}(A-\bmod )$ and $\operatorname{Hot}(B-\bmod )$ are also adjoint.

Similarly, the DG-functor

$$
-\otimes_{B} K: \mathrm{DG}(\bmod -B) \longrightarrow \mathrm{DG}(\bmod -A)
$$

is left adjoint to the DG-functor

$$
\operatorname{Hom}_{A^{\mathrm{op}}}(K,-): \mathrm{DG}(\bmod -A) \longrightarrow \mathrm{DG}(\bmod -B) .
$$

Here the DG-functor $-\otimes_{B} K$ acts on morphisms by the rule $\left(f \otimes_{B} K\right)(l \otimes k)=f(l) \otimes k$ for all $L, M \in \mathrm{DG}(\bmod -B), f \in \operatorname{Hom}_{B^{\text {op }}}(L, M), l \in L$, and $k \in K$. The DG-functor $\operatorname{Hom}_{A^{\text {op }}}(K,-)$ acts on morphisms by the fule $\operatorname{Hom}_{A^{\text {op }}}(K, f)(g)(k)=f(g(k))$ for all $L, M \in \mathrm{DG}(\bmod -A), f \in \operatorname{Hom}_{A^{\text {op }}}(L, M), g \in \operatorname{Hom}_{A^{\text {op }}}(K, L)$, and $k \in K$.

Hence the induced triangulated functors $-\otimes_{B} K$ and $\operatorname{Hom}_{A^{\text {op }}}(K,-)$ between the homotopy categories $\operatorname{Hot}(\bmod -B)$ and $\operatorname{Hot}(\bmod -A)$ are also adjoint.

Now let $(f, a):{ }^{\prime \prime} B=\left({ }^{\prime \prime} B, d^{\prime \prime}, h^{\prime \prime}\right) \longrightarrow{ }^{\prime} B=\left({ }^{\prime} B, d^{\prime}, h^{\prime}\right)$ be a morphism of CDG-rings (as defined in Section 3.2). Let $\left(M, d_{M}^{\prime}\right)$ be a left CDG-module over ('B, $\left.d^{\prime}, h^{\prime}\right)$. The restriction of scalars via the graded ring homomorphism $f:{ }^{\prime \prime} B \longrightarrow{ }^{\prime} B$ defines a structure of graded left " $B$-module on $M$. Put $d_{M}^{\prime \prime}(x)=d_{M}^{\prime}(x)+a x$ for all $x \in M$. Then $\left(M, d_{M}^{\prime \prime}\right)$ is a left CDG-module over $\left({ }^{\prime \prime} B, d^{\prime \prime}, h^{\prime \prime}\right)$. This construction defines the DG-functor DG $\left({ }^{\prime} B\right.$-mod $) \longrightarrow$ DG $\left({ }^{\prime \prime} B\right.$-mod) of restriction of scalars via $(f, a)$.

Let $\left(N, d_{N}^{\prime}\right)$ be a right CDG-module over $\left({ }^{\prime} B, d^{\prime}, h^{\prime}\right)$. The restriction of scalars via the graded ring homomorphism $f:{ }^{\prime \prime} B \longrightarrow{ }^{\prime} B$ defines a structure of graded right " $B$-module on $N$. Put $d_{N}^{\prime \prime}(y)=d_{N}^{\prime}(y)-(-1)^{|y|} y a$ for all $y \in N^{|y|}, \quad|y| \in \mathbb{Z}$. Then $\left(N, d_{N}^{\prime \prime}\right)$ is a right CDG-module over $\left({ }^{\prime \prime} B, d^{\prime \prime}, h^{\prime \prime}\right)$. This construction defines the DG-functor DG $\left({ }^{\prime} B\right.$-mod $) \longrightarrow \mathrm{DG}\left({ }^{\prime \prime} B\right.$-mod) of restriction of scalars via $(f, a)$.

Similarly, let $\left(f, a_{B}\right):{ }^{\prime \prime} B=\left({ }^{\prime \prime} B, d_{B}^{\prime \prime}, h_{B}^{\prime \prime}\right) \longrightarrow{ }^{\prime} B=\left({ }^{\prime} B, d_{B}^{\prime}, h_{B}^{\prime}\right)$ and $\left(g, a_{C}\right):{ }^{\prime \prime} C=$ $\left({ }^{\prime \prime} C, d_{C}^{\prime \prime}, h_{C}^{\prime \prime}\right) \longrightarrow{ }^{\prime} C=\left({ }^{\prime} C, d_{C}^{\prime \prime}, h_{C}^{\prime \prime}\right)$ be two morphisms of CDG-rings. Let $\left(K, d_{K}^{\prime}\right)$ be a CDG-bimodule over $\left({ }^{\prime} B, d_{B}^{\prime}, h_{B}^{\prime}\right)$ and $\left({ }^{\prime} C, d_{C}^{\prime}, h_{C}^{\prime}\right)$. The restriction of scalars via the graded ring homomorphisms $f:{ }^{\prime \prime} B \longrightarrow{ }^{\prime} B$ and $g:{ }^{\prime \prime} C \longrightarrow{ }^{\prime} C$ defines a structure of graded " $B-{ }^{\prime \prime} C$-bimodule on $K$. Put $d_{K}^{\prime \prime}(z)=d_{K}^{\prime}(z)+a_{B} z-(-1)^{|z|} z a_{C}$ for all $z \in K^{|z|}, \quad|z| \in \mathbb{Z}$. Then $\left(K, d_{K}^{\prime \prime}\right)$ is a CDG-bimodule over (" $\left.B, d_{B}^{\prime \prime}, h_{B}^{\prime \prime}\right)$ and $\left({ }^{\prime \prime} C, d_{C}^{\prime \prime}, h_{C}^{\prime \prime}\right)$. All the constructions of the tensor product complex and the tensor 
product and Hom CDG-bimodules above in this section agree with the transformations of CDG-(bi)modules induced by isomorphisms of CDG-rings.

6.2. Nonhomogeneous Koszul complex/CDG-module. Let $R \subset \widetilde{V} \subset \widetilde{A}$ be a 3-left finitely projective weak nonhomogeneous quadratic ring, as defined in Sections 3.1 and 3.3, and let $A=\operatorname{gr}^{F} \widetilde{A}$ be its associated graded ring with respect to the filtration $F$. As in Sections 2 3, we put $V=A_{1}$ and denote by $B$ the 3 -right finitely projective quadratic graded ring quadratic dual to q $A$. Choose a left $R$-submodule of strict generators $V^{\prime} \subset \widetilde{V}=F_{1} \widetilde{A}$, and let $(B, d, h)=\left(B, d^{\prime}, h^{\prime}\right)$ denote the related CDG-ring structure on the graded ring $B$, as constructed in Proposition 3.6.

Denote by $e^{\prime} \in \operatorname{Hom}_{R}(V, R) \otimes_{R} \widetilde{V}=B^{1} \otimes_{R} F_{1} \widetilde{A}$ the element corresponding to the injective left $R$-module map $V \simeq V^{\prime} \hookrightarrow \widetilde{V}$ under the construction of Lemma 2.5. Our aim is to construct the dual nonhomogeneous Koszul $C D G$-module $K^{\vee}(B, \widetilde{A})=$ $K_{e^{\prime}}^{\vee}(B, \widetilde{A})$, which has the form

$$
0 \longrightarrow \widetilde{A} \longrightarrow B^{1} \otimes_{R} \widetilde{A} \longrightarrow B^{2} \otimes_{R} \widetilde{A} \longrightarrow B^{3} \otimes_{R} \widetilde{A} \longrightarrow \cdots
$$

The differential on $K_{e^{\prime}}^{\vee}(B, \widetilde{A})$ does not square to zero when $h \neq 0$. Rather, $K_{e^{\prime}}^{\vee}(B, \widetilde{A})$ is a left CDG-module over the CDG-ring $(B, d, h)$, with the obvious underlying graded left $B$-module structure of the tensor product $B \otimes_{R} \widetilde{A}$. However, the differential on $K_{e^{\prime}}^{\vee}(B, \widetilde{A})$ preserves the right $\widetilde{A}$-module structure of the tensor product $B \otimes_{R} \widetilde{A}$. Summarizing, one can say that $K_{e^{\prime}}^{\vee}(B, \widetilde{A})$ is going to be a CDG-bimodule over the CDG-rings $B=(B, d, h)$ and $\widetilde{A}=(\widetilde{A}, 0,0)$ (where $\widetilde{A}$ is viewed as a graded ring concentrated entirely in degree zero).

When $\widetilde{A}=A=\mathrm{q} A$ is a 3-left finitely projective quadratic graded ring and $V^{\prime}=$ $A_{1} \hookrightarrow F_{1} \widetilde{A}=R \oplus A_{1}$ is the obvious splitting of the direct summand projection $R \oplus A_{1} \longrightarrow A_{1}$ (so the related CDG-ring structure on $B$ is trivial, $d=0=h$ ), the construction of differential $d_{e^{\prime}}$ in the next proposition reduces to that of the differential $d_{e}$ on the dual Koszul complex $K_{e}^{\vee \bullet}=B \otimes_{R} A$ from Section 2.6.

Proposition 6.1. The formula

$$
d_{e^{\prime}}(b \otimes c)=d(b) \otimes c+(-1)^{|b|} b e^{\prime} c
$$

for all $b \in B^{|b|}$ and $c \in \widetilde{A}$, where $e^{\prime} \in B^{1} \otimes_{R} F_{1} \widetilde{A}$ is the element mentioned above, endows the graded left $B$-module $K^{\vee}(B, \widetilde{A})=B \otimes_{R} \widetilde{A}$ with a well-defined structure of left $C D G$-module over the $C D G$-ring $B=(B, d, h)$.

Proof. Neither one of the two summands in the formula (74) produces a well-defined map $B \otimes_{R} \widetilde{A} \longrightarrow B \otimes_{R} \widetilde{A}$. It is only the sum that is well-defined as an endomorphism of the tensor product over $R$. However, the separate summands are well-defined as maps $B \otimes_{\mathbb{Z}} \widetilde{A} \longrightarrow B \otimes_{R} \widetilde{A}$. Indeed, the first summand is obviously well-defined as a map $B \otimes_{\mathbb{Z}} \widetilde{A} \longrightarrow B \otimes_{\mathbb{Z}} \widetilde{A}$, and one can consider the composition with the natural surjection $B \otimes_{\mathbb{Z}} \widetilde{A} \longrightarrow B \otimes_{R} \widetilde{A}$. The grading/filtration components of the second 
summand (without the \pm sign) are the compositions

$$
B^{n} \otimes_{\mathbb{Z}} F_{j} \widetilde{A} \stackrel{e^{\prime}}{\longrightarrow} B^{n} \otimes_{\mathbb{Z}} B^{1} \otimes_{R} F_{1} \widetilde{A} \otimes_{\mathbb{Z}} F_{j} \widetilde{A} \longrightarrow B^{n+1} \otimes_{R} F_{j+1} \widetilde{A} .
$$

Here the map $B^{n} \otimes_{\mathbb{Z}} F_{j} \widetilde{A} \longrightarrow B^{n} \otimes_{\mathbb{Z}} B^{1} \otimes_{R} F_{1} \widetilde{A} \otimes_{\mathbb{Z}} F_{j} \widetilde{A}$ is induced by the abelian group element or map $e^{\prime}: \mathbb{Z} \longrightarrow B^{1} \otimes_{R} F_{1} \widetilde{A}$, while the map $B^{n} \otimes_{\mathbb{Z}} B^{1} \otimes_{R} F_{1} \widetilde{A} \otimes_{\mathbb{Z}} F_{j} \widetilde{A} \longrightarrow$ $B^{n+1} \otimes_{R} F_{j+1} \widetilde{A}$ is the tensor product of two multiplication maps $B^{n} \otimes_{\mathbb{Z}} B^{1} \longrightarrow B^{n+1}$ and $F_{1} \widetilde{A} \otimes_{\mathbb{Z}} F_{j} \widetilde{A} \longrightarrow F_{j+1} \widetilde{A}$.

Let us check that the map $d_{e^{\prime}}$ is well-defined on $B \otimes_{R} \widetilde{A}$, that is, for any $b \in B$, $r \in R$, and $c \in \widetilde{A}$ one has

$$
d(b r) \otimes c+(-1)^{|b|} b r e^{\prime} c=d(b) \otimes r c+(-1)^{|b|} b e^{\prime} r c
$$

in $B \otimes_{R} \widetilde{A}$. In the pairing notation from the proof of Lemma 2.5, by the definition of the element $e^{\prime} \in \operatorname{Hom}_{R}(V, R) \otimes_{R} \widetilde{V}$ we have $\left\langle v, e^{\prime}\right\rangle=v^{\prime}$ for every $v \in V$, where $v^{\prime} \in$ $V^{\prime} \subset \widetilde{V}$ denotes the element corresponding to $v$ under the left $R$-module identification $V \simeq V^{\prime}$. Hence, in particular,

$$
\left\langle v, r e^{\prime}\right\rangle=\left\langle v r, e^{\prime}\right\rangle=(v r)^{\prime}
$$

while

$$
\left\langle v, e^{\prime} r\right\rangle=\left\langle v, e^{\prime}\right\rangle * r=v^{\prime} * r=(v r)^{\prime}+q(v, r)=(v r)^{\prime}+\langle v, d(r)\rangle .
$$

Here, as in Section 3.3, the symbol $*$ denotes the multiplication in $\widetilde{A}$, and in particular, the right action of $R$ in $\widetilde{V}$. The notation $q(v, r)$ was defined in the equation (27), and the equation $q(v, r)=\langle v, d(r)\rangle$ comes from the definition of the differential $d$ on the graded ring $B$ in Proposition 3.6. Therefore,

$$
\left\langle v, e^{\prime} r\right\rangle=\left\langle v, r e^{\prime}\right\rangle+\langle v, d(r)\rangle \quad \text { for all } v \in V .
$$

We have deduced the equation

$$
e^{\prime} r=r e^{\prime}+d(r) \otimes 1
$$

comparing the left and right actions of the ring $R$ on the element $e^{\prime} \in \operatorname{Hom}_{R}(V, R) \otimes_{R}$ $\widetilde{V}$ (where $1 \in R \subset \widetilde{V}$ denotes the unit element). Now we can compute that

$$
\begin{aligned}
d(b r) \otimes c+(-1)^{|b|} b r e^{\prime} c=d(b) r \otimes c+(-1)^{|b|} b d(r) \otimes c+(-1)^{|b|} b r e^{\prime} c & \\
& =d(b) \otimes r c+(-1)^{|b|} b e^{\prime} r c \in B \otimes_{R} \widetilde{A},
\end{aligned}
$$

as desired. So the map $d_{e^{\prime}}: B \otimes_{R} \widetilde{A} \longrightarrow B \otimes_{R} \widetilde{A}$ is well-defined.

It is obvious that $d_{e^{\prime}}$ is a right $\widetilde{A}$-module map. It is also easy to check that $d_{e^{\prime}}$ is an odd derivation of the graded left $B$-module $B \otimes_{R} \widetilde{A}$ compatible with the odd derivation $d$ on the graded ring $B$. Indeed, for all $b, b^{\prime} \in B$ and $c \in \widetilde{A}$ we have

$$
\begin{gathered}
d_{e^{\prime}}\left(b^{\prime} b \otimes c\right)=d\left(b^{\prime} b\right) \otimes c+(-1)^{\left|b^{\prime}\right|+|b|} b^{\prime} b e^{\prime} c \\
=d\left(b^{\prime}\right) b \otimes c+(-1)^{\left|b^{\prime}\right|} b^{\prime} d(b) \otimes c+(-1)^{\left|b^{\prime}\right|+|b|} b^{\prime} b e^{\prime} c \\
=d\left(b^{\prime}\right) b \otimes c+(-1)^{\left|b^{\prime}\right|} b^{\prime}\left(d(b) \otimes c+(-1)^{|b|} b e^{\prime} c\right)=d\left(b^{\prime}\right) b \otimes c+(-1)^{\left|b^{\prime}\right|} b^{\prime} d_{e^{\prime}}(b \otimes c),
\end{gathered}
$$


as desired.

It remains to check the equation

$$
d_{e^{\prime}}^{2}(b \otimes c)=h b \otimes c \quad \text { for all } b \in B \text { and } c \in \widetilde{A} .
$$

For this purpose, we choose two finite collections of elements $v_{\alpha} \in V$ and $u^{\alpha} \in$ $B^{1}=\operatorname{Hom}_{R}(V, R)$, indexed by the same indices $\alpha$, such that $e=\sum_{\alpha} u^{\alpha} \otimes v_{\alpha} \in$ $\operatorname{Hom}_{R}(V, R) \otimes_{R} V$. Then the assertion of Lemma 2.5(b) can be written as the equation

$$
\sum_{\alpha} s u^{\alpha} \otimes v_{\alpha}=\sum_{\alpha} u^{\alpha} \otimes v_{\alpha} s \quad \text { for all } s \in R .
$$

For any element $v \in V$, we have $\sum_{\alpha}\left\langle v, u^{\alpha}\right\rangle v_{\alpha}=v$.

By the definition,

$$
d_{e^{\prime}}(b \otimes c)=d(b) \otimes c+(-1)^{|b|} \sum_{\alpha} b u^{\alpha} \otimes v_{\alpha}^{\prime} * c,
$$

hence

$$
\begin{aligned}
d_{e^{\prime}}^{2}(b \otimes c) & =d^{2}(b) \otimes c+(-1)^{|b|} \sum_{\alpha} d\left(b u^{\alpha}\right) \otimes v_{\alpha}^{\prime} * c \\
& +(-1)^{|b|+1} \sum_{\alpha} d(b) u^{\alpha} \otimes v_{\alpha}^{\prime} * c-\sum_{\alpha} \sum_{\beta} b u^{\alpha} u^{\beta} \otimes v_{\beta}^{\prime} * v_{\alpha}^{\prime} * c \\
& =(h b-b h) \otimes c+\sum_{\alpha} b d\left(u^{\alpha}\right) \otimes v_{\alpha}^{\prime} * c-\sum_{\alpha} \sum_{\beta} b u^{\alpha} u^{\beta} \otimes v_{\beta}^{\prime} * v_{\alpha}^{\prime} * c .
\end{aligned}
$$

So we only need to check that the equation

$$
-h \otimes 1+\sum_{\alpha} d\left(u^{\alpha}\right) \otimes v_{\alpha}^{\prime}-\sum_{\alpha} \sum_{\beta} u^{\alpha} u^{\beta} \otimes v_{\beta}^{\prime} * v_{\alpha}^{\prime}=0
$$

holds in $B^{2} \otimes_{R} F_{2} \widetilde{A}$.

We have $B^{2}=\operatorname{Hom}_{R}(I, R)$. Hence, in order to prove the equation (77), it suffices to check that the pairing of the left-hand side with any element $i \in I$ vanishes as an element of $F_{2} \widetilde{A}$. As in Section 3.3 , we denote by $\widehat{I} \subset V \otimes_{\mathbb{Z}} V$ the full preimage of the $R$-R-subbimodule $I \subset V \otimes_{R} V$ under the natural surjective map $V \otimes_{\mathbb{Z}} V \longrightarrow V \otimes_{R} V$. Consider an element $i \in I$ and choose its preimage $\hat{\imath} \in \widehat{I}$. As in Section 3.3, we write symbolically $i=i_{1} \otimes i_{2}$ and $\hat{\imath}=\hat{\imath}_{1} \otimes \hat{\imath}_{2}$.

Now we deal we the three summands in (77) one by one. Firstly, $\langle i, h\rangle=h(i)$. Secondly,

$$
\begin{aligned}
\left\langle i, \sum_{\alpha} d\left(u^{\alpha}\right) \otimes v_{\alpha}^{\prime}\right\rangle & =\sum_{\alpha}\left\langle i, d\left(u^{\alpha}\right)\right\rangle v_{\alpha}^{\prime} \\
\stackrel{(311)}{=} \sum_{\alpha}\left\langle p\left(\hat{\imath}_{1} \otimes \hat{\imath}_{2}\right), u^{\alpha}\right\rangle v_{\alpha}^{\prime}- & \sum_{\alpha} q\left(\hat{\imath}_{1},\left\langle\hat{\imath}_{2}, u^{\alpha}\right\rangle\right) v_{\alpha}^{\prime} \\
& =p\left(\hat{\imath}_{1} \otimes \hat{\imath}_{2}\right)^{\prime}-\sum_{\alpha} q\left(\hat{\imath}_{1},\left\langle\hat{\imath}_{2}, u^{\alpha}\right\rangle\right) v_{\alpha}^{\prime}
\end{aligned}
$$

according to the formula (31) in Proposition 3.6. Thirdly,

$$
\begin{aligned}
& \left\langle i, \sum_{\alpha} \sum_{\beta} u^{\alpha} u^{\beta} \otimes v_{\beta}^{\prime} * v_{\alpha}^{\prime}\right\rangle=\sum_{\alpha} \sum_{\beta}\left\langle i_{1} \otimes i_{2}, u^{\alpha} u^{\beta}\right\rangle v_{\beta}^{\prime} * v_{\alpha}^{\prime} \\
& \quad=\sum_{\alpha} \sum_{\beta}\left\langle i_{1},\left\langle i_{2}, u^{\alpha}\right\rangle u^{\beta}\right\rangle v_{\beta}^{\prime} * v_{\alpha}^{\prime} \stackrel{(76)}{=} \sum_{\alpha} \sum_{\beta}\left\langle i_{1}, u^{\beta}\right\rangle\left(v_{\beta}\left\langle i_{2}, u^{\alpha}\right\rangle\right)^{\prime} * v_{\alpha}^{\prime}
\end{aligned}
$$




$$
\begin{array}{r}
\stackrel{(27)}{=} \sum_{\alpha} \sum_{\beta}\left\langle\hat{\imath}_{1}, u^{\beta}\right\rangle v_{\beta}^{\prime} *\left\langle\hat{\imath}_{2}, u^{\alpha}\right\rangle * v_{\alpha}^{\prime}-\sum_{\alpha} \sum_{\beta}\left\langle\hat{\imath}_{1}, u^{\beta}\right\rangle q\left(v_{\beta},\left\langle\hat{\imath}_{2}, u^{\alpha}\right\rangle\right) * v_{\alpha}^{\prime} \\
\stackrel{(27)}{=} \sum_{\alpha} \sum_{\beta}\left\langle\hat{\imath}_{1}, u^{\beta}\right\rangle v_{\beta}^{\prime} *\left\langle\hat{\imath}_{2}, u^{\alpha}\right\rangle v_{\alpha}^{\prime}-\sum_{\alpha} \sum_{\beta}\left\langle\hat{\imath}_{1}, u^{\beta}\right\rangle q\left(v_{\beta},\left\langle\hat{\imath}_{2}, u^{\alpha}\right\rangle\right) v_{\alpha}^{\prime} \\
=\hat{\imath}_{1}^{\prime} * \hat{\imath}_{2}^{\prime}-\sum_{\alpha} q\left(\hat{\imath}_{1},\left\langle\hat{\imath}_{2}, u^{\alpha}\right\rangle\right) v_{\alpha}^{\prime}
\end{array}
$$

where the equation (76) is being applied to the expression $e=\sum_{\beta} u^{\beta} \otimes v_{\beta}$ and the elements $s=\left\langle i_{2}, u^{\alpha}\right\rangle \in R$.

Finally, we recall that

$$
-h(i)+p\left(\hat{\imath}_{1} \otimes \hat{\imath}_{2}\right)^{\prime}-\hat{\imath}_{1}^{\prime} * \hat{\imath}_{2}^{\prime}=0
$$

in $F_{2} \widetilde{A}$ by the equation (28). This finishes the proof of the desired equation (77) and of the whole proposition.

Let us show that the left CDG-module $K^{\vee}(B, \widetilde{A})$ which we have constructed does not depend on any arbitrary choices. Let $V^{\prime \prime} \subset \widetilde{V}$ be another left $R$-submodule of strict generators, related to our original choice of the left $R$-submodule $V^{\prime} \subset \widetilde{V}$ by the rule (33). Let ${ }^{\prime} B=\left(B, d^{\prime}, h^{\prime}\right)$ and ${ }^{\prime \prime} B=\left(B, d^{\prime \prime}, h^{\prime \prime}\right)$ denote the CDG-ring structures on the graded ring $B$ corresponding to $V^{\prime} \subset \widetilde{V}$ and $V^{\prime \prime} \subset \widetilde{V}$, as in Proposition 3.9. Let $d_{e^{\prime}}$ and $d_{e^{\prime \prime}}$ denote the related two differentials on the tensor product $B \otimes_{R} \widetilde{A}$, endowing it with the structures of a left CDG-module over ${ }^{\prime} B$ and ${ }^{\prime \prime} B$.

Lemma 6.2. The restriction of scalars (as defined in Section 6.1) with respect to the $C D G$-ring isomorphism (id, $a):$ " $B \longrightarrow$ 'B from Proposition 3.9 transforms the left $C D G$-module $K_{e^{\prime}}^{\vee}(B, \widetilde{A})=\left(B \otimes_{R} \widetilde{A}, d_{e^{\prime}}\right)$ over the $C D G$-ring 'B into the left $C D G$-module $K_{e^{\prime \prime}}^{\vee}(B, \widetilde{A})=\left(B \otimes_{R} \widetilde{A}, d_{e^{\prime \prime}}\right)$ over the $C D G$-ring "B.

Proof. We use the notation of Section 3.5. given an element $v \in V$, the corresponding elements in $V^{\prime}$ and $V^{\prime \prime}$ are denoted by $v^{\prime} \in V^{\prime} \subset \widetilde{V}$ and $v^{\prime \prime} \in V^{\prime \prime} \subset \widetilde{V}$. Let $e^{\prime}$ and $e^{\prime \prime} \in \operatorname{Hom}_{R}(V, R) \otimes_{R} \widetilde{V}$ denote the elements corresponding to the injective $R$-module maps $V \simeq V^{\prime} \hookrightarrow \widetilde{V}$ and $V \simeq V^{\prime \prime} \hookrightarrow \widetilde{V}$. Then for every $v \in V$ we have

$$
\left\langle v, e^{\prime}\right\rangle=v^{\prime} \quad \text { and } \quad\left\langle v, e^{\prime \prime}\right\rangle=v^{\prime \prime}=v^{\prime}+a(v)
$$

by formula (33), hence

$$
e^{\prime \prime}=e^{\prime}+a \otimes 1 \in B^{1} \otimes_{R} F_{1} \widetilde{A} .
$$

Now for all $b \in B$ and $c \in \widetilde{A}$ we can compute that

$$
\begin{aligned}
d_{e^{\prime \prime}}(b \otimes c)= & d^{\prime \prime}(b) \otimes c+(-1)^{|b|} b e^{\prime \prime} c \\
= & d^{\prime}(b) \otimes c+\left(a b-(-1)^{|b|} b a\right) \otimes c+(-1)^{|b|} b e^{\prime} c+(-1)^{|b|} b a \otimes c \\
& =d^{\prime}(b) \otimes c+a b \otimes c+(-1)^{|b|} b e^{\prime} c=d_{e^{\prime}}(b \otimes c)+a b \otimes c,
\end{aligned}
$$

as desired (where the middle equation takes into account the equation (iv) from the definition of a morphism of CDG-rings in Section 3.2). 
Remark 6.3. The dual nonhomogeneous Koszul CDG-module $K_{e^{\prime}}^{\vee}(B, \widetilde{A})$ which we have constructed is a nonhomogeneous generalization of the dual Koszul complex $K_{e}^{\vee}(B, A)$ from Section 2.6. The CDG-(bi)module $K_{e^{\prime}}^{\vee}(B, \widetilde{A})$ will be very convenient to use below in Section 6.5 for the purpose of constructing the nonhomogeneous Koszul duality DG-functors which we are really interested in. But the CDG-module $K_{e^{\prime}}^{\vee}(B, \widetilde{A})$ itself, even when it happens to be a complex, has no good exactness properties (see Remark 2.25). However, applying the functor $\operatorname{Hom}_{\widetilde{A}_{\text {op }}}(-, \widetilde{A})$ to $K_{e^{\prime}}^{\vee}(B, \widetilde{A})$ produces a right CDG-module $\operatorname{Hom}_{\widetilde{A}^{\text {op }}}\left(K_{e^{\prime}}^{\vee}(B, \widetilde{A}), \widetilde{A}\right)$ over $(B, d, h)$ (in fact, a CDG-bimodule over $(\widetilde{A}, 0,0)$ and $(B, d, h))$, whose underlying graded $\widetilde{A}$ - $B$-bimodule is $\widetilde{A} \otimes_{R} \operatorname{Hom}_{R^{\text {op }}}(B, R)$. This CDG-module, which is a nonhomogeneous generalization of the first Koszul complex $K_{\bullet}^{\tau}(B, A)$ from Section 2.5, will appear below in the proof of Theorem 6.7 and the subsequent discussion in Section 6.6.

6.3. Semicoderived category of modules. Let $R$ be an associative ring. The following definition of the coderived category of right $R$-modules goes back to [27, Section 2.1] and [28, Section 3.3] (see [18, Section 2] and [2, Proposition 1.3.6(2)] for an alternative approach).

As a particular case of the notation of Section 6.1, we denote by $\operatorname{Hot}(\bmod -R)$ the homotopy category of (unbounded complexes of infinitely generated) right $R$-modules. Let us consider short exact sequences of complexes of right $R$-modules $0 \longrightarrow L^{\bullet} \longrightarrow M^{\bullet} \longrightarrow N^{\bullet} \longrightarrow 0$. Any such short exact sequence can be viewed as a bicomplex with three rows, and the totalization (the total complex) $\operatorname{Tot}\left(L^{\bullet} \rightarrow M^{\bullet} \rightarrow N^{\bullet}\right)$ of such a bicomplex can be constructed in the usual way. A complex of right $R$-modules is said to be coacyclic if it belongs to the minimal full triangulated subcategory of $\operatorname{Hot}(\bmod -R)$ containing all the totalizations of short exact sequences of complexes of right $R$-modules and closed under infinite direct sums.

The full triangulated subcategory of coacyclic complexes of right $R$-modules is denoted by $\mathrm{Acyc}^{\mathrm{CO}}(\bmod -R) \subset \operatorname{Hot}(\bmod -R)$. The coderived category of right $R$-modules is the triangulated Verdier quotient category of the homotopy category $\operatorname{Hot}(\bmod -R)$ by the triangulated subcategory of coacyclic complexes,

$$
\mathrm{D}^{\mathrm{co}}(\bmod -R)=\operatorname{Hot}(\bmod -R) / \mathrm{Acycl}^{\mathrm{co}}(\bmod -R) .
$$

Obviously, any coacyclic complex of $R$-modules is acyclic, $\mathrm{Acycl}^{\mathrm{co}}(\bmod -R) \subset$ Acycl $(\bmod -R)$; but the converse does not hold in general [28, Examples 3.3]. So the conventional derived category $\mathrm{D}(\bmod -R)$ is a quotient category of $\mathrm{D}^{\mathrm{co}}(\bmod -R)$. All acyclic complexes of right $R$-modules are coacyclic when the right homological dimension of the ring $R$ is finite [27, Remark 2.1].

The following lemma is useful.

Lemma 6.4. Let $0=F_{-1} M^{\bullet} \subset F_{0} M^{\bullet} \subset F_{1} M^{\bullet} \subset \cdots \subset M^{\bullet}$ be a complex of $R$-modules endowed with an exhaustive increasing filtration by subcomplexes of $R$-modules; so $M^{\bullet}=\bigcup_{n \geq 0} F_{n} M^{\bullet}$. Assume that, for every $n \geq 0$, the complex 
of $R$-modules $F_{n} M^{\bullet} / F_{n-1} M^{\bullet}$ is coacyclic. Then the complex of $R$-modules $M^{\bullet}$ is coacyclic, too.

Proof. First one proves by induction in $n$ that the complex of $R$-modules $F_{n} M^{\bullet}$ is coacyclic for every $n \geq 0$, using the fact that the totalizations of short exact sequences $0 \longrightarrow F_{n-1} M^{\bullet} \longrightarrow F_{n} M^{\bullet} \longrightarrow F_{n} M^{\bullet} / F_{n-1} M^{\bullet} \longrightarrow 0$ are coacyclic. Then one concludes that the complex of $R$-modules $\bigoplus_{n=0}^{\infty} F_{n} M^{\bullet}$ is coacyclic. Finally, one deduces coacyclicity of the complex $M^{\bullet}$ from the fact that the totalization of the telescope short exact sequence $0 \longrightarrow \bigoplus_{n=0}^{\infty} F_{n} M^{\bullet} \longrightarrow \bigoplus_{n=0}^{\infty} F_{n} M^{\bullet} \longrightarrow M^{\bullet} \longrightarrow 0$ is a coacyclic complex of $R$-modules.

Now let $R \longrightarrow A$ be a homomorphism of associative rings. The following definition of the $A / R$-semicoderived category of right $A$-modules can be found in [32, Section 5]. The semicoderived category

$$
\mathrm{D}_{R}^{\text {sico }}(\bmod -A)=\operatorname{Hot}(\bmod -A) / \operatorname{Acycl}_{R}^{\text {sico }}(\bmod -A)
$$

is the triangulated Verdier quotient category of the homotopy category of right $A$-modules by its full triangulated subcategory $\mathrm{Acycl}_{R}^{\text {sico }}(\bmod -A)$ consisting of all the complexes of right $A$-modules that are coacyclic as complexes of right $R$-modules.

Obviously, any coacyclic complex of $A$-modules is coacyclic as a complex of $R$-modules; but the converse does not need to be true. So the semicoderived category is intermediate between the derived and the coderived category of $A$-modules: there are natural triangulated Verdier quotient functors $\mathrm{D}^{\mathrm{co}}(\bmod -A) \longrightarrow \mathrm{D}_{R}^{\text {sico }}(\bmod -A) \longrightarrow$ $\mathrm{D}(\bmod -A)$. (We refer to the paper [35] for a more general discussion of such intermediate triangulated quotient categories, called pseudo-coderived categories in 35.) The semicoderived category $\mathrm{D}_{R}^{\text {sico }}(\bmod -A)$ can be thought of as a mixture of the coderived category "along the variables from $R$ " and the conventional derived category "in the direction of $A$ relative to $R$ ".

For example, taking $R=\mathbb{Z}$, one obtains the conventional derived category of $A$-modules

$$
\mathrm{D}_{\mathbb{Z}}^{\text {sico }}(\bmod -A)=\mathrm{D}(\bmod -A)
$$

(because all the acyclic complexes of abelian groups are coacyclic), while taking $R=A$ one obtains the coderived category of $A$-modules

$$
\mathrm{D}_{A}^{\text {sico }}(\bmod -A)=\mathrm{D}^{\mathrm{co}}(\bmod -A) \text {. }
$$

6.4. Coderived category of CDG-comodules. Let $B=(B, d, h)$ be a nonnegatively graded CDG-ring (as defined in Section 3.2). So $B=\bigoplus_{n=0}^{\infty} B^{n}$ is a nonnegatively graded associative ring, $d: B \longrightarrow B$ is an odd derivation of degree 1 , and $h \in B^{2}$ is a curvature element for $d$.

Let $\left(N, d_{N}\right)$ be a right CDG-module over $(B, d, h)$ (as defined in Section 6.1). So $N=\bigoplus_{n \in \mathbb{Z}} N^{n}$ is a graded right $B$-module, $d_{N}: N \longrightarrow N$ is an odd derivation of degree 1 compatible with the derivation $d$ on $B$, and the equation $d_{N}^{2}(y)=-y h$ is satisfied for all $y \in N$. We will say that the CDG-module $\left(N, d_{N}\right)$ is a $C D G$-comodule (or a right $C D G$-comodule) over $(B, d, h)$ if the graded right $B$-module $N$ is a graded right $B$-comodule (in the sense of Section 5.5). 
In other words, a right CDG-comodule $N$ over $(B, d, h)$ is a graded right $B$-comodule endowed with an odd coderivation $d_{N}: N \longrightarrow N$ of degree 1 compatible with the derivation $d$ on $B$ and satisfying the above equation for $d_{N}^{2}$. Here by an odd coderivation of a graded $B$-comodule $N$ (compatible with a given odd derivation $d$ on $B$ ) we mean an odd derivation of $N$ as a graded $B$-module. Equivalently, a right CDG-comodule over $(B, d, h)$ can be simply defined as a graded right $\widehat{B}$-comodule (where $(\widehat{B}, \partial)$ is the quasi-differential graded ring corresponding to $(B, d, h)$, as in Theorem 4.5 and Section 6.1).

The DG-category $\mathrm{DG}(\bmod -B)$ of right $\mathrm{CDG}$-modules over $B=(B, d, h)$ was defined in Section 6.1. The DG-category of right $C D G$-comodules DG(comod-B) over $(B, d, h)$ is defined as the full DG-subcategory of the DG-category DG $(\bmod -B)$ whose objects are the right CDG-comodules over $(B, d, h)$. Obviously, the full DG-subcategory $\mathrm{DG}(\operatorname{comod}-B) \subset \mathrm{DG}(\bmod -B)$ is closed under shifts, twists, and infinite direct sums; in particular, it is closed under the cones of closed morphisms (see [28, Section 1.2] for the terminology).

Let $\operatorname{Hot}(\operatorname{comod}-B)=H^{0} \mathrm{DG}(\bmod -B)$ denote the homotopy category (of the DG-category) of right CDG-comodules over $B$. So Hot $(\operatorname{comod}-B)$ is a full triangulated subcategory in $\operatorname{Hot}(\bmod -B)$. The following definition of the coderived category of right $C D G$-comodules over $B$ is a variation on the definitions in [27, Sections 11.7.1-2] and [28, Sections 3.3 and 4.2].

Let us consider short exact sequences $0 \longrightarrow L \stackrel{i}{\longrightarrow} M \stackrel{p}{\longrightarrow} N \longrightarrow 0$ of right CDG-comodules over $(B, d, h)$. Here $i$ and $p$ are closed morphisms of degree zero in the DG-category DG(comod- $B)$ and the short sequence $0 \longrightarrow L \longrightarrow M \longrightarrow N \longrightarrow 0$ is exact in the abelian category $\operatorname{comod}_{\mathrm{gr}}-B$. Using the construction of the cone of a closed morphism (or the shift, finite direct sum, and twist) in the DG-category DG (comod-B), one can produce the totalization (or the total CDG-comodule) $\operatorname{Tot}(L \rightarrow M \rightarrow N)$ of the finite complex of CDG-comodules $L \longrightarrow M \longrightarrow N$.

A right CDG-comodule over $B$ is said to be coacyclic if it belongs to the minimal full triangulated subcategory of $\operatorname{Hot}(\operatorname{comod}-B)$ containing all the totalizations of short exact sequences of right CDG-comodules over $B$ and closed under infinite direct sums. The full triangulated subcategory of coacyclic CDG-comodules is denoted by $A_{c y c l}{ }^{\circ O}(\operatorname{comod}-B) \subset \operatorname{Hot}(\operatorname{comod}-B)$. The coderived category of right $C D G$-comodules over $B$ is the triangulated Verdier quotient category of the homotopy category Hot (comod- $B$ ) by its triangulated subcategory of coacyclic CDG-comodules,

$$
\mathrm{D}^{\mathrm{co}}(\operatorname{comod}-B)=\operatorname{Hot}(\operatorname{comod}-B) / \mathrm{Acycl}^{\mathrm{co}}(\operatorname{comod}-B) \text {. }
$$

Notice that, generally speaking, the differential $d_{N}$ on a CDG-(co)module $\left(N, d_{N}\right)$ has nonzero square (if $B$ is not a DG-ring, that is $h \neq 0$ in $B$ ). So CDG-(co)modules are not complexes and their cohomology (co)modules are undefined. For this reason, one cannot speak about "acyclic CDG-(co)modules", nor about "quasi-isomorphisms of CDG-(co)modules" in the usual sense of the word "quasi-isomorphism". Therefore, the conventional construction of the derived category of DG-modules is not applicable 
to curved DG-modules or curved DG-comodules. There is only the coderived category and its variations (known as derived categories of the second kind).

Lemma 6.5. Let $B=(B, d, h)$ be a nonnegatively graded $C D G$-ring and $N=\left(N, d_{N}\right)$ be a right $C D G$-comodule over $B$. Let $0=F_{-1} N \subset F_{0} N \subset F_{1} N \subset \cdots \subset N$ be an exhaustive increasing filtration of $N$ by $C D G$-subcomodules, that is $\left(F_{n} N\right) B \subset$ $F_{n} N, \quad d_{N}\left(F_{n} N\right) \subset F_{n} N$, and $N=\bigcup_{n=0}^{\infty} F_{n} N$. Assume that, for every $n \geq 0$, the CDG-comodule $F_{n} N / F_{n-1} N$ is coacyclic over $B$. Then the CDG-comodule $N$ is coacyclic, too.

Proof. This is a generalization of Lemma 6.4, provable by the same argument.

There is an important particular case when the nonnegatively graded ring $B$ has only finitely many grading components, that is $B^{n}=0$ for $n \gg 0$. In this case, all the (ungraded or graded) $B$-modules are (respectively, ungraded or graded) $B$-comodules, so comod $\operatorname{gr}-B=\bmod _{\mathrm{gr}}-B$. Accordingly, the full DG-subcategory of (right) CDG-comodules over $B$ coincides with the whole ambient DG-category of (right) CDG-modules, $\mathrm{DG}(\operatorname{comod}-B)=\mathrm{DG}(\bmod -B)$. In this case, we will write simply $\mathrm{D}^{\mathrm{co}}(\bmod -B)$ instead of $\mathrm{D}^{\mathrm{co}}(\operatorname{comod}-B)$.

6.5. Koszul duality functors for modules and comodules. Let $R \subset \widetilde{V} \subset \widetilde{A}$ be a 3-left finitely projective weak nonhomogeneous quadratic ring, and let $B=(B, d, h)$ be the nonhomogeneous quadratic qual CDG-ring. The dual nonhomogeneous Koszul CDG-module $K^{\vee}(B, \widetilde{A})=K_{e^{\prime}}^{\vee}(B, \widetilde{A})=\left(B \otimes_{R} \widetilde{A}, d_{e^{\prime}}\right)$ constructed in Section 6.2 plays a key role below. We recall from the discussion in Section 6.2 that $K^{\vee}(B, \widetilde{A})$ is a CDG-bimodule over the CDG-rings $B=(B, d, h)$ and $\widetilde{A}=(\widetilde{A}, 0,0)$.

According to Section 6.1, we have the induced pair of adjoint DG-functors between the DG-category $\mathrm{DG}(\bmod -\widetilde{A})$ of complexes of right $\widetilde{A}$-modules and the DG-category $\mathrm{DG}(\bmod -B)$ of right $\mathrm{CDG}$-modules over $(B, d, h)$. Here the left adjoint DG-functor

$$
-\otimes_{B} K^{\vee}(B, \widetilde{A}): \mathrm{DG}(\bmod -B) \longrightarrow \mathrm{DG}(\bmod -\widetilde{A})
$$

takes a right CDG-module $N=\left(N, d_{N}\right)$ over $(B, d, h)$ to the complex of right $\widetilde{A}$-modules whose underlying graded $\widetilde{A}$-module is

$$
N \otimes_{B} K^{\vee}(B, \widetilde{A})=N \otimes_{B}\left(B \otimes_{R} \widetilde{A}\right)=N \otimes_{R} \widetilde{A} .
$$

The right adjoint DG-functor

$$
\operatorname{Hom}_{\widetilde{A}^{\mathrm{op}}}\left(K^{\vee}(B, \widetilde{A}),-\right): \mathrm{DG}(\bmod -\widetilde{A}) \longrightarrow \mathrm{DG}(\bmod -B)
$$

takes a complex of right $\widetilde{A}$-modules $M=M \bullet$ to the right CDG-module over $(B, d, h)$ whose underlying graded $B$-module is

$$
\operatorname{Hom}_{\widetilde{A}^{\mathrm{op}}}\left(K^{\vee}(B, \widetilde{A}), M\right)=\operatorname{Hom}_{\widetilde{A}^{\mathrm{op}}}\left(B \otimes_{R} \widetilde{A}, M\right)=\operatorname{Hom}_{R^{\mathrm{op}}}(B, M) .
$$

Our aim is to restrict this adjoint pair to the DG-subcategory DG(comod- $B) \subset$ $\mathrm{DG}(\bmod -B)$ of $\mathrm{CDG}$-comodules over $(B, d, h)$, as defined in Sections 5.5 and 6.4 .

We recall the notation $\operatorname{Hom}_{R^{\text {op }}}^{\Sigma}(L, M) \subset \operatorname{Hom}_{R^{\text {op }}}(L, M)$ for the direct sum totalization of the bigraded Hom group of two graded right $R$-modules $L$ and $M$, which 
was introduced in Section 5.7. Let $\Gamma_{B}: \bmod _{\mathrm{gr}}-B \longrightarrow \operatorname{comod}_{\mathrm{gr}}-B$ denote the functor assigning to every graded right $B$-module its maximal (graded) submodule which is a (graded) right $B$-comodule (see the proof of Theorem 5.29).

Lemma 6.6. Let $B=\bigoplus_{n=0}^{\infty} B_{n}$ be a nonnegatively graded ring with the degree-zero component $R=B_{0}$. Then for any graded right $R$-module $M$ one has

$$
\Gamma_{B}\left(\operatorname{Hom}_{R^{\mathrm{op}}}(B, M)\right)=\operatorname{Hom}_{R^{\mathrm{op}}}^{\Sigma}(B, M) .
$$

Proof. We have $\operatorname{Hom}_{R^{\text {op }}}^{\Sigma}(B, M)=\bigoplus_{n \in \mathbb{Z}} \operatorname{Hom}_{R^{\text {op }}}\left(B, M_{n}(n)\right)$, where $M \longmapsto M(n)$ denotes the functor of grading shift. Since the nonpositively graded right $B$-module $\operatorname{Hom}_{R^{\text {op }}}(B, L)$ is a graded right $B$-comodule for any ungraded right $R$-module $L$, and the full subcategory of graded right $B$-comodules comod $\operatorname{gr}_{-}-B$ is closed under direct sums in $\bmod _{\mathrm{gr}}-B$, it follows that $\operatorname{Hom}_{R \text { op }}^{\Sigma}(B, M)$ is a graded right $B$-comodule. Hence $\operatorname{Hom}_{R^{\text {op }}}^{\Sigma}(B, M) \subset \Gamma_{B}\left(\operatorname{Hom}_{R^{\text {op }}}(B, M)\right)$. Conversely, let $f \in \operatorname{Hom}_{R^{\text {op }}}(B, M)_{n}$ be a homogeneous element of degree $n$ such that $f B_{\geq m}=0$ for some $m \geq 1$. Then for every $b \in B_{\geq m}$ we have $f(b)=f(b \cdot 1)=(f b)(1)=0$, where $1 \in R \subset B$ is the unit element. It follows immediately that $f \in \operatorname{Hom}_{R^{\text {op }}}^{\Sigma}(B, M)_{n} \subset \operatorname{Hom}_{R^{\text {op }}}(B, M)_{n}$.

For any complex of right $A$-modules $M^{\bullet}$, the graded right $B$-submodule

$$
\operatorname{Hom}_{\widetilde{A}^{\text {op }}}^{\Sigma}\left(K^{\vee}(B, \widetilde{A}), M^{\bullet}\right)=\operatorname{Hom}_{R^{\text {op }}}^{\Sigma}\left(B, M^{\bullet}\right) \subset \operatorname{Hom}_{R^{\text {op }}}\left(B, M^{\bullet}\right)
$$

is preserved by the differential of the CDG-module $\operatorname{Hom}_{\widetilde{A}^{\text {op }}}\left(K^{\vee}(B, \widetilde{A}), M^{\bullet}\right)=$ $\operatorname{Hom}_{R^{\text {op }}}\left(B, M^{\bullet}\right)$ over $(B, d, h)$ (so it is a CDG-submodule). In view of the above discussion and Lemma 6.6, we obtain a pair of adjoint DG-functors between the DG-category $\mathrm{DG}(\bmod -\widetilde{A})$ of complexes of right $\widetilde{A}$-modules and the DG-category $\mathrm{DG}($ comod- $B)$ of right CDG-comodules over $(B, d, h)$.

Here the left adjoint DG-functor

$$
-\otimes_{B} K^{\vee}(B, \widetilde{A}): \mathrm{DG}(\operatorname{comod}-B) \longrightarrow \mathrm{DG}(\bmod -\widetilde{A})
$$

is simply the restriction of the above DG-functor to the full DG-subcategory $\mathrm{DG}(\operatorname{comod}-B) \subset \mathrm{DG}(\bmod -B)$. We denote it by

$$
N \longmapsto N \otimes_{B} K^{\vee}(B, \widetilde{A})=N \otimes_{R}^{\tau^{\prime}} \widetilde{A}
$$

where the placeholder $\tau^{\prime}$ is understood as a notation for the injective left $R$-module $\operatorname{map} \operatorname{Hom}_{R^{\mathrm{op}}}\left(B^{1}, R\right)=F_{1} \widetilde{A} / F_{0} \widetilde{A}=V \simeq V^{\prime} \hookrightarrow \widetilde{V}=F_{1} \widetilde{A}$.

The right adjoint DG-functor

$$
\operatorname{Hom}_{\widetilde{A}^{\text {op }}}^{\Sigma}\left(K^{\vee}(B, \widetilde{A}),-\right): \mathrm{DG}(\bmod -\widetilde{A}) \longrightarrow \mathrm{DG}(\operatorname{comod}-B)
$$

takes a complex of right $\widetilde{A}$-modules $M=M^{\bullet}$ to the right CDG-comodule over $(B, d, h)$ whose underlying graded right $B$-comodule is

$$
\operatorname{Hom}_{\widetilde{A}^{\mathrm{op}}}^{\Sigma}\left(B \otimes_{R} \widetilde{A}, M\right)=\operatorname{Hom}_{R^{\mathrm{op}}}^{\Sigma}(B, M)=M \otimes_{R} \operatorname{Hom}_{R^{\mathrm{op}}}(B, R)=M \otimes_{R} C,
$$

where $C$ is a notation for the graded $R$ - $B$-bimodule (and a graded right $B$-comodule) $C=\operatorname{Hom}_{R^{\text {op }}}(B, R)$. We denote this right CDG-comodule over $(B, d, h)$ by

$$
\operatorname{Hom}_{\widetilde{A}^{\text {op }}}^{\Sigma}\left(K^{\vee}(B, \widetilde{A}), M^{\bullet}\right)=M^{\bullet} \otimes_{R}^{\sigma^{\prime}} \operatorname{Hom}_{R^{\mathrm{op}}}(B, R)=M^{\bullet} \otimes_{R}^{\sigma^{\prime}} C
$$


where $\sigma^{\prime}$ is, for the time being, just a placeholder. The purpose of this placeholder is to remind us of the nontrivial differential $d_{e^{\prime}}$ on $K^{\vee}(B, \widetilde{A})$, which is incorporated into the construction of the differential on the CDG-comodule $M^{\bullet} \otimes_{R}^{\sigma^{\prime}} C$ (cf. the twisting cochain notation in the simpler context of [28, Section 6]). See (88) and Section 8.7 below for our suggested interpretation of $\sigma^{\prime}$.

The pair of adjoint DG-functors that we have constructed induces a pair of adjoint triangulated functors

$$
-\otimes_{B} K^{\vee}(B, \widetilde{A})=-\otimes_{R}^{\tau^{\prime}} \widetilde{A}: \operatorname{Hot}(\operatorname{comod}-B) \longrightarrow \operatorname{Hot}(\bmod -\widetilde{A})
$$

and

$$
\operatorname{Hom}_{\widetilde{A}^{\mathrm{op}}}^{\Sigma}\left(K^{\vee}(B, \widetilde{A}),-\right)=-\otimes_{R}^{\sigma^{\prime}} C: \operatorname{Hot}(\bmod -\widetilde{A}) \longrightarrow \operatorname{Hot}(\operatorname{comod}-B)
$$

between the homotopy category $\operatorname{Hot}(\bmod -\widetilde{A})$ of complexes of right $\widetilde{A}$-modules and the homotopy category Hot(comod- $B)$ of right CDG-comodules over $(B, d, h)$.

6.6. Triangulated equivalence. Let $R \subset \widetilde{V} \subset \widetilde{A}$ be a left finitely projective nonhomogeneous Koszul ring (as defined in Section 4.6), and let $(B, d, h)$ be the corresponding right finitely projective Koszul CDG-ring (as constructed in Proposition 3.6; see also Corollary 4.20). The following theorem is the main result of Section 6.

Theorem 6.7. The pair of adjoint triangulated functors $-\otimes_{R}^{\sigma^{\prime}} C: \operatorname{Hot}(\bmod -\widetilde{A}) \longrightarrow$ $\operatorname{Hot}(\operatorname{comod}-B)$ and $-\otimes_{R}^{\tau^{\prime}} \widetilde{A}: \operatorname{Hot}(\operatorname{comod}-B) \longrightarrow \operatorname{Hot}(\bmod -\widetilde{A})$ defined in Section 6.5 induces a pair of adjoint triangulated functors between the $\widetilde{A} / R$-semicoderived category of right $\widetilde{A}$-modules $\mathrm{D}_{R}^{\text {sico }}(\bmod -\widetilde{A})$, as defined in Section 6.3 , and the coderived category $\mathrm{D}^{\mathrm{co}}(\operatorname{comod}-B)$ of right $C D G$-comodules over $(B, d, h)$, as defined in Section 6.4. Under the above Koszulity assumption, the latter two functors are mutually inverse triangulated equivalences,

$$
\mathrm{D}_{R}^{\text {sico }}(\bmod -\widetilde{A}) \simeq \mathrm{D}^{\mathrm{co}}(\operatorname{comod}-B) .
$$

Proof. This is essentially a generalization of [28, Theorem B.2(a)] and a particular case of [27, Theorem 11.8(b)]. The proof is similar. Let us spell out some details.

Recall from the proof of Theorem 5.29 that a graded right $B$-comodule $N$ is said to be trivial if $N B^{\geq 1}=0$ (where the notation is $B^{\geq m}=\bigoplus_{n \geq m} B^{n} \subset B$ for any integer $m \geq 0)$. A right CDG-comodule $\left(N, d_{N}\right)$ over $(B, d, h)$ is said to be triv$i a l$ if its underlying graded $B$-comodule is trivial. The differential $d_{N}$ on a trivial CDG-comodule $N$ squares to zero, as the curvature element $h$ acts by zero in $N$. The DG-category of trivial right CDG-comodules over $(B, d, h)$ is equivalent to the DG-category of complexes of right $R$-modules.

The ring $\widetilde{A}$ is endowed with an increasing filtration $F$. Let us also introduce an increasing filtration $F$ on the graded $R$-B-bimodule $C=\operatorname{Hom}_{R^{\text {op }}}(B, R)$. We put $F_{n} C=\bigoplus_{i \leq n} \operatorname{Hom}_{R^{\text {op }}}\left(B^{i}, R\right) \subset C$ for every $n \in \mathbb{Z}$. So $F_{-1} C=0$ and $C=\bigcup_{n \geq 0} F_{n} C$. Clearly, $F_{n} C$ is a graded $R$ - $B$-subbimodule in $C$. Notice that both the left $R$-modules $F_{n} \widetilde{A} / F_{n-1} \widetilde{A}$ and the graded left $R$-modules $F_{n} C / F_{n-1} C$ are finitely generated and projective (it is important for the argument below that they are flat). 
Let $M^{\bullet}$ be a complex of right $\widetilde{A}$-modules that is coacyclic as a complex of right $R$-modules. We need to show that the right CDG-comodule $M^{\bullet} \otimes_{R}^{\sigma^{\prime}} C$ is coacyclic over $(B, d, h)$. Let $F$ be the increasing filtration on the tensor product $M \otimes_{R} C$ induced by the increasing filtration $F$ on $C$, that is $F_{n}\left(M \otimes_{R} C\right)=M \otimes_{R} F_{n} C$. Then $F$ is a filtration of the CDG-comodule $M^{\bullet} \otimes_{R}^{\sigma^{\prime}} C$ by CDG-subcomodules over $(B, d, h)$. The successive quotient CDG-comodules $\left(M^{\bullet} \otimes_{R}^{\sigma^{\prime}} F_{n} C\right) /\left(M^{\bullet} \otimes_{R}^{\sigma^{\prime}} F_{n-1} C\right)$ are trivial. Viewed as complexes of right $R$-comodules, these are the tensor products $M^{\bullet} \otimes_{R}\left(F_{n} C / F_{n-1} C\right)$, where $F_{n} C / F_{n-1} C$ is a graded $R$ - $R$-bimodule concentrated in the cohomological degree $-n$ and endowed with the zero differential.

Since the graded left $R$-module $F_{n} C / F_{n-1} C$ is flat, tensoring with it preserves short exact sequences of graded right $R$-modules. The tensor product functor also preserves infinite direct sums. It follows that tensoring with the graded $R$ - $R$-bimodule $F_{n} C / F_{n-1} C$ takes coacyclic complexes of right $R$-modules to coacyclic complexes of right $R$-modules. Clearly, any coacyclic complex of right $R$-modules is also coacyclic as a trivial CDG-comodule over $(B, d, h)$. It remains to make use of Lemma 6.5 in order to conclude that the right CDG-comodule $M^{\bullet} \otimes_{R}^{\sigma^{\prime}} C$ over $(B, d, h)$ is coacyclic.

For any coacyclic right CDG-comodule $N$ over $(B, d, h)$, the complex of right $\widetilde{A}$-modules $N \otimes_{R}^{\tau^{\prime}} \widetilde{A}$ is coacyclic not only as a complex of right $R$-modules, but even as a complex of right $\widetilde{A}$-modules. This follows immediately from the fact that $\widetilde{A}$ is a flat left $R$-module; so the functor $-\otimes_{R}^{\tau^{\prime}} \widetilde{A}$ takes short exact sequences of right $\mathrm{CDG}$-comodules over $(B, d, h)$ to short exact sequences of complexes of right $\widetilde{A}$-modules (and this functor also preserves infinite direct sums).

Let $M^{\bullet}$ be arbitrary complex of right $\widetilde{A}$-modules. We need to show the cone $Y^{\bullet}$ of the natural closed morphism of complexes of right $\widetilde{A}$-modules (the adjunction morphism) $M^{\bullet} \otimes_{R}^{\sigma^{\prime}} C \otimes_{R}^{\tau^{\prime}} \widetilde{A} \longrightarrow M^{\bullet}$ is coacyclic as a complex of right $R$-modules. Endow the graded right $R$-module $M \otimes_{R} C \otimes_{R} \widetilde{A}$ with the increasing filtration $F$ induced by the increasing filtrations $F$ on $C$ and $\widetilde{A}$, that is $F_{n}\left(M \otimes_{R} C \otimes_{R} \widetilde{A}\right)=$ $\sum_{i+j=n} M \otimes_{R} F_{i} C \otimes_{R} F_{j} \widetilde{A} \subset M \otimes_{R} C \otimes_{R} \widetilde{A}$. Then $F$ is an exhaustive filtration of $M^{\bullet} \otimes_{R}^{\sigma^{\prime}} C \otimes_{R}^{\tau^{\prime}} \widetilde{A}$ by subcomplexes of right $R$-modules (but not of right $\widetilde{A}$-modules!). The complex $M^{\bullet}$ is endowed with the trivial filtration $F_{-1} M^{\bullet}=0, \quad F_{0} M^{\bullet}=M^{\bullet}$; and the complex $Y^{\bullet}$ is endowed with the induced increasing filtration $F$.

Then the associated graded complex of right $R$-modules

$$
\operatorname{gr}^{F}\left(M^{\bullet} \otimes_{R}^{\sigma^{\prime}} C \otimes_{R}^{\tau^{\prime}} \widetilde{A}\right)=\bigoplus_{n=0}^{\infty} F_{n}\left(M^{\bullet} \otimes_{R}^{\sigma^{\prime}} C \otimes_{R}^{\tau^{\prime}} \widetilde{A}\right) / F_{n-1}\left(M^{\bullet} \otimes_{R}^{\sigma^{\prime}} C \otimes_{R}^{\tau^{\prime}} \widetilde{A}\right)
$$

is naturally isomorphic to the tensor product $M^{\bullet} \otimes_{R}{ }^{\tau} K_{\bullet}(B, A)$ of the complex of right $R$-modules $M^{\bullet}$ and the complex of (graded) $R$ - $R$-bimodules ${ }^{\tau} K \bullet(B, A)=C \otimes_{R}^{\tau} A$ constructed in Section 2.6 (where it is called "the second Koszul complex"). Here, as usually, $A=\operatorname{gr}^{F} \widetilde{A}$ is the left finitely projective Koszul graded ring associated with $\widetilde{A}$. By Proposition 2.20 or Theorem 2.21(e), every internal degree $n \geq 1$ component of the complex ${ }^{\tau} K_{\bullet}(B, A)$ is a finite acyclic complex of $R$ - $R$-bimodules whose terms are finitely generated and projective left $R$-modules. The internal degree $n=0$ component of the complex ${ }^{\tau} K_{\bullet}(B, A)$ is isomorphic to the $R$ - $R$-bimodule $R$. 
It follows that the associated graded complex $\operatorname{gr}^{F} Y^{\bullet}=\bigoplus_{n \geq 0} F_{n} Y^{\bullet} / F_{n-1} Y^{\bullet}$ is isomorphic to the tensor product $M^{\bullet} \otimes_{R} \operatorname{cone}\left({ }^{\tau} K_{\bullet}(B, A) \rightarrow R\right)$ of the complex of right $R$-modules $M^{\bullet}$ and the cone of the natural morphism of complexes of (graded) $R$ - $R$-bimodules ${ }^{\tau} K_{\bullet}(B, A) \longrightarrow R$. For every $n \in \mathbb{Z}$, the internal degree $n$ component of the complex cone $\left({ }^{\tau} K \cdot(B, A) \rightarrow R\right)$ is a finite acyclic complex of $R$ - $R$-bimodules which are finitely generated and projective as left $R$-modules. Consequently, the complex cone $\left({ }^{\tau} K_{\bullet}(B, A) \rightarrow R\right)$ is "coacyclic with respect to the exact category of left $R$-flat (or even left $R$-projective) $R$ - $R$-bimodules" in the sense of [27, Section 2.1]. Since the functor $M^{\bullet} \otimes_{R}$ - preserves exactness of short exact sequences of left $R$-flat $R$ - $R$-bimodules, we can conclude that $\operatorname{gr}^{F} Y^{\bullet} \simeq M^{\bullet} \otimes_{R} \operatorname{cone}\left({ }^{\tau} K \bullet(B, A) \rightarrow R\right)$ is a coacyclic complex of right $R$-modules. By Lemma 6.4, it follows that $Y^{\bullet}$ is a coacyclic complex of right $R$-modules, as desired.

Let $N=\left(N, d_{N}\right)$ be an arbitrary right CDG-comodule over $(B, d, h)$. We need to show that the cone $Z$ of the natural closed morphism of right CDG-comodules (the adjunction morphism) $N \longrightarrow N \otimes_{R}^{\tau^{\prime}} \widetilde{A} \otimes_{R}^{\sigma^{\prime}} C$ is coacyclic as a CDG-comodule over $(B, d, h)$. Endow the graded right $B$-comodule $N$ with the canonical increasing filtration by graded subcomodules $0=G_{-1} N \subset G_{0} N \subset G_{1} N \subset G_{2} N \subset \cdots \subset N$ as in the proof of Theorem 5.29; the grading component $G_{m} N^{i} \subset N^{i}$ consists of all the elements $y \in N^{i}$ such that $y B^{\geq m+1}=0$ in $N$. We have $d_{N}\left(G_{m} N\right) \subset G_{m} N$, as $d_{N}(y) b=d_{N}(y b)-(-1)^{|y|} y d(b)=0$ for all $y \in G_{m} N^{|y|}$ and $b \in B^{\geq m+1}$. So $G$ is an exhaustive increasing filtration of $N$ by CDG-subcomodules over $(B, d, h)$.

Consider the induced increasing filtrations $G$ of the right CDG-comodule $N \otimes_{R}^{\tau^{\prime}} \widetilde{A} \otimes_{R}^{\sigma^{\prime}} C$ and the right CDG-comodule $Z$ over $(B, d, h)$; so $G_{m}\left(N \otimes_{R}^{\tau^{\prime}} \widetilde{A} \otimes_{R}^{\sigma^{\prime}} C\right)=$ $\left(G_{m} N\right) \otimes_{R}^{\tau^{\prime}} \widetilde{A} \otimes_{R}^{\sigma^{\prime}} C$ and $G_{m} Z=\operatorname{cone}\left(G_{m} N \rightarrow G_{m} N \otimes_{R}^{\tau^{\prime}} \widetilde{A} \otimes_{R}^{\sigma^{\prime}} C\right)$. By Lemma 6.5, it suffices to check that the CDG-comodules $G_{m} Z / G_{m-1} Z$ are coacyclic over $(B, d, h)$ for all $m \geq 0$. Then, since $\widetilde{A}$ and $C$ are flat (graded) left $R$-modules, the CDG-comodule $G_{m} Z / G_{m-1} Z$ is naturally isomorphic to the cone of the adjunction morphism $G_{m} N / G_{m-1} N \longrightarrow\left(G_{m} N / G_{m-1} N\right) \otimes_{R}^{\tau^{\prime}} \widetilde{A} \otimes_{R}^{\sigma^{\prime}} C$.

The successive quotient CDG-comodules $G_{m} N / G_{m-1} N$ are annihilated by $B^{\geq 1}$; so they are trivial right CDG-comodules over $(B, d, h)$ or, which is the same, just complexes of right $R$-modules. Thus we have reduced our problem to the case of a trivial right CDG-comodule $N$, and we can assume that $N=N^{\bullet}$ is simply a complex of right $R$-modules with $B^{\geq 1}$ acting by zero. Then the right CDG-comodule $N \otimes_{R}^{\tau^{\prime}}$ $\widetilde{A} \otimes_{R}^{\sigma^{\prime}} C=N \bullet \otimes_{R} \widetilde{A} \otimes_{R}^{\sigma^{\prime}} C$ over $(B, d, h)$ is simply the tensor product of the complex of right $R$-modules $N^{\bullet}$ and the right CDG-comodule $\widetilde{A} \otimes_{R}^{\sigma^{\prime}} C=\operatorname{Hom}_{\widetilde{A}^{\text {op }}}\left(K_{e^{\prime}}^{\vee}(B, \widetilde{A}), \widetilde{A}\right)$ (which is, in fact, a CDG-bimodule over $\widetilde{A}=(\widetilde{A}, 0,0)$ and $B=(B, d, h)$, hence in particular a CDG-bimodule over $R=(R, 0,0)$ and $B)$.

Endow the graded right $R$-module $N \otimes_{R} \widetilde{A} \otimes_{R} C$ with the increasing filtration $F$ induced by the increasing filtrations $F$ on $\widetilde{A}$ and $C$, that is $F_{n}\left(N \otimes_{R} \widetilde{A} \otimes_{R} C\right)=$ $\sum_{i+j=n} N \otimes_{R} F_{j} \widetilde{A} \otimes_{R} F_{i} C \subset N \otimes_{R} \widetilde{A} \otimes_{R} C$. Then $F$ is an exhaustive filtration of $N^{\bullet} \otimes_{R} \widetilde{A} \otimes_{R}^{\sigma^{\prime}} C$ by CDG-subcomodules over $(B, d, h)$. Let the complex $N^{\bullet}$ be endowed 
with the trivial filtration $F_{-1} N^{\bullet}=0, \quad F_{0} N^{\bullet}=N^{\bullet}$, and let the CDG-comodule $Z=\operatorname{cone}\left(N^{\bullet} \rightarrow N^{\bullet} \otimes_{R} \widetilde{A} \otimes_{R}^{\sigma^{\prime}} C\right)$ be endowed with the induced filtration.

Then the associated graded CDG-comodule

$$
\operatorname{gr}^{F}\left(N^{\bullet} \otimes_{R} \tilde{A} \otimes_{R}^{\sigma^{\prime}} C\right)=\bigoplus_{n=0}^{\infty} F_{n}\left(N^{\bullet} \otimes_{R} \widetilde{A} \otimes_{R}^{\sigma^{\prime}} C\right) / F_{n-1}\left(N^{\bullet} \otimes_{R} \widetilde{A} \otimes_{R}^{\sigma^{\prime}} C\right)
$$

is annihilated by the action of $B^{\geq 1}$; so it is a trivial right CDG-comodule over $(B, d, h)$. As a complex of right $R$-modules, it is naturally isomorphic to the tensor product $N^{\bullet} \otimes_{R} K_{\bullet}^{\tau}(B, A)$ of the complex of right $R$-modules $N^{\bullet}$ and the complex of (graded) $R$-R-bimodules $K_{\bullet}^{\tau}(B, A)=A \otimes_{R}^{\sigma} C$ constructed in Section 2.5 (where it is called "the first Koszul complex"). Here, once again, the superindex $\sigma$ is just a placeholder for the time being (see Section 8.7 below for an explanation). By Proposition 2.19 or Theorem 2.21(d), every internal degree $n \geq 1$ component of the complex $K_{\bullet}^{\tau}(B, A)$ is a finite acyclic complex of $R$ - $R$-bimodules with finitely generated projective underlying left $R$-modules (while the internal degree $n=0$ component is the $R$ - $R$-bimodule $R$ ).

It follows that the associated graded CDG-comodule $\operatorname{gr}^{F} Z=\bigoplus_{n \geq 0} F_{n} Z / F_{n-1} Z$ is a trivial right $\mathrm{CDG}$-comodule over $(B, d, h)$ which, as a complex of right $R$-modules, is naturally isomorphic to the tensor product $N^{\bullet} \otimes_{R} \operatorname{cone}\left(R \rightarrow K_{\bullet}^{\tau}(B, A)\right)$. For every $n \in \mathbb{Z}$, the internal degree $n$ component of the complex cone $\left(R \rightarrow K_{\bullet}^{\tau}(B, A)\right)$ is a finite acyclic complex of $R$ - $R$-bimodules which are finitely generated and projective (hence flat) as left $R$-modules. As above, we can conclude that $\operatorname{gr}^{F} Z \simeq N^{\bullet} \otimes_{R}$ $\operatorname{cone}\left(R \rightarrow K_{\bullet}^{\tau}(B, A)\right)$ is a coacyclic complex of right $R$-modules. Hence $\operatorname{gr}^{F} Z$ is also coacyclic as a right CDG-comodule over $(B, d, h)$. By Lemma 6.5, it follows that $Z$ is a coacyclic CDG-comodule over $(B, d, h)$, too.

Examples 6.8. (1) The free right $R$-module $R$ can be considered as a one-term complex of right $R$-modules, concentrated in the cohomological degree 0 and endowed with the zero differential. This complex, in turn, can be considered as a trivial right CDG-comodule over $(B, d, h)$. The functor $-\otimes_{R}^{\tau^{\prime}} \widetilde{A}=-\otimes_{B} K_{e^{\prime}}^{\vee}(B, \widetilde{A})$ takes this trivial right CDG-comodule $R$ over $(B, d, h)$ to the free right $\widetilde{A}$-module $\widetilde{A}$ (viewed as a one-term complex concentrated in the cohomological degree 0$)$.

(2) Applying the functor $-\otimes_{R}^{\sigma^{\prime}} C=\operatorname{Hom}_{\widetilde{A}^{\text {op }}}\left(K_{e^{\prime}}^{\vee}(B, \widetilde{A}),-\right)$ to the free right $\widetilde{A}$-module $\widetilde{A}$ produces the right CDG-comodule $\widetilde{A} \otimes_{R}^{\sigma^{\prime}} C=\operatorname{Hom}_{\widetilde{A}_{\text {op }}}\left(K_{e^{\prime}}^{\vee}(B, \widetilde{A}), \widetilde{A}\right)$, which was mentioned in the above proof and in Remark 6.3. The left $\widetilde{A}$-module structure on $\widetilde{A}$ induces a left action of $\widetilde{A}$ in $\widetilde{A} \otimes_{R}^{\sigma^{\prime}} C$, making it a CDG-bimodule over $(\widetilde{A}, 0,0)$ and $(B, d, h)$. The adjunction map $R \longrightarrow \widetilde{A} \otimes_{R}^{\sigma^{\prime}} C$ is a closed morphism of right CDG-comodules over $(B, d, h)$ (or, if one wishes, of CDG-bimodules over $(R, 0,0)$ and $(B, d, h))$. According to the above argument, its cone is a coacyclic right CDG-comodule over $(B, d, h)$.

(3) Applying the functor $-\otimes_{R}^{\tau^{\prime}} \widetilde{A}$ to the right CDG-comodule $\widetilde{A} \otimes_{R}^{\sigma^{\prime}} C$ over $(B, d, h)$ produces a complex of right $\widetilde{A}$-modules $\widetilde{A} \otimes_{R}^{\sigma^{\prime}} C \otimes_{R}^{\tau^{\prime}} \widetilde{A}$. The left action of $\widetilde{A}$ in $\widetilde{A} \otimes_{R}^{\sigma^{\prime}} C$ induces a left action of $\widetilde{A}$ in the complex $\widetilde{A} \otimes_{R}^{\sigma^{\prime}} C \otimes_{R}^{\tau^{\prime}} \widetilde{A}$, making it a complex of $\widetilde{A}$ - $\widetilde{A}$-bimodules. The adjunction map $\widetilde{A} \otimes_{R}^{\sigma^{\prime}} C \otimes_{R}^{\tau^{\prime}} \widetilde{A} \longrightarrow \widetilde{A}$ is a morphism of complexes 
of $\widetilde{A}-\widetilde{A}$-bimodules. According to the above proof, its cone is coacyclic as a complex of right $R$-modules (following the details of the argument, one can see that this cone is, in fact, coacyclic as a complex of $\widetilde{A}$-R-bimodules).

In particular, the cone is an acyclic complex; so $\widetilde{A} \otimes_{R}^{\sigma^{\prime}} C \otimes_{R}^{\tau^{\prime}} \widetilde{A}$ is a bimodule resolution of the diagonal $\widetilde{A}-\widetilde{A}$-bimodule $\widetilde{A}$. The terms of this resolution are not projective $\widetilde{A} \otimes_{\mathbb{Z}} \widetilde{A}$-modules, of course; and $\widetilde{A} \otimes_{R} \widetilde{A}$ is, generally speaking, not even a ring! Still one can say that the terms of this resolution are the $\widetilde{A}$ - $\widetilde{A}$-bimodules $\widetilde{A} \otimes_{R} C_{i} \otimes_{R} \widetilde{A}$ induced from the $R$ - $R$-bimodules $C_{i}=\operatorname{Hom}_{R^{\text {op }}}\left(B^{i}, R\right)$ (which are finitely generated and projective as left $R$-modules in our assumptions).

(4) Notice that the bimodule resolution $\widetilde{A} \otimes_{R}^{\sigma^{\prime}} C \otimes_{R}^{\tau^{\prime}} \widetilde{A} \longrightarrow \widetilde{A}$ from (3) is a quasiisomorphism of bounded above complexes of projective left $\widetilde{A}$-modules; hence it is a homotopy equivalence of complexes of left $\widetilde{A}$-modules. It is also a quasi-isomorphism of bounded above complexes of weakly $\widetilde{A} / R$-flat right $\widetilde{A}$-modules in the sense of 32 , Section 5] (cf. Section [2.9). In view of [32, Lemma 5.3(b)], it follows that the tensor product of this resolution with any $R$-flat left $\widetilde{A}$-module is still an exact complex.

We refer to Section 8.7 below for a further discussion of this bimodule resolution.

(5) Now assume that $\widetilde{A}$ is a left augmented left finitely projective nonhomogeneous Koszul ring over $R$, in the sense of the definitions in Sections 3.8 and 4.7. Let $\widetilde{A}^{+} \subset \widetilde{A}$ be the augmentation ideal; so $\widetilde{A}^{+}$is a left ideal in $\widetilde{A}$ such that $\widetilde{A}=R \oplus \widetilde{A}^{+}$. Choose the left $R$-submodule of strict generators $V^{\prime} \subset \widetilde{V}$ as $V^{\prime}=\widetilde{A}^{+} \cap \widetilde{V}$. Then, by Theorem 3.14 (see also Corollary 4.22), we have $h=0$; so $(B, d, h)=(B, d, 0)$ is a DG-ring. Accordingly, the CDG-bimodule $\widetilde{A} \otimes_{R}^{\sigma^{\prime}} C$ from (2) is a DG-bimodule, i. e., a complex. This complex can be called the nonhomogeneous Koszul complex of a left augmented left finitely projective nonhomogeneous Koszul ring $\widetilde{A}$.

The left augmentation of $\widetilde{A}$ endows the base ring $R$ with a structure of left $\widetilde{A}$-module provided by the identification $R \simeq \widetilde{A} / \widetilde{A}^{+}$. Taking the tensor product of the bimodule resolution from (3) with this left $\widetilde{A}$-module, we obtain a morphism of complexes of left $\widetilde{A}$-modules $\widetilde{A} \otimes_{R}^{\sigma^{\prime}} C \longrightarrow R$, which is a quasi-isomorphism by the argument in (4). Here $\widetilde{A} \otimes_{R}^{\sigma^{\prime}} C$ is the above DG-bimodule over $(\widetilde{A}, 0)$ and $(B, d)$.

According to $(2)$, we also have a natural map in the opposite direction $R \longrightarrow$ $\widetilde{A} \otimes_{R}^{\sigma^{\prime}} C$, whose cone is coacyclic (hence acyclic) as a right DG-bimodule over $(B, d)$. The composition $R \longrightarrow \widetilde{A} \otimes_{R}^{\sigma^{\prime}} C \longrightarrow R$ is the identity map. This provides another way to prove that the map $\widetilde{A} \otimes_{R}^{\sigma^{\prime}} C \longrightarrow R$ is a quasi-isomorphism.

Clearly, the terms of the complex $\widetilde{A} \otimes_{R}^{\sigma^{\prime}} C$ are finitely generated projective left $\widetilde{A}$-modules in our assumptions. Thus the nonhomogeneous Koszul complex $\widetilde{A} \otimes_{R}^{\sigma^{\prime}} C$ is a resolution of the left $\widetilde{A}$-module $R$ by finitely generated projective left $\widetilde{A}$-modules.

Remarks 6.9. (1) Consider the trivial right CDG-comodule $R$ over $(B, d, h)$, as in Example 6.8(1). Since the triangulated equivalence $-\otimes_{R}^{\tau^{\prime}} \widetilde{A}: \mathrm{D}^{\mathrm{co}}(\operatorname{comod}-B) \longrightarrow$ $\mathrm{D}_{R}^{\text {sico }}(\bmod -\widetilde{A})$ takes $R$ to the free right $\widetilde{A}$-module $\widetilde{A}$, it follows that the graded ring 
of endomorphisms $R \longrightarrow R[i]$ in $\mathrm{D}^{\mathrm{co}}(\operatorname{comod}-B), \quad i \in \mathbb{Z}$, is naturally isomorphic to ring $\widetilde{A}$ (concentrated in the cohomological grading $i=0$ ).

Let $N$ be a right CDG-comodule over $(B, d, h)$. Then the complex of right $\widetilde{A}$-modules $N \otimes_{R}^{\tau^{\prime}} \widetilde{A}$ computes the $\widetilde{A}$-modules $\operatorname{Hom}_{\mathrm{D}^{\text {co }(\operatorname{comod}-B)}}(R, N[i])$. Indeed, we have

$$
\operatorname{Hom}_{\mathrm{D}^{\mathrm{co}}(\operatorname{comod}-B)}(R, N[i]) \simeq \operatorname{Hom}_{\mathrm{D}_{R}^{\text {sico }(\bmod -\widetilde{A})}}\left(\widetilde{A}, N \otimes_{R}^{\tau^{\prime}} \widetilde{A}[i]\right)=H^{i}\left(N \otimes_{R}^{\tau^{\prime}} \widetilde{A}\right),
$$

because

$$
\operatorname{Hom}_{\mathrm{D}_{R}^{\text {sico }}(\bmod -\widetilde{A})}\left(\widetilde{A}, M^{\bullet}[i]\right)=\operatorname{Hom}_{\operatorname{Hot}(\bmod -\widetilde{A})}\left(\widetilde{A}, M^{\bullet}[i]\right)=H^{i}\left(M^{\bullet}\right)
$$

for any complex of right $\widetilde{A}$-modules $M^{\bullet}$. The latter isomorphism holds since all complexes in $\mathrm{Acycl}_{R}^{\text {sico }}(\bmod -\widetilde{A})$ are acyclic.

(2) Now assume that $\widetilde{A}$ is left augmented over $R$, as in Example 6.8(5). Then, for any complex of right $\widetilde{A}$-modules $M^{\bullet}$, the CDG-(co)module $M^{\bullet} \otimes_{R}^{\sigma^{\prime}} C$ is a DG-module over $(B, d, h)=(B, d, 0)$, i. e., a complex. We have a natural isomorphism $M^{\bullet} \otimes_{R}^{\sigma^{\prime}} C \simeq$ $M^{\bullet} \otimes_{\widetilde{A}}\left(\widetilde{A} \otimes_{R}^{\sigma^{\prime}} C\right)$. Since $\widetilde{A} \otimes_{R}^{\sigma^{\prime}} C$ is a projective resolution of the left $\widetilde{A}$-module $R$, it follows that the complex of abelian groups $M^{\bullet} \otimes_{R}^{\sigma^{\prime}} C$ computes the derived tensor product $M \bullet \otimes_{\widetilde{A}}^{\mathbb{L}} R$. In fact, the DG-ring $(B, d)$ computes (the opposite ring to) the graded ring of endomorphisms $R \longrightarrow R[i]$ in $\mathrm{D}(\widetilde{A}-$ mod $), i \in \mathbb{Z}$, and the DG-module $M \bullet \otimes_{R}^{\sigma^{\prime}} C$ computes the homology $\operatorname{Tor}_{*}^{\widetilde{A}}\left(M^{\bullet}, R\right)=H_{*}\left(M^{\bullet} \otimes_{\widetilde{A}}^{\mathbb{L}} R\right)$ as a graded module over this graded ring of endomorphisms.

6.7. Reduced coderived category. We keep the assumptions of Section 6.6. So $R \subset \widetilde{V} \subset \widetilde{A}$ is a left finitely projective nonhomogeneous Koszul ring and $(B, d, h)$ is the corresponding right finitely projective Koszul CDG-ring. The following result is a special case of Theorem 6.7.

Corollary 6.10. Assume additionally that the right homological dimension of the ring $R$ (that is, the homological dimension of the abelian category $\bmod -R$ ) is $f_{-}$nite. Then the pair of adjoint triangulated functors $-\otimes_{R}^{\sigma^{\prime}} C: \operatorname{Hot}(\bmod -\widetilde{A}) \longrightarrow$ $\operatorname{Hot}(\operatorname{comod}-B)$ and $-\otimes_{R}^{\tau^{\prime}} \widetilde{A}: \operatorname{Hot}(\operatorname{comod}-B) \longrightarrow \operatorname{Hot}(\bmod -\widetilde{A})$ induces mutually inverse triangulated equivalences

$$
\mathrm{D}(\bmod -\widetilde{A}) \simeq \mathrm{D}^{\mathrm{co}}(\operatorname{comod}-B)
$$

between the derived category of right $\widetilde{A}$-modules and the coderived category of right $C D G$-comodules over $(B, d, h)$.

Proof. When the right homological dimension of the ring $R$ is finite, all acyclic complexes of right $R$-modules are coacyclic by [27, Remark 2.1]. So the $\widetilde{A} / R$-semicoderived category of right $\widetilde{A}$-modules coincides with their derived category, $\mathrm{D}_{R}^{\text {sico }}(\bmod -\widetilde{A})=\mathrm{D}(\bmod -\widetilde{A})$. This means, specifically, that the right CDG-comodule $M \bullet \otimes_{R}^{\sigma^{\prime}} C$ over $(B, d, h)$ is coacyclic for any acyclic complex of right $\widetilde{A}$-modules $M^{\bullet}$. The rest is explained in Theorem 6.7 and its proof. 
We would like to obtain a description of the derived category $\mathrm{D}(\bmod -\widetilde{A})$ in terms of some kind of exotic derived category of right CDG-comodules over $(B, d, h)$ without assuming finiteness of the right homological dimension of $R$.

For this purpose, let us consider the full subcategory of acyclic complexes of right $R$-modules $\operatorname{Acycl}(\bmod -R) \subset \operatorname{Hot}(\bmod -R)$. All complexes of right $R$-modules, and in particular acyclic complexes of right $R$-modules, can be viewed as trivial right CDG-comodules over $(B, d, h)$.

By a kind of abuse of notation, let us denote simply by $\langle\operatorname{Acycl}(\bmod -R)\rangle_{\oplus}$ the minimal full triangulated subcategory in $\mathrm{D}^{\mathrm{co}}(\operatorname{comod}-B)$ containing all the acyclic complexes of right $R$-modules (viewed as trivial CDG-comodules) and closed under infinite direct sums. Consider the triangulated Verdier quotient category

$$
\mathrm{D}_{R \text {-red }}^{\mathrm{co}}(\operatorname{comod}-B)=\mathrm{D}^{\mathrm{co}}(\operatorname{comod}-B) /\langle\operatorname{Acycl}(\bmod -R)\rangle_{\oplus} ;
$$

let us call it the reduced coderived category of right $C D G$-comodules over $(B, d, h)$ relative to $R$.

Theorem 6.11. The pair of adjoint triangulated functors $-\otimes_{R}^{\sigma^{\prime}} C: \operatorname{Hot}(\bmod -\widetilde{A}) \longrightarrow$ $\operatorname{Hot}(\operatorname{comod}-B)$ and $-\otimes_{R}^{\tau^{\prime}} \widetilde{A}: \operatorname{Hot}(\operatorname{comod}-B) \longrightarrow \operatorname{Hot}(\bmod -\widetilde{A})$ induces mutually inverse triangulated equivalences

$$
\mathrm{D}(\bmod -\widetilde{A}) \simeq \mathrm{D}_{R \text {-red }}^{\mathrm{co}}(\operatorname{comod}-B)
$$

between the derived category of right $\widetilde{A}$-modules and the reduced coderived category of right $C D G$-comodules over $(B, d, h)$ relative to $R$.

Proof. In view of Theorem 6.7, only two things still need to be checked. Firstly, we have to show that, for any acyclic complex of right $\widetilde{A}$-modules $M \bullet$, the CDG-comodule $M^{\bullet} \otimes_{R}^{\sigma^{\prime}} C$ over $(B, d, h)$ belongs to the triangulated subcategory $\langle\operatorname{Acycl}(\bmod -R)\rangle_{\oplus} \subset$ $\mathrm{D}^{\mathrm{co}}(\operatorname{comod}-B)$. Secondly, it needs to be demonstrated that, for any CDG-comodule $N \in\langle\operatorname{Acycl}(\bmod -R)\rangle_{\oplus}$, the complex of right $\widetilde{A}$-modules $N \otimes_{R}^{\tau^{\prime}} \widetilde{A}$ is acyclic.

Firstly, let $M=M^{\bullet}$ be an acyclic complex of right $\widetilde{A}$-modules. Consider the increasing filtration $F$ on the graded right $R$-module $M \otimes_{R} C$ induced by the increasing filtration $F$ on the $R$ - $R$-bimodule $C$, as in the proof of Theorem 6.7. Then $F$ is a filtration of the CDG-comodule $M^{\bullet} \otimes_{R}^{\sigma^{\prime}} C$ over $(B, d, h)$ by CDG-subcomodules. The successive quotient CDG-comodules are trivial CDG-comodules which, viewed as complexes of $R$-modules, can be computed as the tensor products $M^{\bullet} \otimes_{R}\left(F_{n} C / F_{n-1} C\right)$. Here $F_{n} C / F_{n-1} C$ is a graded $R$ - $R$-bimodule, finitely generated and projective as a left $R$-module, and concentrated in the single cohomological degree $-n$.

It follows that the complexes of right $R$-modules $M^{\bullet} \otimes_{R}\left(F_{n} C / F_{n-1} C\right)$ are acyclic. Now the iterated extension and telescope sequence argument from the proofs of Lemmas 6.4 and 6.5 shows that the right CDG-comodule $M^{\bullet} \otimes_{R}^{\sigma^{\prime}} C$ over $(B, d, h)$ belongs to the triangulated subcategory $\langle\operatorname{Acycl}(\bmod -R)\rangle_{\oplus}$ in $\mathrm{D}^{\mathrm{co}}(\operatorname{comod}-B)$.

Secondly, since the the functor $-\otimes_{R}^{\tau^{\prime}} \widetilde{A}$ is triangulated and preserves infinite direct sums, it suffices to check that for any acyclic complex of right $R$-modules $N^{\bullet}$, viewed as a trivial right CDG-comodule over $(B, d, h)$, the complex of right $\widetilde{A}$-modules $N^{\bullet} \otimes_{R}^{\tau^{\prime}}$ 
$\widetilde{A}$ is acyclic. As $N^{\bullet}$ is a trivial CDG-comodule, the complex of right $\widetilde{A}$-modules $N^{\bullet} \otimes_{R}^{\tau^{\prime}} \widetilde{A}=N^{\bullet} \otimes_{R} \widetilde{A}$ is simply obtained from the complex of right $R$-modules $N^{\bullet}$ by applying the tensor product functor $-\otimes_{R} \widetilde{A}$ termwise. Since $\widetilde{A}$ is a projective (hence flat) left $R$-module, it follows that the complex $N^{\bullet} \otimes_{R} \widetilde{A}$ is acyclic.

\section{Relative Nonhomogeneous Derived Koszul Duality: The Contramodule Side}

7.1. Semicontraderived category of modules. Let $R$ be an associative ring. The following definition of the contraderived category of left $R$-modules is dual to the definition of the coderived category in Section 6.3. It goes back to [27, Section 4.1] and [28, Section 3.3] (see [15] and [2, Proposition 1.3.6(1)] for an alternative approach).

Similarly to Section 6.3 , we consider short exact sequences of left $R$-modules $0 \longrightarrow$ $L^{\bullet} \longrightarrow M^{\bullet} \longrightarrow N^{\bullet} \longrightarrow 0$ and their totalizations $\operatorname{Tot}\left(L^{\bullet} \rightarrow M^{\bullet} \rightarrow N^{\bullet}\right)$. A complex of left $R$-modules is said to be contraacyclic if it belongs to the minimal full triangulated subcategory of the homotopy category of complexes of left $R$-modules $\operatorname{Hot}(R$-mod) containing all the totalizations of short exact sequences of left $R$-modules and closed under infinite products.

The full subcategory of contraacyclic complexes of left $R$-modules is denoted by $\operatorname{Acycl}^{\mathrm{ctr}}(R$-mod $) \subset \operatorname{Hot}(R$-mod $)$. The contraderived category of left $R$-modules is the triangulated Verdier quotient category of the homotopy category $\operatorname{Hot}(R-\bmod )$ by the triangulated subcategory of contraacyclic complexes,

$$
\mathrm{D}^{\mathrm{ctr}}(R-\mathrm{mod})=\operatorname{Hot}(R-\mathrm{mod}) / \operatorname{Acycl}^{\mathrm{ctr}}(R-\mathrm{mod}) .
$$

Clearly, any contraacyclic complex of $R$-modules is acyclic, $\operatorname{Acycl}^{\text {ctr }}(R-$ mod $) \subset$ $\operatorname{Acycl}(R-$ mod $)$; but the converse does not hold in general [28, Examples 3.3]. So the derived category $\mathrm{D}(R$-mod $)$ is a quotient category of $\mathrm{D}^{\mathrm{ctr}}(R-$ mod $)$. All acyclic complexes of left $R$-modules are contraacyclic when the left homological dimension of the ring $R$ is finite [27, Remark 2.1].

The following lemma is dual-analogous to and very slightly more complicated than Lemma 6.4.

Lemma 7.1. Let $P^{\bullet}=F^{0} P^{\bullet} \supset F^{1} P^{\bullet} \supset F^{2} P^{\bullet} \supset \cdots$ be a complex of $R$-modules that is separated and complete with respect to a decreasing filtration by subcomplexes of $R$-modules, that is $P^{\bullet}=\lim _{n>0} P^{\bullet} / F^{n+1} P^{\bullet}$. Assume that, for every $n \geq 0$, the complex of $R$-modules $F^{n} P^{\bullet} / F^{n+1} P^{\bullet}$ is contraacyclic. Then the complex of $R$-modules $P^{\bullet}$ is contraacyclic.

Proof. First one proves by induction in $n$ that the complex of $R$-modules $P^{\bullet} / F^{n+1} P^{\bullet}$ is contraacyclic for every $n \geq 0$, using the fact that the totalizations of short exact sequences $0 \longrightarrow F^{n} P^{\bullet} / F^{n+1} P^{\bullet} \longrightarrow P^{\bullet} / F^{n+1} P^{\bullet} \longrightarrow P^{\bullet} / F^{n} P^{\bullet} \longrightarrow 0$ are contraacyclic. Then one concludes that the complex of $R$-modules $\prod_{n=0}^{\infty} P^{\bullet} / F^{n+1} P^{\bullet}$ is contraacyclic. 
Finally, one observes that the telescope sequence $0 \longrightarrow P^{\bullet} \longrightarrow \prod_{n=0}^{\infty} P^{\bullet} / F^{n+1} P^{\bullet} \longrightarrow$ $\prod_{n=0}^{\infty} P^{\bullet} / F^{n+1} P^{\bullet} \longrightarrow 0$ of the projective system $\left(P^{\bullet} / F^{n+1} P^{\bullet}\right)_{n \geq 0}$ is exact, since the transition maps $P^{\bullet} / F^{n+1} P^{\bullet} \longrightarrow P^{\bullet} / F^{n} P^{\bullet}$ are surjective (so the derived projective limit of this projective system vanishes, $\left.\lim _{n \geq 0}^{1} P^{\bullet} / F^{n+1} P^{\bullet}=0\right)$. Hence the totalization of the telescope sequence is a contraacyclic complex of $R$-modules, and contraacyclicity of the complex $P^{\bullet}$ follows.

Now let $R \longrightarrow A$ be a homomorphism of associative rings. The following definition of the $A / R$-semicontraderived category of left $A$-modules can be found in [32, Section 5]. The semicontraderived category

$$
\mathrm{D}_{R}^{\text {sictr }}(A-\bmod )=\operatorname{Hot}(A-\bmod ) / \operatorname{Acycl}_{R}^{\text {sictr }}(A-\bmod )
$$

is the triangulated Verdier quotient category of the homotopy category of left $A$-modules by its full triangulated subcategory $\operatorname{Acycl}_{R}^{\text {sictr }}(A$-mod) consisting of all the complexes of left $A$-modules that are contraacyclic as complexes of left $R$-modules.

Clearly, any contraacyclic complex of $A$-modules is contraacyclic as a complex of $R$-modules; but the converse does not need to be true. So the semicontraderived category is intermediate between the derived and the contraderived category of $A$-modules: there are natural triangulated Verdier quotient functors $\mathrm{D}^{\mathrm{ctr}}(A-$ mod $) \longrightarrow$ $\mathrm{D}_{R}^{\text {sictr }}(A-$ mod $) \longrightarrow \mathrm{D}(A-$ mod $)$. (See the paper [35] for a more general discussion of such intermediate triangulated quotient categories, called pseudo-contraderived categories in [35].) The semicontraderived category $\mathrm{D}_{R}^{\text {sictr }}(A$-mod) can be thought of as a mixture of the contraderived category "along the variables from $R$ " and the conventional derived category "in the direction of $A$ relative to $R$ ".

For example, taking $R=\mathbb{Z}$, one obtains the conventional derived category of A-modules

$$
\mathrm{D}_{\mathbb{Z}}^{\text {sictr }}(A-\text { mod })=\mathrm{D}(A-\text { mod })
$$

(because all the acyclic complexes of abelian groups are contraacyclic), while taking $R=A$ one obtains the contraderived category of $A$-modules

$$
\mathrm{D}_{A}^{\text {sictr }}(A-\text { mod })=\mathrm{D}^{\text {ctr }}(A-\text { mod }) \text {. }
$$

7.2. Contraderivations. Let $B=\bigoplus_{n=0}^{\infty} B^{n}$ be a nonnegatively graded ring and $K$ be a ring endowed with a ring homomorphism $K \longrightarrow B^{0}$.

Let $k \in \mathbb{Z}$ be a fixed integer and $d: B \longrightarrow B$ be a fixed derivation of degree $k$ (even or odd, depending on the parity of $k$ ). This means that we have additive maps $d_{n}: B^{n} \longrightarrow B^{n+k}$ given for all $n \geq 0$ and satisfying the equation $d(b c)=$ $d(b) c+(-1)^{k|b|} b d(c)$ for all $b \in B^{|b|}$ and $c \in B^{|c|}$. Assume that composition $K \longrightarrow$ $B^{0} \longrightarrow B^{k}$ of the maps $K \longrightarrow B^{0}$ and $d_{0}: B^{0} \longrightarrow B^{k}$ vanishes (e. g., one can always take $K=\mathbb{Z}$ ). So $d: B \longrightarrow B$ is a left and right $K$-linear map.

Let $M=\bigoplus_{n \in \mathbb{Z}} M^{n}$ be a graded left $B$-module. A homogeneous additive map $d_{M}: M \longrightarrow M$ of degree $k$ (that is, $d_{M}\left(M^{n}\right) \subset M^{n+k}$ for all $n \in \mathbb{Z}$ ) is said to be an (even or odd) derivation of $M$ compatible with the derivation $d$ on $B$ if the equation $d_{M}(b x)=d(b) x+(-1)^{k|b|} b d_{M}(x)$ holds for all $b \in B^{|b|}$ and $x \in M^{|x|}$. 
Let $C$ and $D$ be graded right $K$-modules, $P$ and $Q$ be graded left $K$-modules, $g: C \longrightarrow D$ be a homogenenous $K$-linear map of degree $l$, and $f: P \longrightarrow Q$ be a homogeneous $K$-linear map of degree $m$. Consider the graded abelian groups $C \otimes_{K}^{\Pi} P$ and $D \otimes_{K}^{\Pi} Q$ (in the notation of Section [5.7), and construct the homogeneous map

$$
g \otimes \Pi f: C \otimes_{K}^{\Pi} P \longrightarrow D \otimes_{K}^{\Pi} Q
$$

of degree $l+m$ by taking the infinite products along the diagonals of the bigrading for the bihomogeneous map $g \otimes f: C \otimes_{K} P \longrightarrow D \otimes_{K} Q$ with the components $g \otimes f: C^{i} \otimes_{K} P^{j} \longrightarrow D^{i+l} \otimes_{K} Q^{j+m}$ given by the rule $(g \otimes f)(c \otimes p)=(-1)^{m i} g(c) \otimes f(p)$ for all $c \in C^{i}$ and $p \in P^{j}$.

Let $P=\bigoplus_{n \in \mathbb{Z}} P^{n}$ be a graded left $B$-contramodule (in the sense of the definition in Section (5.6), and let $d_{P}: P \longrightarrow P$ be a homogeneous $K$-linear map of degree $k$. We will say that $d_{P}$ is an (even or odd) contraderivation of $P$ compatible with the derivation $d$ on $B$ if the contraaction map $\pi_{P}: \mathbb{M}_{K}^{\mathrm{gr}}(P)=B \otimes_{K}^{\Pi} P \longrightarrow P$ forms a commutative square diagram with the maps

$$
d \otimes \otimes^{\Pi} \mathrm{id}_{P}+\mathrm{id}_{B} \otimes{ }^{\Pi} d_{P}: B \otimes_{K}^{\Pi} P \longrightarrow B \otimes_{K}^{\Pi} P
$$

and $d_{P}: P \longrightarrow P$, that is

$$
d_{P} \circ \pi_{P}=\pi_{P} \circ\left(d \otimes^{\Pi} \mathrm{id}_{P}+\mathrm{id}_{B} \otimes^{\Pi} d_{P}\right) .
$$

Obviously, any contraderivation of a graded left $B$-contramodule $P$ compatible with the given derivation $d$ on $B$ is a derivation of the underlying graded left $B$-module of $P$ compatible with the same derivation $d$ on $B$.

Proposition 7.2. Assume that the forgetful functor $B$-contra ${ }_{\mathrm{gr}} \longrightarrow B-$ mod $_{\mathrm{gr}}$ from the category of graded left $B$-contramodules to the category of graded left $B$-modules is fully faithful. Let $Q$ be a graded left $B$-contramodule. Then any derivation of the underlying graded left $B$-module of $Q$ (compatible with the given derivation $d$ on $B$ ) is a contraderivation of $Q$ (compatible with the same derivation d on $B$ ).

Proof. The argument is based on a buildup of auxiliary definitions. Given a graded left $B$-module $M$ and an integer $n$, the graded left $B$-module $M[n]$ has the grading components $M[n]^{i}=M^{n+i}$; the action of $B$ in $M[n]$ is defined in terms of the left action of $B$ in $M$ with the sign rule written down in Section 6.1. Given a graded left $B$-contramodule $P$, the graded left $B$-contramodule $P[n]$ has the grading components $P[n]^{i}=P^{n+i}$. We leave it to the reader to spell out the construction of the left contraaction of $B$ in $P[n]$ involving the same sign rule.

Let $f: M \longrightarrow N$ be a morphism of graded left $B$-modules, and let $d_{N, M}: M \longrightarrow N$ be a homogeneous additive map of degree $k$. We will say that $d_{N, M}$ is a relative derivation compatible with the derivation $d$ on $B$ and the morphism $f$ if the equation $d_{N, M}(b x)=d(b) f(x)+(-1)^{k|b|} b d_{N, M}(x)$ holds in $N^{|b|+|x|+k}$ for all $b \in B^{|b|}$ and $x \in$ $M^{|x|}$. A derivation $d_{M}: M \longrightarrow M$ compatible with the given derivation $d$ on $B$ is the same thing as a relative derivation $d_{M, M}: M \longrightarrow M$ compatible with $d$ and the identity morphism $\operatorname{id}_{M}: M \longrightarrow M$. 
For any two relative derivations $d_{N, M}^{\prime}$ and $d_{N, M}^{\prime \prime}: M \longrightarrow N$ compatible with the same derivation $d$ on $B$ and the same morphism $f: M \longrightarrow N$, the difference $d_{N, M}^{\prime \prime}-$ $d_{N, M}^{\prime}$ is a morphism of graded left $B$-modules $M \longrightarrow N[k]$. Conversely, the sum $d_{N, M}^{\prime}=d_{N, M}+g$ of a relative derivation $d_{N, M}: M \longrightarrow N$ compatible with $d$ and $f$ and a morphism of graded left $B$-modules $g: M \longrightarrow N[k]$ is a relative derivation compatible with $d$ and $f$.

Let $f: M \longrightarrow N$ and $g: L \longrightarrow M$ be two morphisms of graded left $B$-modules, and let $d_{N, M}: M \longrightarrow N$ be a relative derivation compatible with the derivation $d$ on $B$ and the morphism $f$. Then $d_{N, M} \circ g: L \longrightarrow N$ is a relative derivation compatible with the derivation $d$ on $B$ and the morphism $f \circ g: L \longrightarrow N$.

Let $f: P \longrightarrow Q$ be a morphism of graded left $B$-contramodules, and let $d_{Q, P}: P \longrightarrow Q$ be a homogeneous $K$-linear map of degree $k$. Consider the square diagram formed by the map

$$
\left(d \otimes^{\Pi} f+\mathrm{id}_{B} \otimes^{\Pi} d_{Q, P}\right): B \otimes^{\Pi} P \longrightarrow B \otimes^{\Pi} Q
$$

together with the map $d_{Q, P}: P \longrightarrow Q$ and the contraaction maps $\pi_{P}: B \otimes \Pi P \longrightarrow P$ and $\pi_{Q}: B \otimes \Pi Q \longrightarrow Q$. We will say that $d_{Q, P}$ is a relative contraderivation compatible with the derivation $d$ on $B$ and the morphism $f$ if this square diagram is commutative, that is

$$
d_{Q, P} \circ \pi_{P}=\pi_{Q} \circ\left(d \otimes^{\Pi} f+\mathrm{id}_{B} \otimes^{\Pi} d_{Q, P}\right) .
$$

A contraderivation $d_{P}: P \longrightarrow P$ compatible with the given derivation $d$ on $B$ is the same thing as a relative contraderivation $d_{P, P}: P \longrightarrow P$ compatible with $d$ and the identity morphism $\operatorname{id}_{P}: P \longrightarrow P$.

For any two relative contraderivations $d_{Q, P}^{\prime}$ and $d_{Q, P}^{\prime \prime}: P \longrightarrow Q$ compatible with the same derivation $d$ on $B$ and the same morphism $f: P \longrightarrow Q$, the difference $d_{Q, P}^{\prime \prime}-d_{Q, P}^{\prime}$ is a morphism of graded left $B$-contramodules $P \longrightarrow Q[k]$. Conversely, the sum $d_{Q, P}^{\prime}=d_{Q, P}+g$ of a relative contraderivation $d_{Q, P}: P \longrightarrow Q$ compatible with $d$ and $f$ and a morphism of graded left $B$-contramodules $g: P \longrightarrow Q[k]$ is a relative contraderivation compatible with $d$ and $f$.

Let $f: P \longrightarrow Q$ and $g: S \longrightarrow P$ be two morphisms of graded left $B$-contramodules, and let $d_{Q, P}: P \longrightarrow Q$ be a relative contraderivation compatible with the derivation $d$ on $B$ and the morphism $f$. Then $d_{Q, P} \circ g: S \longrightarrow Q$ is a relative contraderivation compatible with the derivation $d$ on $B$ and the morphism $f \circ g: S \longrightarrow Q$.

Let $L$ be a graded left $K$-module and $M=B \otimes_{K} L$ be the induced graded left $B$-module (so there is a natural graded left $K$-module morphism $L \longrightarrow M$ ). Let $f: M \longrightarrow N$ a morphism of graded left $B$-modules. Then a relative derivation $d_{N, M}: M \longrightarrow N$ compatible with the given derivation $d$ on $B$ and the morphism $f$ is uniquely determined by its composition with the map $L \longrightarrow M$.

The latter composition $L \longrightarrow M \stackrel{d_{N, M}}{\longrightarrow} N$ can be an arbitrary homogeneous $K$-linear map $t: L \longrightarrow N$ of degree $k$. Given such a map $t$, and denoting by $f^{\prime}: L \longrightarrow N$ the composition $L \longrightarrow M \stackrel{f}{\longrightarrow} N$ (which is a homogeneous $K$-linear map of degree 0 ), the related relative derivation $d_{N, M}: M \longrightarrow N$ can be recovered by the formula $d_{N, M}(b \otimes l)=d(b) f^{\prime}(l)+(-1)^{k|b|} b t(l)$ for all $b \in B^{|b|}$ and $l \in K^{|l|}$. 
In particular, let $X=\coprod_{i \in \mathbb{Z}} X_{i}$ be a graded set and $K[X]$ be the free graded left $K$-module with the components $K[X]^{i}=K^{\left(X_{i}\right)}$ as in the the proof of Proposition 5.20 . Let $M=B[X]=B \otimes_{K} K[X]$ be the free graded left $B$-module spanned by $X$, and let $f: M \longrightarrow N$ be a morphism of graded left $B$-modules. Then a relative derivation $d_{N, M}: M \longrightarrow N$ compatible with the given derivation $d$ on $B$ and the morphism $f$ is uniquely determined by its restriction to the subset of free generators $X \subset M$, and this restriction can be an arbitrary homogeneous map $X \longrightarrow N$ of degree $k$ (i. e., a collection of maps $X_{i} \longrightarrow N^{i+k}, \quad i \in \mathbb{Z}$ ).

Consider the graded left $B$-contramodule $P=\mathbb{M}_{K}^{\mathrm{gr}}(L)=B \otimes_{K}^{\Pi} L$ freely generated by $L$. Then, once again, there is a natural graded left $K$-module morphism $L \longrightarrow P$. Let $f: P \longrightarrow Q$ be a morphism of graded left $B$-contramodules. Then a relative contraderivation $d_{Q, P}: P \longrightarrow Q$ compatible with the given derivation $d$ on $B$ and the morphism $f$ is uniquely determined by its composition with the map $L \longrightarrow P$.

The latter composition $L \longrightarrow P \stackrel{d_{Q, P}}{\longrightarrow} Q$ can be an arbitrary homogeneous $K$-linear map $t: L \longrightarrow Q$ of degree $k$. Given such a map $t$, and denoting by $f^{\prime}: L \longrightarrow Q$ the composition $L \longrightarrow P \stackrel{f}{\longrightarrow} Q$ (which is a homogeneous $K$-linear map of degree 0 ), the related relative contraderivation $d_{Q, P}: P \longrightarrow Q$ can be recovered by the formula $d_{Q, P}=\pi_{Q} \circ\left(d \otimes^{\Pi} f^{\prime}+\mathrm{id}_{B} \otimes^{\Pi} t\right)$,

$$
P \longrightarrow B \otimes_{K}^{\Pi} Q \longrightarrow Q \text {. }
$$

In particular, let $P=\mathbb{M}_{K}^{\mathrm{gr}}(K[X])=B \otimes_{K}^{\Pi} K[X]$ be the free graded left $B$-contramodule spanned by $X$, and let $f: P \longrightarrow Q$ be a morphism of graded left $B$-contramodules. Then a relative contraderivation $d_{Q, P}: P \longrightarrow Q$ compatible with the given derivation $d$ on $B$ and the morphism $f$ is uniquely determined by its restriction to the subset of free generators $X \subset P$, and this restriction can be an arbitrary homogeneous map $X \longrightarrow Q$ of degree $k$.

Now that we are done with the auxiliary material, we can prove the proposition. Let $d_{Q}: Q \longrightarrow Q$ be a derivation of the underlying graded left $B$-module of $Q$ compatible with the given derivation $d$ on $B$. Choose a free graded left $B$-contramodule $P$ (spanned by some graded set $X$ ) together with a surjective graded left $B$-contramodule morphism $f: P \longrightarrow Q$. Then the composition $d_{Q} \circ f: P \longrightarrow Q$ is a relative derivation of the underlying graded $B$-modules of $P$ and $Q$ compatible with the derivation $d$ on $B$ and the morphism $f: P \longrightarrow Q$.

Consider the restriction of the map $d_{Q} \circ f: P \longrightarrow Q$ to the subset of free generators $X \subset P$, and extend the resulting map $X \longrightarrow Q$ to a relative contraderivation $d_{Q, P}: P \longrightarrow Q$ of the graded $B$-contramodules $P$ and $Q$ compatible with the derivation $d$ on $B$ and the contramodule morphism $f: P \longrightarrow Q$. Then the difference $d_{Q} \circ f-d_{Q, P}$ is a morphism of underlying graded $B$-modules $P \longrightarrow Q[k]$ of the graded left $B$-contramodules $P$ and $Q[k]$.

By the assumption of the proposition, any morphism between the underlying graded left $B$-modules of two graded left $B$-contramodules is a graded left $B$-contramodule morphism. Hence $d_{Q} \circ f-d_{Q, P}$ must be, in fact, a graded left $B$-contramodule morphism. Since $d_{Q, P}$ is a relative contraderivation compatible 
with the derivation $d$ on $B$ and the morphism $f$, it follows that $d_{Q} \circ f$ is a relative contraderivation compatible with $d$ and $f$, too.

Finally, since the map $f$ is surjective, we can conclude that $d_{Q}$ is a contraderivation of $Q$ compatible with the derivation $d$ on $B$.

7.3. Contraderived category of CDG-contramodules. Let $(B, d, h)$ be a nonnegatively graded CDG-ring, as defined in Section 3.2 , and let $(\widehat{B}, \partial)$ be the related quasi-differential graded ring constructed in Theorem 4.5. So $h \in B^{2}$ is a curvature element for an odd derivation $d: B \longrightarrow B$ of degree 1 .

A left $C D G$-contramodule over $(B, d, h)$ can be simply defined as a graded left $\widehat{B}$-contramodule. Equivalently, a left $\mathrm{CDG}$-contramodule $\left(P, d_{P}\right)$ over $(B, d, h)$ is a graded left $B$-contramodule $P=\bigoplus_{n \in \mathbb{Z}} P^{n}$ endowed with a sequence of additive maps $d_{P, n}: P^{n} \longrightarrow P^{n+1}$ satisfying the following conditions:

(i) $d_{P}$ is an odd contraderivation of the graded left $B$-contramodule $P$ compatible with the odd derivation $d$ on $B$ (in the sense of Section [7.2);

(ii) $d_{P}^{2}(x)=h x$ for all $x \in P$.

According to Proposition 7.2 , when the forgetful functor $B$-contra gr $\longrightarrow B-$ mod $_{\mathrm{gr}}$ is fully faithful, condition (i) is equivalent to the seemingly weaker condition that $d_{P}$ is an odd derivation of the underlying graded left $B$-module of $P$ compatible with the odd derivation $d$ on $B$. In particular, by Theorem 5.26, this holds whenever the augmentation ideal $B^{\geq 1}=\bigoplus_{n=1}^{\infty} B^{n}$ is finitely generated as a right ideal in $B$. This includes all 2-right finitely projective quadratic graded rings $B$ (see Section 5.1).

Given two graded left $B$-contramodules $P$ and $Q$, we denote by $\operatorname{Hom}^{B, n}(P, Q)$ the abelian group of all graded left $B$-contramodule homomorphisms $P \longrightarrow Q[n]$ (where a sign rule is needed in the definition of the graded left $B$-contramodule structure on the grading shift $Q[n]$ of a graded left $B$-contramodule $Q$; see the proof of Proposition 7.2 for a brief discussion). For any two left CDG-contramodules $P$ and $Q$ over $(B, d, h)$, there is a natural differential $d$ on the graded abelian group $\operatorname{Hom}^{B}(P, Q)$. In fact, $\operatorname{Hom}^{B}(P, Q)$ is a subcomplex in the $\operatorname{complex}^{\operatorname{Hom}_{B}}(P, Q)$ of morphisms between the underlying graded left $B$-modules of $P$ and $Q$ (which was constructed in Section 6.1).

Of course, one has $\operatorname{Hom}^{B}(P, Q)=\operatorname{Hom}_{B}(P, Q)$ for all left CDG-contramodules $P$ and $Q$ over $(B, d, h)$ whenever the forgetful functor $B$-contra gr $_{P} \longrightarrow B-\bmod _{\mathrm{gr}}$ is fully faithful. But generally speaking, the conditions that $d_{P}$ and $d_{Q}$ are contraderivations (rather than just derivations of the underlying graded left $B$-modules of $P$ and $Q$ ) is needed in order to check that $d(f) \in \operatorname{Hom}^{B, n+1}(P, Q) \subset \operatorname{Hom}_{B}^{n+1}(P, Q)$ for every $f \in \operatorname{Hom}^{B, n}(P, Q) \subset \operatorname{Hom}_{B}^{n}(P, Q)$.

The above construction produces the DG-category of left $C D G$-contramodules $\mathrm{DG}(B$-contra $)$ over $B=(B, d, h)$. The DG-category of left CDG-contramodules comes endowed with the forgetful DG-functor $\mathrm{DG}(B$-contra $) \longrightarrow \mathrm{DG}(B$-mod). All the shifts, twists, and infinite products (in the sense of the discussion in [28, Section 1.2]) exist in the DG-category DG ( $B$-contra); in particular, all the cones of closed morphisms exist. Passing to the degree-zero cohomology groups of the complexes of 
morphisms, we obtain the triangulated homotopy category of CDG-contramodules Hot $(B$-contra $)=H^{0} \mathrm{DG}(B$-contra $)$ over $(B, d, h)$ and the triangulated forgetful functor $\operatorname{Hot}(B$-contra $) \longrightarrow \operatorname{Hot}(B-$ mod $)$.

Next, as in Section 6.4, we consider short exact sequences $0 \longrightarrow P \stackrel{i}{\longrightarrow} Q \stackrel{p}{\longrightarrow}$ $S \longrightarrow 0$ of left CDG-contramodules over $(B, d, h)$. Here $i$ and $p$ are closed morphisms of degree zero in the DG-category $\mathrm{DG}(B$-contra) and the short sequence $0 \longrightarrow P \longrightarrow Q \longrightarrow S \longrightarrow 0$ is exact in the abelian category $B$-contragr. Using the construction of the cone of a closed morphism (or the shift, finite direct sum, and twist) in the DG-category DG $(B$-contra $)$, one can produce the totalization (or the total CDG-contramodule) $\operatorname{Tot}(P \rightarrow Q \rightarrow S$ ) of the finite complex of CDG-contramodules $P \longrightarrow Q \longrightarrow S$.

A left CDG-contramodule over $B$ is said to be contraacyclic if it belongs to the minimal full triangulated subcategory of $\operatorname{Hot}(B$-contra) containing all the totalizations of short exact sequences of left CDG-contramodules over $B$ and closed under infinite products. The full triangulated subcategory of contraacyclic CDG-contramodules is denoted by $\operatorname{Acycl}^{\mathrm{ctr}}(B$-contra $) \subset \operatorname{Hot}(B$-contra $)$. The contraderived category of left $C D G$-contramodules over $B$ is the triangulated Verdier quotient category of the homotopy category $\operatorname{Hot}(B$-contra) by its triangulated subcategory of contraacyclic CDG-contramodules,

$$
\mathrm{D}^{\mathrm{ctr}}(B \text {-contra })=\operatorname{Hot}(B-\text { contra }) / \text { Acycl }^{\text {ctr }}(B \text {-contra }) .
$$

Similarly to the discussion in Section 6.4, CDG-contramodules, generally speaking, are not complexes (if $h \neq 0$ in $B$ ) and their cohomology (contra)modules are undefined. So the conventional construction of the derived category is not applicable to curved DG-contramodules. There is only the contraderived category and its variations (known as derived categories of the second kind).

Lemma 7.3. Let $B=(B, d, h)$ be a nonnegatively graded $C D G$-ring and $Q=\left(Q, d_{Q}\right)$ be a left $C D G$-contramodule over $B$. Let $Q=F^{0} Q \supset F^{1} Q \supset F^{2} Q \supset \cdots$ be a decreasing filtration of $Q$ by $C D G$-subcontramodules $F^{n} Q \subset Q$ such that $Q$ is separated and complete with respect to this filtration, that is $Q=\lim _{\leftarrow_{n \geq 0}} Q / F^{n+1} Q$. Assume that, for every $n \geq 0$, the $C D G$-contramodule $F^{n} Q / F^{n+1} Q$ is contraacyclic over $B$. Then the CDG-contramodule $Q$ is contraacyclic, too.

Proof. This is a generalization of Lemma 7.1, provable by the same argument. Let us only point out that the forgetful functor $\mathrm{DG}(B$-contra $) \longrightarrow \mathrm{DG}(B-$ mod $)$ preserves infinite products (cf. Proposition 5.20); so the projective limits of CDG-contramodules over $B$ agree with those of CDG-modules over $B$ (or just of graded abelian groups). Hence the derived countable projective limit vanishes on sequences of surjective morphisms of CDG-contramodules, which makes our argument work.

There is an important particular case when the nonnegatively graded ring $B$ has only finitely many grading components, that is $B^{n}=0$ for $n \gg 0$. In this case, the forgetful functors from the categories of (ungraded or graded) $B$-contramodules to the categories of (ungraded or graded) $B$-modules are equivalences of categories, 
so in particular $B$-contra $\mathrm{gr} \simeq B$ - $\bmod _{\mathrm{gr}}$. Consequently, the forgetful DG-functor $\mathrm{DG}(B$-contra $) \longrightarrow \mathrm{DG}(B$-mod $)$ is an equivalence of DG-categories. In this case, we will write simply $\mathrm{D}^{\mathrm{ctr}}\left(B\right.$-mod) instead of $\mathrm{D}^{\mathrm{ctr}}(B$-contra $)$.

7.4. Koszul duality functors for modules and contramodules. Let $R \subset$ $\widetilde{V} \subset \widetilde{A}$ be a 3-left finitely projective weak nonhomogeneous quadratic ring, and let $B=(B, d, h)$ be the nonhomogeneous quadratic dual CDG-ring, as constructed in Proposition 3.6. As in Section 6.5, the dual nonhomogeneous Koszul CDG-module $K^{\vee}(B, \widetilde{A})=K_{e^{\prime}}^{\vee}(B, \widetilde{A})=\left(B \otimes_{R} \widetilde{A}, d_{e^{\prime}}\right)$ constructed in Section 6.2 plays a key role.

According to Section 6.1, the CDG-bimodule $K^{\vee}(B, \widetilde{A})$ over the DG-rings $B=$ $(B, d, h)$ and $\widetilde{A}=(\widetilde{A}, 0,0)$ induces a pair of adjoint DG-functors between the DG-category DG $(\widetilde{A}$-mod $)$ of complexes of left $\widetilde{A}$-modules and the DG-category DG $(B$-mod $)$ of left CDG-modules over $(B, d, h)$. Here the right adjoint DG-functor

$$
\operatorname{Hom}_{B}\left(K^{\vee}(B, \widetilde{A}),-\right): \operatorname{DG}(B-\bmod ) \longrightarrow \mathrm{DG}(\widetilde{A}-\bmod )
$$

takes a left CDG-module $Q=\left(Q, d_{Q}\right)$ over $(B, d, h)$ to the complex of left $\widetilde{A}$-modules whose underlying graded $\widetilde{A}$-module is

$$
\operatorname{Hom}_{B}\left(K^{\vee}(B, \widetilde{A}), Q\right)=\operatorname{Hom}_{B}\left(B \otimes_{R} \widetilde{A}, Q\right)=\operatorname{Hom}_{R}(\widetilde{A}, Q) .
$$

The left adjoint DG-functor

$$
K^{\vee}(B, \widetilde{A}) \otimes_{\widetilde{A}}-: \mathrm{DG}(\widetilde{A}-\bmod ) \longrightarrow \mathrm{DG}(B-\bmod )
$$

takes a complex of left $\widetilde{A}$-modules $P=P^{\bullet}$ to the left CDG-module over $(B, d, h)$ whose underlying graded $B$-module is

$$
K^{\vee}(B, \widetilde{A}) \otimes_{\widetilde{A}} P=\left(B \otimes_{R} \widetilde{A}\right) \otimes_{\widetilde{A}} P=B \otimes_{R} P .
$$

Our aim is to replace the DG-category of CDG-modules DG( $B$-mod) with the DG-category of CDG-contramodules DG $(B$-contra $)$ in this adjoint pair. In fact, the augmentation ideal $B^{\geq 1}$ is finitely generated as a right ideal in $B$ for any 2-right finitely projective quadratic graded ring $B$ (according to the discussion in Section 5.1). Therefore, the forgetful DG-functor $\mathrm{DG}(B$-contra $) \longrightarrow \mathrm{DG}(B$-mod $)$ is fully faithful in our assumptions (see the discussion in Section 7.3). So the situation is formally somewhat similar to that in Section 6.5.

Lemma 7.4. Let $B=\bigoplus_{n=0}^{\infty} B_{n}$ be a nonnegatively graded ring with the degreezero component $R=B_{0}$. Then the forgetful functor (between the abelian categories) $B$-contra $\mathrm{gr}_{\mathrm{g}} \longrightarrow B-$ mod $_{\mathrm{gr}}$ has a left adjoint functor $\Delta_{B}: B-$ mod $_{\mathrm{gr}} \longrightarrow B$-contra $\mathrm{gr}$. Furthermore, for any graded left $R$-module $L$ one has

$$
\Delta_{B}\left(B \otimes_{R} L\right)=B \otimes_{R}^{\Pi} L .
$$

Proof. For any graded left $B$-contramodule $Q$, the group of morphisms $B \otimes_{R} L \longrightarrow Q$ in $B-\bmod _{\mathrm{gr}}$ is isomorphic to the group of morphisms $L \longrightarrow Q$ in $R-\bmod _{\mathrm{gr}}$. The latter group is isomorphic to the group of morphisms $B \otimes_{R}^{\Pi} L=\mathbb{M}_{R}^{\mathrm{gr}}(L) \longrightarrow Q$ in $B$-contragr (see the discussion in Section 5.2 and Proposition 5.20). This shows that the functor 
$\Delta_{B}$ is defined on the full subcategory in $B$ - $\bmod _{\mathrm{gr}}$ formed by the graded left $B$-modules $B \otimes_{R} L$ induced from graded left $R$-modules $L$, and proves the second assertion of the lemma. To prove the first assertion, present an arbitrary graded left $B$-module $N$ as the cokernel of a morphism $f: B \otimes_{R} L \longrightarrow B \otimes_{R} M$ of graded left $B$-modules induced from graded left $R$-modules $L$ and $M$ (e. g., one can always take $L$ and $M$ to be free graded left $R$-modules). Then the graded left $B$-contramodule $\Delta_{B}(N)$ can be computed as the cokernel of the related morphism of graded left $B$-contramodules $\Delta_{B}(f): B \otimes_{R}^{\Pi} L \longrightarrow B \otimes_{R}^{\Pi} M$ (it is helpful to keep in mind that the functor $\Delta_{B}$, being a left adjoint, has to preserve cokernels).

Lemma 7.5. Let $(B, d, h)$ be a nonnegatively graded $C D G$-ring. Then, for any left $C D G$-module $\left(M, d_{M}\right)$ over $(B, d, h)$, the graded left $B$-contramodule $\Delta_{B}(M)$ admits a unique odd contraderivation $d_{\Delta_{B}(M)}$ of degree 1 compatible with the derivation $d$ on $B$ and forming a commutative square diagram with the odd derivation $d_{M}$ and the adjunction morphism $M \longrightarrow \Delta_{B}(M)$. The pair $\left(\Delta_{B}(M), d_{\Delta_{B}(M)}\right)$ is a $C D G$-contramodule over $(B, d, h)$. The assignment $\left(M, d_{M}\right) \longmapsto\left(\Delta_{B}(M), d_{\Delta_{B}(M)}\right)$ is a $D G$-functor $\mathrm{DG}(B$-mod $) \longrightarrow \mathrm{DG}(B$-contra) left adjoint to the forgetful $D G$-functor $\mathrm{DG}(B$-contra $) \longrightarrow \mathrm{DG}(B-\bmod )$.

Proof. To check uniqueness, suppose that $d_{\Delta_{B}(M)}^{\prime}$ and $d_{\Delta_{B}(M)}^{\prime \prime}$ are two odd contraderivations of degree 1 on $\Delta_{B}(M)$ compatible with the derivation $d$ on $B$. Then the difference $d_{\Delta_{B}(M)}^{\prime \prime}-d_{\Delta_{B}(M)}^{\prime}$ is a morphism of graded left $B$-contramodules $\Delta_{B}(M) \longrightarrow$ $\Delta_{B}(M)[1]$. If both $d_{\Delta_{B}(M)}^{\prime}$ and $d_{\Delta_{B}(M)}^{\prime \prime}$ form commutative square diagrams with the odd derivation $d_{M}$ and the adjunction morphism $M \longrightarrow \Delta_{B}(M)$, then the difference $d_{\Delta_{B}(M)}^{\prime \prime}-d_{\Delta_{B}(M)}^{\prime}$ is annihilated by the composition with the adjunction morphism. In view of the adjunction, it follows that $d_{\Delta_{B}(M)}^{\prime \prime}-d_{\Delta_{B}(M)}^{\prime}=0$.

Now let $\left(Q, d_{Q}\right)$ be a left CDG-contramodule over $(B, d, h)$. Then the group of all graded left $B$-contramodule morphisms $\Delta_{B}(M) \longrightarrow Q[n]$ is naturally isomorphic to the group of all graded left $B$-module morphisms $M \longrightarrow Q[n]$ for every $n \in \mathbb{Z}$. Hence we obtain an isomorphism between the underlying graded abelian groups of the complexes $\operatorname{Hom}^{B}\left(\Delta_{B}(M), Q\right)$ and $\operatorname{Hom}_{B}(M, Q)$. Since the adjunction map $M \longrightarrow$ $\Delta_{B}(M)$ commutes with the differentials, it follows that the $\operatorname{Hom}^{B}\left(\Delta_{B}(M), Q\right) \simeq$ $\operatorname{Hom}_{B}(M, Q)$ is an isomorphism of complexes of abelian groups. This establishes the DG-adjunction, which implies the DG-functoriality.

It remains to prove existence of the desired CDG-contramodule structure on $\Delta_{B}(M)$. For this purpose, consider the quasi-differential ring $(\widehat{B}, \partial)$ assigned to the CDG-ring $(B, d, h)$ by the construction of Theorem 4.5. The key observation is that the functors $\Delta_{\widehat{B}}$ and $\Delta_{B}$ agree with each other, that is, they form a commutative square diagram with the functors of restriction of scalars $\widehat{B}-\bmod _{\mathrm{gr}} \longrightarrow B-\bmod _{\mathrm{gr}}$ and $\widehat{B}$ - contra $_{\mathrm{gr}} \longrightarrow B$-contragr. The latter two functors, essentially, assign to a CDG-(contra)module over $(B, d, h)$ its underlying graded $B$-(contra)module.

Since both the functors $\Delta_{\widehat{B}}$ and $\Delta_{B}$ are right exact (while the functors of restriction of scalars are exact), it suffices to check that the two functors agree on free graded $\widehat{B}$-modules. It is helpful to observe that the restriction of scalars $\widehat{B}-\bmod _{\mathrm{gr}} \longrightarrow$ 
$B$ - od $_{\mathrm{gr}}$ takes free graded $\widehat{B}$-modules to free graded $B$-modules. The rest is a straightforward computation based on Lemma 7.4.

Finally, given a CDG-module $\left(M, d_{M}\right)$ over $(B, d, h)$, in order to produce the induced differential $d_{\Delta_{B}(M)}$ on the graded $B$-contramodule $\Delta_{B}(M)$, one simply applies the functor $\Delta_{\widehat{B}}$ to the graded $\widehat{B}$-module $M$.

Now we consider the composition of the pair of adjoint DG-functors $\operatorname{Hom}_{B}\left(K^{\vee}(B\right.$, $\widetilde{A}),-): \operatorname{DG}(B-\bmod ) \longrightarrow \operatorname{DG}(\widetilde{A}-\bmod )$ and $K^{\vee}(B, \widetilde{A}) \otimes_{\widetilde{A}}-: \operatorname{DG}(\widetilde{A}-\bmod ) \longrightarrow$ $\mathrm{DG}(B$-mod) from the above discussion with the pair of adjoint DG-functors $\mathrm{DG}(B$-contra $) \longrightarrow \mathrm{DG}(B$-mod $)$ and $\mathrm{DG}(B$-mod $) \longrightarrow \mathrm{DG}(B$-contra $)$ provided by Lemma 7.5. This produces a pair of adjoint DG-functors between the DG-category $\mathrm{DG}(\widetilde{A}$-mod $)$ of complexes of left $\widetilde{A}$-modules and the DG-category $\mathrm{DG}(B$-contra $)$ of left CDG-contramodules over $(B, d, h)$.

Here the right adjoint DG-functor

$$
\operatorname{Hom}_{B}\left(K^{\vee}(B, \widetilde{A}),-\right): \operatorname{DG}(B-\text { contra }) \longrightarrow \mathrm{DG}(\widetilde{A}-\text { mod })
$$

is the composition $\mathrm{DG}(B$-contra $) \longrightarrow \mathrm{DG}(B-\bmod ) \longrightarrow \mathrm{DG}(\widetilde{A}-$ mod $)$ of the forgetful DG-functor $\mathrm{DG}(B$-contra $) \longrightarrow \mathrm{DG}(B$-mod $)$ with the functor $\operatorname{Hom}_{B}\left(K^{\vee}(B, \widetilde{A}),-\right)$ : $\mathrm{DG}(B-\bmod ) \longrightarrow \mathrm{DG}(\widetilde{A}-$ mod $)$. We denote it by

$$
Q \longmapsto \operatorname{Hom}_{B}\left(K^{\vee}(B, \widetilde{A}), Q\right)=\operatorname{Hom}_{R}^{\tau^{\prime}}(\widetilde{A}, Q),
$$

where $\tau^{\prime}$ is the same placeholder as in Section 6.5.

The left adjoint DG-functor

$$
K^{\vee}(B, \widetilde{A}) \otimes_{\widetilde{A}}^{\Pi}-: \mathrm{DG}(\widetilde{A}-\bmod ) \longrightarrow \mathrm{DG}(B \text {-contra })
$$

is the composition $\mathrm{DG}(\widetilde{A}-\mathrm{mod}) \longrightarrow \mathrm{DG}(B-$ mod $) \longrightarrow \mathrm{DG}(B$-contra $)$ of the DG-functor $K^{\vee}(B, \widetilde{A}) \otimes_{\widetilde{A}}-: \mathrm{DG}(\widetilde{A}$-mod $) \longrightarrow \mathrm{DG}(B$-mod $)$ with the DG-functor $\Delta_{B}: \mathrm{DG}(B$-mod $) \longrightarrow \mathrm{DG}(B$-contra). This DG-functor takes a complex of left $\widetilde{A}$-modules $P=P^{\bullet}$ to the left CDG-contramodule over $(B, d, h)$ whose underlying graded left $B$-contramodule is

$$
K^{\vee}(B, \widetilde{A}) \otimes_{\widetilde{A}}^{\Pi} P=B \otimes_{R}^{\Pi} P=\operatorname{Hom}_{R}\left(\operatorname{Hom}_{R^{\mathrm{op}}}(B, R), P\right)=\operatorname{Hom}_{R}(C, P),
$$

where $C$ is a notation for the graded $R$-B-bimodule $C=\operatorname{Hom}_{R^{\text {op }}}(B, R)$, as in $\operatorname{Sec}$ tion 6.5. We denote this CDG-contramodule over $(B, d, h)$ by

$$
K^{\vee}(B, \widetilde{A}) \otimes_{\widetilde{A}}^{\Pi} P^{\bullet}=\operatorname{Hom}_{R}^{\sigma^{\prime}}\left(\operatorname{Hom}_{R^{\mathrm{op}}}(B, R), P^{\bullet}\right)=\operatorname{Hom}_{R}^{\sigma^{\prime}}\left(C, P^{\bullet}\right),
$$

where $\sigma^{\prime}$ is also the same placeholder as in Section 6.5 (see Section 8.7 below for a further discussion of these placeholders).

Here the computation of $\Delta_{B}\left(B \otimes_{R} P\right)$ as $B \otimes_{R}^{\Pi} P$ is provided by Lemma 7.4. It is only the differential on $K^{\vee}(B, \widetilde{A}) \otimes_{\widetilde{A}} P^{\bullet}=\Delta_{B}\left(K^{\vee}(B, \widetilde{A}) \otimes_{\widetilde{A}} P^{\bullet}\right)$ that remains to be explained. Lemma 7.5 establishes existence of a unique odd contraderivation of degree 1 on $B \otimes_{R}^{\Pi} P=K^{\vee}(B, \widetilde{A}) \otimes_{\widetilde{A}}^{\Pi} P$ compatible with the derivation $d$ on $B$ and agreeing with the differential on $K^{\vee}(B, \widetilde{A}) \otimes_{\widetilde{A}} P^{\bullet}$. Now it is straightforward to check 
that the differential $d_{e^{\prime}} \otimes^{\Pi} \mathrm{id}_{P}+\mathrm{id}_{K^{\vee}(B, \widetilde{A})} \otimes^{\Pi} d_{P} \bullet$ on $K^{\vee}(B, \widetilde{A}) \otimes_{\widetilde{A}}^{\Pi} P^{\bullet}$ satisfies these conditions. Hence we have

$$
d_{\Delta_{B}\left(K^{\vee}(B, \widetilde{A}) \otimes_{\widetilde{A}} P^{\bullet}\right)}=d_{e^{\prime}} \otimes^{\Pi} \mathrm{id}_{P}+\mathrm{id}_{K^{\vee}(B, \widetilde{A})} \otimes^{\Pi} d_{P},
$$

as one would expect (where $d_{e^{\prime}}$ is the differential on the dual nonhomogeneous Koszul CDG-module $K^{\vee}(B, \widetilde{A})$ and $d_{P}$ denotes the differential on the complex $\left.P^{\bullet}\right)$. The placeholder $\sigma^{\prime}$ is supposed to remind us of the first summand in this formula.

The pair of adjoint DG-functors that we have constructed induces a pair of adjoint triangulated functors

$$
\operatorname{Hom}_{B}\left(K^{\vee}(B, \widetilde{A}),-\right)=\operatorname{Hom}_{R}^{\tau^{\prime}}(\widetilde{A},-): \operatorname{Hot}(B-\text { contra }) \longrightarrow \operatorname{Hot}(\widetilde{A}-\bmod )
$$

and

$$
K^{\vee}(B, \widetilde{A}) \otimes \widetilde{A}_{\widetilde{A}}^{\Pi}-=\operatorname{Hom}_{R}^{\sigma^{\prime}}(C,-): \operatorname{Hot}(\widetilde{A}-\bmod ) \longrightarrow \operatorname{Hot}(B-\text { contra })
$$

between the homotopy category $\operatorname{Hot}(\widetilde{A}$-mod) of complexes of left $\widetilde{A}$-modules and the homotopy category Hot $(B$-contra) of left CDG-contramodules over $(B, d, h)$.

7.5. Triangulated equivalence. Let $R \subset \widetilde{V} \subset \widetilde{A}$ be a left finitely projective nonhomogeneous Koszul ring, and let $(B, d, h)$ be the corresponding right finitely projective Koszul CDG-ring (see Sections 3.4 and 4.6 4.7). The following theorem is the main result of Section 7 .

Theorem 7.6. The pair of adjoint triangulated functors $\operatorname{Hom}_{R}^{\sigma^{\prime}}(C,-): \operatorname{Hot}(\widetilde{A}-\bmod )$ $\longrightarrow \operatorname{Hot}(B$-contra $)$ and $\operatorname{Hom}_{R}^{\tau^{\prime}}(\widetilde{A},-): \operatorname{Hot}(B$-contra $) \longrightarrow \operatorname{Hot}(\widetilde{A}$-mod $)$ defined in Section 7.4 induces a pair of adjoint triangulated functors between the $\widetilde{A} / R$-semicontraderived category of left $\widetilde{A}$-modules $\mathrm{D}_{R}^{\text {sictr }}(\widetilde{A}-\bmod )$, as defined in Section $[7.1$, and the contraderived category $\mathrm{D}^{\mathrm{ctr}}(B$-contra) of left $C D G$-contramodules over $(B, d, h)$, as defined in Section 7.3. Under the above Koszulity assumption, the latter two funtors are mutually inverse triangulated equivalences,

$$
\mathrm{D}_{R}^{\text {sictr }}(\widetilde{A}-\mathrm{mod}) \simeq \mathrm{D}^{\text {ctr }}(B-\text { contra }) .
$$

Proof. This is a generalization of [28, Theorem B.2(b)] and a particular case of [27, Theorem 11.8(c)]. The proof is similar to the proofs of these results, and dualanalogous to the proof of Theorem 6.7.

Recall from the discussion in Section 5.6 that any graded left $B$-contramodule $Q$ is endowed with a canonical decreasing filtration by graded $B$-subcontramodules $Q=G^{0} Q \supset G^{1} Q \supset G^{2} Q \supset \cdots$, where $G^{m} Q \subset Q$ is the image of the restriction

$$
B^{\geq m} \otimes_{R}^{\Pi} Q \stackrel{\pi_{Q}}{\longrightarrow} Q
$$

of the contraaction map $\pi_{Q}: B \otimes_{R}^{\Pi} Q \longrightarrow Q$ to the graded submodule $B^{\geq m} \otimes_{R}^{\Pi} Q \subset$ $B \otimes_{R}^{\Pi} Q$. Here, as usually, the notation $B^{\geq m}$ stands for the homogeneous twosided ideal $\bigoplus_{n \geq m} B^{n} \subset B$. The canonical decreasing filtration $G$ on a graded $B$-contramodule $Q$ is always complete, but it does not have to be separated; see Proposition 5.24 and Example 5.25, The successive quotient contramodules 
$G^{m} Q / G^{m+1} Q$ have trivial graded $B$-contramodule structures, in the sense explained in Section 5.6.

A left CDG-contramodule $\left(Q, d_{Q}\right)$ over $(B, d, h)$ is said to be trivial if its underlying graded $B$-contramodule is trivial. The square of the differential $d_{Q}$ on a trivial CDG-contramodule $Q$ is zero, as the curvature element $h \in B^{2}$ acts by zero in $Q$. The DG-category of trivial left CDG-contramodules over $(B, d, h)$ is equivalent to the DG-category of complexes of left $R$-modules. For any left CDG-contramodule $\left(Q, d_{Q}\right)$ over $(B, d, h)$, the canonical decreasing filtration $G$ of the underlying graded $B$-contramodule of $Q$ is a filtration by CDG-subcontramodules with trivial CDG-contramodule successive quotients.

The ring $\widetilde{A}$ is endowed with an increasing filtration $F$, which was defined in Section 3.1. We also endow the graded $R$-B-bimodule $C=\operatorname{Hom}_{R^{\text {op }}}(B, R)$ with the increasing filtration $F$ induced by the grading, as in the proof of Theorem 6.7. It is important for the argument below that both $F_{n} \widetilde{A} / F_{n-1} \widetilde{A}$ and $F_{n} C / F_{n-1} C$ are projective (graded) left $R$-modules for all $n \geq 0$ (in fact, they are finitely generated projective left $R$-modules in our assumptions). In particular, $\widetilde{A}$ is a projective left $R$-module and $C$ is a projective graded left $R$-module. Hence both the functors $\operatorname{Hom}_{R}^{\tau^{\prime}}(\widetilde{A},-)$ and $\operatorname{Hom}_{R}^{\sigma^{\prime}}(C,-)$ preserve short exact sequences (of left CDG-contramodules over $(B, d, h)$ and of complexes of left $\widetilde{A}$-modules, respectively). Both the functors also obviously preserve infinite products.

Let $P^{\bullet}$ be a complex of left $\widetilde{A}$-modules that is contraacyclic as a complex of left $R$-modules. We need to show that the left CDG-contramodule $\operatorname{Hom}_{R}^{\sigma^{\prime}}\left(C, P^{\bullet}\right)$ over $(B, d, h)$ is contraacyclic. Let $F$ be the decreasing filtration on the Hom module $\operatorname{Hom}_{R}(C, P)$ induced by the increasing filtration $F$ on $C$, that is $F^{n} \operatorname{Hom}_{R}(C, P)=$ $\operatorname{Hom}_{R}\left(C / F_{n-1} C, P\right) \subset \operatorname{Hom}_{R}(C, P)$. Then $F$ is a complete, separated filtration of the CDG-contramodule $\operatorname{Hom}_{R}^{\sigma^{\prime}}\left(C, P^{\bullet}\right)$ by CDG-subcontramodules over $(B, d, h)$. The successive quotient CDG-contramodules $F^{n} \operatorname{Hom}_{R}^{\sigma^{\prime}}\left(C, P^{\bullet}\right) / F^{n+1} \operatorname{Hom}_{R}^{\sigma^{\prime}}\left(C, P^{\bullet}\right)$ are trivial. Viewed as complexes of left $R$-modules, these are the Hom complexes $\operatorname{Hom}_{R}\left(F_{n} C / F_{n-1} C, P^{\bullet}\right)$, where $F_{n} C / F_{n-1} C$ is a graded $R$ - $R$-bimodule concentrated in the cohomological degree $-n$ and endowed with the zero differential.

Since the graded left $R$-module $F_{n} C / F_{n-1} C$ is projective, the functor $\operatorname{Hom}_{R}\left(F_{n} C /\right.$ $\left.F_{n-1} C,-\right)$ takes contraacyclic complexes of left $R$-modules to contraacyclic complexes of left $R$-modules. Clearly, any contraacyclic complex of left $R$-modules is also contraacyclic as a CDG-contramodule over $(B, d, h)$ with the trivial contramodule structure. It remains to use Lemma 7.3 in order to conclude that $\operatorname{Hom}_{R}^{\sigma^{\prime}}\left(C, P^{\bullet}\right)$ is a contraacyclic left CDG-contramodule over $(B, d, h)$.

For any contraacyclic left CDG-contramodule $Q$ over $(B, d, h)$, the complex of left $\widetilde{A}$-modules $\operatorname{Hom}_{R}^{\tau^{\prime}}(\widetilde{A}, Q)$ is contraacyclic not only as a complex of left $R$-modules, but even as a complex of left $\widetilde{A}$-modules. This follows immediately from the fact that $\widetilde{A}$ is a projective left $R$-module; so the functor $\operatorname{Hom}_{R}^{\tau^{\prime}}(\widetilde{A},-)$ takes short exact sequences of left CDG-contramodules over $(B, d, h)$ to short exact sequences of complexes of left $\widetilde{A}$-modules (and this functor also preserves infinite products). 
Let $P^{\bullet}$ be an arbitrary complex of left $\widetilde{A}$-modules. We need to show that the cone $Y^{\bullet}$ of the adjunction morphism of complexes of left $\widetilde{A}$-modules $P^{\bullet} \longrightarrow$ $\operatorname{Hom}_{R}^{\tau^{\prime}}\left(\widetilde{A}, \operatorname{Hom}_{R}^{\sigma^{\prime}}\left(C, P^{\bullet}\right)\right)$ is contraacyclic as a complex of left $R$-modules. Endow the graded left $R$-module $\operatorname{Hom}_{R}\left(\widetilde{A}, \operatorname{Hom}_{R}(C, P)\right)$ with the decreasing filtration $F$ induced by the increasing filtrations $F$ on $C$ and $\widetilde{A}$, that is $F^{n} \operatorname{Hom}_{R}\left(\widetilde{A}, \operatorname{Hom}_{R}(C, P)\right)=$ $\sum_{i+j=n-1} \operatorname{Hom}_{R}\left(\widetilde{A} / F_{j} \widetilde{A}, \operatorname{Hom}_{R}\left(C / F_{i} C, P\right)\right) \subset \operatorname{Hom}_{R}\left(\widetilde{A}, \operatorname{Hom}_{R}(C, P)\right)$. Then $F$ is a complete, separated filtration of the complex $\operatorname{Hom}_{R}^{\tau^{\prime}}\left(\widetilde{A}, \operatorname{Hom}_{R}^{\sigma^{\prime}}\left(C, P^{\bullet}\right)\right)$ by subcomplexes of left $R$-modules (but not of left $\widetilde{A}$-modules). The complex $P^{\bullet}$ is endowed with the trivial filtration $F^{0} P^{\bullet}=P^{\bullet}, F^{1} P^{\bullet}=0$; and the complex $Y^{\bullet}$ is endowed with the induced decreasing filtration $F$.

Then the associated graded complex of left $R$-modules

$$
\begin{aligned}
\operatorname{gr}_{F} \operatorname{Hom}_{R}^{\tau^{\prime}}\left(\widetilde{A}, \operatorname{Hom}_{R}^{\sigma^{\prime}}\left(C, P^{\bullet}\right)\right) \\
\quad=\prod_{n=0}^{\infty} F^{n} \operatorname{Hom}_{R}^{\tau^{\prime}}\left(\widetilde{A}, \operatorname{Hom}_{R}^{\sigma^{\prime}}\left(C, P^{\bullet}\right)\right) / F^{n+1} \operatorname{Hom}_{R}^{\tau^{\prime}}\left(\widetilde{A}, \operatorname{Hom}_{R}^{\sigma^{\prime}}\left(C, P^{\bullet}\right)\right)
\end{aligned}
$$

is naturally isomorphic to the Hom complex $\operatorname{Hom}_{R}\left({ }^{\tau} K_{\bullet}(B, A), P^{\bullet}\right)$ from the complex of (graded) $R$-R-bimodules ${ }^{\tau} K_{\bullet}(B, A)=C \otimes_{R}^{\tau} A$ constructed in Section 2.6 into the complex of left $R$-modules $P^{\bullet}$. Here $A=\operatorname{gr}^{F} \widetilde{A}$ is the left finitely projective Koszul graded ring associated with $\widetilde{A}$. Arguing further as in the proof of Theorem 6.7, we conclude that $\operatorname{gr}_{F} Y^{\bullet}=\operatorname{Hom}_{R}\left(\operatorname{cone}\left({ }^{\tau} K \bullet(B, A) \rightarrow R\right)[-1], P^{\bullet}\right)$ is a contraacyclic complex of left $R$-modules. By Lemma 7.1 , it follows that $Y^{\bullet}$ is a contraacyclic complex of left $R$-modules, too.

Let $Q=\left(Q, d_{Q}\right)$ be an arbitrary left CDG-contramodule over $(B, d, h)$. We need to show that the cone $Z$ of the adjunction morphism of left CDG-contramodules $\operatorname{Hom}_{R}^{\sigma^{\prime}}\left(C, \operatorname{Hom}_{R}^{\tau^{\prime}}(\widetilde{A}, Q)\right) \longrightarrow Q$ is contraacyclic as a CDG-contramodule over $(B, d, h)$.

First of all we observe that the class of all left CDG-contramodules $Q$ having the desired property is preserved by the passages to the cokernels of injective closed morphisms of CDG-contramodules (as well as to the kernels of surjective closed morphisms; but we need the cokernels). This holds because the class of all contraacyclic left CDG-contramodules over $(B, d, h)$ is closed under the cokernels of injective closed morphisms (as well as the kernels of surjective closed morphisms).

Consider our CDG-contramodule $Q$ as a graded left contramodule over the graded ring $\widehat{B}$ from Theorem 4.5, and present it as a quotient of a free graded $\widehat{B}$-contramodule. All free graded contramodules are separated, all subcontramodules of separated graded contramodules are separated, and the restriction of scalars takes separated graded $\widehat{B}$-contramodules to separated graded $B$-contramodules (besides, it takes free graded $\widehat{B}$-contramodules to free graded $B$-contramodules). So any subcontramodule of a free graded $\widehat{B}$-contramodule is separated as a graded $B$-contramodule. This allows to present an arbitrary CDG-contramodule $Q$ over $(B, d, h)$ as the cokernel of an injective morphism of CDG-contramodules whose underlying graded $B$-contramodules are separated. Thus we can assume that $Q$ is a separated graded $B$-contramodule. 
Now we endow $Q$ with the canonical decreasing filtration $Q=G^{0} Q \supset G^{1} Q \supset$ $G^{2} Q \supset \cdots$; so, as mentioned above, $G^{m} Q$ are CDG-subcontramodules in $Q$ and $G^{m} Q / G^{m+1} Q$ are trivial CDG-contramodules over $(B, d, h)$. The filtration $G$ is separated and complete. Consider the induced decreasing filtrations $G$ on the left CDG-contramodule $\operatorname{Hom}_{R}^{\sigma^{\prime}}\left(C, \operatorname{Hom}_{R}^{\tau^{\prime}}(\widetilde{A}, Q)\right)$ and the left CDG-contramodule $Z$ over $(B, d, h)$. In particular, $G^{m} \operatorname{Hom}_{R}^{\sigma^{\prime}}\left(C, \operatorname{Hom}_{R}^{\tau^{\prime}}(\widetilde{A}, Q)\right)=\operatorname{Hom}_{R}^{\sigma^{\prime}}\left(C, \operatorname{Hom}_{R}^{\tau^{\prime}}\left(\widetilde{A}, G^{m} Q\right)\right)$. These decreasing filtrations are also separated and complete. The successive quotient CDG-contramodules $\operatorname{Hom}_{R}^{\sigma^{\prime}}\left(C, \operatorname{Hom}_{R}^{\tau^{\prime}}\left(\widetilde{A}, G^{m} Q\right)\right) / \operatorname{Hom}_{R}^{\sigma^{\prime}}\left(C, \operatorname{Hom}_{R}^{\tau^{\prime}}\left(\widetilde{A}, G^{m+1} Q\right)\right)$ are computable as the CDG-contramodules $\operatorname{Hom}_{R}^{\sigma^{\prime}}\left(C, \operatorname{Hom}_{R}^{\tau^{\prime}}\left(\widetilde{A}, G^{m} Q / G^{m+1} Q\right)\right)$, and similarly for the CDG-contramodules $G^{m} Z / G^{m+1} Z$. Arguing as in the proof of Theorem 6.7 and using Lemma 7.3, we reduce the question to the case of a trivial left CDG-contramodule $Q$ over $(B, d, h)$. So we can assume that $Q=Q^{\bullet}$ is simply a complex of left $R$-modules endowed with the trivial graded $B$-contramodule structure.

Next we endow the graded left $R$-module $\operatorname{Hom}_{R}\left(C, \operatorname{Hom}_{R}(\widetilde{A}, Q)\right)$ with the decreasing filtration $F$ induced by the increasing filtrations $F$ on $\widetilde{A}$ and $C$, that is $F^{n} \operatorname{Hom}_{R}\left(C, \operatorname{Hom}_{R}(\widetilde{A}, Q)\right)=\sum_{i+j=n-1} \operatorname{Hom}_{R}\left(C / F_{i} C, \operatorname{Hom}_{R}\left(\widetilde{A} / F_{j} \widetilde{A}, Q\right)\right) \subset$ $\operatorname{Hom}_{R}\left(C, \operatorname{Hom}_{R}(\widetilde{A}, Q)\right)$. Then $F$ is a complete, separated filtration of the CDG-contramodule $\operatorname{Hom}_{R}^{\sigma^{\prime}}\left(C, \operatorname{Hom}_{R}^{\tau^{\prime}}\left(\widetilde{A}, Q^{\bullet}\right)\right)$ by CDG-subcontramodules with trivial CDG-contramodule successive quotients. As a complex of left $R$-modules, the trivial CDG-contramodule $\operatorname{gr}_{F} \operatorname{Hom}_{R}^{\sigma^{\prime}}\left(C, \operatorname{Hom}_{R}^{\tau^{\prime}}\left(\widetilde{A}, Q^{\bullet}\right)\right)$ is naturally isomorphic to the Hom complex $\operatorname{Hom}_{R}\left(K_{\bullet}^{\tau}(B, A), Q^{\bullet}\right)$ from the complex of (graded) $R$ - $R$-bimodules $K_{\bullet}^{\tau}(B, A)=A \otimes_{R}^{\sigma} C$ constructed in Section 2.5 into the complex of left $R$-modules $Q^{\bullet}$. Continuing to argue as in the proof of Theorem 6.7. we conclude that $\operatorname{gr}_{F} Z=$ $\prod_{n=0}^{\infty} F^{n} Z / F^{n+1} Z \simeq \operatorname{Hom}_{R}\left(\operatorname{cone}\left(R \rightarrow K_{\bullet}^{\tau}(B, A)\right)[-1], Q^{\bullet}\right)$ is a contraacyclic complex of left $R$-modules. Here it is important that the complex cone $\left(R \rightarrow K_{\bullet}^{\tau}(B, A)\right)$ is "coacyclic with respect to the exact category of left $R$-projective $R$ - $R$-bimodules" in the sense of [27, Section 2.1]. Hence $\operatorname{gr}_{F} Z$ is also contraacyclic as a left CDG-contramodule over $(B, d, h)$. By Lemma 7.3 , it follows that $Z$ is a contraacyclic CDG-contramodule over $(B, d, h)$ as well.

Examples 7.7. (1) The free left $R$-module $R$ can be considered as a one-term complex of left $R$-modules, concentrated in the cohomological degree 0 and endowed with the zero differential. This one-term complex of left $R$-modules can be then considered as a trivial left CDG-contramodule over $(B, d, h)$. The functor $\operatorname{Hom}_{R}^{\tau^{\prime}}(\widetilde{A},-)=\operatorname{Hom}_{B}\left(K_{\widetilde{e}^{\prime}}^{\vee}(B, \widetilde{A}),-\right)$ takes this trivial left CDG-contramodule $R$ over $(B, d, h)$ to the left $\widetilde{A}$-module $\operatorname{Hom}_{R}(\widetilde{A}, R)$ (viewed as a one-term complex concentrated in the cohomological degree 0$)$.

(2) Assume that $\widetilde{A}$ is a left augmented left finitely projective nonhomogeneous Koszul ring, in the sense of the definitions in Sections 3.8 and 4.7. Then the left augmentation of $\widetilde{A}$ endows the base ring $R$ with a structure of left $\widetilde{A}$-module. 
Furthermore, by Theorem 3.14 we have $h=0$, so $(B, d, h)=(B, d)$ is a DG-ring. As such, $(B, d)$ is naturally a left DG-module over itself. Being nonnegatively cohomologically graded, this DG-module has a unique structure of DG-contramodule over $(B, d)$ compatible with its DG-module structure (see Example [5.22(1)). (Here, by definition, a $D G$-contramodule over a nonnegatively graded DG-ring $(B, d)$ is the same thing as a CDG-contramodule over $(B, d, 0)$.)

In this setting, the functor $\operatorname{Hom}_{R}^{\sigma^{\prime}}(C,-)=K_{e^{\prime}}^{\vee}(B, \widetilde{A}) \otimes \otimes_{\widetilde{A}}^{\Pi}-$ takes the left $\widetilde{A}$-module $R$ to the left DG-contramodule $(B, d)$ over the DG-ring $(B, d)$.

Remark 7.8. Assume that $\widetilde{A}$ is left augmented over $R$, as in Remark 6.9 (2) and Example $7.7(2)$. Then, for any complex of left $\widetilde{A}$-modules $P^{\bullet}$, the CDG-(contra)module $\operatorname{Hom}_{R}^{\sigma^{\prime}}\left(C, P^{\bullet}\right)$ is a DG-module over $(B, d, h)=(B, h)$, i. e., a complex. We have a natural isomorphism of complexes of abelian groups $\operatorname{Hom}_{R}^{\sigma^{\prime}}\left(C, P^{\bullet}\right) \simeq \operatorname{Hom}_{\widetilde{A}}\left(\widetilde{A} \otimes_{R}^{\sigma^{\prime}} C, P^{\bullet}\right)$. Since $\widetilde{A} \otimes_{R}^{\sigma^{\prime}} C$ is a projective resolution of the left $\widetilde{A}$-module $R$ by Example $6.8(5)$, it follows that the complex of abelian groups $\operatorname{Hom}_{R}^{\sigma^{\prime}}\left(C, P^{\bullet}\right)$ computes $\mathbb{R} \operatorname{Hom}_{\widetilde{A}}\left(R, P^{\bullet}\right)$. In fact, the DG-module $\operatorname{Hom}_{R}^{\sigma^{\prime}}\left(C, P^{\bullet}\right)$ computes the cohomology $\operatorname{Ext}_{\widetilde{A}}^{i}\left(R, P^{\bullet}\right)=$ $\operatorname{Hom}_{\mathrm{D}(\widetilde{A}-\text { mod })}\left(R, P^{\bullet}[i]\right), \quad i \in \mathbb{Z}$, as a graded module over the graded ring of endomorphisms of the derived category object $R \in \mathrm{D}(\widetilde{A}-\bmod )$.

7.6. Reduced contraderived category. We keep the assumptions of Section 7.5 (which coincide with the assumptions of Sections 6.6 6.7). The following corollary is a special case of Theorem [7.6.

Corollary 7.9. Assume additionally that the left homological dimension of the ring $R$ (that is, the homological dimension of the abelian category $R$-mod) is finite. Then the pair of adjoint triangulated functors $\operatorname{Hom}_{R}^{\sigma^{\prime}}(C,-): \operatorname{Hot}(\widetilde{A}-\bmod ) \longrightarrow \operatorname{Hot}(B$-contra $)$ and $\operatorname{Hom}_{R}^{\tau^{\prime}}(\widetilde{A},-): \operatorname{Hot}(B-$ contra $) \longrightarrow \operatorname{Hot}(\widetilde{A}-\bmod )$ induces mutually inverse triangulated equivalences

$$
\mathrm{D}(\widetilde{A}-\mathrm{mod}) \simeq \mathrm{D}^{\mathrm{ctr}}(B-\text { contra })
$$

between the derived category of left $\widetilde{A}$-modules and the contraderived category of left $C D G$-contramodules over $(B, d, h)$.

Proof. When the left homological dimension of the $\operatorname{ring} R$ is finite, all acyclic complexes of left $R$-modules are contraacyclic by [27, Remark 2.1]. So the $\widetilde{A} / R$-semicontraderived category of left $\widetilde{A}$-modules coincides with their derived category, $\mathrm{D}_{R}^{\text {sictr }}(\widetilde{A}-$ mod $)=\mathrm{D}(\widetilde{A}-$ mod $)$, and the assertion follows from Theorem [7.6.

We would like to obtain a description of the derived category $\mathrm{D}(\widetilde{A}-$ mod $)$ in terms of some kind of exotic derived category of left CDG-contramodules over $(B, d, h)$ without assuming finiteness of the left homological dimension of $R$.

For this purpose, let us consider the full subcategory of acyclic complexes of left $R$-modules $\operatorname{Acycl}(R$-mod $) \subset \operatorname{Hot}(R$-mod). All complexes of left $R$-modules, and in particular acyclic complexes of left $R$-modules, can be viewed as trivial left CDG-contramodules over $(B, d, h)$. 
By an abuse of notation, let us denote simply by $\langle\operatorname{Acycl}(R-\bmod )\rangle_{\sqcap}$ the minimal full triangulated subcategory in $\mathrm{D}^{\mathrm{ctr}}(B$-contra) containing all the acyclic complexes of left $R$-modules (viewed as trivial CDG-contramodules) and closed under infinite products. Consider the triangulated Verdier quotient category

$$
\mathrm{D}_{R \text {-red }}^{\mathrm{ctr}}(B \text {-contra })=\mathrm{D}^{\mathrm{ctr}}(B-\operatorname{contra}) /\langle\operatorname{Acycl}(R-\mathrm{mod})\rangle_{\sqcap} ;
$$

let us call it the reduced contraderived category of left CDG-contramodules over $(B, d, h)$ relative to $R$.

Theorem 7.10. The pair of adjoint triangulated functors $\operatorname{Hom}_{R}^{\sigma^{\prime}}(C,-): \operatorname{Hot}(\widetilde{A}-\bmod )$ $\longrightarrow \operatorname{Hot}(B$-contra $)$ and $\operatorname{Hom}_{R}^{\tau^{\prime}}(\widetilde{A},-): \operatorname{Hot}(B-$ contra $) \longrightarrow \operatorname{Hot}(\widetilde{A}-\bmod )$ induces mutually inverse triangulated equivalences

$$
\mathrm{D}(\widetilde{A}-\text { mod }) \simeq \mathrm{D}_{R \text {-red }}^{\mathrm{ctr}}(B \text {-contra })
$$

between the derived category of left $\widetilde{A}$-modules and the reduced contraderived category of left $C D G$-contramodules over $(B, d, h)$ relative to $R$.

Proof. This is the dual-analogous assertion to Theorem 6.11. In view of Theorem [7.6, only two things still need to be checked. Firstly, one has to show that, for any acyclic complex of left $\widetilde{A}$-modules $P^{\bullet}$, the CDG-contramodule $\operatorname{Hom}_{R}^{\sigma^{\prime}}\left(C, P^{\bullet}\right)$ over $(B, d, h)$ belongs to the triangulated subcategory $\langle\operatorname{Acycl}(R \text {-mod })\rangle_{\sqcap} \subset \mathrm{D}^{\text {ctr }}(B$-contra $)$. Secondly, it needs to be established that, for any CDG-contramodule $Q \in$ $\langle\operatorname{Acycl}(R-\bmod )\rangle_{\sqcap}$, the complex of left $\widetilde{A}$-modules $\operatorname{Hom}_{R}^{\tau^{\prime}}(\widetilde{A}, Q)$ is acyclic.

Firstly, let $P=P^{\bullet}$ be an acyclic complex of left $\widetilde{A}$-modules. Consider the decreasing filtration $F$ on the graded left $R$-module $\operatorname{Hom}_{R}(C, P)$ induced by the increasing filtration $F$ on the $R$-R-bimodule $C$, that is $F^{n} \operatorname{Hom}_{R}(C, P)=$ $\operatorname{Hom}_{R}\left(C / F_{n-1} C, P\right)$ for all $n \geq 0$. Then $F$ is a complete, separated filtration of the CDG-contramodule $\operatorname{Hom}_{R}^{\sigma^{\prime}}\left(C, P^{\bullet}\right)$ over $(B, d, h)$ by its CDG-subcontramodules. The successive quotient CDG-contramodules $F^{n} \operatorname{Hom}_{R}^{\sigma^{\prime}}(C, P) / F^{n+1} \operatorname{Hom}_{R}^{\sigma^{\prime}}(C, P)$ are trivial CDG-contramodules which, viewed as complexes of left $R$-modules, can be computed as the Hom complexes $\operatorname{Hom}_{R}\left(F_{n} C / F_{n-1} C, P^{\bullet}\right)$.

As in the proof of Theorem 6.11, one can see that the complexes of left $R$-modules $\operatorname{Hom}_{R}\left(F_{n} C / F_{n-1} C, P^{\bullet}\right)$ are acyclic. Now the iterated extension and telescope sequence argument from the proofs of Lemmas 7.1 and 7.3 shows that the left CDG-contramodule $\operatorname{Hom}_{R}^{\sigma^{\prime}}\left(C, P^{\bullet}\right)$ over $(B, d, h)$ belongs to the triangulated subcategory $\langle\operatorname{Acycl}(R-\bmod )\rangle_{\sqcap} \subset \mathrm{D}^{\mathrm{ctr}}(R-\bmod )$.

Secondly, since the functor $\operatorname{Hom}_{R}^{\tau^{\prime}}(\widetilde{A},-)$ is triangulated and preserves infinite products, it suffices to check that for any acyclic complex of left $R$-modules $Q^{\bullet}$, viewed as a trivial left CDG-contramodule over $(B, d, h)$, the complex of left $A$-modules $\operatorname{Hom}_{R}^{\tau^{\prime}}\left(\widetilde{A}, Q^{\bullet}\right)$ is acyclic. As $Q^{\bullet}$ is a trivial CDG-contramodule, the complex of left $\widetilde{A}$-modules $\operatorname{Hom}_{R}^{\tau^{\prime}}\left(\widetilde{A}, Q^{\bullet}\right)=\operatorname{Hom}_{R}\left(\widetilde{A}, Q^{\bullet}\right)$ is simply obtained from the complex of left $R$-modules $Q^{\bullet}$ by applying the Hom functor $\operatorname{Hom}_{R}(\widetilde{A},-)$ termwise. Since $\widetilde{A}$ is a projective left $R$-module, it follows that the complex $\operatorname{Hom}_{R}\left(\widetilde{A}, Q^{\bullet}\right)$ is acyclic. 


\section{The Co-Contra Correspondence}

8.1. Contratensor product. Let $B=\bigoplus_{n=0}^{\infty} B_{n}$ be a nonnegatively graded ring, and let $K$ be a ring endowed with a ring homomorphism $K \longrightarrow B_{0}$. We start from the case of ungraded comodules and contramodules before passing to the graded ones.

Let $N$ be an ungraded right $B$-comodule (in the sense of Section 5.1) and $P$ be an ungraded left $B$-contramodule (in the sense of Section 5.3). The contratensor product $N \odot_{B} P$ is an abelian group constructed as the cokernel of (the difference of) a natural pair of abelian group homomorphisms

$$
N \otimes_{K} \prod_{n=0}^{\infty}\left(B_{n} \otimes_{K} P\right) \rightrightarrows N \otimes_{K} P
$$

Here the first map is simply $N \otimes_{K} \pi_{P}: N \otimes_{K} \prod_{n=0}^{\infty}\left(B_{n} \otimes_{K} P\right) \longrightarrow N \otimes_{K} P$, where $\pi_{P}: \prod_{n=0}^{\infty} B_{n} \otimes_{K} P \longrightarrow P$ is the contraaction map. The second map is constructed using the right (co)action of $B$ in $N$.

Specifically, consider a pair of elements $y \in N$ and $w \in \prod_{n=0}^{\infty} B_{n} \otimes_{K} P$. Choose an integer $m \geq 0$ such that $y B_{\geq m+1}=0$ in $N$. Then the image of $y \otimes w$ under the second map $N \otimes_{K}\left(\prod_{n=0}^{\infty} B_{n} \otimes_{K} P\right) \longrightarrow N \otimes_{K} P$ is, by definition, equal to the image of $y \otimes w$ under the composition

$$
\begin{aligned}
N \otimes_{K} \prod_{n=0}^{\infty}\left(B_{n} \otimes_{K} P\right) \longrightarrow N \otimes_{K} \bigoplus_{n=0}^{m}\left(B_{n} \otimes_{K} P\right) & \\
& =\bigoplus_{n=0}^{m} N \otimes_{K} B_{n} \otimes_{K} P \longrightarrow N \otimes_{K} P .
\end{aligned}
$$

Here the map $N \otimes_{K} \prod_{n=0}^{\infty}\left(B_{n} \otimes_{K} P\right) \longrightarrow N \otimes_{K} \bigoplus_{n=0}^{m}\left(B_{n} \otimes_{K} P\right)$ is the direct summand projection, while the map $\bigoplus_{n=0}^{m} N \otimes_{K} B_{n} \otimes_{K} P \longrightarrow N \otimes_{K} P$ is induced by the right action maps $N \otimes_{K} B_{n} \longrightarrow N$.

Notice that, by construction, the contratensor product $N \odot_{B} P$ is a quotient group of the tensor product $N \otimes_{B} P$. In other words, there is a natural surjective map of abelian groups

$$
N \otimes_{B} P \longrightarrow N \odot_{B} P .
$$

One can also easily see that the contratensor product $N \odot_{B} P$ does not depend on the choice of a ring $K$ (so our notation is unambiguous, and one can take, at one's convenience, $K=\mathbb{Z}$, or $K=B_{0}$, etc.)

Lemma 8.1. Let $E$ be an associative ring and $N$ be an $E$-B-bimodule such that the underlying right $B$-module of $N$ is an (ungraded) right $B$-comodule. Then the functor

$$
\operatorname{Hom}_{E}(N,-): E-\bmod \longrightarrow B \text {-contra }
$$

constructed in Example 5.11 is right adjoint to the contratensor product functor

$$
N \odot_{B}-: B \text {-contra } \longrightarrow E-\bmod .
$$

In other words, for any ungraded left $B$-contramodule $P$ and any left E-module $U$ there is a natural isomorphism of abelian groups

$$
\operatorname{Hom}_{E}\left(N \odot_{B} P, U\right) \simeq \operatorname{Hom}^{B}\left(P, \operatorname{Hom}_{E}(N, U)\right) .
$$


Proof. The claim is that, under the adjunction isomorphism $\operatorname{Hom}_{E}\left(N \otimes_{B} P, U\right) \simeq$ $\operatorname{Hom}_{B}\left(P, \operatorname{Hom}_{E}(N, U)\right)$, the subgroup $\operatorname{Hom}_{E}\left(N \odot_{B} P, U\right) \subset \operatorname{Hom}_{E}\left(N \otimes_{B} P, U\right)$ provided by the surjective left $E$-module map (80) corresponds to the subgroup $\operatorname{Hom}^{B}\left(P, \operatorname{Hom}_{E}(N, U)\right) \subset \operatorname{Hom}_{B}\left(P, \operatorname{Hom}_{E}(N, U)\right)$ provided by the faithful forgetful functor $B$-contra $\longrightarrow B$-mod. One can see this by comparing the above construction of the contratensor product $N \odot_{B} P$ with the construction of the $B$-contramodule structure on the Hom group $\operatorname{Hom}_{E}(N, U)$ in Example 5.11.

Now let $N$ be a graded right $B$-comodule (in the sense of Section 5.5) and $P$ be a graded left $B$-contramodule (in the sense of Section 5.6). Then the contratensor product $N \odot_{B} P$ is a graded abelian group constructed as the cokernel of (the difference of) a natural pair of homomorphisms of graded abelian groups

$$
N \otimes_{K}\left(B \otimes_{K}^{\Pi} P\right) \rightrightarrows N \otimes_{K} P .
$$

Here the first map is $N \otimes_{K} \pi_{P}: N \otimes_{K}\left(B \otimes_{K}^{\Pi} P\right) \longrightarrow N \otimes_{K} P$, where $\pi_{P}: B \otimes_{K}^{\Pi} P=$ $\mathbb{M}_{K}^{\mathrm{gr}}(P) \longrightarrow P$ is the contraaction map. The second map is constructed using the right (co)action of $B$ in $N$.

Specifically, consider a pair of elements $y \in N_{i}$ and $w \in\left(B \otimes_{K}^{\Pi} P\right)_{j}=$ $\prod_{n \in \mathbb{Z}}\left(B_{n} \otimes_{K} P_{j-n}\right), \quad i, j \in \mathbb{Z}$. Choose an integer $m \geq 0$ such that $y B_{\geq m+1}=0$. Then the image of $y \otimes w$ under the second map $N \otimes_{K}\left(B \otimes_{K}^{\Pi} P\right) \longrightarrow N \otimes_{K} P$ is, by definition, equal to the image of $y \otimes w$ under the composition

$$
\begin{aligned}
N_{i} \otimes_{K} \prod_{n=0}^{\infty}\left(B_{n} \otimes_{K} P_{j-n}\right) \longrightarrow N_{i} \otimes_{K} \bigoplus_{n=0}^{m}\left(B_{n} \otimes_{K} P_{j-n}\right) \\
=\bigoplus_{n=0}^{m} N_{i} \otimes_{K} B_{n} \otimes_{K} P_{j-n} \longrightarrow\left(N \otimes_{K} P\right)_{i+j} .
\end{aligned}
$$

Here the map $N_{i} \otimes_{K} \prod_{n=0}^{\infty}\left(B_{n} \otimes_{K} P_{j-n}\right) \longrightarrow N_{i} \otimes_{K} \bigoplus_{n=0}^{m}\left(B_{n} \otimes_{K} P_{j-n}\right)$ is the direct summand projection, while the map $\bigoplus_{n=0}^{m} N_{i} \otimes_{K} B_{n} \otimes_{K} P_{j-n} \longrightarrow\left(N \otimes_{K} P\right)_{i+j}$ is induced by the right action maps $N_{i} \otimes_{K} B_{n} \longrightarrow N_{i+n}$.

By construction, the contratensor product $N \odot_{B} P$ is a homogeneous quotient group of the tensor product $N \otimes_{B} P$. In other words, there is a natural surjective map of graded abelian groups $N \otimes_{B} P \longrightarrow N \odot_{B} P$. One can also easily see that the contratensor product $N \odot_{B} P$ does not depend on the choice of a ring $K$ (so our notation is unambigous).

For any graded left $B$-contramodules $P$ and $Q$, we denote by $\operatorname{Hom}^{B}(P, Q)$ the graded $B$-contramodule Hom group from $P$ to $Q$, that is, the graded abelian group with the components

$$
\operatorname{Hom}^{B}(P, Q)_{n}=\operatorname{Hom}_{B-\text { contragr }}(P, Q(-n)),
$$

where $Q(-n)$ denotes the left $B$-contramodule $Q$ with the shifted grading, $Q(-n)_{j}=$ $Q_{j+n}$ (cf. the notation in Section 5.7).

Lemma 8.2. Let $E=\bigoplus_{n \in \mathbb{Z}} E_{n}$ be a graded associative ring and $N=\bigoplus_{n \in \mathbb{Z}} N_{n}$ be a graded $E$-B-bimodule such that the underlying graded right $B$-module of $N$ is a (graded) right $B$-comodule. Then the functor

$$
\operatorname{Hom}_{E}(N,-): E-\bmod _{149} \longrightarrow B-\text { contra }_{\mathrm{gr}}
$$


constructed in Example 5.23 is right adjoint to the contratensor product functor

$$
N \odot_{B}-: B-\text { contra }_{\mathrm{gr}} \longrightarrow E-\bmod _{\mathrm{gr}} .
$$

In other words, for any graded left B-contramodule $P$ and any graded left E-module $U$ there is a natural isomorphism of graded abelian groups

$$
\operatorname{Hom}_{E}\left(N \odot_{B} P, U\right) \simeq \operatorname{Hom}^{B}\left(P, \operatorname{Hom}_{E}(N, U)\right) .
$$

Proof. Similar to the proof of Lemma 8.1 .

Proposition 8.3. (a) Assume that the forgetful functor $B$-contra $\longrightarrow B$-mod is fully faithful. Then the natural map of abelian groups $N \otimes_{B} P \longrightarrow N \odot_{B} P$ (80) is an isomorphism for any ungraded right $B$-comodule $N$ and any ungraded left $B$-contramodule $P$.

(b) Assume that the forgetful functor $B$-contra ${ }_{\mathrm{gr}} \longrightarrow B-\bmod _{\mathrm{gr}}$ is fully faithful. Then the natural map of graded abelian groups $N \otimes_{B} P \longrightarrow N \odot_{B} P$ is an isomorphism for any graded right $B$-comodule $N$ and any graded left $B$-contramodule $P$.

Proof. We will prove part (b); part (a) is similar. Let $U$ be a graded abelian group. Then we have

$$
\begin{aligned}
& \operatorname{Hom}_{\mathbb{Z}}\left(N \otimes_{B} P, U\right) \simeq \operatorname{Hom}_{B}\left(P, \operatorname{Hom}_{\mathbb{Z}}(N, U)\right), \\
& \operatorname{Hom}_{\mathbb{Z}}\left(N \odot_{B} P, U\right) \simeq \operatorname{Hom}^{B}\left(P, \operatorname{Hom}_{\mathbb{Z}}(N, U)\right) .
\end{aligned}
$$

Applying the functor $\operatorname{Hom}_{\mathbb{Z}}(-, U)$ to the natural map $N \otimes_{B} P \longrightarrow N \odot_{B} P$ produces the map $\operatorname{Hom}^{B}\left(P, \operatorname{Hom}_{\mathbb{Z}}(N, U)\right) \longrightarrow \operatorname{Hom}_{B}\left(P, \operatorname{Hom}_{\mathbb{Z}}(N, U)\right)$ induced by the forgetful functor $B$-contra $\mathrm{gr}_{\mathrm{gr}} \longrightarrow B-\bmod _{\mathrm{gr}}$. Since the latter map is an isomorphism for all graded abelian groups $U$ by assumption, it follows that the former map is an isomorphism, too.

8.2. CDG-contramodules of the induced type. Let $B=\bigoplus_{n=0}^{\infty} B^{n}$ be a nonnegatively graded ring, and let $K$ be a fixed associative ring endowed with a ring homomorphism $K \longrightarrow B^{0}$. Let $L=\bigoplus_{n \in \mathbb{Z}} L^{n}$ be a graded left $K$-module. By the graded left $B$-contramodule induced from (or freely generated by) a graded left $K$-module $L$ we mean the graded left $B$-contramodule $\mathbb{M}_{K}^{\mathrm{gr}}(L)=B \otimes_{K}^{\Pi} L$. These were called the "free modules over the monad $\mathbb{M}=\mathbb{M}_{K}^{\text {gr" }}$ in Section 5.2. Subsequently we described such graded $B$-contramodules in Lemma 7.4.

Assume that $B$ is a flat graded right $K$-module. Then, given a short exact sequence of graded left $K$-modules $0 \longrightarrow L^{\prime} \longrightarrow L \longrightarrow L^{\prime \prime} \longrightarrow 0$, one can apply the functor $\mathbb{M}_{K}^{\mathrm{gr}}$, producing a short exact sequence of graded left $B$-contramodules $0 \longrightarrow \mathbb{M}_{K}^{\text {gr }}\left(L^{\prime}\right) \longrightarrow \mathbb{M}_{K}^{\text {gr }}(L) \longrightarrow \mathbb{M}_{K}^{\text {gr }}\left(L^{\prime \prime}\right) \longrightarrow 0$. The resulting short exact sequence of graded left $B$-contramodules is said to be induced from the original short exact sequence of graded left $K$-modules.

Now let $B=(B, d, h)$ be a nonnegatively graded CDG-ring. We will say that a left CDG-contramodule $\left(Q, d_{Q}\right)$ over $(B, d, h)$ is of the induced type if its underlying graded left $B$-contramodule $Q$ is (isomorphic to a graded left $B$-contramodule) induced from a graded left $K$-module. (Of course, the meaning of this definition 
depends on the choice of a ring $K$ and a ring homomorphism $K \longrightarrow B^{0}$, which we presume to be fixed.)

Similarly, assume that $B$ is a flat graded right $K$-module. Then we will say that a short exact sequence $0 \longrightarrow\left(Q^{\prime}, d_{Q^{\prime}}\right) \longrightarrow\left(Q, d_{Q}\right) \longrightarrow\left(Q^{\prime \prime}, d_{Q^{\prime \prime}}\right) \longrightarrow 0$ of left CDG-contramodules over $(B, d, h)$ (with closed morphisms between them) is a left $C D G$-contramodule short exact sequence of the induced type if the underlying short exact sequence of graded left $B$-contramodules $0 \longrightarrow Q^{\prime} \longrightarrow Q \longrightarrow Q^{\prime \prime} \longrightarrow 0$ is isomorphic to a short exact sequence of graded left $B$-contramodules induced from a short exact sequence of graded left $K$-modules.

As in the proof of Proposition 5.20, by the free graded left $B$-contramodule spanned by a graded set $X$ we mean the graded left $B$-contramodule $\mathbb{M}_{K}^{\text {gr }}(K[X])$ induced from the free graded left $K$-module $K[X]$. The projective graded left $B$-contramodules are the projective objects of the abelian category $B$-contragr; these are the direct summands of the free graded left $B$-contramodules. A CDG-contramodule $\left(P, d_{P}\right)$ over $(B, d, h)$ is said to be of the projective (resp., free) type if its underlying graded left $B$-contramodule is projective (resp., free).

Denote the full DG-subcategories in the DG-category DG $(B$-contra) formed by CDG-contramodules of the induced, free, and projective type by $\mathrm{DG}(B$-contra $K$-ind $)$, $\mathrm{DG}(B$-contra free $)$, and $\mathrm{DG}(B$-contra proj $)$, respectively. Clearly, all the three DG-subcategories are closed under shifts, twists, and finite direct sums; in particular, they are closed under the cones of closed morphisms. Therefore, their homotopy categories $\operatorname{Hot}(B$-contra $K$-ind $)=H^{0} \mathrm{DG}(B$-contra $K$-ind $), \quad \operatorname{Hot}(B$-contra free $)=$ $H^{0} \mathrm{DG}\left(B-\right.$ contra $\left._{\text {free }}\right)$, and $\operatorname{Hot}\left(B-\right.$ contra $\left._{\text {proj }}\right)=H^{0} \mathrm{DG}\left(B-\right.$ contra $\left._{\text {proj }}\right)$ are full triangulated subcategories in $\operatorname{Hot}(B$-contra).

The main results of this Section 8.2 , following below, constitute our version of [27, Theorem 5.5(b)].

Lemma 8.4. The two full triangulated subcategories $\operatorname{Hot}(B$-contrafree $)$ and $\operatorname{Hot}(B$-contra proj $) \subset \operatorname{Hot}(B$-contra $)$ coincide.

Proof. The argument is based on a version of Eilenberg's cancellation trick. Let $\left(Q, d_{Q}\right)$ be a left CDG-contramodule over $(B, d, h)$ whose underlying graded left $B$-contramodule $Q$ is a direct summand of the free graded left $B$-contramodule $B \otimes_{K}^{\Pi} K[X]$ spanned by a graded set $X$. Let $Y=X \times \mathbb{Z}$ be the graded set with the components $Y_{n}=X_{n} \times \mathbb{Z}$. Consider the quasi-differential graded ring $(\widehat{B}, \partial)$ corresponding to the CDG-ring $(B, d, h)$, and let $P=\widehat{B} \otimes_{K}^{\Pi} K[Y]$ be the free graded contramodule over $\widehat{B}$ spanned by the graded set $Y$. Then $P$ can be viewed as a CDG-contamodule $\left(P, d_{P}\right)$ over $(B, d, h)$. Moreover, $\left(P, d_{P}\right)$ is a contractible CDG-contramodule (i. e., it represents a zero object in the homotopy category $\operatorname{Hot}(B$-contra $))$; essentially, the homogeneous map $\partial: P \longrightarrow P$ of degree -1 induced by the differential $\partial$ on $\widehat{B}$ provides a contracting homotopy. As a graded $B$-contramodule, $P$ is isomorphic to $B \otimes_{K}^{\Pi} K[Y] \oplus\left(B \otimes_{K}^{\Pi} K[Y]\right)[-1]$. Hence $P \oplus Q$ is a free graded $B$-contramodule, and the object $\left(Q, d_{Q}\right) \in \operatorname{Hot}(B$-contra proj $)$ is isomorphic to the object $\left(Q, d_{Q}\right) \oplus\left(P, d_{P}\right) \in \operatorname{Hot}(B$-contra free $)$. 
Proposition 8.5. Assume that $B$ is a flat graded right $K$-module and the left homological dimension of the ring $K$ is finite. Then the inclusion $\operatorname{Hot}\left(B-\right.$ contra $\left._{\text {free }}\right) \longrightarrow$ $\operatorname{Hot}(B$-contra $K$-ind $)$ induces a triangulated equivalence between $\operatorname{Hot}(B$-contra free $)$ and the triangulated quotient category of $\operatorname{Hot}\left(B-{ }_{\text {contra }}{ }_{K \text {-ind }}\right)$ by its minimal full triangulated subcategory containing all the totalizations of left CDG-contramodule short exact sequences of the induced type over $(B, d, h)$.

Proof. Essentially, the claim is that the full subcategory of CDG-contramodules of the free type and the minimal full triangulated subcategory containing the totalizations of CDG-contramodule short exact sequences of the induced type form a semiorthogonal decomposition of $\operatorname{Hot}(B$-contra $K$-ind $)$. Firstly, this means that the complex of abelian groups $\operatorname{Hom}^{B}(P, Z)$ is acyclic for any $P \in \operatorname{Hot}(B$-contrafree $)$ and the totalization $Z$ of any CDG-contramodule short exact sequence $0 \longrightarrow Q^{\prime} \longrightarrow Q \longrightarrow Q^{\prime \prime} \longrightarrow 0$ of the induced type. This is a particular case of a standard observation (cf. [28, Theorem 3.5(b)]), and the assumption that the short exact sequence is of the induced type is not needed for it; see the proof of Theorem 8.7 below.

Secondly, it is claimed that for any object $Q \in \operatorname{Hot}\left(B\right.$-contra $\left.{ }_{K \text {-ind }}\right)$ there exists a distinguished triangle $P \longrightarrow Q \longrightarrow Z \longrightarrow P[1]$ in $\operatorname{Hot}(B$-contra $K$-ind $)$ with $P \in \operatorname{Hot}(B$-contra free $)$ and $Z$ belonging to the minimal triangulated subcategory of $\operatorname{Hot}(B$-contra $K$-ind $)$ containing the totalizations of CDG-contramodule short exact sequences of the induced type. This is provable by a variation of the standard argument from [28, proof of Theorem 3.6].

Let $\left(Q, d_{Q}\right)$ be a CDG-contramodule structure on the graded left $B$-contramodule $Q=B \otimes_{K}^{\Pi} L$ induced from a graded left $K$-module $L$. Choose a free graded left $K$-module $G=K[X]$ together with a surjective morphism of graded left $K$-modules $g: G \longrightarrow L$. Consider the free graded left $\widehat{B}$-contramodule $F=\widehat{B} \otimes_{K}^{\Pi} G$ induced from $G$. The CDG-contramodule $\left(Q, d_{Q}\right)$ over $(B, d, h)$ can be also viewed as a graded $\widehat{B}$-contramodule. Let $f: F \longrightarrow Q$ be the graded left $\widehat{B}$-contramodule morphism corresponding to the composition of graded left $K$-module maps $G \longrightarrow L \longrightarrow Q$. Then the restriction of $f$ to the graded $B$-subcontramodule $B \otimes_{K}^{\Pi} G \subset \widehat{B} \otimes_{K}^{\Pi} G$ is the graded $B$-contramodule map $B \otimes_{K}^{\Pi} G \longrightarrow B \otimes_{K}^{\Pi} L=Q$ obtained by applying the functor $B \otimes_{K}^{\Pi}$ - to the graded left $K$-module map $g: G \longrightarrow L$.

The key observation is that the whole map $f: F \longrightarrow Q$, viewed as a morphism of graded left $B$-contramodules, is induced from a morphism of graded left $K$-modules. Put $F^{\prime}=B \otimes_{K}^{\Pi} G \subset \widehat{B} \otimes_{K}^{\Pi} G=F$. Then the quotient graded left $B$-contramodule $F^{\prime \prime}=F / F^{\prime}$ is isomorphic to $F^{\prime}[-1]$; so it is also a free graded left $B$-contramodule. Hence the short exact sequence of graded left $B$-contramodules $0 \longrightarrow F^{\prime} \longrightarrow F \longrightarrow F^{\prime \prime} \longrightarrow 0$ splits; we can choose a splitting and consider $F^{\prime \prime}$ as a graded left $B$-subcontramodule in $F$. Put $f^{\prime}=\left.f\right|_{F^{\prime}}: F^{\prime} \longrightarrow Q$ and $f^{\prime \prime}=\left.f\right|_{F^{\prime \prime}}: F^{\prime \prime} \longrightarrow Q$; so $f=\left(f^{\prime}, f^{\prime \prime}\right): F^{\prime} \oplus F^{\prime \prime} \longrightarrow Q$. Then $f^{\prime}=B \otimes_{K}^{\Pi} g$ is a surjective morphism of graded left $B$-contramodules (as $g: G \longrightarrow L$ is a surjective morphism of graded left $K$-modules). Since $F^{\prime \prime}$ is a projective graded left $B$-contramodule, there exists a graded left $B$-contramodule morphism $t: F^{\prime \prime} \longrightarrow F^{\prime}$ such that $f^{\prime \prime}=f^{\prime} \circ t$. Using the map $t$, one can construct an automorphism 
of the graded left $B$-contramodule $F=F^{\prime} \oplus F^{\prime \prime}$ such that the composition $F^{\prime} \oplus F^{\prime \prime} \longrightarrow F^{\prime} \oplus F^{\prime \prime} \stackrel{f}{\longrightarrow} Q$ is equal to the map $\left(f^{\prime}, 0\right): F^{\prime} \oplus F^{\prime \prime} \longrightarrow Q$.

Put $F_{0}=F$ and $G_{0}=G \oplus G[-1]$; and denote by $Q_{1}$ the kernel of the graded left $\widehat{B}$-contramodule morphism $f: F_{0} \longrightarrow Q$. Then $0 \longrightarrow Q_{1} \longrightarrow F_{0} \longrightarrow Q \longrightarrow 0$ is a short exact sequence of graded left $\widehat{B}$-contramodules. We have shown that, as a short exact sequence of graded left $B$-contramodules, it is induced from a short exact sequence of graded left $K$-modules $0 \longrightarrow L_{1} \longrightarrow G_{0} \longrightarrow L \longrightarrow 0$ (where $L_{1}$ is the kernel of the morphism $\left.(g, 0): G_{0}=G \oplus G[-1] \longrightarrow L\right)$. Now we can consider $0 \longrightarrow Q_{1} \longrightarrow F_{0} \longrightarrow Q \longrightarrow 0$ as a CDG-contramodule short exact sequence of the induced type over $(B, d, h)$.

It remains to iterate this construction, applying it to the CDG-contramodule $Q_{1}$ in place of $Q$, etc. Proceeding in this way, we produce an exact sequence of CDG-contramodules $\cdots \longrightarrow F_{2} \longrightarrow F_{1} \longrightarrow F_{0} \longrightarrow Q \longrightarrow 0$ such that its underlying exact sequence of graded left $B$-contramodules is induced from an exact sequence of graded left $K$-modules $\cdots \longrightarrow G_{2} \longrightarrow G_{1} \longrightarrow G_{0} \longrightarrow L \longrightarrow 0$. Here $G_{i}$ are free graded left $K$-modules by construction. Since the left homological dimension of the $\operatorname{ring} K$ is finite by assumption, there exists $k \geq 0$ such that the image $L_{k}$ of the morphism $G_{k} \longrightarrow G_{k-1}$ is a projective graded left $K$-module.

Consider the finite exact sequence of CDG-contramodules $0 \longrightarrow Q_{k} \longrightarrow$ $F_{k-1} \longrightarrow \cdots \longrightarrow F_{0} \longrightarrow Q \longrightarrow 0$ over $(B, d, h)$, where $Q_{k}$ is the image of the CDG-contramodule morphism $F_{k} \longrightarrow F_{k-1}$. Denote by $\left(Z, d_{Z}\right)$ the total CDG-contramodule of this finite exact sequence of CDG-contramodules; and denote by $\left(P, d_{P}\right)$ the total CDG-contramodule of the complex of CDG-contramodules $Q_{k} \longrightarrow F_{k-1} \longrightarrow \cdots \longrightarrow F_{0}$. Then we have a distinguished triangle $P \longrightarrow Q \longrightarrow$ $Z \longrightarrow P[1]$ in $\operatorname{Hot}\left(B-\right.$ contra $\left._{K \text {-ind }}\right)$.

The underlying exact sequence of graded left $B$-contramodules $0 \longrightarrow Q_{k} \longrightarrow$ $F_{k-1} \longrightarrow \cdots \longrightarrow F_{0} \longrightarrow Q \longrightarrow 0$ is induced from the exact sequence of graded left $K$-modules $0 \longrightarrow L_{k} \longrightarrow G_{k-1} \longrightarrow \cdots \longrightarrow G_{0} \longrightarrow L \longrightarrow 0$. Hence it follows that the CDG-contramodule $\left(Z, d_{Z}\right)$ over $(B, d, h)$ belongs to the minimal triangulated subcategory in $\operatorname{Hot}(B$-contra $K$-ind $)$ containing the totalizations of CDG-contramodule short exact sequences of the induced type. Finally, the underlying graded left $B$-contramodule $P$ of the CDG-contramodule $\left(P, d_{P}\right)$ is projective, since the graded left $B$-contramodule $Q_{k}$ is induced from the projective graded left $K$-module $L_{k}$. By Lemma 8.4, the CDG-contramodule $\left(P, d_{P}\right)$ is homotopy equivalent to a CDG-contramodule of the free type over $(B, d, h)$.

Proposition 8.6. Assume that the right $K$-module $B^{n}$ is finitely generated and projective for every $n \geq 0$. Then the inclusion $\operatorname{Hot}\left(B\right.$-contra $\left._{K \text {-ind }}\right) \longrightarrow \operatorname{Hot}(B$-contra $)$ induces a triangulated equivalence between the contraderived category $\mathrm{D}^{\mathrm{ctr}}(B$-contra) (as defined in Section 7.3) and the triangulated quotient category of the homotopy category $\operatorname{Hot}(B$-contra $K$-ind $)$ by its intersection with the full triangulated subcategory Acycl $^{\text {ctr }}$ (B-contra) of contraacyclic $C D G$-contramodules in $\mathrm{Hot}(B$-contra),

$$
\operatorname{Hot}(B \text {-contra } K \text {-ind }) /\left(\operatorname{Acycl}^{\mathrm{ctr}}(B \text {-contra }) \cap \operatorname{Hot}(B \text {-contra } K \text {-ind })\right) \simeq \mathrm{D}^{\mathrm{ctr}}(B \text {-contra }) \text {. }
$$


Proof. In view of a well-known lemma ([27, Lemma 2.6] or [28, Lemma 1.6(a)]), it suffices to show that for any left CDG-contramodule $\left(S, d_{S}\right)$ over $(B, d, h)$ there exists a left CDG-contramodule $\left(Q, d_{Q}\right)$ of the induced type together with a closed morphism of CDG-contramodules $\left(Q, d_{Q}\right) \longrightarrow\left(S, d_{S}\right)$ whose cone is a contraacyclic CDG-contramodule over $(B, d, h)$. A standard argument (cf. [28, Theorem 3.8]) reduces the question to showing that countable products of free graded left $B$-contramodules are induced graded left $B$-contramodules.

Let us spell out some details. Consider the CDG-contramodule $\left(S, d_{S}\right)$ as a graded left $\widehat{B}$-contramodule. Let $\cdots \longrightarrow P_{2} \longrightarrow P_{1} \longrightarrow P_{0} \longrightarrow S \longrightarrow 0$ be a resolution of the object $S \in \widehat{B}$ - contra $_{\text {gr }}$ by free graded left $\widehat{B}$-contramodules $P_{i}$. Consider this resolution as a complex of left CDG-contramodules $\cdots \longrightarrow P_{2} \longrightarrow P_{1} \longrightarrow P_{0} \longrightarrow 0$ over $(B, d, h)$ and totalize it by taking infinite products along the diagonals. We refer to [28, Section 1.2] for a discussion of totalizations of complexes in DG-categories. Notice that the infinite products in $B$-contragr agree with those in $K-\bmod _{\mathrm{gr}}$, so there is no ambiguity in how the totalization construction needs to be applied. Let $\left(Q, d_{Q}\right)$ be the resulting total CDG-contramodule over $(B, d, h)$. Then the underlying graded left $B$-contramodule $Q$ of $\left(Q, d_{Q}\right)$ is simply the countable product $Q=\prod_{i=0}^{\infty} P_{i}[i]$. Once again we recall that the forgetful functor $\widehat{B}$-contra gr $\longrightarrow B$-contra gr $_{\text {rakes free }}$ graded left $\widehat{B}$-contramodules to free graded left $B$-contramodules.

The cone $\left(Y, d_{Y}\right)$ of the natural closed morphism of CDG-contramodules $\left(Q, d_{Q}\right)$ $\longrightarrow\left(S, d_{S}\right)$ is the infinite product totalization of the exact complex $\cdots \longrightarrow P_{2} \longrightarrow$ $P_{1} \longrightarrow P_{0} \longrightarrow S \longrightarrow 0$. As such, it admits a complete, separated decreasing filtration $F$ by the totalizations of the subcomplexes of canonical filtration of the complex $\cdots \longrightarrow P_{2} \longrightarrow P_{1} \longrightarrow P_{0} \longrightarrow S \longrightarrow 0$. The successive quotient CDG-contramodules $F^{n} Y / F^{n+1} Y$ are contractible. In fact, the CDG-contramodules $F^{n} Y / F^{n+1} Y$ are the cones of identity endomorphisms of the CDG-contramodules of cocycles of our acyclic complex of CDG-contramodules (up to some shifts). By Lemma 7.3, it follows that the CDG-contramodule $\left(Y, d_{Y}\right)$ is contraacyclic.

It remains to check that the class of all induced graded left $B$-contramodules is

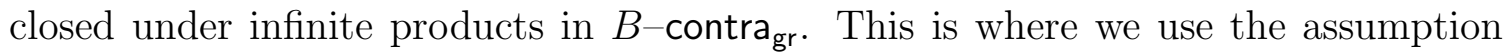
that $B^{n}$ is a finitely generated projective right $K$-module for every $n \geq 0$. Under this assumption, one can easily see that the functor $B \otimes_{K}^{\Pi}-$ (viewed as a functor $K-\bmod _{\mathrm{gr}} \longrightarrow K-\bmod _{\mathrm{gr}}$ or $K-\bmod _{\mathrm{gr}} \longrightarrow B$-contra $\left.{ }_{\mathrm{gr}}\right)$ preserves infinite products. In fact, it suffices that $B^{n}$ be a finitely presentable right $K$-module for every $n \geq 0$.

Theorem 8.7. Assume that $B^{n}$ is a finitely generated projective right $K$-module for every $n \geq 0$ and the left homological dimension of the ring $K$ is finite. Then the composition of the fully faithful inclusion $\operatorname{Hot}\left(B\right.$-contra $\left.{ }_{\text {proj }}\right) \longrightarrow \operatorname{Hot}(B$-contra $)$ with the triangulated quotient functor $\operatorname{Hot}(B$-contra $) \longrightarrow \mathrm{D}^{\text {ctr }}(B$-contra $)$ is a triangulated equivalence

$$
\operatorname{Hot}\left(B-\text { contra }_{\text {proj }}\right) \simeq \mathrm{D}^{\mathrm{ctr}}(B \text {-contra })
$$

between the homotopy category of left $C D G$-contramodules of the projective type over $(B, d, h)$ and the contraderived category of left $C D G$-contramodules over $(B, d, h)$. 
Furthermore, the intersection of two full subcategories

$$
\operatorname{Acycl}^{\operatorname{ctr}}(B \text {-contra }) \cap \operatorname{Hot}(B \text {-contra } K \text {-ind }) \subset \operatorname{Hot}(B \text {-contra })
$$

coincides with the minimal full triangulated subcategory in $\operatorname{Hot}\left(B\right.$-contra $\left.{ }_{K \text {-ind }}\right)$ containing all the totalizations of $C D G$-contramodule short exact sequences of the induced type over $(B, d, h)$.

Proof. In the first assertion of the theorem, the claim is that the full subcategory of CDG-contramodules of the projective type $\operatorname{Hot}\left(B-\right.$ contra $\left._{\text {proj }}\right)$ and the full subcategory of contraacyclic CDG-contramodules $\operatorname{Acycl}^{\text {ctr }}(B$-contra) form a semiorthogonal decomposition of the homotopy category $\operatorname{Hot}(B$-contra). First of all, this means that the complex of abelian groups $\operatorname{Hom}^{B}(P, Z)$ is acyclic for any $P \in \operatorname{Hot}(B$-contra proj $)$ and $Z \in \mathrm{Acycl}^{\mathrm{ctr}}$ ( $B$-contra). A particular case of this observation was already mentioned in the proof of Proposition 8.5.

Indeed, let us fix a CDG-contramodule $\left(P, d_{P}\right) \in \operatorname{Hot}(B$-contraproj $)$. Then the class of all CDG-contramodules $\left(Z, d_{Z}\right) \in \operatorname{Hot}(B$-contra) for which the complex $\operatorname{Hom}^{B}(P, Z)$ is acyclic is closed under shifts, cones, and infinite products in Hot $(B$-contra $)$. Thus it remains to check that the totalization $Z=\operatorname{Tot}\left(Q^{\prime} \rightarrow\right.$ $\left.Q \rightarrow Q^{\prime \prime}\right)$ of any short exact sequence of CDG-contramodules $0 \longrightarrow\left(Q^{\prime}, d_{Q^{\prime}}\right) \longrightarrow$ $\left(Q, d_{Q}\right) \longrightarrow\left(Q^{\prime \prime}, d_{Q^{\prime \prime}}\right) \longrightarrow 0$ over $(B, d, h)$ has the desired property. Here it suffices to notice that the complex of abelian groups $\operatorname{Hom}^{B}(P, Z)$ is the totalization of the bicomplex with three rows $\operatorname{Hom}^{B}\left(P, Q^{\prime}\right) \longrightarrow \operatorname{Hom}^{B}(P, Q) \longrightarrow \operatorname{Hom}^{B}\left(P, Q^{\prime \prime}\right)$. The short sequence of complexes of abelian groups $0 \longrightarrow \operatorname{Hom}^{B}\left(P, Q^{\prime}\right) \longrightarrow$ $\operatorname{Hom}^{B}(P, Q) \longrightarrow \operatorname{Hom}^{B}\left(P, Q^{\prime \prime}\right) \longrightarrow 0$ is exact, since the graded left $B$-contramodule $P$ is projective. The totalization of any short exact sequence of complexes of abelian groups is an exact complex.

Now we can prove the second assertion of the theorem. Let $\left(Z, d_{Z}\right)$ be a left CDG-contramodule of the induced type over $(B, d, h)$ which is contraacyclic as a left CDG-contramodule over $(B, d, h)$. Then, according to the above argument, the complex $\operatorname{Hom}^{B}(P, Z)$ is acyclic for any left CDG-contramodule $\left(P, d_{P}\right)$ of the free type over $(B, d, h)$. In view of the semiorthogonal decomposition of the category $\operatorname{Hot}(B$-contra $K$-ind $)$ constructed in the proof of Proposition 8.5 , it follows that $\left(Z, d_{Z}\right)$ belongs to the minimal triangulated subcategory of $\operatorname{Hot}(B$-contra $K$-ind $)$ containing the totalizations of CDG-contramodule short exact sequences of the induced type.

Finally, the assertion that the functor $\operatorname{Hot}(B$-contra proj $) \longrightarrow \mathrm{D}^{\mathrm{ctr}}(B$-contra $)$ is a triangulated equivalence can be now obtained by comparing the results of Lemma 8.4, Proposition 8.5, and Proposition 8.6. This also implies the semiorthogonal decomposition promised in the beginning of this proof (given that the semiorthogonality of the two subcategories in question has been established already).

8.3. CDG-comodules of the coinduced type. Let $B=\bigoplus_{n=0}^{\infty} B^{n}$ be a nonnegatively graded ring, and let $K$ be a fixed ring endowed with a ring homomorphism $K \longrightarrow B^{0}$. Similarly to the definition of graded right $B$-comodules in Section 5.5, one can define graded left $B$-comodules. So a graded left $B$-module $M=\bigoplus_{n \in \mathbb{Z}} M^{n}$ 
is said to be a $B$-comodule (or a graded left $B$-comodule) if for every element $x \in M^{j}$, $j \in \mathbb{Z}$, there exists an integer $m \geq 0$ such that $B^{\geq m+1} x=0$. We denote the full subcategory of graded left $B$-comodules by $B$-comod ${ }_{\text {gr }} \subset B$ - mod $_{\text {gr }}$.

Let $L=\bigoplus_{n \in \mathbb{Z}} L^{n}$ be a graded left $K$-module. By the graded left $B$-comodule coinduced from (or cofreely cogenerated by) a graded left $K$-module $L$ we mean the graded left $B$-comodule $\operatorname{Hom}_{K}^{\Sigma}(B, L)$, in the notation of Section 5.7 (cf. Lemma 6.6).

Assume that $B$ is a projective graded left $K$-module. Then, given a short exact sequence of graded left $K$-modules $0 \longrightarrow L^{\prime} \longrightarrow L \longrightarrow L^{\prime \prime} \longrightarrow 0$, one can apply the functor $\operatorname{Hom}_{K}^{\Sigma}(B,-)$, producing a short exact sequence of graded left $B$-comodules $0 \longrightarrow \operatorname{Hom}_{K}^{\Sigma}\left(B, L^{\prime}\right) \longrightarrow \operatorname{Hom}_{K}^{\Sigma}(B, L) \longrightarrow \operatorname{Hom}_{K}^{\Sigma}\left(B, L^{\prime \prime}\right) \longrightarrow 0$. The resulting short exact sequence of graded left $B$-comodules is said to be coinduced from the original short exact sequence of graded left $K$-modules.

Now let $B=(B, d, h)$ be a nonnegatively graded CDG-ring. Similarly to the definition of right CDG-comodules over $(B, d, h)$ in Section 6.4, one can define left CDG-comodules over $(B, d, h)$. So a left CDG-module $\left(M, d_{M}\right)$ over $(B, d, h)$ is said to be a $C D G$-comodule (or a left $C D G$-comodule) over $(B, d, h)$ if the graded left $B$-module $M$ is a graded left $B$-comodule. The full DG-subcategory in DG $(B$-mod $)$ whose objects are the left CDG-comodules over $(B, d, h)$ is called the $D G$-category of left $C D G$-comodules over $(B, d, h)$ and denoted by $\mathrm{DG}(B$-comod $)$. Its triangulated homotopy category is denoted by $\operatorname{Hot}(B$-comod $)=H^{0} \mathrm{DG}(B$-comod $)$.

We will say that a left CDG-comodule $\left(M, d_{M}\right)$ over $(B, d, h)$ is of the coinduced type if its underlying graded left $B$-comodule $M$ is (isomorphic to a graded left $B$-comodule) coinduced from a graded left $K$-module. (The meaning of this definition depends on the choice of a ring $K$ and a ring homomorphism $K \longrightarrow B^{0}$, which we presume to be fixed.)

Assume that $B$ is a projective graded left $K$-module. Then we will say that a short exact sequence $0 \longrightarrow\left(M^{\prime}, d_{M^{\prime}}\right) \longrightarrow\left(M, d_{M}\right) \longrightarrow\left(M^{\prime \prime}, d_{M^{\prime \prime}}\right) \longrightarrow 0$ of left CDG-comodules over $(B, d, h)$ (with closed morphisms between them) is a left $C D G$-comodule short exact sequence of the coinduced type if the underlying short exact sequence of graded left $B$-comodules $0 \longrightarrow M^{\prime} \longrightarrow M \longrightarrow M^{\prime \prime} \longrightarrow 0$ is isomorphic to a short exact sequence of graded left $B$-comodules coinduced from a short exact sequence of graded left $K$-modules.

By the cofree graded left $B$-comodule spanned by a graded set $X$ we mean the graded left $B$-comodule $\operatorname{Hom}_{K}^{\Sigma}\left(B, K[X]^{+}\right)$coinduced from the cofree graded left $K$-module $K[X]^{+}$. Here $F^{+}$is the notation for the graded character group $\operatorname{Hom}_{\mathbb{Z}}(F, \mathbb{Q} / \mathbb{Z})$ of an abelian group $F$ (see Section 5.7). The injective graded left $B$-comodules are the injective objects of the abelian category $B$ - $\operatorname{comod}_{\mathrm{gr}}$; these are the direct summands of the cofree graded left $B$-comodules (see the discussion in the proof of Theorem 5.29).

A CDG-comodule $\left(J, d_{J}\right)$ over $(B, d, h)$ is said to be of the injective (resp., cofree) type if its underlying graded left $B$-comodule is injective (resp., cofree). Denote the full DG-subcategories in the DG-category $\mathrm{DG}(B$-comod) formed by CDG-comodules of the coinduced, cofree, and injective type by $\mathrm{DG}\left(B-\operatorname{comod}_{K \text {-coind }}\right)$, 
$D G\left(B-\right.$ comod $\left._{\text {cofr }}\right)$, and $\mathrm{DG}\left(B-\right.$ comod $\left._{\text {inj }}\right)$, respectively. All the three DG-subcategories are closed under shifts, twists, and finite direct sums; in particular, they are closed under the cones of closed morphisms. Therefore, their homotopy categories $\operatorname{Hot}\left(B-\operatorname{comod}_{K \text {-coind }}\right)=H^{0} \mathrm{DG}\left(B-\operatorname{comod}_{K \text {-coind }}\right), \quad \operatorname{Hot}\left(B-\operatorname{comod}_{\text {cofr }}\right)=$ $H^{0} \mathrm{DG}\left(B-\right.$ comod $\left._{\text {cofr }}\right)$, and $\operatorname{Hot}\left(B-\right.$ comod $\left._{\text {inj }}\right)=H^{0} \mathrm{DG}\left(B-\right.$ comod $\left._{\text {inj }}\right)$ are full triangulated subcategories in $\operatorname{Hot}(B$-comod).

The main results of this Section [8.3, following below, form our version of [27, Theorem 5.5(a)].

Lemma 8.8. The two full triangulated subcategories $\operatorname{Hot}\left(B-\operatorname{comod}_{\mathrm{cofr}}\right)$ and $\operatorname{Hot}\left(B-\operatorname{comod}_{\text {inj }}\right) \subset \operatorname{Hot}(B-\operatorname{comod})$ coincide.

Proof. This is a dual-analogous version of Lemma 8.4, provable by a similar argument using the cancellation trick.

Proposition 8.9. Assume that $B$ is a projective graded left $K$-module and the left homological dimension of the ring $K$ is finite. Then the inclusion $\operatorname{Hot}\left(B-\operatorname{comod}_{\text {cofr }}\right) \longrightarrow$ $\operatorname{Hot}\left(B-\operatorname{comod}_{K \text {-coind }}\right)$ induces a triangulated equivalence between $\operatorname{Hot}\left(B-\operatorname{comod}_{\text {cofr }}\right)$ and the triangulated quotient category of $\operatorname{Hot}\left(B-\operatorname{comod}_{K \text {-coind }}\right)$ by its minimal full triangulated subcategory containing all the totalizations of left $C D G$-comodule short exact sequences of the coinduced type over $(B, d, h)$.

Proof. Essentially, the claim is that the full subcategory of CDG-comodules of the cofree type and the minimal full triangulated subcategory containing the totalizations of CDG-comodule short exact sequences of the coinduced type form a semiorthogonal decomposition of $\operatorname{Hot}\left(B-\operatorname{comod}_{K \text {-coind }}\right)$. Firstly, this means that the complex of abelian groups $\operatorname{Hom}_{B}(Z, J)$ is acyclic for any $J \in \operatorname{Hot}\left(B-\operatorname{comod}_{\text {cofr }}\right)$ and the totalization $Z$ of any CDG-comodule short exact sequence $0 \longrightarrow M^{\prime} \longrightarrow M \longrightarrow M^{\prime \prime} \longrightarrow 0$ of the coinduced type. This is a particular case of a standard observation (cf. [28, Theorem 3.6(a)]), and the assumption that the short exact sequence is of the coinduced type is not needed for it; see the proof of Theorem 8.11 below.

Secondly, it is claimed that for any object $N \in \operatorname{Hot}\left(B-\operatorname{comod}_{K \text {-coind }}\right)$ there exists a distinguished triangle $Z \longrightarrow N \longrightarrow J \longrightarrow Z[1]$ in $\operatorname{Hot}\left(B-\operatorname{comod}_{K \text {-coind }}\right)$ with

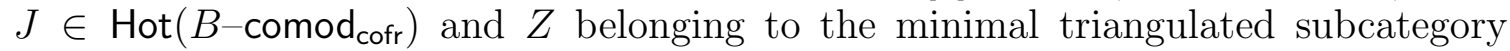
of $\operatorname{Hot}(B$-comod $K$-coind $)$ containing the totalizations of CDG-comodule short exact sequences of the coinduced type. This is provable by a variation of the standard argument form [28, proof of Theorem 3.6]. We skip the further details, which are very similar to the proof of the dual-analogous result in Proposition 8.5.

Proposition 8.10. Assume that the left $K$-module $B^{n}$ is finitely generated for every $n \geq 0$. Then the inclusion $\operatorname{Hot}\left(B-\operatorname{comod}_{K \text {-coind }}\right) \longrightarrow \operatorname{Hot}(B$-comod $)$ induces a triangulated equivalence between the coderived category $\mathrm{D}^{\mathrm{co}}(B$-comod) (as defined in Section (6.4) and the triangulated quotient category of the homotopy category $\operatorname{Hot}\left(B-\operatorname{comod}_{K \text {-coind }}\right)$ by its intersection with the full triangulated subcategory $\mathrm{Acycl}^{\mathrm{CO}}(B$-comod) of coacyclic $C D G$-comodules in $\operatorname{Hot}(B$-comod),

$$
\operatorname{Hot}\left(B-\operatorname{comod}_{K \text {-coind }}\right) /\left(\operatorname{Acycl}^{\mathrm{co}}(B-\operatorname{comod}) \cap \operatorname{Hot}\left(B-\operatorname{comod}_{K \text {-coind }}\right) \simeq \mathrm{D}^{\mathrm{co}}(B-\operatorname{comod})\right. \text {. }
$$


Proof. In view of [27, Lemma 2.6] or [28, Lemma 1.6(b)], it suffices to show that for any left CDG-comodule $\left(M, d_{M}\right)$ over $(B, d, h)$ there exists a left CDG-comodule $\left(N, d_{N}\right)$ of the coinduced type together with a closed morphism of CDG-comodules $\left(M, d_{M}\right) \longrightarrow\left(N, d_{N}\right)$ whose cone is a coacyclic CDG-comodule over $(B, d, h)$. A standard argument using Lemma 6.5 (cf. [28, proof of Theorem 3.7]) reduces the question to showing that countable direct sums of cofree graded left $B$-comodules are coinduced graded left $B$-comodules. We skip the details, which are very similar to the ones in the proof of the dual-analogous result in Proposition 8.6.

Hence it remains to check that the class of all coinduced graded left $B$-comodules is closed under infinite direct sums in $B$ - comod $_{\mathrm{gr}}$. This is where we use the assumption that $B^{n}$ is a finitely generated left $K$-module for every $n \geq 0$. Under this assumption, one can easily see that the functor $\operatorname{Hom}_{K}^{\Sigma}(B,-)$ (viewed as a functor $K-\bmod _{\mathrm{gr}} \longrightarrow$ $K-\bmod _{\mathrm{gr}}$ or $K-\bmod _{\mathrm{gr}} \longrightarrow B-$ comod $\left._{\mathrm{gr}}\right)$ preserves infinite direct sums.

Theorem 8.11. Assume that $B^{n}$ is a finitely generated projective left $K$-module for every $n \geq 0$ and the left homological dimension of the ring $K$ is finite. Then the composition of the fully faithful inclusion $\operatorname{Hot}\left(B-\operatorname{comod}_{i n j}\right) \longrightarrow \operatorname{Hot}(B-\operatorname{comod})$ with the triangulated quotient functor $\operatorname{Hot}(B$-comod $) \longrightarrow \mathrm{D}^{\mathrm{co}}(B$-comod $)$ is a triangulated equivalence

$$
\operatorname{Hot}\left(B-\operatorname{comod}_{\text {inj }}\right) \simeq \mathrm{D}^{\mathrm{co}}(B-\operatorname{comod})
$$

between the homotopy category of left $C D G$-comodules of the injective type over $(B, d, h)$ and the coderived category of left $C D G$-comodules over $(B, d, h)$. Furthermore, the intersection of two full subcategories

$$
\operatorname{Acycl}^{\mathrm{co}}(B \text {-comod }) \cap \operatorname{Hot}\left(B-\operatorname{comod}_{K \text {-coind }}\right) \subset \operatorname{Hot}(B \text {-comod })
$$

coincides with the minimal full triangulated subcategory in $\operatorname{Hot}\left(B-\operatorname{comod}_{K \text {-coind }}\right)$ containing all the totalizations of $C D G$-comodule short exact sequences of the coinduced type over $(B, d, h)$.

Proof. In the first assertion of the theorem, the claim is that the full subcategory of CDG-comodules of the injective type $\operatorname{Hot}\left(B-\operatorname{comod}_{i n j}\right)$ and the full subcategory of coacyclic CDG-comodules $\mathrm{Acyc}^{\mathrm{co}}{ }^{\mathrm{C}}(B$-comod) form a semiorthogonal decomposition of the homotopy category $\operatorname{Hot}(B$-comod). First of all, this means that the complex of abelian groups $\operatorname{Hom}_{B}(Z, J)$ is acyclic for any $Z \in \operatorname{Acycl}^{\mathrm{co}}(B$-comod) and $J \in$ $\operatorname{Hot}\left(B-\right.$ comodinj $\left._{i}\right)$. A particular case of this observation was already mentioned in the proof of Proposition 8.9 .

The proof of the theorem is based on Lemma 8.8, Proposition 8.9, and Proposition 8.10, We skip the details, which are very similar to the proof of the dualanalogous Theorem 8.7 .

8.4. The diagonal CDG-bicomodule. Let $B=\bigoplus_{n=0}^{\infty} B^{n}$ be a nonnegatively graded ring, and let $K$ be a ring endowed with a ring homomorphism $K \longrightarrow B^{0}$. Put $C_{n}=C^{-n}=\operatorname{Hom}_{K^{\text {op }}}\left(B^{n}, K\right)$. We will use the pairing notation

$$
\langle c, b\rangle=c(b) \in K \quad \text { for all } c \in C_{n}, b \in B^{n}, n \geq 0,
$$




$$
\langle c, b\rangle=0 \quad \text { for all } c \in C_{j}, b \in B^{i}, i \neq j .
$$

The graded abelian group $C=\bigoplus_{n \leq 0} C^{n}$ has a natural structure of graded $K$ - $B$-bimodule, with the left action of $K$ given by the rule

$$
\langle k c, b\rangle=k\langle c, b\rangle \quad \text { for all } k \in K, c \in C^{-n}, b \in B^{n}
$$

and the right action of $B$ given by the rule

$$
\left\langle c b, b^{\prime}\right\rangle=\left\langle c, b b^{\prime}\right\rangle \text { for all } c \in C, b, b^{\prime} \in B .
$$

Furthermore, assume that $B^{n}$ is a finitely generated projective right $K$-module for every $n \geq 0$. Then a straightforward generalization of the construction from Remark 2.8 endows $C$ with the structure of a graded coring over $K$. (The discussion in Remark 2.8 presumes the base ring $K=R=B^{0}$.) The grading components $\mu_{i, j}: C_{i+j} \longrightarrow C_{i} \otimes_{K} C_{j}, i, j \geq 0$, of the comultiplication map $\mu: C \longrightarrow C \otimes_{K} C$ are obtained by applying the functor $\operatorname{Hom}_{K^{\mathrm{op}}}(-, K)$ to the multiplication maps $B^{j} \otimes_{K}$ $B^{i} \longrightarrow B^{i+j}$ and taking into account the natural isomorphisms of Lemma 1.1(b). In the pairing notation,

$$
\left\langle\mu_{i, j}(c), b^{\prime \prime} \otimes b^{\prime}\right\rangle=\left\langle c, b^{\prime \prime} b^{\prime}\right\rangle \quad \text { for all } c \in C_{i+j}, b^{\prime} \in B^{i}, b^{\prime \prime} \in B^{j},
$$

where the pairing $\langle\rangle:, C_{i} \otimes_{K} C_{j} \otimes_{K} B^{j} \otimes_{K} B^{i} \longrightarrow K$ is defined by the rule

$$
\left\langle c^{\prime} \otimes c^{\prime \prime}, b^{\prime \prime} \otimes b^{\prime}\right\rangle=\left\langle c^{\prime}\left\langle c^{\prime \prime}, b^{\prime \prime}\right\rangle, b^{\prime}\right\rangle=\left\langle c^{\prime},\left\langle c^{\prime \prime}, b^{\prime \prime}\right\rangle b^{\prime}\right\rangle .
$$

The counit map $\varepsilon: C_{0} \longrightarrow K$ is obtained by applying the functor $\operatorname{Hom}_{K^{\text {op }}}(-, K)$ to the ring homomorphism $K \longrightarrow B^{0}$,

$$
\varepsilon(c)=\langle c, 1\rangle \text { for all } c \in C,
$$

where $1 \in B^{0}$ is the unit element.

Generalizing Remark 2.8 again, we put ${ }^{\#} B^{n}=\operatorname{Hom}_{K^{\text {op }}}\left(C_{n}, K\right)$ for every $n \geq 0$. The related pairing notation is

$$
\begin{gathered}
\left\langle{ }^{\#} b, c\right\rangle={ }^{\#} b(c) \quad \text { for all }{ }^{\#} b \in{ }^{\#} B^{n}, c \in C_{n}, n \geq 0, \\
\left\langle{ }^{\#} b, c\right\rangle=0 \quad \text { for all }{ }^{\#} b \in{ }^{\#} B^{i}, c \in C_{j}, i \neq j .
\end{gathered}
$$

Then ${ }^{\#} B^{n}$ is a $K$ - $K$-bimodule with the left and right actions of $K$ given by the usual rules

$$
\left\langle k^{\#} b, c\right\rangle=k\left\langle^{\#} b, c\right\rangle, \quad\left\langle{ }^{\#} b k, c\right\rangle=\left\langle{ }^{\#} b, k c\right\rangle \quad \text { for all } k \in K,{ }^{\#} b \in{ }^{\#} B^{n}, c \in C_{n} .
$$

Moreover, ${ }^{*} B=\bigoplus_{n=0}^{\infty}{ }^{\#} B^{n}$ is a nonnegatively graded ring with the multiplication given by the rule

$$
\left\langle{ }^{\#} b^{\prime \#} b^{\prime \prime}, c\right\rangle=\left\langle \#^{\prime} \otimes{ }^{\#} b^{\prime \prime}, \mu(c)\right\rangle \text { for all }{ }^{\#} b^{\prime},{ }^{\#} b^{\prime \prime} \in{ }^{\#} B, c \in C,
$$

where the pairing $\langle\rangle:,{ }^{\#} B^{j} \otimes_{K}{ }^{\#} B^{i} \otimes_{K} C_{i} \otimes_{K} C_{j} \longrightarrow K$ is defined by

$$
\left\langle \# b^{\prime \prime} \otimes{ }^{\#} b^{\prime}, c^{\prime} \otimes c^{\prime \prime}\right\rangle=\left\langle{ }^{\# \prime} b^{\prime \prime}\left\langle{ }^{\#} b^{\prime}, c^{\prime}\right\rangle, c^{\prime \prime}\right\rangle=\left\langle{ }^{\#} b^{\prime \prime},\left\langle{ }^{\#} b^{\prime}, c^{\prime}\right\rangle c^{\prime \prime}\right\rangle \text {. }
$$

Applying the functor $\operatorname{Hom}_{K^{\mathrm{op}}}(-, K)$ to the counit map $C_{0} \longrightarrow K$, we obtain a ring homomorphism $K \longrightarrow{ }^{\#} B^{0}$ (which, together with the graded ring structure on 
${ }^{\#} B$, induces the $K$ - $K$-bimodule structures on ${ }^{\#} B^{n}$ mentioned above). In the pairing notation, the formula

defines the unit element $1 \in{ }^{\#} B^{0}$

$$
\langle 1, c\rangle=\varepsilon(c) \quad \text { for all } c \in C
$$

Furthermore, the graded left $K$-module structure on $C=\bigoplus_{n>0} C^{n}$ extends naturally to a graded left ${ }^{\#} B$-module structure, making $C$ a graded ${ }^{\#} B$ - $B$-bimodule (as it was mentioned already in Remark 2.8). In the pairing notation, the right action of $B$ in $C$ (defined above) can be expressed in terms of the comultiplication in $C$ by the formula

$$
c b=\left\langle\mu_{i, j}(c), b\right\rangle \quad \text { for all } c \in C_{i+j} \text { and } b \in B^{j},
$$

where $\langle$,$\rangle denotes the map C_{i} \otimes_{K} C_{j} \otimes_{K} B^{j} \longrightarrow C_{i}$ induced by the pairing $C_{j} \otimes_{K}$ $B^{j} \longrightarrow K$. Similarly, the left action of ${ }^{\#} B$ in $C$ is defined by the formula

$$
{ }^{\#} b c=\left\langle{ }^{\#} b, \mu_{j, i}(c)\right\rangle \quad \text { for all } c \in C_{i+j} \text { and }{ }^{\#} b \in{ }^{*} B^{j},
$$

where $\langle$,$\rangle denotes the map { }^{\#} B^{j} \otimes_{K} C_{j} \otimes_{K} C_{i} \longrightarrow C_{i}$ induced by the pairing ${ }^{\#} B^{j} \otimes_{K}$ $C_{j} \longrightarrow K$.

By construction, we know that $C_{n}$ is a finitely generated projective left $K$-module for every $n \geq 0$. Now we make an additional assumption.

Proposition 8.12. Assume additionally that $C_{n}$ is a finitely generated projective right $K$-module for every $n \geq 0$. Then there is a natural bijective correspondence between $C D G$-ring structures $(B, d, h)$ on the graded ring $B$, viewed up to change-ofconnection isomorphisms $(i d, a):\left(B, d^{\prime}, h^{\prime}\right) \longrightarrow(B, d, h)$, and $C D G$-ring structures $\left({ }^{\#} B,{ }^{\#} d,{ }^{\#} h\right)$ on the graded ring ${ }^{\#} B$, viewed up to change-of-connection isomorphisms $\left(i d,{ }^{\#} a\right):\left({ }^{\#} B,{ }^{\#} d^{\prime},{ }^{\#} h^{\prime}\right) \longrightarrow\left({ }^{\#} B,{ }^{\#} d,{ }^{\#} h\right)$.

Proof. We use the result of Theorem 4.5 and identify CDG-ring structures $(B, d, h)$ on $B$ with quasi-differential structures $(\widehat{B}, \partial)$ (that is, quasi-differential graded rings $(\widehat{B}, \partial)$ endowed with an injective graded ring homomorphism $B \longrightarrow \widehat{B}$ with the image equal to ker $\partial \subset \widehat{B})$. Similarly, we identify CDG-ring structures $\left({ }^{{ }^{*}} B,{ }^{{ }^{*}} d,{ }^{{ }^{*}} h\right)$ on the graded ring ${ }^{\#} B$ with quasi-differential structures $\left({ }^{\#} \widehat{B}, \partial\right)$ on ${ }^{\#} B$, and construct a bijection between the isomorphism classes of $(\widehat{B}, \partial)$ and $(\# \widehat{B}, \partial)$.

Let $(\widehat{B}, \partial)$ be a quasi-differential structure on $B$. Recall that the differential $\partial: \widehat{B}^{n} \longrightarrow \widehat{B}^{n-1}$ is a morphism of $B^{0}-B^{0}$-bimodules ( since $\partial\left(B^{0}\right) \subset B^{-1}=0$ ), hence also a morphism of $K$-K-bimodules. Therefore, in view of the short exact sequences $0 \longrightarrow B^{n} \longrightarrow \widehat{B}^{n} \stackrel{\partial}{\longrightarrow} B^{n-1} \longrightarrow 0$, the graded right $K$-module $\widehat{B}^{n}$ is also finitely generated and projective for every $n \geq 0$.

Applying the above construction to the graded ring $\widehat{B}$, we produce a graded coring $\widehat{C}$ with the components $\widehat{C}_{n}=\widehat{C}^{-n}=\operatorname{Hom}_{K^{\text {op }}}\left(\widehat{B}^{n}, K\right)$. Moreover, the differential $\partial: \widehat{B}^{n} \longrightarrow \widehat{B}^{n-1}$ induces a differential $\partial: \widehat{C}_{n-1} \longrightarrow \widehat{C}_{n}, n \geq 0$, which we define with the sign rule so that

$$
\langle\partial(c), b\rangle+(-1)^{|c|}\langle c, \partial(b)\rangle=0 \quad \text { for all } c \in \widehat{C}^{|c|} \text { and } b \in \widehat{B}^{|b|} .
$$


By construction, $\partial: \widehat{C} \longrightarrow \widehat{C}$ is a homogeneous $K$ - $K$-bimodule map. As explained in the second proof of Theorem 4.19 in Section 4.6, it is also an odd coderivation of the coring $\widehat{C}$ (of degree -1 in the upper indices). Since $\partial^{2}=0$ on $\widehat{B}$, we also have $\partial^{2}=0$ on $\widehat{C}$. The inclusion of graded rings $B \longrightarrow \widehat{B}$ induces a graded coring homomorphism $\widehat{C} \longrightarrow C$, whose composition with the odd coderivation $\partial$ on $\widehat{C}$ obviously vanishes.

Furthermore, since the short exact sequences of $K$ - $K$-bimodules $0 \longrightarrow B^{n} \longrightarrow$ $\widehat{B}^{n} \stackrel{\partial}{\longrightarrow} B^{n-1} \longrightarrow 0$ are split exact as short sequences of right $K$-modules, we have short exact sequences of $K$ - $K$-bimodules $0 \longrightarrow C_{n-1} \longrightarrow \widehat{C}_{n} \longrightarrow C_{n} \longrightarrow 0$. So the graded coring homomorphism $\widehat{C} \longrightarrow C$ is surjective and its kernel is equal to the image of the odd coderivation $\partial$ on $C$, as well as to the kernel of the odd coderivation $\partial$.

Continuing to apply the above construction, we produce a graded ring $\# \widehat{B}$ with the components ${ }^{*} \widehat{B}^{n}=\operatorname{Hom}_{K^{\text {op }}}\left(\widehat{C}_{n}, K\right)$. The differential $\partial: \widehat{C}_{n-1} \longrightarrow \widehat{C}_{n}$ induces a differential $\partial:{ }^{\#} \widehat{B}^{n} \longrightarrow{ }^{\#} \widehat{B}^{n-1}$, which we define using the sign rule

$$
\left\langle\partial\left({ }^{\#} b\right), c\right\rangle+(-1)^{\left|\#_{b}\right|}\left\langle \#_{b}, \partial(c)\right\rangle=0 \quad \text { for all }{ }^{\#} b \in{ }^{\#} \widehat{B}^{\#_{b} \mid} \text { and } c \in \widehat{C}^{|c|} \text {. }
$$

The map $\partial:{ }^{\#} \widehat{B} \longrightarrow{ }^{\#} \widehat{B}$ is an odd derivation of degree -1 on the graded ring ${ }^{\#} \widehat{B}$. Since $\partial^{2}=0$ on $\widehat{C}$, we also have $\partial^{2}=0$ on $\# \widehat{B}$. The surjective homomorphism of graded corings $\widehat{C} \longrightarrow C$ induces an injective homomorphism of graded rings ${ }^{\#} B \longrightarrow$ $\# \widehat{B}$, whose composition with the coderivation $\partial$ on $\# \widehat{B}$ vanishes.

By the additional assumption, all the $K$ - $K$-bimodules in the short exact sequence $0 \longrightarrow C_{n-1} \longrightarrow \widehat{C}_{n} \longrightarrow C_{n} \longrightarrow 0$ are finitely generated and projective as right $K$-modules, so the sequence splits as a short exact sequence of right $K$-modules. Hence we have short exact sequnces of $K$ - $K$-bimodules $0 \longrightarrow{ }^{*} B^{n} \longrightarrow{ }^{*} \widehat{B}^{n} \longrightarrow$ ${ }^{\#} B^{n-1} \longrightarrow 0$. Thus the kernel of the odd derivation $\partial$ on ${ }^{\#} \widehat{B}$ is equal to the image of the injective ring homomorphism ${ }^{*} B \longrightarrow{ }^{*} \widehat{B}$, as well as the to the image of the odd derivation $\partial:{ }^{\#} \longrightarrow{ }^{\#} \widehat{B}$.

We have constructed the quasi-differential graded ring $(\# \widehat{B}, \partial)$ corresponding to a quasi-differential graded ring $(\widehat{B}, \partial)$. To check that this is a bijective correspondence, it suffices to reverse the construction, recovering the graded coring $\widehat{C}$ with its odd coderivation $\partial$ as the graded Hom bimodule $\widehat{C}=\operatorname{Hom}_{K}\left({ }^{\sharp} \widehat{B}, K\right)$, and the graded ring $\widehat{B}$ with its odd derivation $\partial$ as the graded Hom group/bimodule $\widehat{B}=\operatorname{Hom}_{K}(\widehat{C}, K)$.

Lemma 8.13. In the context of Proposition 8.12, for any pair of CDG-ring structures $B=(B, d, h)$ and ${ }^{*} B=\left({ }^{\#} B,{ }^{*} d,{ }^{*} h\right)$ corresponding to each other under the construction of the proposition, there is a natural structure of CDG-bimodule over ${ }^{\#} B$ and $B$ on the graded ${ }^{\#} B$-B-bimodule $C$.

Proof. Saying that $B=(B, d, h)$ and ${ }^{\#} B=\left({ }^{\#} B,{ }^{\#} d,{ }^{*} h\right)$ correspond to each other under the construction of Proposition 8.12 means the following. Let $(\widehat{B}, \partial)$ and $\left({ }^{\#} \widehat{B}, \partial\right)$ be a pair of quasi-differential graded rings corresponding to each other under the construction from the proof of the proposition. (This presumes that $\widehat{B}$ and ${ }^{\#} \widehat{B}$ 
are nonnegatively graded, and that we are given ring homomorphisms $K \longrightarrow \widehat{B}^{0}$ and $K \longrightarrow{ }^{\#} \widehat{B}^{0}$ such that $\widehat{B}^{n}$ is a finitely generated projective right $K$-module and ${ }^{\#} \widehat{B}^{n}$ is a finitely generated projective left $K$-module for every $n \geq 0$.)

Choose arbitrary (unrelated) elements $\delta \in \widehat{B}^{1}$ such that $\partial(\delta)=1$ in $\widehat{B}^{0}$ and ${ }^{\#} \delta \in{ }^{\#} \widehat{B}^{1}$ such that $\partial\left({ }^{\#} \delta\right)=1$ in ${ }^{\#} \widehat{B}^{0}$. Define the differential $d$ on $B=\operatorname{ker} \partial \subset \widehat{B}$ and the curvature element $h \in B^{2}$ as in the proof of Theorem 4.5,

$$
d(b)=[\delta, b] \text { for all } b \in B, \quad \text { and } \quad h=\delta^{2},
$$

where the bracket $[$,$] denotes the graded commutator. Similarly, consider the graded$ ring ${ }^{\#} B=\operatorname{ker} \partial \subset{ }^{\#} \widehat{B}$, and put

$$
{ }^{\#} d\left({ }^{\#} b\right)=\left[{ }^{\#} \delta,{ }^{\#} b\right] \text { for all }{ }^{\#} b \in{ }^{\#} B, \text { and }{ }^{\#} h={ }^{\#} \delta^{2} .
$$

In this context, we define the differential $d_{\widehat{C}}$ on the graded $\# \widehat{B}$ - $\widehat{B}$-bimodule $\widehat{C}$ by the rule

$$
d_{\widehat{C}}(c)={ }^{\#} \delta c-(-1)^{|c|} c \delta \quad \text { for all } c \in \widehat{C} .
$$

The claim is that the map $d_{\widehat{C}}: \widehat{C} \longrightarrow \widehat{C}$ induces a well-defined map $d_{C}: C \longrightarrow C$. In other words, there exists an (obviously unique) homogeneous map $d_{C}: C \longrightarrow C$ (of degree 1 in the upper indices) forming a commutative square diagram with the map $d_{\widehat{C}}$ and the surjective coring homomorphism $\widehat{C} \longrightarrow C$.

In order to prove this assertion, we will check that $d_{\widehat{C}}$ preserves the kernel $\partial(C)$ of the surjective map $\widehat{C} \longrightarrow C$, that is $d_{\widehat{C}}(\partial(C)) \subset \partial(C)$. Moreover, we will show that the two differentials $\partial$ and $d_{\widehat{C}}$ on $\widehat{C}$ anti-commute, that is

$$
\partial \circ d_{\widehat{C}}+d_{\widehat{C}} \circ \partial=0 \text { on } \widehat{C} \text {. }
$$

First of all, let us check that the differential $\partial$ on the graded right $\widehat{B}$-module $\widehat{C}$ is an odd derivation compatible with the odd derivation $\partial$ on $\widehat{B}$, that is

$$
\partial(c b)=\partial(c) b+(-1)^{|c|} c \partial(b) \in \widehat{C} \quad \text { for all } c \in \widehat{C}^{|c|} \text { and } b \in \widehat{B}^{|b|} .
$$

Indeed, for any $b^{\prime} \in \widehat{B}$ we have

$$
\begin{gathered}
\left\langle\partial(c b), b^{\prime}\right\rangle=-(-1)^{|c|+|b|}\left\langle c b, \partial\left(b^{\prime}\right)\right\rangle=-(-1)^{|c|+|b|}\left\langle c, b \partial\left(b^{\prime}\right)\right\rangle \\
=-(-1)^{|c|}\left\langle c, \partial\left(b b^{\prime}\right)\right\rangle+(-1)^{|c|}\left\langle c, \partial(b) b^{\prime}\right\rangle \\
=\left\langle\partial(c), b b^{\prime}\right\rangle+(-1)^{|c|}\left\langle c, \partial(b) b^{\prime}\right\rangle=\left\langle\partial(c) b, b^{\prime}\right\rangle+(-1)^{|c|}\left\langle c \partial(b), b^{\prime}\right\rangle .
\end{gathered}
$$

Similarly one can check that the differential $\partial$ on the graded left $\# \widehat{B}$-module $\widehat{C}$ is also an odd derivation compatible with the odd derivation $\partial$ on ${ }^{*} \widehat{B}$, that is

$$
\partial\left(^{\#} b c\right)=\partial\left({ }^{\#} b\right) c+(-1)^{\left|{ }^{\#} b\right| \#_{b}} \partial(c) \in \widehat{C} \quad \text { for all }{ }^{\#} b \in{ }^{\#} \widehat{B}^{\left|{ }^{\#}\right|} \text { and } c \in \widehat{C}^{|c|} \text {. }
$$

Now for any $c \in \widehat{C}$ we have

$$
\partial\left({ }^{\#} \delta c\right)=\partial\left({ }^{\#} \delta\right) c-{ }^{\#} \delta \partial(c)=c-{ }^{\#} \delta \partial(c)
$$

and

$$
\partial(c \delta)=\partial(c) \delta+(-1)^{|c|} c \partial(\delta)=(-1)^{|c|} c+\partial(c) \delta
$$


hence

$$
\partial\left(d_{\widehat{C}}(c)\right)=\partial\left({ }^{\#} \delta c-(-1)^{|c|} c \delta\right)=-{ }^{\#} \delta \partial(c)-(-1)^{|c|} \partial(c) \delta=-d_{\widehat{C}}(\partial(c)),
$$

and the equation (81) is deduced.

Thus the desired differential $d_{C}: C \longrightarrow C$ is well-defined. Checking that $d_{C}$ is an odd derivation on the graded left ${ }^{\#} B$-module $C$ and the graded right $B$-module $C$ compatible with the odd derivations ${ }^{\#} d=\left[{ }^{\#} \delta,-\right]$ on ${ }^{\#} B$ and $d=[\delta,-]$ on $B$ is easy. The same applies to checking the equation $d_{C}^{2}(c)={ }^{\#} h c-c h$ for all $c \in C$.

8.5. Comodule-contramodule correspondence. We formulate two lemmas of rather general character before specializing to the situation we are interested in.

Lemma 8.14. Let $B=\bigoplus_{n=0}^{\infty} B^{n}$ be a nonnegatively graded ring, and let $K$ be an associative ring endowed with a ring homomorphism $K \longrightarrow B^{0}$. Then

(a) For any graded left $K$-module $L$ and any graded left $B$-comodule $M$, the graded abelian group of graded left $B$-comodule (or graded left $B$-module) Hom from $M$ to the coinduced graded left B-comodule $\operatorname{Hom}_{K}^{\Sigma}(B, L)$ can be computed as

$$
\operatorname{Hom}_{B}\left(M, \operatorname{Hom}_{K}^{\Sigma}(B, L)\right) \simeq \operatorname{Hom}_{K}(L, M) .
$$

(b) For any graded left $K$-module $L$ and any graded right $B$-comodule $N$, the contratensor product of $N$ with the induced graded left $B$-contramodule $\mathbb{M}_{K}^{\text {gr }}(L)=$ $B \otimes_{K}^{\Pi} L$ can be computed as

$$
N \odot_{B}\left(B \otimes_{K}^{\Pi} L\right) \simeq N \otimes_{K} L .
$$

Proof. The natural isomorphism of graded abelian groups in part (a) follows from the similar natural isomorphism for the graded Hom group of graded $B$-modules, $\operatorname{Hom}_{B}\left(M, \operatorname{Hom}_{K}(B, L)\right) \simeq \operatorname{Hom}_{K}(L, M)$, together with Lemma 6.6.

To construct the natural isomorphism of graded abelian groups in part (b), let $U$ be a graded abelian group. Then we have natural isomorphisms

$$
\begin{aligned}
& \operatorname{Hom}_{\mathbb{Z}}\left(N \odot_{B}\left(B \otimes_{K}^{\Pi} L\right), U\right) \stackrel{8.2}{\stackrel{8.2}{\simeq}} \operatorname{Hom}^{B}\left(B \otimes_{K}^{\Pi} L, \operatorname{Hom}(N, U)\right) \\
& \stackrel{7.4}{\sim} \operatorname{Hom}_{K}(L, \operatorname{Hom}(N, U)) \simeq \operatorname{Hom}_{K}\left(N \otimes_{K} L, U\right) \text {, }
\end{aligned}
$$

where the first isomorphism is provided by Lemma 8.2 (applied to the graded $\operatorname{ring} E=$ $\mathbb{Z}$ concentrated in degree 0 ), while the second one holds in view of the discussion in Section 5.2 or by Lemma 7.4. It remains to say that any isomorphism of representable functors comes from a unique isomorphism of the representing objects. (In fact, the isomorphism we have constructed is provided by the map $N \otimes_{K} L \longrightarrow N \odot_{B}\left(B \otimes_{K} L\right)$ induced by the unit element of $B$.)

Lemma 8.15. Let $E=\left(E, d_{E}, h_{E}\right)$ be a $C D G$-ring and $B=(B, d, h)$ be a nonnegatively graded $C D G$-ring. Let $N=\left(N, d_{N}\right)$ be a $C D G$-bimodule over $E$ and $B$ whose underlying graded right $B$-module is a graded right $B$-comodule. Then

(a) for any left $C D G$-module $M=\left(M, d_{M}\right)$ over $E$, the graded left B-contramodule $\operatorname{Hom}_{E}(N, M)$ constructed in Example 5.23 endowed with the differential of the left 
$C D G$-module $\operatorname{Hom}_{E}(N, M)$ over $B$ constructed in Section 6.1 is a left CDG-contramodule over $B$ in the sense of the definition in Section 7.3.

(b) for any left $C D G$-contramodule $\left(Q, d_{Q}\right)$ over $B$, the differential of the left $C D G$-module $N \otimes_{B} Q$ over $E$ constructed in Section 6.1 induces a well-defined differential on the graded quotient E-module $N \odot_{B} Q$ of $N \otimes_{B} Q$ constructed in Section 8.1, making $N \odot_{B} Q$ a left $C D G$-module over $E$.

Proof. The proofs of both (a) and (b) are straightforward.

Now we assume that $B=(B, d, h)$ and ${ }^{*} B=\left({ }^{*} B,{ }^{*} d,{ }^{*} h\right)$ are two CDG-rings corresponding to each other under the construction of Proposition 8.12, This means, in particular, that $B=\bigoplus_{n=0}^{\infty} B^{n}$ and ${ }^{\#} B=\bigoplus_{n=0}^{\infty}{ }^{\#} B^{n}$ are nonnegatively graded, that we are given a ring $K$ together with ring homomorphisms $K \longrightarrow B^{0}$ and $K \longrightarrow{ }^{\#} B^{0}$, that the right $K$-module $B^{n}$ is finitely generated and projective for every $n \geq 0$, and that the left $K$-module ${ }^{\#} B^{n}$ is finitely generated and projective for every $n \geq 0$.

The following theorem is the first main result of Section 8. It is a generalization of [28, Theorem B.3]. It also solves a particular case of the problem posed in [27, Question at the end of Section 11].

Theorem 8.16. Assume additionally that the left homological dimension of the ring $K$ is finite. Then there is a natural triangulated equivalence between the coderived category of left $C D G$-comodules over ${ }^{\#} B=\left({ }^{\#} B,{ }^{*} d,{ }^{\#} h\right)$ and the contraderived category of left $C D G$-contramodules over $(B, d, h)$,

$$
\mathbb{R} \operatorname{Hom}_{\#_{B}}(C,-): \mathrm{D}^{\mathrm{co}}\left({ }^{\#} B-\text { comod }\right) \simeq \mathrm{D}^{\text {ctr }}(B \text {-contra }): C \odot_{B}^{\mathbb{L}}-,
$$

provided by derived functors of comodule Hom and contratensor product with the $C D G$-bi(co)module $\left(C, d_{C}\right)$ over ${ }^{\#} B$ and $B$ constructed in Lemma 8.13.

Proof. First of all, the CDG-bimodule $\left(C, d_{C}\right)$ is nonpositively cohomologically graded. According to the discussion in Section [5.5, it follows that $C$ is a graded left ${ }^{\#} B$-comodule and a graded right $B$-comodule.

The DG-functor

$$
\operatorname{Hom}_{\#_{B}}(C,-): \mathrm{DG}\left({ }^{\#} B-\text { comod }\right) \longrightarrow \mathrm{DG}(B-\text { contra })
$$

assigns to a left CDG-comodule $M$ over $\left({ }^{\#} B,{ }^{*} d,{ }^{\#} h\right)$ the graded left $B$-contramodule $\operatorname{Hom}_{\#_{B}}(C, M)$, as constructed in Example 5.23, endowed with the structure of a left CDG-contramodule over $(B, d, h)$ described in Lemma 8.15(a). Since $\operatorname{Hom}_{\#_{B}}(C,-)$ is a DG-functor DG( ${ }^{\#} B$-mod $) \longrightarrow \mathrm{DG}(B$-mod) according to the discussion in Section 6.1, it follows that it is also a DG-functor $\mathrm{DG}\left({ }^{\#} B\right.$-comod $) \longrightarrow \mathrm{DG}(B$-contra $)$.

The DG-functor

$$
C \odot_{B}-: \mathrm{DG}(B \text {-contra }) \longrightarrow \mathrm{DG}\left({ }^{\#} B \text {-comod }\right)
$$

assigns to a left CDG-contramodule $Q$ over $(B, d, h)$ the graded abelian group of the contratensor product $C \odot_{B} Q$, as constructed in Section 8.1, endowed with the obvious graded left ${ }^{\#} B$-comodule structure induced by the graded left ${ }^{\#} B$-comodule structure on $C$ and with the structure of a left CDG-comodule over $\left({ }^{*} B,{ }^{\#} d,{ }^{*} h\right)$ described 
in Lemma 8.15(b). Since $C \otimes_{B}-$ is a DG-functor $\mathrm{DG}(B-\bmod ) \longrightarrow \mathrm{DG}\left({ }^{\#} B\right.$-mod $)$ according to the discussion in Section 6.1, it follows that $C \odot_{B}-$ is a DG-functor $\mathrm{DG}(B$-contra $) \longrightarrow \mathrm{DG}\left({ }^{\#} B\right.$-comod $)$.

The adjunction of Lemma 8.2 makes the DG-functors (83) and (84) adjoint to each other. Hence the induced triangulated functors between the homotopy categories

$$
\operatorname{Hom}_{\#_{B}}(C,-): \operatorname{Hot}\left({ }^{\#} B-\text { comod }\right) \longrightarrow \operatorname{Hot}(B-\text { contra })
$$

and

$$
C \odot_{B}-: \operatorname{Hot}(B-\text { contra }) \longrightarrow \operatorname{Hot}\left({ }^{\#} B-\text { comod }\right)
$$

are also adjoint.

In order to construct the derived functor $\mathbb{R} \operatorname{Hom}_{\#_{B}}(C,-): \mathrm{D}^{\mathrm{co}}\left({ }^{\#} B\right.$-comod $) \longrightarrow$ $\mathrm{D}^{\mathrm{ctr}}\left(B\right.$-contra), we restrict the triangulated functor $\operatorname{Hom}_{\#_{B}}(C,-)$ (85) to the full triangulated subcategory of CDG-comodules of the coinduced type

$$
\operatorname{Hot}\left({ }^{\#} B \text {-comod } K \text {-coind }\right) \subset \operatorname{Hot}\left({ }^{\#} B \text {-comod }\right) \text {, }
$$

which was defined in Section 8.3. Similarly, in order to construct the derived functor $C \odot_{B}^{\mathbb{L}}-: \mathrm{D}^{\mathrm{ctr}}(B$-contra $) \longrightarrow \mathrm{D}^{\mathrm{co}}(B$-comod $)$, we restrict the triangulated functor $C \odot_{B}-(86)$ to the full triangulated subategory of CDG-contramodules of the induced type

$$
\operatorname{Hot}\left(B \text { - } \text { contra }_{K \text {-ind }}\right) \subset \operatorname{Hot}(B \text {-contra }),
$$

which was defined in Section 8.2 .

Now it is claimed that the functors $\operatorname{Hom}_{\#_{B}}(C,-)$ and $C \odot_{B}-$ take the full subcategories $\operatorname{Hot}\left({ }^{\#} B\right.$-comod $K$-coind $) \subset \operatorname{Hot}\left({ }^{\#} B\right.$-comod $)$ and $\operatorname{Hot}(B$-contra $K$-ind $) \subset$ Hot $(B$-contra) into each other and induce mutually inverse triangulated equivalences between them

$$
\operatorname{Hom}_{\#_{B}}(C,-): \operatorname{Hot}\left({ }^{\#} B-\operatorname{comod}_{K \text {-coind }}\right) \simeq \operatorname{Hot}\left(B-\text { contra }_{K \text {-ind }}\right): C \odot_{B}-.
$$

Moreover, we have mutually inverse DG-equivalences

$$
\operatorname{Hom}_{\#_{B}}(C,-): \mathrm{DG}\left({ }^{\#} B-\text { comod }_{K \text {-coind }}\right) \simeq \mathrm{DG}(B \text {-contra } K \text {-ind }): C \odot_{B}-.
$$

It suffices to check the latter assertion on the level of the full subcategory of coinduced graded comodules ${ }^{\#} B$ - comod $_{K \text {-coind }} \subset{ }^{\#} B$-comod in the abelian category of graded left ${ }^{\#} B$-comodules ${ }^{\#} B$-comod and the full subcategory of induced graded contramodules $B$-contra $K$-ind $\subset B$-contra in the abelian category of graded left contramodules $B$-contra. So we need to check that the adjunction of Lemma 8.2 restricts to a pair of mutually inverse equivalences of additive categories

$$
\operatorname{Hom}_{\#_{B}}(C,-):{ }^{\#} B-\text { comod }_{K \text {-coind }} \simeq B \text {-contra } K \text {-ind }: C \odot_{B}-\text {. }
$$

This amounts to a simple computation based on Lemma 8.14. To show that two adjoint functors are mutually inverse equivalences, it suffices to check that the adjunction morphisms (i. e., the adjunction unit and counit) are isomorphisms. 
Let $L$ be a graded left $K$-module, $\operatorname{Hom}_{K}^{\Sigma}\left({ }^{\#} B, L\right)$ be the graded left ${ }^{\#} B$-comodule coinduced from $L$, and $B \otimes_{K}^{\Pi} L$ be the graded left $B$-contramodule induced from $L$. Then we have

$$
\operatorname{Hom}_{\#_{B}}\left(C, \operatorname{Hom}_{K}^{\Sigma}\left({ }^{\#} B, L\right)\right) \stackrel{8.14}{\simeq} \text { a) } \operatorname{Hom}_{K}(C, L) \simeq \operatorname{Hom}_{K}(C, K) \otimes_{K}^{\Pi} L=B \otimes_{K}^{\Pi} L,
$$
since $C^{-n}=\operatorname{Hom}_{K^{\mathrm{op}}}\left(B^{n}, K\right)$ is a finitely generated projective left $K$-module for every $n \geq 0$. Similarly,

$$
\left.C \odot_{B}\left(B \otimes_{K}^{\Pi} L\right) \stackrel{8.14}{\simeq} \mathrm{b}\right) \quad C \otimes_{K} L \simeq \operatorname{Hom}_{K}^{\Sigma}\left(\operatorname{Hom}_{K^{\mathrm{op}}}(C, K), L\right)=\operatorname{Hom}_{K}^{\Sigma}\left({ }^{\#} B, L\right),
$$

since $C^{-n}=\operatorname{Hom}_{K}\left({ }^{\#} B^{n}, K\right)$ is a finitely generated projective right $K$-module for every $n \geq 0$. This finishes the proof of the triangulated equivalence (87).

Finally, in order to show that the triangulated equivalence (87) descends to the desired triangulated equivalence (82), we use the descriptions of the coderived category $\mathrm{D}^{\mathrm{co}}\left({ }^{\#} B\right.$-comod) and the contraderived category $\mathrm{D}^{\mathrm{ctr}}(B$-contra $)$ provided by Theorems 8.11 and 8.7 together with Propositions 8.10 and 8.6 .

Specifically, by Proposition 8.10 and the second assertion of Theorem 8.11, the coderived category $\mathrm{D}^{\mathrm{co}}\left({ }^{\#} B\right.$-comod) is equivalent to the triangulated quotient category of $\operatorname{Hot}\left({ }^{\#} B-\right.$ comod $\left._{K \text {-coind }}\right)$ by its minimal triangulated subcategory containing all the totalizations of left CDG-comodule short exact sequences of the coinduced type over $\left({ }^{\#} B,{ }^{\#} d,{ }^{\#} h\right)$. Similarly, by Proposition 8.6 and the second assertion of Theorem 8.7, the contraderived category $\mathrm{D}^{\mathrm{ctr}}(B$-contra) is equivalent to the triangulated quotient category of $\operatorname{Hot}(B$-contra $K$-ind $)$ by its minimal triangulated subcategory containing all the totalizations of left CDG-contramodule short exact sequences of the induced type over $(B, d, h)$.

In view of these results, it remains to observe that the DG-functors $\operatorname{Hom}_{\#_{B}}(C,-)$ and $C \odot_{B}$ - take CDG-comodule short exact sequences of the coinduced type over $\left({ }^{\#} B,{ }^{\#} d,{ }^{\#} h\right)$ to $C D G$-contramodule short exact sequences of the induced bype over $(B, d, h)$ and back. This is also an assertion on the level of the additive categories ${ }^{\#} B$ - comod $_{K \text {-coind }}$ and $B$-contra $K$-ind, and it follows from the above computation based on Lemma 8.14.

Let us recall that, whenever the forgetful functor $B$-contra $\mathrm{gr}_{\mathrm{gr}} \longrightarrow B-\bmod _{\mathrm{gr}}$ is fully faitfhul, the contratensor product functor $C \odot_{B}$ - appearing in the above theorem is isomorphic to the tensor product functor $C \otimes_{B}-$ by Proposition 8.3(b). In particular, by Theorem 5.26, this holds whenever the augmentation ideal $B^{\geq 1}=\bigoplus_{n=1}^{\infty} B^{n}$ is finitely generated as a right ideal in $B$.

8.6. Two-sided finitely projective Koszul rings. Let $A=\bigoplus_{n=0}^{\infty} A_{n}$ be a nonnegatively graded ring with the degree-zero component $R=A_{0}$. We will say that $A$ is two-sided finitely projective Koszul if it is both left finitely projective Koszul and right finitely projective Koszul (in the sense of Section 2.10).

Let $A$ be a two-sided finitely projective Koszul graded ring. Denote by $B$ the right finitely projective Koszul graded ring quadratic dual to the left finitely projective Koszul ring $A$, and denote by ${ }^{\#} B$ the left finitely projective Koszul gradedring 
quadratic dual to the right finitely projective Koszul ring $A$ (as in Section 1 and Proposition 2.23). So we have

$$
B=\operatorname{Ext}_{A}(R, R)^{\mathrm{op}} \quad \text { and } \quad{ }^{\#} B=\operatorname{Ext}_{A^{\mathrm{op}}}(R, R),
$$

where $\operatorname{Ext}_{A}$ is taken in the category of graded left $A$-modules and $\operatorname{Ext}_{A^{\text {op }}}$ in the category of graded right $A$-modules, as usually.

Lemma 8.17. For any two-sided finitely projective Koszul graded ring $A$, the nonnegatively graded rings $B$ and ${ }^{\#} B$ are related to each other by the construction of Remark 2.8 and Section 8.4 (with $K=R$ ). In other words, the grading components of $B$ and ${ }^{\#} B$ can be obtained from each other as the $R$ - $R$-bimodules

$$
{ }^{\#} B^{n}=\operatorname{Hom}_{R^{\text {op }}}\left(\operatorname{Hom}_{R^{\text {op }}}\left(B^{n}, R\right), R\right) \quad \text { and } \quad B^{n}=\operatorname{Hom}_{R}\left(\operatorname{Hom}_{R}\left({ }^{\#} B^{n}, R\right), R\right)
$$

for every $n \geq 0$, and the multiplicative structures are related accordingly.

Proof. Put $V=A_{1}$, and denote by $I \subset V \otimes_{R} V$ the kernel of the multiplication map $A_{1} \otimes_{R} A_{1} \longrightarrow A_{2}$. The graded $R$ - $R$-bimodule $C$ with the components

$$
C_{n}=\operatorname{Tor}_{n}^{A}(R, R)=\operatorname{Tor}_{n, n}^{A}(R, R)
$$

plays a key role. By Proposition 2.1(b), we have $C_{0}=R, C_{1}=V, C_{2}=I$, and

$$
C_{n}=\bigcap_{k=1}^{n-1} V^{\otimes_{R} k-1} \otimes_{R} I \otimes_{R} V^{\otimes_{R} n-k-1} \subset V^{\otimes_{R} n}, \quad n \geq 2 .
$$

Since $A$ is a left finitely projective Koszul graded ring, Theorem 2.21(c) tells, in particular, that $C_{n}$ is a finitely generated projective left $R$-module for every $n \geq 0$. As $A$ is also a right finitely projective Koszul graded ring, $C_{n}$ is also a finitely generated projective right $R$-module.

According to Remark 2.3, $C$ has a natural graded coring structure. The grading components of the comultiplication map $\mu_{i, j}: C_{i+j} \longrightarrow C_{i} \otimes_{R} C_{j}, i, j \geq 0$, can be described explicitly as the $R$ - $R$-subbimodule inclusions

$$
\begin{aligned}
C_{i+j}=\bigcap_{k=1}^{i+j-1} V^{\otimes_{R} k-1} & \otimes_{R} I \otimes_{R} V^{\otimes_{R} i+j-k-1} \\
& \longrightarrow \bigcap_{1 \leq k \leq i+j-1}^{k \neq i} V^{\otimes_{R} k-1} \otimes_{R} I \otimes_{R} V^{\otimes_{R} i+j-k-1} \simeq C_{i} \otimes_{R} C_{j},
\end{aligned}
$$

where the latter isomorphism holds by Lemma 2.15)(b,d,e).

Computing the Ext in terms of the relative cobar complex as in Proposition 2.2 , one obtains the isomorphisms

$$
B^{n}=\operatorname{Ext}_{A}^{n}(R, R)=\operatorname{Ext}_{A}^{n, n}(R, R) \simeq \operatorname{Hom}_{R}\left(C_{n}, R\right)
$$

and

$$
{ }^{\#} B^{n}=\operatorname{Ext}_{A^{\text {op }}}^{n}(R, R)=\operatorname{Ext}_{A^{\text {op }}}^{n, n}(R, R) \simeq \operatorname{Hom}_{R^{\text {op }}}\left(C_{n}, R\right),
$$

and these isomorphisms are compatible with the multiplicative structures. 
Now let $(\widetilde{A}, F)$ be an associative ring with an increasing filtration $0=F_{-1} \widetilde{A} \subset$ $R=F_{0} \widetilde{A} \subset \widetilde{V}=F_{1} \widetilde{A} \subset F_{2} \widetilde{A} \subset \ldots$. We recall that the filtered $\operatorname{ring}(\widetilde{A}, F)$ is said to be left finitely projective nonhomogeneous Koszul if the graded $\operatorname{ring} A=\operatorname{gr}^{F} \widetilde{A}=$ $\bigoplus_{n=0}^{\infty} F_{n} \widetilde{A} / F_{n-1} \widetilde{A}$ is left finitely projective Koszul (see Section 4.6).

Dually, let us say that $(\widetilde{A}, F)$ is right finitely projective nonhomogeneous Koszul if $\left(\widetilde{A}^{\text {op }}, F^{\text {op }}\right.$ ) is left finitely projective nonhomogeneous Koszul, that is, in other words, the graded ring $A$ is right finitely projective Koszul. We will say that $(\widetilde{A}, F)$ is twosided finitely projective nonhomogeneous Koszul if it is both left and right finitely projective nonhomogeneous Koszul; in other words, this means that the graded ring $A$ is two-sided finitely projective Koszul.

Let $B$ and ${ }^{\#} B$ be the Koszul dual rings to $A$ on the two sides, as in Lemma 8.17. Recall the notation $\tau^{\prime}$ introduced in Section 6.5 for a left $R$-linear splitting

$$
\operatorname{Hom}_{R^{\mathrm{op}}}\left(B^{1}, R\right) \simeq F_{1} \widetilde{A} / F_{0} \widetilde{A}=V \hookrightarrow \widetilde{V}=F_{1} \widetilde{A}
$$

of the surjective $R$ - $R$-bimodule map $\widetilde{V}=F_{1} \widetilde{A} \longrightarrow F_{1} \widetilde{A} / F_{0} \widetilde{A}=V$. Such a splitting was first considered (under the assumption of projectivity of the left $R$-module $V$, guaranteeing its existence) in Section 3.3.

For a two-sided finitely projective nonhomogeneous Koszul ring $\widetilde{A}$, one can also choose a right $R$-linear splitting $V \longrightarrow \widetilde{V}$ of the surjective $R$ - $R$-bimodule map $\widetilde{V} \longrightarrow$ $\widetilde{V} / R=V$. Denote by $\sigma^{\prime}$ the resulting right $R$-module map

$$
\operatorname{Hom}_{R}\left({ }^{\#} B^{1}, R\right) \simeq F_{1} \widetilde{A} / F_{0} \widetilde{A}=V \hookrightarrow \widetilde{V}=F_{1} \widetilde{A} .
$$

Having chosen a one-sided splitting $\tau^{\prime}$, one can apply the construction of Sections 3.3 3.4 to the left finitely projective nonhomogeneous Koszul ring $\widetilde{A}$, and produce a CDG-ring structure $(B, d, h)$ on the right finitely projective Koszul graded ring $B$. Having chosen a splitting $\sigma^{\prime}$ on the other side, one can apply the opposite version of the same construction (with the left and right sides switched) to the right finitely projective nonhomogeneous Koszul ring $\widetilde{A}$, and produce a CDG-ring structure $\left({ }^{\#} B,{ }^{\#} d,{ }^{\#} h\right)$ on the left finitely projective Koszul graded ring ${ }^{\#} B$.

Lemma 8.18. For any two-sided finitely projective nonhomogeneous Koszul ring $\widetilde{A}$, the nonnegatively graded $C D G$-rings $(B, d, h)$ and $\left({ }^{*} B,{ }^{*} d,{ }^{*} h\right)$ are related by the construction of Proposition 8.12 (with $K=R$ ).

Proof. We use the interpretation of nonhomogeneous quadratic duality in terms of quasi-differential corings, as formulated in Theorem 4.17. The graded ring $\widehat{A}=$ $\bigoplus_{n=0}^{\infty} \widetilde{A}_{n}$ is left finitely projective Koszul by Lemma 4.2 and Theorem 4.3 . By the opposite assertion, the graded ring $\widehat{A}$ is also right finitely projective Koszul.

Let $\widehat{B}$ be the right finitely projective Koszul graded ring quadratic dual to the left finitely projective Koszul graded ring $\widehat{A}$, and let ${ }^{\widehat{B}}$ be the left finitely projective Koszul graded ring quadratic dual to the right finitely projective Koszul graded ring $\widehat{A}$. By Lemma 8.17] applied to the two-sided finitely projective Koszul graded 
ring $\widehat{A}$, the nonnegatively graded rings $\widehat{B}$ and $\# \widehat{B}$ are related to each other by the construction from the beginning of Section 8.4.

It remains to observe that the acyclic odd derivations $\partial$ of degree -1 on the graded rings $\widehat{B}$ and $\# \widehat{B}$, both originating from the canonical central element $t \in \widehat{A}_{1}$ via the construction of Lemma 4.11(b) and its opposite version, correspond to each other under the construction from the proof of Proposition 8.12.

8.7. Bimodule resolution revisited. Now, under the more restrictive assumption of two-sided (rather than just left) finite projective Koszulity, we can offer an explanation of the "twisting cochain $\sigma^{\prime}$ notation" of Sections 6.5 and 7.4. Let us start with the "twisting cochain $\sigma$ ", which is the homogeneous Koszul version (it was briefly mentioned in the proofs of Theorems 6.7 and 7.6).

Let $A$ be a left finitely projective Koszul graded ring and $B$ be the quadratic dual right finitely projective Koszul graded ring. In this context, in the discussion of homogeneous Koszul complexes in Sections 2.5 2.6, the symbol $\tau$ was used as a notation for the structure isomorphism of quadratic duality

$$
\tau: \operatorname{Hom}_{R^{\mathrm{op}}}\left(B^{1}, R\right) \stackrel{\simeq}{\longrightarrow} A_{1} .
$$

Assume that $A$ is also a right finitely projective Koszul graded ring, and let ${ }^{\#} B$ be the quadratic dual left finitely projective Koszul graded ring. Denote by $\sigma$ the structure isomorphism

$$
\sigma: \operatorname{Hom}_{R}\left({ }^{\#} B^{1}, R\right) \stackrel{\simeq}{\longrightarrow} A_{1} .
$$

Moreover, in the context of the discussion of the dual Koszul complex $K_{e}^{\vee} \bullet(B, A)=$ $\left(B \otimes_{R} A, d_{e}\right)$ in Section 2.6, we denoted by $e \in B^{1} \otimes_{R} A_{1}$ the element corresponding to the map $\tau$ under the construction of Lemma 2.5. Let us now denote by $u \in$ $A_{1} \otimes_{R}{ }^{\#} B^{1}$ the element corresponding to the map $\sigma$ under the opposite version of the same construction. Denote the related dual Koszul complex by $K_{u}^{\vee} \cdot\left(A,{ }^{\#} B\right)=$ $\left(A \otimes_{R}{ }^{\#} B, d_{u}\right)$.

For any left $A$-module $M$ and any graded right $B$-module $N$ (which we would prefer to assume additionally to be a $B$-comodule) we define the complex $N \otimes_{R}^{\tau} M$ as the tensor product

$$
N \otimes_{R}^{\tau} M=N \otimes_{B} K_{e}^{\vee \bullet}(B, A) \otimes_{A} M
$$

with the differential induced by the differential $d_{e}$ on $K_{e}^{\vee \bullet}$. So $N \otimes_{R}^{\tau} M$ is a complex of abelian groups whose underlying graded abelian group is $N \otimes_{R} M$. Additional structures on a left $A$-module $M$ and a graded right $B$-comodule $N$ may induce additional structures on the complex $N \otimes_{R}^{\tau} M$, as usually.

Similarly, for any right $A$-module $M$ and any graded left ${ }^{\#} B$-module $N$ (which we would rather assume to be a graded left ${ }^{\#} B$-comodule) we define the complex $M \otimes_{R}^{\sigma} N$ as the tensor product

$$
M \otimes_{R}^{\sigma} N=M \otimes_{A} K_{u}^{\vee} \cdot\left(A,{ }^{\#} B\right) \otimes_{\#_{B}} N
$$

with the differential induced by the differential $d_{u}$ on $K_{u}^{\vee \bullet}$. So $M \otimes_{R}^{\sigma} N$ is a complex of abelian groups whose underlying graded abelian group is $M \otimes_{R} N$. 
Now we recall the notation $C$ for the graded $R$ - $R$-bimodule (in fact, a graded coring) $\operatorname{Tor}^{A}(R, R)$ which was used and discussed in the proof of Lemma 8.17 and the references therein. In particular, according to Remark 2.8 and Section 8.4, $C$ is a graded ${ }^{\#} B$ - $B$-bimodule (in fact, even a comodule on both sides, as explained in the beginning of the proof of Theorem 8.16).

With these preparations, we can interpret the second Koszul complex ${ }^{\tau} K_{\bullet}(B, A)=$ $\left(\operatorname{Hom}_{R^{\mathrm{op}}}(B, R) \otimes_{R} A,{ }^{\tau} \partial\right)(21)$ as

$$
{ }^{\tau} K_{\bullet}(B, A)=C \otimes_{R}^{\tau} A .
$$

The first Koszul complex $K_{\bullet}^{\tau}(B, A)=\left(\operatorname{Hom}_{R^{\text {op }}}(B, A), \partial^{\tau}\right)=\left(A \otimes_{R} \operatorname{Hom}_{R^{\text {op }}}(B, R), \partial^{\tau}\right)$ (19) gets interpreted as

$$
K_{\bullet}^{\tau}(B, A)=A \otimes_{R}^{\sigma} C,
$$

as mentioned in the proofs of Theorems 6.7 and 7.6, Looking on $A$ as primarily a right finitely projective Koszul graded ring rather than a left one, the first and second Koszul complexes switch their roles, of course.

Let us turn to the nonhomogeneous situation. Let $(\widetilde{A}, F)$ be a two-sided finitely projective nonhomogeneous Koszul ring, and let $(B, d, h)$ and $\left({ }^{{ }^{*}} B,{ }^{*} d,{ }^{\#} h\right)$ be its two nonhomogeneous quadratic dual CDG-rings on the right and left sides, as in Section 8.6. The notation $\sigma^{\prime}$ for the right $R$-linear map between $R$ - $R$-bimodules $\operatorname{Hom}_{R}\left({ }^{\#} B^{1}, R\right) \longrightarrow F_{1} \widetilde{A}$ was already introduced in (88) .

Denote by $u^{\prime} \in F_{1} \widetilde{A} \otimes_{R}{ }^{\#} B^{1}$ the element corresponding to $\sigma^{\prime}$ under the opposite version of the construction of Lemma 2.5. The opposite version of the construction of Section 6.2 produces the dual nonhomogeneous Koszul CDG-module $K^{\vee}\left(\widetilde{A},{ }^{\#} B\right)=$ $K_{u^{\prime}}^{\vee}\left(\widetilde{A},{ }^{\#} B\right)$, which has the form

$$
0 \longrightarrow \widetilde{A} \longrightarrow \widetilde{A} \otimes_{R}{ }^{*} B^{1} \longrightarrow \widetilde{A} \otimes_{R}{ }^{*} B^{2} \longrightarrow \cdots
$$

The differential on $K_{u^{\prime}}^{\vee}\left(\widetilde{A},{ }^{\#} B\right)$ does not square to zero when ${ }^{\#} \neq 0$. Rather, $K_{u^{\prime}}^{\vee}\left(\widetilde{A},{ }^{\#} B\right)$ is a right CDG-module over $\left({ }^{\#} B,{ }^{\#} d,{ }^{\#} h\right)$, and in fact a CDG-bimodule over $(\widetilde{A}, 0,0)$ and $\left({ }^{\#} B,{ }^{\#} d,{ }^{\#} h\right)$. The underlying graded $\widetilde{A}$ - ${ }^{*} B$-bimodule of $K_{u^{\prime}}^{\vee}\left(\widetilde{A},{ }^{\#} B\right)$ is the bimodule tensor product $\widetilde{A} \otimes_{R}{ }^{\#} B$.

Now let $D=\left(D, d_{D}, h_{D}\right)$ and $E=\left(E, d_{E}, h_{E}\right)$ be two CDG-rings. Let $M$ be a CDG-bimodule over $(\widetilde{A}, 0,0)$ and $\left(E, d_{E}, h_{E}\right)$, and let $N$ be a CDG-bimodule over $\left(D, d_{D}, h_{D}\right)$ and $(B, d, h)$. We would prefer to assume $N$ to be a graded right $B$-comodule rather than an arbitrary graded right $B$-module. In this setting, we define the CDG-bimodule $N \otimes_{R}^{\tau^{\prime}} M$ over $D$ and $E$ as the tensor product of CDG-bimodules

$$
N \otimes_{R}^{\tau^{\prime}} M=N \otimes_{B} K_{e^{\prime}}^{\vee}(B, \widetilde{A}) \otimes_{\widetilde{A}} M .
$$

The underlying graded $D$-E-bimodule of $N \otimes_{R}^{\tau^{\prime}} M$ is the bimodule tensor product $N \otimes_{R} M$. If $D$ is nonnegatively graded and $N$ is a $D$-comodule, or if $E$ is nonnegatively graded and $M$ is an $E$-comodule, then the tensor product $N \otimes_{R} M$ is obviously a comodule on the respective side. 
Similarly, let $M$ be a CDG-bimodule over $\left(E, d_{E}, h_{E}\right)$ and $(\widetilde{A}, 0,0)$, and let $N$ be a CDG-bimodule over $\left({ }^{\#} B,{ }^{\#} d,{ }^{\#} h\right)$ and $\left(D, d_{D}, h_{D}\right)$. We would rather assume the graded left ${ }^{\#} B$-module $N$ to be a ${ }^{\#} B$-comodule. Then we define the CDG-bimodule $M \otimes_{R}^{\sigma^{\prime}} N$ over $E$ and $D$ as the tensor product of CDG-bimodules

$$
M \otimes_{R}^{\sigma^{\prime}} N=M \otimes_{\widetilde{A}} K_{u^{\prime}}^{\vee}\left(\widetilde{A},{ }^{\#} B\right) \otimes_{\#_{B}} N .
$$

The underlying graded $E$-D-bimodule of $M \otimes_{R}^{\sigma^{\prime}} N$ is the bimodule tensor product $M \otimes_{R} N$. If $D$ is nonnegatively graded and $N$ is a $D$-comodule, or if $E$ is nonnegatively graded and $M$ is an $E$-comodule, then the tensor product $M \otimes_{R} N$ is obviously a comodule on the respective side.

Now we recall that, by Lemma 8.13 (which is applicable to the situation at hand in view of Lemma 8.18), there is a natural differential $d_{C}$ on the graded ${ }^{\#} B$ - $B$-bimodule $C$, making $\left(C, d_{C}\right)$ a CDG-bimodule over $\left({ }^{\#} B,{ }^{*} d,{ }^{\#} h\right)$ and $(B, d, h)$. With these preparations, we can interpret the nonhomogeneous Koszul duality functors

$$
M^{\bullet} \longmapsto M^{\bullet} \otimes_{R}^{\sigma^{\prime}} C \text { and } N \longmapsto N \otimes_{R}^{\tau^{\prime}} \widetilde{A}
$$

of Section 6.5 as particular cases of the above contsructions of twisted tensor products. This explains the meaning of the "placeholder $\sigma^{\prime}$ " in Section 6.5.

Let $M$ be a CDG-bimodule over $(\widetilde{A}, 0,0)$ and $\left(D, d_{D}, h_{D}\right)$, and let $Q$ be a left CDG-module over $(B, d, h)$. We would prefer to assume $Q$ to be a left CDG-contramodule over $(B, d, h)$. In this setting, we define the left CDG-module $\operatorname{Hom}_{R}^{\tau^{\prime}}(M, Q)$ over $\left(D, d_{D}, h_{D}\right)$ as the Hom CDG-module

$$
\operatorname{Hom}_{R}^{\tau^{\prime}}(M, Q)=\operatorname{Hom}_{B}\left(K_{e^{\prime}}^{\vee}(B, \widetilde{A}) \otimes_{\widetilde{A}} M, Q\right)=\operatorname{Hom}_{\widetilde{A}}\left(M, \operatorname{Hom}_{B}\left(K_{e^{\prime}}^{\vee}(B, \widetilde{A}), Q\right)\right) .
$$

The underlying graded left $D$-module of $\operatorname{Hom}_{R}^{\tau^{\prime}}(M, Q)$ is the Hom module $\operatorname{Hom}_{R}(M, Q)$ with the left $D$-module structure induced by the right $D$-module structure on $M$. If $D$ is nonnegatively graded and $M$ is a $D$-comodule, then $\operatorname{Hom}_{R}^{\tau^{\prime}}(M, Q)$ is a left CDG-contramodule over $\left(D, d_{D}, h_{D}\right)$ by Lemma 8.15)(a).

Let $N$ be a CDG-bimodule over $\left({ }^{\#} B,{ }^{\#} d,{ }^{\#} h\right)$ and $\left(D, d_{D}, h_{D}\right)$, and let $P=P \bullet$ be a complex of left $\widetilde{A}$-modules. We would rather assume the graded left ${ }^{*} B$-module $N$ to be a ${ }^{\#} B$-comodule. Then we define the left CDG-module $\operatorname{Hom}_{R}^{\sigma^{\prime}}\left(N, P^{\bullet}\right)$ over $\left(D, d_{D}, h_{D}\right)$ as the Hom CDG-module

$\operatorname{Hom}_{R}^{\sigma^{\prime}}\left(N, P^{\bullet}\right)=\operatorname{Hom}_{\widetilde{A}}\left(K_{u^{\prime}}^{\vee}\left(\widetilde{A},{ }^{\#} B\right) \otimes_{\#_{B}} N, P^{\bullet}\right)=\operatorname{Hom}_{\#_{B}}\left(N, \operatorname{Hom}_{\widetilde{A}}\left(K_{u^{\prime}}^{\vee}\left(\widetilde{A},{ }^{\#} B\right), P^{\bullet}\right)\right)$.

The underlying graded left $D$-module of $\operatorname{Hom}_{R}^{\sigma^{\prime}}\left(N, P^{\bullet}\right)$ is the Hom module $\operatorname{Hom}_{R}(N, P)$ with the left $D$-module structure induced by the right $D$-module structure on $N$. If $D$ is nonnegatively graded and $N$ is a $D$-comodule, then $\operatorname{Hom}_{R}^{\sigma^{\prime}}\left(N, P^{\bullet}\right)$ is a left CDG-contramodule over $\left(D, d_{D}, h_{D}\right)$ by Lemma 8.15(a).

With these preparations, we can interpret the nonhomogeneous Koszul duality functors

$$
P^{\bullet} \longmapsto \operatorname{Hom}_{R}^{\sigma^{\prime}}\left(C, P^{\bullet}\right) \quad \text { and } \quad Q \longmapsto \operatorname{Hom}_{R}^{\tau^{\prime}}(\widetilde{A}, Q)
$$

of Section 7.4 as particular cases of the above constructions of twisted Hom CDG-modules. This explains the meaning of the "placeholder $\sigma^{\prime}$ " in Section 7.4 . 
To conclude, let us say a few words about the bimodule resolution $\widetilde{A} \otimes_{R}^{\sigma^{\prime}} C \otimes_{R}^{\tau^{\prime}} \widetilde{A}$ of the diagonal $\widetilde{A}$ - $\widetilde{A}$-bimodule $\widetilde{A}$, which was constructed in Examples 6.8 (3-4). It was explained in Examples 6.8 why this is indeed a bimodule resolution, but the construction itself was decidedly not left-right symmetric, and the meaning of the placeholder $\sigma^{\prime}$ remained mysterious.

Now this is explained, and the symmetry is restored (under the additional assumption that $\widetilde{A}$ is two-sided finitely projective nonhomogeneous Koszul). As a corollary of the above discussion, we have

$$
\widetilde{A} \otimes_{R}^{\sigma^{\prime}} C \otimes_{R}^{\tau^{\prime}} \widetilde{A}=K_{u^{\prime}}^{\vee}\left(\widetilde{A},{ }^{\#} B\right) \otimes_{\#_{B}} C \otimes_{B} K_{e^{\prime}}^{\vee}(B, \widetilde{A}),
$$

where $C$ is a CDG-bimodule over $\left({ }^{\#} B,{ }^{*} d,{ }^{\#} h\right)$ and $(B, d, h)$, as explained above, and the tensor products in the right-hand side are the CDG-bimodule tensor products of Section 6.1.

8.8. Koszul triality. A "Koszul triality" is a commutative triangle diagram of triangulated equivalences between a derived category of modules, a coderived category of comodules, and a contraderived category of contramodules. This phenomenon was first observed in [28] (see [28, Sections 6.3-6.5]; cf. [28, Sections 6.7-6.8]). It is not supposed to manifest itself in the more general setting of [27, Chapter 11], which has coalgebra variables in the base (it is expected to be a "quadrality" there; cf. Section 9.7). But it still exists in the context of the present paper, under the assumptions listed in Theorem 8.21 in this section.

Let $R \subset \widetilde{V} \subset \widetilde{A}$ be a right finitely projective nonhomogeneous Koszul ring, and let ${ }^{\#} B=\left({ }^{\#} B,{ }^{\#} d,{ }^{\#} h\right)$ be the corresponding left finitely projective Koszul CDG-ring under the opposite version of the construction of Proposition 3.6 and Corollary 4.20. Then, as a particular case of the discussion in Section 8.7, there are DG-functors

$$
\widetilde{A} \otimes_{R}^{\sigma^{\prime}}-: \mathrm{DG}\left({ }^{\#} B-\mathrm{comod}\right) \longrightarrow \mathrm{DG}(\widetilde{A}-\mathrm{mod})
$$

and

$$
C \otimes{ }_{R}^{\tau^{\prime}}-: \operatorname{DG}(\widetilde{A}-\bmod ) \longrightarrow \mathrm{DG}\left({ }^{\#} B-\text { comod }\right) .
$$

By the opposite version of the discussion in Section 6.5, the DG-functor $\widetilde{A} \otimes_{R}^{\sigma^{\prime}}-$ is left adjoint to the DG-functor $C \otimes_{R}^{\tau^{\prime}}-$. Hence the induced triangulated functors between the homotopy categories,

$$
\widetilde{A} \otimes_{R}^{\sigma^{\prime}}-: \operatorname{Hot}\left({ }^{\#} B-\text { comod }\right) \longrightarrow \operatorname{Hot}(\widetilde{A}-\bmod )
$$

and

are also adjoint.

$$
C \otimes_{R}^{\tau^{\prime}}-: \operatorname{Hot}(\widetilde{A}-\bmod ) \longrightarrow \operatorname{Hot}\left({ }^{\#} B-\text { comod }\right),
$$

The following assertions are the opposite versions of Theorem 6.7 and Corollary 6.10.

Theorem 8.19. The above pair of adjoint triangulated functors $C \otimes_{R}^{\tau^{\prime}}-$ and $\widetilde{A} \otimes_{R}^{\sigma^{\prime}}-$ induces a pair of adjoint triangulated functors between the $\widetilde{A} / R$-semicoderived category of left $\widetilde{A}$-modules $\mathrm{D}_{R}^{\text {sico }}(\widetilde{A}$-mod $)$ and the coderived category $\mathrm{D}^{\mathrm{co}}\left({ }^{\#} B\right.$-comod $)$ of 
left $C D G$-comodules over $\left({ }^{\#} B,{ }^{*} d,{ }^{*} h\right)$, which are mutually inverse triangulated equivalences

$$
\mathrm{D}_{R}^{\text {sico }}(\widetilde{A}-\text { mod }) \simeq \mathrm{D}^{\mathrm{co}}\left({ }^{\#} B-\text { comod }\right) .
$$

Corollary 8.20. Assume additionally that the left homological dimension of the ring $R$ is finite. Then the pair of adjoint triangulated functors $\widetilde{A} \otimes_{R}^{\sigma^{\prime}}-$ : $\operatorname{Hot}\left({ }^{\#} B-\right.$ comod $) \longrightarrow \operatorname{Hot}(\widetilde{A}-\bmod )$ and $C \otimes_{R}^{\tau^{\prime}}-: \operatorname{Hot}(\widetilde{A}-\bmod ) \longrightarrow \operatorname{Hot}\left({ }^{\#} B-\operatorname{comod}\right)$ induces mutually inverse triangulated equivalences

$$
\mathrm{D}(\widetilde{A}-\bmod ) \simeq \mathrm{D}^{\mathrm{co}}\left({ }^{\#} B-\text { comod }\right)
$$

between the derived category of left $\widetilde{A}$-modules and the coderived category of left $C D G$-comodules over $\left.{ }^{\#} B,{ }^{\#} d,{ }^{\#} h\right)$.

The next theorem is the second main result of Section 8 ,

Theorem 8.21. Let $R \subset \widetilde{V} \subset \widetilde{A}$ be a two-sided finitely projective nonhomogeneous Koszul ring, let $B=(B, d, h)$ be the right finitely projective Koszul $C D G$-ring nonhomogeneous quadratic dual to $\widetilde{A}$, and let ${ }^{\#} B=\left({ }^{\#} B,{ }^{*} d,{ }^{\#} h\right)$ be the left finitely projective Koszul CDG-ring nonhomogeneous quadratic dual to $\widetilde{A}$, as in Lemma 8.18. Assume that the left homological dimension of the ring $R$ is finite. Then the triangulated equivalence of derived nonhomogeneous Koszul duality on the comodule side (89) of Corollary 8.20 (cf. the right (co)module side version in (178) of Corollary 6.10),

$$
C \otimes_{R}^{\tau^{\prime}}-: \mathrm{D}(\widetilde{A}-\mathrm{mod}) \simeq \mathrm{D}^{\mathrm{co}}\left({ }^{\#} B-\operatorname{comod}\right): \widetilde{A} \otimes_{R}^{\sigma^{\prime}}-,
$$

the triangulated equivalence of derived nonhomogeneous Koszul duality on the contramodule side (79) of Corollary 7.9 ,

$$
\operatorname{Hom}_{R}^{\sigma^{\prime}}(C,-): \mathrm{D}(\widetilde{A}-\mathrm{mod}) \simeq \mathrm{D}^{\mathrm{ctr}}(B-\text { contra }): \operatorname{Hom}_{R}^{\tau^{\prime}}(\widetilde{A},-),
$$

and the triangulated equivalence of derived comodule-contramodule correspondence (82) of Theorem 8.16,

$$
\mathbb{R} \operatorname{Hom}_{\#_{B}}(C,-): \mathrm{D}^{\mathrm{co}}\left({ }^{\#} B-\text { comod }\right) \simeq \mathrm{D}^{\mathrm{ctr}}(B-\text { contra }): C \odot_{B}^{\mathbb{L}}-
$$

form a commutative triangle diagram of triangulated category equivalences,

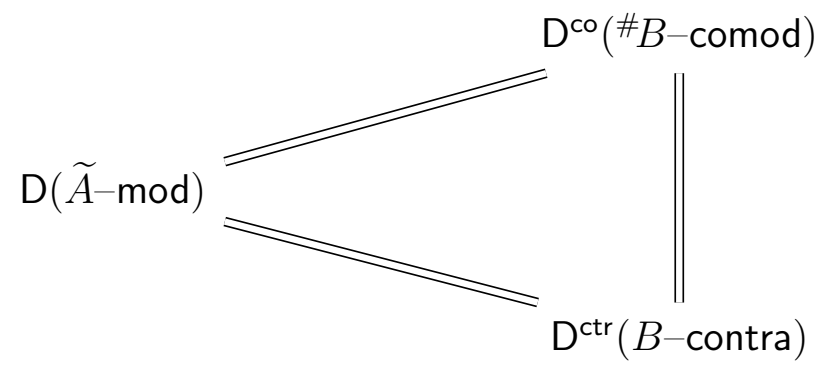


Proof. As a preliminary observation, it is worth pointing out that the contratensor product functor $C \odot_{B}$ - appearing in the third displayed formula is isomorphic to the tensor product functor $C \otimes_{B}-$ in our present assumptions. This is the result of Proposition 8.3(b), which is applicable in view of Theorem 5.26.

To prove the theorem, we notice first of all that, for any complex of left $\widetilde{A}$-modules $M=M^{\bullet}$, one has

$$
\mathbb{R} \operatorname{Hom}_{\#_{B}}\left(C, C \otimes_{R}^{\tau^{\prime}} M^{\bullet}\right)=\operatorname{Hom}_{\#_{B}}\left(C, C \otimes_{R}^{\tau^{\prime}} M^{\bullet}\right)
$$

and similarly,

$$
C \odot_{B}^{\mathbb{L}} \operatorname{Hom}_{R}^{\sigma^{\prime}}\left(C, M^{\bullet}\right)=C \odot_{B} \operatorname{Hom}_{R}^{\sigma^{\prime}}\left(C, M^{\bullet}\right),
$$

that is, the derived functors $\mathbb{R} \operatorname{Hom}_{\#_{B}}(C,-)$ and $C \odot_{B}^{\mathbb{L}}-$ coincide with the respective underived functors on these objects. Indeed, the graded left ${ }^{*} B$-module $C \otimes_{R} M=\operatorname{Hom}_{R}^{\Sigma}\left({ }^{\#} B, M\right)$ is coinduced (from the graded left $R$-module $M$ ), and the graded left $B$-contramodule $\operatorname{Hom}_{R}(C, M)=B \otimes_{R}^{\Pi} M$ is induced (from the graded left $R$-module $M)$. So the objects in question are adjusted to the respective functors in view of the construction of the derived functors in the proof of Theorem 8.16.

Finally, the main claim is that, for any complex of left $\widetilde{A}$-modules $M=M^{\bullet}$, there is a natural isomorphism of left CDG-contramodules over $(B, d, h)$

$$
\operatorname{Hom}_{\#_{B}}\left(C, C \otimes_{R}^{\tau^{\prime}} M^{\bullet}\right) \simeq \operatorname{Hom}_{R}^{\sigma^{\prime}}\left(C, M^{\bullet}\right),
$$

as well as a natural isomorphism of left CDG-comodules over $\left({ }^{\#} B,{ }^{\#} d,{ }^{\#} h\right)$

$$
C \odot_{B} \operatorname{Hom}_{R}^{\sigma^{\prime}}\left(C, M^{\bullet}\right) \simeq C \otimes_{R}^{\tau^{\prime}} M^{\bullet} .
$$

Indeed, on the level of the underlying graded co/contramodules we have

$$
\operatorname{Hom}_{\#_{B}}\left(C, C \otimes_{R} M\right) \simeq \operatorname{Hom}_{\#_{B}}\left(C, \operatorname{Hom}_{R}^{\Sigma}\left({ }^{\#} B, M\right)\right) \simeq \operatorname{Hom}_{R}(C, M)
$$

and

$$
C \odot_{B} \operatorname{Hom}_{R}(C, M) \simeq C \odot_{B}\left(B \otimes_{R}^{\Pi} M\right) \simeq C \otimes_{R} M
$$

(cf. Lemma 8.14 and the computation in the proof of Theorem 8.16). We leave it to the reader to check that the differentials agree.

\section{Koszul Duality and Conversion Functor}

9.1. Relatively Frobenius rings. Let $K$ be an associative ring and $B$ be a $K$ - $K$-bimodule. Assume that $B$ is a finitely generated projective left $K$-module. Then for any left $K$-module $L$ there is a natural isomorphism of left $K$-modules $\operatorname{Hom}_{K}(B, L) \simeq \operatorname{Hom}_{K}(B, K) \otimes_{K} L$.

Let $T$ be another $K$ - $K$-bimodule which is finitely generated and projective as a left $K$-module. Then there is a natural morphism of $K$ - $K$-bimodules $K \longrightarrow$ $\operatorname{Hom}_{K}(T, T) \simeq \operatorname{Hom}_{K}(T, K) \otimes_{K} T$ induced by the right action of $K$ in $T$. There is also a natural evaluation morphism of $K$ - $K$-bimodules $T \otimes_{K} \operatorname{Hom}_{K}(T, K) \longrightarrow K$ given by the rule $t \otimes f \longmapsto f(t)$ for all $t \in T$ and $f \in \operatorname{Hom}_{K}(T, K)$. The pairing notation $\langle t, f\rangle=f(t)$ is convenient. 
We will say that a $K$-K-bimodule $T$ is invertible if $T$ is projective and finitely generated as a left $K$-module, and both the above morphisms $K \longrightarrow \operatorname{Hom}_{K}(T, K) \otimes_{K}$ $T$ and $T \otimes_{K} \operatorname{Hom}_{K}(T, K) \longrightarrow K$ are isomorphisms. In this case, the functors $T \otimes_{K}-$ : $K-\bmod \longrightarrow K-\bmod$ and $\operatorname{Hom}_{K}(T,-)=\operatorname{Hom}_{K}(T, K) \otimes_{K}-: K-\bmod \longrightarrow K-\bmod$ are mutually inverse auto-equivalences of the category of left $K$-modules. Similarly, the functors $-\otimes_{K} T: \bmod -K \longrightarrow \bmod -K$ and $-\otimes_{K} \operatorname{Hom}_{K}(T, K): \bmod -K \longrightarrow$ mod- $K$ are mutually inverse auto-equivalences of the category of right $K$-modules. It follows that $T$ is also a finitely generated projective right $K$-module, and the notion of an invertible $K$ - $K$-bimodule is left-right symmetric. Moreover, we obtain a natural isomorphism of $K$ - $K$-bimodules $\operatorname{Hom}_{K}(T, K) \simeq \operatorname{Hom}_{K^{\mathrm{op}}}(T, K)$.

Furthermore, for any invertible $K-K$-bimodule $T$ and any finitely generated projective left $K$-module $L$ the natural evaluation map $L \longrightarrow \operatorname{Hom}_{K^{\text {op }}}\left(\operatorname{Hom}_{K}(L, T), T\right)$ is an isomorphism of left $K$-modules (as one can see, e. g., by reducing the question to the case of a free module with one generator $L=K$ ).

Let $B^{\prime}$ and $B^{\prime \prime}$ be $K$ - $K$-bimodules, $T$ be an invertible $K$ - $K$-bimodule, and $\phi: B^{\prime} \otimes_{K}$ $B^{\prime \prime} \longrightarrow T$ be a $K$-K-bimodule morphism. We will say that $\phi$ is a perfect pairing if $B^{\prime}$ is a finitely generated projective left $K$-module and the $K$ - $K$-bimodule map $\check{\phi}: B^{\prime \prime} \longrightarrow \operatorname{Hom}_{K}\left(B^{\prime}, T\right) \simeq \operatorname{Hom}_{K}\left(B^{\prime}, K\right) \otimes_{K} T$ induced by $\phi$ is an isomorphism. Then $\operatorname{Hom}_{K}\left(B^{\prime}, K\right)$ is a finitely generated projective right $K$-module, and it follows that $B^{\prime \prime}$ is a finitely generated projective right $K$-module. Furthermore, the $K$-K-bimodule map $\hat{\phi}: B^{\prime} \longrightarrow \operatorname{Hom}_{K^{\mathrm{op}}}\left(B^{\prime \prime}, T\right) \simeq T \otimes_{K} \operatorname{Hom}_{K^{\mathrm{op}}}\left(B^{\prime \prime}, K\right)$ induced by $\phi$ is an isomorphism, because $\hat{\phi} \simeq \operatorname{Hom}_{K^{\text {op }}}(\check{\phi}, T)$ (while $\check{\phi} \simeq \operatorname{Hom}_{K}(\hat{\phi}, T)$ ). Therefore, the notion of a perfect pairing $\phi$ is left-right symmetric as well.

Let $K \longrightarrow B$ be a ring homomorphism, $T$ be an invertible $K$ - $K$-bimodule, and $t: B \longrightarrow T$ be a morphism of $K-K$-bimodules. Take $\phi: B \otimes_{K} B \longrightarrow K$ to be equal to the composition of the multiplication map $B \otimes_{K} B \longrightarrow B$ with the map $t$. Then the map $\check{\phi}: B \longrightarrow \operatorname{Hom}_{K}(B, T)$ is a morphism of left $B$-modules, while the map $\hat{\phi}: B \longrightarrow$ $\operatorname{Hom}_{K^{\mathrm{op}}}(B, T)$ is a morphism of right $B$-modules. We will say that the $\operatorname{ring} B$ is relatively Frobenius over $K$ (with respect to the map $t$ ) if the pairing $\phi: B \otimes_{K} B \longrightarrow T$ induced by the map $t: B \longrightarrow T$ is perfect. Following the above discussion, any relatively Frobenius ring $B$ over $K$ is a finitely generated and projective left and right $K$-module, and both the maps $\check{\phi}$ and $\hat{\phi}$ are isomorphisms in this case.

Let $B$ be a relatively Frobenius ring over $K$ with respect to a $K$ - $K$-bimodule morphism $t: B \longrightarrow T$. Then for any left $K$-module $L$ there is a natural isomorphism of left $B$-modules $B \otimes_{K} L \simeq \operatorname{Hom}_{K}(B, T) \otimes_{K} L \simeq \operatorname{Hom}_{K}(B, K) \otimes_{K} T \otimes_{K} L \simeq$ $\operatorname{Hom}_{K}\left(B, T \otimes_{K} L\right)$. Consequently, for any left $K$-module $M$ there is a natural isomorphism of left $B$-modules $\operatorname{Hom}_{K}(B, M) \simeq B \otimes_{K} \operatorname{Hom}_{K}(T, M)$. So the class of left $B$-modules induced from left $K$-modules coincides with that of left $B$-modules coinduced from left $K$-modules.

9.2. Relatively Frobenius graded rings. Let $B=\bigoplus_{n=0}^{\infty} B^{n}$ be a nonnegatively graded ring, and let $K \longrightarrow B^{0}$ be a ring homomorphism. Let $T$ be an invertible $K$-K-bimodule. Assume that there is an integer $m \geq 0$ such that $B^{n}=0$ for all $n>m$, and a morphism of $K$-K-bimodules $t: B^{m} \longrightarrow T$ is given. 
Then we will say that $B$ is a relatively Frobenius graded ring over $K$ (with respect to the map $t$ ) if the underlying ungraded ring of $B$ is relatively Frobenius, in the sense of the definition in Section 9.1, with respect to the composition of the direct summand projection $B=\bigoplus_{n=0}^{m} B^{n} \longrightarrow B^{m}$ with the map $t$. Equivalently, this means that the composition $\phi_{n}: B^{n} \otimes_{K} B^{m-n} \longrightarrow T$ of the multiplication map $B^{n} \otimes_{K} B^{m-n} \longrightarrow B^{m}$ with the map $t: B^{m} \longrightarrow T$ is a perfect pairing (in the sense of the definition in Section 9.1) for every $0 \leq n \leq m$. In this case, $B^{n}$ is a finitely generated projective left and right $K$-module for every $0 \leq n \leq m$.

Since $B^{n}=0$ for all $n>m$, all (graded or ungraded, right or left) $B$-modules are $B$-comodules, as mentioned at the end of Section 6.4. So, in particular, we have $B-$ comod $_{\mathrm{gr}}=B-\bmod _{\mathrm{gr}}$, in the notation of Section 8.3 , and therefore we will use $\mathrm{D}^{\mathrm{co}}(B$-mod $)$ instead of $\mathrm{D}^{\mathrm{co}}(B$-comod $)$ as the notation for the coderived category of left CDG-(co)modules over $B$ when $B$ is endowed with a CDG-ring structure $B=(B, d, h)$. Similarly, the forgetful functors from the categories of (graded or ungraded) $B$-contamodules to the similar categories of $B$-modules are category equivalences, as mentioned at the end of Section 7.3. So we will use $\mathrm{D}^{\mathrm{ctr}}(B-\mathrm{mod})$ instead of $\mathrm{D}^{\mathrm{ctr}}(B$-contra) as the notation for the contraderived category of left CDG-(contra)modules over $B$ when $B$ is endowed with a CDG-ring structure.

The definitions of the induced graded contramodules and coinduced graded comodules given in Sections 8.2 and 8.3 simplify similarly: for any graded left $K$-module $L$ one has $B \otimes_{K}^{\Pi} L=B \otimes_{K} L$ and $\operatorname{Hom}_{K}^{\Sigma}(B, L)=\operatorname{Hom}_{K}(B, L)$. So we will speak of induced and coinduced graded left $B$-modules instead of induced graded left $B$-contramodules and coinduced graded left $B$-comodules.

Finally, since $B$ is a relatively Frobenius graded ring over $K$, the class of left graded $B$-modules induced from graded left $K$-modules coincides with that of graded left $B$-modules coinduced from graded left $K$-modules, essentially as explained in Section 9.1. More precisely, for any graded left $K$-modules $L$ and $M$ there are natural isomorphisms of graded left $B$-modules $B \otimes_{K} L \simeq \operatorname{Hom}_{K}\left(B, T \otimes_{K} L[-m]\right)$ and $\operatorname{Hom}_{K}(B, M) \simeq B \otimes_{K} \operatorname{Hom}_{K}(T, M[m])$, where $[i], i \in \mathbb{Z}$, denotes the usual (cohomological) grading shift.

9.3. Relatively Frobenius co-contra correspondence. Let $B=(B, d, h)$ be a nonnegatively graded CDG-ring, so $B=\bigoplus_{n=0}^{\infty} B^{n}$, and let $K \longrightarrow B^{0}$ be a ring homomorphism. We will say that the $\mathrm{CDG}$-ring $(B, d, h)$ is relatively Frobenius over $K$ if the graded ring $B$ is relatively Frobenius (with respect to some morphism of $K$-K-bimodules $\left.t: B^{m} \longrightarrow T\right)$.

Let $(B, d, h)$ be a relatively Frobenius CDG-ring over $K$. Similarly to Sections 8.2 and 8.3, one can speak of left $C D G$-modules of the induced type and left $C D G$-modules of the coinduced type over $(B, d, h)$. Moreover, following the discussion in Section 9.2 , these two classes of left CDG-modules coincide.

Furthermore, similarly to Sections 8.2 and 8.3, one can speak of left CDG-module short exact sequences of the induced type and of the coinduced type over $(B, d, h)$. Once again, following the discussion in Section 9.2 , these two clases of short exact sequences of left CDG-modules over $(B, d, h)$ coincide. 
Hence we arrive to the following theorem, which is the first main result of Section 9 . It is to be compared with [28, Theorems 3.9-3.10], and it is a generalization of [28, Theorem B.3]. It is also a simplified version of Theorem 8.16 above.

Theorem 9.1. Let $B=(B, d, h)$ be a relatively Frobenius $C D G$-ring over a ring $K$. Assume that the left homological dimension of $K$ is finite. Then there is a natural triangulated equivalence between the coderived and contraderived categories of left $C D G$-modules over $(B, d, h)$,

$$
\mathrm{D}^{\mathrm{co}}(B-\bmod ) \simeq \mathrm{D}^{\mathrm{ctr}}(B-\bmod ) .
$$

Proof. By Proposition 8.10 and the second assertion of Theorem 8.11, the coderived category $\mathrm{D}^{\mathrm{co}}(B$-mod $)$ is equivalent to the triangulated quotient category of the full triangulated subcategory in $\operatorname{Hot}(B$-mod) formed by left CDG-modules of the coinduced type over $(B, d, h)$ by its minimal triangulated subcategory containing all the totalizations of left CDG-module short exact sequences of the coinduced type. Similarly, by Proposition 8.6 and the second assertion of Theorem 8.7, the contraderived category $\mathrm{D}^{\mathrm{ctr}}(B$-mod) is equivalent to the triangulated quotient category of the full subcategory in $\operatorname{Hot}(B$-mod) formed by left CDG-modules of the induced type over $(B, d, h)$ by its minimal triangulated subcategory containing all the totalizations of left CDG-module short exact sequences of the induced type. Following the above discussion, it is one and the same triangulated quotient category.

9.4. Relatively Frobenius Koszul graded rings. Let $A=\bigoplus_{n=0}^{\infty} A_{n}$ be a left finitely projective Koszul graded ring with the degree-zero component $R=A_{0}$, and let $B=\bigoplus_{n=0}^{\infty} B^{n}$ be the quadratic dual right finitely projective Koszul graded ring, as per the construction of Section 1 and Proposition 2.23.

Assume that there is an integer $m \geq 0$ such that $B^{n}=0$ for $n>m$, the $R$-R-bimodule $T=B^{m}$ is invertible, and the graded ring $B$ is relatively Frobenius over the ring $K=R$ with respect to the identity morphism of $R$ - $R$-bimodules $B^{m} \longrightarrow T$. In other words, this means that the multiplication map $B^{n} \otimes_{R} B^{m-n} \longrightarrow$ $B^{m}$ is a perfect pairing for every $0 \leq n \leq m$.

Consider the dual Koszul complex $K_{e}^{\vee \bullet}(B, A)$ (20) from Section 2.6. (92) $0 \longrightarrow A \longrightarrow B^{1} \otimes_{R} A \longrightarrow \cdots \longrightarrow B^{m-1} \otimes_{R} A \longrightarrow B^{m} \otimes_{R} A \longrightarrow 0$, and consider also the second Koszul complex ${ }^{\tau} K_{\bullet}(B, A)=C \otimes_{B} K_{e}^{\vee \bullet}(B, A)=C \otimes_{R}^{\tau} A$ from formula (21) and Remark 2.8 (where $C=\operatorname{Hom}_{R^{\mathrm{op}}}(B, R)$ ).

According to Theorem 2.21(e), the complex ${ }^{\tau} K_{\bullet}(B, A)$ is a graded right $A$-module resolution of the graded right $A$-module $R$. In view of the isomorphism of graded right $B$-modules $B \simeq \operatorname{Hom}_{R^{\text {op }}}(B, T) \simeq T \otimes_{R} \operatorname{Hom}_{R^{\text {op }}}(B, R)[-m]=T \otimes_{R} C[-m]$ (see Sections 9.1 9.2), the dual Koszul complex (92) is a graded right $A$-module resolution of the graded right $A$-module $T=B^{m}$ (placed in the cohomological degree $m$ ). In other words, the complex (92) is acyclic at every term except the rightmost one, and at the rightmost term $B^{m} \otimes_{R} A$ its cohomology module is the internal grading component $B^{m} \otimes_{R} A_{0}=T$. 
9.5. Two-sided Koszul CDG-rings. Let $B=\bigoplus_{n=0}^{\infty} B^{n}$ be a two-sided finitely projective Koszul graded ring (in the sense of Section 8.6) with the degree-zero component $R=B^{0}$. Denote by $A$ the left finitely projective Koszul graded ring quadratic dual to the right finitely projective Koszul ring $B$, and denote by $A^{\#}$ the right finitely projective Koszul graded ring quadratic dual to the left finitely projective Koszul ring $B$. So, according to Proposition 2.23, we have

$$
A=\operatorname{Ext}_{B^{\mathrm{op}}}(R, R) \text { and } A^{\#}=\operatorname{Ext}_{B}(R, R)^{\mathrm{op}},
$$

where $\operatorname{Ext}_{B^{\text {op }}}$ is taken in the category of graded right $B$-modules and $\operatorname{Ext}_{B}$ is taken in the category of graded left $B$-modules. Following Lemma 8.17, there are natural isomorphisms of $R$ - $R$-bimodules

$$
A_{n}^{\#}=\operatorname{Hom}_{R}\left(\operatorname{Hom}_{R}\left(A_{n}, R\right), R\right) \quad \text { and } \quad A_{n}=\operatorname{Hom}_{R^{\mathrm{op}}}\left(\operatorname{Hom}_{R^{\mathrm{op}}}\left(A_{n}^{\#}, R\right), R\right)
$$

for all $n \geq 0$, and the multiplicative structures on $A$ and $A^{\#}$ are related by the construction of Remark 2.8 and Section 8.4 .

Now let $(B, d, h)$ be a nonnegatively graded CDG-ring. We recall that $(B, d, h)$ is said to be right finitely projective Koszul if the graded ring $B=\bigoplus_{n=0}^{\infty} B^{n}$ is right finitely projective Koszul. Dually, let us say that $(B, d, h)$ is left finitely projective Koszul if the graded ring $B$ is left finitely projective Koszul. We will say that a CDG-ring $(B, d, h)$ is two-sided finitely projective Koszul if the graded ring $B$ is twosided finitely projective Koszul.

Let $(B, d, h)$ be a two-sided finitely projective Koszul CDG-ring. Applying the Poincaré-Birkhoff-Witt Theorem 4.19 (see also Corollary 4.20) to the right finitely projective Koszul CDG-ring $(B, d, h)$, we obtain a left finitely projective nonhomogeneous Koszul ring $\widetilde{A}$ with an increasing filtration $0=F_{-1} \widetilde{A} \subset R=F_{0} \widetilde{A} \subset$ $\widetilde{V}=F_{1} \widetilde{A} \subset F_{2} \widetilde{A} \subset \cdots$ and a natural isomorphism of graded rings $A \simeq \operatorname{gr}^{F} \widetilde{A}=$ $\bigoplus_{n=0}^{\infty} F_{n} \widetilde{A} / F_{n-1} \widetilde{A}$. The filtered ring $\widetilde{A}$ comes together with a left $R$-linear splitting

$$
\operatorname{Hom}_{R^{\text {op }}}\left(B^{1}, R\right) \simeq F_{1} \widetilde{A} / F_{0} \widetilde{A} \simeq A_{1}=V \hookrightarrow \widetilde{V}=F_{1} \widetilde{A},
$$

which we denote by $\tau^{\prime}$, as in Sections 6.5 and 7.4.

Applying the opposite version of Theorem 4.19 and Corollary 4.20 to the left finitely projective Koszul CDG-ring $(B, d, h)$, we obtain a right finitely projective nonhomogeneous Koszul ring $\widetilde{A}^{\#}$ with an increasing filtration $0=F_{-1} \widetilde{A}^{\#} \subset R=$ $F_{0} \widetilde{A}^{\#} \subset \widetilde{V}^{\#}=F_{1} \widetilde{A}^{\#} \subset F_{2} \widetilde{A}^{\#} \subset \cdots$ and a natural isomorphism of graded rings $A^{\#} \simeq \operatorname{gr}^{F} \widetilde{A}^{\#}=\bigoplus_{n=0}^{\infty} F_{n} \widetilde{A}^{\#} / F_{n-1} \widetilde{A}^{\#}$. The filtered ring $\widetilde{A}^{\#}$ comes together with a right $R$-linear splitting

$$
\operatorname{Hom}_{R}\left(B^{1}, R\right) \simeq F_{1} \widetilde{A}^{\#} / F_{0} \widetilde{A}^{\#} \simeq A_{1}^{\#}=V^{\#} \hookrightarrow \widetilde{V}^{\#}=F_{1} \widetilde{A}^{\#},
$$

which we will denote by $\rho^{\prime}$.

The dual nonhomogeneous Koszul CDG-module $K^{\vee}(B, \widetilde{A})$ was constructed in Section 6.2. We recall that $K^{\vee}(B, \widetilde{A})$ is a CDG-bimodule over the CDG-rings $B=$ $(B, d, h)$ and $\widetilde{A}=(\widetilde{A}, 0,0)$ whose underlying graded $B$ - $\widetilde{A}$-bimodule is $B \otimes_{R} \widetilde{A}$. The opposite construction produces a CDG-bimodule $K^{\vee}\left(\widetilde{A}^{\#}, B\right)$ over the CDG-rings 
$\widetilde{A}^{\#}=\left(\widetilde{A}^{\#}, 0,0\right)$ and $B=(B, d, h)$. So the underlying graded $\widetilde{A}^{\#-B}$-bimodule of $K^{\vee}\left(\widetilde{A}^{\#}, B\right)$ is $\widetilde{A}^{\#} \otimes_{R} B$.

From now on we will assume for simplicity that $B^{n}=0$ for $n \gg 0$; so there is no difference between $B$-modules and $B$-comodules, and $B$ is a finitely generated projective graded left and right $R$-module. Let $D=\left(D, d_{D}, h_{D}\right)$ and $E=\left(E, d_{E}, h_{E}\right)$ be two CDG-rings. Let $M$ be a CDG-bimodule over $(\widetilde{A}, 0,0)$ and $\left(E, d_{E}, h_{E}\right)$, and let $N$ be a CDG-bimodule over $\left(D, d_{D}, h_{D}\right)$ and $(B, d, h)$. Following the constructions of Sections 6.5 and 8.7, the CDG-bimodule $N \otimes_{R}^{\tau^{\prime}} M$ over $D$ and $E$ is defined as the tensor product of CDG-bimodules

$$
N \otimes_{R}^{\tau^{\prime}} M=N \otimes_{B} K^{\vee}(B, \widetilde{A}) \otimes_{\widetilde{A}} M .
$$

Furthermore, let $M$ be a CDG-bimodule over $\left(E, d_{E}, h_{E}\right)$ and $(\widetilde{A}, 0,0)$. Then the CDG-bimodule $M \otimes_{R}^{\sigma^{\prime}} C$ over $\left(E, d_{E}, h_{E}\right)$ and $(B, d, h)$ is defined as the Hom CDG-bimodule

$$
M \otimes_{R}^{\sigma^{\prime}} C=\operatorname{Hom}_{\widetilde{A}^{\mathrm{op}}}\left(K^{\vee}(B, \widetilde{A}), M\right),
$$

where $\sigma^{\prime}$ is the placeholder introduced in Section 6.5 and explained in Section 8.7, while $C$ is the graded coring $C=\operatorname{Hom}_{R^{\mathrm{op}}}(B, R)$.

Let us introduce notation for the opposite constructions. Let $M$ be a CDG-bimodule over $\left(E, d_{E}, h_{E}\right)$ and $\left(\widetilde{A}^{\#}, 0,0\right)$, and let $N$ be a CDG-bimodule over $(B, d, h)$ and $\left(D, d_{D}, h_{D}\right)$. We define the CDG-bimodule $M \otimes_{R}^{\rho^{\prime}} N$ over $E$ and $D$ as the tensor product of CDG-bimodules

$$
M \otimes_{R}^{\rho^{\prime}} N=M \otimes_{\widetilde{A}^{\#}} K^{\vee}\left(\widetilde{A}^{\#}, B\right) \otimes_{B} N .
$$

So the underlying graded $E$-D-bimodule of $M \otimes_{R}^{\rho^{\prime}} N$ is $M \otimes_{R} N$.

Furthermore, let $M$ be a CDG-bimodule over $\left(\widetilde{A}^{\#}, 0,0\right)$ and $\left(E, d_{E}, h_{E}\right)$. Then the CDG-bimodule $C^{\#} \otimes_{R}^{\pi^{\prime}} M$ over $(B, d, h)$ and $\left(E, d_{E}, h_{E}\right)$ is defined as the Hom CDG-bimodule

$$
C^{\#} \otimes_{R}^{\pi^{\prime}} M=\operatorname{Hom}_{\widetilde{A}^{\#}}\left(K^{\vee}\left(\widetilde{A}^{\#}, B\right), M\right),
$$

where $C^{\#}$ is the graded coring $C^{\#}=\operatorname{Hom}_{R}(B, R)$ over $R$, while $\pi^{\prime}$ is another placeholder. The underlying graded $B$-E-bimodule of $C^{\#} \otimes_{R}^{\pi^{\prime}} M$ is $\operatorname{Hom}_{\widetilde{A}^{\#}}\left(\widetilde{A}^{\#} \otimes_{R} B, M\right) \simeq$ $\operatorname{Hom}_{R}(B, M) \simeq C^{\#} \otimes_{R} M$.

9.6. Conversion bimodule. Let $B=(B, d, h)$ be a two-sided finitely projective Koszul nonnegatively graded CDG-ring (in the sense of Section 9.5 ) with the degreezero component $R=B^{0}$. We will say that $(B, d, h)$ is a relatively Frobenius Koszul $C D G$-ring if the graded ring $B$ is relatively Frobenius over $R$ in the sense of Section 9.4. This means that there is an integer $m \geq 0$ such that $B^{n}=0$ for $n>m$, the $R$ - $R$-bimodule $T=B^{m}$ is invertible, and the multiplication map $B^{n} \otimes_{R} B^{m-n} \longrightarrow B^{m}$ is a perfect pairing for every $0 \leq n \leq m$ (in the sense of Section 9.1).

Let $(B, d, h)$ be a relatively Frobenius Koszul CDG-ring. We will use the notation of Section 9.5. In particular, $\widetilde{A}$ is the left finitely projective nonhomogeneous Koszul ring corresponding to the right finitely projective Koszul CDG-ring $(B, d, h)$, while 
$\widetilde{A}^{\#}$ is the right finitely projective nonhomogeneous Koszul ring correponding to the left finitely projective Koszul CDG-ring $(B, d, h)$.

Lemma 9.2. (a) There is a natural morphism $T[-m] \longrightarrow B \otimes_{R}^{\tau^{\prime}} \widetilde{A}=K^{\vee}(B, \widetilde{A})$ of left $C D G$-modules over $(B, d, h)$, where the one-term complex of left $R$-modules $T[-m]$ is endowed with the trivial structure of left $C D G$ - $($ co)module over $(B, d, h)$. The cone of this morphism is a coacyclic left $C D G$-(co)module over $(B, d, h)$.

(b) There is a natural morphism $T[-m] \longrightarrow \widetilde{A}^{\#} \otimes_{R}^{\rho^{\prime}} B=K^{\vee}\left(\widetilde{A}^{\#}, B\right)$ of right $C D G$-modules over $(B, d, h)$, where the one-term complex of right $R$-modules $T[-m]$ is endowed with the trivial structure of right $C D G$-(co)module over $(B, d, h)$. The cone of this morphism is a coacyclic right $C D G$-(co)module over $(B, d, h)$.

Proof. Let us prove part (a); part (b) is the opposite version. The left finitely projective nonhomogeneous Koszul ring $\widetilde{A}$ is endowed with an increasing filtration $F$. Define a decreasing filtration $F$ on the graded ring $B$ by the rule $F^{n} B=\bigoplus_{i \geq n} B^{i}[-i] \subset B$; so $F$ is the decreasing filtration on $B$ induced by the grading. Define an increasing filtration $F$ on $B$ by the rule $F_{-n} B=F^{n} B$; so $0=F_{-m-1} B \subset F_{-m} B \subset \cdots \subset F_{0} B=B$. Consider the increasing filtration $F$ on $K^{\vee}(B, \widetilde{A})=B \otimes_{R} \widetilde{A}$ induced by the increasing filtrations $F$ on $B$ and $\widetilde{A}$, i. e., $F_{n}\left(B \otimes_{R} \widetilde{A}\right)=\sum_{i+j=n} F_{i} B \otimes_{R} F_{j} \widetilde{A}$.

It is clear from the definition of the differential $d_{e^{\prime}}$ on $K^{\vee}(B, \widetilde{A})$ that $F_{n} K^{\vee}(B, \widetilde{A})$ is a CDG-submodule of the left CDG-module $K^{\vee}(B, \widetilde{A})$ over $(B, d, h)$ for every $-m \leq$ $n<\infty$. Furthermore, the CDG-submodule $F_{-m} K^{\vee}(B, \widetilde{A})=B^{m}[-m] \otimes_{R} F_{0} A=$ $B^{m}[-m] \subset K^{\vee}(B, \widetilde{A})$ is isomorphic to $T[-m]$; hence the desired (injective) morphism of CDG-modules $T[-m] \longrightarrow K^{\vee}(B, \widetilde{A})$. In view of Lemma [6.5, in order to show that the cone (equivalently, the cokernel) of this morphism is coacyclic, it suffices to check that the CDG-(co)module $F_{n} K^{\vee}(B, \widetilde{A}) / F_{n-1} K^{\vee}(B, \widetilde{A})$ over $(B, d, h)$ is coacyclic for every $n \geq-m+1$.

In fact, $F_{n} K^{\vee}(B, \widetilde{A}) / F_{n-1} K^{\vee}(B, \widetilde{A})$ is a trivial CDG-comodule over $(B, d, h)$, that is, the ideal $B^{\geq 1} \subset B$ acts by zero in this quotient CDG-module. The whole associated graded CDG-module $\operatorname{gr}^{F} K^{\vee}(B, \widetilde{A})=\bigoplus_{n=-m}^{\infty} F_{n} K^{\vee}(B, \widetilde{A}) / F_{n-1} K^{\vee}(B, \widetilde{A})$ is the complex of left $R$-modules $K_{e}^{\vee} \cdot(B, A)=B \otimes_{R} A$ (20) from Section 2.6, viewed as a left CDG-module over $(B, d, h)$ with the trivial CDG-(co)module structure. In view of the discussion in Section 9.4, this means that, for every $n \geq-m+1$, the complex of left $R$-modules $F_{n} K^{\vee}(B, \widetilde{A}) / F_{n-1} K^{\vee}(B, \widetilde{A})$ is acyclic. This is a finite complex of finitely generated projective left $R$-modules; so it is contractible as a complex of left $R$-modules, hence it is also contractible (and consequently, coacyclic) as a trivial left CDG-(co)module over $(B, d, h)$.

Lemma 9.3. (a) For any complex of right $\widetilde{A}^{\#}$-modules $M^{\bullet}$, there is a natural quasiisomorphism of complexes of right $R$-modules

$$
M^{\bullet} \otimes_{R} T[-m] \longrightarrow M^{\bullet} \otimes_{R}^{\rho^{\prime}} B \otimes_{R}^{\tau^{\prime}} \tilde{A},
$$


where $M^{\bullet} \otimes_{R}^{\rho^{\prime}} B \otimes_{R}^{\tau^{\prime}} \widetilde{A}=M^{\bullet} \otimes_{\widetilde{A}^{\#}} K^{\vee}\left(\widetilde{A}^{\#}, B\right) \otimes_{B} K^{\vee}(B, \widetilde{A})$ is the complex of right $\widetilde{A}$-modules provided by the constructions of Section 9.5 .

(b) For any complex of left $\widetilde{A}$-modules $L \cdot$, there is a natural quasi-isomorphism of complexes of left $R$-modules

$$
T \otimes_{R} L \cdot[-m] \longrightarrow \widetilde{A}^{\#} \otimes_{R}^{\rho^{\prime}} B \otimes_{R}^{\tau^{\prime}} L^{\bullet}
$$

where $\widetilde{A}^{\#} \otimes_{R}^{\rho^{\prime}} B \otimes_{R}^{\tau^{\prime}} L^{\bullet}=K^{\vee}\left(\widetilde{A}^{\#}, B\right) \otimes_{B} K^{\vee}(B, \widetilde{A}) \otimes_{\widetilde{A}} L^{\bullet}$ is the complex of left $\widetilde{A}^{\#}$-modules provided by the constructions of Section 9.5.

Proof. Let us explain part (a); part (b) is opposite. The desired morphism is obtaned by applying the functor $M^{\bullet} \otimes_{R}^{\rho^{\prime}}-$ to the morphism of CDG-modules in Lemma 0.2 (a). It is important here that the latter morphism is right $R$-linear; so it is, in fact, a morphism of CDG-bimodules over $(B, d, h)$ and $(R, 0,0)$. Furthermore, following the proof of Lemma 9.2 (a), the cone of the morphism $T[-m] \longrightarrow B \otimes_{R}^{\tau^{\prime}} \widetilde{A}$ is coacyclic as a CDG-bimodule over $(B, d, h)$ and $(R, 0,0)$, and in fact even as a CDG-bimodule over $(B, d, h)$ and $(R, 0,0)$ with a projective underlying graded left $R$-module (because any finite acyclic complex of $R$ - $R$-bimodules is coacyclic). Hence the tensor product functor $M^{\bullet} \otimes_{R}^{\rho^{\prime}}-$ preserves coacyclicity of the cone, so the cone of the resulting morphism $M^{\bullet} \otimes_{R} T[-m] \longrightarrow M^{\bullet} \otimes_{R}^{\rho^{\prime}} B \otimes_{R}^{\tau^{\prime}} \widetilde{A}$ is not only acyclic but even coacyclic as a complex of right $R$-modules.

We are interested in the finite complex of $\widetilde{A}^{\#}-\widetilde{A}$-bimodules $\widetilde{A}^{\#} \otimes_{R}^{\rho^{\prime}} B \otimes_{R}^{\tau^{\prime}} \widetilde{A}$, or more specifically, in its top cohomology bimodule $E=H^{m}\left(\widetilde{A}^{\#} \otimes_{R}^{\rho^{\prime}} B \otimes_{R}^{\tau^{\prime}} \widetilde{A}\right)$. According to Lemma 9.3 applied to the one-term complexes $M^{\bullet}=\widetilde{A}^{\#}$ and $L^{\bullet}=\widetilde{A}$, we have $H^{n}\left(\widetilde{A}^{\#} \otimes_{R}^{\rho^{\prime}} B \otimes_{R}^{\tau^{\prime}} \widetilde{A}\right)=0$ for all $n \neq m$, while the $\widetilde{A}^{\#}-\widetilde{A}$-bimodule $E$ is naturally isomorphic to $\widetilde{A}^{\#} \otimes_{R} T$ as an $\widetilde{A}^{\#}-R$-bimodule and naturally isomorphic to $T \otimes_{R} \widetilde{A}$ as an $R$ - $\widetilde{A}$-bimodule. The following theorem tells that $E$ is a Morita equivalence bimodule for the rings $A$ and $A^{\#}$.

Theorem 9.4. Let $(B, d, h)$ be a relatively Frobenius Koszul CDG-ring, and let $\widetilde{A}$ and $\widetilde{A}^{\#}$ be the two nonhomogeneous Koszul rings quadratic dual to $B$ on the two sides, as above. Then

(a) the tensor product and Hom functors

$$
E \otimes_{\widetilde{A}}-: \widetilde{A}-\bmod \simeq \widetilde{A}^{\#}-\bmod : \operatorname{Hom}_{\widetilde{A}^{\#}}(E,-)
$$

are mutually inverse equivalences of abelian categories;

(b) the tensor product and Hom functors

$$
-\otimes_{\widetilde{A}^{\#}} E: \bmod -\widetilde{A}^{\#} \simeq \bmod -\widetilde{A}: \operatorname{Hom}_{\widetilde{A}^{\text {op }}}(E,-)
$$

are mutually inverse equivalences of abelian categories.

Proof. The left $\widetilde{A}^{\#}$-module $E \simeq \widetilde{A}^{\#} \otimes_{R} T$ is a finitely generated projective generator

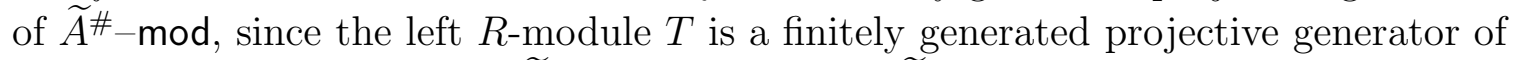
$R$-mod. Similarly, the right $\widetilde{A}$-module $E \simeq T \otimes_{R} \widetilde{A}$ is a finitely generated projective 
generator of $\bmod -\widetilde{A}$, since the right $R$-module $T$ is a finitely generated projective generator of $\bmod -R$. Finally, one computes that $\operatorname{Hom}_{\widetilde{A}^{\#}}(E, E) \simeq \operatorname{Hom}_{\widetilde{A}^{\#}}\left(\widetilde{A}^{\#} \otimes_{R} T\right.$, $E) \simeq \operatorname{Hom}_{R}(T, E) \simeq \operatorname{Hom}_{R}\left(T, T \otimes_{R} \widetilde{A}\right) \simeq \operatorname{Hom}_{R}(T, T) \otimes_{R} \widetilde{A} \simeq \widetilde{A^{\text {op }}}$, and similarly $\operatorname{Hom}_{\widetilde{A}^{\text {op }}}(E, E) \simeq \widetilde{A}^{\#}$. These observations imply the assertions of the theorem.

The result of Theorem 9.4 can be rephrased as follows. For any left $\widetilde{A}$-module $L$, the tensor product $T \otimes_{R} L$ has a natural left $\widetilde{A}^{\#}$-module structure. For any right $\widetilde{A}^{\#}$-module $M$, the tensor product $M \otimes_{R} T$ has a natural right $\widetilde{A}$-module structure. For any left $\widetilde{A}^{\#}$-module $N$, the left $R$-module $\operatorname{Hom}_{R}(T, N)$ has a natural left $\widetilde{A}$-module structure. For any right $\widetilde{A}$-module $N$, the right $R$-module $\operatorname{Hom}_{R^{\text {op }}}(T, N)$ has a natural right $\widetilde{A}^{\#}$-module structure.

Remark 9.5. When $(B, d, h)$ is a graded commutative DG-ring (so the graded ring $B$ is graded commutative and $h=0$ ), the two filtered rings $\widetilde{A}$ and $\widetilde{A}^{\#}$ are naturally opposite to each other, that is $\widetilde{A}^{\#} \simeq \widetilde{A}^{\text {op }}$. This isomorphism is induced by the natural identity isomorphism between the CDG-ring $B=(B, d, h)$ and its opposite CDG-ring $B^{\mathrm{op}}=\left(B^{\mathrm{op}}, d^{\mathrm{op}},-h^{\mathrm{op}}\right)$. So the Morita equivalence bimodule $E$ can be viewed as having two right $\widetilde{A}$-module structures which commute with each other, and Theorem 9.4 provides an equivalence between the categories of left and right $\widetilde{A}$-modules, $\widetilde{A}-\bmod \simeq \bmod -\widetilde{A}$, in this case.

Here, for an arbitrary CDG-ring $(B, d, h)$, the CDG-ring $\left(B^{\mathrm{op}}, d^{\mathrm{op}},-h^{\mathrm{op}}\right)$ is defined as follows. As a graded abelian group, $B^{\text {op }}$ is identified with $B$ by the map denoted by $b \longmapsto b^{\mathrm{op}}: B^{n} \longrightarrow B^{\mathrm{op}, n}, \quad n \in \mathbb{Z}$. The multiplication in $B^{\mathrm{op}}$ is given by the formula $b^{\mathrm{op}} c^{\mathrm{op}}=(-1)^{|b||c|}(c b)^{\mathrm{op}}$ for all $b, c \in B$, the differential is $d^{\mathrm{op}}\left(b^{\mathrm{op}}\right)=$ $d(b)^{\mathrm{op}}$, and the curvature element is $-h^{\mathrm{op}} \in B^{\mathrm{op}, 2}$. The construction of the opposite CDG-ring ( $B^{\mathrm{op}}, d^{\mathrm{op}},-h^{\mathrm{op}}$ ) has the expected property that the DG-category of right CDG-modules over $(B, d, h)$ is equivalent to the DG-category of left CDG-modules over $\left(B^{\mathrm{op}}, d^{\mathrm{op}},-h^{\mathrm{op}}\right)$ and vice versa. Notice that the formulas above imply that the map $b \longmapsto b^{\text {op }}: B \longrightarrow B^{\text {op }}$ is not an isomorphism of CDG-rings when the $\operatorname{ring} B$ is graded commutative, but $2 h \neq 0$ in $B^{2}$. Therefore, there is no natural isomorphism between the rings $\widetilde{A}^{\#}$ and $\widetilde{A}^{\text {op }}$ in the situation with a nonzero curvature in characteristic different from 2 (generally speaking).

9.7. Relatively Frobenius Koszul quadrality. We keep the assumptions and notation of Sections 9.5 9.6. So $B=(B, d, h)$ is a relatively Frobenius Koszul CDG-ring, $\widetilde{A}$ is the left finitely projective nonhomogeneous Koszul ring nonhomogeneous quadratic dual to the right finitely projective Koszul CDG-ring $(B, d, h)$, and $\widetilde{A}^{\#}$ is the right finitely projective nonhomogeneous Koszul ring nonhomogeneous quadratic dual to the left finitely projective Koszul CDG-ring $(B, d, h)$. The $\widetilde{A}^{\#}$ - $\widetilde{A}$-bimodule $E$ was constructed in the paragraph before Theorem 9.4 .

Following the discussion in Section 9.5, there are DG-functors

$$
\widetilde{A}^{\#} \otimes_{R}^{\rho^{\prime}}-: \mathrm{DG}(B-\mathrm{mod}) \longrightarrow \mathrm{DG}\left(\widetilde{A}^{\#}-\text { mod }\right)
$$


and

$$
C^{\#} \otimes_{R}^{\pi^{\prime}}-: \mathrm{DG}\left(\widetilde{A}^{\#}-\text { mod }\right) \longrightarrow \mathrm{DG}(B-\bmod )
$$

between the DG-category of complexes of left $\widetilde{A}^{\#}$-modules and the DG-category of left CDG-modules over $(B, d, h)$. Following the discussion in the beginning of Section 8.8, the DG-functor $\widetilde{A}^{\#} \otimes_{R}^{\rho^{\prime}}-$ is left adjoint to the DG-functor $C^{\#} \otimes_{R}^{\pi^{\prime}}-$. Hence the induced triangulated functors between the homotopy categories,

$$
\widetilde{A}^{\#} \otimes_{R}^{\rho^{\prime}}-: \operatorname{Hot}(B-\bmod ) \longrightarrow \operatorname{Hot}\left(\widetilde{A}^{\#}-\bmod \right)
$$

and

$$
C^{\#} \otimes_{R}^{\pi^{\prime}}-: \operatorname{Hot}\left(\widetilde{A}^{\#}-\bmod \right) \longrightarrow \operatorname{Hot}(B-\bmod ),
$$

are also adjoint on the respective sides.

Lemma 9.6. (a) For any complex of right $\widetilde{A}^{\#}{ }_{-}$modules $M^{\bullet}$, there is a natural closed isomorphism of right $C D G$-modules over $(B, d, h)$

$$
M^{\bullet} \otimes_{\widetilde{A}^{\#}} E[-m] \otimes_{R}^{\sigma^{\prime}} C \simeq M^{\bullet} \otimes_{R}^{\rho^{\prime}} B .
$$

(b) For any complex of left $\widetilde{A}$-modules $L^{\bullet}$, there is a natural closed isomorphism of left $C D G$-modules over $(B, d, h)$

$$
C^{\#} \otimes_{R}^{\pi^{\prime}} E[-m] \otimes_{\widetilde{A}} L^{\bullet} \simeq B \otimes_{R}^{\tau^{\prime}} L^{\bullet}
$$

Proof. Let us prove part (b); part (a) is opposite. We will construct a closed isomorphism $B \otimes_{R}^{\tau^{\prime}} \widetilde{A} \longrightarrow C^{\#} \otimes_{R}^{\pi^{\prime}} E[-m]$ of CDG-bimodules over $(B, d, h)$ and $(\widetilde{A}, 0,0)$. Then the desired closed isomorphism of left CDG-modules in (b) will be produced by applying the DG-functor $-\otimes_{\widetilde{A}} L^{\bullet}$.

Recall that, by the definition, the $\widetilde{A}^{\#}-\widetilde{A}$-bimodule $E$ is constructed as $E=$ $H^{m}\left(\widetilde{A}^{\#} \otimes_{R}^{\rho^{\prime}} B \otimes_{R}^{\tau^{\prime}} \widetilde{A}\right)$. Moreover, there is a natural quasi-isomorphism of finite complexes of $\widetilde{A}^{\#}-\widetilde{A}$-bimodules $\widetilde{A}^{\#} \otimes_{R}^{\rho^{\prime}} B \otimes_{R}^{\tau^{\prime}} \widetilde{A} \longrightarrow E[-m]$. Applying the DG-functor $C^{\#} \otimes_{R}^{\pi^{\prime}}-$, we obtain a closed morphism

$$
C^{\#} \otimes_{R}^{\pi^{\prime}} \widetilde{A}^{\#} \otimes_{R}^{\rho^{\prime}} B \otimes_{R}^{\tau^{\prime}} \widetilde{A} \longrightarrow C^{\#} \otimes_{R}^{\pi^{\prime}} E[-m]
$$

of CDG-bimodules over $(B, d, h)$ and $(\widetilde{A}, 0,0)$. In fact, the cone of the closed morphism (93) is a coacyclic CDG-bimodule (since $C^{\#}$ is a projective graded right $R$-module), but we will not need to use this observation.

For any left CDG-module $N$ over $(B, d, h)$, there is a natural adjunction morphism $N \longrightarrow C^{\#} \otimes_{R}^{\pi^{\prime}} \widetilde{A}^{\#} \otimes_{R}^{\rho^{\prime}} N$, which is a closed morphism of left CDG-modules over $(B, d, h)$. In particular, we are interested in the adjunction morphism

$$
B \otimes_{R}^{\tau^{\prime}} \widetilde{A} \longrightarrow C^{\#} \otimes_{R}^{\pi^{\prime}} \widetilde{A}^{\#} \otimes_{R}^{\rho^{\prime}} B \otimes_{R}^{\tau^{\prime}} \widetilde{A},
$$

which is a closed morphism of CDG-bimodules over $(B, d, h)$ and $(\widetilde{A}, 0,0)$. The composition of (94) and (93) is the desired closed morphism of CDG-bimodules

$$
B \otimes_{R}^{\tau^{\prime}} \widetilde{A} \longrightarrow C^{\#} \otimes_{R}^{\pi^{\prime}} E[-m] .
$$


In order to show that (95) is an isomorphism, one can define filtrations on the left-hand and the right-hand side and check that the associated graded map is an isomorphism. The nonhomogeneous Koszul rings $\widetilde{A}$ and $\widetilde{A}^{\#}$ are endowed with increasing filtrations $F$. A finite increasing filtration $F$ on the graded $\operatorname{ring} B$ was defined in the proof of Lemma 9.2. Hence one obtains an induced increasing filtration on the complex of $\widetilde{A}^{\#}-\widetilde{A}$-bimodules $\widetilde{A}^{\#} \otimes_{R}^{\rho^{\prime}} B \otimes_{R}^{\tau^{\prime}} \widetilde{A}$, and consequently on its cohomology $\widetilde{A}^{\#}$ - $\widetilde{A}$-bimodule $E$. Finally, a finite increasing filtration $F$ on the graded coring $C$ was defined in the proof of Theorem 6.7 (as the filtration induced by the grading); a filtration $F$ on the graded coring $C^{\#}$ is constructed similarly.

Both the left-hand and the right-hand sides of the maps (93) and (94) acquire induced filtrations, which are preserved by both the maps. It is straightforward to check that the composition (95) becomes an isomorphism after the passage to the associated graded bimodules with respect to $F$.

The following assertion is a restatement of Corollary 8.20 with $\widetilde{A}$ replaced by $\widetilde{A}^{\#}$ and ${ }^{\#} B$ replaced by $B$, and with the notation taking into account the assumption that $B^{n}=0$ for $n>m$. (Recall that Corollary 8.20, in turn, is the opposite version of Corollary 6.10.)

Corollary 9.7. Assume that the left homological dimension of the ring $R$ is $f_{\text {- }}$ nite. Then the pair of adjoint triangulated functors $\widetilde{A}^{\#} \otimes_{R}^{\rho^{\prime}}-: \operatorname{Hot}(B-\bmod ) \longrightarrow$ $\operatorname{Hot}\left(\widetilde{A}^{\#-\bmod }\right)$ and $C^{\#} \otimes_{R}^{\pi^{\prime}}-: \operatorname{Hot}\left(\widetilde{A}^{\#}-\bmod \right) \longrightarrow \operatorname{Hot}(B-\bmod )$ induces mutually inverse triangulated equivalences

$$
\mathrm{D}\left(\widetilde{A}^{\#}-\mathrm{mod}\right) \simeq \mathrm{D}^{\mathrm{co}}(B-\bmod )
$$

between the derived category of left $\widetilde{A}^{\#}$-modules and the coderived category of left $C D G$-modules over $(B, d, h)$.

The next theorem is the second main result of Section 9 ,

Theorem 9.8. Let $B=(B, d, h)$ be a relatively Frobenius Koszul CDG-ring whose degree-zero component $R=B^{0}$ is a ring of finite left homological dimension. Let $\tilde{A}$ and $\widetilde{A}^{\#}$ be two nonhomogeneous Koszul rings quadratic dual to $B$ on the two sides, as above. Then there is a commutative square diagram of triangulated equivalences

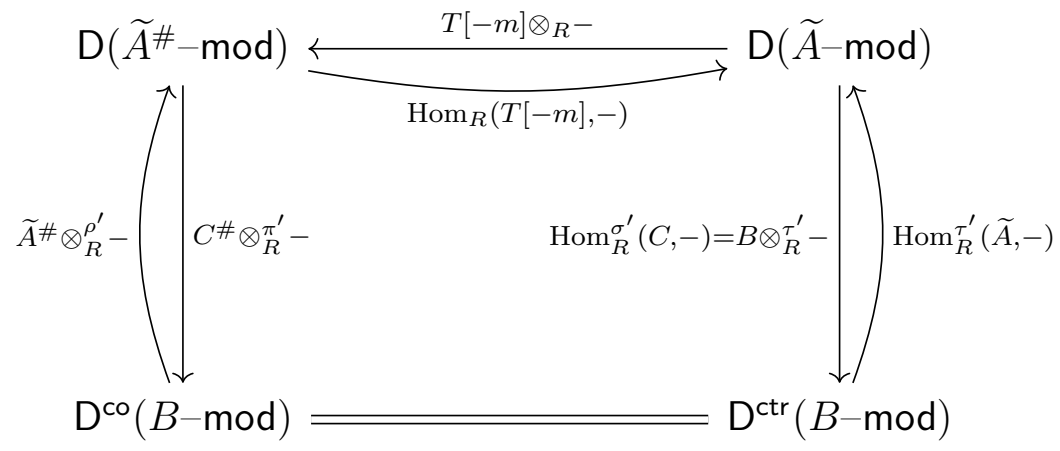


Here the triangulated equivalence in the upper line is induced by the equivalence of abelian categories from Theorem 9.4(a) (up to the cohomological shift by $[-m]$ ). The triangulated equivalence in the leftmost column is the assertion of Corollary 9.7. The triangulated equivalence in the rightmost column is a particular case of Corollary 7.9. The triangulated equivalence in the lower line is provided by Theorem 9.1.

Proof. Recall that, by the definition, for any complex of left $\widetilde{A}$-modules $L^{\bullet}$ we have $\operatorname{Hom}_{R}^{\sigma^{\prime}}\left(C, L^{\bullet}\right)=K_{e^{\prime}}^{\vee}(B, \widetilde{A}) \otimes_{\widetilde{A}} L^{\bullet}=B \otimes_{R}^{\tau^{\prime}} L^{\bullet}$, since $B^{n}=0$ for $n>m$ (see Sections 7.4 and 8.7). Hence the equality in the label at the rightmost straight arrow in the diagram. We also have $B$-contra $=B$ - $\bmod =B$-comod because $B^{n}=0$ for $n>m$.

It remains to explain why the diagram is commutative. It is convenient to check commutativity of the diagram of triangulated functors between the homotopy categories formed by the three straight arrows and the equality in the lower horizontal line. This follows from the natural closed isomorphism of left CDG-modules in Lemma 9.6(b). Then one observes that both sides of the latter closed isomorphism are CDG-modules of the induced and coinduced type; so they are adjusted to the derived functors in the construction of the triangulated equivalence in Theorem 9.1 .

\section{EXAMPLES}

10.1. Symmetric and exterior algebras. Let us start with the tensor ring. Let $R$ be an associative ring and $V$ be an $R$ - $R$-bimodule. Consider the tensor ring $T_{R}(V)=\bigoplus_{n=0}^{\infty} V^{\otimes_{R} n}$, as defined in Section 1 (see the notation in Section 2.2). By the definition, the graded ring $A=T_{R}(V)$ is quadratic.

It is clear from, e. g., Theorem 2.18(b) that the graded ring $A$ is left flat Koszul whenever $V$ is a flat left $R$-module. By Theorem 2.21(a), the graded ring $A$ is left finitely projective Koszul whenever $V$ is a finitely generated projective left $R$-module. In the latter case, the quadratic dual right finitely projective Koszul graded ring $B$ has the components $B_{0}=R, B_{1}=\operatorname{Hom}_{R}(V, R)$, and $B_{n}=0$ for all $n \geq 2$.

Now let $R$ be a commutative ring and $V$ be an $R$-module, viewed as an $R$ - $R$-bimodule in which the left and right actions of $R$ coincide. Then $T_{R}(V)$ is a graded $R$-algebra (or in other words, the degree-zero component $R=T_{R, 0}(V)$ lies in the center of $\left.T_{R}(V)\right)$. By the definition, the symmetric algebra $\operatorname{Sym}_{R}(V)=\bigoplus_{n=0}^{\infty} \operatorname{Sym}_{R}^{n}(V)$ is the largest commutative quotient algebra of $T_{R}(V)$. Equivalently, $\operatorname{Sym}_{R}(V)=$ $T_{R}(V) /(I)$ is the quadratic algebra over $R$ with the submodule of quadratic relations $I \subset V \otimes_{R} V$ spanned by all the tensors $v \otimes w-w \otimes v \in V \otimes_{R} V$ with $v, w \in V$.

The exterior algebra $\Lambda_{R}(V)=\bigoplus_{n=0}^{\infty} \Lambda_{R}^{n}(V)$ is the largest strictly graded commutative quotient algebra of $T_{R}(V)$, that is, the largest quotient algebra of $T_{R}(V)$ in which the identities $a b=(-1)^{|a||b|} b a$ and $c^{2}=0$ hold for all elements $a$ of degree $|a|, \quad b$ of degree $|b|$, and $c$ of odd degree. Equivalently, $\Lambda_{R}(V)=T_{R}(V) /(I)$ is the quadratic algebra over $R$ with the submodule of quadratic relations $I \subset V \otimes_{R} V$ spanned by all the tensors $v \otimes v \in V \otimes_{R} V$ with $v \in V$. When the element $2 \in R$ is invertible, the submodule $I \subset V \otimes_{R} V$ is also spanned by the tensors $v \otimes w+w \otimes v$ with $v, w \in V$. 
When $V$ is a (finitely generated) free $R$-module, the grading components of the quadratic algebras $\operatorname{Sym}_{R}(V)$ and $\Lambda_{R}(V)$ are also (finitely generated) free $R$-modules with explicit basises which are easy to construct. It follows by passing to retracts that the $R$-modules $\operatorname{Sym}_{R}^{n}(V)$ and $\Lambda_{R}^{n}(V)$ are (finitely generated) projective whenever the $R$-module $V$ is (finitely generated) projective. Passing to the filtered direct limits, one can see that the grading components of $\operatorname{Sym}_{R}(V)$ and $\Lambda_{R}(V)$ are flat $R$-modules whenever $V$ is a flat $R$-module.

It is worth noticing that the naïve definition of an exterior algebra as the quotient algebra of the tensor algebra by the relations $v \otimes w+w \otimes v$ with $v, w \in V$ (equivalently, the maximal quotient algebra of $T_{R}(V)$ in which the identity $a b=(-1)^{|a||b|} b a$ holds) does not have these flatness/projectivity properties. For a counterexample, it suffices to take $R$ to be the ring of integers $\mathbb{Z}$ or the ring of 2 -adic integers $\mathbb{Z}_{2}$, and $V$ to be the free $R$-module with one generator.

Let $V$ be a finitely generated projective $R$-module, and let $V^{\vee}=\operatorname{Hom}_{R}(V, R)$ denote the dual finitely generated projective $R$-module. Then the quadratic graded rings $A=\operatorname{Sym}_{R}(V)$ and $B=\Lambda_{R}\left(V^{\vee}\right)$ are quadratic dual to each other (in the sense of Propositions 1.2 and 1.3). Moreover, both the quadratic algebras $\operatorname{Sym}_{R}(V)$ and $\Lambda_{R}\left(V^{\vee}\right)$ are (left and right) finitely projective Koszul, as one can see, e. g., from exactness of the Koszul complex associated with any chosen basis in the module of generators $V$ of the polynomial algebra $\operatorname{Sym}_{R}(V)$ (use Theorem 2.21(d) or (e)).

As the class of (left or right) flat Koszul graded rings is closed under filtered direct limits, it follows that the quadratic graded rings $\operatorname{Sym}_{R}(V)$ and $\Lambda_{R}(V)$ are (left and right) flat Koszul for any flat $R$-module $V$.

Let $V$ be a finitely generated projective $R$-module everywhere of rank $m$ (i. e., for every prime ideal $\mathfrak{p}$ in $R$, the localization $V_{\mathfrak{p}}$ is a free $R_{\mathfrak{p}}$-module of rank $m$ ). Then one has $\Lambda_{R}^{n}(V)=0$ for $n>m$, and $\Lambda_{R}(V)$ is a relatively Frobenius Koszul graded ring over $R$ in the sense of Section 9.4. In other words, $\Lambda_{R}^{m}(V)$ is an invertible $R$-module and the multiplication map $\Lambda_{R}^{n}(V) \otimes_{R} \Lambda_{R}^{m-n}(V) \longrightarrow \Lambda_{R}^{m}(V)$ is a perfect pairing for every $0 \leq n \leq m$ (in the sense of Section 9.1).

10.2. Algebraic differential operators. Let $X$ be a smooth affine algebraic variety over a field $k$ (which we will eventually assume to have characteristic 0 in this section). Denote by $O(X)$ the finitely generated commutative $k$-algebra of regular functions on $X$. Differential operators on $X$ (in the sense of Grothendieck) form a subring in the ring $\operatorname{End}_{k}(O(X))$ of $k$-linear endomorphisms of the $k$-vector space $O(X)$.

The differential operators of order 0 are the $O(X)$-linear endomorphisms of $O(X)$, that is the operators of multiplication with regular functions $f \in O(X)$. A $k$-linear map $D: O(X) \longrightarrow O(X)$ is said to be a differential operator of order $\leq n$ if, for any $f \in O(X)$, the operator $[D, f]=D \circ f-f \circ D: O(X) \longrightarrow O(X)$ is a differential operator of order $\leq n-1$. A differential operator $D: O(X) \longrightarrow O(X)$ is a map which is a differential operator of some finite order $n \geq 0$.

We denote the subspace of differential operators by $\operatorname{Diff}(X) \subset \operatorname{End}_{k}(O(X))$ and the subspace of differential operators of order $\leq n$ by $F_{n} \operatorname{Diff}(X) \subset \operatorname{Diff}(X)$ for every integer $n \geq 0$. It is straightforward to check that $\operatorname{Diff}(X)$ is a subring in 
$\operatorname{End}_{k}(O(X))$ (with respect to the composition multiplication of linear operators) and $F$ is a multiplicative increasing filtration on $\operatorname{Diff}(X)=\bigcup_{n} F_{n} \operatorname{Diff}(X)$.

Let $T$ denote the tangent bundle to $X$ and $T^{*}$ denote the cotangent bundle. The global sections of the tangent bundle are called the vector fields on $X$ and the global sections of the cotangent bundle are called the differential 1-forms.

The $k$-vector space of vector fields $T(X)$ can be constructed as the subspace $T(X) \subset F_{1} \operatorname{Diff}(X)$ of all differential operators $v$ of order $\leq 1$ on $X$ which annihilate the constant functions, that is $v(1)=0$. Equivalently, $T(X)$ is the space of all derivations of the $k$-algebra $O(X)$, that is $k$-linear maps $v: O(X) \longrightarrow O(X)$ such that $v(f g)=v(f) g+f v(g)$ for all $f, g \in O(X)$. The subspace $T(X) \subset F_{1} \operatorname{Diff}(X)$ is preserved by the left (but not right) multiplications of differential operators by the functions; so the rule $(f v)(g)=f(v(g))$ defines an $O(X)$-module structure on $T(X)$.

The $O(X)$-module of differential 1 -forms $T^{*}(X)$ is produced by the construction of Kähler differentials. Consider the free $O(X)$-module spanned by the symbols $d(f)$ with $f \in O(X)$ and take its quotient module by the submodule spanned by all elements of the form $d(f g)-f d(g)-g d(f)$ with $f, g \in O(X)$ and $d(a)$ with $a \in k$; this quotient module is $T^{*}(X)$.

Since we are assuming that $X$ is smooth, both $T(X)$ and $T^{*}(X)$ are finitely generated projective $O(X)$-modules (of the rank equal to the dimension of $X$ over $k$ ). They are also naturally dual to each other: one has $T(X)=\operatorname{Hom}_{O(X)}\left(T^{*}(X), O(X)\right)$.

We are interested in the symmetric powers of the $O(X)$-module $T(X)$ and the exterior powers of the $O(X)$-module $T^{*}(X)$. Following the notation in Section 10.1, the former are denoted by $\operatorname{Sym}_{O(X)}^{n} T(X)$ and the latter by $\Lambda_{O(X)}^{n}\left(T^{*}(X)\right)=\Omega^{n}(X)$, where $n \geq 0$ (so, in particular, $\operatorname{Sym}_{O(X)}^{0} T(X)=O(X)=\Omega^{0}(X), \operatorname{Sym}_{O(X)}^{1} T(X)=$ $T(X)$, and $\left.\Omega^{1}(X)=T^{*}(X)\right)$. These are the global sections of the symmetric/exterior powers of the vector bundles $T$ and $T^{*}$ on $X$. The elements of $\Omega^{n}(X)$ are called the differential $n$-forms on $X$.

There exists a unique odd derivation $d$ of degree 1 with $d^{2}=0$ on the graded algebra $\Omega(X)$ whose restriction to $\Omega^{0}(X)=O(X)$ is the map $d: O(X) \longrightarrow \Omega^{1}(X)$ appearing in the above construction of the $O(X)$-module $\Omega^{1}(X)=T^{*}(X)$ as the module of Kähler differentials. The differential $d: \Omega^{n}(X) \longrightarrow \Omega^{n+1}(X), n \geq 0$, is called the de Rham differential. So $(\Omega(X), d)$ is a DG-ring. More precisely, it is a DG-algebra over $k$; it is called the de Rham DG-algebra.

When the characteristic of $k$ is equal to 0 , the associated graded $\operatorname{ring} \operatorname{gr}^{F} \operatorname{Diff}(X)=$ $\bigoplus_{n=0}^{\infty} F_{n} \operatorname{Diff}(X) / F_{n-1} \operatorname{Diff}(X)$ is naturally isomorphic to the graded $O(X)$-algebra $\operatorname{Sym}_{O(X)} T(X)$. Since the latter is left (and right) finitely projective Koszul, the filtered ring $(\operatorname{Diff}(X), F)$ is left finitely projective nonhomogeneous Koszul in the sense of Section 4.6. Furthermore, the ring $\operatorname{Diff}(X)$ is left augmented over its subring $O(X)$ in the sense of Section 3.8. The natural left action of $\operatorname{Diff}(X)$ in $O(X)$ (by the differential operators) provides the augmentation.

One can check that the left augmented left finitely projective nonhomogeneous Koszul ring $\operatorname{Diff}(X)$ (with the above filtration and augmentation) corresponds to the 
right finitely projective Koszul DG-ring $\Omega(X)$ (with the de Rham differential) under the anti-equivalence of categories from Corollary 4.22.

Notice that the commutative ring $R=O(X)$ has finite homological dimension (since $X$ is a smooth algebraic variety by assumption). Furthermore, the graded ring $\Omega(X)$ has only finitely many grading components (indeed, $\Omega^{n}(X)=0$ for $n>$ $\left.\operatorname{dim}_{k} X\right)$; so there is no difference between graded comodules, graded contramodules, and the conventional graded modules over $\Omega(X)$. Hence the results of Sections 6 and 7 lead to the following theorem.

Theorem 10.1. For any smooth affine algebraic variety $X$ over a field $k$ of characteristic 0 , the construction of Corollary 6.10 provides a triangulated equivalence between the derived category of right modules over the ring of algebraic differential operators $\operatorname{Diff}(X)$ and the coderived category of right DG-modules over the de Rham $D G$-algebra $(\Omega(X), d)$,

$$
\mathrm{D}(\bmod -\operatorname{Diff}(X)) \simeq \mathrm{D}^{\mathrm{co}}(\bmod -(\Omega(X), d)) .
$$

In the same context, the construction of Corollary 7.9 provides an equivalence between the derived category of left $\operatorname{Diff}(X)$-modules and the contraderived category of left $D G$-modules over $(\Omega(X), d)$,

$$
\mathrm{D}(\operatorname{Diff}(X)-\bmod ) \simeq \mathrm{D}^{\mathrm{ctr}}((\Omega(X), d)-\bmod ) .
$$

The abelian categories of left and right modules over the ring Diff $(X)$ are naturally equivalent (see the discussion in Section 0.11). The results of Section 9 provide a square diagram of triangulated equivalences connecting (98) with (99).

Theorem 10.2. For any smooth affine algebraic variety $X$ over a field $k$ of characteristic 0 , the constructions of Theorem 9.8 (with Remark 9.5 taken into account) provide a commutative square diagram of triangulated equivalences

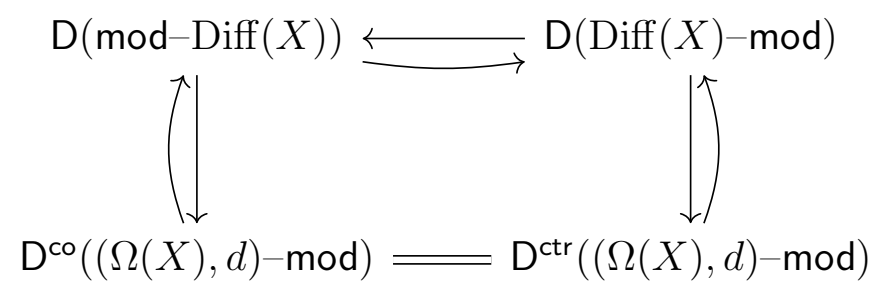

where the equivalence of derived categories in the upper line is induced by the conversion equivalence of abelian categories $\bmod -\operatorname{Diff}(X) \simeq \operatorname{Diff}(X)-\bmod ($ up to a cohomological shift by $\left.\left[-\operatorname{dim}_{k} X\right]\right)$, while the co-contra correspondence in the lower line is the result of Theorem 9.1. The vertical equivalences are (98) and (99).

Modules over the ring of differential operators Diff $(X)$ (or more generally, sheaves of modules over the sheaf of rings of differential operators over a nonaffine smooth algebraic variety $X$ ) are known colloquially as "D-modules" [5, 10, 3]. An approach to the theory of $D$-modules based on DG-modules over the de Rham DG-algebra was developed in the paper [41]. 
10.3. Crystalline differential operators. Let $X$ be a smooth affine algebraic variety over a field $k$ of arbitrary characteristic. The ring of crystalline differential operators $\operatorname{Diff}^{\mathrm{cr}}(X)$ is defined by generators and relations as follows [6, Section 1.2].

The generators are the elements of the ring of functions $O(X)$ and the $O(X)$-module of vector fields $T(X)$. The sum of any two elements of $O(X)$ in $\operatorname{Diff}^{\mathrm{cr}}(X)$ equals their sum in $O(X)$; and the sum of any two elements of $T(X)$ in $\operatorname{Diff}^{\text {cr }}(X)$ equals their sum in $T(X)$. Concerning the product, let us use the notation of Section 3.3 and denote by $*$ the product of any two elements in $\operatorname{Diffc}^{\mathrm{cr}}(X)$, to be distinguished from their product in $O(X)$ or $T(X)$. Then the relations

$$
\begin{gathered}
f * g=f g \quad \text { for all } f, g \in O(X), \\
f * v=f v \quad \text { for all } f \in O(X) \text { and } v \in T(X), \\
v * f=f v+v(f) \quad \text { for all } f \in O(X) \text { and } v \in T(X)
\end{gathered}
$$

are imposed. Here $f g \in O(X)$ denotes the product in $O(X)$ and $f v \in T(X)$ denotes the action of elements of $O(X)$ in the $O(X)$-module $T(X)$, while $v(f) \in O(X)$ denotes the action of vector fields by differential operators (or more precisely derivations) on the functions; so $v(f)$ is "the derivative of $f$ along $v$ ".

Finally, we need to recall that derivations form a Lie algebra: for any vector fields $v$ and $w \in T(X)$, there exists a unique vector field $[v, w] \in T(X)$, called the commutator of $v$ and $w$, such that $[v, w](f)=v(w(f))-w(v(f))$ for all $f \in O(X)$. The relation

$$
v * w-w * v=[v, w] \quad \text { for all } v, w \in T(X)
$$

is also imposed in $\operatorname{Diff}^{\mathrm{cr}}(X)$.

One considers the increasing filtration $F$ on the ring $\operatorname{Diff}^{\mathrm{cr}}(X)$ generated by $F_{1} \operatorname{Diff}^{\text {cr }}(X)=O(X) \oplus T(X)$ over $F_{0} \operatorname{Diff}^{\text {cr }}(X)=O(X)$ (cf. the discussion of generated filtrations in Section 3.1). Then, irrespectively of the characteristic of $k$, the associated graded ring $\operatorname{gr}^{F} \operatorname{Diff}^{\mathrm{cr}}(X)=\bigoplus_{n=0}^{\infty} F_{n} \operatorname{Diff}^{\mathrm{cr}}(X) / F_{n-1} \operatorname{Diff}^{\mathrm{cr}}(X)$ is naturally isomorphic to the symmetric algebra $\operatorname{Sym}_{O(X)} T(X)$ (this is provable as a particular case of Theorem 4.19).

There is a natural homomorphism of filtered rings $\operatorname{Diff}^{\mathrm{cr}}(X) \longrightarrow \operatorname{Diff}(X)$ uniquely defined by the condition that it acts by the identity maps on the subring $O(X) \subset$ $\operatorname{Diff}^{c r}(X)$ (taking it to the subring $\left.O(X) \subset \operatorname{Diff}(X)\right)$ and on the subspace $T(X) \subset$ $\operatorname{Diff}^{\text {cr }}(X)$ (taking it to the subspace $\left.T(X) \subset \operatorname{Diff}(X)\right)$. Over a field $k$ of characteristic 0 , this is a ring isomorphism, and in fact an isomorphism of filtered rings. But it is neither surjective nor injective in prime characteristic.

To give an example of noninjectivity, let $Y$ be the affine line over a field $k$ of prime characteristic $p$; so $O(Y)=k[y]$ is the ring of polynomials in one variable. Then $d / d y: k[y] \longrightarrow k[y]$ is a vector field on $Y$. One would expect $(d / d y)^{p}: k[y] \longrightarrow k[y]$ to be a differential operator of order $p$, but in fact it is a zero map. So $(d / d y)^{p} \in \operatorname{Diff}^{\mathrm{cr}}(Y)$ is an element of $F_{p} \operatorname{Diff}^{\text {cr }}(Y)$ not belonging to $F_{p-1} \operatorname{Diff}^{\mathrm{cr}}(Y)$, but belonging to the kernel of the ring homomorphism $\operatorname{Differ}^{\text {cr }}(Y) \longrightarrow \operatorname{Diff}(Y)$.

To give an example of nonsurjectivity, consider the ring of polynomials with integer coefficients $R=\mathbb{Z}[y]$. Then, for any element $f \in \mathbb{Z}[y]$, the element $f^{(p)}(y)=$ 
$d^{p} f / d y^{p} \in \mathbb{Z}[y]$ is divisible by $p$; so $\frac{1}{p}(d / d y)^{p}$ is a well-defined map $R \longrightarrow R$. Taking the tensor product $k \otimes_{\mathbb{Z}}-$, one obtains a differential operator of order $p$ on $k[y]$ which can be denoted by " $\frac{1}{p}(d / d y)^{p} ": k[y] \longrightarrow k[y]$. This is an element of $F_{p} \operatorname{Diff}(Y)$ not belonging to the sum of $F_{p-1} \operatorname{Diff}(Y)$ with the image of the ring homomorphism $\operatorname{Diff}^{\mathrm{cr}}(Y) \longrightarrow \operatorname{Diff}(Y)$.

To give another example, let $Z$ be the punctured affine line over $k$; so $O(Z)=$ $k\left[z, z^{-1}\right]$. Then $z \frac{d}{d z}$ is a vector field on $Z$. One would expect $\left(z \frac{d}{d z}\right)^{p}: k\left[z, z^{-1}\right] \longrightarrow$ $k\left[z, z^{-1}\right]$ to be a differential operator of order $p$, but in fact it is the same map as $z \frac{d}{d z}$. So $\left(z \frac{d}{d z}\right)^{p}-z \frac{d}{d z} \in \operatorname{Diff}^{\text {cr }}(Z)$ is an element of $F_{p} \operatorname{Diffc}^{c r}(Z)$ not belonging to $F_{p-1} \operatorname{Diff}^{\text {cr }}(Z)$, but belonging to the kernel of the ring homomorphism $\operatorname{Diff}^{\text {cr }}(Z) \longrightarrow$ $\operatorname{Diff}(Z)$. Similarly to the construction above, one can define a differential operator " $\frac{1}{p}\left(\left(z \frac{d}{d z}\right)^{p}-z \frac{d}{d z}\right) ": k\left[z, z^{-1}\right] \longrightarrow k\left[z, z^{-1}\right]$. This is an element of $F_{p} \operatorname{Diff}(Z)$ not belonging to the sum of $F_{p-1} \operatorname{Diff}(Z)$ with the image of the ring homomorphism $\operatorname{Diff}^{\mathrm{cr}}(Z) \longrightarrow \operatorname{Diff}(Z)$.

Returning to the general case, let point out that the map $F_{1} \operatorname{Diff}^{\mathrm{cr}}(X) \longrightarrow$ $F_{1} \operatorname{Diff}(X)$ is still an isomorphism, for any smooth affine variety $X$ over a field $k$ of any characteristic. In fact, the map $F_{p-1} \operatorname{Diff}^{\mathrm{cr}}(X) \longrightarrow F_{p-1} \operatorname{Diff}(X)$ is an isomorphism when $k$ has characteristic $p$. But the $\operatorname{ring} \operatorname{Diff}(X)$ is not generated by $F_{1} \operatorname{Diff}(X)$ in the latter case: the subring in $\operatorname{Diff}(X)$ generated by $F_{1} \operatorname{Diff}(X)$ does not contain $F_{p} \operatorname{Diff}(X)$ (whenever the dimension of $X$ is more than zero); moreover, the ring $\operatorname{Diff}(X)$ is not finitely generated [43, Section 3]. The ring $\operatorname{Diff}^{\mathrm{cr}}(X)$ has very different properties: over a field $k$ of prime characteristic, $\operatorname{Diff}^{\mathrm{cr}}(X)$ is finitely generated as a module over its center [6, Sections 1.3 and 2].

On the other hand, $\left(\operatorname{Diff}^{c r}(X), F\right)$ is a left finitely projective nonhomogeneous Koszul ring in the sense of Section 4.6, irrespectively of the characteristic of $k$. Composing the action of $\operatorname{Diff}(X)$ in $O(X)$ with the ring homomorphism $\operatorname{Diff}^{\text {cr }}(X) \longrightarrow$ $\operatorname{Diff}(X)$, one defines a left action of $\operatorname{Diff}^{\mathrm{cr}}(X)$ in $O(X)$ making $\operatorname{Diff}^{\mathrm{cr}}(X)$ a left augmented ring over its subring $O(X)$. The left augmented left finitely projective nonhomogeneous Koszul ring of crystalline differential operators $\operatorname{Diff}^{\mathrm{cr}}(X)$ (with the above filtration and augmentation) corresponds to the right finitely projective Koszul DG-ring of differential forms $\Omega(X)$ (with the above differential) under the anti-equivalence of categories from Corollary 4.22 .

Similarly to the characteristic 0 case of Section 10.2, the ring of functions $R=O(X)$ on any smooth affine algebraic variety over a field $k$ of any characteristic has finite homological dimension, and the graded ring $\Omega(X)$ has only finitely many grading components. Hence we have the following generalization of Theorem 10.1

Theorem 10.3. For any smooth affine algebraic variety $X$ over a field $k$, the construction of Corollary 6.10 provides a triangulated equivalence between the derived category of right modules over the ring of crystalline differential operators $\operatorname{Diff}^{\mathrm{cr}}(X)$ and the coderived category of right DG-modules over the de Rham DG-algebra $(\Omega(X), d)$,

$$
\mathrm{D}\left(\bmod -\operatorname{Diff}^{\mathrm{cr}}(X)\right) \simeq \mathrm{D}^{\mathrm{co}}(\bmod -(\Omega(X), d)) .
$$


In the same context, the construction of Corollary 7.9 provides an equivalence between the derived category of left $\operatorname{Diff}^{\mathrm{cr}}(X)$-modules and the contraderived category of left $D G$-modules over $(\Omega(X), d)$,

$$
\mathrm{D}\left(\operatorname{Diff}{ }^{\mathrm{cr}}(X)-\bmod \right) \simeq \mathrm{D}^{\mathrm{ctr}}((\Omega(X), d)-\bmod ) .
$$

Similarly to Section 10.2, there is a natural equivalence between the abelian categories of left and right modules over $\operatorname{Diff}^{\mathrm{cr}}(X)$, provided by the mutually inverse functors of tensor product with $\Omega^{m}(X)$ and $\Lambda_{O(X)}^{m}(T(X))$ over $O(X)$ (where $\left.m=\operatorname{dim}_{k} X\right)$. The results of Section 9 provide a comparison between (103) and (104).

Theorem 10.4. For any smooth affine algebraic variety $X$ over a field $k$, the constructions of Theorem 9.8 (with Remark 9.5 taken into account) provide a commutative square diagram of triangulated equivalences

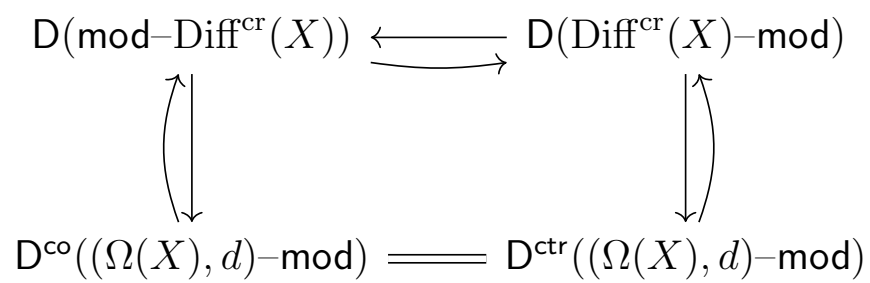

where the equivalence of derived categories in the upper line is induced by the conversion equivalence of abelian categories $\bmod -\operatorname{Diff}^{\mathrm{cr}}(X) \simeq \operatorname{Diff}^{\mathrm{cr}}(X)-\bmod$ (up to a cohomological shift by $\left.\left[-\operatorname{dim}_{k} X\right]\right)$, while the co-contra correspondence in the lower line is the result of Theorem 9.1. The vertical equivalences are (103) and (104).

Proof. Notice that $\Omega(X)$ is a relatively Frobenius Koszul graded ring (as per the discussion at the end of Section 10.1); so Theorem 9.8 is indeed applicable.

10.4. Differential operators in a vector bundle. Let $E$ be a vector bundle over a smooth affine algebraic variety $X$ (over a field $k$ ). Then the space of global sections $E(X)$ of the vector bundle $E$ is a finitely generated projective $O(X)$-module.

Given two vector bundles $E^{\prime}$ and $E^{\prime \prime}$ over $X$, one can consider differential operators acting from $E^{\prime}(X)$ to $E^{\prime \prime}(X)$. The space of such differential operators $\operatorname{Diff}\left(X, E^{\prime}, E^{\prime \prime}\right)$ is a filtered $k$-vector subspace in $\operatorname{Hom}_{k}\left(E^{\prime}(X), E^{\prime \prime}(X)\right)$.

Specifically, the differential operators of order 0 are the $O(X)$-linear maps $E^{\prime}(X)$ $\longrightarrow E^{\prime \prime}(X)$. A $k$-linear map $D: E^{\prime}(X) \longrightarrow E^{\prime \prime}(X)$ is said to be a differential operator of order $\leq n$ if, for every regular function $f \in O(X)$, the map $[D, f]=D \circ f-$ $f \circ D: E^{\prime}(X) \longrightarrow E^{\prime \prime}(X)$ is a differential operator of order $\leq n-1$. Here $f$ acts in $E^{\prime}(X)$ and $E^{\prime \prime}(X)$ as in $O(X)$-modules. A differential operator $E^{\prime}(X) \longrightarrow E^{\prime \prime}(X)$ is a map which is a differential operator of some finite order $n \geq 0$.

We denote the subspace of differential operators of order $\leq n$ by $F_{n} \operatorname{Diff}\left(X, E^{\prime}, E^{\prime \prime}\right)$ $\subset \operatorname{Diff}\left(X, E^{\prime}, E^{\prime \prime}\right)$. When the two vector bundles are the same, $E^{\prime}=E=E^{\prime \prime}$, we write simply $\operatorname{Diff}(X, E)$ instead of $\operatorname{Diff}\left(X, E^{\prime}, E^{\prime \prime}\right)$. The $k$-vector space $\operatorname{Diff}(X, E)$ with its increasing filtration $F$ is a filtered ring and a subring in $\operatorname{End}_{k}(E(X))$. 
The subring of differential operators of order 0 in $\operatorname{Diff}(X, E)$ is $F_{0} \operatorname{Diff}(X, E)=$ $\operatorname{Hom}_{O(X)}(E(X), E(X))$; it can be described as the ring of global sections $\operatorname{End}(E)(X)$ of the vector bundle $\operatorname{End}(E)$ of endomorphisms of the vector bundle $E$ over $X$.

Assuming that the characteristic of $k$ is equal to 0 , the associated graded ring $\operatorname{gr}^{F} \operatorname{Diff}(X, E)$ is naturally isomorphic to the tensor product of the $O(X)$-algebra $\operatorname{End}(E)(X)$ and the graded $O(X)$-algebra $\operatorname{Sym}_{O(X)}(T(X))$,

$$
\operatorname{gr}^{F} \operatorname{Diff}(X, E) \simeq \operatorname{End}(E)(X) \otimes_{O(X)} \operatorname{Sym}_{O(X)}(T(X)) .
$$

Both $\operatorname{End}(E)(X)$ and the grading components of $\operatorname{Sym}_{O(X)}(T(X))$ are finitely generated projective $O(X)$-modules, while the filtration components of the ring $\operatorname{Diff}(X, E)$ have the left and the right $O(X)$-module structures, induced by the inclusion $O(X) \longrightarrow \operatorname{End}(E)(X)=F_{0} \operatorname{Diff}(X, E)$. In particular, we have a short exact sequence of $O(X)-O(X)$-bimodules

(106)

$$
0 \longrightarrow \operatorname{End}(E)(X) \longrightarrow F_{1} \operatorname{Diff}(X, E) \longrightarrow \operatorname{End}(E)(X) \otimes_{O(X)} T(X) \longrightarrow 0 \text {. }
$$

In fact, this is even a short exact sequence of bimodules over the (noncommutative) $O(X)$-algebra End $(E)(X)$, which exists irrespectively of the characteristic of $k$ (cf. the discussion in Section 10.3).

A connection $\nabla$ in a vector bundle $E$ is a splitting of (106) as a short exact sequence of left $\operatorname{End}(E)(X)$-modules. All the three terms of (106) are projective as left (as well as right) $\operatorname{End}(E)(X)$-modules; so a connection in a vector bundle $E$ over a smooth affine algebraic variety $X$ always exists. In fact, the connections in $E$ form an affine space (in a different language, a principal homogenenous space) over the vector space $T^{*}(X) \otimes_{O(X)} \operatorname{End}(E)(X)$. In other words, the difference $\nabla^{\prime \prime}-\nabla^{\prime}$ of any two connections in $E$ is an element of $T^{*}(X) \otimes_{O(X)} \operatorname{End}(E)(X)$, and conversely, to any connection $\nabla$ one can add any element of $T^{*}(X) \otimes_{O(X)} \operatorname{End}(E)(X)$ and obtain a new connection in $E$.

The inclusion of rings $O(X) \longrightarrow \operatorname{End}(E)(X)$ induces an inclusion of $O(X)$-modules $T(X) \longrightarrow \operatorname{End}(E)(X) \otimes_{O(X)} T(X)$. Taking the pull-back of the short exact sequence (106) with respect to the latter map, we obtain a short exact sequence of $O(X)$ - $O(X)$-bimodules

$$
0 \longrightarrow \operatorname{End}(E)(X) \longrightarrow \bar{F}_{1} \operatorname{Diff}(X, E) \longrightarrow T(X) \longrightarrow 0 .
$$

Here $\bar{F}_{1} \operatorname{Diff}(X, E)$ is a certain $O(X)$ - $O(X)$-subbimodule in $F_{1} \operatorname{Diff}(X, E)$. A splitting of (106) as a short exact sequence of left $\operatorname{End}(E)(X)$-modules is equivalent to a left $O(X)$-linear splitting of (107).

We arrive to the definition of a connection $\nabla$ in $E$ as a map assigning to every vector field $v \in T(X)$ a $k$-linear operator $\nabla_{v}: E(X) \longrightarrow E(X)$ in such a way that the following two equations are satisfied:

(i) $\nabla_{f v}(e)=f \nabla_{v}(e)$ for all $f \in O(X), v \in T(X)$, and $e \in E(X)$;

(ii) $\nabla_{v}(f e)=v(f) e+f \nabla_{v}(e)$ for all $f \in O(X), v \in T(X)$, and $e \in E(X)$.

Here $v(f) \in O(X)$ is the derivative of $f$ along $v$. 
A connection $\nabla$ in $E$ can be also interpreted as a $k$-linear map $E(X) \longrightarrow$ $\Omega^{1}(X) \otimes_{O(X)} E(X)$ defined by the rule $\langle v, \nabla(e)\rangle=\nabla_{v}(e)$, where $\langle$,$\rangle denotes$ the $O(X)$-linear map $T(V) \otimes_{O(X)} T^{*}(X) \otimes_{O(X)} E(X) \longrightarrow E(X)$ induced by the natural pairing $T(X) \otimes_{O(X)} T^{*}(X) \longrightarrow O(X)$. Then the identity (ii) takes the form $\nabla(f e)=d(f) \otimes e+f \nabla(e)$, where $d$ denotes the de Rham differential $d: O(X) \longrightarrow \Omega^{1}(X)$.

If vector bundles $E^{\prime}$ and $E^{\prime \prime}$ over $X$ are endowed with connections $\nabla^{\prime}$ and $\nabla^{\prime \prime}$, then any vector bundle produced naturally from $E^{\prime}$ and $E^{\prime \prime}$, such as $E^{\prime} \oplus E^{\prime \prime}$ and $E^{\prime} \otimes E^{\prime \prime}$, acquires an induced connection. In particular, the vector bundle $E^{\prime} \otimes E^{\prime \prime}$ is defined by the rule $\left(E^{\prime} \otimes E^{\prime \prime}\right)(X)=E^{\prime}(X) \otimes_{O(X)} E^{\prime \prime}(X)$, and the induced connection $\nabla$ on $E^{\prime} \otimes E^{\prime \prime}$ is given by the rule $\nabla_{v}\left(e^{\prime} \otimes e^{\prime \prime}\right)=\nabla_{v}^{\prime}\left(e^{\prime}\right) \otimes e^{\prime \prime}+e^{\prime} \otimes \nabla_{v}^{\prime \prime}\left(e^{\prime \prime}\right)$ for all $e^{\prime} \in E^{\prime}(X), \quad e^{\prime \prime} \in E^{\prime \prime}(X)$, and $v \in T(X)$. Similarly, the vector bundle $\operatorname{Hom}\left(E^{\prime}, E^{\prime \prime}\right)$ is defined by the rule $\operatorname{Hom}\left(E^{\prime}, E^{\prime \prime}\right)(X)=\operatorname{Hom}_{O(X)}\left(E^{\prime}(X), E^{\prime \prime}(X)\right)$, and the induced connection $\nabla$ on $\operatorname{Hom}\left(E^{\prime}, E^{\prime \prime}\right)$ is given by the rule $\nabla_{v}(g)\left(e^{\prime}\right)=\nabla_{v}^{\prime \prime}\left(g\left(e^{\prime}\right)\right)-g\left(\nabla_{v}^{\prime}\left(e^{\prime}\right)\right)$ for all $g \in \operatorname{Hom}_{O(X)}\left(E^{\prime}(X), E^{\prime \prime}(X)\right), e^{\prime} \in E^{\prime}(X)$ and $v \in T(X)$.

Let $\nabla=\nabla_{E}$ be a connection in a vector bundle $E$ over $X$. Consider the graded left $\Omega(X)$-module $\Omega(X) \otimes_{O(X)} E(X)$ of differential forms on $X$ with the coefficients in $E$. Then the connection $\nabla$ on $E$ induces an odd derivation $d_{\nabla}$ on the graded module $\Omega(X) \otimes_{O(X)} E(X)$ compatible with the odd derivation $d$ (the de Rham differential) on the graded ring $\Omega(X)$, in the sense of Section 6.1. The map $d_{\nabla}: \Omega^{n}(X) \otimes_{O(X)} E(X)$ $\longrightarrow \Omega^{n+1}(X) \otimes_{O(X)} E(X)$ is given by the formula

$$
d_{\nabla}(\omega \otimes e)=d(\omega) \otimes e+(-1)^{n} \omega \wedge \nabla(e)
$$

for all $\omega \in \Omega^{n}(X)$ and $e \in E(X)$. Here the wedge $\wedge$ denotes the map $\Omega^{n}(X) \otimes_{k}$ $\Omega^{1}(X) \otimes_{O(X)} E(X) \longrightarrow \Omega^{n+1}(X) \otimes_{O(X)} E(X)$ induced by the multiplication map $\Omega^{1}(X) \otimes_{k} \Omega^{n}(X) \longrightarrow \Omega^{n+1}(X)$. One needs to check that the map $d_{\nabla}=d_{\nabla_{E}}$ is well-defined, that is $d_{\nabla}(f \omega \otimes e)=d_{\nabla}(\omega \otimes f e)$ for all $f \in O(X)$.

The square $d_{\nabla}^{2}$ of the differential $d_{\nabla}$ on $\Omega(X) \otimes_{O(X)} E(X)$ is an $\Omega(X)$-linear map. Hence there exists an element $h_{\nabla} \in \Omega^{2}(X) \otimes_{O(X)} \operatorname{End}(E)(X)$ such that $d_{\nabla}^{2}(\phi)=h(\phi)$ for all $\phi \in \Omega(X) \otimes_{O(X)} E(X)$. Here the left action of $\Omega(X) \otimes_{O(X)} \operatorname{End}(E)(X)$ in $\Omega(X) \otimes_{O(X)} E(X)$ is induced by the multiplication in $O(X)$ and the left action of $\operatorname{End}(E)(X)$ in $E(X)$. The element $h_{\nabla}=h_{\nabla_{E}} \in \Omega^{2}(X) \otimes_{O(X)} \operatorname{End}(E)(X)$ is called the curvature of the connection $\nabla$ in a vector bundle $E$.

The tensor product $\Omega(X) \otimes_{O(X)} \operatorname{End}(E)(X)$ of the graded $O(X)$-algebra $\Omega(X)$ with the $O(X)$-algebra $\operatorname{End}(E)(X)$ has a natural structure of graded $O(X)$-algebra. The connection $\nabla=\nabla_{E}$ on the vector bundle $E$ induces a connection $\nabla_{\operatorname{End}(E)}$ on the vector bundle $\operatorname{End}(E)$ on $X$, as explained above. The induced differential $d_{\nabla_{\operatorname{End}(E)}}$ on the graded $\Omega(X)$-module $\Omega(X) \otimes_{O(X)} \operatorname{End}(E)(X)$ is, in fact, an odd derivation of the graded algebra $\Omega(X) \otimes_{O(X)} \operatorname{End}(E)(X)$ (since the connection $\nabla_{\operatorname{End}(E)}$ is compatible with the composition multiplication on $\operatorname{End}(E)$, in the appropriate sense). The triple

$$
\left(\Omega(X) \otimes_{O(X)} \operatorname{End}(E)(X), d_{\nabla_{\operatorname{End}(E)}}, h_{\nabla_{E}}\right)
$$


is a curved DG-ring. The pair

$$
\left(\Omega(X) \otimes_{O(X)} E(X), d_{\nabla_{E}}\right)
$$

is a left CDG-module over (109).

The graded ring $A=\operatorname{End}(E)(X) \otimes_{O(X)} \operatorname{Sym}_{O(X)}(T(X))$ is left (and right) finitely projective Koszul (over its degree-zero component $R=\operatorname{End}(E)(X)$ ). The quadratic dual right (and left) finitely projective Koszul graded ring to $A$, as per the construction of Propositions 1.2 and [1.3, is $B=\Omega(X) \otimes_{O(X)} \operatorname{End}(E)(X)$.

Assume that the characteristic of $k$ is equal to 0 . Then the filtered ring (Diff $(X, E), F)$ is a left finitely projective nonhomogeneous Koszul ring. The choice of a connection $\nabla$ on $E$ means the choice of a left $\operatorname{End}(E)(X)$-linear splitting of the short exact sequence (106); in the terminology of Section 3.3 , this is the choice of a submodule of strict generators for $\operatorname{Diff}(X, E)$. The corresponding right finitely projective Koszul CDG-ring produced by the construction of Proposition 3.6 is the CDG-ring $\Omega(X) \otimes_{O(X)} \operatorname{End}(E)(X)$ (109). Replacing a connection $\nabla_{E}$ in $E$ with another connection $\nabla_{E}^{\prime}$ leads to a CDG-ring $\left(\Omega(X) \otimes_{O(X)} \operatorname{End}(E)(X), d_{\nabla_{\operatorname{End}(E)}^{\prime}}, h_{\nabla_{E}^{\prime}}\right)$ connected with (109) by a natural change-of-connection isomorphism of CDG-rings, as per the discussion in Sections 3.2 and 3.5 .

The ring of endomorphisms $R=\operatorname{End}(E)(X)$ of any vector bundle $E$ on a smooth affine algebraic variety $X$ has finite left and right homological dimensions; in fact, assuming that $E$ is nonzero on all the connected components of $X$, both the abelian categories of left and right $R$-modules are equivalent to $O(X)$-mod. Furthermore, just as in Section 10.2, the graded ring $\Omega(X) \otimes_{O(X)} \operatorname{End}(E)(X)$ has only finitely many grading components. Hence we obtain the following theorem.

Theorem 10.5. For any smooth affine algebraic variety $X$ over a field $k$ of characteristic 0 and any vector bundle $E$ over $X$ with a chosen connection $\nabla_{E}$, the construction of Corollary 6.10 provides a triangulated equivalence between the derived category of right modules over the ring of differential operators $\operatorname{Diff}(X, E)$ acting in the sections of $E$ and the coderived category of right $C D G$-modules over the $C D G$-algebra (109) of differential forms on $X$ with the coefficients in $\operatorname{End}(E)$,

$$
\mathrm{D}(\bmod -\operatorname{Diff}(X, E)) \simeq \mathrm{D}^{\mathrm{co}}\left(\bmod -\left(\Omega(X) \otimes_{O(X)} \operatorname{End}(E)(X), d_{\nabla_{\operatorname{End}(E)}}, h_{\nabla_{E}}\right)\right) .
$$

In the same context, the construction of Corollary 7.9 provides an equivalence between the derived category of left $\operatorname{Diff}(X, E)$-modules and the contraderived category of left $C D G$-modules over the CDG-ring (109)

$$
\mathrm{D}(\operatorname{Diff}(X, E)-\mathrm{mod}) \simeq \mathrm{D}^{\mathrm{ctr}}\left(\left(\Omega(X) \otimes_{O(X)} \operatorname{End}(E)(X), d_{\nabla_{\operatorname{End}(E)}}, h_{\nabla_{E}}\right)-\bmod \right) .
$$

For example, the equivalence of categories (112) assigns the left CDG-module $\left(\Omega(X) \otimes_{O(X)} E(X), d_{\nabla_{E}}\right)$ (110) over the CDG-ring (109) to the left $\operatorname{Diff}(X, E)$-module $E(X)$. The result of Theorem 10.5 is the affine, characteristic 0 particular case of the $\mathcal{D}-\Omega$ duality theorem of [28, Theorem B.2]. 
10.5. Twisted differential operators. Let us start with specializing the discussion in Section 10.4 to the case of a line bundle $E=L$. In this case $L(X)$ in an invertible finitely generated projective $O(X)$-module; specifically, one has $L(X) \otimes_{O(X)} L^{*}(X) \simeq$ $O(X)$, where $L^{*}$ is the dual line bundle to $L$. Notice that for any vector bundle $E$ one has $\operatorname{End}(X)=E \otimes E^{*}$, so $\operatorname{End}(E)(X)=E(X) \otimes_{O(X)} E^{*}(X)$ (where $E^{*}$ is the dual vector bundle to $E$, so $\left.E^{*}(X)=\operatorname{Hom}_{O(X)}(E(X), O(X))\right)$. For a line bundle $L$, this means that $\operatorname{End}(L)$ is the trivial line bundle, $\operatorname{End}(L)(X)=O(X)$.

Choose a connection $\nabla=\nabla_{L}$ in $L$. Then the induced connection $\nabla_{\operatorname{End}(L)}$ is the trivial (canonical) connection in the trivial line bundle $\operatorname{End}(L)$. Therefore, the graded ring $\Omega(X) \otimes_{O(X)}$ End $(L)(X)$ is simply the ring of differential forms $\Omega(X)$, and the differential $d_{\nabla_{\operatorname{End}(L)}}$ in the CDG-ring (109) is equal to the standard de Rham differential, $d_{\nabla_{\text {End }(L)}}=d$. However, the curvature form $h_{\nabla_{L}} \in \Omega^{2}(X)$ of the connection $\nabla_{L}$ in $L$ can well be nontrivial. It is always a closed differential form: $d\left(h_{\nabla_{L}}\right)=0$ in $\Omega^{3}(X)$ (cf. the equation (iii) in the definition of a CDG-ring in Section 3.2).

Replacing $\nabla_{L}$ with another connection $\nabla_{L}^{\prime}$ in $L$ replaces the differential 2-form $h_{\nabla_{L}}$ with $h_{\nabla_{L}^{\prime}}=h_{\nabla_{L}}+d(\alpha)$, where $\alpha=\nabla_{L}^{\prime}-\nabla_{L} \in \Omega^{1}(X)$ is the change-of-connection 1-form (see the discussion in Section 10.4, cf. Section 3.5). So the cohomology class of the 2-form $h_{\nabla_{L}}$, viewed as an element of the cohomology ring of the DG-ring $(\Omega(X), d)$, does not depend on a connection $\nabla_{L}$, but only on the line bundle $L$ itself. It is called the first Chern class of $L$ and denoted by $c_{1}(L) \in H^{2}(\Omega(X), d)$.

For any integer $n \in \mathbb{Z}$, one can consider the line bundle $L^{\otimes n}$ over $X$, defined in the obvious way for $n \geq 0$ and by the rule $L^{\otimes n}=L^{* \otimes-n}$ for $n \leq 0$. The curvature form of the induced connection $\nabla_{L \otimes n}$ in $L^{\otimes n}$ is given by the rule $h_{\nabla_{L} \otimes n}=n h_{\nabla_{L}} \in \Omega^{2}(X)$.

Now assume for a moment that $k$ is a field of characteristic 0 , and let $z \in k$ be any element. Then there is no such thing as "a line bundle $L^{\otimes z}$ over $X$ ". However, one can define a filtered ring $\operatorname{Diff}\left(X, L^{\otimes z}\right)$ of "differential operators acting in the sections of $L^{\otimes z} "$. For this purpose, one simply chooses a connection $\nabla$ in $L$, considers the right finitely projective Koszul CDG-ring $\left(\Omega(X), d, z h_{\nabla}\right)$, and constructs $\operatorname{Diff}\left(X, L^{\otimes z}\right)$ as the left finitely projective nonhomogeneous Koszul ring corresponding to $\left(\Omega(X), d, z h_{\nabla}\right)$ under the anti-equivalence of categories from Corollary 4.20.

Replacing the connection $\nabla$ with another connection $\nabla^{\prime}$ in $L$ corresponds to a natural change-of-connection isomorphism between the CDG-rings $\left(\Omega(X), d, h_{\nabla}\right)$ and $\left(\Omega(X), d, h_{\nabla^{\prime}}\right)$, and consequently also a change-of-connection isomorphism between the CDG-rings $\left(\Omega(X), d, z h_{\nabla}\right)$ and $\left(\Omega(X), d, z h_{\nabla^{\prime}}\right)$. This simply means that one has $h_{\nabla_{L}^{\prime}}=h_{\nabla_{L}}+d(\alpha)$, and consequently $z h_{\nabla_{L}^{\prime}}=z h_{\nabla_{L}}+d(z \alpha)$, where $\alpha=\nabla_{L}^{\prime}-\nabla_{L}$ (as $\alpha^{2}=0$ in $\Omega^{2}(X)$ ). An isomorphism of right finitely projective Koszul CDG-rings induces an isomorphism of the corresponding left finitely projective nonhomogeneous Koszul rings; so the filtered ring $\left(\operatorname{Diff}\left(X, L^{\otimes z}\right), F\right)$ is defined uniquely up to a natural isomorphism. Similarly, given two line bundles $L_{1}$ and $L_{2}$ over $X$ and two scalars $z_{1}$ and $z_{2} \in k$, one can define a filtered ring $\operatorname{Diff}\left(X, L_{1}^{\otimes z_{1}} \otimes L_{2}^{\otimes z_{2}}\right)$, etc.

Quite generally, let $X$ be a smooth affine variety over a field $k$ of arbitrary characteristic, and let $h \in \Omega^{2}(X)$ be a closed 2-form (i. e., $\left.d(h)=0\right)$. Then the triple $(\Omega(X), d, h)$, with the standard de Rham differential $d$ and the curvature element $h$, 
is a curved DG-ring (because the graded ring $\Omega(X)$ is graded commutative, so in particular $h$ is a central element in $\Omega(X)$ and the equation (ii) of Section 3.2 is satisfied). The curved DG-ring $(\Omega(X), d, h)$ is right finitely projective Koszul. The corresponding left finitely projective nonhomogeneous Koszul ring under the antiequivalence of categories from Corollary 4.20 is denoted by $\operatorname{Diff}^{\mathrm{cr}}(X, h)$ and called the ring of twisted crystalline differential operators on $X$ (twisted by $h$ ). (See [3, Section 2] or [10, Chapter II] for a much more abstract discussion of twisted differential operators over nonaffine varieties.)

Explicitly, $\operatorname{Diff}^{\mathrm{cr}}(X, h)$ is the filtered ring generated by the functions on $X$ (placed in the filtration component $F_{0}$ ) and the vector fields on $X$ (placed in the filtration component $F_{1}$ ), subject to the same relations as in Section 10.3, except that the relation (102) is replaced with

$$
v * w-w * v=[v, w]+\langle v \wedge w, h\rangle,
$$

where $\langle$,$\rangle denotes the natural pairing \Lambda_{O(X)}^{2}(T(X)) \otimes_{O(X)} \Lambda_{O(X)}^{2}\left(T^{*}(X)\right) \longrightarrow O(X)$ given by the rule $\langle v \wedge w, \alpha \wedge \beta\rangle=\langle v, \alpha\rangle\langle w, \beta\rangle-\langle v, \beta\rangle\langle w, \alpha\rangle$ for $v, w \in T(X)$ and $\alpha$, $\beta \in T^{*}(X)$ (cf. formula (28) in Section 3.3). So $[v, w] \in T(X)$ is a vector field and $\langle v \wedge w, h\rangle \in O(X)$ is a function on $X$. It is claimed, based on Theorem 4.19, that the associated graded ring $\operatorname{gr}^{F} \operatorname{Diff}^{\mathrm{cr}}(X, h)=\bigoplus_{n=0}^{\infty} F_{n} \operatorname{Diff}^{\mathrm{cr}}(X, h) / F_{n-1} \operatorname{Diff}^{\mathrm{cr}}(X, h)$ is naturally isomorphic to the symmetric algebra $\operatorname{Sym}_{O(X)}(T(X))$. The assumption that $d(h)=0$ is needed for this to be true.

When two closed 2-forms $h^{\prime}$ and $h^{\prime \prime} \in \Omega^{2}(X)$ represent the same de Rham cohomology class in $H^{2}(\Omega(X), d)$, the related filtered rings of twisted (crystalline) differential operators $\operatorname{Diff}^{\mathrm{cr}}\left(X, h^{\prime}\right)$ and $\operatorname{Diff}^{\mathrm{cr}}\left(X, h^{\prime \prime}\right)$ are isomorphic, but not yet naturally isomorphic. The choice of a 1 -form $\alpha \in \Omega^{1}(X)$ such that $h^{\prime \prime}-h^{\prime}=d(\alpha)$ leads to a concrete isomorphism of filtered rings $\operatorname{Diff}^{\mathrm{cr}}\left(X, h^{\prime}\right) \simeq \operatorname{Diff}^{\mathrm{cr}}\left(X, h^{\prime \prime}\right)$.

The results of Sections 6 and 7 lead to the following theorem.

Theorem 10.6. For any smooth affine algebraic variety $X$ over a field $k$ and any closed differential 2-form $h \in \Omega^{2}(X), d(h)=0$, the construction of Corollary 6.10 provides a triangulated equivalence between the derived category of right modules over the ring of twisted (crystalline) differential operators $\operatorname{Diff}^{\mathrm{cr}}(X, h)$ and the coderived category of right $C D G$-modules over the de Rham $C D G$-algebra $(\Omega(X), d, h)$,

$$
\mathrm{D}\left(\bmod -\operatorname{Diff}^{\mathrm{cr}}(X, h)\right) \simeq \mathrm{D}^{\mathrm{co}}(\bmod -(\Omega(X), d, h)) .
$$

In the same context, the construction of Corollary 7.9 provides an equivalence between the derived category of left $\operatorname{Diff}^{\mathrm{cr}}(X, h)$-modules and the contraderived category of left $C D G$-modules over $(\Omega(X), d, h)$,

$$
\mathrm{D}\left(\operatorname{Diff}^{\mathrm{cr}}(X, h)-\mathrm{mod}\right) \simeq \mathrm{D}^{\mathrm{ctr}}((\Omega(X), d, h)-\bmod ) .
$$

For any closed 2-form $h$ on $X$, there is a natural equivalence between the abelian categories of left modules over $\operatorname{Diff}^{\text {cr }}(X, h)$ and right modules over $\operatorname{Diffcr}^{\mathrm{cr}}(X,-h)$, provided by the mutually inverse functors of tensor product with the $O(X)$-modules 
modules of top differential forms and top polyvector fields on $X$. This Morita equivalence can be obtained as a particular case of Theorem 9.4 with Remark 9.5. The results of Section 9 provide a comparison between (114) and (115).

Theorem 10.7. For any smooth affine algebraic variety $X$ over a field $k$ and any closed differential 2-form $h \in \Omega^{2}(X)$, the constructions of Theorem 9.8 (with Remark 9.5) provide a commutative square diagram of triangulated equivalences

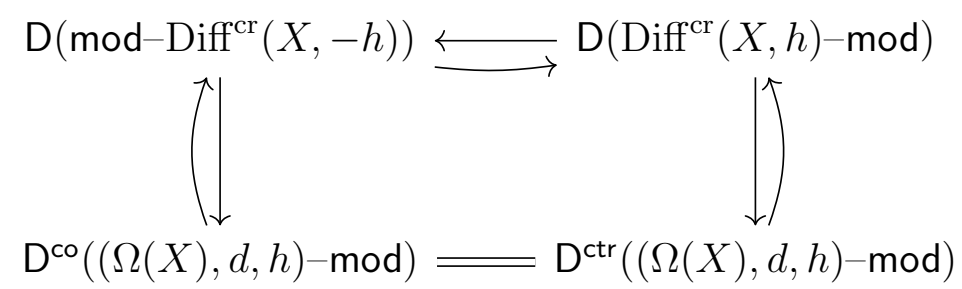

where the equivalence of derived categories in the upper line is induced by the conversion equivalence of abelian categories $\bmod -\operatorname{Diff}^{\mathrm{cr}}(X,-h) \simeq \operatorname{Diff}^{\mathrm{cr}}(X, h)$-mod (up to a shift by $\left.\left[-\operatorname{dim}_{k} X\right]\right)$, while the co-contra correspondence in the lower line is the result of Theorem 9.1. The vertical equivalences are (114) and (115).

10.6. Smooth differential operators. Let $X$ be a smooth compact real manifold. Denote by $O(X)$ the ring of smooth global functions $X \longrightarrow \mathbb{R}$.

We will consider smooth locally trivial vector bundles $E$ on $X$. Then the $\mathbb{R}$-vector space of smooth global sections $E(X)$ has a natural $O(X)$-module structure. Moreover, the $O(X)$-module $E(X)$ is finitely generated and projective. The correspondence $E \longmapsto E(X)$ is an equivalence between the category of (smooth locally trivial) vector bundles on $X$ and the category of finitely generated projective $O(X)$-modules. In particular, any short exact sequence of vector bundles over $X$ splits (as one can show using a partition of unity on $X$ ). In this sense, smooth compact real manifolds are analogues of affine algebraic varieties.

Given a vector bundle $E$ on $X$, the module of global sections of the dual vector bundle $E^{*}$ can be obtained as the dual finitely generated projective module, $E^{*}(X)=\operatorname{Hom}_{O(X)}(E(X), O(X))$. Given two vector bundles $E^{\prime}$ and $E^{\prime \prime}$, the global sections of the tensor product bundle $E^{\prime} \otimes E^{\prime \prime}$ are the tensor product of the global sections, $\left(E^{\prime} \otimes E^{\prime \prime}\right)(X)=E^{\prime}(X) \otimes_{O(X)} E^{\prime \prime}(X)$. Similarly, the global sections of the symmetric and exterior powers of a vector bundle $E$ are computable as the symmetric and exterior powers of the global sections, $\left(\operatorname{Sym}^{n} E\right)(X)=\operatorname{Sym}_{O(X)}^{n}(E(X))$ and $\left(\Lambda^{n} E\right)(X)=\Lambda_{O(X)}^{n}(E(X))$.

In particular, the tangent bundle $T$ and its dual (cotangent) bundle $T^{*}$ are smooth locally trivial vector bundles on $X$. In a local coordinate system $x_{1}, \ldots, x_{m}$ defined on an open subset $U \subset X$, the sections of $T$ (called the vector fields) are represented by expressions like $\sum_{i=1}^{m} f_{i} \partial / \partial x_{i}$ and the sections of $T^{*}$ (called the differential 1-forms) are represented by expressions like $\sum_{i=1}^{m} f_{i} d x_{i}$, where $f_{i}: U \longrightarrow \mathbb{R}$ are local functions. The natural map $d: O(X) \longrightarrow T^{*}(X)$ (the differential) is defined locally by the rule $d(f)=\sum_{i=1}^{m} \partial f / \partial x_{i} d x_{i}$, and the action of vector fields in the functions (the 
derivative $v(f)$ of a function $f$ along a vector field $v$ ) is given locally by the formula $\left(\sum_{i=1}^{m} f_{i} \partial / \partial x_{i}\right)(f)=\sum_{i=1}^{m} f_{i} \partial f / \partial x_{i}$.

Let $E^{\prime}$ and $E^{\prime \prime}$ be two vector bundles on $X$ of ranks (the dimensions of the fibers) $r^{\prime}$ and $r^{\prime \prime} \geq 0$. Then a differential operator $D: E^{\prime}(X) \longrightarrow E^{\prime \prime}(X)$ of order $\leq n$ is an $\mathbb{R}$-linear map such that, for any open subset $U \subset X$ with a coordinate system $x_{1}, \ldots, x_{m}$ and any chosen trivializations of $E^{\prime}$ and $E^{\prime \prime}$ over $U$, the operator $D$ can be expressed locally over $U$ as a linear combination of compositions of at most $n$ partial derivatives $\partial / \partial x_{i}$ (acting in vector functions $U \longrightarrow \mathbb{R}^{r^{\prime}}$ component-wise) with $r^{\prime \prime} \times r^{\prime}$-matrices of smooth functions $U \longrightarrow \mathbb{R}$ as the coefficients. In particular, a differential operator $D: O(X) \longrightarrow O(X)$ of order $\leq n$ is represented locally over $U$ as a linear combination of compositions of at most $n$ partial derivatives $\partial / \partial x_{i}$, $1 \leq i \leq m$, with smooth local functions $U \longrightarrow \mathbb{R}$ as the coefficients. A differential operator $E^{\prime}(X) \longrightarrow E^{\prime \prime}(X)$ of order 0 is the same thing as a global section of the vector bundle $\operatorname{Hom}\left(E^{\prime}, E^{\prime \prime}\right)$, or an $O(X)$-linear map $E^{\prime}(X) \longrightarrow E^{\prime \prime}(X)$.

We denote the $\mathbb{R}$-vector space of smooth differential operators $E^{\prime}(X) \longrightarrow E^{\prime \prime}(X)$ by $\operatorname{Diff}\left(X, E^{\prime}, E^{\prime \prime}\right)$, and the subspace of differential operators of order $\leq n$ by $F_{n} \operatorname{Diff}\left(X, E^{\prime}, E^{\prime \prime}\right) \subset \operatorname{Diff}\left(X, E^{\prime}, E^{\prime \prime}\right) ;$ so $\operatorname{Diff}\left(X, E^{\prime}, E^{\prime \prime}\right)=\bigcup_{n=0}^{\infty} F_{n} \operatorname{Diff}\left(X, E^{\prime}, E^{\prime \prime}\right)$. When the two vector bundles $E^{\prime}=E=E^{\prime \prime}$ are the same, we write $\operatorname{simply} \operatorname{Diff}(X, E)$. Then $\operatorname{Diff}(X, E)$ is a subalgebra in the $\mathbb{R}$-algebra $\operatorname{End}_{\mathbb{R}}(E(X))$ of all $\mathbb{R}$-linear endomorphisms of the vector space $E(X)$ (with respect to the composition). Furthermore, $\operatorname{Diff}(X, E)$ is a filtered ring with an increasing filtration $F$. When $E$ is the trivial line bundle on $X$ (so $E(X)=O(X)$ ), we denote simply by $\operatorname{Diff}(X)$ the filtered ring of differential operators $O(X) \longrightarrow O(X)$.

Global sections of the exterior power $\Lambda^{n}\left(T^{*}\right)$ of the cotangent bundle $T^{*}$ are called the differential $n$-forms on $X$. The vector space (in fact, $O(X)$-module) of differential $n$-forms is denoted by $\Omega^{n}(X)=\Lambda_{O(X)}^{n}\left(T^{*}(X)\right)$. There is a natural differential operator $d: \Omega^{n}(X) \longrightarrow \Omega^{n+1}(X)$ of order 1 , defined for every $n \geq 0$ and called the de Rham differential. The operator $d$ is uniquely defined by the property of being and odd derivation of the graded algebra $\Omega(X)$ with $d^{2}=0$ whose restriction to the ring of functions, $d: O(X) \longrightarrow \Omega^{1}(X)=T^{*}(X)$, is the map mentioned above.

The left action of $\operatorname{Diff}(X)$ by differential operators in $O(X)$ makes $\operatorname{Diff}(X)$ a left augmented ring over its subring $O(X)=F_{0} \operatorname{Diff}(X)$, in the sense of Section 3.8. The associated graded ring $\operatorname{gr}^{F} \operatorname{Diff}(X)=\bigoplus_{n=0}^{\infty} F_{n} \operatorname{Diff}(X) / F_{n-1} \operatorname{Diff}(X)$ is naturally isomorphic to the graded ring $\operatorname{Sym}_{O(X)}(T(X))$, which is left and right finitely projective Koszul (according to the discussion in Section 10.1). Similarly to Sections 10.2 10.3, the anti-equivalence of categories from Corollary 4.22 assigns the right finitely projective Koszul DG-ring $(\Omega(X), d)$ to the left augmented left finitely projective nonhomogeneous Koszul ring $\operatorname{Diff}(X)$ (with the filtration $F$ ).

Hence the results of Sections 6 and 7 lead us to the following theorem. It is a rather rough algebraic version of derived relative nonhomogeneous Koszul duality for the ring of smooth differential operators $\operatorname{Diff}(X)$, in that the base ring of smooth functions $O(X)$ is considered as an abstract ring without any additional structures (such as a Banach space metric or a topology). Notice that, similarly to the algebraic 
examples above, the graded ring $\Omega(X)$ has only finitely many grading components $\left(\right.$ as $\Omega^{n}(X)=0$ for $\left.n>\operatorname{dim}_{\mathbb{R}} X\right)$.

Theorem 10.8. For any smooth compact real manifold $X$, the construction of Theorem 6.7 provides a triangulated equivalence between the $\operatorname{Diff}(X) / O(X)$-semicoderived category of right modules over the ring of smooth differential operators $\operatorname{Diff}(X)$ and the coderived category of right DG-modules over the de Rham DG-algebra $(\Omega(X), d)$,

$$
\mathrm{D}_{O(X)}^{\text {sico }}(\bmod -\operatorname{Diff}(X)) \simeq \mathrm{D}^{\mathrm{co}}(\bmod -(\Omega(X), d)),
$$

while Theorem 6.11 establishes a triangulated equivalence between the derived category of right $\operatorname{Diff}(X)$-modules and the reduced coderived category of right DG-modules over $(\Omega(X), d)$ relative to $O(X)$,

$$
\mathrm{D}(\bmod -\operatorname{Diff}(X)) \simeq \mathrm{D}_{O(X)-\operatorname{red}}^{\mathrm{co}}(\bmod -(\Omega(X), d)) .
$$

In the same context, the construction of Theorem 7.6 provides a triangulated equivalence between the $\operatorname{Diff}(X) / O(X)$-semicontraderived category of left modules over the ring $\operatorname{Diff}(X)$ and the contraderived category of left DG-modules over the DG-algebra $(\Omega(X), d)$,

$$
\mathrm{D}_{O(X)}^{\mathrm{sictr}}(\operatorname{Diff}(X)-\mathrm{mod}) \simeq \mathrm{D}^{\mathrm{ctr}}((\Omega(X), d)-\bmod )
$$

while Theorem 7.10 establishes a triangulated equivalence between the derived category of left $\operatorname{Diff}(X)$-modules and the reduced contraderived category of left DG-modules over $(\Omega(X), d)$ relative to $O(X)$,

$$
\mathrm{D}(\operatorname{Diff}(X)-\bmod ) \simeq \mathrm{D}_{O(X)-\operatorname{red}}^{\mathrm{ctr}}((\Omega(X), d)-\bmod ) .
$$

There is also a classical natural equivalence between the abelian categories of left and right $\operatorname{Diff}(X)$-modules, $\operatorname{Diff}(X)-\bmod \simeq \bmod -\operatorname{Diff}(X)$, which was discussed in Section 0.11. It can be obtained as a particular case of Theorem 9.4 with Remark 9.5 (since in fact $\Omega(X)$ is a relatively Frobenius Koszul graded ring).

More generally, the associated graded ring $\operatorname{gr}^{F} \operatorname{Diff}(X, E)=\bigoplus_{n=0}^{\infty} F_{n} \operatorname{Diff}(X, E) /$ $F_{n-1} \operatorname{Diff}(X, E)$ is naturally isomorphic to the tensor product $\operatorname{End}(E)(X) \otimes_{O(X)}$ $\operatorname{Sym}_{O(X)}(T(X))$ of the $O(X)$-algebra $\operatorname{End}(E)(X)=\operatorname{Hom}_{O(X)}(E(X), E(X))$ of sections of the vector bundle $\operatorname{End}(E)$ and the graded $O(X)$-algebra $\operatorname{Sym}_{O(X)}(T(X))$. This graded ring is left and right finitely projective Koszul over its degree-zero component $\operatorname{End}(E)(X)$. So the filtered $\operatorname{ring}(\operatorname{Diff}(X, E), F)$ is left finitely projective nonhomogeneous Koszul. In order to describe the corresponding CDG-ring, the notion of a connection in a smooth vector bundle $E$ over $X$ is needed.

A (smooth) connection $\nabla$ in a smooth vector bundle $E$ is a map assigning to every smooth vector field $v \in T(X)$ and any smooth section $e \in E(X)$ a smooth section $\nabla_{v}(e) \in E(X)$ such that the equations (i) and (ii) from Section 10.4 are satisfied. Using a partition of unity, one can show that a smooth connection exists in any smooth vector bundle over a smooth manifold. 
Similarly to the algebraic case discussed in Section 10.4, a connection $\nabla$ in $E$ can be interpreted as an $\mathbb{R}$-linear map $E(X) \longrightarrow \Omega^{1}(X) \otimes_{O(X)} E(X)$ defined by the rule $\langle v, \nabla(e)\rangle=\nabla_{v}(e)$. In fact, it is a differential operator $E(X) \longrightarrow\left(T^{*} \otimes E\right)(X)$ of order 1 . The trivial line bundle on $X$ has a canonical trivial connection defined by the rule $\nabla_{v}(f)=v(f)$ for all $v \in T(X)$ and $f \in O(X)$. If vector bundles $E^{\prime}$ and $E^{\prime \prime}$ over $X$ are endowed with connections $\nabla^{\prime}$ and $\nabla^{\prime \prime}$, then the vector bundles $E^{\prime} \oplus E^{\prime \prime}$, $E^{\prime} \otimes E^{\prime \prime}$, and $\operatorname{Hom}\left(E^{\prime}, E^{\prime \prime}\right)$ acquire the induced connections.

Similarly to Section 10.4, a connection $\nabla$ in a smooth vector bundle $E$ induces an odd derivation $d_{\nabla}$ of degree 1 on the graded left $\Omega(X)$-module $\Omega(X) \otimes_{O(X)} E(X)$ compatible with the de Rham differential $d$ on the graded ring $\Omega(X)$, in the sense of Section 6.1. The map $d_{\nabla}: \Omega^{n}(X) \otimes_{O(X)} E(X) \longrightarrow \Omega^{n+1}(X) \otimes_{O(X)} E(X), \quad n \geq 1$ (in fact, a differential operator of order 1 acting between the global sections of the vector bundles $\Lambda^{n}\left(T^{*}\right) \otimes E$ and $\Lambda^{n+1}\left(T^{*}\right) \otimes E$ on $\left.X\right)$ is given by the formula (108).

The curvature 2-form $h_{\nabla}$ of a smooth connection $\nabla$ in $E$ is an element of the vector space (or $O(X)$-module) $\Omega^{2}(X) \otimes_{O(X)} \operatorname{End}(E)(X)$ of differential 2-forms on $X$ with the coefficients in $\operatorname{End}(E)$, that is, global sections of the vector bundle $\Lambda^{2}\left(T^{*}\right) \otimes$ $\operatorname{End}(E)$ on $X$. The element $h_{\nabla} \in \Omega^{2}(X) \otimes_{O(X)} \operatorname{End}(E)$ is defined by the identity

$$
\nabla_{v}\left(\nabla_{w}(e)\right)-\nabla_{w}\left(\nabla_{v}(e)\right)=\nabla_{[v, w]}(e)+\langle v \wedge w, h\rangle(e)
$$

holding for all $v, w \in T(X)$ and $e \in E(X)$. Here $[v, w] \in T(X)$ is the commutator of the vector fields $v$ and $w$ on $X$ (defined as in Section 10.3), while $\langle$,$\rangle denotes the$ $O(X)$-linear map $\Lambda_{O(X)}^{2}(T(X)) \otimes_{O(X)} \Omega^{2}(X) \otimes_{O(X)} \operatorname{End}(E)(X) \longrightarrow \operatorname{End}(E)(X)$ induced by the pairing $\Lambda_{O(X)}^{2}(T(X)) \otimes_{O(X)} \Lambda_{O(X)}^{2}\left(T^{*}(X)\right) \longrightarrow O(X)$ from Section 10.5.

The choice of a smooth connection $\nabla=\nabla_{E}$ in $E$ defines a left $\operatorname{End}(E)(X)$-submodule of strict generators in $F_{1} \operatorname{Diff}(X, E)$ (in the sense of Section 3.3) consisting of all the $\mathbb{R}$-linear combinations of differential operators of the form $g \nabla_{v}: E(X) \longrightarrow E(X)$, where $g \in \operatorname{End}(E)(X)$ and $v \in T(X)$. Let $\nabla_{\operatorname{End}(E)}$ denote the connection in the vector bundle $\operatorname{End}(E)$ induced by the connection $\nabla$ in $E$.

Similarly to the algebraic case considered in Section 10.4, the anti-equivalence of categories from Corollary 4.20 assigns the right finitely projective Koszul CDG-ring

$$
\left(\Omega(X) \otimes_{O(X)} \operatorname{End}(E)(X), d_{\nabla_{\operatorname{End}(E)}}, h_{\nabla_{E}}\right)
$$

as in the formula (109) to the left finitely projective nonhomogeneous Koszul ring $\operatorname{Diff}(X, E)$ with the filtration $F$. Hence we obtain the following Koszul duality theorem.

Theorem 10.9. For any smooth compact real manifold $X$ and vector bundle $E$ on $X$, the construction of Theorem 6.7 provides a triangulated equivalence between the $\operatorname{Diff}(X, E) / \operatorname{End}(E)(X)$-semicoderived category of right modules over the ring of smooth differential operators $\operatorname{Diff}(X, E)$ acting in the sections of $E$ and the coderived category of right CDG-modules over the CDG-algebra (121) of differential forms on $X$ with the coefficients in $\operatorname{End}(E)$,

$$
\mathrm{D}_{\operatorname{End}(E)(X)}^{\text {sico }}(\bmod -\operatorname{Diff}(X, E))
$$




$$
\simeq \mathrm{D}^{\mathrm{co}}\left(\bmod -\left(\Omega(X) \otimes_{O(X)} \operatorname{End}(E)(X), d_{\nabla_{\operatorname{End}(E)}}, h_{\nabla_{E}}\right)\right)
$$

while Theorem 6.11 establishes a triangulated equivalence between the derived category of right $\operatorname{Diff}(X, E)$-modules and the reduced coderived category of right $C D G$-modules over the $C D G$-ring (121) relative to the ring $\operatorname{End}(E)(X)$,

$$
\begin{aligned}
\mathrm{D}(\bmod -\operatorname{Diff}(X, E)) & \\
& \simeq \mathrm{D}_{\operatorname{End}(E)(X) \text {-red }}^{\mathrm{co}}\left(\bmod -\left(\Omega(X) \otimes_{O(X)} \operatorname{End}(E)(X), d_{\nabla_{\operatorname{End}(E)}}, h_{\nabla_{E}}\right)\right) .
\end{aligned}
$$

In the same context, the construction of Theorem 7.6 provides a triangulated equivalence between the $\operatorname{Diff}(X, E) / \operatorname{End}(E)(X)$-semicontraderived category of left modules over the ring $\operatorname{Diff}(X, E)$ and the contraderived category of left $C D G$-modules over the $C D G$-algebra (121),

$$
\begin{aligned}
\mathrm{D}_{\operatorname{End}(E)(X)}^{\operatorname{sict}}(\operatorname{Diff}(X, E)-\bmod ) & \\
& \simeq \mathrm{D}^{\operatorname{ctr}}\left(\left(\Omega(X) \otimes_{O(X)} \operatorname{End}(E)(X), d_{\nabla_{\operatorname{End}(E)}}, h_{\nabla_{E}}\right)-\bmod \right),
\end{aligned}
$$

while Theorem 7.10 establishes a triangulated equivalence between the derived category of left $\operatorname{Diff}(X, E)$-modules and the reduced contraderived category of left $C D G$-modules over the CDG-ring (121) relative to the ring $\operatorname{End}(E)(X)$,

$$
\begin{aligned}
& \mathrm{D}(\operatorname{Diff}(X, E)-\bmod ) \\
& \simeq \mathrm{D}_{\operatorname{End}(E)(X)-\operatorname{crd}}^{\mathrm{ctr}}\left(\left(\Omega(X) \otimes_{O(X)} \operatorname{End}(E)(X), d_{\nabla_{\operatorname{End}(E)}}, h_{\nabla_{E}}\right)-\bmod \right)
\end{aligned}
$$

For example, the equivalences of categories (124 125) assign the left CDG-module $\left(\Omega(X) \otimes_{O(X)} E(X), d_{\nabla_{E}}\right) \quad$ (as in (110) ) over the CDG-ring (121) to the left $\operatorname{Diff}(X, E)$-module $E(X)$.

Furthermore, similarly to Section 10.5, for any closed 2-form $h \in \Omega^{2}(X)$ (that is a smooth differential 2-form such that $d(h)=0)$, the triple $(\Omega(X), d, h)$ is a right finitely projective Koszul CDG-ring. The corresponding left finitely projective nonhomogeneous Koszul filtered ring $\operatorname{Diff}(X, h)$, defined by the relations (101) and (113), is called the ring of twisted smooth differential operators on $X$ (twisted by $h$ ). It is claimed, on the basis of Theorem 4.19, that the associated graded ring $\operatorname{gr}^{F} \operatorname{Diff}^{\mathrm{cr}}(X, h)=\bigoplus_{n=0}^{\infty} F_{n} \operatorname{Diff}^{\mathrm{cr}}(X, h) / F_{n-1} \operatorname{Diff}^{\mathrm{cr}}(X, h)$ is naturally isomorphic to the symmetric algebra $\operatorname{Sym}_{O(X)}(T(X))$.

Theorem 10.10. For any smooth compact real manifold $X$ and any closed differential 2 -form $h \in \Omega^{2}(X), d(h)=0$, the construction of Theorem 6.7 provides a triangulated equivalence between the $\operatorname{Diff}(X, h) / O(X)$-semicoderived category of right modules over the ring of twisted smooth differential operators $\operatorname{Diff}(X, h)$ and the coderived category of right CDG-modules over the de Rham CDG-algebra $(\Omega(X), d, h)$,

$$
\mathrm{D}_{O(X)}^{\text {sico }}(\bmod -\operatorname{Diff}(X, h)) \simeq \mathrm{D}^{\mathrm{co}}(\bmod -(\Omega(X), d, h)),
$$

while Theorem 6.11 establishes a triangulated equivalence between the derived category of right $\operatorname{Diff}(X, h)$-modules and the reduced coderived category of right CDG-modules 
over $(\Omega(X), d, h)$ relative to $O(X)$,

$$
\mathrm{D}(\bmod -\operatorname{Diff}(X, h)) \simeq \mathrm{D}_{O(X)-\operatorname{red}}^{\mathrm{co}}(\bmod -(\Omega(X), d, h)) .
$$

In the same context, the construction of Theorem 7.6 provides a triangulated equivalence between the $\operatorname{Diff}(X, h) / O(X)$-semicontraderived category of left modules over the ring $\operatorname{Diff}(X, h)$ and the contraderived category of left $C D G$-modules over the $C D G$-algebra $(\Omega(X), d, h)$,

$$
\mathrm{D}_{O(X)}^{\mathrm{sictr}}(\operatorname{Diff}(X, h)-\bmod ) \simeq \mathrm{D}^{\mathrm{ctr}}((\Omega(X), d, h)-\bmod ),
$$

while Theorem 7.10 establishes a triangulated equivalence between the derived category of left $\operatorname{Diff}(X, h)$-modules and the reduced contraderived category of left $C D G$-modules over $(\Omega(X), d, h)$ relative to $O(X)$,

$$
\mathrm{D}(\operatorname{Diff}(X, h)-\bmod ) \simeq \mathrm{D}_{O(X)-\operatorname{red}}^{\mathrm{ctr}}((\Omega(X), d, h)-\bmod ) .
$$

Similarly to Section 10.5, there is a natural equivalence between the abelian categories of left modules over $\operatorname{Diff}(X, h)$ and right modules over $\operatorname{Diff}(X,-h)$, provided by the mutually inverse functors of tensor product with the $O(X)$-modules $\Omega^{m}(X)$ and $\Lambda_{O(X)}^{m}(T(X))$, where $m=\operatorname{dim}_{\mathbb{R}} X$. This equivalence can be obtained as a particular case of the construction of Theorem 9.4 with Remark 9.5 .

10.7. Dolbeault differential operators. For a reference on the basics of complex analytic geometry, see, e. g., [45, Section I.2].

In this section, we denote by $i$ a chosen imaginary unit (a square root of -1 ) in the field of complex numbers $\mathbb{C}$. The complex conjugation map is denoted by $z=x+i y \longmapsto \bar{z}=x-i y($ where $x, y \in \mathbb{R})$.

For any vector space $V$ over $\mathbb{C}$, we denote by $V_{\mathbb{R}}$ the underlying real vector space of $V$. So $\operatorname{dim}_{\mathbb{R}} V_{\mathbb{R}}=2 \operatorname{dim}_{\mathbb{C}} V$. Similarly, for any vector space $U$ over $\mathbb{R}$, we denote by $U_{\mathbb{C}}$ the $\mathbb{C}$-vector space $U_{\mathbb{C}}=\mathbb{C} \otimes_{\mathbb{R}} U$. So $\operatorname{dim}_{\mathbb{C}} U_{\mathbb{C}}=\operatorname{dim}_{\mathbb{R}} U$. We denote the elements of $U_{\mathbb{C}}$ by $u+i v$, where $u, v \in U$ and $i$ is the imaginary unit.

We also denote by $\bar{V}$ the vector space $V$ with the conjugate complex structure. Denoting by $\bar{v} \in \bar{V}$ the element corresponding to an element $v \in V$, the $\mathbb{C}$-vector space structure on $\bar{V}$ is defined by the rule $\bar{z} \bar{v}=\overline{z v}$ for all $z \in \mathbb{C}$ and $v \in V$, where $z \longmapsto \bar{z}: \mathbb{C} \longrightarrow \mathbb{C}$ is the complex conjugation. The vector space $U_{\mathbb{C}}$ is endowed with a natural isomorphism of $\mathbb{C}$-vector spaces $\overline{U_{\mathbb{C}}} \simeq U_{\mathbb{C}}$; the isomorphism is provided by the complex conjugation map $w \longmapsto \bar{w}: U_{\mathbb{C}} \longrightarrow U_{\mathbb{C}}$ defined by the obvious rule $\overline{u+i v}=u-i w$ for all $u, v \in U$.

For any $\mathbb{C}$-vector space $V$, the $\mathbb{C}$-vector space $\left(V_{\mathbb{R}}\right)_{\mathbb{C}}$ decomposes canonically into a direct sum of two $\mathbb{C}$-vector spaces, one of them isomorphic naturally to $V$ and the other one to $\bar{V}$. Specifically, denote by $J: V \longrightarrow V$ the action of the imaginary unit $i \in \mathbb{C}$ in $V$. Let $V^{(1,0)} \subset\left(V_{\mathbb{R}}\right)_{\mathbb{C}}$ denote the set of all elements of the form $v-i J v$, where $v \in V$, and let $V^{(0,1)} \subset\left(V_{\mathbb{R}}\right)_{\mathbb{C}}$ denote the set of all elements of the form $v+i J v$, where $v \in V$. Then both $V^{(1,0)}$ and $V^{(0,1)}$ are $\mathbb{C}$-vector subspaces in $\left(V_{\mathbb{R}}\right)_{\mathbb{C}}$, and $\left(V_{\mathbb{R}}\right)_{\mathbb{C}}=V^{(1,0)} \oplus V^{(0,1)}$. Furthermore, there are natural isomorphisms of $\mathbb{C}$-vector 
spaces $v \longmapsto v-i J v: V \longrightarrow V^{(1,0)}$ and $\bar{v} \longmapsto v+i J v: \bar{V} \longrightarrow V^{(0,1)}$. The complex conjugation map $w \longmapsto \bar{w}:\left(V_{\mathbb{R}}\right)_{\mathbb{C}} \longrightarrow\left(V_{\mathbb{R}}\right)_{\mathbb{C}}$ switches the two sides of this direct sum decomposition, taking $V^{(1,0)}$ into $V^{(0,1)}$ and $V^{(0,1)}$ into $V^{(1,0)}$.

For any $\mathbb{C}$-vector space $V$, one can consider the dual $\mathbb{C}$-vector space $\operatorname{Hom}_{\mathbb{C}}(V, \mathbb{C})$. One can also consider the dual $\mathbb{R}$-vector space $\operatorname{Hom}_{\mathbb{R}}(V, \mathbb{R})$. Let us endow the $\mathbb{R}$-vector space $\operatorname{Hom}_{\mathbb{R}}(V, \mathbb{R})$ with a $\mathbb{C}$-vector space structure by the rule $\langle v, J f\rangle=\langle J v, f\rangle$ for all $v \in V$ and $f \in \operatorname{Hom}_{\mathbb{R}}(V, \mathbb{R})$ (where $\langle\rangle:, V \otimes_{\mathbb{R}} \operatorname{Hom}_{\mathbb{R}}(V, \mathbb{R}) \longrightarrow \mathbb{R}$ is the natural pairing). Then there is a natural isomorphism of $\mathbb{C}$-vector $\operatorname{spaces}^{\operatorname{Hom}_{\mathbb{R}}}(V, \mathbb{R}) \simeq$ $\operatorname{Hom}_{\mathbb{C}}(V, \mathbb{C})$ given by the rule $\operatorname{Hom}_{\mathbb{R}}(V, \mathbb{R}) \ni f \longmapsto \hat{f} \in \operatorname{Hom}_{\mathbb{C}}(V, \mathbb{C})$ with $\langle\langle v, \hat{f}\rangle\rangle=$ $\langle v, f\rangle-i\langle J v, f\rangle \in \mathbb{C}$ for all $v \in V$ (where $\langle\langle\rangle\rangle:, V \otimes_{\mathbb{C}} \operatorname{Hom}_{\mathbb{C}}(V, \mathbb{C}) \longrightarrow \mathbb{C}$ is the notation for the $\mathbb{C}$-valued pairing).

Let $X$ be a complex manifold (which we will eventually assume to be compact). We denote the underlying smooth real manifold of $X$ by $X_{\mathbb{R}}$, and refer to the previous Section 10.6 for the discussion of differential operators and differential forms on $X_{\mathbb{R}}$. For any vector bundle $V$ on $X_{\mathbb{R}}$ and a point $s \in X$, we denote by $V_{s}$ the fiber of $V$ at $s$. So $V_{s}$ is a finite-dimensional (real or complex) vector space.

Let $T$ and $T^{*}$ denote the tangent and cotangent bundles to $X_{\mathbb{R}}$; so $T_{s}$ is the tangent space to $X_{\mathbb{R}}$ at $s$ and $T_{s}^{*}=\operatorname{Hom}_{\mathbb{R}}\left(T_{s}, \mathbb{R}\right)$ is the cotangent space for every $s \in X$. For every point $s \in X$, the $\mathbb{R}$-vector space $T_{s}$ has a natural $\mathbb{C}$-vector space structure, which is constructed as follows.

Let $z_{1}, \ldots, z_{m}$ be a holomorphic local coordinate system in an open subset $U \subset X$, $s \in U$. Denote by $x_{k}$ and $y_{k}$ the real and imaginary parts of the complex variable $z_{k}$, $1 \leq k \leq m$; so $z_{k}=x_{k}+i y_{k}$ and $x_{k}, y_{k}$ are $\mathbb{R}$-valued functions on $U$. Let $w_{1}, \ldots, w_{m}$ be another holomorphic coordinate system in $U$; put $w_{l}=u_{l}+i v_{l}$, where $u_{l}, v_{l}$ are $\mathbb{R}$-valued local functions for all $1 \leq l \leq m$.

Then, for every fixed point $s \in U \subset X$, the $2 m \times 2 m$-matrix with real entries

$$
\left(\begin{array}{ll}
\frac{\partial u_{l}}{\partial x_{k}}(s) & \frac{\partial u_{l}}{\partial y_{k}}(s) \\
\frac{\partial v_{l}}{\partial x_{k}}(s) & \frac{\partial v_{l}}{\partial y_{k}}(s)
\end{array}\right)
$$

can be obtained by applying the functor $V \longmapsto V_{\mathbb{R}}$ to the $m \times m$-matrix with complex entries $\left(\frac{\partial w_{l}}{\partial z_{k}}(s)\right)$. Consequently, the $\mathbb{C}$-vector space structure on the vector space $T_{s}$ defined by the rules $J\left(\frac{\partial}{\partial x_{k}}\right)=\frac{\partial}{\partial y_{k}}$ and $J\left(\frac{\partial}{\partial y_{k}}\right)=-\frac{\partial}{\partial x_{k}}$ does not depend on the choice of a holomorphic coordinate system $\left(z_{k}\right)_{k=1}^{m}$ in a neighborhood of $s$ in $X$.

Following the above rule for the passage to the dual vector space, there is the induced $\mathbb{C}$-vector space structure on the $\mathbb{R}$-vector space $T_{s}^{*}$ for every $s \in X$, defined in holomorphic local coordinates $z_{k}$ by the formulas $J\left(d x_{k}\right)=-d y_{k}$ and $J\left(d y_{k}\right)=d x_{k}$. Notice that $T_{s}^{*}$ is the vector space of all differentials $d_{s} f$ at the point $s \in X$ of realvalued smooth functions $f: X \longrightarrow \mathbb{R}$; still it has a natural $\mathbb{C}$-vector space structure. If $g: X \longrightarrow \mathbb{C}$ is a complex-valued smooth function, then the differential $d_{s} g$ belongs to the complexified cotangent space $T_{s, \mathbb{C}}^{*}=\mathbb{C} \otimes_{\mathbb{R}} T_{s}^{*}$.

According to the above discussion, there is a natural direct sum decomposition $T_{s, \mathbb{C}}^{*}=T_{s}^{*(1,0)} \oplus T_{s}^{*(0,1)}$. Here the holomorphic cotangent space $T_{s}^{*(1,0)}$ to $X$ at $s$ is a $\mathbb{C}$-vector space with a basis $d z_{k}=d x_{k}+i d y_{k}, 1 \leq k \leq m$. It consists of the 
differentials $d_{s} h$ of holomorphic local functions $h: U \longrightarrow \mathbb{C}$ at the point $s \in U$. The anti-holomorphic cotangent space $T_{s}^{*(0,1)}$ is a $\mathbb{C}$-vector space with a basis $d \bar{z}_{k}=$ $d x_{k}-i d y_{k}$. It consists of the differentials $d_{s} \bar{h}$ of anti-holomorphic local functions $\bar{h}: U \longrightarrow \mathbb{C}$ (where $h$ is holomorphic).

The complexified cotangent space $T_{s, \mathbb{C}}^{*}$ is the dual $\mathbb{C}$-vector space to the complexified tangent space $T_{s, \mathbb{C}}=\mathbb{C} \otimes_{\mathbb{R}} T_{s}$. The natural pairing $\langle\rangle:, T_{s, \mathbb{C}} \otimes_{\mathbb{C}} T_{s, \mathbb{C}}^{*} \longrightarrow$ $\mathbb{C}$ is constructed as the unique $\mathbb{C}$-linear extension of the natural $\mathbb{R}$-linear pairing $\langle\rangle:, T_{s} \otimes_{\mathbb{R}} T_{s}^{*} \longrightarrow \mathbb{R}$. The dual basis to $\left(d z_{k}, d \bar{z}_{k} \in T_{s, \mathbb{C}}^{*}\right)_{k=1}^{m}$ with respect to this pairing is denoted by $\left(\partial / \partial z_{k}, \partial / \partial \bar{z}_{k} \in T_{s, \mathbb{C}}\right)_{k=1}^{m}$; so $\left\langle\partial / \partial z_{k}, d z_{l}\right\rangle=\delta_{k, l}=\left\langle\partial / \partial \bar{z}_{k}, d \bar{z}_{l}\right\rangle$ and $\left\langle\partial / \partial z_{k}, d \bar{z}_{l}\right\rangle=0=\left\langle\partial / \partial \bar{z}_{k}, d z_{l}\right\rangle$ for $1 \leq k, l \leq m$. Then one has $d g=$ $\sum_{k=1}^{m} \frac{\partial g}{\partial z_{k}} d z_{k}+\sum_{k=1}^{m} \frac{\partial g}{\partial \bar{z}_{k}} d \bar{z}_{k}$ for any smooth function $g: U \longrightarrow \mathbb{C}$. Explicitly, $\partial / \partial z_{k}=$ $\frac{1}{2}\left(\partial / \partial x_{k}-i \partial / \partial y_{k}\right)$ and $\partial / \partial \bar{z}_{k}=\frac{1}{2}\left(\partial / \partial x_{k}+i \partial / \partial y_{k}\right)$.

According to the same discussion above, there is also a natural direct sum decomposition $T_{s, \mathbb{C}}=T_{s}^{(1,0)} \oplus T_{s}^{(0,1)}$. Here the holomorphic tangent space $T_{s}^{(1,0)}$ to $X$ at $s$ is a $\mathbb{C}$-vector space with a basis $\left(\partial / \partial z_{k}\right)_{k=1}^{m}$ and the anti-holomorphic tangent space $T_{s}^{(0,1)}$ is a $\mathbb{C}$-vector space with a basis $\left(\partial / \partial \bar{z}_{k}\right)_{k=1}^{m}$. Smooth sections of the vector bundle $T^{(1,0)}$ on $X$ are called $(1,0)$-vector fields, and smooth sections of the vector bundle $T^{(0,1)}$ on $X$ are called $(0,1)$-vector fields. Holomorphic local functions $h: U \longrightarrow \mathbb{C}$ are distinguished by the condition that $v(h)=0$ in $U$ for any $(0,1)$-vector field $v \in T^{(0,1)}(X)$. Similarly, anti-holomorphic local functions $\bar{h}: U \longrightarrow \mathbb{C}$ are characterized by the condition that $u(\bar{h})=0$ in $U$ for any $(1,0)$-vector field $u \in T^{(1,0)}(X)$.

For every $n \geq 0$, the complexified space of exterior forms $\Lambda_{\mathbb{R}}^{n}\left(T_{s}^{*}\right)_{\mathbb{C}}$ at a point $s$ in $X_{\mathbb{R}}$ decomposes naturally as

$$
\begin{aligned}
\mathbb{C} \otimes_{\mathbb{R}} \Lambda_{\mathbb{R}}^{n}\left(T_{s}^{*}\right) \simeq \Lambda_{\mathbb{C}}^{n}\left(T_{s, \mathbb{C}}^{*}\right) \simeq \Lambda_{\mathbb{C}}^{n}\left(T_{s}^{*(1,0)} \oplus T_{s}^{*(0,1)}\right) & \\
& \simeq \bigoplus_{p+q=n}^{p, q \geq 0} \Lambda_{\mathbb{C}}^{p}\left(T_{s}^{*(1,0)}\right) \otimes_{\mathbb{C}} \Lambda_{\mathbb{C}}^{q}\left(T_{s}^{*(0,1)}\right) .
\end{aligned}
$$

This is a direct sum decomposition of the smooth complex vector bundle $\Lambda^{n}\left(T^{*}\right)_{\mathbb{C}}$ on $X_{\mathbb{R}}$. The space of global sections decomposes accordingly,

$$
\Omega^{n}\left(X_{\mathbb{R}}\right)_{\mathbb{C}} \simeq \Lambda^{n}\left(T^{*}\right)_{\mathbb{C}}\left(X_{\mathbb{R}}\right) \simeq \bigoplus_{p+q=n}^{p, q \geq 0} \Omega^{(p, q)}(X)
$$

This is a decomposition of the $\mathbb{C}$-vector space $\Omega^{n}\left(X_{\mathbb{R}}\right)_{\mathbb{C}}$ of smooth $\mathbb{C}$-valued differential $n$-forms on $X_{\mathbb{R}}$ into the direct sum of the spaces of differential $(p, q)$-forms. In holomorphic local coordinates $z_{k}$, a differential $(p, q)$-form is an expression like

$$
\sum_{1 \leq k_{1}<\cdots<k_{p} \leq m} \sum_{1 \leq l_{1}<\cdots<l_{q} \leq m} f_{k_{1}, \ldots, k_{p} ; l_{1}, \ldots, l_{q}} d z_{k_{1}} \wedge \cdots \wedge d z_{k_{p}} \wedge d \bar{z}_{l_{1}} \wedge \cdots \wedge d \bar{z}_{l_{q}}
$$

with $p$ holomorphic differentials $d z_{k_{1}}, \ldots, d z_{k_{p}}$ and $q$ anti-holomorphic differentials $d \bar{z}_{l_{1}}, \ldots, d \bar{z}_{l_{q}}$ in the exterior product. Here the coefficients $f_{k_{1}, \ldots, k_{p} ; l_{1}, \ldots, l_{q}}$ are smooth complex-valued local functions $U \longrightarrow \mathbb{C}$. In fact, this is even a direct sum decomposition of $\Omega^{n}\left(X_{\mathbb{R}}\right)_{\mathbb{C}}$ as a module over the ring $O\left(X_{\mathbb{R}}\right)_{\mathbb{C}}$ of smooth complex-valued global functions on $X_{\mathbb{R}}$. This direct sum decomposition makes $\Omega\left(X_{\mathbb{R}}\right)_{\mathbb{C}}$ a bigraded algebra over the ring $O\left(X_{\mathbb{R}}\right)_{\mathbb{C}}$. 
The de Rham differential $d$ on the graded algebra of differential forms $\Omega\left(X_{\mathbb{R}}\right)$ was discussed in Section 10.6. Taking the tensor product with $\mathbb{C}$ over $\mathbb{R}$, we obtain an odd derivation of degree 1 on the graded algebra of $\mathbb{C}$-valued differential forms $\Omega\left(X_{\mathbb{R}}\right)_{\mathbb{C}}$; we denote this differential also by $d$. With respect to the direct sum decomposition (130), the differential $d: \Omega^{n}\left(X_{\mathbb{R}}\right)_{\mathbb{C}} \longrightarrow \Omega^{n+1}\left(X_{\mathbb{R}}\right)_{\mathbb{C}}$ has two components:

$$
\partial: \Omega^{p, q}(X) \longrightarrow \Omega^{p+1, q}(X) \text { and } \bar{\partial}: \Omega^{p, q}(X) \longrightarrow \Omega^{p, q+1}(X),
$$

making $\Omega^{\bullet \bullet \bullet}(X)$ a bicomplex. In particular, the differential $d: O\left(X_{\mathbb{R}}\right)_{\mathbb{C}} \longrightarrow$ $\Omega^{1}\left(X_{\mathbb{R}}\right)_{\mathbb{C}} \simeq \Omega^{(1,0)}(X) \oplus \Omega^{(0,1)}(X)$ decomposes as $d=\partial+\bar{\partial}$, where in holomorphic local coordinates $z_{k}$ one has $\partial(f)=\sum_{k=1}^{m} \frac{\partial f}{\partial z_{k}} d z_{k}$ and $\bar{\partial}(f)=\sum_{k=1}^{m} \frac{\partial f}{\partial \bar{z}_{k}} d \bar{z}_{k}$ for any smooth complex-valued function $f$.

The $p=0$ part of the bicomplex $\Omega^{p, q}(X)$

$$
0 \longrightarrow \Omega^{0,0}(X) \stackrel{\bar{\partial}}{\longrightarrow} \Omega^{0,1}(X) \stackrel{\bar{\partial}}{\longrightarrow} \cdots \stackrel{\bar{\partial}}{\longrightarrow} \Omega^{0, m}(X) \longrightarrow 0
$$

is called the Dolbeault complex of a complex manifold $X$. Here $m=\operatorname{dim}_{\mathbb{C}} X$ is the dimension of $X$ as a complex manifold. The Dolbeault complex $\Omega^{0, \bullet}(X)$ is a DG-algebra over the field $\mathbb{C}$.

The degree-zero component $\Omega^{0,0}(X)$ is the $\mathbb{C}$-algebra $O\left(X_{\mathbb{R}}\right)_{\mathbb{C}}$ of smooth $\mathbb{C}$-valued functions on $X_{\mathbb{R}}$. The kernel $H_{\bar{\partial}}^{0}\left(\Omega^{0, \bullet}(X)\right)=O(X)$ of the differential $\bar{\partial}: \Omega^{0,0}(X) \longrightarrow$ $\Omega^{0,1}(X)$ in the Dolbeault complex is the subalgebra $O(X) \subset O\left(X_{\mathbb{R}}\right)_{\mathbb{C}}$ of holomorphic global functions $X \longrightarrow \mathbb{C}$. For comparison, the kernel $H_{d}^{0}\left(\Omega^{\bullet}\left(X_{\mathbb{R}}\right)\right)$ of the differential $d: \Omega^{0}\left(X_{\mathbb{R}}\right) \longrightarrow \Omega^{1}\left(X_{\mathbb{R}}\right)$ in the de Rham complex is the subalgebra of locally constant global functions in the $\mathbb{R}$-algebra $O\left(X_{\mathbb{R}}\right)$ of smooth functions $X_{\mathbb{R}} \longrightarrow \mathbb{R}$.

Consider the ring (or $\mathbb{R}$-algebra) of differential operators $\operatorname{Diff}\left(X_{\mathbb{R}}\right)$ on the smooth real manifold $X_{\mathbb{R}}$, as discussed in Section 10.6. The tensor product $\mathbb{C} \otimes_{\mathbb{R}} \operatorname{Diff}\left(X_{\mathbb{R}}\right)=$ $\operatorname{Diff}\left(X_{\mathbb{R}}\right)_{\mathbb{C}}$ is a $\mathbb{C}$-algebra acting naturally in the $\mathbb{C}$-vector space of smooth complexvalued global functions $O\left(X_{\mathbb{R}}\right)_{\mathbb{C}}$. We are interested in the following subring (or $\mathbb{C}$-subalgebra) $\operatorname{Diff}^{\bar{\partial}}(X) \subset \operatorname{Diff}\left(X_{\mathbb{R}}\right)_{\mathbb{C}}$, which we call the ring of $\bar{\partial}$-differential operators (or Dolbeault differential operators) on a complex manifold $X$.

By the definition, Diff $\bar{\partial}(X)$ is the subring generated by the subring of smooth $\mathbb{C}$-valued functions $O\left(X_{\mathbb{R}}\right)_{\mathbb{C}} \subset \operatorname{Diff}\left(X_{\mathbb{R}}\right)_{\mathbb{C}}$ and the left $O\left(X_{\mathbb{R}}\right)_{\mathbb{C} \text {-submodule of smooth }}$ $(0,1)$-vector fields $T^{(0,1)}(X) \subset T(X)_{\mathbb{C}} \subset \operatorname{Diff}\left(X_{\mathbb{R}}\right)_{\mathbb{C}}$ in the ring of smooth $\mathbb{C}$-valued differential operators $\operatorname{Diff}\left(X_{\mathbb{R}}\right)_{\mathbb{C}}$ on $X_{\mathbb{R}}$. The increasing filtration $F$ by the order of differential operators on $\operatorname{Diff}^{\bar{\partial}}(X)$ is induced by the filtration $F$ on $\operatorname{Diff}\left(X_{\mathbb{R}}\right)_{\mathbb{C}}$ (which, in turn, is induced by the filtration $F$ on $\operatorname{Diff}\left(X_{\mathbb{R}}\right)$ ).

The main property of the ring $\operatorname{Diff}^{\bar{\partial}}(X)$ is that its action in the ring of smooth complex-valued functions $O\left(X_{\mathbb{R}}\right)_{\mathbb{C}}$ commutes with the operators of multiplication by holomorphic functions. This holds true because holomorphic functions have zero derivatives along $(0,1)$-vector fields.

More generally, a smooth complex vector bundle $E$ over $X_{\mathbb{R}}$ is said to be holomorphic (or "have a holomorphic structure") if the notion of a holomorphic local section of $E$ is defined. For example, the $(1,0)$-cotangent bundle $T^{*(1,0)}$ on $X$ has a natural holomorphic structure in which $\mathbb{C}$-linear combinations of the expressions $f d(g)$ with 
holomorphic local functions $f, g: U \longrightarrow \mathbb{C}$ are the holomorphic local sections (these are called the holomorphic differential 1-forms on $X)$. Similarly, the (1,0)-tangent bundle $T^{(1,0)}$ on $X$ has a natural holomorphic structure in which the expressions $\sum_{k=1}^{m} f_{k} \frac{\partial}{\partial z_{k}}$ with holomorphic local functions $f_{k}: U \longrightarrow \mathbb{C}$ are the holomorphic local sections (one has to check that the class of holomorphic vector fields, defined in this way, does not depend on the choice of a local coordinate system $z_{k}$ ). We denote the $\mathbb{C}$-vector space of smooth sections of a holomorphic vector bundle $E$ on $X$ by $E\left(X_{\mathbb{R}}\right)$ and the subspace of holomorphic sections by $E(X) \subset E\left(X_{\mathbb{R}}\right)$.

For any holomorphic vector bundle $E$ over $X$, the ring of $\bar{\partial}$-differential operators $\operatorname{Diff}^{\bar{\partial}}(X)$ acts naturally in the $\mathbb{C}$-vector space of smooth sections $E\left(X_{\mathbb{R}}\right)$; so $E\left(X_{\mathbb{R}}\right)$ is a left $\operatorname{Diff}^{\bar{\partial}}(X)$-module. This action is constructed as follows. The ring of smooth $\mathbb{C}$-valued functions $O\left(X_{\mathbb{R}}\right)_{\mathbb{C}}$ acts in $E\left(X_{\mathbb{R}}\right)$ by the usual multiplications of smooth sections of a vector bundle with smooth functions. To define the action of $(0,1)$-vector fields in $E\left(X_{\mathbb{R}}\right)$, choose a local basis of holomorphic sections $e_{l} \in E(U), 1 \leq l \leq n$. This means that $e_{l}$ are holomorphic sections of $E$ over $U$ and for every point $s \in U$ the vectors $e_{l}(s), 1 \leq l \leq n$, form a basis of the $\mathbb{C}$-vector space $E_{s}$.

Let $e=\sum_{l=1}^{n} f_{l} e_{l} \in E\left(U_{\mathbb{R}}\right)$ be a smooth section of $E$ over $U$; so $f_{l} \in O\left(U_{\mathbb{R}}\right)_{\mathbb{C}}$ are smooth complex-valued local functions. Furthermore, let $v=\sum_{k=1}^{m} g_{k} \partial / \partial \bar{z}_{k}$ be a smooth $(0,1)$-vector field in $U$; so $g_{k}: U \longrightarrow \mathbb{C}$ are smooth complex-valued local functions as well. Then we put

$$
v(e)=\sum_{l=1}^{n} v\left(f_{l}\right) e_{l}=\sum_{l=1}^{n} \sum_{k=1}^{m} g_{k} \frac{\partial f_{l}}{\partial \bar{z}_{k}} e_{l} \in E\left(U_{\mathbb{R}}\right) .
$$

Using the fact that $v(h)=0$ for any holomorphic local function $h: U \longrightarrow \mathbb{C}$, one can check that the above definition of $v(e)$ does not depend on the choice of a local basis of holomorphic sections $e_{l}$ of $E$ over $U$; so the local constructions glue together to a well-defined differential operator of order 1 providing the action of $v$ in $E\left(X_{\mathbb{R}}\right)$.

Similarly one can show that, for any morphism $E^{\prime} \longrightarrow E^{\prime \prime}$ of holomorphic vector bundles over $X$ (that is, a morphism of smooth compex vector bundes taking holomorphic local sections to holomorphic local sections), the induced map of the spaces of smooth sections $E^{\prime}\left(X_{\mathbb{R}}\right) \longrightarrow E^{\prime \prime}\left(X_{\mathbb{R}}\right)$ is a morphism of left $\operatorname{Diff}^{\bar{\partial}}(X)$-modules. This generalizes the above assertion about the commutativity of the action of $\operatorname{Diff}^{\bar{\partial}}(X)$ in $E\left(X_{\mathbb{R}}\right)$ with the multiplications by holomorphic functions.

Now let us assume that $X$ is a compact complex manifold. Then, according to the discussion in Section 10.6, the category of smooth real vector bundles over $X_{\mathbb{R}}$ is equivalent to the category of finitely generated projective modules over $O\left(X_{\mathbb{R}}\right)$. Similarly, the category of smooth complex vector bundles over $X_{\mathbb{R}}$ is equivalent to the category of finitely generated projective modules over the ring $O\left(X_{\mathbb{R}}\right)_{\mathbb{C}}$.

The associated graded ring $\operatorname{gr}^{F} \operatorname{Diff}^{\bar{\partial}}(X)=\bigoplus_{n=0}^{\infty} F_{n} \operatorname{Diff}^{\bar{\partial}}(X) / F_{n-1} \operatorname{Diff}^{\bar{\partial}}(X)$ is isomorphic to the symmetric algebra $A=\operatorname{Sym}_{O\left(X_{\mathbb{R}}\right)}\left(T^{(0,1)}\left(X_{\mathbb{R}}\right)\right)$ of the finitely generated projective module $T^{(0,1)}\left(X_{\mathbb{R}}\right)$ of smooth $(0,1)$-vector fields on $X$ over the ring $O\left(X_{\mathbb{R}}\right)_{\mathbb{C}}$ of smooth complex-valued functions. According to Section 10.1, this graded ring is left and right finitely projective Koszul. Hence the filtered ring $\operatorname{Diff}^{\bar{\partial}}(X)$ is 
a left finitely projective nonhomogeneous Koszul ring. Furthermore, the natural left action of $\operatorname{Diff}^{\bar{\partial}}(X)$ in $O\left(X_{\mathbb{R}}\right)_{\mathbb{C}}$ makes $\operatorname{Diff}^{\bar{\partial}}(X)$ a left augmented ring over its subring $F_{0} \operatorname{Diff}^{\bar{\partial}}(X)=O\left(X_{\mathbb{R}}\right)_{\mathbb{C}}$. So $\left(\operatorname{Diff}^{\bar{\partial}}(X), F\right)$ is a left augmented left finitely projective nonhomogeneous Koszul ring.

The graded ring $B=\bigoplus_{q=0}^{m} \Omega^{0, q}(X)=\Lambda_{O\left(X_{\mathbb{R}}\right)}\left(T^{*(0,1)}\left(X_{\mathbb{R}}\right)\right)$ is left and right finitely projective Koszul as well. It is also the right finitely projective Koszul ring quadratic dual to the left finitely projective Koszul ring $A$, in the sense of Corollary 2.24. The (right finitely projective Koszul) Dolbeault DG-ring $\left(\Omega^{0, \bullet}(X), \bar{\partial}\right)$ (131) corresponds to the (left augmented left finitely projective Koszul) filtered ring ( $\left.\operatorname{Diff}^{\bar{\partial}}(X), F\right)$ under the anti-equivalence of categories from Corollary 4.22.

Similarly to the smooth real manifold case of Theorem 10.8, we obtain the following rough algebraic version of Koszul duality for the ring of $\bar{\partial}$-differential operators $\operatorname{Diff}^{\bar{\partial}}(X)$.

Theorem 10.11. For any compact complex manifold $X$, the construction of Theorem 6.7 provides a triangulated equivalence between the $\operatorname{Diff}^{\bar{\partial}}(X) / O\left(X_{\mathbb{R}}\right)_{\mathbb{C}^{-} \text {-semico- }}$ derived category of right modules over the ring of $\bar{\partial}$-differential operators $\operatorname{Diff}^{\bar{\partial}}(X)$ and the coderived category of right DG-modules over the Dolbeault DG-algebra $\left(\Omega^{0, \bullet}(X), \bar{\partial}\right)$,

$$
\mathrm{D}_{O\left(X_{\mathbb{R}}\right)_{\mathbb{C}}}^{\text {sico }}\left(\bmod -\operatorname{Diff}^{\bar{\partial}}(X)\right) \simeq \mathrm{D}^{\mathrm{co}}\left(\bmod -\left(\Omega^{0, \bullet}(X), \bar{\partial}\right)\right),
$$

while Theorem 6.11 establishes a triangulated equivalence between the derived category of right $\operatorname{Diff}^{\bar{\partial}}(X)$-modules and the reduced coderived category of right DG-modules over $\left(\Omega^{0, \bullet}(X), \bar{\partial}\right)$ relative to $O\left(X_{\mathbb{R}}\right)_{\mathbb{C}}$,

$$
\mathrm{D}\left(\bmod -\operatorname{Diff}^{\bar{\partial}}(X)\right) \simeq \mathrm{D}_{O\left(X_{\mathbb{R}}\right)_{\mathbb{C}}-\operatorname{red}}^{\mathrm{co}}\left(\bmod -\left(\Omega^{0, \bullet}(X), \bar{\partial}\right)\right) .
$$

In the same context, the construction of Theorem 7.6 provides a triangulated equivalence between the $\operatorname{Diff}^{\bar{\partial}}(X) / O\left(X_{\mathbb{R}}\right)_{\mathbb{C}}$-semicontraderived category of left modules over the ring $\operatorname{Diff}^{\bar{\partial}}(X)$ and the contraderived category of left $D G$-modules over the $D G$-algebra $\left(\Omega^{0, \bullet}(X), \bar{\partial}\right)$,

$$
\mathrm{D}_{O\left(X_{\mathbb{R}}\right) \mathbb{C}}^{\text {sictr }}\left(\operatorname{Difff}^{\bar{\partial}}(X)-\bmod \right) \simeq \mathrm{D}^{\mathrm{ctr}}\left(\left(\Omega^{0, \bullet}(X), \bar{\partial}\right)-\bmod \right),
$$

while Theorem 7.10 establishes a triangulated equivalence between the derived category of left $\operatorname{Diff}^{\bar{\partial}}(X)$-modules and the reduced contraderived category of left DG-modules over $\left(\Omega^{0, \bullet}(X), \bar{\partial}\right)$ relative to $O\left(X_{\mathbb{R}}\right)_{\mathbb{C}}$,

$$
\mathrm{D}\left(\operatorname{Diff}^{\bar{\partial}}(X)-\bmod \right) \simeq \mathrm{D}_{O\left(X_{\mathbb{R}}\right)_{\mathbb{C}}-\mathrm{red}}^{\mathrm{ctr}}\left(\left(\Omega^{0, \bullet}(X), \bar{\partial}\right)-\bmod \right) .
$$

In particular, for every holomorphic vector bundle $E$ over $X$, we have the left $\operatorname{Diff}^{\bar{\partial}}(X)$-module $E\left(X_{\mathbb{R}}\right)$, as explained above. The corresponding left DG-module over the Dolbeault DG-ring (131), under the equivalences of categories (134-135), is the Dolbeault complex $\left(\Omega^{0, \bullet}(X) \otimes_{O\left(X_{\mathbb{R}}\right)_{\mathbb{C}}} E\left(X_{\mathbb{R}}\right), \bar{\partial}\right)$ with the coefficients in a holomorphic vector bundle $E$ on $X$. The the $\mathbb{C}$-vector spaces $H_{\bar{\partial}}^{*}\left(\Omega^{0, \bullet}(X) \otimes_{O\left(X_{\mathbb{R}}\right)_{\mathbb{C}}} E\left(X_{\mathbb{R}}\right)\right)$ of 
cohomology of the latter complex are the sheaf cohomology spaces $H^{*}(X, \mathcal{E})$ of the sheaf of holomorphic local sections $\mathcal{E}$ of the vector bundle $E$.

Furthermore, there is a natural equivalence between the abelian categories of left and right modules over the ring $\operatorname{Diff}^{\partial}(X)$, provided by the mutually inverse functors of tensor product with the invertible modules $\Omega^{0, m}(X)$ and $\Lambda_{O\left(X_{\mathbb{R}}\right)_{\mathbb{C}}}\left(T^{(0,1)}(X)\right)$ over the ring $O\left(X_{\mathbb{R}}\right)_{\mathbb{C}}$ (where $\left.m=\operatorname{dim}_{\mathbb{C}} X\right)$. This Morita equivalence can be obtained as a particular case of the construction of Theorem 9.4 with Remark 9.5 (since $\Omega^{0, \bullet}(X)$ is a relatively Frobenius Koszul graded ring).

10.8. Relative differential operators. Let $\iota: S \longrightarrow R$ be a homomorphism of commutative rings. We will consider DG-rings $(B, d)$ endowed with a ring homomorphism $R \longrightarrow B^{0}$ such that the following conditions are satisfied:

(i) the graded ring $B=\bigoplus_{n \in \mathbb{Z}} B^{n}$ is strictly graded commutative (in the sense of Section 10.1);

(ii) $B$ is a DG-algebra over $S$, that is, in other words, the image of the composition $S \longrightarrow R \longrightarrow B^{0}$ is annihilated by the differential $d_{0}: B^{0} \longrightarrow B^{1}$.

The initial object in the category of all such DG-rings $(B, d)$ with ring homomorphisms $R \longrightarrow B^{0}$ is called the strictly graded commutative $D G$-algebra over $S$ freely generated by $R$ and denoted by $\left(\Omega_{R / S}, d\right)$. The other name for $\left(\Omega_{R / S}, d\right)$ is the de Rham $D G$-algebra of $R$ over $S$ [13, Tag $0 \mathrm{FKF}]$.

Clearly, the graded ring $\Omega_{R / S}$ is generated by the images of elements from $R$ and their differentials. It follows that one has $\Omega_{R / S}^{n}=0$ for $n<0$ and $\Omega_{R / S}^{0}=R$. Furthermore, the graded ring $\Omega_{R / S}$ is generated by its first-degree component $\Omega_{R / S}^{1}$ over $\Omega_{R / S}^{0}=R$. The $R$-module $\Omega_{R / S}^{1}$ can be constructed as the module of Kähler differentials of $R$ over $S$ [13, Tag 00RM] (cf. the discussion of the particular case when $S$ is a field in Section 10.2 above). Specifically, $\Omega_{R / S}^{1}$ is the $R$-module generated by the symbols $d(r)$ with $r \in R$ subject to the relations $d(f g)=f d(g)-g d(f)$ for all $f, g \in R$ and $d(\iota(s))=0$ for all $s \in S$.

The key observation is that the graded ring $\Omega_{R / S}$ is, in fact, the exterior algebra of the $R$-module $\Omega_{R / S}^{1}$, that is $\Omega_{R / S}=\Lambda_{R}\left(\Omega_{R / S}^{1}\right)$. In other words, this means that there exists a well-defined odd derivation $d$ of degree 1 on the graded ring $\Lambda_{R}\left(\Omega_{R / S}^{1}\right)$ whose restriction to $R=\Lambda_{R}^{0}\left(\Omega_{R / S}^{1}\right)$ is the natural map $d: R \longrightarrow \Omega_{R / S}^{1}$ and whose square vanishes. Such a derivation is clearly unique, because the exterior algebra $\Lambda_{R}^{0}\left(\Omega_{R / S}^{1}\right)$ is generated by $\Omega_{R / S}^{1}$ over $R$ and the action of $d$ on $\Omega_{R / S}^{1}$ is computable as $d(f d(g))=d(f) \wedge d(g)+f d^{2}(g)=d(f) \wedge d(g)$.

Concerning the existence, one needs to check that odd derivations defined on the generators and satisfying obvious compatibilities extend well to freely generated strictly graded commutative rings (cf. the discussion in the last paragraph of the proof of Proposition 3.6 and Lemma 3.7). In particular, one can compute that $d\left(c^{2}\right)=d(c) c-c d(c)=0$ since $c$ commutes with $d(c)$ for any element $c$ of odd degree in $\Lambda_{R}\left(\Omega_{R / S}^{1}\right)$ (as it should be). Furthermore, one has $d^{2}(a b)=d(d(a) b+$ $\left.(-1)^{|a|} a d(b)\right)=d^{2}(a) b+(-1)^{|a|+1} d(a) d(b)+(-1)^{|a|} d(a) d(b)+a d^{2}(b)=0$ provided 
that $d^{2}(a)=0=d^{2}(b)$ for a given pair of elements $a, b \in \Lambda_{R}\left(\Omega_{R / S}^{1}\right)$; so the square of an odd derivation vanishes whenever it vanishes on the generators.

A simpler approach may be to construct $\Omega_{R / S}$ as the graded commutative graded ring generated by the symbols $f$ of degree 0 and $d(f)$ of degree 1 for all $f \in R$, with the relations that the addition and multiplication of the symbols $f$ in $\Omega_{R / S}$ agrees with their addition and multiplication in $R$, and also $d(f+g)=d(f)+d(g)$, $d(f g)=d(f) g+f d(g)$ for all $f, g \in R$, and $d(\iota(s))=0$ for all $s \in S$. Then the isomorphism $\Omega_{R / S}=\Lambda_{R}\left(\Omega_{R / S}^{1}\right)$ follows simply from the fact that all the relations imposed have degrees 0 or 1 . Having observed that, one needs to convince oneself that there exists an (obviously unique) odd derivation $d$ on $\Omega_{R / S}$ taking $f$ to $d(f)$ and $d(f)$ to 0 for all $f \in R$. Indeed, $d(d(f g)-d(f) g-f d(g))=0+d(f) d(g)-d(f) d(g)=0$, as it should be; so the relation is preserved by the desired odd derivation.

When 2 is invertible in $R$ (so there is no difference between graded commutativity and strict graded commutativity for $B$ ), the condition (i) can be replaced by its weaker form

$\left(\mathrm{i}^{\prime}\right)$ the image of the map $R \longrightarrow B^{0}$ is contained in the (graded) center of $B$.

The universal graded commutative DG-ring $(E, d)$ with a map $R \longrightarrow E^{0}$ satisfying $\left(\mathrm{i}^{\prime}\right)$ and (ii) is the same as the universal graded commutative DG-ring with a similar map satisfying (i) and (ii).

Indeed, the graded ring $E$, being universal, is clearly generated by $E^{0}=R$ and $d(R) \subset E^{1}$. So it remains to check that $d(f) d(g)+d(g) d(f)=0$ in $B$ under (i') for all $f, g \in R$. For this purpose, it suffices to compute that $d(f) d(g)+d(g) d(f)=$ $d(f d(g)-d(g) f)=d(0)=0$. More generally, one shows that the graded center $Z$ of any graded ring $B$ is preserved by any odd derivation $d$ on $B$, as one has $d(z) b=d(z b)-(-1)^{|z|} z d(b)=(-1)^{|z||b|} d(b z)-(-1)^{|z||b|} d(b) z=(-1)^{(|z|+1)|b|} b d(z)$ for all $b \in B$ and $z \in Z$.

Elements of the $R$-module $T_{R / S}=\operatorname{Hom}_{R}\left(\Omega_{R / S}^{1}, R\right)$ are interpreted as derivations of the $S$-algebra $R$ (i. e., $S$-linear maps $v: R \longrightarrow R$ such that $v(f g)=v(f) g+f v(g)$ for all $f, g \in R$ ). Specifically, given an $R$-linear map $v: \Omega_{R / S}^{1} \longrightarrow R$, the action of $v$ on $R$ is defined by the rule $v(f)=v(d f) \in R$. In fact, this rule defines a natural $R$-module isomorphism between $T_{R / S}$ and the $R$-module of all $S$-linear derivations of $R$. Consequently, the underlying $S$-module of $T_{R / S}$ acquires the structure of a Lie algebra over $S$ : the bracket $[v, w]$ of two elements $v, w \in T_{R / S}$ is defined by the usual rule $[v, w](f)=v(w(f))-w(v(f))$.

The construction of the de Rham DG-algebra $\Omega_{R / S}$ is well-behaved for smooth morphisms of rings $S \longrightarrow R$. In order not to delve into the intricacies of various definitions of a smooth morphism, let us define and impose the minimal condition that we will actually need. Let us say that a morphism of commutative rings $S \longrightarrow R$ is weakly smooth of relative dimension $m$ if $\Omega_{R / S}^{1}$ is a finitely generated projective $R$-module everywhere of rank $m$ (in the sense of Section 10.1). In this case, $T_{R / S}$ is also a finitely generated projective $R$-module everywhere of rank $m$.

Notice that the relative dimension of a weakly smooth morphism can exceed the (relative) Krull dimension. For example, given a field $k$, denote by $k(x)$ the field 
of rational functions in one variable $x$ with the coefficients in $k$. Then the natural inclusion $k \longrightarrow k(x)$ is a weakly smooth morphism of relative dimension 1. Moreover, for a field $k$ of prime characteristic $p$, the inclusion $k\left(x^{p}\right) \longrightarrow k(x)$ is a weakly smooth morphism of relative dimension 1 in the sense of our definition (even though it is a finite, algebraic field extension).

The ring of relative crystalline differential operators $\operatorname{Diff}_{R / S}^{c r}$ is now defined similarly to Section 10.3. The ring $\operatorname{Diff}_{R / S}^{\text {cr }}$ is generated by elements of the ring $R$ and the $R$-module $T_{R / S}$, subject to the relations (101) imposed for all $f, g \in R$ and $v$,

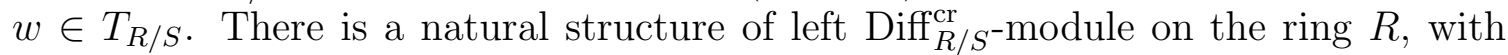
the elements $g \in R \subset \operatorname{Diff}_{R / S}^{\mathrm{cr}}$ acting in $R$ by the multiplication maps $f \longmapsto g f$ and the elements $v \in T_{R / S} \subset \operatorname{Diff}_{R / S}^{c r}$ acting in $R$ by the derivations $f \longmapsto v(f)$. The operators with which the ring $\operatorname{Diff}_{R / S}^{\mathrm{cr}}$ acts in $R$ are $S$-linear, but not $R$-linear. There is also a natural increasing filtration $F$ on the ring $\operatorname{Diff} R / S$ generated by $F_{1} \operatorname{Diff}_{R / S}^{c r}=$ $R \oplus T_{R / S} \subset \operatorname{Diff}_{R / S}^{\mathrm{cr}}$ over $F_{0} \operatorname{Diff}_{R / S}^{\mathrm{cr}}=R$.

Assume that the morphism of commutative rings $S \longrightarrow R$ is weakly smooth. Then the filtered ring Diff $R / S$ together with its action in $R$ can be also defined as the left augmented left finitely projective projective nonhomogeneous Koszul ring corresponding to the right finitely projective Koszul DG-ring $\left(\Omega_{R / S}, d\right)$ under the antiequivalence of categories from Corollary 4.22. By the Poincaré-Birkhoff-Witt Theorem 4.19, the associated graded ring $\operatorname{gr}^{F} \operatorname{Diff}_{R / S}^{c r}=\bigoplus_{n=0}^{\infty} F_{n} \operatorname{Diff}_{R / S}^{c r} / F_{n-1} \operatorname{Diff}_{R / S}^{c r}$ is naturally isomorphic to the symmetric algebra $\operatorname{Sym}_{R}\left(T_{R / S}\right)$ of the $R$-module $T_{R / S}$.

Hence the results of Sections 6 and 7 specialize to the following theorem.

Theorem 10.12. For any weakly smooth morphism of commutative rings $S \longrightarrow$ $R$, the construction of Theorem 6.7 provides a triangulated equivalence between the $\operatorname{Diff}_{R / S}^{c r} / R$-semicoderived category of right modules over the ring of relative crystalline differential operators Differ ${ }_{R / S}$ and the coderived category of right $D G$-modules over the relative de Rham DG-algebra $\left(\Omega_{R / S}, d\right)$,

$$
\mathrm{D}_{R}^{\text {sico }}\left(\bmod -\mathrm{Diff}{ }_{R / S}^{\mathrm{cr}}\right) \simeq \mathrm{D}^{\mathrm{co}}\left(\bmod -\left(\Omega_{R / S}, d\right)\right),
$$

while Theorem 6.11 establishes a triangulated equivalence between the derived category of right Diffcr $R_{R}$-modules and the reduced coderived category of right DG-modules over $\left(\Omega_{R / S}, d\right)$,

$$
\mathrm{D}\left(\bmod -\operatorname{Diff}_{R / S}^{\mathrm{cr}}\right) \simeq \mathrm{D}_{R \text {-red }}^{\mathrm{co}}\left(\bmod -\left(\Omega_{R / S}, d\right)\right) .
$$

In the same context, the construction of Theorem 7.6 provides a triangulated equivalence between the Diffcr $R / S / R$-semicontraderived category of left modules over the ring $\operatorname{Diff}_{R / S}^{\mathrm{cr}}$ and the contraderived category of left DG-modules over the DG-algebra $\left(\Omega_{R / S}, d\right)$,

$$
\mathrm{D}_{R}^{\mathrm{sictr}}\left(\operatorname{Diff}_{R / S}^{\mathrm{cr}}-\mathrm{mod}\right) \simeq \mathrm{D}^{\mathrm{ctr}}\left(\left(\Omega_{R / S}, d\right)-\bmod \right),
$$

while Theorem 7.10 establishes a triangulated equivalence between the derived category of left $\operatorname{Diff}_{R / S}^{\mathrm{cr}}$-modules and the reduced contraderived category of left DG-modules over 
$\left(\Omega_{R / S}, d\right)$,

$$
\mathrm{D}\left(\operatorname{Diff}_{R / S}^{\mathrm{cr}}-\mathrm{mod}\right) \simeq \mathrm{D}_{R \text {-red }}^{\mathrm{ctr}}\left(\left(\Omega_{R / S}, d\right)-\mathrm{mod}\right) .
$$

Furthermore, whenever the ring $R$ has finite homological dimension, Corollaries 6.10 and 7.9 are applicable; so there is no difference between (136) and (137), and similarly there is no difference between (138) and (139). Moreover, the results of Section 9 lead to the following theorem in this case. Here the natural equivalence between the abelian categories of left and right Diff $R / S$-modules is provided by the mutually inverse functors of tensor product with the invertible $R$-modules $\Omega_{R / S}^{m}$ and $\Lambda_{R}^{m}\left(T_{R / S}\right)$, as described in Theorem 9.4 .

Theorem 10.13. For any weakly smooth morphism of commutative rings $S \longrightarrow$ $R$ of relative dimension $m$ such that the ring $R$ has finite homological dimension, the constructions of Theorem 9.8 (with Remark 9.5) provide a commutative square diagram of triangulated equivalences

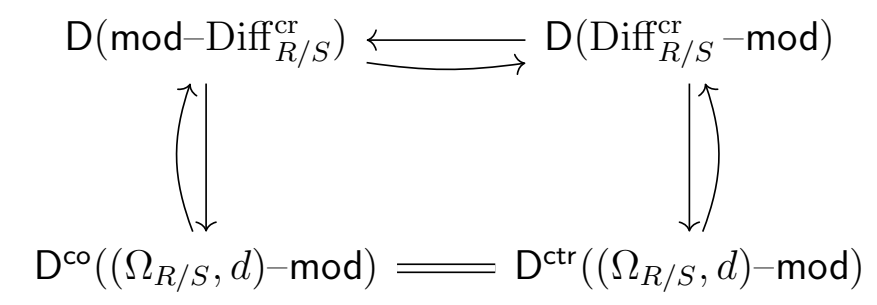

where the equivalence of derived categories in the upper line is induced by the conversion equivalence of abelian categories $\bmod -\mathrm{Diff}_{R / S}^{\mathrm{cr}} \simeq \mathrm{Diff}_{R / S}^{\mathrm{cr}}-\bmod$ (up to a cohomological shift by $[-m]$ ), while the co-contra correspondence in the lower line is the result of Theorem [.1. The vertical equivalences are (136-137) and (138-139).

Given a closed relative 2 -form $h$, i. e., an element $h \in \Omega_{R / S}^{2}$ such that $d(h)=0$, one can also consider the right finitely projective Koszul CDG-ring $\left(\Omega_{R / S}, d, h\right)$ and construct its nonhomogeneous quadratic dual left finitely projective nonhomogeneous Koszul filtered ring Diff $R / S, h$ of twisted relative crystalline differential operators, using Theorem 4.19 or Corollary 4.20. The results of Theorems 10.12 and 10.13 can be then extented to the twisted case (similarly to Section 10.5).

10.9. Lie algebroids. The setting in this section is a common generalizations of Sections 10.210 .3 and 10.610 .8 .

A Lie algebroid (known also as a Lie-Rinehart algebra) [40, 22] is a pair of abelian groups $(R, \mathfrak{g})$ endowed with the following structures:

- $R$ is a commutative ring (with unit);

- $\mathfrak{g}$ is a Lie algebra over $\mathbb{Z}$, i. e., it is endowed with an additive map of Lie bracket $[-,-]: \Lambda_{\mathbb{Z}}^{2} \mathfrak{g} \longrightarrow \mathfrak{g}$ satisfying the Jacobi identity;

- $\mathfrak{g}$ is an $R$-module, so a commutative ring action map $R \otimes_{\mathbb{Z}} \mathfrak{g} \longrightarrow \mathfrak{g}$ is given, denoted by $a \otimes x \longmapsto a x$ for all $a \in R$ and $x \in \mathfrak{g}$; 
- $R$ is a $\mathfrak{g}$-module, so a Lie action map $\mathfrak{g} \otimes_{\mathbb{Z}} R \longrightarrow R$ is given, denoted by $x \otimes a \longmapsto x(a)$ for all $a \in R$ and $x \in \mathfrak{g}$.

In addition to the usual Jacobi identity on the bracket in $\mathfrak{g}$, the identity involved in the notion of a $\mathfrak{g}$-module, the associativity and commutativity equations on the multiplication in $R$, and the associativity equation involved in the notion of an $R$-module, the listed structures must also satisfy the following equations:

(i) $\mathfrak{g}$ acts in $R$ by derivations of the commutative multiplication, that is

$$
x(a b)=x(a) b+a x(b) \quad \text { for all } x \in \mathfrak{g} \text { and } a, b \in R ;
$$

(ii) the identity

$$
(a x)(b)=a x(b) \quad \text { for all } x \in \mathfrak{g} \text { and } a, b \in R
$$

holds in $R$, where $a x \in \mathfrak{g}, \quad(a x)(b) \in R, \quad x(b) \in R$, and $a x(b) \in R$ are the elements obtained using the action of $R$ in $\mathfrak{g}$, the action of $\mathfrak{g}$ in $R$, the action of $\mathfrak{g}$ in $R$, and the multiplication in $R$, respectively;

(iii) the identity

$$
[x, a y]=x(a) y+a[x, y] \quad \text { for all } x, y \in \mathfrak{g} \text { and } a \in R
$$

holds in $\mathfrak{g}$, where $x(a) \in R$ is the element obtained using the action of $\mathfrak{g}$ in $R$, ay and $x(a) y \in \mathfrak{g}$ are the elements obtained using the action of $R$ in $\mathfrak{g}$, $[x, y]$ and $[x, a y] \in \mathfrak{g}$ are the Lie brackets in $\mathfrak{g}$, and $a[x, y] \in \mathfrak{g}$ is the element obtained using the action of $R$ in $\mathfrak{g}$.

Notice that $\mathfrak{g}$ is not a Lie algebra over $R$, as the Lie bracket in $\mathfrak{g}$ is not $R$-linear. The identity (iii) describes the obstacle term to $R$-linearity of the bracket in $\mathfrak{g}$.

For example, for any smooth affine algebraic variety $X$ over a field $k$, the commutative ring of functions $R=O(X)$ and the Lie algebra of vector fields $\mathfrak{g}=T(X)$ form a Lie algebroid with $O(X)$ acting in $T(X)$ as in the module of sections of a vector bundle (namely, the tangent bundle) on $X$ and $T(X)$ acting in $O(X)$ by the derivations of functions along vector fields (cf. Section 10.3). More generally, for any homomorphism of commutative rings $S \longrightarrow R$, the ring $R$ and the Lie algebra $\mathfrak{g}=T_{R / S}$ of $S$-linear derivations of $R$ (as in Section 10.8) form a Lie algebroid.

Furthermore, for any smooth real manifold $X$, the ring $R=O(X)$ of smooth $\mathbb{R}$-valued functions on $X$ together with the Lie algebra $\mathfrak{g}=T(X)$ of smooth vector fields on $X$ (as in Section 10.6) form a Lie algebroid. Similarly, for any real manifold $X$, the ring $R=O(X)_{\mathbb{C}}$ of smooth $\mathbb{C}$-valued functions on $X$ together with the Lie algebra $\mathfrak{g}^{\prime}=T(X)_{\mathbb{C}}$ of smooth $\mathbb{C}$-valued vector fields on $X$ form a Lie algebroid. For a complex manifold $X$, the same ring of smooth $\mathbb{C}$-valued functions $R=O\left(X_{\mathbb{R}}\right)_{\mathbb{C}}$ together with the Lie subalgebra $\mathfrak{g}=T^{(0,1)}(X) \subset \mathfrak{g}^{\prime}=T\left(X_{\mathbb{R}}\right)_{\mathbb{C}}$ of smooth $(0,1)$-vector fields on $X$ form a Lie algebroid as well (see Section 10.7).

The universal enveloping ring $U(R, \mathfrak{g})$ of a Lie algebroid $(R, \mathfrak{g})$ is an associative ring defined by generators and relations as follows (see [40, 22] for a differently worded, but equivalent version of this construction). The set of generators $R \sqcup \mathfrak{g}$ is the disjoint union of $R$ and $\mathfrak{g}$. The sum of any two elements of $R$ in $U(R, \mathfrak{g})$ equals their sum in $R$, and the sum of any two elements of $\mathfrak{g}$ in $U(R, \mathfrak{g})$ equals their sum in $\mathfrak{g}$. Denoting 
the product in $U(R, \mathfrak{g})$ by $*$, one imposes the multiplicative relations similar to the ones in Section 10.3.

$$
\begin{gathered}
a * b=a b \quad \text { for all } a, b \in R, \\
a * x=a x \quad \text { for all } a \in R \text { and } x \in \mathfrak{g}, \\
x * a=a x+x(a) \quad \text { for all } a \in R \text { and } x \in \mathfrak{g}
\end{gathered}
$$

and

$$
x * y-y * x=[x, y] \quad \text { for all } x, y \in \mathfrak{g} .
$$

The commutative ring $R$ has a natural structure of left module over the associative ring $U(R, \mathfrak{g})$. To define this action, one lets the generators $b \in R$ of the $\operatorname{ring} U(R, \mathfrak{g})$ act in $R$ by the multiplication maps $a \longmapsto b a$ and the generators $x \in \mathfrak{g}$ of the ring $U(R, \mathfrak{g})$ act in $R$ as the Lie algebra $\mathfrak{g}$ acts in $R$, that is $a \longmapsto x(a)$. Then one needs to check that the assignment of such endomorphisms of the abelian group $R$ to the generators of $U(R, \mathfrak{g})$ respects the relations (141 142), so the resulting action of $U(R, \mathfrak{g})$ in $R$ is well-defined.

Let $(R, \mathfrak{g})$ be a Lie algebroid such that the $R$-module $\mathfrak{g}$ is projective and finitely generated. Denote by $\mathfrak{g}^{\vee}=\operatorname{Hom}_{R}(\mathfrak{g}, R)$ the dual finitely generated projective $R$-module. Consider the symmetric algebra $A=\operatorname{Sym}_{R}(\mathfrak{g})$ of the $R$-module $\mathfrak{g}$ and the exterior algebra $B=\Lambda_{R}\left(\mathfrak{g}^{\vee}\right)$. According to Section [10.1, $A$ and $B$ are left and right finitely projective Koszul graded rings over $R$. Furthermore, the right finitely projective Koszul graded ring $B$ is quadratic dual to the left finitely projective Koszul graded ring $A$, in the sense of Section 1 and Proposition 2.23.

Put $\widetilde{A}=U(R, \mathfrak{g})$, and endow the ring $\widetilde{A}$ with the increasing filtration $F$ generated by $F_{1} \widetilde{A}=\operatorname{im}(R \oplus \mathfrak{g} \rightarrow \widetilde{A})$ over $F_{0} \widetilde{A}=\operatorname{im}(R \rightarrow \widetilde{A})$. Consider the associated graded $\operatorname{ring} \operatorname{gr}^{F} \widetilde{A}=\bigoplus_{n=0}^{\infty} F_{n} \widetilde{A} / F_{n-1} \widetilde{A}$. Then there is a unique homomorphism of graded rings $A \longrightarrow \operatorname{gr}^{F} \widetilde{A}$ forming commutative triangle diagrams with the natural isomorphisms $R \simeq A_{0}$ and $\mathfrak{g} \simeq A_{1}$ and the natural surjective maps $R \longrightarrow F_{0} \widetilde{A}$ and $\mathfrak{g} \longrightarrow F_{1} \widetilde{A} / F_{0} \widetilde{A}$. This graded ring homomorphism is obviously surjective.

We would like to show, as a particular case of the Poincaré-Birkhoff-Witt Theorem 4.19, that the graded ring homomorphism $A \longrightarrow \operatorname{gr}^{F} \widetilde{A}$ is, in fact, an isomorphism. In particular, it will follow that the natural surjective maps $R \longrightarrow F_{0} \widetilde{A}$ and $R \oplus \mathfrak{g} \longrightarrow F_{1} \widetilde{A}$ are, in fact, bijective. Let us emphasize that these assertions in the conclusion of the Poincaré-Birkhoff-Witt theorem only have a chance to hold due to (i-iii) and other equations imposed on the structure maps of a Lie algebroid (such as the Jacobi identity for the bracket in $\mathfrak{g}$ ).

Using the notation of Section 3.3, put $V=\mathfrak{g}=A_{1}$ and let $I \subset V \otimes_{R} V$ denote the kernel of the multiplication map $A_{1} \otimes_{R} A_{1} \longrightarrow A_{2}$. So $I$ is the $R$-submodule of skew-symmetric tensors in $V \otimes_{R} V$. Put $q(x, a)=x(a)$ for every $x \in V=\mathfrak{g}$ and $a \in R$. Then the relations (141) take the form (27).

Following further the notation of Section 3.3. denote by $\widehat{I} \subset V \otimes_{\mathbb{Z}} V$ the full preimage of the submodule $I \subset V \otimes_{R} V$ under the natural surjective map $V \otimes_{\mathbb{Z}} V \longrightarrow$ $V \otimes_{R} V$. So we get a surjective map $\widehat{I} \longrightarrow I$. 
The abelian group $\widehat{I}$ is spanned by the tensors of the form $x \otimes y-y \otimes x$ and $a x \otimes y-x \otimes a y$, where $x, y \in \mathfrak{g}$ and $a \in R$. The rules $p(x \otimes y-y \otimes x)=[x, y] \in \mathfrak{g}$ and $p(a x \otimes y-x \otimes a y)=-x(a) y \in \mathfrak{g}$ define an abelian group homomorphism $p: \widehat{I} \longrightarrow \mathfrak{g}$. Let $h: \widehat{I} \longrightarrow R$ be the zero map, $h=0$. Then the relation (142) takes the form (28) for all $\hat{\imath}=x \otimes y-y \otimes x \in \widehat{I}$, and it follows from the relations (141) that (28) also holds for all $\hat{\imath}=a x \otimes y-x \otimes a y \in \widehat{I}$, i. e., $a x * y-x * a y=-x(a) y$ in $U(R, \mathfrak{g})$.

Using the equations imposed on the structure maps of a Lie algebroid, one can check that the maps $q, p$, and $h$ satisfy the equations $(\mathrm{a}-\mathrm{k})$ in Proposition 3.5. Following the proof of Proposition 3.6, one can then conclude that the formulas (30 31) define an odd derivation $d: B \longrightarrow B$ of degree 1 with zero square, $d^{2}=0$. So one obtains a DG-ring $(B, d)$, called the cohomological Chevalley-Eilenberg complex of a Lie algebroid $(R, \mathfrak{g})$ (with trivial coefficients). Alternatively, it may be easier to check directly from the definition of a Lie algebroid that the formulas (30 31) define a DG-ring structure on the graded ring $B=\Lambda_{R}\left(\mathfrak{g}^{\vee}\right)$.

Now Theorem 4.19 is applicable to the right finitely projective Koszul DG-ring $(B, d)$, and the filtered $\operatorname{ring}(\widetilde{A}, F)$ together with the above action of $\widetilde{A}=U(R, \mathfrak{g})$ in $R$ is the left augmented left finitely projective nonhomogeneous Koszul ring corresponding to $(B, d)$ under the anti-equivalence of categories from Corollary 4.22 . Hence the desired isomorphism $A \simeq \operatorname{gr}^{F} \widetilde{A}$. A more general version of this result can be found in the classical paper [40, Theorem 3.1].

According to Example 7.7(2) and Remark 7.8, the Chevalley-Eilenberg complex $\left(\Lambda_{R}\left(\mathfrak{g}^{\vee}\right), d\right)$ computes the Ext groups/ring $\operatorname{Ext}_{U(R, \mathfrak{g})}^{*}(R, R)$ (cf. [40, Section 4]).

The results of Sections 6 and 7 specialize to the following derived nonhomogeneous Koszul duality theorem.

Theorem 10.14. For any Lie algebroid $(R, \mathfrak{g})$ such that the $R$-module $\mathfrak{g}$ is projective and finitely generated, the construction of Theorem 6.7 provides a triangulated equivalence between the $U(R, \mathfrak{g}) / R$-semicoderived category of right modules over the universal enveloping ring $U(R, \mathfrak{g})$ and the coderived category of right DG-modules over the Chevalley-Eilenberg DG-ring $\left(\Lambda_{R}\left(\mathfrak{g}^{\vee}\right), d\right)$,

$$
\mathrm{D}_{R}^{\text {sico }}(\bmod -U(R, \mathfrak{g})) \simeq \mathrm{D}^{\mathrm{co}}\left(\bmod -\left(\Lambda_{R}\left(\mathfrak{g}^{\vee}\right), d\right)\right)
$$

while Theorem 6.11 establishes a triangulated equivalence between the derived category of right $U(R, \mathfrak{g})$-modules and the reduced coderived category of right DG-modules over $\left(\Lambda_{R}\left(\mathfrak{g}^{\vee}\right), d\right)$,

$$
\mathrm{D}(\bmod -U(R, \mathfrak{g})) \simeq \mathrm{D}_{R \text {-red }}^{\mathrm{co}}\left(\bmod -\left(\Lambda_{R}\left(\mathfrak{g}^{\vee}\right), d\right)\right) .
$$

In the same context, the construction of Theorem 7.6 provides a triangulated equivalence between the $U(R, g) / R$-semicontraderived category of left modules over the ring $U(R, \mathfrak{g})$ and the contraderived category of left $D G$-modules over the DG-algebra $\left(\Lambda_{R}\left(\mathfrak{g}^{\vee}\right), d\right)$,

$$
\mathrm{D}_{R}^{\text {sictr }}(U(R, \mathfrak{g})-\bmod ) \simeq \mathrm{D}^{\mathrm{ctr}}\left(\left(\Lambda_{R}\left(\mathfrak{g}^{\vee}\right), d\right)-\bmod \right)
$$


while Theorem 7.10 establishes a triangulated equivalence between the derived category of left $U(R, \mathfrak{g})$-modules and the reduced contraderived category of left DG-modules over $\left(\Lambda_{R}\left(\mathfrak{g}^{\vee}\right), d\right)$,

$$
\mathrm{D}(U(R, \mathfrak{g})-\bmod ) \simeq \mathrm{D}_{R \text {-red }}^{\mathrm{ctr}}\left(\left(\Lambda_{R}\left(\mathfrak{g}^{\vee}\right), d\right)-\bmod \right)
$$

Whenever the ring $R$ has finite homological dimension, Corollaries 6.10 and 7.9 are applicable. So there is no difference between (143) and (144), and similarly there is no difference between (145) and (146) in this case.

Assume that the finitely generated projective $R$-module $\mathfrak{g}$ is everywhere of the same rank $m \geq 0$ (in the sense of the definition at the end of Section 10.1). Then there is a natural equivalence between the abelian categories of left and right $U(R, \mathfrak{g})$-modules, provided by the mutually inverse functors of tensor product with the invertible $R$-modules $T=\Lambda_{R}^{m}\left(\mathfrak{g}^{\vee}\right)$ and $\operatorname{Hom}_{R}(T, R)=\Lambda_{R}^{m}(\mathfrak{g})$, as described in Theorem 9.4. Moreover, the results of Section 9 lead to the following relative nonhomogeneous Koszul quadrality theorem.

Theorem 10.15. For any Lie algebroid $(R, \mathfrak{g})$ such that $\mathfrak{g}$ is a finitely generated projective $R$-module everywhere of rank $m$ and the homological dimension of $R$ is finite, the constructions of Theorem 9.8 (with Remark 9.5) provide a commutative square diagram of triangulated equivalences

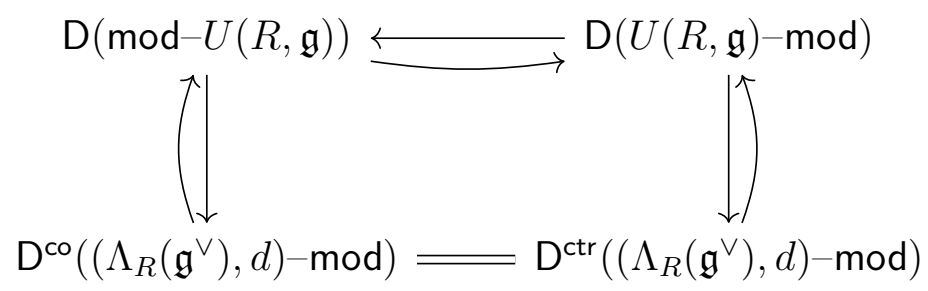

where the equivalence of derived categories in the upper line is induced by the conversion equivalence of abelian categories $\bmod -U(R, \mathfrak{g}) \simeq U(R, \mathfrak{g})-\bmod ($ up to a cohomological shift by $[-m]$ ), while the co-contra correspondence in the lower line is the result of Theorem 9.1. The vertical equivalences are (143-144) and (145-146).

10.10. Noncommutative differential forms. Let $\iota: S \longrightarrow R$ be a homomorphism of associative rings. We will consider DG-rings $(B, d)$ endowed with a ring homomorphism $R \longrightarrow B^{0}$ such that the image of the composition $S \longrightarrow R \longrightarrow B^{0}$ is annihilated by the differential $d_{0}: B^{0} \longrightarrow B^{1}$.

The initial object in the category of all such DG-rings $(B, d)$ with ring homomorphisms $R \longrightarrow B^{0}$ is called the $D G$-ring over $S$ freely generated by $R$ and denoted by $\left(N C_{R / S}, d\right)$. The other name for $\left(N C_{R / S}, d\right)$ is the $D G$-ring of noncommutative differential forms for $R$ over $S$ (cf. [, proof of Proposition II.1]).

This definition is the noncommutative version of the one in the beginning of Section 10.8. The difference is that, even when the rings $S$ and $R$ happen to be commutative, the DG-rings $(B, d)$ considered in this section do not need to be graded 
commutative. In other words, we are dropping the condition (i) or (i') of Section 10.8 and keeping only the condition (ii).

As in Section 10.8, it is clear that the graded ring $N C_{R / S}$ is generated by the images of elements from $R$ and their differentials. It follows that one has $N C_{R / S}^{n}=0$ for $n<0$ and $N C_{R / S}^{0}=R$. Furthermore, the graded ring $N C_{R / S}$ is generated by its first-degree component $N C_{R / S}^{1}$ over $N C_{R / S}^{0}=R$. The next lemma provides a precise, explicit description.

Lemma 10.16. The maps

$$
R \otimes_{S} R / \iota(S) \otimes_{S} R / \iota(S) \otimes_{S} \cdots \otimes_{S} R / \iota(S) \longrightarrow N C_{R / S}^{n}
$$

and

$$
R / \iota(S) \otimes_{S} R / \iota(S) \otimes_{S} \cdots \otimes_{S} R / \iota(S) \otimes_{S} R \longrightarrow N C_{R / S}^{n}
$$

given by the formulas $f \otimes \bar{g}_{1} \otimes \cdots \otimes \bar{g}_{n} \longmapsto f d\left(g_{1}\right) d\left(g_{2}\right) \cdots d\left(g_{n}\right)$ and $\bar{g}_{1} \otimes \cdots \otimes \bar{g}_{n} \otimes$ $f \longmapsto d\left(g_{1}\right) d\left(g_{2}\right) \cdots d\left(g_{n}\right) f$ are isomorphisms of $S$-S-bimodules for all $n \geq 0$. More precisely, the map (148) is an isomorphism of $R$-S-bimodules, while the map (149) is an isomorphism of $S$-R-bimodules.

Here the left-hand side of (148) is the tensor product of one factor $R$ and $n$ factors $R / \iota(S)$. The left-hand side of (149) is the tensor product of $n$ factors $R / \iota(S)$ and one factor $R$. All the tensor products signs in (148 149) mean tensor products of $S$-S-bimodules. The notation is $f, g_{i} \in R$ for all $1 \leq i \leq n$ and $\bar{g}_{i} \in R / \iota(S)$ is the image of $g_{i}$ under the natural surjection $R \longrightarrow R / \iota(S)$.

Proof. The maps (148 149) are well-defined due to the condition that $d(\iota(s))=0$ in $N C_{R / S}^{1}$ for all $s \in S$. Having observed that, one can split the assertions of the lemma in two parts. Firstly, it is claimed that the maps $R \otimes_{S} R / \iota(S) \longrightarrow N C_{R / S}^{1} \longleftarrow$ $R / \iota(S) \otimes_{S} R$ given by the formulas $f \otimes \bar{g} \longmapsto f d(g)$ and $\bar{g} \otimes f \longmapsto d(g) f$ are isomorphisms (where $f, g \in R$ and $\bar{g} \in R / \iota(S)$ is the image of $g$ ).

Secondly, notice that

$$
\begin{aligned}
\left(R \otimes_{S} R / \iota(S)\right) \otimes_{R}\left(R \otimes_{S} R / \iota(S)\right) & \otimes_{R} \cdots \otimes_{R}\left(R \otimes_{S} R / \iota(S)\right) \\
& \simeq R \otimes_{S} R / \iota(S) \otimes_{S} R / \iota(S) \otimes_{S} \cdots \otimes_{S} R / \iota(S) .
\end{aligned}
$$

So, in order to deduce the assertions of the lemma for an arbitrary $n \geq 1$ from such assertions for $n=1$, one needs to show that the multiplication map

$$
N C_{R / S}^{1} \otimes_{R} \cdots \otimes_{R} N C_{R / S}^{1} \longrightarrow N C_{R / S}^{n}
$$

( $n$ factors in the left-hand side) is an isomorphism for $n \geq 1$. In other words, this means that the graded ring $N C_{R / S}$ is the tensor ring of the $R$ - $R$-bimodule $N C_{R / S}^{1}$, that is $N C_{R / S} \simeq T_{R}\left(N C_{R / S}^{1}\right)$.

Concerning the first part, one observes that the $R$ - $R$-bimodule $N C_{R / S}^{1}$ is spanned by the symbols $d(f), f \in R$, with the relations $d(f g)=d(f) g+f d(g)$ for $f, g \in R$ and $d(\iota(s))=0$ for $s \in S$. It follows immediately that the maps $R \otimes_{S} R / \iota(S) \longrightarrow$ $N C_{R / S}^{1} \longleftarrow R / \iota(S) \otimes_{S} R$ are surjective. In order to show that these maps are 
isomorphisms, it suffices to define $R$-R-bimodule structures on $R \otimes_{S} R / \iota(S)$ and $R / \iota(S) \otimes_{S} R$ in such a way that the above relations are satisfied.

Concerning the second part, in order to prove that the maps (150) are isomorphisms, it suffices to show that the map $d: R=N C_{R / S}^{0} \longrightarrow N C_{R / S}^{1}$ extends to a welldefined odd derivation with zero square on the tensor ring $T_{R}\left(N C_{R / S}^{1}\right)$. Lemma 3.7 is a suitable tool here.

Similarly to Section 10.8, a simpler alternative approach might be to define $N C_{R / S}$ as the graded associative ring generated by the symbols $f$ and $d(f)$ with $f \in R$, subject to the relations that the addition and multiplication of elements $f$ in $R$ agrees with their addition and multiplication in $N C_{R / S}$, and also $d(f+g)=d(f)+d(g)$, $d(f g)=d(f) g+f d(g)$ for $f, g \in R$, and $d(\iota(s))=0$ for $s \in S$. Then the isomorphism $N C_{R / S} \simeq T_{R}\left(N C_{R / S}^{1}\right)$ follows simply from the fact that all the relations imposed have degrees 0 or 1 . Subsequently one needs to see that there exists an odd derivation $d$ on $N C_{R / S}$ taking $f$ to $d(f)$ and $d(f)$ to 0 for all $f \in R$.

Now let us assume that the map $\iota: S \longrightarrow R$ is injective and the right $S$-module $R / \iota(S)$ is finitely generated and projective. Then it is clear from the isomorphism (149) that the right $R$-module $N C_{R / S}^{n}$ is finitely generated and projective for every $n \geq 1$. Moreover, $N C_{R / S}^{n} \simeq T_{R}\left(N C_{R / S}^{1}\right)$ is the tensor algebra of the $R$ - $R$-bimodule $N C_{R / S}^{1}$, as we have seen in (150). Thus the DG-ring of noncommutative differential forms $\left(N C_{R / S}, d\right)$ is right finitely projective Koszul.

Consider the following filtered $\operatorname{ring}(\widetilde{A}, F)$. Let $\widetilde{A}=\operatorname{Hom}_{S^{\mathrm{op}}}(R, R)$ be the ring of endomorpisms of the right $S$-module $R$; so $R$ is a left $\widetilde{A}$-module. This is our "ring of noncommutative differential operators acting $S$-linearly in $R$ ". The left action of $R$ in itself defines a natural ring inclusion $R \longrightarrow \widetilde{A}$. So $\widetilde{A}$ is a left augmented ring over its subring $R$, in the sense of Section 3.8, Let $\widetilde{A}^{+} \subset \widetilde{A}$ be the augmentation ideal, that is, the subgroup of all elements in $\widetilde{A}$ whose action annhilates the unit element $1 \in R$. So $\widetilde{A}^{+}$is a left ideal in $\widetilde{A}$, and the left $R$-module $\widetilde{A}$ decomposes naturally as the direct sum $\widetilde{A}=R \oplus_{\widetilde{A}} \widetilde{A}^{+}$.

Put $F_{0} \widetilde{A}=R$ and $F_{n} \widetilde{A}=\widetilde{A}$ for all $n \geq 1$. Then the underlying left $R$-module of the $R$ - $R$-bimodule $F_{1} \widetilde{A} / F_{0} \widetilde{A}$ is isomorphic to $\widetilde{A}^{+}$. One easily computes this left $R$-module as $\widetilde{A}^{+} \simeq \operatorname{Hom}_{S^{\text {op }}}(R / \iota(S), R) \simeq \operatorname{Hom}_{R^{\text {op }}}\left(R / \iota(S) \otimes_{S} R, R\right)$. In our assumptions, this is a finitely generated projective left $R$-module. It follows that the associated graded $\operatorname{ring} A=\operatorname{gr}^{F} \widetilde{A}=F_{0} \widetilde{A} \oplus F_{1} \widetilde{A} / F_{0} \widetilde{A}$ is left finitely projective Koszul and quadratic dual to the right finitely projective Koszul graded ring $B=N C_{R / S}$ (in the sense of Section 1 and Proposition 2.23).

The right finitely projective Koszul DG-ring $\left(N C_{R / S}, d\right)$ corresponds to the left augmented left finitely projective nonhomogeneous Koszul filtered ring $(\widetilde{A}, F)$ under the equivalence of categories from Corollary 4.22,

The results of Sections 6 and 7 specialize to the following theorem. Notice that the graded ring of noncommutative differential forms $N C_{R / S}$ usually has infinitely many nonzero grading components in our assumptions. So one has to distinguish 
$N C_{R / S^{-}}$comodules and $N C_{R / S^{-}}$contramodules (in the sense of Section 5 ) from objects of their ambient categories of arbitrary $N C_{R / S}$-modules.

We use the natural terminology "DG-comodules" and "DG-contramodules" for CDG-comodules and CDG-contramodules, in the sense of Sections 6.4 and 7.3, over a DG-ring (i. e., a CDG-ring with a vanishing curvature element $h$ ).

Theorem 10.17. For any injective morphism of associative rings $\iota: S \longrightarrow R$ such that $R / \iota(S)$ is a finitely generated projective right $S$-module, the construction of Theorem 6.7 provides a triangulated equivalence between the $\operatorname{Hom}_{S^{\circ \mathrm{p}}}(R, R) / R$-semicoderived category of right modules over the endomorphism ring $\operatorname{Hom}_{S^{\mathrm{op}}}(R, R)$ and the coderived category of right DG-comodules over the nonnegatively graded $D G$-ring of noncommutative differential forms $\left(N C_{R / S}, d\right)$,

$$
\mathrm{D}_{R}^{\text {sico }}\left(\bmod -\operatorname{Hom}_{S^{\text {op }}}(R, R)\right) \simeq \mathrm{D}^{\mathrm{co}}\left(\operatorname{comod}-\left(N C_{R / S}, d\right)\right),
$$

while Theorem 6.11 establishes a triangulated equivalence between the derived category of right $\operatorname{Hom}_{S^{\mathrm{op}}}(R, R)$-modules and the reduced coderived category of right $D G$-comodules over $\left(N C_{R / S}, d\right)$,

$$
\mathrm{D}\left(\bmod -\operatorname{Hom}_{S \text { op }}(R, R)\right) \simeq \mathrm{D}_{R \text {-red }}^{\text {co }}\left(\operatorname{comod}-\left(N C_{R / S}, d\right)\right) .
$$

In the same context, the construction of Theorem 7.6 provides a triangulated equivalence between the $\operatorname{Hom}_{S^{\mathrm{op}}}(R, R) / R$-semicontraderived category of left modules over the ring $\operatorname{Hom}_{S \text { op }}(R, R)$ and the contraderived category of left $D G$-contramodules over the nonnegatively graded $D G$-ring $\left(N C_{R / S}, d\right)$,

$$
\mathrm{D}_{R}^{\text {sictr }}\left(\operatorname{Hom}_{S^{\mathrm{op}}}(R, R)-\mathrm{mod}\right) \simeq \mathrm{D}^{\mathrm{ctr}}\left(\left(N C_{R / S}, d\right)-\text { contra }\right),
$$

while Theorem 7.10 establishes a triangulated equivalence between the derived category of left $\operatorname{Hom}_{S^{\mathrm{op}}}(R, R)$-modules and the reduced contraderived category of left $D G$-contramodules over $\left(N C_{R / S}, d\right)$,

$$
\mathrm{D}\left(\operatorname{Hom}_{S^{\mathrm{op}}}(R, R)-\mathrm{mod}\right) \simeq \mathrm{D}_{R \text {-red }}^{\mathrm{ctr}}\left(\left(N C_{R / S}, d\right)-\text { contra }\right) .
$$

Furthermore, whenever the ring $R$ has finite homological dimension, Corollaries 6.10 and 7.9 are applicable; so there is no difference between (151) and (152), and similarly there is no difference between (153) and (154).

\section{REFERENCES}

[1] S. M. Arkhipov. Semi-infinite cohomology of associative algebras and bar-duality. Internat. Math. Research Notices 1997, \#17, p. 833-863. arXiv:q-alg/9602013

[2] H. Becker. Models for singularity categories. Advances in Math. 254, p. 187-232, 2014. arXiv:1205.4473 [math.CT]

[3] A. Beilinson, J. Bernstein. A proof of Jantzen conjectures. In: "I. M. Gelfand Seminar", Advances in Soviet Math. 16, part 1, American Math. Society, 1993, p. 1-50.

[4] A. Beilinson, V. Drinfeld. Quantization of Hitchin's integrable system and Hecke eigensheaves. February 2000. Available from http://www.math.utexas.edu/ benzvi/Langlands.html 
[5] J. Bernstein. Algebraic theory of D-modules. Available from http://www.math.uchicago.edu/ ${ }^{\sim m i t y a / l a n g l a n d s . h t m l . ~}$

[6] R. Bezrukavnikov, I. Mirković, D. Rumynin. Localization of modules for a semisimple Lie algebra in prime characteristic. Annals of Math. 167, \#3, p. 945-991, 2008. arXiv:math.RT/0205144

[7] J. Chuang, A. Lazarev, W. Mannan. Cocommutative coalgebras: homotopy theory and Koszul duality. Homology, Homotopy and Appl. 18, \#2, p. 303-336, 2016. arXiv:1403.0774 [math.AT]

[8] A. Connes. Noncommutative differential geometry. Publications Math. de l'IHES 62, \#2, p. 257-360, 1985.

[9] P. C. Eklof, J. Trlifaj. How to make Ext vanish. Bulletin of the London Math. Society 33, \#1, p. $41-51,2001$.

[10] V. Ginzburg. Lectures on D-modules. Available from http://people.math.harvard.edu/ raitsgde/grad2009/Ginzburg.pdf.

[11] V. Hinich. DG coalgebras as formal stacks. Journ. of Pure and Appl. Algebra 162, \#2-3, p. 209-250, 2001. arXiv:math. AG/9812034

[12] J. Hirsh, J. Millès. Curved Koszul duality theory. Math. Annalen 354, \#4, p. 1465-1520, 2012. arXiv:1008.5368 [math.KT]

[13] A. J. de Jong et al. The Stacks Project. Available from https://stacks . math . columbia.edu/

[14] B. Jónsson. Distributive sublattices of a modular lattice. Proceedings of the Amer. Math. Soc. 6, \#5, 682-688, 1955.

[15] P. Jørgensen. The homotopy category of complexes of projective modules. Advances in Math. 193, \#1, p. 223-232, 2005. arXiv:math.RA/0312088

[16] M. Kapranov. On DG-modules over the de Rham complex and the vanishing cycles functor. In: Algebraic Geometry (Chicago, 1989), Lecture Notes in Math. 1479, 1991, p. 57-86.

[17] B. Keller. Koszul duality and coderived categories (after K. Lefèvre). October 2003. Available from http://webusers.imj-prg.fr/ bernhard.keller/publ/index.html

[18] H. Krause. The stable derived category of a noetherian scheme. Compositio Math. 141, \#5, p. 1128-1162, 2005. arXiv:math.AG/0403526

[19] K. Lefèvre-Hasegawa. Sur les $A_{\infty}$-catégories. Thèse de doctorat, Université Denis Diderot Paris 7, November 2003. arXiv:math.CT/0310337. Corrections, by B. Keller. Available from http://webusers.imj-prg.fr/ bernhard.keller/lefevre/publ.html

[20] S. Mac Lane. Categories for the working mathematician. Second edition. Graduate Texts in Mathematics, 5. Springer-Verlag, New York, 1998.

[21] J. Maunder. Koszul duality and homotopy theory of curved Lie algebras. Homology, Homotopy and Appl. 19, \#1, p. 319-340, 2017. arXiv:1512.01975 [math.AT]

[22] I. Moerdijk, J. Mrčun. On the universal enveloping algebra of a Lie algebroid. Proceedings of the Amer. Math. Soc. 138, \#9, p. 3135-3145, 2010. arXiv:0801.3929 [math.QA]

[23] R. Musti, E. Buttafuoco. Sui subreticoli distributivi dei reticoli modulari. Bolletino dell'Unione Matem. Italiana (3) 11, \#4, 584-587, 1956.

[24] A. Polishchuk, L. Positselski. Quadratic algebras. University Lecture Series, 37. American Math. Society, Providence, RI, 2005.

[25] L. Positselski. Nonhomogeneous quadratic duality and curvature. Functional Analysis and its Appl. 27, \#3, p. 197-204, 1993. arXiv:1411.1982 [math.RA]

[26] L. Positselski. Koszul property and Bogomolov's conjecture. Internat. Math. Research Notices 2005, \#31, p. 1901-1936. arXiv: 1405.0965 [math.KT]

[27] L. Positselski. Homological algebra of semimodules and semicontramodules: Semi-infinite homological algebra of associative algebraic structures. Appendix $\mathrm{C}$ in collaboration with D. Rumynin; Appendix D in collaboration with S. Arkhipov. Monografie Matematyczne, vol. 70, Birkhäuser/Springer Basel, 2010. xxiv+349 pp. arXiv:0708.3398 [math.CT] 
[28] L. Positselski. Two kinds of derived categories, Koszul duality, and comodule-contramodule correspondence. Memoirs of the Amer. Math. Soc. 212, \#996, 2011, vi+133 pp. arXiv:0905. 2621 [math.CT]

[29] L. Positselski. Weakly curved $\mathrm{A}_{\infty}$-algebras over a topological local ring. Mémoires de la Société Mathématique de France 159, 2018. vi+206 pp. arXiv:1202.2697 [math.CT]

[30] L. Positselski. Contramodules. Electronic preprint arXiv:1503.00991 [math.CT], to appear in Confluentes Math.

[31] L. Positselski. Dedualizing complexes and MGM duality. Journ. of Pure and Appl. Algebra 220, \#12, p. 3866-3909, 2016. arXiv: 1503.05523 [math.CT]

[32] L. Positselski. Coherent rings, fp-injective modules, dualizing complexes, and covariant SerreGrothendieck duality. Selecta Math. (New Ser.) 23, \#2, p. 1279-1307, 2017. arXiv: 1504.00700 [math.CT]

[33] L. Positselski. Koszulity of cohomology $=K(\pi, 1)$-ness + quasi-formality. Journ. of Algebra 483, p. 188-229, 2017. arXiv:1507.04691 [math.KT]

[34] L. Positselski. Contraadjusted modules, contramodules, and reduced cotorsion modules. Moscow Math. Journ. 17, \#3, p. 385-455, 2017. arXiv:1605.03934 [math.CT]

[35] L. Positselski. Pseudo-dualizing complexes and pseudo-derived categories. Rendiconti Seminario Matematico Univ. Padova 143, p. 153-225, 2020. arXiv:1703.04266 [math.CT]

[36] L. Positselski. Abelian right perpendicular subcategories in module categories. Electronic preprint arXiv:1705.04960 [math.CT].

[37] L. Positselski. Flat ring epimorphisms of countable type. Glasgow Math. Journ. 62, \#2, p. 383439, 2020. arXiv: 1808.00937 [math.RA]

[38] L. Positselski, J. Rosický. Covers, envelopes, and cotorsion theories in locally presentable abelian categories and contramodule categories. Journ. of Algebra 483, p. 83-128, 2017. arXiv:1512.08119 [math.CT]

[39] L. Positselski, J. Štovíček. The tilting-cotilting correspondence. Internat. Math. Research Notices 2021, \#1, p. 189-274, 2021. arXiv:1710.02230 [math.CT]

[40] G. S. Rinehart. Differential forms on general commutative algebras. Transactions of the Amer. Math. Soc. 108, p. 195-222, 1963.

[41] S. Rybakov. DG-modules over de Rham DG-algebra. European Journ. of Math. 1, \#1, p. 25-53, 2015. arXiv:1311.7503 [math.AG]

[42] A.-M. Simon. Approximations of complete modules by complete big Cohen-Macaulay modules over a Cohen-Macaulay local ring. Algebras and Represent. Theory 12, \#2-5, p. 385-400, 2009.

[43] S. P. Smith. Differential operators on commutative algebras. In: Ring Theory (Antwerp, 1985), Lecture Notes in Math. 1197, 1986, p. 165-177.

[44] B. Stenström. Rings of quotients. An introduction to methods of ring theory. Springer-Verlag, Berlin-Heidelberg-New York, 1975.

[45] C. Voisin. Hodge theory and complex algebraic geometry, vol. I. Translated by L. Schneps. Cambridge Studies in Advanced Math., 76, Cambridge Univ. Press, 2002.

[46] R. Vyas, A. Yekutieli. Weak proregularity, weak stability, and the noncommutative MGM equivalence. Journ. of Algebra 513, p. 265-325, 2018. arXiv:1608.03543 [math.RA]

[47] A. Yekutieli. On flatness and completion for infinitely generated modules over noetherian rings. Communicat. in Algebra 39, \#11, p. 4221-4245, 2011. arXiv:0902.4378 [math.AC]

Institute of Mathematics of the Czech ACAdemy of Sciences, Žitná 25, 11567 Praha 1 (Czech Republic); AND

Laboratory of Algebra and Number Theory, Institute for Information TransmisSion Problems, Moscow 127051 (Russia)

Email address: positselski@math.cas.cz 